\title{
Development of a membrane resistance based modeling framework for comparison of ultrafiltration processes
}

David Albert Masciola

West Virginia University

Follow this and additional works at: https://researchrepository.wvu.edu/etd

\section{Recommended Citation}

Masciola, David Albert, "Development of a membrane resistance based modeling framework for comparison of ultrafiltration processes" (2000). Graduate Theses, Dissertations, and Problem Reports. 2325.

https://researchrepository.wvu.edu/etd/2325

This Dissertation is protected by copyright and/or related rights. It has been brought to you by the The Research Repository @WVU with permission from the rights-holder(s). You are free to use this Dissertation in any way that is permitted by the copyright and related rights legislation that applies to your use. For other uses you must obtain permission from the rights-holder(s) directly, unless additional rights are indicated by a Creative Commons license in the record and/ or on the work itself. This Dissertation has been accepted for inclusion in WVU Graduate Theses, Dissertations, and Problem Reports collection by an authorized administrator of The Research Repository @ WVU.

For more information, please contact researchrepository@mail.wvu.edu. 
Development of a Membrane Resistance Based Modeling Framework for Comparison of Ultrafiltration Processes

\author{
David A. Masciola
}

Dissertation submitted to the

\title{
College of Engineering and Mineral Resources
}

At West Virginia University

In partial fulfillment of the requirements

For the degree of

\section{Doctor of Philosophy \\ In}

Civil and Environmental Engineering

\author{
Roger C. Viadero, Jr., Ph.D., Chair \\ Pierre R. Bérubé, Ph.D. \\ Ronald H. Fortney, Ph.D. \\ Donald D. Gray, Ph.D., P.E. \\ Kenneth J. Semmens, Ph.D.
}

Department of Civil and Environmental Engineering

\author{
Morgantown, West Virginia \\ 2000
}

Keywords: Ultrafiltration, Membrane Modules, Resistance, Permeate Flux, Modeling

Copyright 2000 David A. Masciola 


\title{
ABSTRACT \\ Development of a Membrane Resistance Based Modeling Framework for Comparison of Ultrafiltration Processes
}

\author{
David A. Masciola
}

A parametric study was conducted to develop a more general modeling framework as a basis for comparison of ultrafiltration membrane modules and separation processes. Discrete experiments were performed to examine the effects of operating parameters on permeate flux behavior in the tubular and high-shear rotary ultrafiltration (HSRUF) systems using a synthetic metal working (MW) fluid as a surrogate feed stream due to its relevance in industrial process and treatment applications. The conventional thin-film model was successfully applied to permeate flux data collected at low feed oil concentrations; however, model predictions at high concentrations were physically meaningless due to the formation of a two phase limiting permeate flux. Application of the resistance-in-series (RIS) approach was examined as an alternative to the conventional model and was successful in predicting permeate flux values at all oil concentrations examined in this study. The RIS model was then modified by postulating an explicit form of the resistance index in terms of operating parameters characteristic of all ultrafiltration (UF) processes. Due to the general nature of the modified RIS approach, the relationships developed in this study were utilized as a means of comparison between the tubular and HSRUF modules resulting in the development of a technical operating scheme for a hybrid tubular-HSRUF separation system. The general form of the modified RIS approach enables application of the procedures presented in this study as a means for comparison between a wide range of UF modules. Further, the 
approach developed is a general foundation for future investigations of emerging focus areas in separations research, such as hybrid schemes. 


\section{TABLE OF CONTENTS}

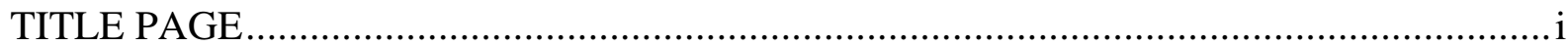

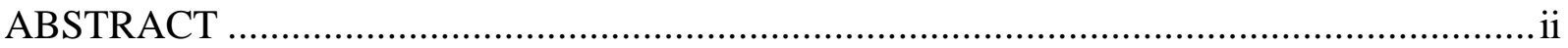

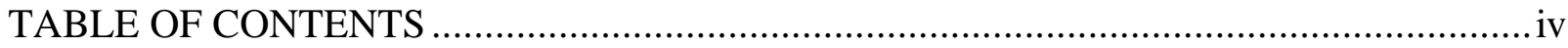

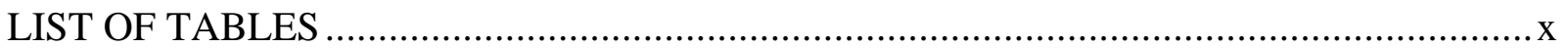

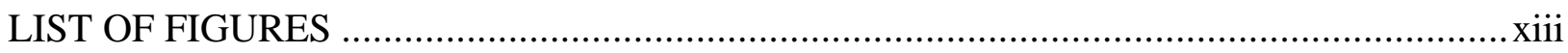

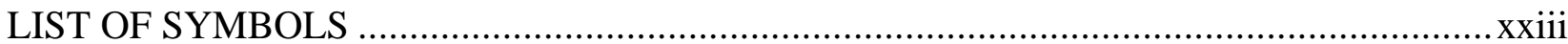

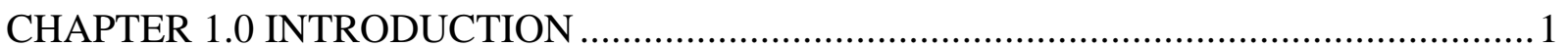

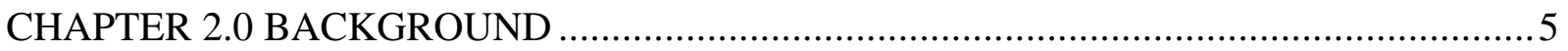

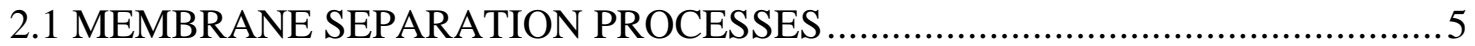

2.1.1 General Process Description ...............................................................

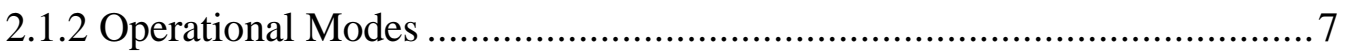

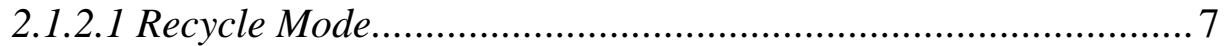

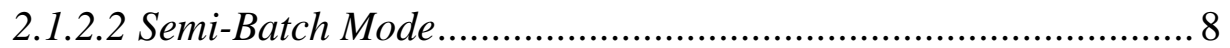

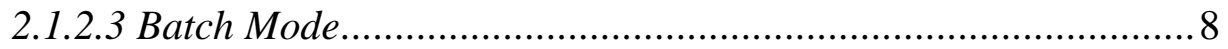

2.1.3 General Types of Membrane Separation Processes ................................... 10

2.1.3.1 Reverse Osmosis and Nanofiltration ......................................... 10

2.1.3.2 Ultrafiltration and Microfiltration ........................................... 12

2.1.4 Factors Controlling Ultrafiltration Permeate Flux ................................. 14

2.1.4.1 Concentration Polarization ....................................................... 14

2.1.4.2 Membrane Fouling .............................................................. 18

2.1.4.3 Membrane Resistance .............................................................20

2.2 CONVENTIONAL CROSS-FLOW ULTRAFILTRATION SYSTEMS ................21

2.2.1 Configurations of Conventional Membrane Modules................................. 21

2.2.1.1 Tubular Membrane Modules ...................................................21

2.2.1.2 Hollow Fiber Membrane Modules.............................................22

2.2.1.3 Spiral Wound Membrane Modules ............................................24

2.2.1.4 Plate and Frame Membrane Modules ……….........................25

2.2.2 Limitations of Conventional Cross-Flow Ultrafiltration Systems .............26 
2.3.1 Hydraulic Turbulence in the High-Shear Rotary Ultrafiltration System.

2.3.2 Permeate Backpressure in the High-Shear Rotary Ultrafiltration System. 30

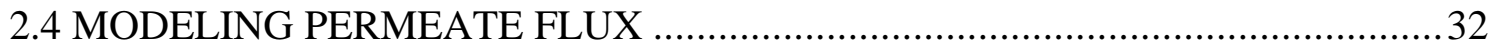

2.4.1 Pressure-Controlled Region - Hagen-Poiseuille Model .............................. 32

2.4.2 Pressure Independent Region - The Thin-Film Model................................ 34

2.4.2.1 Thin-Film Model Case Studies .................................................. 38

2.4.2.2 Limitations Of the Thin-Film Model ...........................................39

2.4.3 The Resistance-In-Series Model ....................................................... 41

2.5 MEMBRANE TREATMENT OF OILY WASTEWATERS..................................4

2.5.1 Characteristics of Oil-In-Water $(\mathrm{O} / \mathrm{W})$ Emulsions and Oily

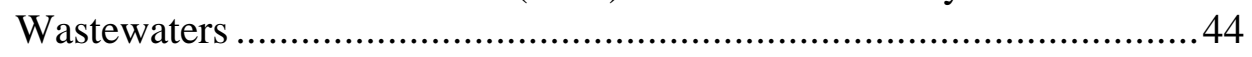

2.5.2 Overview of Membrane Treatment of Oily Wastewaters .......................... 49

2.5.2.1 Conventional Ultrafiltration Systems.........................................49

2.5.2.2 Mechanically Enhanced Ultrafiltration Systems ........................52

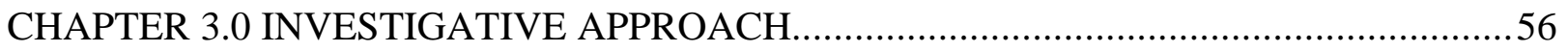

3.1 BASELINE METAL WORKING FLUID CHARACTERISTICS ........................56

3.1.1 Metal Working Fluid Density ................................................................56

3.1.2 Metal Working Fluid Absolute Viscosity.................................................56

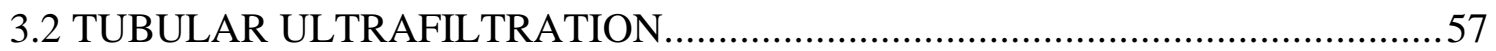

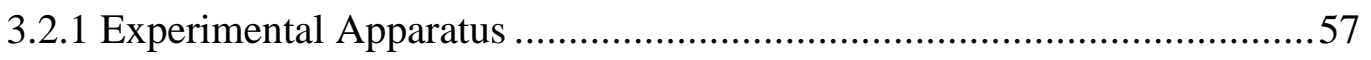

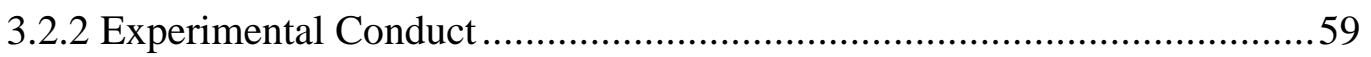

3.2.2.1 Metal-Working Fluid Experiments …………….......................59

3.2.2.2 Membrane Cleaning Procedure ………………......................... 63

3.2.2.3 Quality Assurance/Quality Control ............................................ 64

3.2.3 Permeate Flux Temperature Correction .....................................................65

3.3 HIGH-SHEAR ROTARY ULTRAFILTRATION ..............................................6

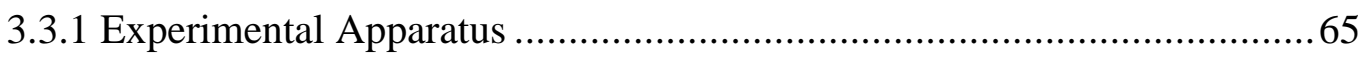

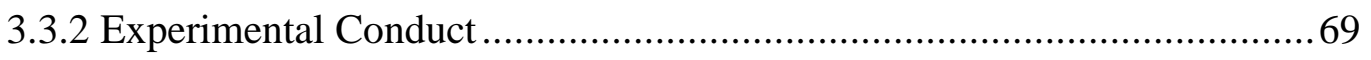

3.3.2.1 Metal-Working Fluid Experiments ...........................................69 
3.3.2.2 Membrane Cleaning Procedure ………………….................. 72

3.3.2.3 Quality Assurance/Quality Control ............................................74

3.3.3 Waste Metal Working Fluid Experiment................................................... 74

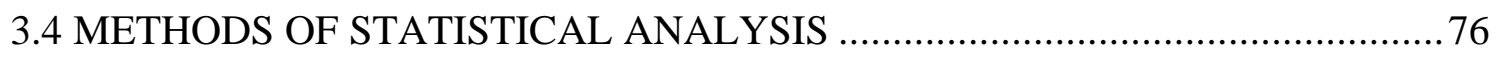

3.4.1 Two-way Analysis of Variance................................................................

3.4.2 Regression Analysis .......................................................................... 79

3.4.2.1 Incremental Regression Analysis ............................................ 80

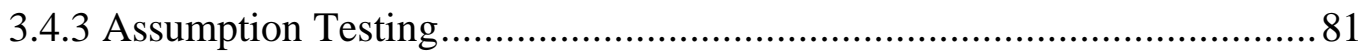

3.4.3.1 Kolmogorov-Smirnov Test...................................................... 82

3.4.3.2 Levene Median Test ............................................................. 82

3.4.3.3 Spearman Rank Correlation....................................................... 83

CHAPTER 4.0 RESULTS AND PRELIMINARY DATA REDUCTION ……….................... 85

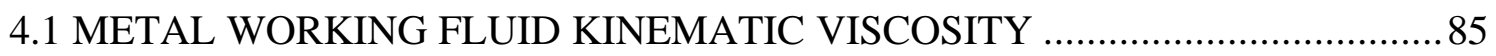

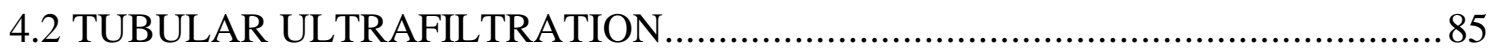

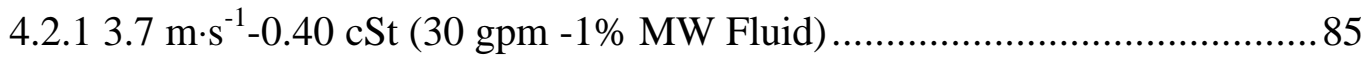

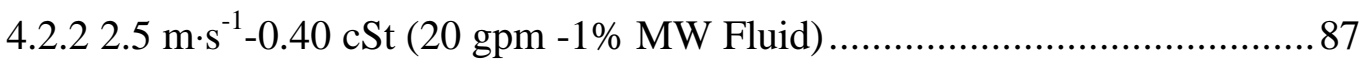

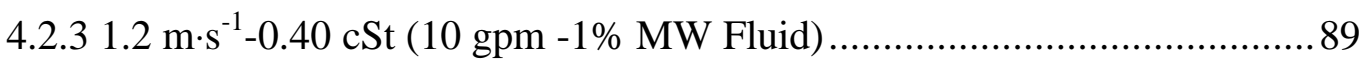

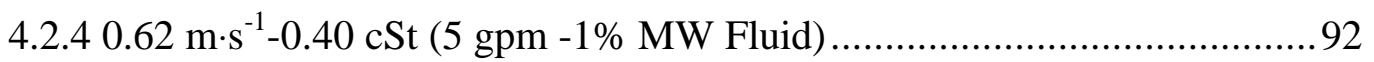

4.2.5 3.7 m. $\mathrm{s}^{-1}-0.46 \mathrm{cSt}$ (30 gpm -5\% MW Fluid) ........................................... 94

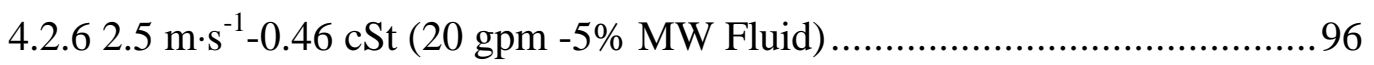

4.2.7 1.2 $\mathrm{m} \cdot \mathrm{s}^{-1}-0.46 \mathrm{cSt}(10 \mathrm{gpm}-5 \%$ MW Fluid) ......................................... 98

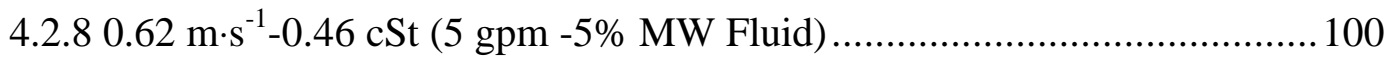

4.2.9 3.7 m.s ${ }^{-1}-0.64 \mathrm{cSt}$ (30 gpm -10\% MW Fluid) ........................................ 102

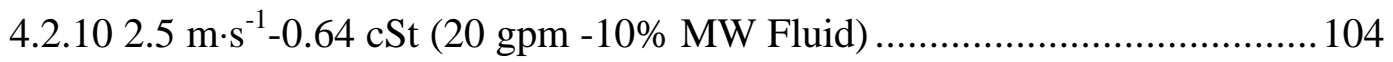

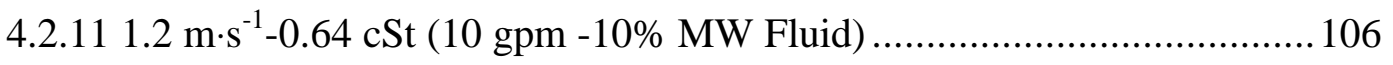

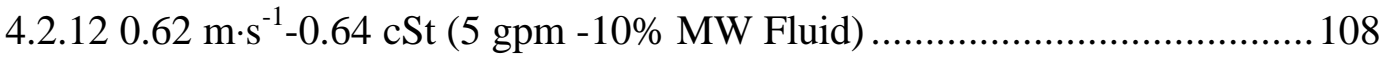

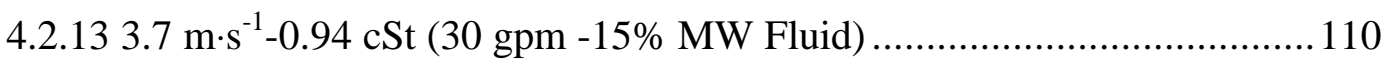

4.2.14 2.5 $\mathrm{m} \cdot \mathrm{s}^{-1}-0.94 \mathrm{cSt}(20 \mathrm{gpm}-15 \%$ MW Fluid) ….................................... 110

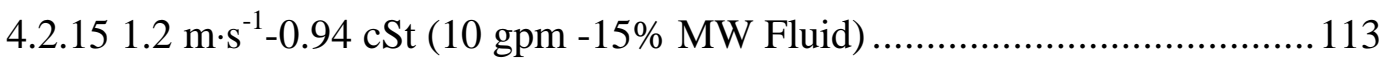

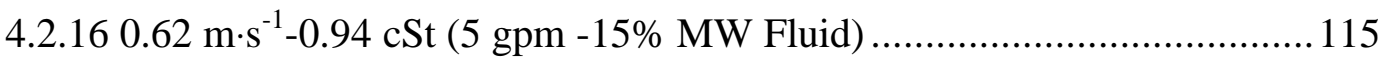


4.2.17 3.7 $\mathrm{m} \cdot \mathrm{s}^{-1}-1.2 \mathrm{cSt}(30 \mathrm{gpm}-20 \%$ MW Fluid) ............................................. 117

4.2.18 2.5 $\mathrm{m} \cdot \mathrm{s}^{-1}-1.2 \mathrm{cSt}(20 \mathrm{gpm}-20 \%$ MW Fluid) ......................................... 119

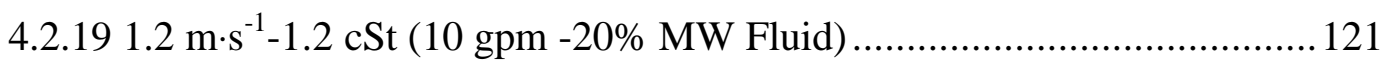

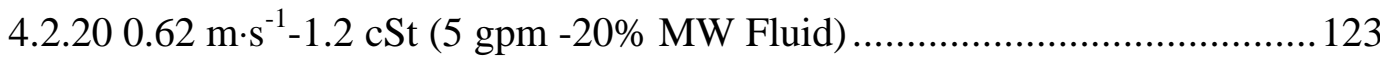

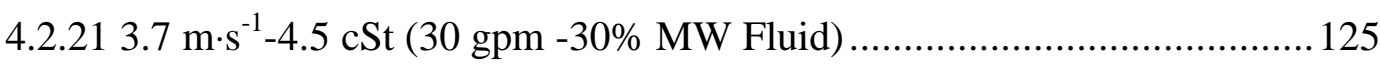

4.2.22 2.5 $\mathrm{m} \cdot \mathrm{s}^{-1}-4.5 \mathrm{cSt}(20 \mathrm{gpm}-30 \%$ MW Fluid) ......................................... 127

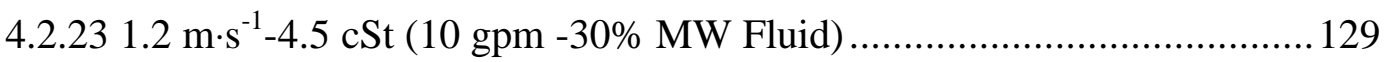

4.2.24 $0.62 \mathrm{~m} \cdot \mathrm{s}^{-1}-4.5 \mathrm{cSt}(5 \mathrm{gpm}-30 \% \mathrm{MW}$ Fluid) ......................................... 131

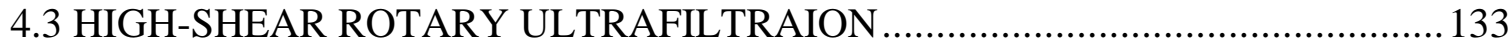

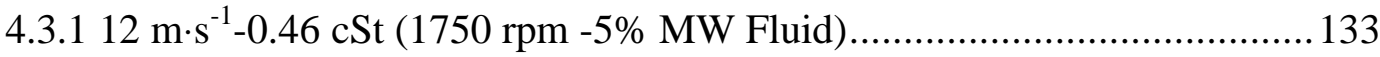

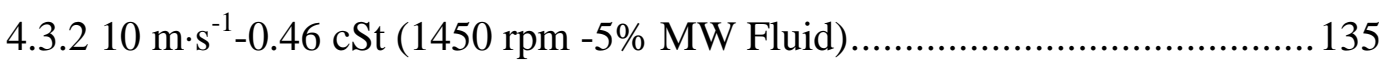

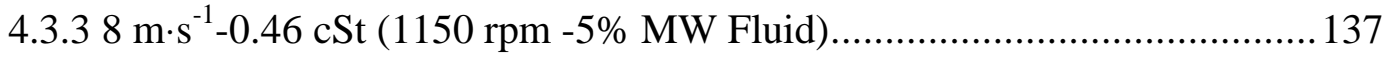

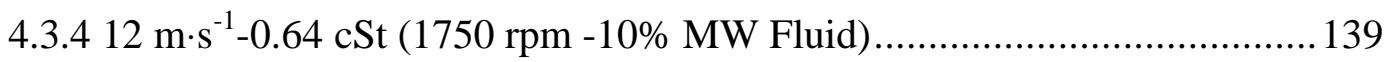

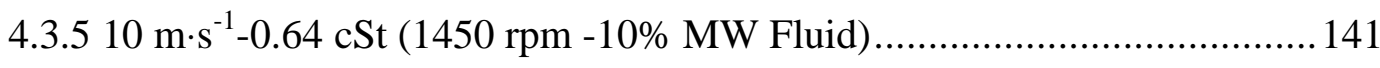

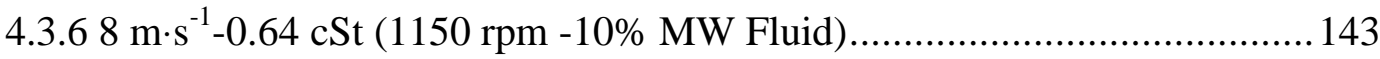

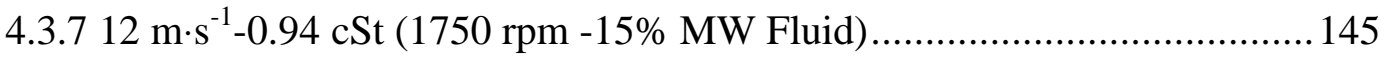

4.3.8 $10 \mathrm{~m} \cdot \mathrm{s}^{-1}-0.94 \mathrm{cSt}(1450 \mathrm{rpm}-15 \%$ MW Fluid) ........................................ 147

$4.3 .98 \mathrm{~m} \cdot \mathrm{s}^{-1}-0.94 \mathrm{cSt}(1150 \mathrm{rpm}-15 \%$ MW Fluid).......................................... 149

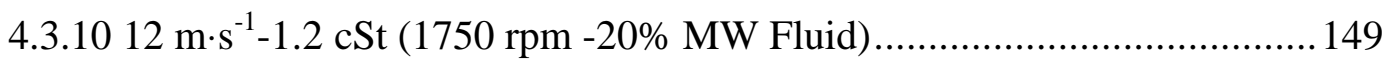

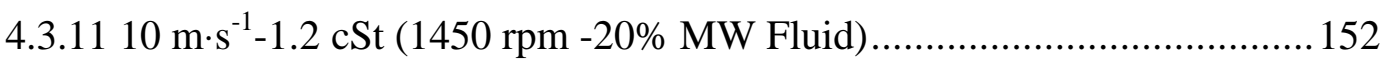

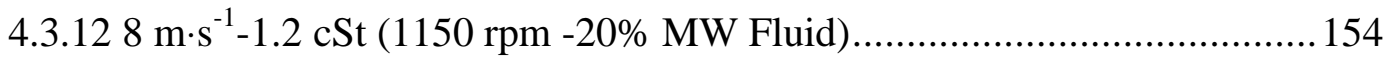

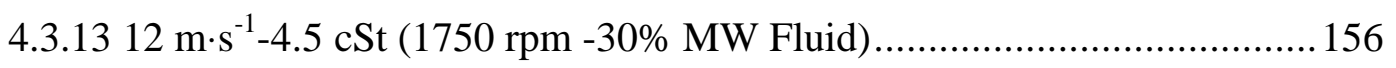

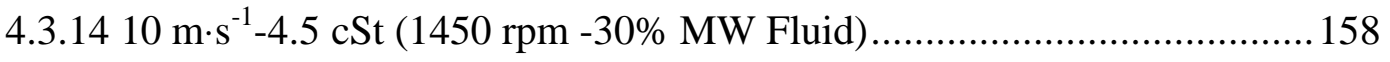

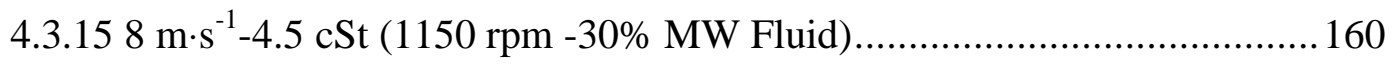

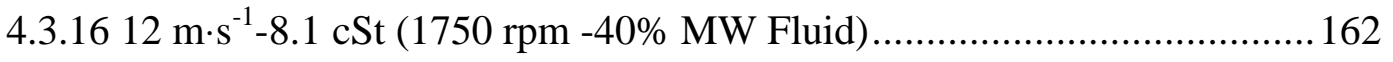

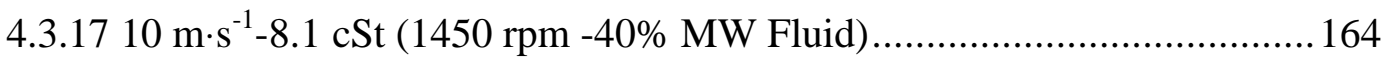

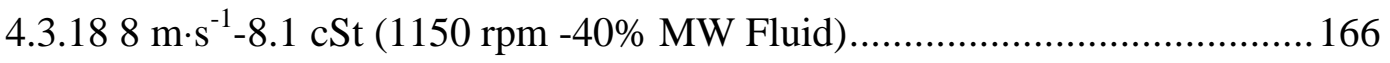

4.3.19 $12 \mathrm{~m} \cdot \mathrm{s}^{-1}-45.6 \mathrm{cSt}$ (1750 rpm -50\% MW Fluid) .................................... 168

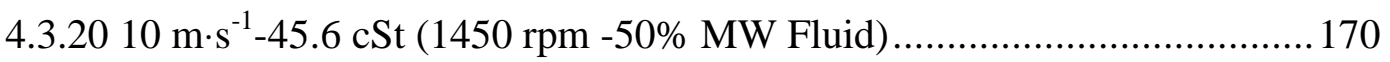


4.3.21 $8 \mathrm{~m} \cdot \mathrm{s}^{-1}-45.6 \mathrm{cSt}(1150 \mathrm{rpm}-50 \%$ MW Fluid) .......................................... 170

4.3.22 $12 \mathrm{~m} \cdot \mathrm{s}^{-1}-121 \mathrm{cSt}(1750 \mathrm{rpm}-60 \%$ MW Fluid) ..................................... 172

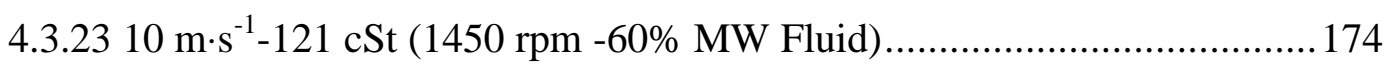

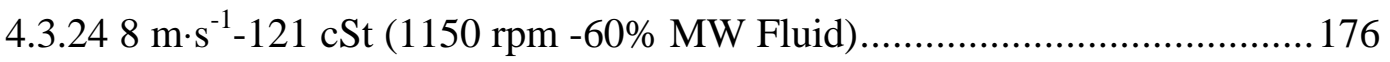

4.4 WASTE METAL WORKING FLUID EXPERIMENT ……………………….... 178

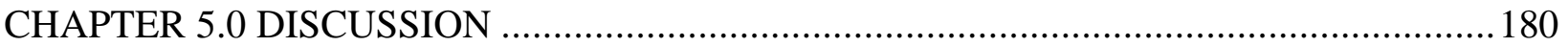

5.1 THE EFFECTS OF OPERATING PARAMETERS ON PERMEATE FLUX .........180

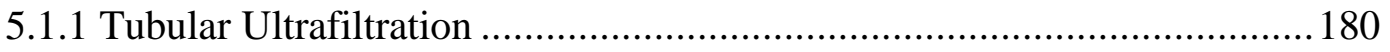

5.1.2 High-Shear Rotary Ultrafiltration ........................................................ 182

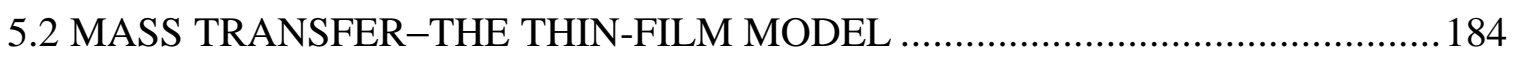

5.2.1 Gel Layer Oil Concentration................................................................. 184

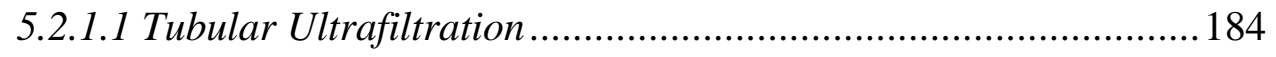

5.2.1.2 High-Shear Rotary Ultrafiltration ............................................. 187

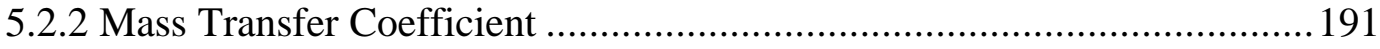

5.2.2.1 Tubular Ultrafiltration ............................................................... 191

5.2.2.2 High-Shear Rotary Ultrafiltration .............................................. 192

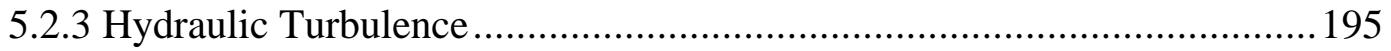

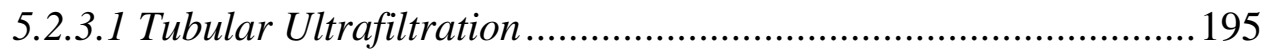

5.2.3.2 High-Shear Rotary Ultrafiltration ............................................... 197

5.2.4 Two Region Limiting Flux ..................................................................2200

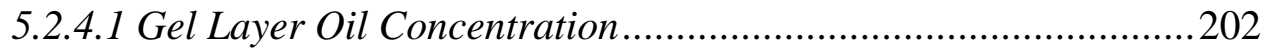

5.2.4.2 Mass Transfer Coefficient .......................................................... 203

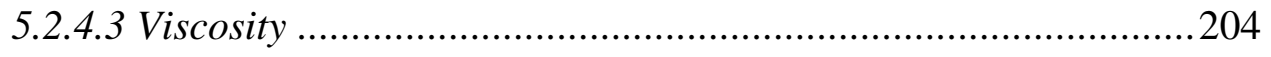

5.2.4.4 Consequences of Two Region Limiting Flux .............................. 207

5.3 SERIES RESISTANCES IN ULTRAFILTRATION PROCESSES ……….............208

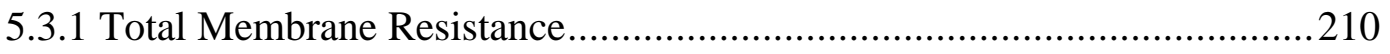

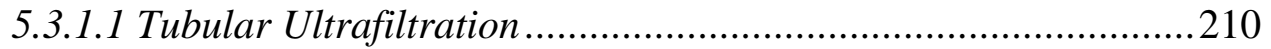

5.3.1.2 High-Shear Rotary Ultrafiltration ............................................212

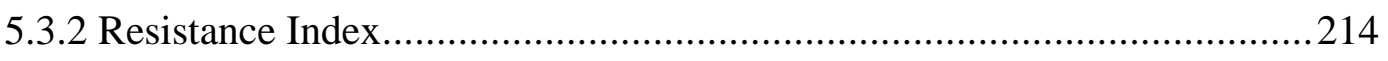

5.3.2.1 Tubular Ultrafiltration ..............................................................2. 215

5.3.2.2 High-Shear Rotary Ultrafiltration ............................................2217 
5.3.3 Modification of the Resistance in Series Model .....................................219

5.3.3.1 Tubular Ultrafiltration .............................................................220

5.3.3.2 High-Shear Rotary Ultrafiltration ............................................223

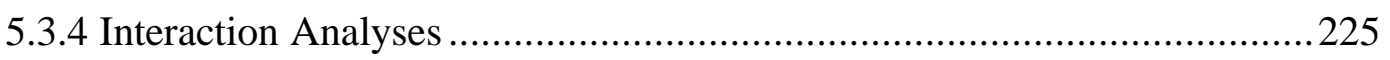

5.3.4.1 Tubular Ultrafiltration .............................................................225

5.3.4.2 High-Shear Rotary Ultrafiltration ..............................................226

5.3.5 Significance of the Modified Resistance-In-Series Model .........................229

5.3.6 Waste Permeate Flux Prediction ...........................................................2232

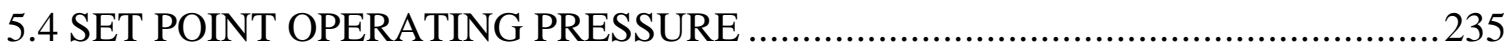

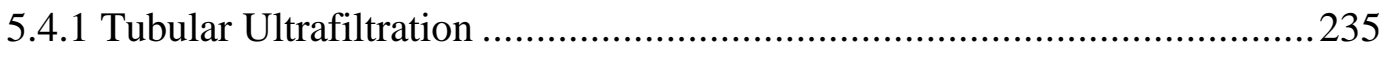

5.4.2 High-Shear Rotary Ultrafiltration .........................................................2236

5.4.3 Significance of the Set Point Operating Pressure …...................................237

5.5 HYBRID MEMBRANE-MEMBRANE SEPARATION ………...........................238

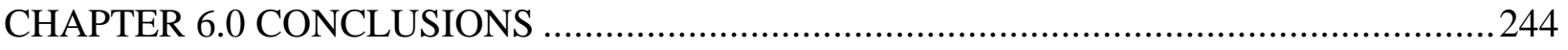

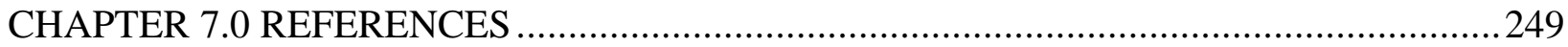

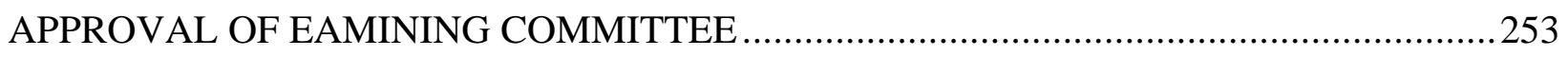




\section{LIST OF TABLES}

TABLE

PAGE

Table 2.1 Typical membrane resistances for micro and ultrafiltration membranes.

Table 2.2 A summary of typical Cg Values for several non-oily and process streams.

Table 2.3 Typical $\mathrm{O} / \mathrm{G}$ concentration ranges for selected industrial wastewaters.

Table 3.1 A summary of the polymeric membrane characteristics.

Table 3.2 Summary of operational condition applied to each discrete tubular experiment.

Table 3.3 Summary of tubular UF QA/QC replicate experiments.

Table 3.4 Average and maximum permeate backpressures.

Table 3.5 Summary of the ceramic membrane characteristics.

Table 3.6 Experimental matrix for surrogate waste experiments conducted using high-shear rotary ultrafiltration.

Table 3.7 Summary of HSRUF QA/QC replicate experiments.

Table 3.8 A hypothetical two-way ANOVA data set.

Table 3.9 A hypothetical two-variable data set.

Table 3.10 Ranked values for the Spearman Rank Correlation.

Table 5.1 Gel layer oil concentrations and mass transfer coefficients for each v/P combination examined in the tubular unit.

Table 5.2 Gel layer oil concentrations and mass transfer coefficients for each $\mathrm{v} / \mathrm{P}$ combination examined in the high-shear rotary unit.

Table 5.3 Summary of regression parameters for HSRUF synthetic MW fluid experiments

Table 5.4 Summary of regression parameters for HSRUF waste MW fluid experiment. 
Table 5.5 Summary of maximum, average, and minimum radial Re at transitional oil concentrations for HSRUF synthetic and waste MW fluid experiments.

Table 5.6 Number of observations, degrees of freedom, and highest model order examined for each dependent variable.

Table 5.7 Incremental analysis on tubular $\Phi$ versus v data at $4.5 \mathrm{cSt}$ $(25.5 \%$ oil $)$

Table 5.8 Incremental analysis on tubular $\Phi$ versus v data at $1.2 \mathrm{cSt}$ (17.0\% oil)

Table 5.9 Incremental analysis on tubular $\Phi$ versus v data at $0.94 \mathrm{cSt}$ (12.8\% oil)

Table 5.10 Incremental analysis on tubular $\Phi$ versus v data at $0.64 \mathrm{cSt}$ (8.5\% oil)

Table 5.11 Incremental analysis on tubular $\Phi$ versus v data at $0.46 \mathrm{cSt}$ (4.3\% oil)

Table 5.12 Incremental analysis on tubular $\Phi$ versus v data at $0.40 \mathrm{cSt}$ $(0.85 \%$ oil $)$

Table 5.13 Incremental analysis on tubular $\Phi$ versus $\eta$ data at $3.7 \mathrm{~m} \cdot \mathrm{s}^{-1}$ (30 gpm).

Table 5.14 Incremental analysis on tubular $\Phi$ versus $\eta$ data at $2.5 \mathrm{~m} \cdot \mathrm{s}^{-1}(20$ gpm).

Table 5.15 Incremental analysis on tubular $\Phi$ versus $\eta$ data at $1.2 \mathrm{~m} \cdot \mathrm{s}^{-1}(10$ gpm).

Table 5.16 Incremental analysis on tubular $\Phi$ versus $\eta$ data at $0.62 \mathrm{~m} \cdot \mathrm{s}^{-1}(5$ gpm).

Table 5.17 Incremental analysis on HSR $\Phi$ versus $\eta$ data at $12 \mathrm{~m} \cdot \mathrm{s}^{-1}(1750$ rpm).

Table 5.18 Incremental analysis on HSR $\Phi$ versus $\eta$ data at $10 \mathrm{~m} \cdot \mathrm{s}^{-1}(1450$ rpm).

Table 5.19 Incremental analysis on HSR $\Phi$ versus $\eta$ data at $8 \mathrm{~m} \cdot \mathrm{s}^{-1}(1150$ rpm). 
Table 5.21 Results from regression interaction analysis of the tubular UF model.

Table 5.21 Results from regression interaction analysis of the HSRUF model. 


\section{LIST OF FIGURES}

Figure 2.1 A schematic of a dead-end filtration process.

Figure 2.2 A schematic of a cross-flow filtration process.

Figure 2.3 A schematic of semi-batch and batch operational modes.

Figure 2.4 The useful ranges of the four general membrane separation processes.

Figure 2.5 A schematic of the phenomena of osmosis and reverse osmosis

Figure 2.6 The theoretical relationship between permeate flux, transmembrane pressure, feed concentration, and hydraulic turbulence.

Figure 2.7 A schematic of the transport phenomena occurring at the membrane surface.

Figure 2.8 A schematic of a tubular membrane module.

Figure 2.9 A schematic of a hollow fiber membrane module.

Figure 2.10 A schematic of a spiral wound membrane module.

Figure 2.11 A schematic of a plate and frame membrane module.

Figure 2.12 A schematic of the high-shear rotary ultrafiltration pressure vessel.

Figure 2.13 A schematic of an emulsified oil droplet.

Figure 3.1 A schematic of the tubular ultrafiltration experimental apparatus. $\quad 58$

Figure 3.2 A schematic of the high-shear rotary ultrafiltration experimental apparatus.

Figure 4.1 Kinematic viscosity versus oil concentration for the synthetic waste metal working fluids.

Figure 4.2 Permeate flux versus time for the $3.7 \mathrm{~m} \cdot \mathrm{s}^{-1}-0.40 \mathrm{cSt}$ experiment. 
Figure 4.3 Average steady state permeate flux versus average transmembrane pressure for the $3.7 \mathrm{~m} \cdot \mathrm{s}^{-1}-0.40 \mathrm{cSt}$ experiment.

Figure 4.4 Permeate flux versus operational time for the $2.5 \mathrm{~m} \cdot \mathrm{s}^{-1}-0.40 \mathrm{cSt}$ experiment.

Figure 4.5 Average steady state permeate flux versus average transmembrane pressure for the $2.5 \mathrm{~m} \cdot \mathrm{s}^{-1}-0.40 \mathrm{cSt}$ experiment.

Figure 4.6 Permeate flux versus operational time for the $1.2 \mathrm{~m} \cdot \mathrm{s}^{-1}-0.40 \mathrm{cSt}$ experiment.

Figure 4.7 Average steady state permeate flux versus average transmemebrane pressure for the $1.2 \mathrm{~m} \cdot \mathrm{s}^{-1}-0.40 \mathrm{cSt}$ experiment.

Figure 4.8 Permeate flux versus operational time for the $0.62 \mathrm{~m} \cdot \mathrm{s}^{-1}-0.40 \mathrm{cSt}$ experiment.

Figure 4.9 Average steady state permeate flux versus average transmembrane pressure for the $0.62 \mathrm{~m} \cdot \mathrm{s}^{-1}-0.40 \mathrm{cSt}$ experiment.

Figure 4.10 Permeate flux versus operational time for the $3.7 \mathrm{~m} \cdot \mathrm{s}^{-1}-0.46 \mathrm{cSt}$ experiment.

Figure 4.11 Average steady state permeate flux versus average transmembrane pressure for the $3.7 \mathrm{~m} \cdot \mathrm{s}^{-1}-0.46 \mathrm{cSt}$ experiment.

Figure 4.12 Permeate flux versus operational time for the $2.5 \mathrm{~m} \cdot \mathrm{s}^{-1}-0.46 \mathrm{cSt}$ experiment.

Figure 4.13 Average steady state permeate flux versus average transmembrane pressure for the $2.5 \mathrm{~m} \cdot \mathrm{s}^{-1}-0.46 \mathrm{cSt}$ experiment.

Figure 4.14 Permeate flux versus operational time for the $1.2 \mathrm{~m} \cdot \mathrm{s}^{-1}-0.46 \mathrm{cSt}$ experiment.

Figure 4.15 Average steady state permeate flux versus average transmembrane pressure for the $1.2 \mathrm{~m} \cdot \mathrm{s}^{-1}-0.46 \mathrm{cSt}$ experiment.

Figure 4.16 Permeate flux versus operational time for the $0.62 \mathrm{~m} \cdot \mathrm{s}^{-1}-0.46 \mathrm{cSt}$ experiment.

Figure 4.17 Average steady state permeate flux versus average transmembrane pressure for the $0.62 \mathrm{~m} \cdot \mathrm{s}^{-1}-0.46 \mathrm{cSt}$ experiment. 
Figure 4.18 Permeate flux versus operational time for the $3.7 \mathrm{~m} \cdot \mathrm{s}^{-1}-0.64 \mathrm{cSt}$ experiment.

Figure 4.19 Average steady state permeate flux versus average transmembrane pressure for the $3.7 \mathrm{~m} \cdot \mathrm{s}^{-1}-0.64 \mathrm{cSt}$ experiment.

Figure 4.20 Permeate flux versus operational time for the $2.5 \mathrm{~m} \cdot \mathrm{s}^{-1}-0.64 \mathrm{cSt}$ experiment.

Figure 4.21 Average steady state permeate flux versus average transmembrane pressure for the $2.5 \mathrm{~m} \cdot \mathrm{s}^{-1}-0.64 \mathrm{cSt}$ experiment.

Figure 4.22 Permeate flux versus operational time for the $1.2 \mathrm{~m} \cdot \mathrm{s}^{-1}-0.64 \mathrm{cSt}$ experiment.

Figure 4.23 Average steady state permeate flux versus average transmembrane pressure for the $1.2 \mathrm{~m} \cdot \mathrm{s}^{-1}-0.64 \mathrm{cSt}$ experiment.

Figure 4.24 Permeate flux versus operational time for the $0.62 \mathrm{~m} \cdot \mathrm{s}^{-1}-0.64 \mathrm{cSt}$ experiment.

Figure 4.25 Average steady state permeate flux versus average transmembrane pressure for the $0.62 \mathrm{~m} \cdot \mathrm{s}^{-1}-0.64 \mathrm{cSt}$ experiment.

Figure 4.26 Permeate flux versus operational time for the $3.7 \mathrm{~m} \cdot \mathrm{s}^{-1}-0.94 \mathrm{cSt}$ experiment.

Figure 4.27 Average steady state permeate flux versus average transmembrane pressure for the $3.7 \mathrm{~m} \cdot \mathrm{s}^{-1}-0.94 \mathrm{cSt}$ experiment.

Figure 4.28 Permeate flux versus operational time for the $2.5 \mathrm{~m} \cdot \mathrm{s}^{-1}-0.94 \mathrm{cSt}$ experiment.

Figure 4.29 Average steady state permeate flux versus average transmembrane pressure for the $2.5 \mathrm{~m} \cdot \mathrm{s}^{-1}-0.94 \mathrm{cSt}$ experiment.

Figure 4.30 Permeate flux versus operational time for the $1.2 \mathrm{~m} \cdot \mathrm{s}^{-1}-0.94 \mathrm{cSt}$ experiment.

Figure 4.31 Average steady state permeate flux versus average transmembrane pressure for the $1.2 \mathrm{~m} \cdot \mathrm{s}^{-1}-0.94 \mathrm{cSt}$ experiment.

Figure 4.32 Permeate flux versus operational time for the $0.62 \mathrm{~m} \cdot \mathrm{s}^{-1}-0.94 \mathrm{cSt}$ experiment. 
Figure 4.33 Average steady state permeate flux versus average transmembrane pressure for the $0.62 \mathrm{~m} \cdot \mathrm{s}^{-1}-0.94 \mathrm{cSt}$ experiment.

Figure 4.34 Permeate flux versus operational time for the $3.7 \mathrm{~m} \cdot \mathrm{s}^{-1}-1.2 \mathrm{cSt}$ experiment.

Figure 4.35 Average steady state permeate flux versus average transmembrane pressure for the $0.62 \mathrm{~m} \cdot \mathrm{s}^{-1}-1.2 \mathrm{cSt}$ experiment.

Figure 4.36 Permeate flux versus operational time for the $2.5 \mathrm{~m} \cdot \mathrm{s}^{-1}-1.2 \mathrm{cSt}$ experiment.

Figure 4.37 Average steady state permeate flux versus average transmembrane pressure for the $2.5 \mathrm{~m} \cdot \mathrm{s}^{-1}-1.2 \mathrm{cSt}$ experiment.

Figure 4.38 Permeate flux versus operational time for the $1.2 \mathrm{~m} \cdot \mathrm{s}^{-1}-1.2 \mathrm{cSt}$ experiment.

Figure 4.39 Average steady state permeate flux versus average transmembrane pressure for the $1.2 \mathrm{~m} \cdot \mathrm{s}^{-1}-1.2 \mathrm{cSt}$ experiment.

Figure 4.40 Permeate flux versus operational time for the $0.62 \mathrm{~m} \cdot \mathrm{s}^{-1}-1.2 \mathrm{cSt}$ experiment.

Figure 4.41 Average steady state permeate flux versus average transmembrane pressure for the $0.62 \mathrm{~m} \cdot \mathrm{s}^{-1}-1.2 \mathrm{cSt}$ experiment.

Figure 4.42 Permeate flux versus operational time for the $3.7 \mathrm{~m} \cdot \mathrm{s}^{-1}-4.5 \mathrm{cSt}$ experiment.

Figure 4.43 Average steady state permeate flux versus average transmembrane pressure for the $3.7 \mathrm{~m} \cdot \mathrm{s}^{-1}-4.5 \mathrm{cSt}$ experiment.

Figure 4.44 Permeate flux versus operational time for the $2.5 \mathrm{~m} \cdot \mathrm{s}^{-1}-4.5 \mathrm{cSt}$ experiment.

Figure 4.45 Average steady state permeate flux versus average transmembrane pressure for the $2.5 \mathrm{~m} \cdot \mathrm{s}^{-1}-4.5 \mathrm{cSt}$ experiment.

Figure 4.46 Permeate flux versus operational time for the $1.2 \mathrm{~m} \cdot \mathrm{s}^{-1}-4.5 \mathrm{cSt}$ experiment.

Figure 4.47 Average steady state permeate flux versus average transmembrane pressure for the $1.2 \mathrm{~m} \cdot \mathrm{s}^{-1}-4.5 \mathrm{cSt}$ experiment. 
Figure 4.48 Permeate flux versus operational time for the $0.62 \mathrm{~m} \cdot \mathrm{s}^{-1}-4.5 \mathrm{cSt}$ experiment.

Figure 4.49 Average steady state permeate flux versus average transmembrane pressure for the $0.62 \mathrm{~m} \cdot \mathrm{s}^{-1}-4.5 \mathrm{cSt}$ experiment.

Figure 4.50 Permeate flux versus operational time for the $12 \mathrm{~m} \cdot \mathrm{s}^{-1}-0.46 \mathrm{cSt}$ experiment.

Figure 4.51 Average steady state permeate flux versus average transmembrane pressure for the $12 \mathrm{~m} \cdot \mathrm{s}^{-1}-0.46 \mathrm{cSt}$ experiment.

Figure 4.52 Permeate flux versus operational time for the $10 \mathrm{~m} \cdot \mathrm{s}^{-1}-0.46 \mathrm{cSt}$ experiment.

Figure 4.53 Average steady state permeate flux versus average transmembrane pressure for the $10 \mathrm{~m} \cdot \mathrm{s}^{-1}-0.46 \mathrm{cSt}$ experiment.

Figure 4.54 Permeate flux versus operational time for the $8 \mathrm{~m} \cdot \mathrm{s}^{-1}-0.46 \mathrm{cSt}$ experiment.

Figure 4.55 Average steady state permeate flux versus average transmembrane pressure for the $8 \mathrm{~m} \cdot \mathrm{s}^{-1}-0.46 \mathrm{cSt}$ experiment.

Figure 4.56 Permeate flux versus operational time for the $12 \mathrm{~m} \cdot \mathrm{s}^{-1}-0.64 \mathrm{cSt}$ experiment.

Figure 4.57 Average steady state permeate flux versus average transmembrane pressure for the $12 \mathrm{~m} \cdot \mathrm{s}^{-1}-0.64 \mathrm{cSt}$ experiment.

Figure 4.58 Permeate flux versus operational time for the $10 \mathrm{~m} \cdot \mathrm{s}^{-1}-0.64 \mathrm{cSt}$ experiment.

Figure 4.59 Average steady state permeate flux versus average transmembrane pressure for the $10 \mathrm{~m} \cdot \mathrm{s}^{-1}-0.64 \mathrm{cSt}$ experiment.

Figure 4.60 Permeate flux versus operational time for the $8 \mathrm{~m} \cdot \mathrm{s}^{-1}-0.64 \mathrm{cSt}$ experiment.

Figure 4.61 Average steady state permeate flux versus average transmembrane pressure for the $8 \mathrm{~m} \cdot \mathrm{s}^{-1}-0.64 \mathrm{cSt}$ experiment.

Figure 4.62 Permeate flux versus operational time for the $12 \mathrm{~m} \cdot \mathrm{s}^{-1}-0.94 \mathrm{cSt}$ experiment. 
Figure 4.63 Average steady state permeate flux versus average transmembrane pressure for the $12 \mathrm{~m} \cdot \mathrm{s}^{-1}-0.94 \mathrm{cSt}$ experiment.

Figure 4.64 Permeate flux versus operational time for the $10 \mathrm{~m} \cdot \mathrm{s}^{-1}-0.94 \mathrm{cSt}$ experiment.

Figure 4.65 Average steady state permeate flux versus average transmembrane pressure for the $\mathrm{m} \cdot \mathrm{s}^{-1}-0.94 \mathrm{cSt}$ experiment.

Figure 4.66 Permeate flux versus operational time for the $8 \mathrm{~m} \cdot \mathrm{s}^{-1}-0.94 \mathrm{cSt}$ experiment.

Figure 4.67 Average steady state permeate flux versus average transmembrane pressure for the $8 \mathrm{~m} \cdot \mathrm{s}^{-1}-0.94 \mathrm{cSt}$ experiment.

Figure 4.68 Permeate flux versus operational time for the $12 \mathrm{~m} \cdot \mathrm{s}^{-1}-1.2 \mathrm{cSt}$ experiment.

Figure 4.69 Average steady state permeate flux versus average transmembrane pressure for the $12 \mathrm{~m} \cdot \mathrm{s}^{-1}-1.2 \mathrm{cSt}$ experiment.

Figure 4.70 Permeate flux versus operational time for the $10 \mathrm{~m} \cdot \mathrm{s}^{-1}-1.2 \mathrm{cSt}$ experiment.

Figure 4.71 Average steady state permeate flux versus average transmembrane pressure for the $10 \mathrm{~m} \cdot \mathrm{s}^{-1}-1.2 \mathrm{cSt}$ experiment.

Figure 4.72 Permeate flux versus operational time for the $8 \mathrm{~m} \cdot \mathrm{s}^{-1}-1.2 \mathrm{cSt}$ experiment.

Figure 4.73 Average steady state permeate flux versus average transmembrane pressure for the $8 \mathrm{~m} \cdot \mathrm{s}^{-1}-1.2 \mathrm{cSt}$ experiment.

Figure 4.74 Permeate flux versus operational time for the $12 \mathrm{~m} \cdot \mathrm{s}^{-1}-4.5 \mathrm{cSt}$ experiment.

Figure 4.75 Average steady state permeate flux versus average transmembrane pressure for the $12 \mathrm{~m} \cdot \mathrm{s}^{-1}-4.5 \mathrm{cSt}$ experiment.

Figure 4.76 Permeate flux versus operational time for the $10 \mathrm{~m} \cdot \mathrm{s}^{-1}-4.5 \mathrm{cSt}$ experiment.

Figure 4.77 Average steady state permeate flux versus average transmembrane pressure for the $10 \mathrm{~m} \cdot \mathrm{s}^{-1}-4.5 \mathrm{cSt}$ experiment. 
Figure 4.78 Permeate flux versus operational time for the $8 \mathrm{~m} \cdot \mathrm{s}^{-1}-4.5 \mathrm{cSt}$ experiment.

Figure 4.79 Average steady state permeate flux versus average transmembrane pressure for the $8 \mathrm{~m} \cdot \mathrm{s}^{-1}-4.5 \mathrm{cSt}$ experiment.

Figure 4.80 Permeate flux versus operational time for the $12 \mathrm{~m} \cdot \mathrm{s}^{-1}-8.1 \mathrm{cSt}$ experiment.

Figure 4.81 Average steady state permeate flux versus average transmembrane pressure for the $12 \mathrm{~m} \cdot \mathrm{s}^{-1}-8.1 \mathrm{cSt}$ experiment.

Figure 4.82 Permeate flux versus operational time for the $10 \mathrm{~m} \cdot \mathrm{s}^{-1}-8.1 \mathrm{cSt}$ experiment.

Figure 4.83 Average steady state permeate flux versus average transmembrane pressure for the $10 \mathrm{~m} \cdot \mathrm{s}^{-1}-8.1 \mathrm{cSt}$ experiment.

Figure 4.84 Permeate flux versus operational time for the $8 \mathrm{~m} \cdot \mathrm{s}^{-1}-8.1 \mathrm{cSt}$ experiment.

Figure 4.85 Average steady state permeate flux versus average transmembrane pressure for the $8 \mathrm{~m} \cdot \mathrm{s}^{-1}-8.1 \mathrm{cSt}$ experiment.

Figure 4.86 Permeate flux versus operational time for the $12 \mathrm{~m} \cdot \mathrm{s}^{-1}-45.6 \mathrm{cSt}$ experiment.

Figure 4.87 Average steady state permeate flux versus average transmembrane pressure for the $12 \mathrm{~m} \cdot \mathrm{s}^{-1}-45.6 \mathrm{cSt}$ experiment.

Figure 4.88 Permeate flux versus operational time for the $10 \mathrm{~m} \cdot \mathrm{s}^{-1}-45.6 \mathrm{cSt}$ experiment.

Figure 4.89 Average steady state permeate flux versus average transmembrane pressure for the $10 \mathrm{~m} \cdot \mathrm{s}^{-1}-45.6 \mathrm{cSt}$ experiment.

Figure 4.90 Permeate flux versus operational time for the $8 \mathrm{~m} \cdot \mathrm{s}^{-1}-45.6 \mathrm{cSt}$ experiment.

Figure 4.91 Average steady state permeate flux versus average transmembrane pressure for the $8 \mathrm{~m} \cdot \mathrm{s}^{-1}-45.6 \mathrm{cSt}$ experiment.

Figure 4.92 Permeate flux versus operational time for the $12 \mathrm{~m} \cdot \mathrm{s}^{-1}-121 \mathrm{cSt}$ experiment. 
Figure 4.93 Average steady state permeate flux versus average transmembrane pressure for the $12 \mathrm{~m} \cdot \mathrm{s}^{-1}-121 \mathrm{cSt}$ experiment.

Figure 4.94 Permeate flux versus operational time for the $10 \mathrm{~m} \cdot \mathrm{s}^{-1}-121 \mathrm{cSt}$ experiment.

Figure 4.95 Average steady state permeate flux versus average transmembrane pressure for the $10 \mathrm{~m} \cdot \mathrm{s}^{-1}-121 \mathrm{cSt}$ experiment.

Figure 4.96 Permeate flux versus operational time for the $8 \mathrm{~m} \cdot \mathrm{s}^{-1}-121 \mathrm{cSt}$ experiment.

Figure 4.97 Average steady state permeate flux versus average transmembrane pressure for the $8 \mathrm{~m} \cdot \mathrm{s}^{-1}-121 \mathrm{cSt}$ experiment.

Figure 4.98 Permeate flux and feed concentration factor versus time for the waste MW fluid experiment.

Figure 4.99 Average permeate flux versus concentration factor for the waste MW fluid experiment.

Figure 5.1 Average permeate flux versus average transmembrane pressure for experiments conducted at $3.7 \mathrm{~m} \cdot \mathrm{s}^{-1}$.

Figure 5.2 Average permeate flux versus average transmembrane pressure for the experiments conducted at $4.5 \mathrm{cSt}$.

Figure 5.3 Average permeate flux versus average transmembrane pressure for experiments conducted at $12 \mathrm{~m} \cdot \mathrm{s}^{-1}$

Figure 5.4 Average permeate flux versus average transmembrane pressure for experiments conducted at $4.5 \mathrm{cSt}$.

Figure 5.5 Average permeate flux versus feed oil concentration for experiments conducted at $2.5 \mathrm{~m} \cdot \mathrm{s}^{-1}$.

Figure 5.6 Average permeate flux versus feed oil concentration for experiments conducted at $1.2 \mathrm{~m} \cdot \mathrm{s}^{-1}$.

Figure 5.7 Average permeate flux versus feed oil concentration for experiments conducted at $0.62 \mathrm{~m} \cdot \mathrm{s}^{-1}$.

Figure 5.8 Average permeate flux versus feed oil concentration for experiments conducted at $12 \mathrm{~m} \cdot \mathrm{s}^{-1}$. 
Figure 5.9 Average permeate flux versus feed oil concentration for experiments conducted at $10 \mathrm{~m} \cdot \mathrm{s}^{-1}$.

Figure 5.10 Average permeate flux versus feed oil concentration for experiments conducted at $8 \mathrm{~m} \cdot \mathrm{s}^{-1}$.

Figure 5.11 Plots of experimental data and thin-film model predictions of limiting flux versus feed oil concentration for the tubular UF system.

Figure 5.12 Experimental data and thin-film model predictions of limiting permeate flux versus feed oil concentration for the high-shear rotary unit.

Figure 5.13 Limiting permeate flux versus Reynolds Number for experiments conducted with the tubular unit.

Figure 5.14 Limiting permeate flux versus Reynolds Number for experiments conducted with the high-shear rotary unit.

Figure 5.15 Limiting permeate flux versus feed oil concentration for the high-shear rotary synthetic metal-working fluid experiments conducted at $379 \mathrm{kPa}$.

Figure 5.16 Limiting permeate flux versus feed oil concentration for the high-shear rotary waste metal-working fluid experiment.

Figure 5.17 Data reduction flow chart for modified RIS model development.

Figure 5.18 Total membrane resistance versus average relative velocity for each feed viscosity examined in the tubular unit.

Figure 5.19 Total membrane resistance versus average relative velocity for each feed viscosity examined in the high-shear rotary unit.

Figure 5.20 Resistance Index versus average relative velocity for each feed viscosity examined in the tubular unit.

Figure 5.21 Resistance Index versus average relative velocity for each feed viscosity examined in the high-shear rotary unit.

Figure 5.22 Permeate flux versus average transmembrane pressure for experiments conducted at $3.7 \mathrm{~m} \cdot \mathrm{s}^{-1}$.

Figure 5.23 Permeate flux versus average transmembrane pressure for experiments conducted at $2.5 \mathrm{~m} \cdot \mathrm{s}^{-1}$. 
Figure 5.24 Permeate flux versus average transmembrane pressure for experiments conducted at $1.2 \mathrm{~m} \cdot \mathrm{s}^{-1}$.

Figure 5.25 Permeate flux versus average transmembrane pressure for experiments conducted at $0.62 \mathrm{~m} \cdot \mathrm{s}^{-1}$.

Figure 5.26 Permeate flux versus average transmembrane pressure for experiments conducted at 0.46 through $8.1 \mathrm{cSt}-12 \mathrm{~m} \cdot \mathrm{s}^{-1}$.

Figure 5.27 Permeate flux versus average transmembrane pressure for experiments conducted at 0.46 through $8.1 \mathrm{cSt}-10 \mathrm{~m} \cdot \mathrm{s}^{-1}$.

Figure 5.28 Permeate flux versus average transmembrane pressure for experiments conducted at 0.46 through $8.1 \mathrm{cSt}-8 \mathrm{~m} \cdot \mathrm{s}^{-1}$.

Figure 5.29 Permeate flux versus average transmembrane pressure for experiments conducted at 45.6 and $121 \mathrm{cSt}-12 \mathrm{~m} \cdot \mathrm{s}^{-1}$.

Figure 5.30 Permeate flux versus average transmembrane pressure for experiments conducted at 45.6 and $121 \mathrm{cSt}-10 \mathrm{~m} \cdot \mathrm{s}^{-1}$.

Figure 5.31 Permeate flux versus average transmembrane pressure for experiments conducted at 45.6 and $121 \mathrm{cSt}-10 \mathrm{~m} \cdot \mathrm{s}^{-1}$.

Figure 5.32 Average permeate flux versus feed oil concentration for the waste MW fluid experiment.

Figure 5.33 Set point operating pressure versus feed kinematic viscosity for each average relative velocity examined in the tubular unit.

Figure 5.34 Set point operating pressure versus feed kinematic viscosity for each average relative velocity examined in the high-shear rotary unit.

Figure 5.35 Plots of tubular and high-shear rotary resistance index versus

Figure 5.36 A plot of feed kinematic viscosity versus average relative velocity. 


\section{LIST OF SYMBOLS}

A $\quad$ Cross-sectional area of the membrane tube $\left(\mathrm{m}^{2}\right)$

3.2

$A_{p} \quad$ Membrane permeability coefficient

2.28

C Solute concentration (\%)

$2.18,2.19,2.20,2.21$

$\mathrm{C}_{\mathrm{b}} \quad$ Solute concentration in the bulk solution (\%)

$2.2,2.17,2.20,2.22$, $2.23,2.27$

$\mathrm{C}_{\mathrm{g}} \quad$ Solute gel layer concentration (\%)

$\mathrm{C}_{\mathrm{m}} \quad$ Solute concentration at the membrane surface (\%)

$2.21,2.22,2.23$

$\mathrm{C}_{\mathrm{P}} \quad$ Solute concentration in the permeate (\%)

$2.19,2.22$

$\mathrm{CF}_{\mathrm{sb}} \quad$ Concentration factor during semi-batch operation (X)

2.1

$\mathrm{CF}_{\mathrm{b}} \quad$ Concentration factor during batch operation (X)

2.2

D Diffusion coefficient

2.16, 2.19, 2.22, 2.23, $2.24,2.25,2.26,5.1$, 5.2

$\mathrm{DF}_{\text {Error }} \quad$ Error degrees of freedom

$3.13,3.15$

$\mathrm{DF}_{\text {Factor }} \quad$ Degrees of freedom for the factor

$3.8,3.10,3.12$

$\mathrm{DF}_{\mathrm{Res}} \quad$ Residual degrees of freedom

$3.18,3.20$

$\mathrm{d}_{\mathrm{h}} \quad$ Hydraulic diameter of the membrane tube (m)

$2.25,2.26,5.1,5.2$

$\mathrm{F}_{\mathrm{A}} \quad$ F-test statistic calculated in ANOVA analyses

$\mathrm{F}_{\mathrm{R}} \quad$ F-test statistic calculated in regression analyses

I Radius of gyration (cm)

$2.7,2.8,2.11,3.5,3.6$

J Pressure-dependent permeate flux $\left(\mathrm{m}^{3} \cdot \mathrm{m}^{-2} \cdot \mathrm{d}^{-1}\right)$

$2.4,2.15,2.16,2.17$, 2.19, 2.22, 2.23, 2.28, $2.29,2.31,2.34,5.3$

$\mathrm{J}_{\mathrm{R}}$

High-shear rotary permeate flux $\left(\mathrm{m}^{3} \cdot \mathrm{m}^{-2} \cdot \mathrm{d}^{-1}\right)$

$5.20,5.21$ 


\begin{tabular}{|c|c|c|}
\hline $\mathrm{J}_{\mathrm{s}}$ & Solute flux $\left(\mathrm{m}^{3} \cdot \mathrm{m}^{-2} \cdot \mathrm{d}^{-1}\right)$ & $2.17,2.18$ \\
\hline $\mathrm{J}_{\mathrm{T}}$ & Tubular permeate flux $\left(\mathrm{m}^{3} \cdot \mathrm{m}^{-2} \cdot \mathrm{d}^{-1}\right)$ & $5.17,5.18$ \\
\hline $\mathrm{J}^{*}$ & Pressure-independent permeate flux $\left(\mathrm{m}^{3} \cdot \mathrm{m}^{-2} \cdot \mathrm{d}^{-1}\right)$ & $2.9,2.27$ \\
\hline $\mathrm{k}_{\mathrm{sv}}$ & Kolmogorov-Smirnov test statistic & 3.22 \\
\hline $\mathrm{k}_{\mathrm{s}}$ & Mass transfer coefficient $\left(\mathrm{m}^{3} \cdot \mathrm{m}^{-2} \cdot \mathrm{d}^{-1}\right)$ & $\begin{array}{c}2.24,2.25,2.26,2.27 \\
5.1,5.2\end{array}$ \\
\hline $\mathrm{L}$ & Membrane module channel length $(\mathrm{cm})$ & $2.26,5.2$ \\
\hline $\mathrm{MS}_{\text {Error }}$ & Error mean square & $3.7,3.13$ \\
\hline $\mathrm{MS}_{\text {Factor }}$ & Mean square for the factor & $3.7,3.8$ \\
\hline $\mathrm{MS}_{\text {Reg }}$ & Regression mean square & 3.17 \\
\hline $\mathrm{MS}_{\text {Res }}$ & Residual mean square & $3.17,3.18$ \\
\hline $\mathrm{N}$ & Number of observations in the sample population & $3.21,3.22$ \\
\hline $\mathrm{P}$ & Average transmembrane pressure $(\mathrm{kPa})$ & $\begin{array}{c}2.4,2.13,2.15,2.16, \\
2.28,2.29,2.31,2.33, \\
2.34,3.1,3.3,5.3,5.5\end{array}$ \\
\hline $\mathrm{P}_{\text {applied }}$ & Applied pressure $(\mathrm{kPa})$ & $2.13,2.14,3.3$ \\
\hline $\mathrm{P}_{\text {back }}$ & Permeate back pressure $(\mathrm{kPa})$ & 2.10 \\
\hline$P_{\text {back-avg }}$ & Average permeate back pressure $(\mathrm{kPa})$ & $2.11,2.13,3.3$ \\
\hline$P_{\text {back-max }}$ & Maximum permeate back pressure $(\mathrm{kPa})$ & $2.12,2.14$ \\
\hline$P_{\text {in }}$ & Upstream pressure $(\mathrm{kPa})$ & 3.1 \\
\hline $\mathrm{P}_{\text {out }}$ & Downstream pressure $(\mathrm{kPa})$ & 3.1 \\
\hline $\mathrm{Q}_{\mathrm{r}}$ & Concentrate return flow $\left(\mathrm{m}^{3} \cdot \mathrm{s}^{-1}\right)$ & 3.2 \\
\hline $\mathrm{R}$ & Total resistance to permeate flow $\left(\mathrm{kPa} \cdot \mathrm{h} \cdot \mathrm{m}^{-1}\right)$ & $2.29,2.30$ \\
\hline $\mathrm{R}^{2}$ & Coefficient of determination & 3.16 \\
\hline
\end{tabular}




\begin{tabular}{|c|c|c|}
\hline $\mathrm{R}_{\mathrm{F}}$ & Fouling resistance $\left(\mathrm{kPa} \cdot \mathrm{h} \cdot \mathrm{m}^{-1}\right)$ & $2.30,2.31,2.32,5.4$ \\
\hline $\mathrm{R}_{\mathrm{m}}$ & Intrinsic membrane resistance $\left(\mathrm{kPa} \cdot \mathrm{h} \cdot \mathrm{m}^{-1}\right)$ & $\begin{array}{c}2.4,2.5,2.30,2.31 \\
2.32,5.4\end{array}$ \\
\hline $\mathrm{R}_{\mathrm{m}}^{\prime}$ & Total membrane resistance $\left(\mathrm{kPa} \cdot \mathrm{h} \cdot \mathrm{m}^{-1}\right)$ & $\begin{array}{c}2.32,2.34,2.35,5.3 \\
5.4\end{array}$ \\
\hline $\mathrm{R}_{\mathrm{m}}^{\prime}(\mathrm{R})$ & High-shear rotary total membrane resistance $\left(\mathrm{kPa} \cdot \mathrm{h} \cdot \mathrm{m}^{-1}\right)$ & 5.20 \\
\hline $\mathrm{R}_{\mathrm{m}}^{\prime}(\mathrm{T})$ & Tubular Total membrane resistance $\left(\mathrm{kPa} \cdot \mathrm{h} \cdot \mathrm{m}^{-1}\right)$ & 5.17 \\
\hline $\mathrm{R}_{\mathrm{p}}$ & Polarization resistance $\left(\mathrm{kPa} \cdot \mathrm{h} \cdot \mathrm{m}^{-1}\right)$ & $2.30,2.31,2.33,5.5$ \\
\hline $\mathrm{Ri}$ & Inner radius of the membrane disk $(\mathrm{cm})$ & $2.7,3.6$ \\
\hline $\mathrm{R}_{\mathrm{o}}$ & Outer radius of the membrane disk $(\mathrm{cm})$ & $2.7,2.12,3.6$ \\
\hline $\mathrm{r}$ & Membrane disk radius $(\mathrm{cm})$ & $2.6,2.10,3.4$ \\
\hline$r_{\mathrm{p}}$ & Mean pore radius $(\mathrm{cm})$ & $2.15,2.16$ \\
\hline $\mathrm{r}_{\mathrm{s}}$ & Spearman rank correlation coefficient & 3.23 \\
\hline $\operatorname{Re}$ & Reynolds number & 2.9 \\
\hline $\operatorname{Re}_{\mathrm{r}}$ & Radial Reynolds number & 2.6 \\
\hline $\operatorname{Re}_{\mathrm{r}-\mathrm{avg}}$ & Average radial Reynolds number & 2.7 \\
\hline $\mathrm{SS}_{\text {Error }}$ & Error sum of squares & $3.13,3.14$ \\
\hline $\mathrm{SS}_{\text {Factor }}$ & Sum of squares for the factor & $3.8,3.9,3.11$ \\
\hline $\mathrm{SS}_{\text {Res }}$ & Residual sum of squares & $3.18,3.19$ \\
\hline $\mathrm{T}$ & Temperature $\left({ }^{\circ} \mathrm{C}\right)$ & 2.3 \\
\hline $\mathrm{V}$ & Volume $\left(\mathrm{m}^{3}\right)$ & $2.1,2.2$ \\
\hline
\end{tabular}



5.10, 5.11, 5.12, 5.14, $5.15,5.16,5.17,5.18$, 5.19, 5.20, 5.21, 5.23, 5.24, 5.26, 5.27, 5.28

$\begin{array}{ll}\Delta \mathrm{P}_{\text {set }} & \text { Set point operating pressure }(\mathrm{kPa}) \\ \Delta \mathrm{P}_{\text {set( } \mathrm{R})} & \text { High-shear rotary set point operating pressure }(\mathrm{kPa}) \\ \Delta \mathrm{P}_{\mathrm{set}(\mathrm{T})} & \text { Tubular set point operating pressure }(\mathrm{kPa}) \\ \Delta \mathrm{x} & \text { Membrane thickness }(\mathrm{cm}) \\ \Phi & \text { Resistance index }\left(\mathrm{h} \cdot \mathrm{m}^{-1}\right) \\ \Phi_{\mathrm{R}} & \text { High-shear rotary UF resistance index }\left(\mathrm{h} \cdot \mathrm{m}^{-1}\right) \\ \Phi_{\mathrm{T}} & \text { Tubular UF resistance index }\left(\mathrm{h} \cdot \mathrm{m}^{-1}\right) \\ \Pi & \text { Osmotic Pressure } \\ \alpha & \text { Exponent in the Reynolds number power law relationship } \\ \delta & \text { Thickness of the concentration boundary layer (cm) } \\ \varepsilon & \text { Membrane surface porosity } \\ \eta & \text { Kinematic viscosity }(\mathrm{cSt})\end{array}$

$2.35,5.22$

2.33, 2.34, 2.35, 5.3, 5.5, 5.7, 5.8, 5.12, $5.13,5.22$

5.14, 5.15, 5.16, 5.19, 5.25

$5.9,5.10,5.11,5.25$

$2.21,2.22,2.23,2.24$

$2.15,2.16$

2.3, 2.6, 2.7, 2.25, 5.1, 5.8, 5.9, 5.10, $5.11,5.13,5.14,5.16$, $5.17,5.18,5.20,5.21$, $5.23,5.24,5.26,5.28$

$\mu \quad$ Absolute viscosity (cP)

$2.4,2.5,2.16,2.28$

$\rho \quad$ Density $\left(\mathrm{kg} / \mathrm{m}^{3}\right)$

$2.10,2.11,2.12$

$\omega \quad$ Membrane rotational speed (rpm)

2.6, 2.7, 2.10, 2.11, $2.12,3.4,3.5$ 


\section{CHAPTER 1.0 INTRODUCTION}

The successful treatment of metal working (MW) fluids using ultrafiltration (UF) membrane separation processes has been reported in a number of studies (Reed et al. 1997a and b; Cheryan and Rajagopalan 1998; Viadero and Reed 1999; Viadero et al. 1999 and 2000). Traditionally, conventional cross-flow technologies such as tubular module configurations have been employed for the treatment of oily wastewaters. In cross-flow separation systems, high recirculating velocities $\left(\sim 4.6 \mathrm{~m} \cdot \mathrm{s}^{-1} ; 15 \mathrm{ft} \cdot \mathrm{s}^{-1}\right)$ are used to create a hydraulic cleaning action which minimizes the accumulation of rejected species at the membrane surface. However, as feed streams are concentrated it becomes difficult to maintain high velocities in conventional cross-flow systems due to a resultant increase in waste viscosity. A subsequent decrease in permeate flux is observed.

In the high-shear rotary (HSR) UF system, disk membranes are rotated at speeds up to $1750 \mathrm{rpm}$ to generate hydraulic turbulence, which scours the membrane surface. Unlike conventional cross-flow systems, the hydraulic cleaning action is decoupled from the feed recirculation/pressurization; thus, an acceptable permeate flux can be maintained as wastes are concentrated. In this mechanically enhanced system, the pump is required only to provide transmembrane pressure and a small amount of recirculation. In treatment applications where high feed concentrations are desired, hybrid membranemembrane processes have been proposed, where a high-shear system is used to treat conventional cross-flow residual. Presently, little is known about the technical operating scheme of hybrid membrane-membrane separation processes; thus, it is necessary to develop a general approach to compare the performance of different UF module 
configurations as well as develop an optimum set of operational parameters for transition between modules in a hybrid treatment application.

The objective of this research was to perform a parametric study examining the effects of operating parameters on permeate flux in the tubular and high-shear rotary ultrafiltration of oily feed streams. A synthetic MW fluid was selected as a surrogate feed stream due to its relevance in industrial process and treatment applications as well as its interest to the United States Department of Energy and the metal-working industry. Additionally, oily waste streams are often defined as "difficult to treat" due to the characteristically deformable nature of an oil droplet and the wide particle size range associated with such solutions.

The conventional thin-film model was employed to describe pressure-independent permeate flux behavior in UF processes; however, a two-region flux has been reported in which the rate of change in the pressure-independent "limiting" permeate flux, $J^{*}$, decreases at high feed solute concentrations (Pritchard et al. 1995; Viadero et al. 2000). Thus, the ability of the thin-film model to predict permeate flux behavior at high feed concentrations is in question.

An alternative to the thin-film model is the resistance-in-series (RIS) approach, which has been shown to be successful in predicting both pressure-dependent and pressure-independent permeate flux behavior in UF applications (Chiang and Cheryan 1986; Viadero et al. 1999). Viadero et al. (1999) presented a modified form of the RIS model for the HSRUF of a synthetic MW fluid in terms of membrane rotational speed and feed MW fluid concentration. The RIS model can be further modified to include operating parameters common to all UF modules; thus, the modified RIS approach was 
applied to data collected from the tubular and HSRUF experiments conducted in this study. Further, the RIS model was modified by postulating a specific form of the resistance index in terms of average relative velocity and feed kinematic viscosity.

Through the use of general operating parameters common to both the tubular and HSRUF systems, the relationships determined in the modified RIS approach were used as a comparison of the two separation systems. Additionally, the modified RIS relationships were employed for the development of a technical operating scheme in a hybrid tubularHSRUF separation system. The specific goals of this research were to:

1) Determine the ability of the thin-film model to predict permeate flux behavior in the tubular and HSRUF systems.

2) Investigate the formation of a two-phase limiting flux when applying the thinfilm model to high feed oil concentrations in the HSRUF of a synthetic and waste MW fluid.

3) Apply the resistance-in-series model to data collected from the tubular and HSRUF treatment of a synthetic MW fluid in order to:

- Develop a more general form of the RIS model in terms of average relative velocity and feed kinematic viscosity,

- Examine application of the modified RIS model to high feed oil concentrations in the tubular and HSRUF of the synthetic and waste MW fluid.

4) Investigate application of the modified RIS model to the HSRUF of a waste MW fluid.

5) Study the modified RIS models for use in developing a technical operating scheme for a tubular-HSRUF separation system by developing a procedure to determine a matrix of operational set points for transition between the two modules when operated in a hybrid configuration.

Both research and application oriented field engineers can benefit from the conclusions drawn from these experiments. It is envisioned that the model developed in this study can be used as a means to compare the performance of a wide range of membrane separation systems. Further, with the emergence of hybrid separation systems 
in treatment applications where high feed concentrations are desired, the approach developed in this study can be used as a foundation to facilitate the future investigation of hybrid membrane-membrane separations systems. 


\section{CHAPTER 2.0 \\ BACKGROUND}

\subsection{MEMBRANE SEPARATION PROCESSES}

\subsubsection{General Process Description}

Membrane filtration is a fluid/fluid and particle/fluid separation technology used to remove dissolved substances and fine particles from water and process streams. In membrane separation processes, a thin semi-permeable membrane acts as a selective barrier to separate particles based on size. In general, water and constituents smaller than the membrane pore size are able to pass through the membrane as "permeate" while particles and molecules larger than the membrane pore size are retained as "concentrate." "Permeate flux" is the term used to describe how fast permeate passes through a membrane. Flux is generally reported as volume/area-time. Permeate flux generally depends upon the individual membrane characteristics (i.e., membrane pore size, materials and surface properties), the characteristics of the feed stream (i.e., viscosity and solute particle size), and operating parameters (i.e., transmembrane pressure, feed temperature and velocity).

Most full-scale membrane separation systems used in industrial applications are operated in a cross-flow feed configuration as opposed to dead-end filtration. A schematic of a dead-end filtration process is presented in Figure 2.1. In dead-end filtration the feed stream is introduced perpendicular to the membrane surface, which is favorable to the accumulation of rejected species. A subsequent reduction of permeate flux is observed. A schematic of a cross-flow filtration process is presented in Figure 2.2. In cross-flow filtration, feed flow is parallel to the membrane surface, which provides 


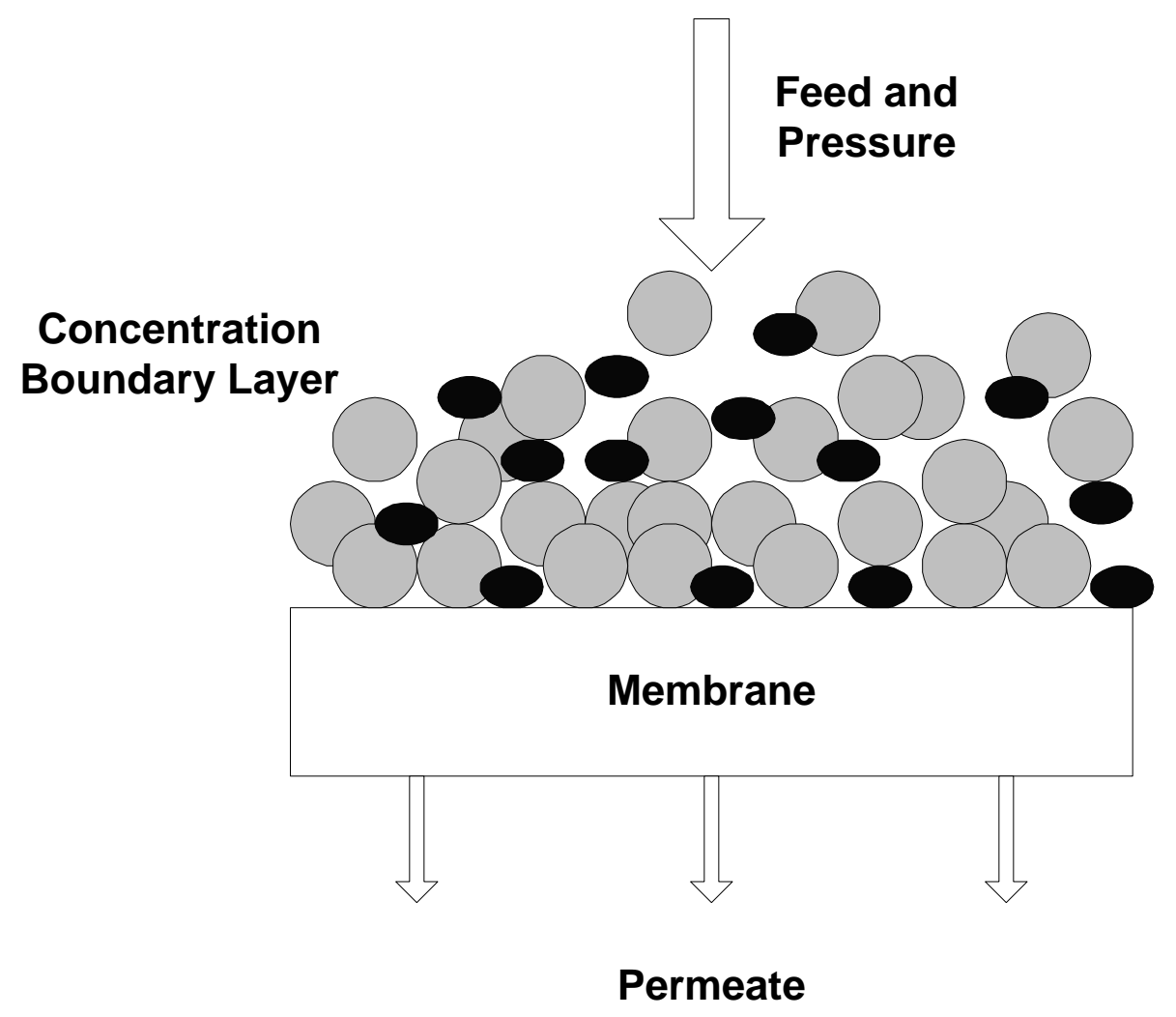

Figure 2.1 A schematic of a dead-end filtration process.

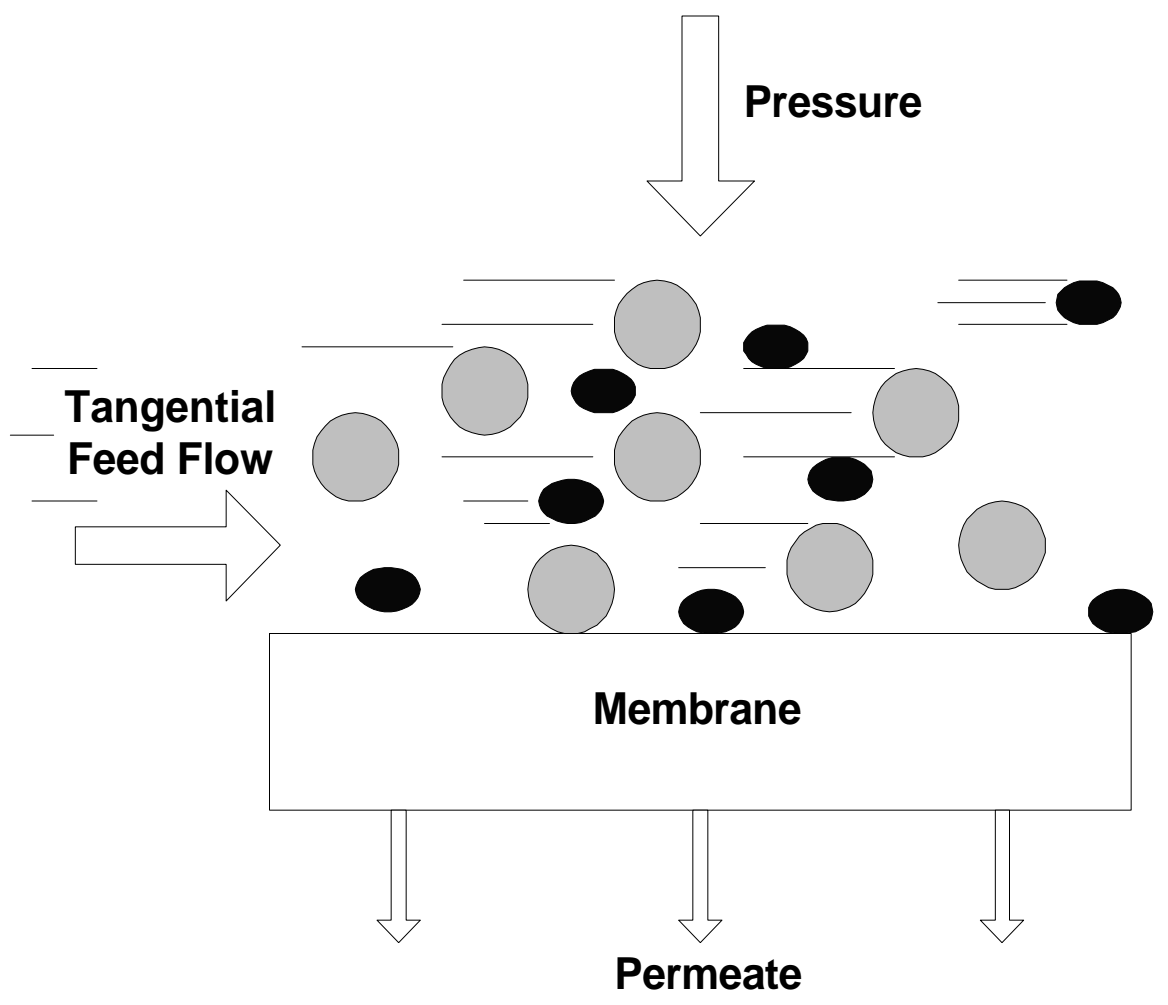

Figure 2.2 A schematic of a cross-flow filtration process. 
hydraulic turbulence to minimize the accumulation of rejected species and the subsequent loss of permeate flux.

Membrane separation processes are gaining acceptance as viable alternatives to other treatment technologies such as dissolved air flotation and biological treatment. For example, membrane separation systems can be used to recover valuable materials from waste streams and to reduce the volume of waste sent for further treatment or disposal. Additional industrial applications of membrane separation systems include the treatment of metal-working, pulp and paper, and commercial laundry wastewaters; the treatment of landfill leachates; the production of high quality water; as well as use in various production applications (Cheryan 1998).

\subsubsection{Operational Modes}

Membrane separation systems are typically operated in one of three main filtration modes: (1) recycle mode, (2) semi-batch ("modified batch") mode, or (3) batch mode. Since membrane separation technologies are volume reduction processes, the feed concentration factor $(\mathrm{CF})$ is an important system efficiency parameter. The concentration factor is defined as the ratio of feed concentration at a given time to the initial concentration. Concentration factors are typically expressed as $1 \mathrm{X}, 2 \mathrm{X}$, etc., and increase with treatment time. Concentration factors in excess of $100 \mathrm{X}$ are possible with membrane separation technologies.

\subsubsection{Recycle Mode}

In recycle mode, feed is pumped from the feed tank into the membrane module. Permeate is forced through the membrane under pressure and is returned to the feed tank; thus, the concentration of the solution in the feed tank remains constant over time. Since 
the feed solution is not concentrated over time, recycle mode is not used in industrial waste and process applications. However, membrane separation systems are frequently operated in recycle mode in laboratory studies when it is advantageous to maintain a constant feed concentration.

\subsubsection{Semi-Batch Mode}

In field applications, membrane separation systems are typically operated in either semi-batch or batch mode. A schematic of semi-batch and batch operational modes is presented in Figure 2.3. In semi-batch mode, fresh feed solution (at $1 \mathrm{X}$ ) is added to the feed tank at the same rate permeate is produced and the feed solution is concentrated over time. The concentration of the feed solution in semi-batch operation is given in Equation 2.1.

$$
\mathrm{CF}_{\mathrm{sb}}=1+\frac{\mathrm{V}_{\text {perm }}}{\mathrm{V}_{\text {feed }}}
$$

where $\mathrm{CF}_{\mathrm{sb}}=$ concentration factor during semi-batch operation, $\mathrm{V}_{\text {perm }}=$ volume of permeate produced, and $\mathrm{V}_{\text {feed }}=$ volume of solution in feed tank (constant during semibatch operation). Semi-batch operation is the most frequently used in large scale continuous operation applications.

\subsubsection{Batch Mode}

A schematic of batch operation mode was presented previously in Figure 2.3. In batch mode, which typically occurs at the end of semi-batch operation, fresh 1X solution is not added to the feed tank while permeate is removed. Thus, the feed volume is reduced and the residual feed solution remaining at the end of semi-batch operation is

concentrated further. The concentration factor during batch operation is given in Equation 2.2. 
$\llbracket$ Feed

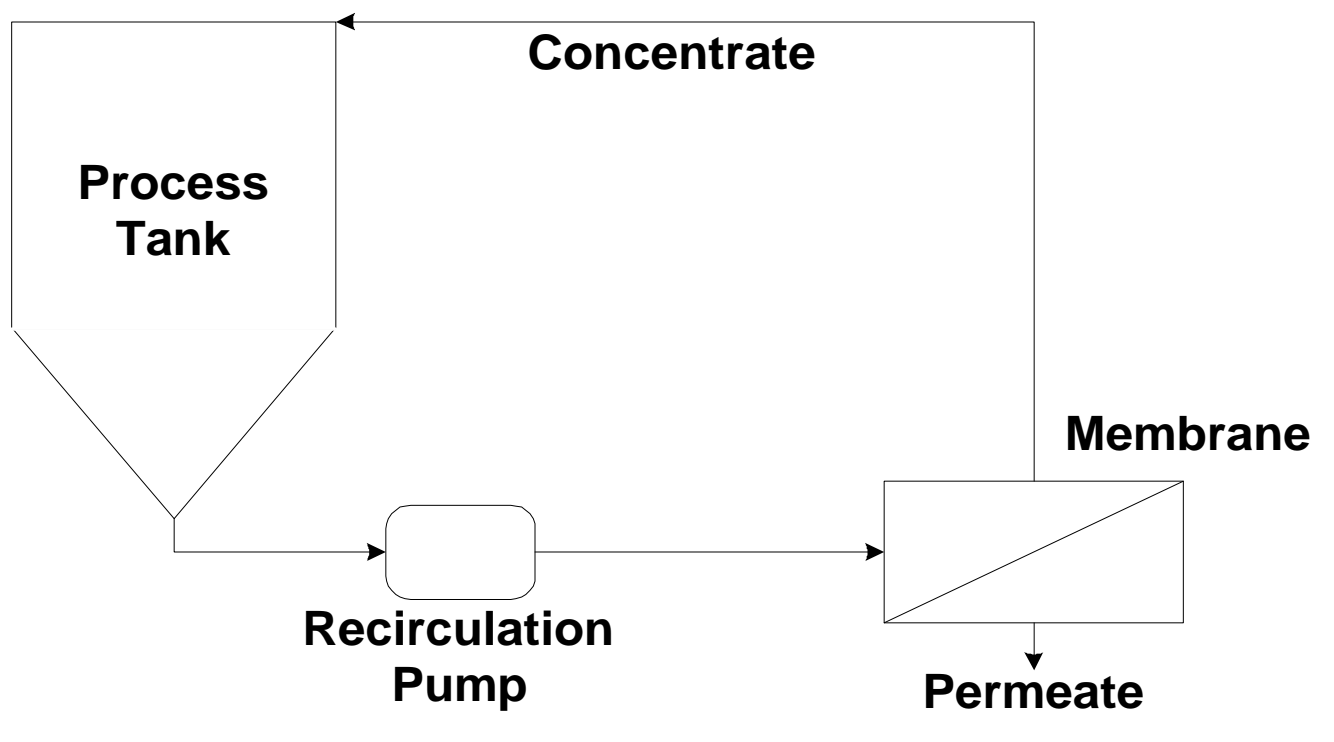

Semi-Batch

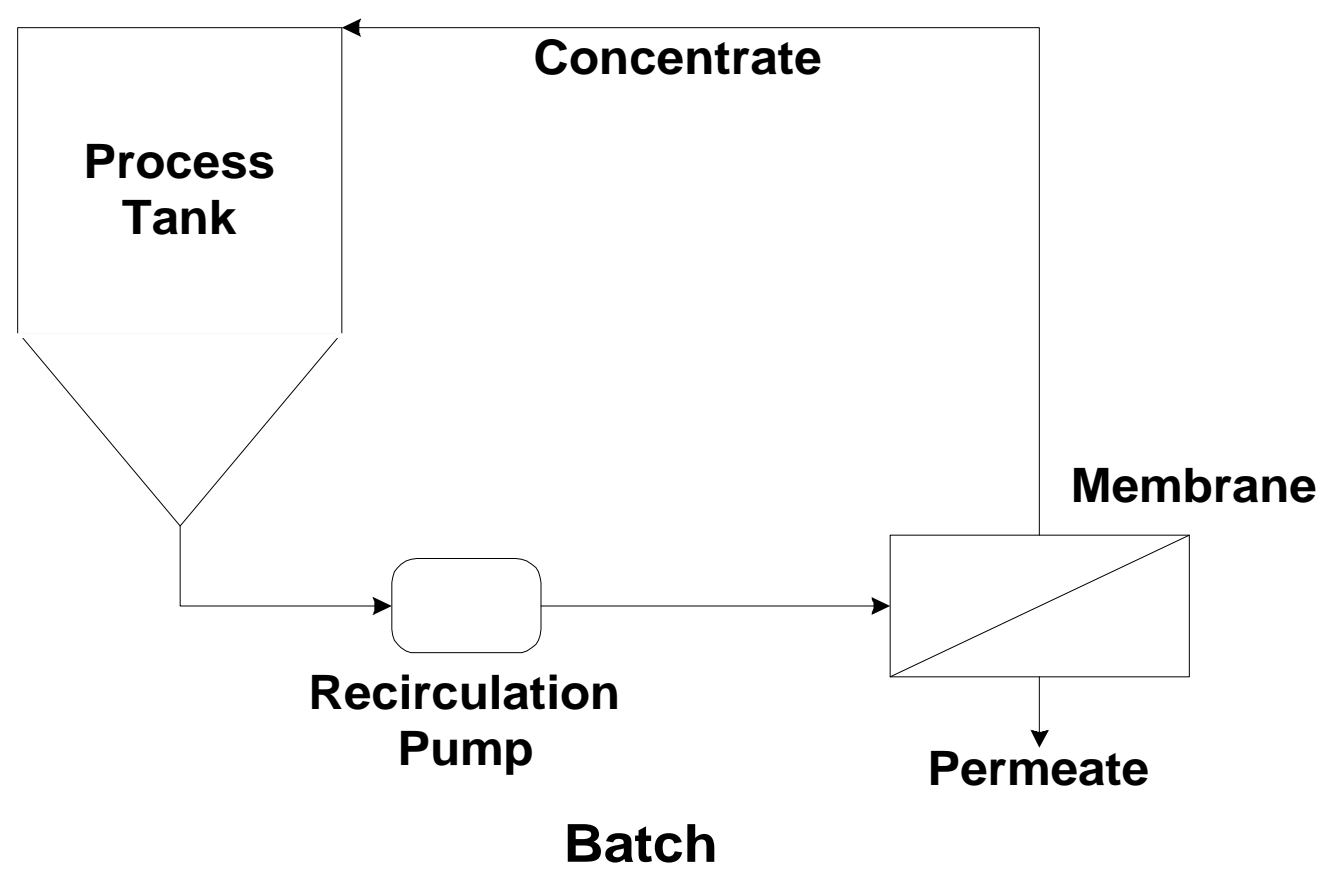

Figure 2.3 A schematic of semi-batch and batch operational modes. 


$$
\mathrm{CF}_{\mathrm{b}}=\mathrm{CF}_{\mathrm{i}} \times\left[\frac{\mathrm{V}_{\text {feed }}}{\left(\mathrm{V}_{\text {feed }}-\mathrm{V}_{\text {perm }}\right)}\right]
$$

where $\mathrm{CF}_{\mathrm{b}}=$ the concentration factor during batch operation and $\mathrm{CF}_{\mathrm{i}}=$ the concentration factor at the initiation of batch mode.

\subsubsection{General Types of Membrane Separation Processes}

Membrane processes are differentiated by the size of particles they separate. The four principle membrane separation techniques used to treat industrial waste and process waters are: reverse osmosis (RO), nanofiltration (NF), ultrafiltration (UF), and microfiltration (MF). The useful ranges of the four general membrane separation processes are presented in Figure 2.4 (after Cheryan (1986) and Perry and Green (1997)).

\subsubsection{Reverse Osmosis and Nanofiltration}

Membranes are characterized by the diameter of a particle or molecular weight of a molecule that is retained (known as the molecular weight cutoff (MWCO)) by the membrane. Additional details regarding membrane characteristics are presented later in Chapter 2.0. Reverse osmosis (RO) is a membrane separation technique used to remove materials with diameters from $\sim 10^{-4}$ to $10^{-3} \mu \mathrm{m}$ (less than $\sim 200$ to 2,000 MWCO), which generally include dissolved salts and ions. The phenomena of osmosis and reverse osmosis are presented schematically in Figure 2.5.

Osmosis is the flow of solvent (usually water) through a semi-permeable membrane from a region of low chemical potential to a region of higher chemical potential. For example, when a salt solution and pure water are separated by a semipermeable membrane, a net flow from the pure water to the saline side of the membrane will occur. This flow is due to differences in the chemical potential (and thus, the 


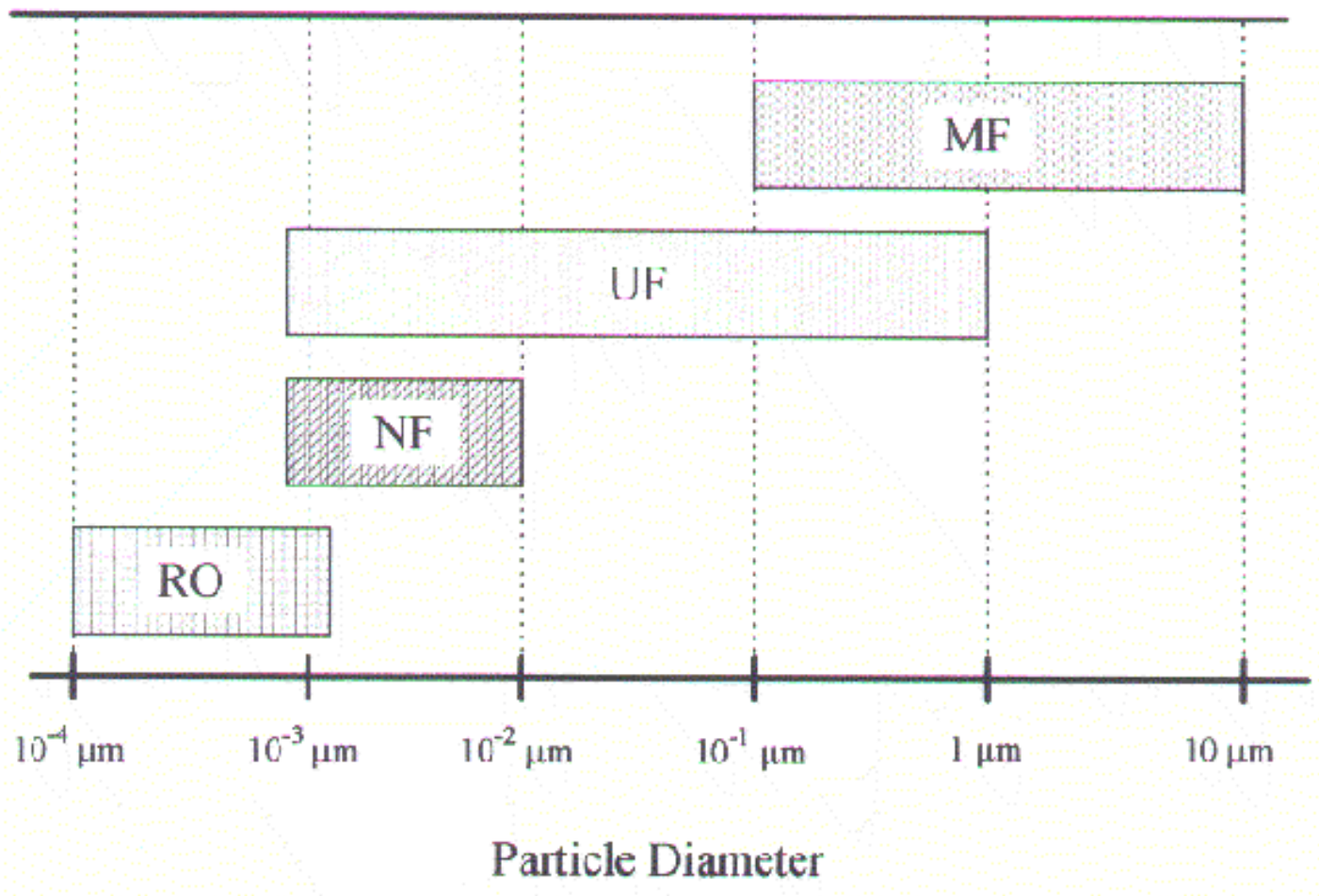

Figure 2.4 The useful ranges of the four general membrane separation processes (Cheryan 1998; Perry and Green 1997).
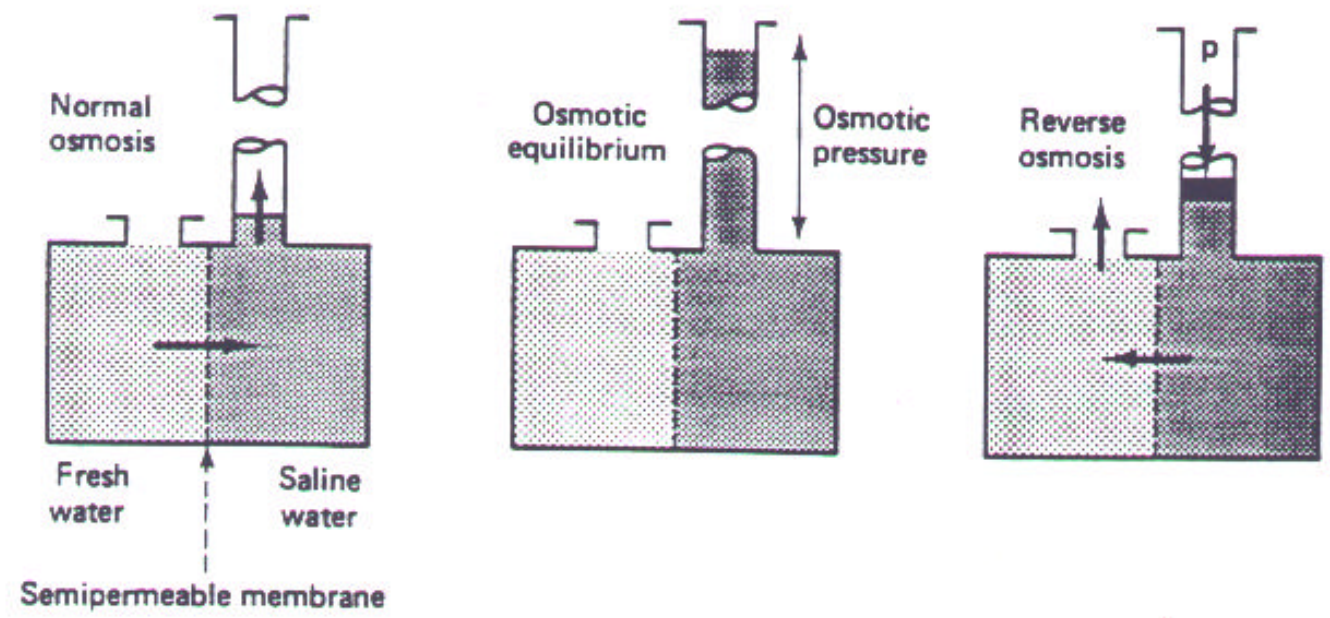

Figure 2.5 The phenomena of osmosis and reverse osmosis (Cheryan 1998). 
osmotic pressure) of the two solutions. The salt solution will rise to a point at which the head is equal to the osmotic pressure head, as presented in Figure 2.5. In RO, the pressure on the salt solution is raised above the osmotic pressure so the net movement of water is from the more concentrated saline-side to the pure water-side of the semipermeable membrane. Transmembrane pressures ranging from 3450 to $13,800 \mathrm{kPa}$ (500 to $2,000 \mathrm{psi}$ ) are common in RO applications.

Reverse osmosis has been used in the production of potable water, most prominently in the Middle East and on islands where the demand for potable water exceeds the natural supply. Small RO units have also been incorporated into multistage flash distillation systems to provide potable water sources on ocean vessels. Additionally, RO is used to create ultra high quality water in for a number of manufacturing processes.

Nanofiltration (NF) systems operate under the same basic principle as reverse osmosis. However, nanofiltration is generally used to remove particles in the $\sim 10^{-3}$ to 10 $-2 \mu \mathrm{m}$ range $(\sim 2,000$ to $20,000 \mathrm{MWCO})$. Nanofiltration is often referred to as "loose RO" because nanofiltration operates on the same basic principle as RO but is applied to larger particle sizes. Smaller constituents of a waste or process stream (e.g., metal ions and dissolved salts) which would typically be removed using RO may pass through a nanofiltration membrane. Nanofiltration is often used to dewater pesticide and herbicide solutions (Perry and Green, 1997).

\subsubsection{Ultrafiltration and Microfiltration}

Ultrafiltration (UF) is a pressure-driven membrane separation technique based on a "sieving mechanism" in which particles are removed from solution based on size. UF 
is used to separate material in the $\sim 10^{-3}$ to $1 \mu \mathrm{m}$ size range $(\sim 2,000$ to $500,000 \mathrm{MWCO})$. Microfiltration (MF) is a similar membrane separation technique which is used to separate particles in the $\sim 10^{-1}$ to $10 \mu \mathrm{m}$ size range $(\sim 100,000$ to $>500,000 \mathrm{MWCO})$. Similarly to the case of reverse osmosis and nanofiltration, there is an overlap in the size range over which UF and MF are generally applied (Perry and Green, 1997). Thus, ultra and microfiltration are discussed as primarily one technique with the main distinction being the larger membrane pore size used in MF. Transmembrane pressures ranging from 35 to $1035 \mathrm{kPa}$ (5 to $150 \mathrm{psi}$ ) are common in UF and MF applications.

Ultrafiltration has been successfully applied to the treatment of concentrated oily wastes from metal-working processes such as sheet rolling and die cutting. UF has been used to treat wastewaters high in oil, grease, and solids while reducing the volume of waste sent for disposal, while at the same time recovering alkaline process cleaners for re-use (Cheryan and Rajagopalan 1998; Viadero et al. 1999; Masciola et al. 2000). Additionally, Cheryan (1998) summarized the results from several studies in which UF was demonstrated to be an effective treatment method for a wide variety of waste and process streams including: (1) the dairy industry for skim, whole milk, and cottage cheese whey processing; (2) biotechnology/bioengineering for protein fractionation and recovery of valuable microbes; (3) food processing for protein and juice extraction; and (4) for the recovery of ink in the printing industry.

Similarly to UF, microfiltration has also been applied to the treatment of oily wastewaters. MF is used in water treatment to remove cryptosporidium and giardia cysts. Additionally, MF is frequently used in the dairy industry (1) as a non-thermal method to remove bacteria from milk and protein products, (2) to separate fat from milk or dairy 
streams, and (3) to recover caustic agents used to clean dairy evaporators (Perry and Green, 1997)

\subsubsection{Factors Controlling Ultrafiltration Permeate Flux}

\subsubsection{Concentration Polarization}

The theoretical relationship between permeate flux, transmembrane pressure, feed concentration, and hydraulic turbulence is presented in Figure 2.6. Under conditions of low transmembrane pressure, low feed concentration, and high hydraulic turbulence, permeate flux is linearly proportional to transmembrane pressure. The linear permeate flux/transmembrane pressure relationship is idealized in the case of a clean water feed. When a clean water feed is ultrafiltered there are theoretically no contaminants to either (1) plug the membrane pores or (2) accumulate on the membrane surface.

The buildup of solute at the membrane surface is known as "concentration polarization" and is largely responsible for the deviation of the product flux from the linear flux-pressure relationship. Primarily, three competing solute transport mechanisms occur in ultrafiltration: (1) convective transport of solute to the membrane surface (2) shear induced back diffusion of accumulated solute to the bulk solution, and (3) solute permeation through the membrane, as presented schematically in Figure 2.7. Solute is convectively transported to the porous membrane surface and a fraction of the solvent is removed as permeate which results in a locally higher solute concentration at the membrane surface relative to the bulk solute concentration. As the transmembrane pressure is increased, feed concentration is increased, or hydraulic turbulence is decreased, the permeate flux becomes independent of pressure. 


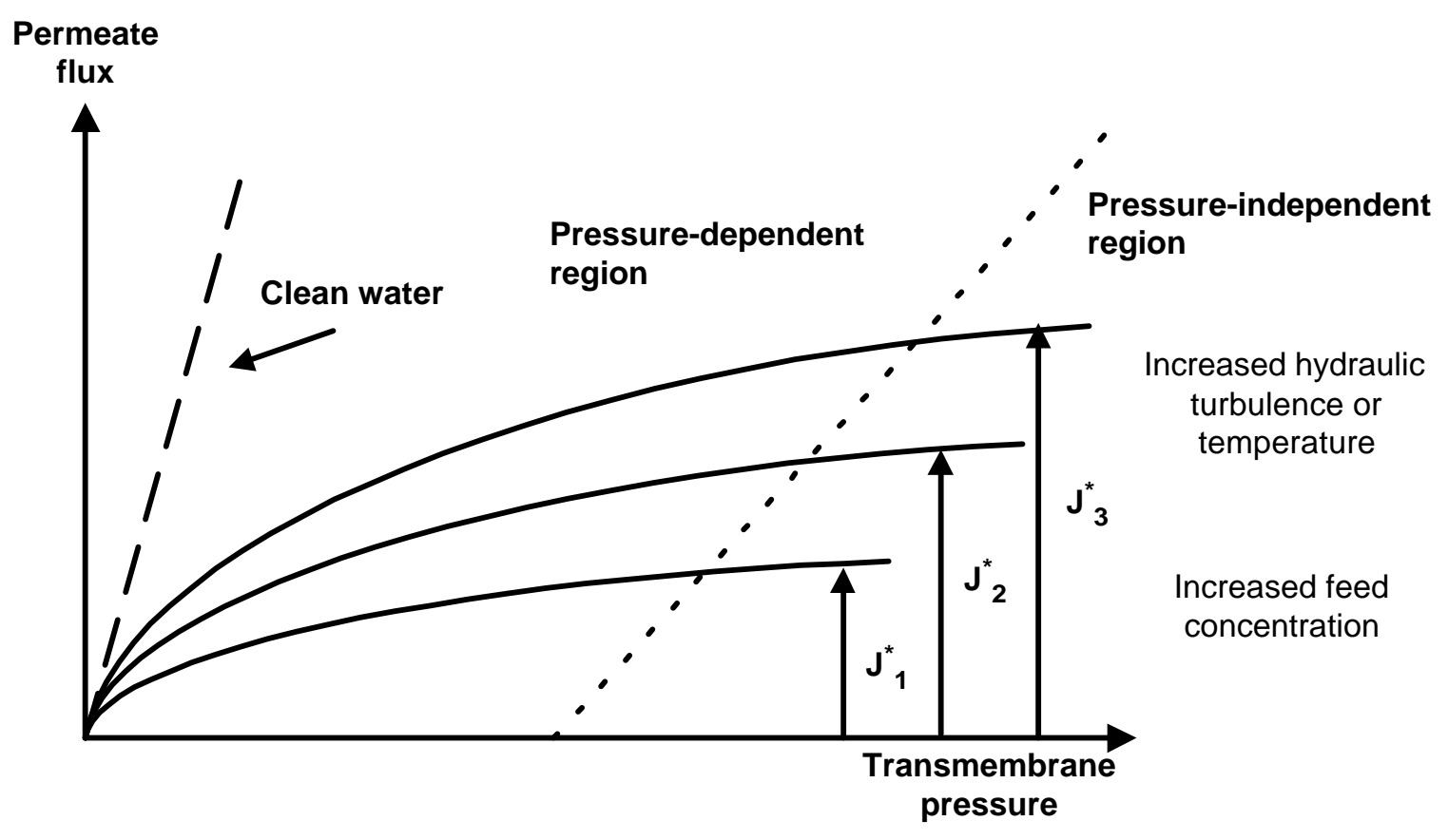

Figure 2.6 The theoretical relationship between permeate flux, transmembrane pressure, feed concentration and hydraulic turbulence (Cheryan 1998).

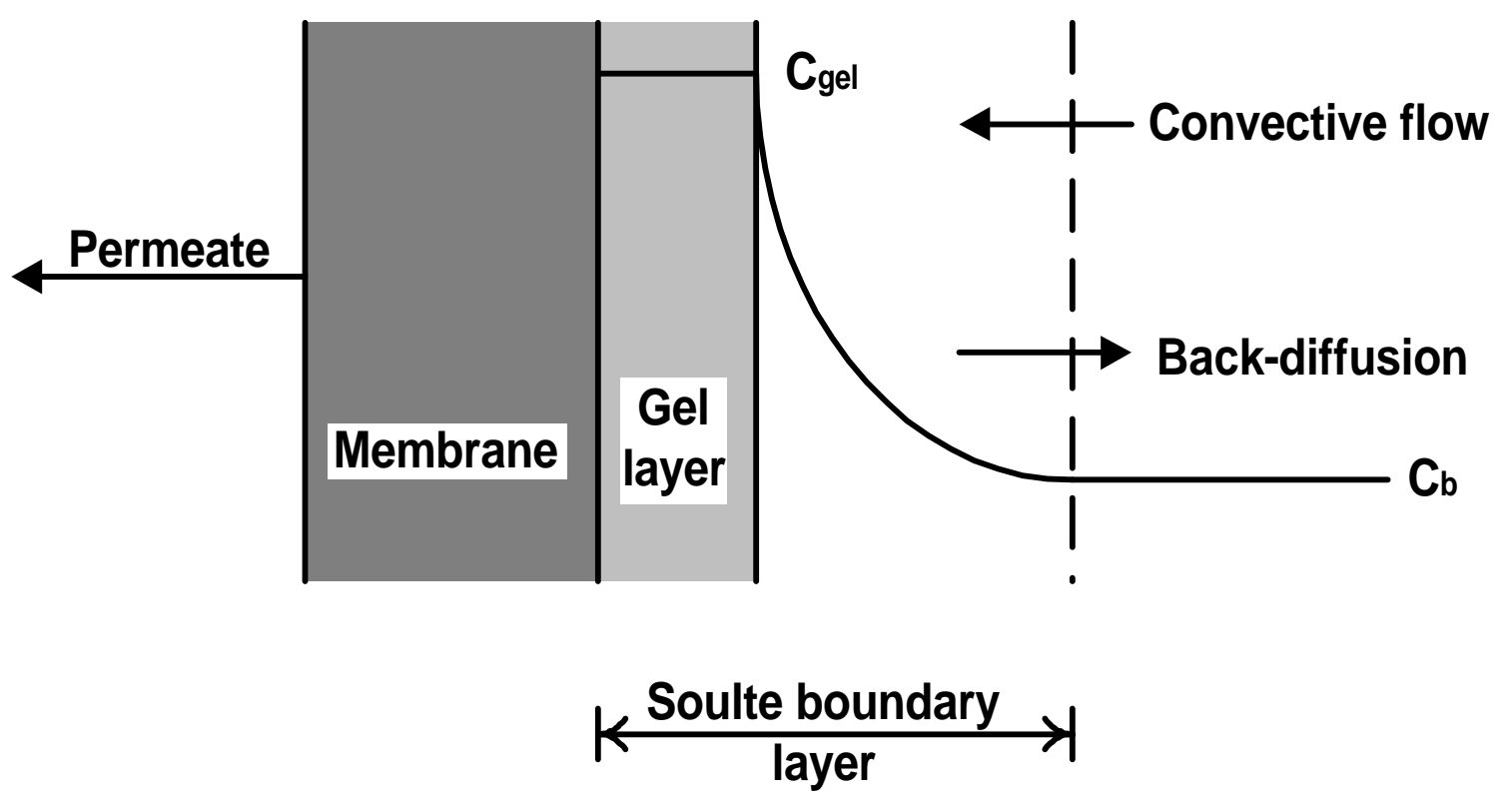

Figure 2.7 A schematic of the transport phenomena at the membrane surface (Cheryan 1998). 
In general, there are four operational and feed solution parameters which contribute to the formation of the polarized solute concentration boundary layer and subsequent asymptotic permeate flux response to increased transmembrane pressure: (1) transmembrane pressure, (2) feed concentration, (3) hydraulic turbulence, and (4) feed viscosity/temperature. Transmembrane pressure is the principle driving force in membrane separation systems. However, as transmembrane pressure is increased, there is a corresponding increase in convective transport of solute molecules to the membrane surface. A thicker solute boundary layer forms as a result of the enhanced convective transport of solute molecules to the membrane surface and the permeate flux becomes independent of transmembrane pressure.

Solute concentration in the feed is another parameter which limits permeate flux. As the solute concentration in the feed increases, permeate flux decreases due to the accumulation of solute molecules at the membrane surface. At high feed solute concentrations, it becomes difficult to pump a feed solution at high enough velocities to minimize the thickness of the polarized concentration boundary layer. Thus, the permeate flux decreases as the solute concentration is increased. The hydraulic turbulence in membrane separation systems also plays an important role in maintaining a satisfactory permeate flux. As hydraulic turbulence is increased, the permeate flux should theoretically also increase due to a decrease in the thickness of the polarized concentration boundary layer. Similarly, as the temperature of the feed solution is increased, permeate flux increases due to a decrease in the viscosity of the feed solution and the permeate as well as an increase in solute diffusivity which results in an increased diffusion of solute molecules from the membrane surface back to the bulk solution 
(Cheryan, 1998). Assuming temperature affects only the viscosity of the permeate, the resultant increase in permeate flux can be estimated by Equation 2.3 (Blanck and Brady).

$$
\operatorname{Flux}_{\mathrm{T} 1}=\left(\frac{\dot{\mathrm{A}}_{\mathrm{T} 2}}{\hat{\mathrm{A}}_{\mathrm{T} 1}}\right) \text { Flux }_{\mathrm{T} 2}
$$

where Flux $\mathrm{x}_{\mathrm{T} 1}=$ the permeate flux at temperature $\mathrm{T}_{1}$, corrected for the change in viscosity, Flux $_{\mathrm{T} 2}=$ the permeate at temperature $\mathrm{T}_{2}, \eta_{\mathrm{T} 1}$ and $\eta_{\mathrm{T} 2}$ are the kinematic viscosities of water at temperatures $T_{1}$ and $T_{2}$, respectively.

Cheryan (1977) noted three operational characteristics which can be used to judge the extent of concentration polarization in a UF system: (1) deviation from the pure water line, (2) relatively rapid attainment of pressure-independent flux behavior, and (3) marked hysteresis effect observed on lowering the pressure. Although the deviation from the pure water flux may be attributed to a decrease in the driving force due to increased osmotic pressure, Cheryan believed the primary mechanism of flux decline was the increased hydrodynamic resistance in the concentration boundary layer. Cheryan supported the argument in favor of flux decline due to the formation of a polarized gel layer based on prior knowledge of the gel characteristics of soybean proteins and the gellike behavior of highly structured macromolecules (at concentrations in excess of $\sim 10 \mathrm{wt}$. $\%$ ) which were in good agreement with experimental values. Additionally, the hysteresis effects observed as pressure was varied in these experiments supported the hypothesis that interactions had occurred between solute molecules which were approaching a consolidated "close packed" configuration. Citing the marked differences in the mass transfer properties of feed solutions, Cheryan suggested that mass transfer models will 
have to be supplemented with experimental data to be used in UF process design (Cheryan, 1977; Cheryan, 1998).

\subsubsection{Membrane Fouling}

Membrane fouling can also result in a decrease in permeate flux over time. Fouling is a term used to describe the loss of permeate throughput of a membrane device as it becomes chemically or physically changed by a process fluid. Fouling is different from concentration polarization. Both fouling and concentration polarization result in a reduced membrane system output and the resulting resistances due to both phenomena are additive. For example, when the flow rate in a cross-flow membrane separation system is reduced, permeate flux will decrease. If the decrease in permeate flux is due only to the effects of concentration polarization (i.e., the accumulation of solute molecules at the membrane surface), the permeate flux should return to the initial value when the flow rate is raised. However, if the permeate flux does not increase to the initial value when the flow rate is increased, membrane fouling is the operative flux decline phenomena.

Membrane fouling is primarily due to (1) pore plugging and/or (2) solute adsorption onto the membrane surface. In the case of pore plugging, solute molecules or other minor constituents of a feed stream (e.g., bacteria) are forced into the membrane pores resulting in a loss of permeate flow channels and thus a decrease in permeate flux. Pore plugging becomes more prevalent under operating conditions such as low hydraulic turbulence and high transmembrane pressure. At low hydraulic turbulence, a thicker solute layer accumulates on the membrane surface. When combined with high transmembrane pressure, the potential for forcing solute molecules into the membrane 
pores increases. Additionally, if solute molecules are small enough to enter the membrane pores, some solute may be adsorbed onto the pore walls. The adsorbed molecules decrease the effective pore diameter. As the effective pore diameter is decreased, the overall resistance to hydraulic flow increases and a decrease in permeate flux is observed. Solute adsorption is due principally to physical and/or chemical properties of the feed solution (Jonsson and Tragardh, 1990).

The proper selection of operating conditions, feed pretreatment, and cleaning methods are important aspects to minimizing flux loss due to membrane fouling. For example, the transmembrane pressure should be balanced to provide maximum permeate flux at the minimum transmembrane pressure. Thus, an understanding of the fluxpressure relationship presented previously in Figure 2.6 is important to the minimization of membrane fouling and concentration polarization. Specifically, a membrane separation system should not be run at transmembrane pressures in excess of the value necessary to achieve a pressure-independent permeate flux (Cheryan 1998).

Feed pretreatment is often stream or site-specific and has received little attention in the literature. However, steps such as removing free oil from a waste or process stream prior to the application of membrane separation are often used to minimize potential membrane fouling. The primary method of restoring permeate flux lost to membrane fouling is membrane cleaning. Fouling which can be removed through membrane cleaning is called "reversible fouling" and fouling which cannot be removed through cleaning is called "irreversible fouling." Cleaning procedures are highly streamspecific. Although cleaning is used to restore permeate flux, the use of aggressive chemicals such as concentrated acidic and caustic solutions often results in shortened 
membrane life due to negative affects on membrane surface properties (Perry and Green, 1997).

\subsubsection{Membrane Resistance}

Membrane resistance is a measure of the hydraulic resistance to flow through a pore channel and is an intrinsic material property of the membrane. The membrane resistance can be determined by applying Darcy's Law for a virgin membrane with a clean water feed:

$$
J=\frac{P}{\mu R_{m}}
$$

where, $\mathrm{J}=$ permeate flux, $\mathrm{P}=$ transmembrane pressure, $\mu=$ water viscosity (absolute), and $\mathrm{R}_{\mathrm{m}}=$ membrane resistance. The assumption of well-developed laminar flow through the membrane pore channels is made when using Equation 2.4. The relationship between permeate flux and transmembrane pressure for a clean water feed was presented previously in Figure 2.6. When clean water is used as the feed, the slope of a regression line through the origin on the plot of permeate flux versus transmembrane pressure is inversely proportional to the product of the feed viscosity and the resistance of the membrane, $R_{m}$ (Cheryan 1998). In this case, the membrane resistance is:

$$
\mathrm{R}_{\mathrm{m}}=\frac{1}{\mu * \text { slope }}
$$

A summary of typical resistances for several ceramic (aluminum oxide) micro and ultrafiltration membranes studied by Nazzal and Wiesner (1996) is presented in Table 2.1. Additionally, a resistance of $\sim 10^{12} \mathrm{~m}^{-1}$ was measured by Lipp et al. (1988) for a polymeric membrane used to treat oil-in-water emulsions in a stirred cell UF unit. The 
MF and UF membrane resistances reported by Nazzal and Wiesner (1996) and Lipp et al. (1988) were comparable to values measured by other researchers (Gutman, 1987; Bhave, 1991).

Table 2.1. Typical Membrane Resistances for Micro and Ultrafiltration Membranes (Nazzal and Wiesner 1996).

\begin{tabular}{|c|c||}
\hline \hline Pore diameter $(\mu \mathbf{m})$ & Membrane resistance $\left(\mathbf{m}^{-1}\right)$ \\
\hline \hline 0.8 & $1.5 \times 10^{11}$ \\
\hline 0.5 & $2.0 \times 10^{11}$ \\
\hline 0.2 & $5.0 \times 10^{11}$ \\
\hline 0.05 & $6.0 \times 10^{11}$ \\
\hline
\end{tabular}

\subsection{CONVENTIONAL CROSS-FLOW ULTRAFILTRATION SYSTEMS}

\subsubsection{Configurations of Conventional Membrane Modules}

\subsubsection{Tubular Membrane Modules}

A schematic of a tubular membrane module is presented in Figure 2.8 (MacNeil and McCoy, 1989). A tubular membrane consists of a porous membrane material cast on a permeable support matrix housed inside a rigid hollow tube. Feed is recirculated through the tube at a velocity high enough to produce turbulence at the membrane surface. Permeate is forced radially through the membrane under pressure and is collected through a port in the rigid outer casing (Belfort, 1988). Tubular membranes are available in single or multi-tube configurations. Typical commercial tubular membranes can be operated at pressures up to $620 \mathrm{kPa}(90 \mathrm{psig})$. These modules are generally 3.048 $\mathrm{m}(10 \mathrm{ft})$ long and have inner tube diameters ranging from 1.27 to $2.54 \mathrm{~cm}$ (0.5 to 1 in). Typical flow rates necessary to achieve a sufficient shear in a $2.54 \mathrm{~cm}(1 \mathrm{in})$ tubular membrane range from $\sim 114$ to $151 \mathrm{Lpm}$ ( 30 to $40 \mathrm{gpm})$. Additionally, Reynolds numbers up to 100,000 are commonly observed in tubular UF modules. 


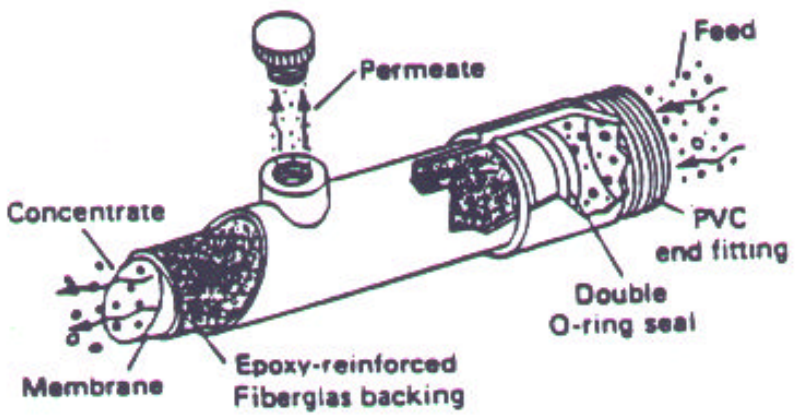

Figure 2.8 A schematic of a tubular membrane module (MacNeil and McCoy 1989). Due to large channel sizes (typically 1.27 to $2.54 \mathrm{~cm} ; 0.5$ to $1 \mathrm{inch}$ ), tubular membranes are commonly used to treat waste and process streams with high solids concentrations. Additionally, the large flow channels enable mechanical and chemical cleaning techniques to be more effective at removing accumulated solute molecules from the membrane surface (MacNeil and McCoy, 1989). The disadvantages of tubular membrane modules include a relatively low packing density (membrane area per unit volume) and a large footprint (volume hold-up per unit area of membrane) when compared with hollow fiber and spiral wound membrane modules (Belfort, 1988).

\subsubsection{Hollow Fiber Membrane Modules}

A schematic of a hollow fiber membrane module is presented in Figure 2.9 (MacNeil and McCoy, 1989). Hollow fiber membrane modules consist of a bundle of 


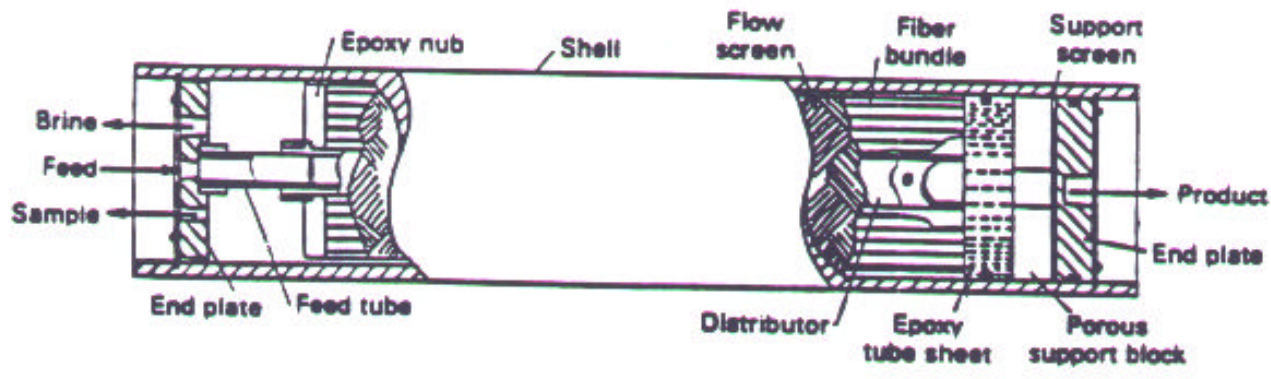

Figure 2.9 A schematic of a hollow fiber membrane module (MacNeil and McCoy 1989).

fine tubes (typical inside diameters range from $\sim 20$ to $106 \mathrm{~mm}$ ) made of the membrane material bound at each end of the module by epoxy discs. Unlike tubular membranes, hollow fiber membranes do not have an underlying support structure. Feed is forced into the membrane module radially under pressure through a perforated tube in the center of the housing. Permeate flows through the individual fibers and is collected through epoxy tube sheets at the end of the module (MacNeil and McCoy, 1989). Transmembrane pressures of up to $\sim 276 \mathrm{kPa}(\sim 40 \mathrm{psig}$ ) are common in hollow fiber modules (Belfort, 1988).

Hollow fiber membrane modules have a higher packing density than tubular modules but are more subject to fouling effects. Further, hollow fiber membranes cannot be mechanically cleaned (MacNeil and McCoy, 1989). However, the direction of 
permeate flow can be changed to effectively back-flush the membrane, removing fouling materials from the surface (Belfort, 1988).

\subsubsection{Spiral Wound Membrane Modules}

A schematic of a spiral wound membrane module is presented in Figure 2.10. A spiral wound membrane module consists of alternating layers of porous membranes, permeate carriers, and spacers, wrapped around a central hollow permeate collection tube. The feed solution flows axially through the wound membrane module. Permeate is forced through the porous membrane and is collected through the hollow center tube while rejected feed solution exits at the end of the module. Spiral wound membrane modules range from $\sim 5$ to $20 \mathrm{~cm}$ in diameter and can be operated at pressures up to $\sim 1035 \mathrm{kPa}(\sim 150 \mathrm{psig})$.

Typically, spiral wound membranes foul more easily than tubular membranes due to smaller waste flow channels (common spacer thickness ranges from 28 to $80 \mathrm{~mm}$ ). This factor coupled with the fact that spiral wound modules cannot be mechanically cleaned limits the application of these membrane configurations to waste and process streams with low solids concentrations (MacNeil and McCoy, 1989). However, spiral wound membranes have a significantly higher packing density than tubular and hollow fiber configurations, making spiral wound membranes applicable to the treatment of streams with high volume flow rates and low solids concentrations (Eringis et al. 1993). Spiral wound units have primarily been used in laboratory-scale filtration units to recover

proteins in the biotechnology field and have been considered for large-scale commercial bioprocessing applications (Belfort, 1988). 


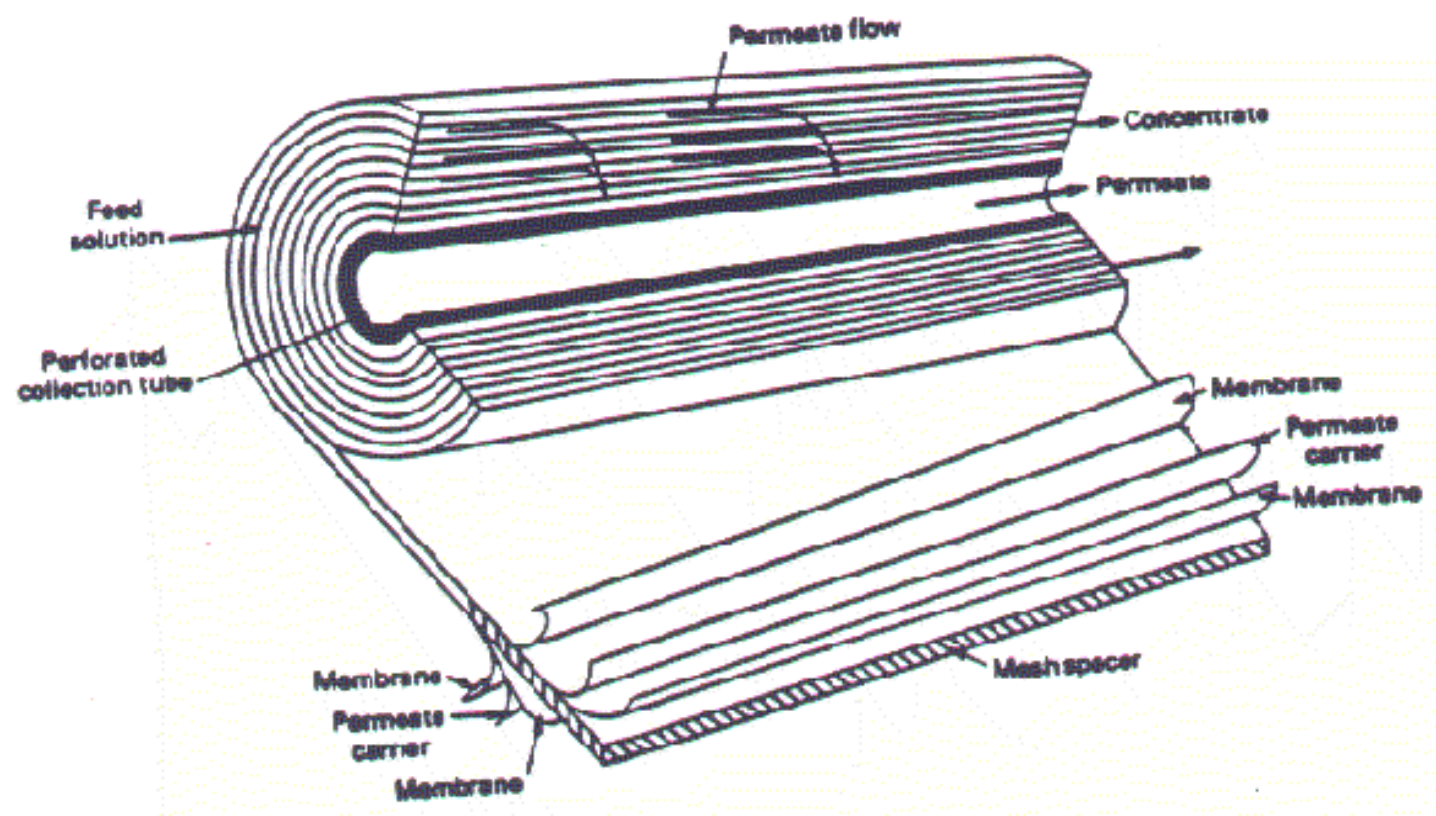

Figure 2.10 A schematic of a spiral wound membrane module (MacNeil and McCoy 1989).

\subsubsection{Plate and Frame Membrane Modules}

A schematic of a plate and frame membrane module is presented in Figure 2.11 (MacNeil and McCoy, 1989). In these systems, hollow disc-shaped spacers located between porous membranes are used to direct the feed radially along the membrane surface. A stack of multiple membranes separated by spacers is placed in series or in parallel inside a cylindrical housing. Permeate is forced through the membrane under pressure and is collected at the outer edges of the membrane disc plate. Concentrated solute is collected at the top of the stack.

The advantages of this configuration include a relatively low packing density, and a thin channel height (typically 0.3 to $0.6 \mathrm{~mm}$ ) which permits these units to process 


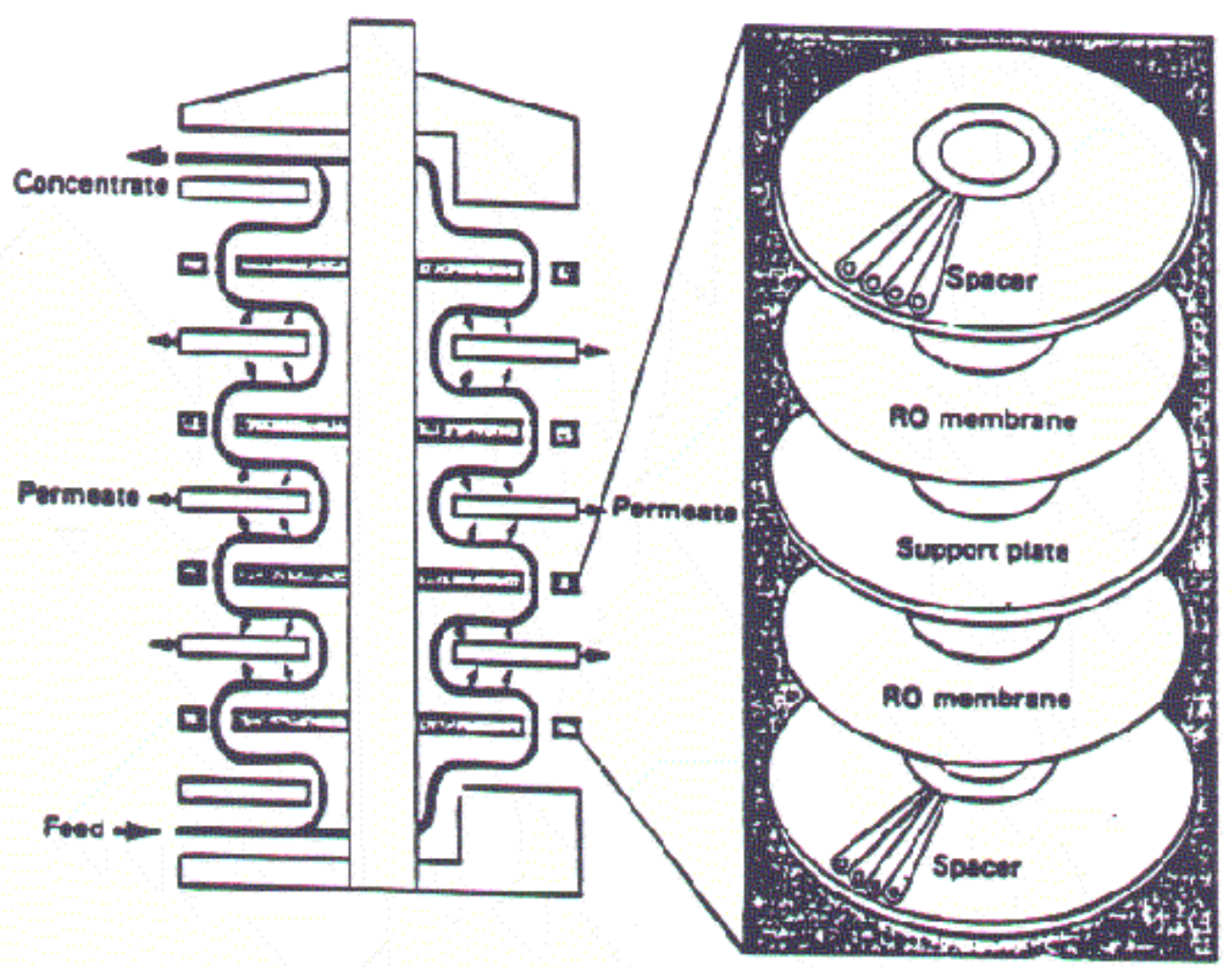

Figure 2.11 A schematic of a plate and frame membrane module (MacNeil and McCoy 1989).

highly viscous solutions. The disadvantages of this membrane configuration include susceptibility to channel plugging and difficulties in mechanical cleaning (Belfort, 1988). Additionally, the commercial application of plate and frame membrane configurations has been limited due to high capital costs relative to other filtration units (MacNeil and McCoy, 1989).

\subsubsection{Limitations of Conventional Cross-Flow Ultrafiltration Systems}

A major limitation in the application of conventional UF membrane modules/systems (e.g., tubular, hollow fiber, etc.) for the treatment of industrial wastewaters has been the low permeate flux observed at high solute concentrations. With time, a solute boundary layer forms as solute molecules accumulate on the membrane surface. The accumulation of solute molecules at the membrane surface is referred to as 
concentration polarization and was presented schematically in Figure 2.7. If the accumulated solute layer is sufficiently thick, a decrease in permeate flow rate will occur.

The buildup of solute molecules at the membrane surface is reduced by back diffusion of solute molecules from the region of high concentration near the membrane surface to the region of lower solute concentration in the bulk solution. The solute boundary layer thickness can be further reduced by providing enhanced hydraulic turbulence to "scour" the accumulated solute from the membrane surface. In conventional membrane systems the hydraulically induced turbulence is provided by recycling a large portion $(\sim 98 \%)$ of the concentrate back to the membrane unit producing high liquid velocities $\left(\sim 5 \mathrm{~m} \cdot \mathrm{s}^{-1}\right)$ near the membrane surface. These large velocities increase turbulence, which reduces the thickness of the solute concentration boundary layer through a subsequent increase in the back diffusion of solute molecules away from the membrane surface. However, conventional systems are unable to maintain the high velocities necessary to minimize the deleterious effects of the solute boundary layer on permeate flux because of the difficulty in pumping viscous liquids at high flow rates as

the feed solution is concentrated with treatment time (Murkes and Carlsson 1988; Cheryan 1998).

\subsection{HIGH SHEAR ROTARY ULTRAFILTRATION SYSTEMS}

Membrane fouling and the subsequent decline in permeate flux is a major barrier preventing the wider application of membrane technology. Although significant progress has been made to improve the chemical, mechanical, and thermal properties of membrane materials, there has not been a corresponding development of methods to combat the accumulation of solute molecules at the membrane surface and subsequent fouling of the 
membranes. As a consequence, the performance of new membranes rapidly degrades to that of conventional membrane filter systems. To address the problem of flux decline due to accumulation of solute molecules at the membrane surface, several mechanically enhanced UF modules have been designed including rotary as well as vibratory membrane systems.

In conventional membrane systems, maximum surface velocities of approximately $15 \mathrm{ft} / \mathrm{s}(4.6 \mathrm{~m} / \mathrm{s})$ are possible while surface velocities of $60 \mathrm{ft} / \mathrm{s}(18 \mathrm{~m} / \mathrm{s})$ are typical in rotary systems. It is possible to treat extremely concentrated wastes with high-shear rotary UF systems because the cleaning action is effectively decoupled from the pressurization/feed recirculation by rotating the membrane surface at high speeds (Reed et al. 1997b; Viadero and Reed 1999; Viadero et al. 1999). In high-shear rotary UF systems, the pump is needed only to provide transmembrane pressure (the driving force) and a small amount of recirculation while the membrane rotation induces turbulence needed to minimize the thickness of the concentration boundary layer (Murkes and Carlsson 1988, Reed et al. 1997b; Viadero and Reed 1999; Viadero et al. 1999).

In rotary disk systems, the membrane-containing plates have traditionally remained stationary while the solid spacer disks are rotated due to difficulties in module design and operation such as devising an adequate sealing system for permeate collection through a rotating body. One recent variation on the rotary disk design is the SpinTek high-shear rotary ultrafiltration (HSRUF) unit, which has been studied by Reed et al. (1997b), Viadero and Reed (1999), Viadero et al. (1999 and 2000). In the SpinTek HSRUF system, a round membrane disk pack is set on a hollow rotating shaft inside a 


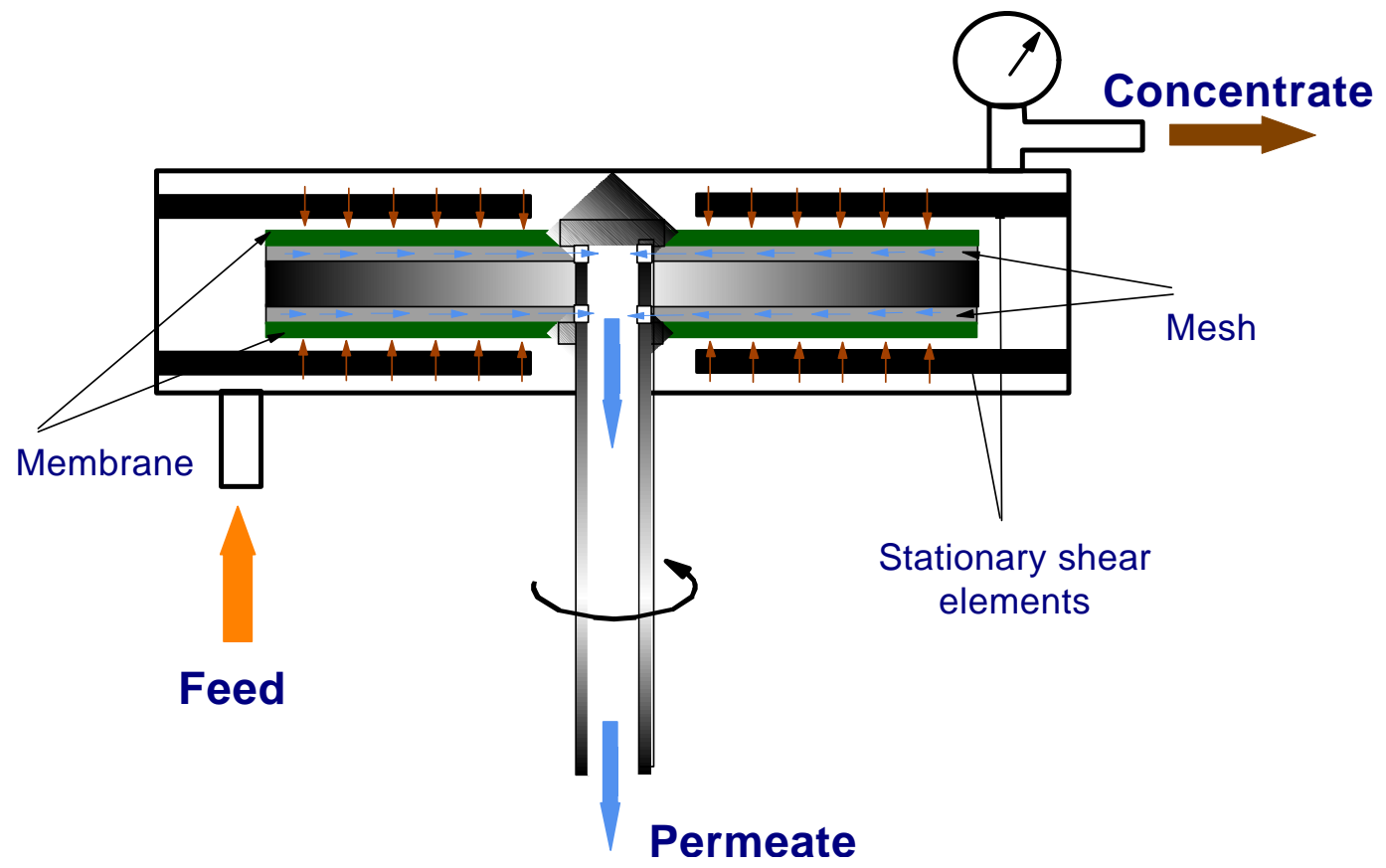

Figure 2.12 A schematic of the high-shear rotary ultrafiltration pressure vessel.

cylindrical housing. A schematic of a high-shear rotary UF pressure vessel is presented in Figure 2.12. The fluid stream enters the membrane chamber under pressure and is distributed across the membrane surface. Permeate is forced through the membrane and is collected as well as discharged through the hollow rotating shaft. The concentrate exits at the edge of the membrane pack and is returned to the feed tank to be further concentrated.

In the HSRUF unit, the rotation of the membrane disk is used to induce the hydraulic turbulence required to minimize the thickness of the concentration boundary layer. To enhance the effect of hydraulic turbulence, static turbulence promoters ("wagon wheels") are located on both sides of the membrane disk pack to prevent vortex formation. 


\subsubsection{Hydraulic Turbulence in the High-Shear Rotary Ultrafiltration System}

In the HSRUF system, a high-shear is achieved by rotating the membrane surface. The flow in the rotary disk system can be characterized by the Reynolds Number in the radial direction, which is given as:

$$
\operatorname{Re}_{\mathrm{r}}=\frac{\omega \mathrm{r}^{2}}{\eta}
$$

where $\omega=$ rotational speed, $r=$ membrane radius, and $\eta=$ kinematic viscosity of feed solution. For a given feed solution, the maximum Reynolds Number occurs at the outer radius of the rotating disk and increases with rotational speed (Murkes and Carlsson 1988; Viadero et al. 2000). However, since the Reynolds Number is radially distributed, the radius of gyration, an average radial dimension of a rotating body, for the membrane disk was selected as a representative radial property of the HSRUF unit (Viadero et al. 2000). Thus, the Reynolds Number can be expressed by the following equation:

$$
\operatorname{Re}_{\mathrm{r}-\mathrm{avg}}=\frac{\omega \mathrm{I}^{2}}{\eta}
$$

where $\mathrm{I}=$ radius of gyration for a flat rotating ring. The explicit form of the radius of gyration term for the rotating ring is (Weast 1976):

$$
I=\sqrt{\frac{R_{i}^{2}+R_{0}^{2}}{2}}
$$

where $R_{i}$ is the inner radius and $R_{o}$ is the outer radius of the membrane disk.

In high-shear as well as conventional cross-flow membrane separation systems, the relationship between the hydraulic turbulence represented by the Reynolds Number and the pressure-independent "limiting" permeate flux can be represented as (Cheryan 1998, Viadero and Reed 1999):

$$
\mathrm{J}^{*}=f(\operatorname{Re})^{\alpha}
$$


where $\mathrm{J}^{*}$ is the pressure-independent "limiting" permeate flux, and $\alpha$ is determined as the slope of a line through the data in a log-log plot of limiting permeate flux versus Reynolds Number.

\subsubsection{Permeate Backpressure in the High-Shear Rotary Ultrafiltration System}

In the HSRUF system illustrated previously in Figure 2.12, the central aim is to use membrane rotation to induce turbulence at the membrane surface to reduce the thickness of the flux-defeating solute concentration boundary layer. However, in addition to providing turbulence in the system, membrane rotation has an impact on the net transmembrane pressure. A non-uniformly distributed backpressure results from centrifugal force exerted on permeate due to membrane rotation. The permeate backpressure for a given membrane rotational speed and radius is expressed as:

$$
\mathrm{P}_{\text {back }}=\frac{\rho \omega \mathrm{r}^{2}}{2}
$$

where $\omega=$ membrane rotational speed, $r=$ the membrane radius, and $\rho=$ feed solution density. The maximum and minimum permeate backpressures are found at the maximum and minimum membrane radii, respectively. The average backpressure due to centrifugal force on the permeate in the HSRUF system is determined at radius of gyration for a flat rotating ring:

$$
\mathrm{P}_{\text {back-avg }}=\frac{\rho \omega \mathrm{I}^{2}}{2}
$$

where I, the radius of gyration is calculated from Equation 2.8. The maximum permeate backpressure, $\mathrm{P}_{\text {back-max }}$, is observed along the outer radius of the rotating disk, $\mathrm{R}_{\mathrm{o}}$ :

$$
\mathrm{P}_{\text {back-max }}=\frac{\rho \omega \mathrm{R}_{\mathrm{o}}^{2}}{2}
$$


In the high-shear rotary UF system, the average transmembrane pressure, $\mathrm{P}$, is the difference between the applied pressure, $\mathrm{P}_{\text {applied }}$, and the average permeate back pressure, Pack-avg:

$$
\mathrm{P}=\mathrm{P}_{\text {applied }}-\mathrm{P}_{\text {back-avg }}
$$

An important aspect of the pressure/rotation relationship in the HSRUF system is the need to set system operating parameters to ensure a positive net transmembrane pressure:

$$
\mathrm{P}_{\text {applied }}>\mathrm{P}_{\text {back-max }}
$$

If the condition in Equation 2.14 is not met, the driving force will reverse, and fluid from the permeate side of the membrane can flow back to the concentrate side. Serious operational difficulties such as membrane delamination and a loss in operating efficiency may result as a consequence of flow reversal.

\subsection{MODELING PERMEATE FLUX}

\subsubsection{Pressure-Controlled Region - Hagen-Poiseuille Model}

Many attempts at modeling permeate flux have been presented in the literature, though none of which have been entirely satisfactory at predicting flux response to changes in operational parameters (feed concentration, pressure, temperature, etc.) The theoretical relationship between permeate flux, transmembrane pressure, and feed concentration was presented previously in Figure 2.6. At low feed concentrations and low transmembrane pressures, the Hagen-Poiseuille model for streamline flow through channels in an ideal membrane system is generally accepted as the best description of fluid flow through a semi-permeable membrane: 


$$
J=\varepsilon r_{p}^{2} \frac{P-\Delta \Pi}{8 \mu \Delta x}
$$

where $\mathbf{J}$ is the permeate flux, $\varepsilon$ is the membrane surface porosity, $r_{p}$ is the mean pore radius, $\mathrm{P}$ is the average transmembrane pressure, $\Delta \Pi$ is the osmotic pressure difference between the feed and the permeate, $\mu$ is the absolute viscosity of fluid permeating the membrane, and $\Delta \mathrm{x}$ is the membrane thickness. The osmotic pressure term is generally small compared to the applied pressure in ultrafiltration due to the relatively high molecular weights of the retained solutes (Cheryan 1998). In this case, $\Delta \Pi \ll<\Delta \mathrm{P}_{\mathrm{T}}$ and Equation 2.15 can be simplified:

$$
\mathrm{J}=\frac{\varepsilon \mathrm{r}_{\mathrm{p}}^{2} \mathrm{P}}{8 \mu \Delta \mathrm{x}}
$$

Several assumptions are made when using the Hagen-Poiseuille model (Bird et al. 1960):

(1) The flow through the membrane pore channels is laminar (i.e., $\operatorname{Re}<4,000$ ). This assumption was validated statistically and experimentally (Cheryan 1998). (2) Permeate fluid is incompressible (3) Permeate flow is independent of time ("steady state" conditions) (4) The permeate fluid is Newtonian (i.e., the shear force per unit area is linearly proportional to the negative of the velocity gradient). (5) End-effects are negligible (i.e., the flow is "fully developed"). Additionally, the fluid flowing through the pores is generally considered to be contaminant-free water. Therefore, it is customary to use the viscosity of water as the $\mu$ term in Equation 2.16 when modeling permeate flux as a function of applied pressure (Cheryan 1998).

In the Hagen-Poiseuille model, the permeate flux is controlled directly by the transmembrane pressure and inversely by the permeate fluid viscosity. Since the 
viscosity of a fluid is inversely proportional to the fluid temperature, an increase in temperature will result in an increased permeate flux. As the transmembrane pressure or feed concentration are increased, the permeate flux will become independent of pressure due to the effects of a buildup of solute molecules at the membrane surface. This situation is not accounted for in Equation 2.16 and thus, is a limitation of the HagenPoiseuille model.

\subsubsection{Pressure-Independent Region - The Thin-Film Model}

As the transmembrane pressure or the feed concentration are increased, or the hydraulic turbulence in the system is decreased, the flux becomes independent of pressure due to a buildup of rejected species at the membrane surface. The thin-film model is used to describe the pressure-independent permeate flux. In the thin-film model, the concept of concentration polarization is used as a basis to describe the asymptotic flux-pressure relationship shown schematically in Figure 2.6. As a feed solution is ultrafiltered, solute particles are transported to the membrane surface at a rate $\mathrm{J}_{\mathrm{s}}$, given by the following equation (Cheryan 1998; Viadero 1997):

$$
\mathrm{J}_{\mathrm{s}}=\mathrm{JC}_{\mathrm{b}}
$$

where $\mathrm{J}$ is the permeate flux and $\mathrm{C}_{\mathrm{b}}$ is the concentration of the bulk feed solution. The resultant concentration gradient in the boundary layer causes back diffusion of particles into the bulk solution (see Figure 2.7), which is expressed as:

$$
\mathrm{J}_{\mathrm{s}}=\mathrm{D} \frac{\mathrm{dC}}{\mathrm{dx}}
$$

where $\mathrm{D}$ is the diffusion coefficient and $\mathrm{dC} / \mathrm{dx}$ is the concentration gradient over a differential element at the membrane-feed solution interface. Using Equations 2.17 and 
2.18 as well as accounting for solute permeation through the membrane, a steady-state mass flux balance over a the differential volume is given as:

$$
0=\mathrm{JC}-\mathrm{D} \frac{\mathrm{dC}}{\mathrm{dx}}-\mathrm{JC}_{\mathrm{p}}
$$

where $\mathrm{C}$ is the solute concentration at a given position, $\mathrm{x}, \mathrm{D}$ is the solute diffusion coefficient, $\mathrm{J}$ is the permeate flux, and $\mathrm{C}_{\mathrm{p}}$ is the solute concentration in the permeate. The general form of the thin-film model, presented in Equation 2.22, is obtained by rearranging and integrating Equation 2.19 and using the boundary conditions (presented in Equations 2.20 and 2.21).

$$
\begin{aligned}
& C=C_{b}, x=0 \\
& C=C_{m}, x=\delta \\
& J=\frac{D}{\delta} \ln \frac{C_{m}-C_{p}}{C_{b}-C_{p}}
\end{aligned}
$$

where $\delta$ is the concentration boundary layer thickness and $\mathrm{C}_{\mathrm{m}}$ is the solute concentration at the membrane surface. Assuming the solute concentration in the permeate is approximately zero, Equation 2.22 can be simplified to the following relationship.

$$
\mathrm{J}=\frac{\mathrm{D}}{\delta} \ln \frac{\mathrm{C}_{\mathrm{m}}}{\mathrm{C}_{\mathrm{b}}}
$$

In the thin-film model, a lumped parameter approach, based on mass transfer considerations, is used to estimate the permeate flux. The solute boundary layer is idealized as a thin liquid film in which mass transport takes place under steady state conditions, where resistance to mass transfer is governed by molecular diffusion normal to the membrane surface (Lipp et al. 1984). The solute mass transfer coefficient, $\mathrm{k}_{\mathrm{s}}$, is 
directly related to the molecular mass diffusivity, $\mathrm{D}$, and inversely proportional to the film thickness, $\delta$ :

$$
\mathrm{k}_{\mathrm{s}}=\frac{\mathrm{D}}{\delta}
$$

A basic assumption when applying the thin film model is that the mass transfer coefficient is constant as the feed concentration is increased; thus, a semi-logarithmic plot of $\mathrm{J}^{*}$ versus feed oil concentration should be linear. However, it has been reported that the mass transfer coefficient can vary with changes in feed solution properties (Cheryan, 1998). For example, the following two representations of the mass transfer coefficient have been reported as a function of solution properties for laminar and turbulent hydraulic conditions in tubular UF separation systems:

Turbulent flow:

$$
\mathrm{k}_{\mathrm{s}}=0.023\left(\frac{\mathrm{D}^{0.67} \mathrm{v}^{0.8}}{\mathrm{~d}_{\mathrm{h}}^{0.2} \eta^{0.47}}\right)
$$

Laminar flow:

$$
\mathrm{k}_{\mathrm{s}}=1.86\left(\frac{\mathrm{D}^{0.67} \mathrm{v}^{0.33}}{\mathrm{~d}_{\mathrm{h}}^{0.33} \mathrm{~L}^{0.33}}\right)
$$

where $\mathrm{v}$ is the fluid velocity, $\mathrm{d}_{\mathrm{h}}$ is the hydraulic diameter of the tubular membrane module, $\eta$ is the kinematic viscosity of the feed solution, and $L$ is the membrane module channel length (Cheryan 1998). Thus, under conditions of turbulent hydraulic flow, the solute mass transfer coefficient and the limiting flux decrease as the concentration is increased due to a corresponding increase in viscosity as presented in Equation 2.25. However, as the viscosity is increased, a transition from turbulent to laminar conditions can occur. Under laminar flow conditions, the mass transfer coefficient is no longer a 
function of viscosity (Equation 2.26); thus, the limiting flux decreases little when compared with the relatively rapid increase in viscosity as feed solutions are concentrated. Consequently, a two-region permeate flux can be observed (Pritchard et al. 1995; Cheryan 1998).

As the solute concentration at the membrane surface increases, a viscous "gel layer" may form. The "gel layer" can be an actual gel or the "closest-packed" arrangement of solute molecules/particles without phase separation resulting from the restricted mobility of solute molecules near the membrane surface. In the case of gel layer formation the permeate flux becomes independent of pressure and the following form of the thin-film model is obtained:

$$
\mathrm{J}^{*}=\mathrm{k}_{\mathrm{s}} \ln \frac{\mathrm{C}_{\mathrm{g}}}{\mathrm{C}_{\mathrm{b}}}
$$

where $\mathrm{J}^{*}$ is the pressure-independent "limiting" permeate flux and $\mathrm{C}_{\mathrm{g}}$ is the solute gel layer concentration. Before the gel layer has formed, the permeate flux is a weak function of the bulk solute concentration, $\mathrm{C}_{\mathrm{b}}$, because the solute concentration at the membrane surface increases with $C_{b}$. However, once the gel layer has formed, the permeate flux decreases logarithmically with increasing bulk solute concentration. The gel layer concentration can be determined by extrapolating the linear portion of the flux versus $\log \left(\mathrm{C}_{\mathrm{b}}\right)$ plot to zero flux. The application of the thin-film model is restricted to the pressure independent region where the concentration boundary layer plays the primary role in limiting flux. Cheryan (1998) noted several common flaws in experiments performed to determine gel layer concentrations. In particular, Cheryan cited the need to maintain constant flow rates and/or turbulence in a system, particularly 
at high feed concentrations. Additionally, individual experiments should be performed at discrete feed concentrations as data from batch or semi-batch experiments can include effects of feed aging, solute denaturation due to heat or shear, and fouling.

\subsubsection{Thin-Film Model Case Studies}

A summary of typical $C_{g}$ values for several non-oily waste and process streams is presented in Table 2.3 (Cheryan 1998). A gel layer concentration of $35 \%$ was determined by Lipp et al. (1984) for a metal-working lubricant treated using a conventional tubular UF system. Lee et al. (1984) reported a gel layer concentration of $\sim 40 \%(\mathrm{v} / \mathrm{v})$ for a mineral oil-based coolant treated in a stirred cell UF unit. The gel layer concentration in these experiments was independent of the applied pressure and the initial oil concentration, as anticipated in the pressure-independent region.

Table 2.2--A Summary of Typical $C_{g}$ Values For Several Non-Oily Waste And Process Streams (Cheryan 1998).

\begin{tabular}{||c|c|c||}
\hline \hline Feed Solution & $\mathbf{C}_{\mathbf{g}}$ & Reference \\
\hline \hline Whole milk (3.5\% fat) & $9-11 \%$ protein & Yan et al. 1979 \\
\hline Gelatin & $20-30 \%$ protein & Porter and Michaels 1972 \\
\hline Kraft black liquor & $30-34 \%$ lignin & Woerner 1983 \\
\hline Egg-white & $40 \%$ protein & Porter and Michaels 1972 \\
\hline Styrene-Butadiene & $70 \%$ & Porter 1979 \\
\hline Soybean water extracts & $10 \%$ protein & Cheryan 1977 \\
\hline Human blood & $28.7 \%$ protein & Isaacson et al. 1980 \\
\hline
\end{tabular}

A substantially higher gel layer concentration of 60 to $65 \%$ was reported by Reed et al. (1997b) for a metal-working lubricant treated using the high-shear rotary disk ultrafiltration system. Additionally, the transitional gel layer concentration (the concentration at which the permeate flux decreases exponentially with increasing bulk feed concentration, signifying the transition between the pressure dependent and pressure independent regions) observed by Reed et al. (1997b) of 23\% was substantially higher 
than the value of $\sim 15 \%$ reported by Lipp et al. (1987). The solute mass transfer coefficient is equal to the absolute value of the slope of the limiting flux versus $\ln$ (concentration) plot. The units of $\mathrm{k}_{\mathrm{s}}$ are the same as the units of permeate flux (volume/area-time). Reed et al. determine $\mathrm{k}_{\mathrm{S}}$ values of ranging from 14.2 to $18.7 \mathrm{~m}^{3} \cdot \mathrm{m}^{-}$ ${ }^{2} \cdot \mathrm{d}^{-1}\left(347\right.$ to $\left.457 \mathrm{gal} \cdot \mathrm{ft}^{-2} \cdot \mathrm{d}^{-1}\right)$ when treating a spent metal-working fluid using a ceramic membrane with a $0.11 \mu \mathrm{m}$ average pore size and a 100k MWCO PVDF membrane in a high-shear rotary UF system. In the pressure independent region, any attempt to increase permeate flux "without providing a compensating mechanism to increase the rate of back-transport will be self-defeating," as emphasized by Cheryan (1986). Thus, the permeate flux can only be improved by providing some mechanism to enhance the solute mass transfer coefficient $\mathrm{k}_{\mathrm{s}}$ as defined in Equation 2.24 (e.g., raise the feed temperature to increase $\mathrm{D}$ or increase the hydraulic turbulence to decrease $\delta$ ).

\subsubsection{Limitations Of The Thin-Film Model}

Jonsson and Boesen (1984) discussed the limitations of the thin-film model and reported that the central assumption of a gel layer with a well-defined concentration, $C_{g}$, and variable thickness determined by the bulk fluid concentration and hydraulic turbulence is not an accurate description of the phenomenon occurring at the membrane surface. A study was cited in which the gel layer concentration was measured for a polyvinyl alcohol membrane treating ovalbumin (Jonsson and Boesen 1984). In the study, the gel layer concentration was found to increase with increasing bulk concentrations and decreasing feed velocities. Additionally, Jonsson and Boesen cited a study in which the osmotic pressures of concentrated solutions were comparable to typical applied pressures used in UF. It was suggested that the permeate flux was likely 
limited by a combination of both the osmotic pressure and the formation of a gel layer, depending on the properties of the feed solutions. An additional limitation of the thinfilm model is the assumption that the concentration of solute in the permeate is approximately zero which may not be true in all cases. Cheryan (1986) suggested using Equation 2.22 to account for solute concentration in the permeate prior to making calculations of gel layer concentrations. However, Reed et al. (1997b) and Viadero and Reed (1999) both presented the successful application of the thin-film model to the HSRUF of oil-in-water emulsions. In both of these studies, the concentration of oil in the permeate $\left(\mathrm{C}_{\mathrm{p}}\right)$ was assumed to be equal to zero.

The issue of flux reduction due to a decrease in driving force caused by a local increase in osmotic pressure was also addressed by Cheryan (1977), who noted that it was often difficult to differentiate between the flux reduction due to an increase in osmotic pressure and the flux reduction due to an increase in hydrodynamic resistance of the consolidated concentration boundary layer. Cheryan ultrafiltered soy proteins in a pilot-scale hollow fiber UF unit with equipped with an anisotropic noncellulosic membrane (50k MWCO) and concluded that the highly structured macromolecules displayed a gel-like behavior at concentrations $>10 \mathrm{wt} \%$ as suggested by Blatt et al. (1970). Cheryan calculated a $C_{g}$ value of $10 \%$ protein obtained when applying Equation 2.22 to the experimental data obtained in the ultrafiltration of soy protein. The value of $10 \%$ protein was in good agreement with the known gel characteristics of soybean proteins as presented by Smith and Circle (1972). Additionally, Lee et al. (1992) investigated gel layer formation in the treatment of an oil-in-water emulsion (initial oil concentrations of 1 to 5 vol.\%) using a 20k MWCO polymeric membrane in an Amicon 
S4OC stirred UF cell. To visualize the accumulation of oil droplets at the membrane surface, a red coloring agent ("Cerol") which is soluble in oil but not in water, was added to the feed. In the experiments, the color of the concentrated "gel" remained opaque white. Thus, the layer on the membrane surface was a concentrated oil-in-water emulsion and not a true oil gel. The oil content of the concentrated solute layer (measured using a Beckman KF-4B aquameter), was $\sim 40$ vol.\% oil, which is in agreement with the gel layer value determined by applying Equation 2.23 to experimental flux data. In the case of the oil-in-water emulsion, the gel layer can be described not as a true gel, but as the "closest packed" arrangement of solute molecules. In contrast, the application of the thin-film model to non-gelling protein solutions resulted in a gel layer concentration below the known value. The lower than predicted gel layer concentration was explained by an osmotic pressure difference across the concentration boundary layer which exceeded the hydrostatic driving force.

\subsubsection{The Resistance-In-Series Model}

In the resistance-in-series (RIS) model, Darcy's Law is used as a basis for describing permeate flux decline in terms of a system's overall resistance to hydraulic flow. Using this approach, the concentrated solution of macromolecules at the membrane surface is viewed as a physical barrier to hydraulic flow. The resistance of this boundary layer can be divided into and treated as the sum of several individual resistances, analogous to the "resistance in series" concept applied in heat transfer. The following expression for permeate flux can be obtained:

$$
\mathrm{J}=\mathrm{A}_{\mathrm{p}} \frac{\mathrm{P}}{\mu}
$$


where $A_{p}$ is the membrane permeability coefficient and $\mu$ is the absolute viscosity of the permeate. For a particular feed solution, viscosity can be grouped together with A and expressed as $1 / \mathrm{R}$ where $\mathrm{R}$ is the total resistance to permeate flow:

$$
\mathrm{J}=\frac{\mathrm{P}}{\mathrm{R}}
$$

In ultrafiltration, $\mathrm{R}$ is composed of three individual resistances:

$$
\mathrm{R}=\mathrm{R}_{\mathrm{m}}+\mathrm{R}_{\mathrm{f}}+\mathrm{R}_{\mathrm{p}}
$$

where $R_{m}$ is the intrinsic membrane resistance; $R_{f}$ is the fouling resistance; and $R_{p}$ is the resistance of the polarization layer. Substituting Equation 2.30 into Equation 2.29, yields the following expression for permeate flux:

$$
J=\frac{P}{R_{m}+R_{f}+R_{p}}
$$

$\mathrm{R}_{\mathrm{m}}$ is determined for a virgin membrane using pure water as the feed solution (Cheryan, 1998). Typically, $R_{f}$ is caused by solute adsorption onto the membrane surface and/or membrane pore plugging. Since fouling leads to alterations in the physical makeup of the membrane, $R_{m}$ and $R_{f}$ are grouped together and considered as the total membrane resistance, $R_{m}{ }^{\prime}$ :

$$
\mathrm{R}_{\mathrm{m}}{ }^{\prime}=\mathrm{R}_{\mathrm{m}}+\mathrm{R}_{\mathrm{f}}
$$

$\mathrm{R}_{\mathrm{p}}$ is a function of the density, permeability and thickness of the concentration boundary layer, which is a function of the average transmembrane pressure; thus:

$$
\mathrm{R}_{\mathrm{p}}=\Phi \cdot \mathrm{P}
$$

where $\Phi$ is the resistance index, which is a function of system mass transfer properties (e.g., feed viscosity and hydraulic turbulence). After substituting Equations 2.32 and 
2.33 into Equation 2.31, the RIS model becomes (Cheryan, 1998; Johnsson and Tagardh, 1990; Chiang and Cheryan, 1986):

$$
J=\frac{P}{R_{m}{ }^{\prime}+\Phi \cdot P}
$$

Unlike the Hagen-Poiseuille and thin-film models (used to describe the pressuredependent and pressure-independent permeate flux, respectively), both the pressure dependent and pressure independent regions can be predicted using the RIS approach. In the pressure dependent region the concentration polarization layer is not very thick; thus, $R_{p} \ll<R_{m}{ }^{\prime}$ and $J$ is linearly related to $P$. In the pressure independent region, the thickness or density of the concentration polarization layer increases and $R_{p} \gg R_{m}^{\prime}$. Under such conditions, $\mathrm{J}$ becomes independent of $\mathrm{P}$ at a limiting flux of $1 / \Phi$. In order to increase the pressure independent permeate flux, it is necessary to reduce $\Phi$ by decreasing the feed concentration or increasing hydraulic turbulence (Chiang and Cheryan 1986; Cheryan 1998; Viadero et al. 1999).

Additionally, it is possible to quantify individual resistances, thus identifying the different flux decline constituents and determining the magnitude of the effect that each resistance has on the permeate flux. UF operation can then be optimized by determining which resistances are the largest under a given set of operating conditions and taking measures to reduce the magnitude of the resistance by altering operational parameters accordingly (Chiang and Cheryan 1986; Cheryan 1998; Viadero et al. 1999). Further, Cheryan (1998) presented a set-point for UF operation, $\Delta \mathrm{P}_{\text {set }}$, as the pressure at which polarization effects are balanced against the total membrane resistance: 


$$
\Delta \mathrm{P}_{\mathrm{set}}=\frac{\mathrm{R}_{\mathrm{m}}{ }^{\prime}}{\Phi}
$$

The resistance-in-series approach has been successfully applied to membrane separation processes in a number of studies. A model was developed using the resistance-in-series concept to determine the effect of operating parameters on permeate flux in the hollow fiber UF of skim milk (Chiang and Cheryan 1986). Both pressuredependent and pressure-independent permeate flux data were adequately predicted; however, the model was process specific. Further, the relationship developed was not used to examine the optimization of operational parameters. Viadero et al. (1999) used the resistance-in-series concept to model the relationship between operating parameters and permeate flux in the high-shear rotary (HSR) UF of a synthetic MW fluid. Set point operating conditions were determined; however, application of the model was limited to the HSR UF of synthetic MW fluids.

\subsection{MEMBRANE TREATMENT OF OILY WASTEWATERS}

\subsubsection{Characteristics of Oil-In-Water (O/W) Emulsions and Oily Wastewaters}

The metalworking industry produces large volumes of oily wastewaters. Examples of metalworking processes which utilize large quantities of emulsified oils for lubrication, cooling, and quenching include metal rolling, forming, grinding, and cutting

operations. Concentrations of oil and grease $(\mathrm{O} / \mathrm{G})$ vary widely across the metalworking industry. A summary of typical $\mathrm{O} / \mathrm{G}$ concentration ranges for selected industrial wastewaters is presented in Table 2.4 (Bennett 1988). 
Table 2.4 Typical O/G Concentration Ranges For Selected Industrial Wastewaters (Bennett 1988).

\begin{tabular}{|c|c|}
\hline Industry & O/G concentration range (mg/L) \\
\hline \hline Food processing & $100-1,000$ \\
\hline Textile (wool processing ) & $10-50$ \\
\hline Petroleum refining & $100-1,000$ \\
\hline Metal rinse water & $10-1,000$ \\
\hline Metal fabrication & $10,000-150,000$ \\
\hline Metal rolling & $4,000-50,000$ \\
\hline Commercial laundry & $100-2,000$ \\
\hline
\end{tabular}

Oil is typically found in three forms in industrial processes: 1) free oil consisting of discrete oil droplets, usually $5 \mu \mathrm{m}$ in diameter or larger; 2) agglomerated oil made up of discrete oil droplets $5 \mu \mathrm{m}$ or less in diameter; and 3) emulsified oil in which fine globules of oil are dispersed in water by the addition of a chemical "emulsifier" (Laemmle 1992). Free oil can be removed through gravity separation. Unstable emulsions containing agglomerated oil can be chemically broken. However, stable emulsions require increasingly sophisticated treatment techniques to meet more stringent effluent discharge guidelines. Traditionally, these wastewaters are treated using chemical addition followed by gravity separation techniques. Often, the effluent from chemical addition solid-liquid separation processes required additional treatment prior to discharge. In contrast, pressure-driven membrane separation systems can typically treat the waste in a single step and are gaining acceptance as an alternative treatment technology (Cheryan and Rajagopalan 1998; Reed et al. 1997a; Burke 1991).

Emulsions are mixtures of either simple or compounded oils with water, stabilized by the use of emulsifiers and other additives such as secondary stabilizers/surfactants. Emulsified oils are widely used when both cooling and lubricating properties are required, whereas straight oils are used when lubrication is the main concern and cooling 
capacity is a minor factor. A schematic of an emulsified oil droplet is presented in Figure 2.13 (after Burke, 1991). In an oil-in-water emulsion, oil is said to be the "dispersed phase" and water is the "continuous phase." It is possible for an emulsion to have a net positive or negative charge, depending upon the properties of the emulsifier. However, anionic emulsifiers are typically used in the cooling/lubricating fluids common in the metal-working industry. In a typical $\mathrm{O} / \mathrm{W}$ emulsion, oil globules are sequestered by a polar emulsifier which consists of an oil soluble end and a negatively charged oleophobic/hydrophilic end. Typical oil globule sizes range from $\sim 0.2$ to $10 \mu \mathrm{m}$ (Laemmle 1992). However, the size of oil globules in an $\mathrm{O} / \mathrm{W}$ emulsion is not discrete throughout a solution but varies over some distribution which depends primarily on the ratio of oil to emulsifier, rather than on the oil content (Lipp, et al. 1988). Since the typical size of the emulsified oil droplet is similar in size to, or greater than, the wavelength of visible light, these emulsions appear milky white.

The stability and lubricating characteristics of an O/W emulsion used in metalworking processes are carefully balanced to optimize the operational properties of the solution. $\mathrm{O} / \mathrm{W}$ mixtures are thermodynamically unstable (i.e., state of lowest free energy is total phase separation). As a result, oil tends to separate from water in solution. To keep the oil droplets sequestered, emulsifiers and secondary stabilizers (chemicals which concentrate at the oil-water interface and prevent oil globule coalescence) are used. Since the hydrophilic end of the O/W emulsion is negatively charged, the net surface charge of the emulsified oil droplet is negative. The emulsified oil droplets tend to stay dispersed due to electrostatic repulsion between the emulsified oil droplets. The emulsion is then said to be kinetically stable because the state of lowest free energy of the 


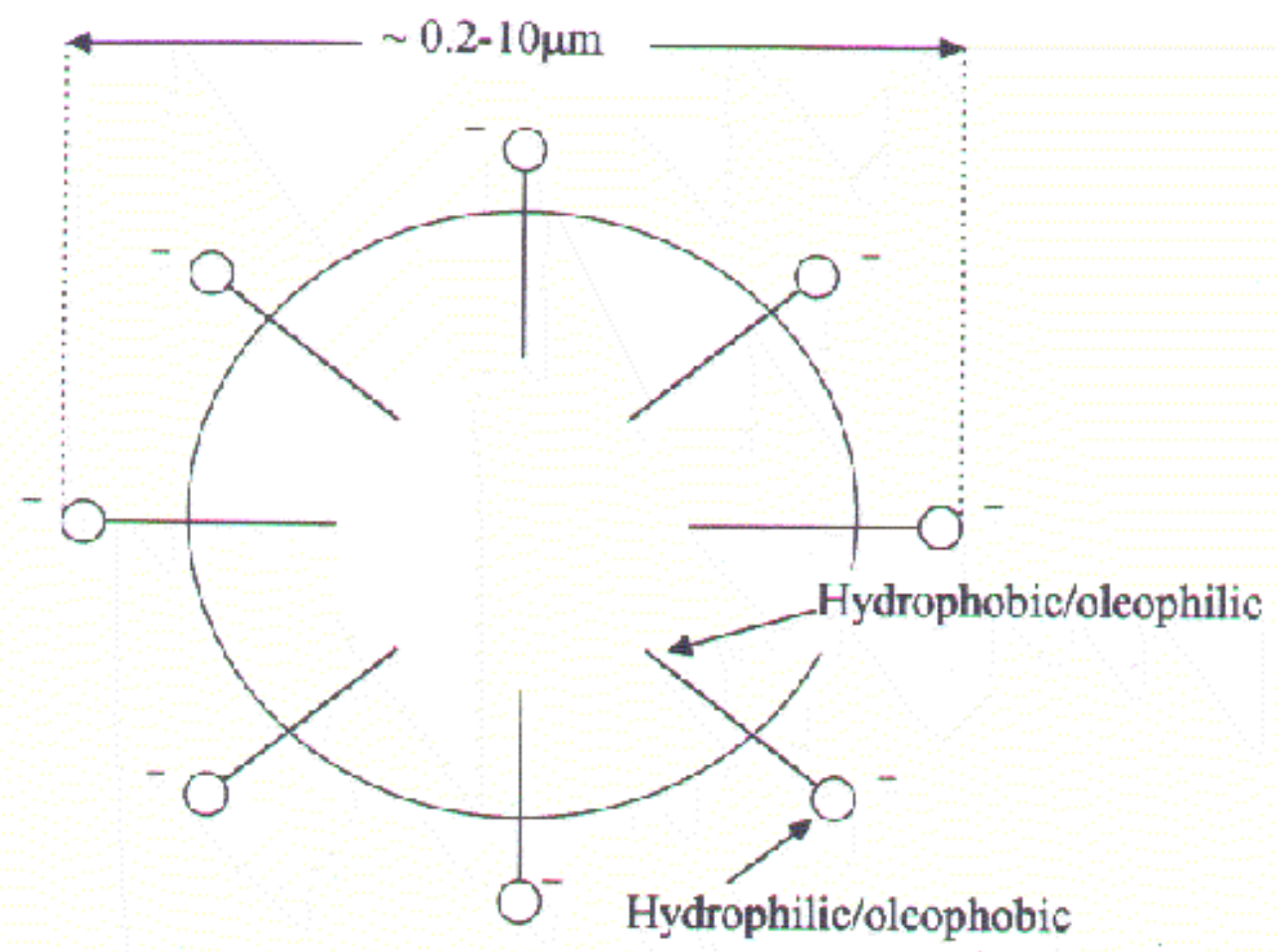

Figure 2.13 A schematic of an emulsified oil droplet (Burke 1991).

emulsion is still total separation. In general, stable emulsions tend to have a smaller average globule size distribution than unstable emulsions. Emulsion stability can vary over a wide range and is affected by the chemical nature of the oil, the concentrations of emulsifier and additives in the solution, and the age of the emulsion.

The ability of an $\mathrm{O} / \mathrm{W}$ emulsion to provide sufficient lubrication is tied to the availability of the oil phase to lubricate (lubrication improves with oil availability). Oil availability is controlled by both the emulsion stability and oil content. Typically, oil availability increases as oil content increases and as emulsion stability decreases. Less stable emulsions are subject to rapid, often unpredictable, declines in stability over short periods of time. The transient behavior can lead to severe operating problems in metal- 
working processes such as rolling mill operations. Additionally, as the oil content in an emulsion increases, cooling capacity decreases because there is less water available for cooling. As a result, the stability and oil concentration of an emulsified metal-working fluid must be balanced to meet both the lubricating and cooling needs of each specific metal-working process, leading to the heterogeneous nature of wastewaters from the metal-working industry.

Since O/W emulsions are kinetically stable but not thermodynamically stable, the "batch life" of the coolant/lubricant package must also be taken into consideration. New emulsions are typically the most stable and have the least oil available for lubrication. Over time, the maximum performance of the emulsion is reached as emulsifiers are (1) depleted through biological degradation and (2) as debris generated in the emulsion provides nucleation sites for oil globule coalescence. However, as the emulsion is degraded further, the emulsion becomes so unstable as to be rendered useless and a new batch of emulsion must be introduced into the process. Emulsions that are initially more stable generally have a longer batch life than emulsions which are initially less stable (Laemmle 1992).

Typical $\mathrm{O} / \mathrm{W}$ emulsions used in rolling mill operations consist of complex proprietary mixtures of fatty acids and mineral oils, emulsifiers, biocides, stabilizers, anti-foaming agents, and rust inhibitors. The main oil and grease $(\mathrm{O} / \mathrm{G})$ components of these rolling mill oils are fatty acids and mineral oils. Wastewaters in rolling mill processes contain $\mathrm{O} / \mathrm{G}$ from coolant sprayed on metal strips to dissipate heat and lubricate the material sheets. Other contributions to $\mathrm{O} / \mathrm{G}$ concentrations in these wastewaters come from non-emulsified "tramp oils" which leak into the waste stream 
from ancillary mechanical operations (e.g., hydraulic fluids). Typical coolant/lubricant solutions used in metal-working operations contain approximately 5 to $10 \%$ emulsified oil and approximately 90 to $95 \%$ water (Laemmle 1992). A common base oil consists of $\sim 80 \%$ mineral oil and $\sim 20 \%$ additives (e.g., emulsifiers, anti-foaming agents, biocides, etc.) (Lee et al. 1984).

\subsubsection{Overview of Membrane Treatment of Oily Wastewaters}

Ultrafiltration has been successfully applied to the treatment of concentrated oily wastes from metal-working processes such as sheet rolling and die cutting (Lee et al. 1984; Lipp et al. 1988; Burke 1991; Cheryan and Rajagopalan 1998; Reed et al. 1997a and b; Viadero and Reed 1999; Viadero et al. 1999 and 2000; Masciola et al. 2000). UF has been used to treat wastewaters high in oil, grease, and solids while reducing the volume of waste sent for disposal, while at the same time recovering alkaline process cleaner for re-use. The advantages of UF treatment of oily wastewaters over more traditional methods such as chemical addition solid/liquid separation include: (1) chemical additives are not necessary; (2) higher oil removal efficiencies; and (3) relative ease of use over other techniques (Lee et al. 1984; Burke 1991; Cheryan and Rajagopalan 1998).

\subsubsection{Conventional Ultrafiltration Systems}

Pinto (1978) reported that UF treatment of a waste cutting oil emulsions reduced the volume of wastewater by 95 to $98 \%$ and concentrated solids and oil as much as $60 \%$. Cheryan (1998) summarized the application of UF technology to the treatment of a mixed oily wastewater containing mineral oils, paraffin wax, oleic acid, and other oily constituents. The mixed waste stream was stabilized to minimize membrane fouling by 
adding a nonionic surfactant. The waste was then treated using Abocor tubular membranes $\left(72 \mathrm{~m}^{2}, 776 \mathrm{ft}^{2}\right.$ membrane area). The membrane pore size/molecular weight cut off (MWCO) was not specified. At an average pressure of $\sim 2.5$ atm (37 psi), an average permeate flux of $41 \mathrm{~L} / \mathrm{m}^{2}-\mathrm{h}\left(24 \mathrm{gal} / \mathrm{ft}^{2}-\mathrm{d}\right)$ was observed while maintaining a permeate water quality acceptable for direct discharge to the sanitary sewer. Oil and grease concentrations were reduced from $3,530 \mathrm{mg} / \mathrm{L}$ in the feed to $35 \mathrm{mg} / \mathrm{L}$ in the effluent. Further, a 94\% reduction in chemical oxygen demand (COD) was observed after UF treatment of the oily wastewater.

Due to the heterogeneous nature of oily wastewaters, the type of membrane material and the molecular weight cutoff (MWCO) used to treat oily wastes varies widely. It was reported that, in general, oily wastewaters are not very demanding on membrane materials since the $\mathrm{pH}$ of the feed solution is typically less than 10 . However, since the exact composition of oily wastewaters can vary widely, a membrane material should ideally be able to withstand the wide range of $\mathrm{pHs}$ which may be encountered in the aggressive caustic and acidic cleaning procedures necessary to restore permeate flux. Common membrane materials used in the treatment of oily wastewaters include (1) polymeric (e.g., polyvinyliden fluoride (PVDF), polyamide, and polysulfone), (2) cellulose, and (3) ceramic.

Lipp et al. (1988) reported on the UF treatment of oil-in-water emulsions in which oil rejections of $99.9 \%$ were observed. Regenerated cellulose (5 and 30k MWCO), polysulfone (30k MWCO), polyacrylic (10k MWCO), and polyamide (100k MWCO) membranes were used in the experiments. Oil rejection was independent of membrane material and operating pressure. Total organic carbon (TOC) rejections ranging from 96 
to $98 \%$ were observed. Elevated TOC concentrations in the permeate, relative to the concentration of oil in the permeate, were attributed to the presence of lower molecular weight soluble additives (e.g., surfactants and stabilizers) that passed through the membrane. Although oil rejections of $99.9 \%$ were observed for each membrane material, Lipp et al. (1988) reported that regenerated cellulose membranes were the most easily cleaned. In contrast, Lipp et al. (1988) reported that the polysulfone membranes were not able to be cleaned effectively. Additionally, Canepa et al. (1988) used a tubular polysulfone membrane with a 20k MWCO to concentrate an oily waste down to $30 \%$ of its original volume. However, Canepa et al. did not discuss the durability/cleanability of the polysulfone membrane after treating the oily wastewater.

Mahdi and Skold (1991) used a tubular fluropolymer membrane with a MWCO of 20k to reduce the effluent mineral oil content of a synthetic water-based metal grinding coolant from $44 \mathrm{mg} / \mathrm{L}$ prior to processing to $1 \mathrm{mg} / \mathrm{L}$ after $\mathrm{UF}$ treatment. Bodzek and Konieczy (1992) used polyacrylonitrile (PAN) and polyvinyl chloride (PVC) tubular membranes with MWCOs ranging from 20k to 50k to treat a waste oil emulsion. Bodzek and Konieczy reported that COD retentions of greater than $95 \%$ were observed using these membranes. Additionally, polymeric membranes with MWCOs ranging from 5k to 50k were used by Zaidi et al. (1992) to remove oil successfully from oilfield brine. Reed et al. (Sep. Sci. \& Tech. 1997) reported the effective use of a 100k MWCO PVDF tubular membrane to concentrate a waste metal-working fluid to a maximum of 116 times its initial oil concentration of $\sim 0.2$ to $0.5 \%$.

Oil volume reductions of 95 to $99 \%$ were reported by Bodzek and Konieczny (1992) for the treatment of a metal-working lubricant using polyacrylonitrile and 
polyvinyl chloride tubular membranes with MWCOs ranging from 20k to 50k in a pilotscale UF setup. Reed et al. (1997a) reported on the pilot-scale treatment of an aluminum manufacturer's $\sim 0.2$ to $0.5 \%$ oil and grease wastewater using tubular PVDF UF membranes. Volume reductions ranging from 94 to $99 \%$ were reported in these experiments.

Membrane surface properties can also affect permeate flux and quality. Electrostatic repulsion between the membrane and the contaminant can be used to enhance waste solute retention and increase flux. For example, a membrane with a negative surface charge can be used to treat a waste containing a negatively charged contaminant. Reed et al. (1997a) reported that a negatively charged tubular membrane with a MWCO of $120 \mathrm{k}$ had a higher flux and similar permeate quality when compared with a membrane having a neutral surface charge and an MWCO of 100k when treating a $\sim 0.2$ to $0.5 \% \mathrm{O} / \mathrm{G}$ wastewater. In this case, Reed et al. hypothesized that the electrostatic repulsion between the negative charge of the membrane surface and the negative charge of the emulsified oil droplets decreased the solute boundary layer thickness and oil adsorption on the membrane surface which improved the membrane performance.

\subsubsection{Mechanically Enhanced Ultrafiltration Systems}

Reed et al. (1997b) reported on an applied study of the treatment of an aluminum manufacturers oily wastewater using a high-shear rotary UF system. A comparison of a $100 \mathrm{k}(\sim 0.01 \mu \mathrm{m}$ pore size $)$ polyvinlyden fluoride membrane with a ceramic $\left(\mathrm{TiO}_{2} / \mathrm{Al}_{3} \mathrm{O}_{2}\right.$, $0.11 \mu \mathrm{m}$ average pore size) membrane in a high-shear rotary UF system was conducted and it was concluded that the performance of the ceramic membrane was superior to that of the PVDF with regard to flux quantity and quality as well as membrane 
cleaning/durability. In the experiments, an oily wastewater was concentrated from 5 to as high as $70 \%$ using a $0.11 \mu \mathrm{m}$ ceramic membrane in the high-shear rotary UF system.

Reed et al. (1997b) also reported on the effects of feed temperature on permeate flux. When the feed temperature was increased from 100 to $140{ }^{0} \mathrm{~F}$, for a $20 \%$ waste metal-working fluid feed treated in a high-shear rotary operated at $1,750 \mathrm{rpm}$, permeate flux increased by $\sim 45 \%$. However, the theoretically anticipated flux increase, based on the change in permeate viscosity, was $\sim 21 \%$. Reed et al. attributed the larger than anticipated increase in permeate flux to an increase in solute molecule diffusivity at elevated temperature. Thus, using the ceramic membrane, the researchers were able to make better use of a strong relationship between feed temperature and permeate flux by operating the system at higher temperatures than would have been possible with a PVDF membrane. Additionally, the ceramic membrane was able to be cleaned effectively (using $\mathrm{NaOH} /$ surfactant and acid solutions) and reused after treating concentrated oily wastewaters (up to $70 \%$ oil in the feed), whereas it was not possible to fully recover a satisfactory permeate flux when treating the same type of wastewater using the PVDF membrane. In this case, a new PVDF membrane was required for each experiment.

Reed et al. (1997b) also reported on the effects of membrane rotational speed and hydraulic turbulence on permeate flux in a high-shear rotary UF system. The n value was consistent with the range of 0.8 to 1.2 for turbulent hydraulic flow condition reported by Cheryan (1986). Reed et al. noted that when the membrane rotational speed was decreased from 1,750 to $1,000 \mathrm{rpm}$, permeate flux decreased. It was hypothesized that the decrease in permeate flux was due to an increase in the thickness of the solute concentration boundary layer at the membrane surface. When the membrane rotational 
speed was increased from 1,000 to 1,500 an increase in permeate flux was observed. The authors hypothesized that the increase in permeate flux was due to a decrease in the thickness of the solute concentration boundary layer. A decrease in permeate flux was observed when the rotational speed was decreased from 1,500 to 1,250 rpm (similarly to the first decrease in rotational speed from 1,750 to $1,000 \mathrm{rpm}$ ). When the membrane rotational speed was increased from 1,250 to the pre-excursion value of $1,750 \mathrm{rpm}$, the permeate flux increased. However, the permeate flux did not recover to the initial preexcursion level. Reed et al. hypothesized that the gel layer was sufficiently stable to reduce the effects of increased hydraulic turbulence as the membrane rotational speed was raised. Thus, Reed et al. recommended running the high-shear rotary UF system at the highest rotational speed possible.

Viadero and Reed (1999) conducted a study investigating the application of the thin-film model to data collected from the HSRUF of a synthetic MW fluid. The thinfilm model adequately described the limiting flux data collected in this study. A gel layer concentration of $39 \%$ was reported, which was comparable to many other values reported in the literature for oil-in-water emulsions. Further, the gel layer was shown to be independent of operating parameters (i.e., average transmembrane pressure and membrane rotational speed). Through an examination of the limiting permeate fluxReynolds Number relationship and comparison with other studies presented in the literature, it was determined that the HSRUF system was more effective than conventional units at minimizing the thickness of the solute boundary layer and promoting mass transfer at the membrane surface. Thus, Viadero and Reed (1999) 
concluded that it is possible to treat higher feed concentrations with the HSRUF system than with conventional UF modules.

Viadero et al. (1999) conducted a study examining application of the resistancein-series approach to permeate flux modeling to data collected from the HSRUF of a synthetic MW fluid. The RIS model was successful in describing permeate flux behavior observed in this study. It was determined that the total membrane and the fouling resistances were independent of membrane rotational speed and feed oil concentration. Additionally, the potential for membrane pore plugging or solute adsorption onto the membrane surface was minimal due to the low fouling resistance observed in the HSRUF system. It was also shown that polarization was the predominant rate controlling resistance in the HSRUF of synthetic MW fluids. Viadero et al. discussed how the high permeate flux and low fouling and polarization resistances obtained by operating at high membrane rotational speeds must be weighed against the corresponding increase in operating costs as a feed stream is concentrated.

Viadero et al. (1999) also presented a modified form of the RIS model based on an explicit form of the resistance index in terms of membrane rotational speed and feed MW fluid concentration. The modified RIS model presented by Viadero et al. was successful in predicting permeate flux versus pressure data collected in this study. Additionally, a set-point operating pressure was determined as a function of membrane rotational speed and feed MW fluid concentration. Viadero et al. described how fouling and polarization resistances can be minimized by maintaining the optimum pressure developed in this study as wastes are concentrated; thus, enhanced operating efficiency, operational cost savings, and an extension of membrane life can be achieved. 


\section{CHAPTER 3.0 INVESTIGATIVE APPROACH}

In this chapter, the experimental design, materials and methods, and quality assurance/quality control measures used in this study are described. Average transmembrane pressures are designated as $\mathrm{P}$, average relative velocities as $\mathrm{v}$, and metalworking (MW) fluid kinematic viscosities are designated as $\eta$.

\subsection{BASELINE METAL WORKING FLUID CHARACTERISTICS}

\subsubsection{Metal Working Fluid Density}

The densities of each concentration of MW fluid examined were determined at $43.3 \pm 1{ }^{\circ} \mathrm{C}\left(110 \pm 2{ }^{\circ} \mathrm{F}\right)$ by measuring the mass of $100 \mathrm{ml}$ of a stock solution in a tarred volumetric flask using a Denver Instrument Company Model 100A analytical balance (accurate to $+0.0001 \mathrm{~g}$ ). Each MW fluid concentration was heated to $43.3{ }^{\circ} \mathrm{C}$ on a laboratory heating plate. The temperature was measured using a Fisher Scientific digital temperature probe. A $100 \mathrm{ml}$ sample of each heated MW fluid concentration was transferred into a tarred $100 \mathrm{ml}$ volumetric flask and its mass was measured. The temperature of the solution was then re-measured to determine if the temperature had deviated from the $43.3 \pm 1{ }^{\circ} \mathrm{C}$ range. If the temperature was outside the specified range after measuring the mass, a new sample was measured. Additionally, analytical triplicates (i.e., three separate $100 \mathrm{ml}$ samples from the same $1 \mathrm{~L}$ stock solution) were performed for each MW fluid concentration.

\subsubsection{Metal Working Fluid Absolute Viscosity}

The absolute viscosities of the MW fluid concentrations examined in this study were determined to enable comparison of applications treating a wide variety of feed 
streams. Viscosities were determined at $43.3 \pm 1{ }^{\circ} \mathrm{C}\left(110 \pm 2{ }^{\circ} \mathrm{F}\right)$ using a digital Brookfield model DV-III rheometer equipped with an ultra low (UL) absolute viscosity adapter and a built-in temperature probe. A $500 \mathrm{ml}$ sample of each MW fluid concentration was heated to $43.3{ }^{\circ} \mathrm{C}$ in a $600 \mathrm{ml}$ beaker using a laboratory heating plate (a $600 \mathrm{ml}$ sample cell was recommended by Brookfield Engineering Laboratories). The rheometer's built-in temperature probe was used to monitor the MW fluid temperature. The rheometer was positioned above the sample and heating plate, leveled, and immersed in the MW fluid up to the designated mark on the UL adapter. The absolute viscosity of each MW fluid was then measured at a shear rate of $5 \mathrm{rpm}$. After analyzing the six MW fluids, stock solutions of 45, 47, and 48\% MW fluid were prepared and analyzed to obtain additional absolute viscosity data due to a large increase in absolute viscosity observed between 40 and 50\% MW fluid. To check the accuracy of the rheometer, the absolute viscosity of deionized water was measured at $43.3{ }^{\circ} \mathrm{C}$ and $5 \mathrm{rpm}$. The average absolute viscosity of the water sample was $0.56 \mathrm{cP}$ and ranged from 0.48 to $0.60 \mathrm{cP}$. All absolute viscosity measurements were performed in triplicate.

\subsection{TUBULAR ULTRAFILTRATION}

\subsubsection{Experimental Apparatus}

A schematic of the tubular ultrafiltration experimental apparatus is presented in Figure 3.1. The feed solution was transferred from the feed tank and pressurized using a Grundfos model CR4-50U positive displacement pump capable of a maximum feed pressure of $745 \mathrm{kPa}(108 \mathrm{psi})$ at a zero gpm outlet flowrate. Feed solutions were prepared and processed in a 200L Nalgene tank, while all cleanings were performed from a similar 60L tank. 


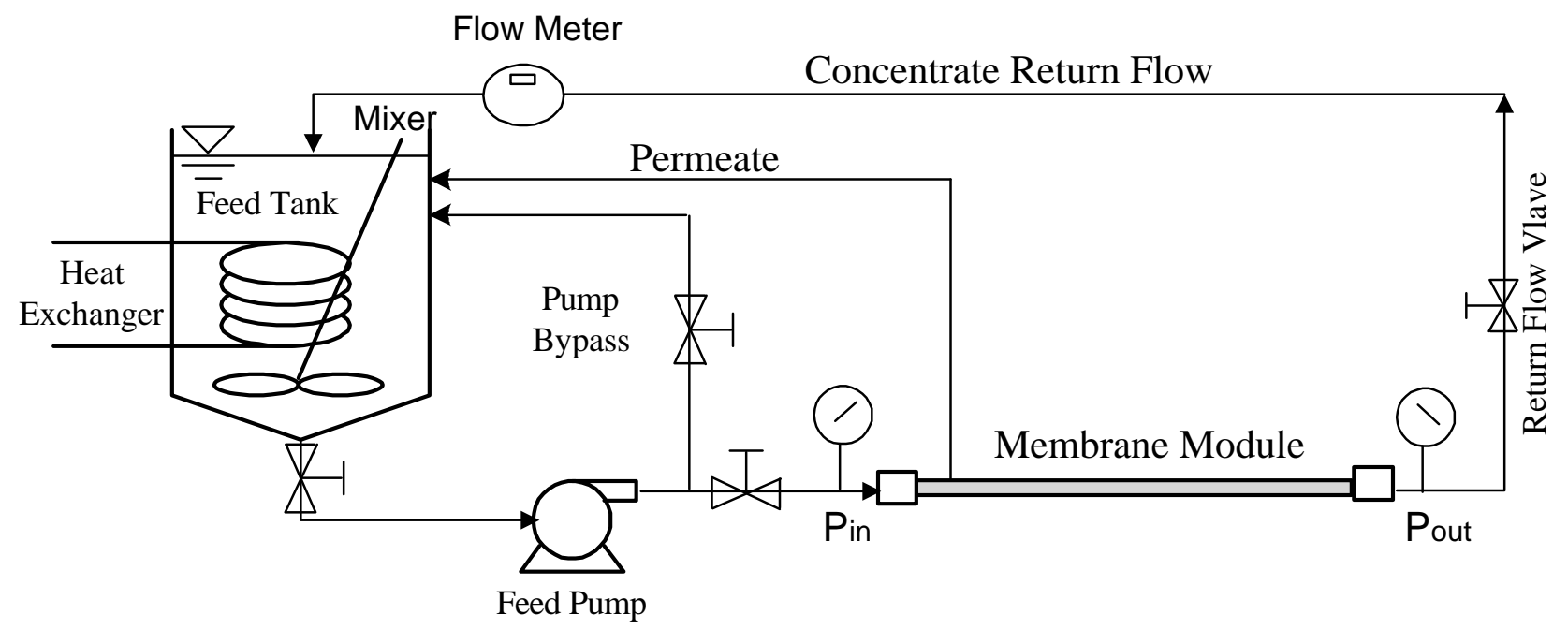

Figure 3.1. A schematic of the tubular ultrafiltration experimental apparatus.

The feed flow and pressure to the membrane tube were controlled using a bypass valve installed on the pump. The inlet and outlet pressures to the membrane tube were measured using pressure gauges with a $690 \mathrm{kPa}(100 \mathrm{psi})$ maximum. The pressure gauges, manufactured by Weksler Glass Thermometer Corp., were graduated in $7 \mathrm{kPa}$ (1 psi) increments. The average transmembrane pressure was calculated as the average of the inlet and outlet pressures:

$$
\mathrm{P}=\frac{\mathrm{P}_{\text {in }}+\mathrm{P}_{\text {out }}}{2}
$$

The concentrate return flowrate was measured with a Signet 8512 paddle wheel flow meter, which was controlled by adjusting the return flow valve located on the concentrate return line as presented in Figure 3.1. The return flow rate could also be measured manually using a graduated cylinder and a stopwatch. This procedure was used to periodically check the calibration of the flow meter. Velocity relative to a stationary reference frame (v) was calculated for each return flow examined in the tubular UF unit as a basis for comparison with the high-shear rotary (HSR) UF system. Tubular UF average relative velocity was calculated as: 


$$
\mathrm{v}=\frac{\mathrm{Q}_{\mathrm{r}}}{\mathrm{A}}
$$

where $\mathrm{Q}_{\mathrm{r}}$ is the concentrate return flow rate, and $\mathrm{A}$ is the cross-sectional area of the membrane tube $\left(5 \mathrm{~cm}^{2}, 0.8 \mathrm{in}^{2}\right)$. The concentrate return flowrate was varied from 5 to 30 gpm corresponding to average relative velocities ranging from 0.62 to $3.70 \mathrm{~m} \cdot \mathrm{s}^{-1}$. The feed and permeate temperatures were measured with certified Fisher Scientific digital temperature probes, and the feed temperature was controlled by passing tap water through a copper coil heat exchanger located in the process tank. Additionally, a mixer located in the process tank was used to maintain feed homogeneity and to aid in temperature control.

Reed et al. (1997a) conducted a study investigating the pilot-scale treatment of a waste MW fluid using different tubular UF membranes. A polyvinylidene fluoride (PVDF) membrane with a net negative surface charge and a 120,000 MWCO was shown to be most successful in treating the MW fluid. The feed stream examined by Reed et al. (1997) was a waste version of the synthetic MW fluid studied in this research; thus, a similar polymeric membrane (Koch Membrane Systems, Inc.) was selected for use in the tubular UF experiments conducted in this study. A summary of the polymeric membrane characteristics is presented in Table 3.1 .

\subsubsection{Experimental Conduct}

\subsubsection{Metal-Working Fluid Experiments}

Twenty-four experiments at discrete average relative velocity/feed viscosity combinations were conducted using a synthetic MW fluid as a surrogate waste stream. Each individual experiment was conducted under constant $v-\eta$ conditions. A summary of operational conditions applied in each discrete experiment is presented in Table 3.2. 
Table 3.1 A summary of the polymeric membrane characteristics.

\begin{tabular}{|c|c|}
\hline Manufacturer & Koch Membrane Systems, Inc. \\
\hline Designation & HFP-276-FNO \\
\hline Composition & Polyvinylidene fluoride (PVDF) \\
\hline Molecular Weight Cutoff (MWCO) & Negative \\
\hline Surface Charge & $120,5-10.5$ \\
\hline Housing Material & $49^{\circ} \mathrm{C}\left(120^{\circ} \mathrm{F}\right)$ \\
\hline $\mathrm{pH}$ range & $586 \mathrm{kPa}(85 \mathrm{psi})$ \\
\hline Maximum temperature & $4.2 \mathrm{~cm}(1.6 \mathrm{in})$ \\
\hline Maximum inlet pressure & $2.54 \mathrm{~cm}(1 \mathrm{in})$ \\
\hline Outer diameter & $3.05 \mathrm{~m}(10 \mathrm{ft})$ \\
\hline Inner (flow channel) diameter & $0.20 \mathrm{~m}^{2}\left(2.2 \mathrm{ft}^{2}\right)$ \\
\hline Length & \\
\hline Total membrane area & \\
\hline
\end{tabular}

Feed Preparation: A base MW fluid was used to make the 1 to $30 \%$ metalworking fluid solutions used in these experiments. The base MW fluid contained $85 \%$ mineral oil and $15 \%$ proprietary additives (i.e., emulsifying agents, film strength additives, etc.). Distilled water used to make the feed oil package and cleaning solutions was obtained from West Virginia University Civil and Environmental Engineering Laboratory's house supply. The $\mathrm{pH}$ and conductivity of the distilled water were measured at room temperature and compared with previously observed values to judge the quality of the water prior to being used in these experiments. The $\mathrm{pH}$ and conductivity ranges of distilled water used to make the feed MW fluid solutions were 6.69 to 7.27 and 1.17 to $6.88 \mu \mathrm{S} / \mathrm{cm}$, respectively, and did not vary greatly; thus, bias in the results due to changes in the characteristics of the distilled water were discarded. In each experiment, $100 \mathrm{~L}$ of feed solution was freshly prepared in the feed tank by adding the appropriate volume of base MW fluid to distilled water. Freshly prepared feed emulsions were used in each experiment to minimize changes in feed properties due to high heat and/or shear, as recommended by Cheryan (1998). 
Table 3.2 Summary of operational conditions applied to each discrete tubular experiment.

\begin{tabular}{|c|c|c|c|c|c|c|}
\hline $\begin{array}{l}\text { Feed Viscosity } \\
\qquad \begin{array}{c}\rightarrow \\
\text { Cross-Flow }\end{array} \\
\text { Velocity } \\
\downarrow\end{array}$ & $\begin{array}{c}0.40 \text { cSt } \\
\text { (0.85\% oil, 1\% } \\
\text { MW Fluid) }\end{array}$ & $\begin{array}{c}\text { 0.46 cSt } \\
\text { (4.3\% oil, 5\% } \\
\text { MW Fluid) }\end{array}$ & $\begin{array}{c}0.64 \text { cSt } \\
\text { (8.5\% oil, 10\% } \\
\text { MW Fluid) }\end{array}$ & $\begin{array}{c}\text { 0.94 cSt } \\
\text { (12.8\% oil, 15\% } \\
\text { MW Fluid) }\end{array}$ & $\begin{array}{c}1.2 \mathrm{cSt} \\
(17.0 \% \text { oil, } 20 \% \\
\text { MW Fluid) }\end{array}$ & $\begin{array}{c}4.5 \text { cSt } \\
(25.5 \% \text { oil, } 30 \% \\
\text { MW Fluid) }\end{array}$ \\
\hline $\begin{array}{l}3.7 \mathrm{~m} \cdot \mathrm{s}^{-1} \\
(30 \mathrm{gpm})\end{array}$ & $\begin{array}{c}35-310 \mathrm{kPa} \\
(5-45 \mathrm{psi})\end{array}$ & $\begin{array}{c}35-414 \mathrm{kPa} \\
(5-60 \mathrm{psi})\end{array}$ & $\begin{array}{c}35-207 \mathrm{kPa} \\
(5-30 \mathrm{psi})\end{array}$ & $\begin{array}{c}35-207 \mathrm{kPa} \\
(5-30 \mathrm{psi})\end{array}$ & $\begin{array}{l}69-207 \mathrm{kPa} \\
(10-30 \mathrm{psi})\end{array}$ & $\begin{array}{l}69-207 \mathrm{kPa} \\
(10-30 \mathrm{psi})\end{array}$ \\
\hline $\begin{array}{l}2.5 \mathrm{~m} \cdot \mathrm{s}^{-1} \\
(20 \mathrm{gpm})\end{array}$ & $\begin{array}{c}35-517 \mathrm{kPa} \\
(5-75 \mathrm{psi})\end{array}$ & $\begin{array}{c}35-414 \mathrm{kPa} \\
(5-60 \mathrm{psi})\end{array}$ & $\begin{array}{c}35-310 \mathrm{kPa} \\
(5-45 \mathrm{psi})\end{array}$ & $\begin{array}{c}35-310 \mathrm{kPa} \\
(5-45 \mathrm{psi})\end{array}$ & $\begin{array}{c}35-207 \mathrm{kPa} \\
(5-30 \mathrm{psi})\end{array}$ & $\begin{array}{l}\text { 69-207 kPa } \\
(10-30 \mathrm{psi})\end{array}$ \\
\hline $\begin{array}{l}1.2 \mathrm{~m} \cdot \mathrm{s}^{-1} \\
(10 \mathrm{gpm})\end{array}$ & $\begin{array}{c}104-517 \mathrm{kPa} \\
(15-75 \mathrm{psi})\end{array}$ & $\begin{array}{l}104-507 \mathrm{kPa} \\
(15-73.5 \mathrm{psi})\end{array}$ & $\begin{array}{c}104-503 \mathrm{kPa} \\
(15-73 \mathrm{psi})\end{array}$ & $\begin{array}{l}104-493 \mathrm{kPa} \\
(15-71.5 \mathrm{psi})\end{array}$ & $\begin{array}{l}76-310 \mathrm{kPa} \\
(11-45 \mathrm{psi})\end{array}$ & $\begin{array}{c}69-207 \mathrm{kPa} \\
(10-30 \mathrm{psi})\end{array}$ \\
\hline $\begin{array}{c}0.62 \mathrm{~m} \cdot \mathrm{s}^{-1} \\
(5 \mathrm{gpm})\end{array}$ & $\begin{array}{c}104-372 \mathrm{kPa} \\
(15-54 \mathrm{psi})\end{array}$ & $\begin{array}{l}104-362 \mathrm{kPa} \\
(15-52.5 \mathrm{psi})\end{array}$ & $\begin{array}{c}104-372 \mathrm{kPa} \\
(15-54 \mathrm{psi})\end{array}$ & $\begin{array}{c}104-372 \mathrm{kPa} \\
(15-54 \mathrm{psi})\end{array}$ & $\begin{array}{l}107-352 \mathrm{kPa} \\
(15.5-51 \mathrm{psi})\end{array}$ & $\begin{array}{c}117-310 \mathrm{kPa} \\
(17-45 \mathrm{psi})\end{array}$ \\
\hline
\end{tabular}


System Operation: Prior to starting the system, the metal-working fluid solution was heated to $29.4 \pm 1{ }^{\circ} \mathrm{C}\left(85 \pm 2{ }^{\circ} \mathrm{F}\right)$ and the mixer was run to homogenize the feed solution and to aid in heating. The system was then started, the applied pressure was set at the minimum value and the concentrate return flow rate was stabilized at the value to be examined by adjusting the feed pump bypass and concentrate return flow valves. In all experiments, operation was conducted in recycle mode (i.e., permeate and concentrate were returned to the feed tank so that the concentration factor of the feed solution remained constant). Throughout each experiment, $\mathrm{P}$ was increased from the lowest to highest value in a stepwise fashion to avoid biasing flux determination by initially operating under conditions which were favorable to the accumulation of oil at the membrane surface. The system was operated at each pressure condition until a quasi steady state permeate flux (flux decline $<10 \%$ over 30 minutes of operation) was achieved, at which point the pressure was increased to the next condition to be examined.

Once all operational parameters were stabilized, the permeate flux as well as permeate and concentrate conductivity; $\mathrm{pH}$; and temperature were measured regularly at each applied pressure condition. Permeate and feed $\mathrm{pH}$ and conductivity were measured at $29.4 \pm 1{ }^{\circ} \mathrm{C}\left(85 \pm 2{ }^{\circ} \mathrm{F}\right)$ as a real time check of permeate quality. Flux measurements were made every two minutes for the first half-hour of operation and then every ten minutes for the remainder of the experiment. The permeate flow was measured by disconnecting the permeate line from the feed tank and measuring the volume of permeate collected over a given period of time using a graduated cylinder and a stopwatch. The $\mathrm{pH}$, conductivity, and turbidity of the permeate sample were also measured. The permeate temperature was measured using a Fisher Scientific digital 
temperature probe placed in the graduated cylinder as a permeate sample was being collected. To obtain a representative reading of the permeate temperature, the thermometer probe was agitated in the cylinder prior to measuring the temperature. The concentrate $\mathrm{pH}$ and conductivity were measured every twenty minutes, immediately after measuring permeate parameters. Since each experiment was conducted in recycle mode (i.e., constant feed concentration), all permeate and concentrate samples were returned to the feed tank after measuring the flux, temperature, $\mathrm{pH}$ and conductivity.

System Shutdown: Upon completing the final applied pressure condition of each discrete $v-\eta$ experiment, the pump was shut off, the pump and lines were drained of any residual feed solution and the cleaning procedure detailed in section 3.2.2.2 was applied. Following system cleaning, the experimental procedure was repeated at the next discrete experiment.

\subsubsection{Membrane Cleaning Procedure}

Upon completing each discrete $v-\eta$ experiment, the system was stopped and the feed tank and lines were drained. The membrane was then cleaned with a solution of "Koch liquid detergent" (KLD; a proprietary alkaline surfactant manufactured by Koch Membrane Systems, Inc. for cleaning of the PVDF membranes) and distilled water to dissolve oils accumulated on the membrane surface. Additionally, spongeballs (manufactured by Koch Membrane Systems, Inc.) were used to mechanically clean any residual oil from the membrane surface (Masciola et al., 2000). The membrane cleaning procedure is summarized below:

1. The system was flushed with $190 \mathrm{~L}(50 \mathrm{gal})$ of tap water $\left(\sim 29.4{ }^{\circ} \mathrm{C} ; 85\right.$ $\left.{ }^{\circ} \mathrm{F}\right)$.

2. The cleaning solution was prepared as follows: 
a) $200 \mathrm{~mL}$ of $\mathrm{KLD}$ was added to $40 \mathrm{~L}$ (7.8 gal) of distilled water $(0.5 \%$ $\mathrm{KLD} v / \mathrm{v} ; \mathrm{pH} \approx 10.8$ ).

b) The cleaning solution was then heated to $48.9 \pm 1{ }^{\circ} \mathrm{C}\left(120 \pm 2{ }^{\circ} \mathrm{F}\right)$. using the copper coil heat exchanger.

3. The membrane was cleaned with the KLD solution for 30 minutes at 1.9 $\mathrm{m} \cdot \mathrm{s}^{-1}(15 \mathrm{gpm}), 48.9 \pm 1{ }^{\circ} \mathrm{C}\left(120 \pm 2{ }^{\circ} \mathrm{F}\right), 276 \mathrm{kPa}(40 \mathrm{psi})$.

4. The membrane was then mechanically cleaned with spongeballs (3 spongeballs, 3 passes each through the membrane tube.

5. Upon completion of the spongeball sequence, the KLD cleaning solution was recycled through the system for an additional 30 minutes using the same operating parameters presented in step 3.

6. Cleaning procedure steps 3-5 were repeated until no further increase in permeate flux was observed from the end of step 3 to the beginning of step 5. The cleaning procedure was conducted for a total of 1 operational hour when no flux decline was observed.

7. The system was drained and flushed with $190 \mathrm{~L}$ (50 gal) of tap water $\left(\sim 29.4^{\circ} \mathrm{C} ; 85^{\circ} \mathrm{F}\right) . \quad 190 \mathrm{~L}$ was determined to be the volume necessary to reduce the $\mathrm{pH}$ of the effluent flush water to the $\mathrm{pH}$ of tap water after cleaning with KLD.

\subsubsection{Quality Assurance/Quality Control}

After the entire matrix of experiments presented previously in Table 3.2 was completed, 3 of the $24(\sim 13 \%)$ discrete experiments were replicated to ascertain whether changes in membrane characteristics (due to pore plugging, fouling, or chemical/surface changes) had an influence on the determination of permeate flux. Permeate flux data for initial and replicate experiments are presented in Table 3.3. Limiting fluxes at each of the replicate experiments deviated less than 5\% from values calculated with initial experimental data; thus, it was concluded that the data collected in this study were not biased by changes in membrane characteristics. 
Table 3.3 Summary of tubular UF QA/QC replicate experiments.

\begin{tabular}{|c|c|c|c|}
\hline Experiment ID & $\begin{array}{c}\text { Initial limiting } \\
\text { flux, } \\
\mathbf{m}^{3} \cdot \mathbf{m}^{-2} \cdot \mathbf{d}^{-1} \\
\left(\mathrm{gal}^{-1} \mathbf{f t}^{-2} \cdot \mathbf{d}^{-1}\right)\end{array}$ & $\begin{array}{c}\text { Replicate limiting } \\
\text { flux, } \\
\mathbf{m}^{3} \cdot \mathbf{m}^{-2} \cdot \mathbf{d}^{-1} \\
\left(\mathrm{gal}^{-1} \mathbf{f t}^{-2} \cdot \mathbf{d}^{-1}\right)\end{array}$ & $\begin{array}{c}\% \\
\text { Difference }\end{array}$ \\
\hline $\begin{array}{c}1.2 \mathrm{~m} \cdot \mathrm{s}^{-1}-0.64 \mathrm{cSt} \\
(10 \mathrm{gpm}-10 \% \text { MW Fluid) }\end{array}$ & $1.61(40)$ & $1.61(40)$ & 0 \\
\hline $\begin{array}{c}0.62 \mathrm{~m} \cdot \mathrm{s}^{-1}-0.46 \mathrm{cSt} \\
(5 \mathrm{gpm}-5 \% \text { MW Fluid })\end{array}$ & $1.35(33)$ & $1.33(32)$ & 0.02 \\
\hline $\begin{array}{c}0.62 \mathrm{~m} \cdot \mathrm{s}^{-1}-0.64 \mathrm{cSt} \\
(5 \mathrm{gpm}-10 \% \text { MW Fluid) }\end{array}$ & $0.83(20)$ & $0.79(19)$ & 4.8 \\
\hline
\end{tabular}

\subsubsection{Permeate Flux Temperature Correction}

Tubular UF experiments were conducted at a constant feed temperature $29.4 \pm 1$ ${ }^{\circ} \mathrm{C}\left(85 \pm 2{ }^{\circ} \mathrm{F}\right)$ in an attempt to closely mimic the operating parameters of typical fullscale tubular UF treatment applications. However, absolute viscosity measurements were taken at $43.3 \pm 1{ }^{\circ} \mathrm{C}\left(110 \pm 2{ }^{\circ} \mathrm{F}\right)$ (see section 3.1.2). Further, high-shear rotary (HSR) UF experiments were also conducted at $43.3 \pm 1{ }^{\circ} \mathrm{C}\left(110 \pm 2{ }^{\circ} \mathrm{F}\right)$ (see section 3.3.2.1); thus, tubular UF data were temperature corrected from 29.4 to $43.3{ }^{\circ} \mathrm{C}$ using Equation 2.3. All results and data reduction presented in the proceding sections of this paper were conducted with corrected tubular data in order to allow for comparison with the HSRUF system.

\subsection{HIGH-SHEAR ROTARY ULTRAFILTRATION}

\subsubsection{Experimental Apparatus}

A single disk high-shear rotary ultrafiltration unit (manufactured by SpinTek Systems, Inc.) was used in this study. A schematic of the HSRUF experimental apparatus is presented in Figure 3.2. A schematic of the HSRUF pressure vessel was presented previously in Figure 2.12. In the system, one 8 inch $(20 \mathrm{~cm})$ round membrane disk pack 


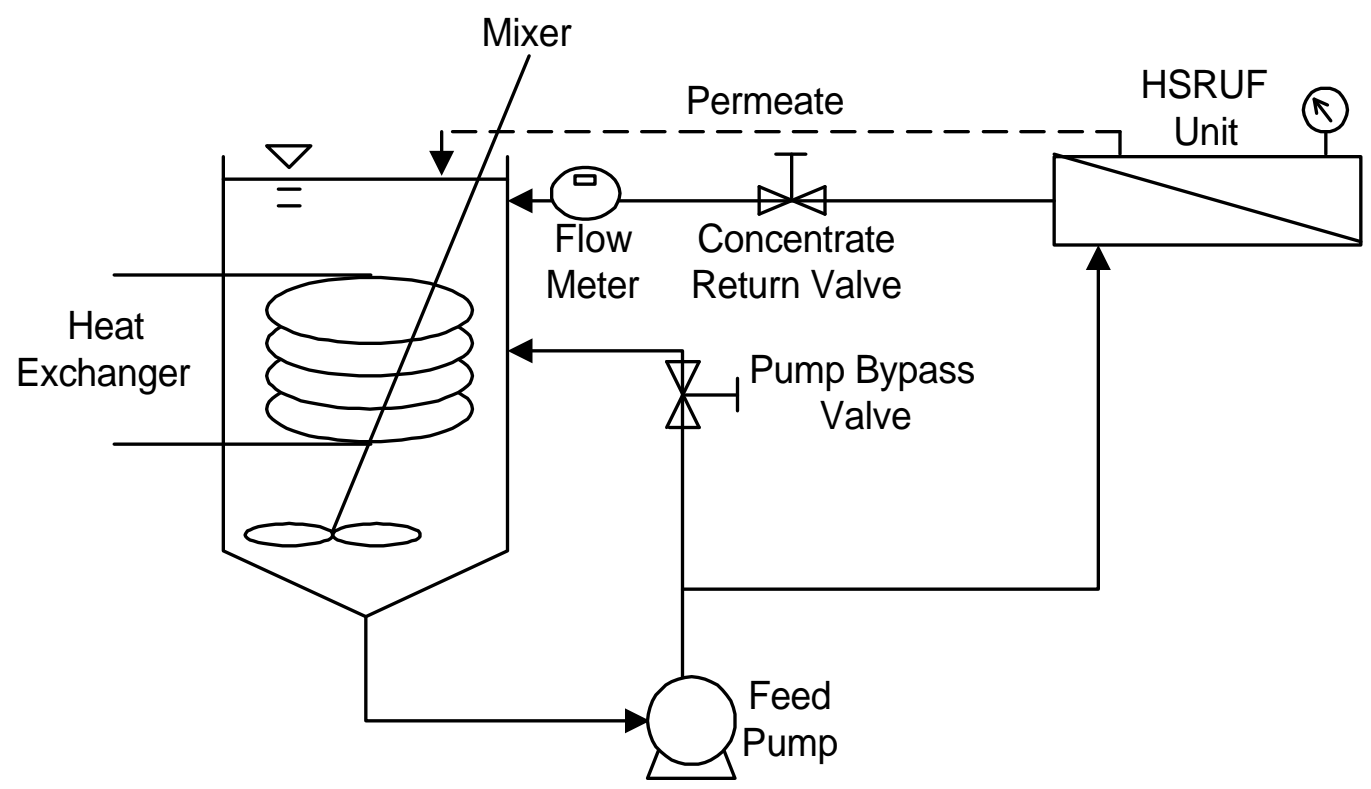

\section{Figure 3.2 A schematic of the high-shear rotary ultrafiltration experimental apparatus.}

was set on a hollow rotating shaft inside the vessel housing. The feed stream entered the membrane chamber under pressure, which was supplied by a Grundfos model CRN2 centrifugal pump capable of a maximum feed pressure of $\sim 540 \mathrm{kPa}$ (78 psi) at zero gpm outlet flow rate. Feed and cleaning solutions were prepared and processed in a $60 \mathrm{~L}$ Nalgene tank. The feed flow and pressure to the vessel were controlled using a bypass valve installed on the pump. The applied pressure was measured using a $690 \mathrm{kPa}(100$ psi) maximum pressure gauge installed on top of the pressure vessel (see Figure 3.2). The pressure gauge, manufactured by McDaniel Controls, Inc., was graduated in $7 \mathrm{kPa}$ (1 psi) increments. The average and maximum permeate backpressures over the range of membrane rotational speeds investigated in this study were accounted for to ensure a positive transmembrane pressure across the entire membrane disk. Average and maximum permeate backpressures at membrane rotational speeds investigated in this study are presented in Table 3.4. 
Table 3.4. Average and maximum permeate backpressures.

\begin{tabular}{||c|c|c||}
\hline $\begin{array}{c}\text { Membrane Rotational Speed, } \\
\mathrm{R}_{\mathrm{j}}(\mathrm{rpm})\end{array}$ & Average $\mathrm{p}_{\text {back }}(\mathrm{psi})$ & Maximum pack $(\mathrm{psi})$ \\
\hline 1,750 & 11.1 & 20.6 \\
\hline 1,450 & 7.6 & 14.1 \\
\hline 1,150 & 4.8 & 9.0 \\
\hline
\end{tabular}

The average transmembrane pressure was calculated as:

$$
\mathrm{P}=\mathrm{P}_{\text {applied }}-\mathrm{P}_{\text {back-avg }}
$$

where $\mathrm{P}$ is the average transmembrane pressure, $\mathrm{P}_{\text {applied }}$ is the applied pressure and $\mathrm{P}_{\text {back- }}$ avg is the average permeate backpressure (calculated by equation 2.14).

The concentrate return flow rate was measured using a Signet 8511 turbine flow meter, as presented in Figure 3.2, and was maintained at about $0.5 \mathrm{~m} \cdot \mathrm{s}^{-1}(\sim 1.0 \mathrm{gpm})$ throughout these experiments by adjusting return flow valve installed on the concentrate return line. Additionally, a shut-off ball valve was added to the concentrate return line so the return flow rate could be measured manually using a graduated cylinder and a stopwatch. This procedure was used to periodically check the calibration of the turbine flow meter. As with the tubular UF unit, velocity relative to a stationary reference frame (v) was calculated for each membrane rotational speed examined in the HSR UF system as a basis for comparison of the two membrane separation systems. As presented earlier, the concentrate return flow in the HSR UF system was held constant at $\sim 0.5 \mathrm{~m} \cdot \mathrm{s}^{-1}$, which represents nominal fluid flow through the membrane vessel when compared to membrane velocities of 8 to $12 \mathrm{~m} \cdot \mathrm{s}^{-1}$ (1150 to $\left.1750 \mathrm{rpm}\right)$. Thus, it was assumed that the fluid velocity was negligible and the average relative velocity in the HSR UF system was calculated as: 


$$
\mathrm{v}=\omega \cdot \mathrm{r}
$$

where $\omega$ is the membrane rotational speed and $r$ is the membrane radius. In the HSR UF system, average relative velocity is radially distributed; thus, the radius of gyration (an average radial dimension of a rotating body) for the membrane disk was selected as a representative property:

$$
\mathrm{V}=\omega \cdot \mathrm{I}
$$

where I, the radius of gyration for the membrane disk is calculated as:

$$
I=\sqrt{\frac{R_{i}^{2}+R_{o}^{2}}{2}}
$$

where $R_{i}$ and $R_{o}$ are the inner and outer radii of the membrane disk, respectively (see Table 3.5). The rotation of the membrane disk could be varied from 1,150 to $1,750 \mathrm{rpm}$ corresponding to average relative velocities ranging from 8 to $12 \mathrm{~m} \cdot \mathrm{s}^{-1}$. These limiting values were preset by the manufacturer and could not be altered on-site. The feed and permeate temperatures were measured with certified Fisher Scientific digital temperature probes, and the feed temperature was controlled by passing tap water through a copper coil heat exchanger located in the process tank. Additionally, a mixer located in the process tank was used to maintain feed homogeneity and to aid in temperature control.

A number of studies have been conducted showing the successful treatment of a feed stream identical to the synthetic MW fluid examined in this study using a ceramic membrane in HSRUF system (Viadero and Reed 1999; Viadero et al. 1999; Viadero et al. 2000). Further, Reed et al. (1997b) presented the successful treatment of a waste MW fluid using a ceramic membrane in the HSRUF system. The feed stream examined by Reed et al. (1997b) was a waste version of the synthetic MW fluid studied in this research; thus, a ceramic membrane was chosen for use in the HSRUF system 
experiments conducted in this study. A summary of the ceramic membrane characteristics is presented in Table 3.5.

Table 3.5 Summary of the ceramic membrane characteristics.

\begin{tabular}{|c|c||}
\hline Manufacturer & TRUMEM \\
\hline Lot number & CRM-011M-TA \\
\hline Chemical composition & $\mathrm{TiO}_{2} / \mathrm{Al}_{2} \mathrm{O}_{3}$ \\
\hline Mean pore size & $0.11 \mu \mathrm{m}$ \\
\hline Maximum pore size & $0.70 \mu \mathrm{m}$ \\
\hline Total membrane area & $0.53 \mathrm{ft}^{2}$ \\
\hline Maximum operating temperature & $>158{ }^{0} \mathrm{~F}$ \\
\hline Maximum operating pressure & $>150 \mathrm{psi}$ \\
\hline Thickness & $240 \mu \mathrm{m}$ \\
\hline Inner radius & $0.975 \mathrm{in}$. \\
\hline Outer radius & $3.625 \mathrm{in}$. \\
\hline Radius of gyration & $2.654 \mathrm{in}$. \\
\hline \hline
\end{tabular}

\subsubsection{Experimental Conduct}

\subsubsection{Metal-Working Fluid Experiments}

Twenty-four experiments at discrete average relative velocity/feed viscosity combinations were conducted using a synthetic MW fluid as a surrogate waste stream. Each individual experiment was conducted under constant $v-\eta$ conditions. A summary of operational conditions applied in each discrete experiment is presented in Table 3.6. Each discrete $v-\eta$ experiment was conducted in recycle mode, with a total feed volume of $40 \mathrm{~L}$, at a feed temperature of $43.3 \pm 1^{\circ} \mathrm{C}\left(110 \pm 2{ }^{\circ} \mathrm{F}\right)$, and a return flow rate of $3.75 \pm$ $0.75 \mathrm{~L} \cdot \mathrm{m}^{-1}(1.0 \pm 0.2 \mathrm{gpm})$ 
Table 3.6 Experimental Matrix for Surrogate Waste Experiments conducted using High-Shear Rotary Ultrafiltration.

\begin{tabular}{|c|c|c|c|c|c|c|c|c|}
\hline $\begin{array}{c}\text { Average Relative } \\
\text { Velocity } \\
\downarrow\end{array}$ & $\begin{array}{c}\text { 0.46 cSt } \\
\text { (4.3\% oil, } \\
5 \% \text { MW } \\
\text { Fluid) }\end{array}$ & $\begin{array}{l}\text { 0.64 cSt } \\
\text { (8.5\% oil, } \\
\text { 10\% MW } \\
\text { Fluid) }\end{array}$ & $\begin{array}{l}0.94 \text { cSt } \\
\text { (12.8\% oil, } \\
15 \% \text { MW } \\
\text { Fluid) }\end{array}$ & $\begin{array}{c}1.2 \mathrm{cSt} \\
(17.0 \% \text { oil, } \\
20 \% \mathrm{MW} \\
\text { Fluid) }\end{array}$ & $\begin{array}{l}4.5 \mathrm{cSt} \\
\text { (25.5\% oil, } \\
30 \% \mathrm{MW} \\
\text { Fluid) }\end{array}$ & $\begin{array}{c}8.1 \text { cSt } \\
\text { (34.0\% oil, } \\
\text { 40\% MW } \\
\text { Fluid) }\end{array}$ & $\begin{array}{c}45.6 \mathrm{cSt} \\
\text { (42.5\% oil, } \\
50 \% \mathrm{MW} \\
\text { Fluid) }\end{array}$ & $\begin{array}{l}121 \mathrm{cSt} \\
\text { (51.0\% oil, } \\
\text { 60\% MW } \\
\text { Fluid) }\end{array}$ \\
\hline $\begin{array}{c}12 \mathrm{~m} \cdot \mathrm{s}^{-1} \\
(1750 \mathrm{rpm})\end{array}$ & $\begin{array}{c}173-517 \\
\mathrm{kPa} \\
(25-75 \mathrm{psi})\end{array}$ & $\begin{array}{c}173-517 \\
\mathrm{kPa} \\
(25-75 \mathrm{psi})\end{array}$ & $\begin{array}{c}138-517 \\
\mathrm{kPa} \\
(20-75 \mathrm{psi})\end{array}$ & $\begin{array}{c}138-483 \\
\mathrm{kPa} \\
(20-70 \mathrm{psi})\end{array}$ & $\begin{array}{c}173-483 \\
\mathrm{kPa} \\
(25-70 \mathrm{psi})\end{array}$ & $\begin{array}{c}173-483 \\
\mathrm{kPa} \\
(25-70 \mathrm{psi})\end{array}$ & $\begin{array}{c}173-483 \\
\mathrm{kPa} \\
(25-70 \mathrm{psi})\end{array}$ & $\begin{array}{c}173-483 \\
\mathrm{kPa} \\
(25-70 \mathrm{psi})\end{array}$ \\
\hline $\begin{array}{c}10 \mathrm{~m} \cdot \mathrm{s}^{-1} \\
(1450 \mathrm{rpm})\end{array}$ & $\begin{array}{c}173-517 \\
\mathrm{kPa} \\
(25-75 \mathrm{psi})\end{array}$ & $\begin{array}{c}173-517 \\
\mathrm{kPa} \\
(25-75 \mathrm{psi})\end{array}$ & $\begin{array}{c}173-483 \\
\mathrm{kPa} \\
(25-70 \mathrm{psi})\end{array}$ & $\begin{array}{c}104-483 \\
\mathrm{kPa} \\
(15-70 \mathrm{psi})\end{array}$ & $\begin{array}{c}173-483 \\
\mathrm{kPa} \\
(25-70 \mathrm{psi})\end{array}$ & $\begin{array}{c}138-483 \\
\mathrm{kPa} \\
(20-70 \mathrm{psi})\end{array}$ & $\begin{array}{c}138-483 \\
\mathrm{kPa} \\
(20-70 \mathrm{psi})\end{array}$ & $\begin{array}{c}138-483 \\
\mathrm{kPa} \\
(20-70 \mathrm{psi})\end{array}$ \\
\hline $\begin{array}{c}8 \mathrm{~m} \cdot \mathrm{s}^{-1} \\
(1150 \mathrm{rpm})\end{array}$ & $\begin{array}{c}173-517 \\
\mathrm{kPa} \\
(25-75 \mathrm{psi})\end{array}$ & $\begin{array}{c}173-517 \\
\mathrm{kPa} \\
(25-75 \mathrm{psi})\end{array}$ & $\begin{array}{c}104-483 \\
\mathrm{kPa} \\
(15-70 \mathrm{psi})\end{array}$ & $\begin{array}{c}104-483 \\
\mathrm{kPa} \\
(15-70 \mathrm{psi})\end{array}$ & $\begin{array}{c}104-483 \\
\mathrm{kPa} \\
(15-70 \mathrm{psi})\end{array}$ & $\begin{array}{c}104-483 \\
\mathrm{kPa} \\
(15-70 \mathrm{psi})\end{array}$ & $\begin{array}{c}104-483 \\
\mathrm{kPa} \\
(15-70 \mathrm{psi})\end{array}$ & $\begin{array}{c}68.9-448 \\
\mathrm{kPa}(10-65 \\
\mathrm{psi})\end{array}$ \\
\hline
\end{tabular}


Feed Preparation: A base MW fluid was used to make the 5 to $60 \%$ metalworking fluid solutions in these experiments as presented previously for the tubular UF unit. Distilled water to make the feed oil package solution and cleaning solutions was obtained from West Virginia University Civil and Environmental Engineering Laboratory's house supply. $40 \mathrm{~L}$ feed solutions were freshly prepared in the feed tank by adding the appropriate volume of base MW fluid to distilled water. Freshly prepared feed emulsions were used in each experiment to minimize changes in feed properties due to high heat and/or shear, as recommended by Cheryan (1998).

System Operation: Prior to starting the system, the metal-working fluid solution was heated to $43.3 \pm 1{ }^{\circ} \mathrm{C}\left(110 \pm 2{ }^{\circ} \mathrm{F}\right)$ and the mixer was turned on to homogenize the feed solution and to aid in heating. The system was then started and the membrane rotational speed was set. The applied pressure was set at the minimum value and the concentrate return flow rate was stabilized at $3.75 \pm 0.75 \mathrm{~L} / \mathrm{min}(1.0 \pm 0.2 \mathrm{gpm})$ by adjusting the feed pump bypass and concentrate return flow valves. Throughout each experiment, $\mathrm{P}$ was increased from the lowest to highest value in a stepwise fashion to avoid biasing flux determination by initially operating under conditions which were favorable to the accumulation of oil at the membrane surface. The system was operated at each pressure condition until a quasi steady state permeate flux was achieved, at which point the pressure was increased. Periodically, the concentrate return flow rate was measured using a graduated cylinder and a stopwatch as a check on the flow meter calibration.

Once all operational parameters were stabilized, the permeate flux as well as permeate and feed conductivity, $\mathrm{pH}$, and temperature were measured every five minutes 
for the first fifteen minutes at each pressure condition and then every ten to fifteen minutes thereafter. Permeate and feed $\mathrm{pH}$ and conductivity were measured at $43.3 \pm 1{ }^{\circ} \mathrm{C}$ $\left(110 \pm 2{ }^{\circ} \mathrm{F}\right)$ as a real time check of permeate quality. The permeate flow was measured by disconnecting the permeate line from the feed tank and measuring the volume of permeate collected over a set period of time using a graduated cylinder and a stopwatch. The permeate temperature was measured using a Fisher Scientific digital temperature probe placed in the graduated cylinder as a permeate sample was being collected. To obtain a representative measurement of the permeate temperature, the thermometer probe was agitated in the cylinder prior to measuring the temperature. Additionally, the concentrate $\mathrm{pH}$ and conductivity were measured every fifteen minutes immediately after measuring permeate parameters. Since each experiment was conducted in recycle mode (i.e., constant feed concentration), all permeate and concentrate samples were returned to the feed tank after the measurements were taken.

System Shutdown: Upon completion of the final applied pressure condition in each discrete v- $\eta$ experiment, the membrane rotational speed was turned to the lowest setting of $150 \mathrm{rpm}$, the pump was shut off, and the power to the unit was disconnected. The vessel was opened and physical observations of the membrane's condition were made. Particular attention was paid to the accumulation/formation of an oily ring or a gel-like layer on the membrane surface. Additionally, the membrane was inspected for scratches and tears. The pump and lines were drained of any residual feed solution and the cleaning procedure detailed in section 3.3.2.1 was applied. Following system cleaning, the entire experimental procedure was repeated for the next discrete $v-\eta$ combination examined. 


\subsubsection{Membrane Cleaning Procedure}

Upon completing each discrete $v-\eta$ experiment, the system was stopped and the feed tank and lines were drained. At this point, the vessel was opened and physical observations were made paying particular attention to the presence of a residual oily layer as well as scratches or tears on the membrane surface. The membrane was then reinstalled and the cleaning procedure initiated. Dawn dishwashing detergent, an EDTAcontaining surfactant, was added to the base cleaning solution to dissolve oils accumulated on the membrane surface (Reed et al. 1997; Viadero and Reed 1997). The membrane cleaning procedure is summarized below:

1 The system was flushed with $190 \mathrm{~L}$ (50 gal) of hot tap water $\left(\sim 50{ }^{\circ} \mathrm{C} ; 122\right.$ $\left.{ }^{\circ} \mathrm{F}\right)$ (The membrane was not rotated at this time).

2 A base/surfactant cleaning solution was prepared:

a) $19 \mathrm{~L} \mathrm{(5} \mathrm{gal)} \mathrm{of} \mathrm{distilled} \mathrm{water} \mathrm{was} \mathrm{adjusted} \mathrm{to} \mathrm{pH} 11.8$ using $\sim 200 \mathrm{~mL}$ of $\mathrm{NaOH}$ from a $1 \mathrm{~N}$ stock solution stored with a soda scrubber.

b) $30 \mathrm{~mL}$ of Dawn detergent was mixed into the water/ $\mathrm{NaOH}$ solution.

c) The base/surfactant cleaning solution was then heated to $57.2 \pm 1{ }^{\circ} \mathrm{C}$ $\left(135 \pm 2{ }^{\circ} \mathrm{F}\right)$ using the copper coil heat exchanger.

3 The membrane was cleaned with the base/surfactant cleaning solution for 30 minutes at $12 \mathrm{~m} \cdot \mathrm{s}^{-1}(1,750 \mathrm{rpm}), 57.2 \pm 1^{\circ} \mathrm{C}\left(135 \pm 2^{\circ} \mathrm{F}\right)$, and $276 \mathrm{kPa}$ (40 psig).

4 The system was drained and flushed with $190 \mathrm{~L}$ (50 gal) of hot tap water $\left(\sim 50{ }^{\circ} \mathrm{C} ; 122^{\circ} \mathrm{F}\right)(190 \mathrm{~L}$ was determined to be the volume necessary to reduce the $\mathrm{pH}$ of the effluent flush water to the $\mathrm{pH}$ of tap water after cleaning with $\mathrm{NaOH}$ ).

5 The acid cleaning solution was prepared:

a) $19 \mathrm{~L}$ (5 gal) of distilled water was $\mathrm{pH}$ adjusted 2.2 using a stock $1 \mathrm{M}$ $\mathrm{H}_{2} \mathrm{SO}_{4}$ solution. $\sim 185 \mathrm{~mL}$ of $1 \mathrm{M} \mathrm{H}_{2} \mathrm{SO}_{4}$ was necessary to reduce the $\mathrm{pH}$ of the distilled water to 2.2 .

b) The acid cleaning solution was then heated to $57.2 \pm 1{ }^{\circ} \mathrm{C}\left(135 \pm 2{ }^{\circ} \mathrm{F}\right)$ using the copper coil heat exchanger.

6 The membrane was cleaned with the acid cleaning solution for 30 minutes at $12 \mathrm{~m} \cdot \mathrm{s}^{-1}(1,750 \mathrm{rpm}), 57.2 \pm 1{ }^{\circ} \mathrm{C}\left(135 \pm 2{ }^{\circ} \mathrm{F}\right)$, and $276 \mathrm{kPa}(40 \mathrm{psig})$. 
7 The system was drained and flushed with $190 \mathrm{~L}$ (50 gal) of hot tap water $\left(\sim 50{ }^{\circ} \mathrm{C} ; 122^{\circ} \mathrm{F}\right) .190 \mathrm{~L}$ was determined to be the volume necessary to raise the $\mathrm{pH}$ of the effluent flush water to the $\mathrm{pH}$ of tap water after cleaning with $\mathrm{H}_{2} \mathrm{SO}_{4}$.

8. The membrane vessel was opened and physical observations were made, as described earlier.

\subsubsection{Quality Assurance/Quality Control}

After the entire matrix of experiments presented previously in Table 3.6 was completed, 3 of the $24(\sim 13 \%) \mathrm{v}-\eta$ combinations were replicated to ascertain whether changes in membrane characteristics (due to pore plugging, fouling, or chemical/surface changes) had an influence on permeate flux determination. A summary of HSRUF QA/AC replicate experiments is presented in Table 3.7. In each replicate, limiting flux values deviated less than $7 \%$ from values determined in initial experiments; thus, it was determined that the data collected in this study were not biased by changes in membrane characteristics.

Table 3.7 Summary of HSRUF QA/QC replicate experiments.

\begin{tabular}{|c|c|c|c|}
\hline Experiment ID & $\begin{array}{l}\text { Initial limiting flux, } \\
\mathrm{m}^{3} / \mathrm{m}^{2} \cdot \mathrm{d}\left(\mathrm{gal} / \mathrm{ft}^{2} \cdot \mathrm{d}\right)\end{array}$ & $\begin{array}{c}\text { Replicate limiting flux, } \\
\mathrm{m}^{3} / \mathrm{m}^{2} \cdot \mathrm{d}\left(\mathrm{gal} / \mathrm{ft}^{2} \cdot \mathrm{d}\right)\end{array}$ & $\begin{array}{c}\% \\
\text { difference }\end{array}$ \\
\hline $\begin{array}{c}10 \mathrm{~m} \cdot \mathrm{s}^{-1}-0.64 \mathrm{cSt} \\
(10 \% \mathrm{MW} \text { fluid-1,450 rpm) }\end{array}$ & $13.4(330)$ & $14.3(352)$ & $6.7 \%$ \\
\hline $\begin{array}{c}10 \mathrm{~m} \cdot \mathrm{s}^{-1}-4.5 \mathrm{cSt} \\
(30 \% \mathrm{MW} \text { fluid-1,750 rpm) }\end{array}$ & $3.95(97)$ & $3.87(95)$ & $2.0 \%$ \\
\hline $\begin{array}{c}8 \mathrm{~m} \cdot \mathrm{s}^{-1}-8.2 \mathrm{cSt} \\
(40 \% \mathrm{MW} \text { fluid- } 1,150 \mathrm{rpm})\end{array}$ & $0.90(22)$ & $0.90(22)$ & $0 \%$ \\
\hline
\end{tabular}

\subsubsection{Waste Metal Working Fluid Experiment}

A waste MW fluid was acquired from a commercial metal rolling facility and was treated using the HSRUF system. The waste MW fluid had an initial concentration of $4.3 \%$ oil. The experiment was conducted under the following conditions: $\mathrm{v}=12 \mathrm{~m} \cdot \mathrm{s}^{-1}$ 
$(1750 \mathrm{rpm})$; feed temperature $=43 \pm 1^{\circ} \mathrm{C}$; and applied pressure $=290 \mathrm{kPa}(42 \mathrm{psig})$. Unlike experiments conducted using the synthetic MW fluid, flux versus feed oil concentration data were collected in one continuous experiment (through semi-batch and batch operation) to mimic typical operating conditions used in field applications.

A total volume of $190 \mathrm{~L}$ (50 gallons) of the waste MW fluid was treated with biocide and pumped into a $208 \mathrm{~L}$ (55 gallon) Nalgene tank referred to as the waste water storage tank. Additionally, a total of $38 \mathrm{~L}$ (10 gallons) of waste was pumped from the waste drum into two calibrated $19 \mathrm{~L}$ (5 gallon) containers. Prior to being pumped into the waste water storage tank and the calibrated buckets, the waste was passed through a \#40 sieve (0.425 mm opening) in order to remove large solids, which may result in physical damage to the membrane. Further, care was taken to extract MW fluid from the middle of the drum, to avoid collecting excess solids from the bottom and free oil from the top.

The feed tank contents were heated to $41.9^{\circ} \mathrm{C}\left(107.4{ }^{\circ} \mathrm{F}\right)$ using hot water through the heating coils. The system was started in recycle mode and the temperature was allowed to increase to $43.3^{\circ} \mathrm{C}\left(110{ }^{\circ} \mathrm{F}\right)$ using hot water through the heating coils as well as energy from the pump and the rotating membrane. After reaching $43.3{ }^{\circ} \mathrm{C}$, the temperature was maintained at $43.3 \pm 1{ }^{\circ} \mathrm{C}$ by running cold tap water through the copper coil heat exchanger. Once the feed temperature had reached $43.3{ }^{\circ} \mathrm{C}$, the system was run in recycle mode for an additional 2 hours to establish quasi steady state operational conditions. Feed viscosity measurements were taken during this time. After 2 hours of operation in recycle mode, semi-batch mode was initiated. Approximately 38 L (10 gallons) of permeate were collected to increase the feed concentration by a factor of 2 , 
while pumping MW fluid from the waste water storage tank into the feed tank at a rate equal to the permeate flow rate. The system was operated in recycle mode at each whole number concentration factor. Once quasi steady state operational conditions were again established, semi-batch or batch operation was reinitiated. Operational parameters were measured every 10-15 minutes during each recycle mode.

After the first 50 gallons of sieved waste had been pumped into the feed tank, an additional 50 gallons were pumped through the sieve into the wastewater storage tank, totaling 100 gallons of initial coolant. The system waste was then concentrated from 1 to 10X through semi-batch operation, and from 10 to $14.1 \mathrm{X}$ in batch mode (7 gallons of concentrate were remaining in the feed tank at the conclusion of the experiment). At the conclusion of this experiment, the system was cleaned using the standardized procedure outlined in Section 3.3.2.1.

\subsection{METHODS OF STATISTICAL ANALYSIS}

\subsubsection{Two-way Analysis of Variance}

A two-way analysis of variance (ANOVA) is a parametric test used to measure the contribution of two experimental factors (independent variables) to the prediction of a dependent variable and can be used to check for interactions between the independent variables. In ANOVA evaluations, the F-test statistic $\left(\mathrm{F}_{\mathrm{A}}\right)$ is used to measure the contribution of each independent variable in predicting the dependent variable. If $\mathrm{F}_{\mathrm{A}} \approx 1$, the independent variable does not contribute significantly to the prediction of the dependent variable; however, if $\mathrm{F}_{\mathrm{A}} \gg$, the independent variable has a statistically significant influence on the determination of the dependent variable (Hayter, 1996). The

F-test statistic is calculated as: 


$$
\mathrm{F}_{\mathrm{A}}=\frac{\mathrm{MS}_{\text {Factor }}}{\mathrm{MS}}
$$

where $\mathrm{MS}_{\text {Factor }}$ is the mean square for each "factor" (i.e., independent variable) and $\mathrm{MS}_{\text {Error }}$ is the error mean square. The mean square for each factor is an estimate of the variance of the population for an individual independent variable:

$$
\mathrm{MS}_{\text {Factor }}=\frac{\mathrm{SS}_{\text {Factor }}}{\mathrm{DF}_{\text {Factor }}}
$$

where $\mathrm{SS}_{\text {Factor }}$ and $\mathrm{DF}_{\text {Factor }}$ are the sum of squares and the degrees of freedom, respectively, for the independent variable. A hypothetical two-way ANOVA data set is presented in Table 3.8. The data set presented in Table 3.8 represents a study examining the effects of two independent variables (Factors A and B) on a dependent variable. The values of $\mathrm{y}_{\mathrm{ij}}$ represent the observed value of the dependent variable at when factor $\mathrm{A}$ is held at level "i" and factor B is held at level " $\mathrm{j}$ ".

Table 3.8 A hypothetical two-way ANOVA data set.

\begin{tabular}{|c|c|c|c|c|c|c||}
\hline \multirow{4}{*}{ Factor } & \multicolumn{7}{|c|}{ Factor B } \\
\cline { 2 - 7 } & & $\mathbf{1}$ & $\mathbf{2}$ & $\mathbf{3}$ & $\mathbf{4}$ & Total \\
\cline { 2 - 7 } & $\mathbf{1}$ & $\mathrm{y}_{11}$ & $\mathrm{y}_{12}$ & $\mathrm{y}_{13}$ & $\mathrm{y}_{14}$ & $\mathbf{y}_{\mathbf{1 j}}$ \\
\cline { 2 - 7 } & $\mathbf{2}$ & $\mathrm{y}_{21}$ & $\mathrm{y}_{22}$ & $\mathrm{y}_{23}$ & $\mathrm{y}_{24}$ & $\mathbf{y}_{\mathbf{2}}$ \\
\cline { 2 - 7 } & $\mathbf{3}$ & $\mathrm{y}_{31}$ & $\mathrm{y}_{32}$ & $\mathrm{y}_{33}$ & $\mathrm{y}_{34}$ & $\mathbf{y}_{3 \mathbf{j}}$ \\
\cline { 2 - 7 } & Total & $\mathbf{y}_{\mathbf{i} 1}$ & $\mathbf{y}_{\mathbf{i} 2}$ & $\mathbf{y}_{\mathbf{i} 3}$ & $\mathbf{y}_{\mathbf{i} 4}$ & $\mathbf{y}_{\mathbf{i j}}$ \\
\hline
\end{tabular}

For the data set presented in Table 3.8, the sum of squares for Factor A is calculated as:

$$
\mathrm{SS}_{\mathrm{A}}=\frac{1}{\mathrm{~J}} \sum_{\mathrm{i}=1}^{\mathrm{I}}\left(\mathrm{y}_{\mathrm{i} .}^{2}\right)-\frac{1}{\mathrm{IJ}} \mathrm{y}_{\mathrm{ij}}
$$

where $y_{i}$ i is the summation of observed values of the dependent variable obtained when factor A is held at level "i," I is the number of levels in the Factor A population and $\mathrm{J}$ is the number of levels in the Factor B population. The degrees of freedom for Factor A are calculated as: 


$$
\mathrm{DF}_{\mathrm{A}}=\mathrm{I}-1
$$

Similarly, the sum of squares and degrees of freedom for Factor B are calculated by equations 3.11 and 3.12 , respectively:

$$
\begin{aligned}
& \mathrm{SS}_{\mathrm{B}}=\frac{1}{\mathrm{I}} \sum_{\mathrm{j}=1}^{\mathrm{J}}\left(\mathrm{y}_{. \mathrm{j}}^{2}\right)-\frac{1}{\mathrm{IJ}} \mathrm{y}_{\mathrm{ij}}^{2} \\
& \mathrm{DF}_{\mathrm{B}}=\mathrm{J}-1
\end{aligned}
$$

where $y_{. j}$ is the summation of observed values of the dependent variable when factor B is held at level " $\mathrm{j}$." The error mean square is calculated as:

$$
\mathrm{MS}_{\text {Error }}=\frac{\mathrm{SS}_{\text {Error }}}{\mathrm{DF}_{\text {Error }}}
$$

where $\mathrm{SS}_{\mathrm{Errror}}$ the error sum of squares and $\mathrm{DF}_{\mathrm{Error}}$, the error degrees of freedom are calculated by equations 3.13 and 3.14, respectively.

$$
\begin{aligned}
& \mathrm{SS}_{\text {Error }}=\sum_{\mathrm{i}=1}^{\mathrm{I}} \sum_{\mathrm{j}=1}^{\mathrm{J}}\left(\mathrm{y}_{\mathrm{ij}}-\overline{\mathrm{y}}_{\mathrm{i} .}-\overline{\mathrm{y}}_{. \mathrm{j}}+\overline{\mathrm{y}}_{\mathrm{ij}}\right) \\
& \mathrm{DF}_{\text {Error }}=(\mathrm{I}-1)(\mathrm{J}-1)
\end{aligned}
$$

where $\bar{y}_{i}$ is the average of observed values of the dependent variable obtained when factor A is held at level "i," $\bar{y}_{. j}$ is the average of observed values of the dependent variable obtained when factor B is held at level " $\mathrm{j}$," and $\bar{y}_{\mathrm{ij}}$ is the grand average of observed values of the dependent variable.

The $\mathrm{P}$ value calculated in ANOVA analysis $\left(\mathrm{P}_{\mathrm{A}}\right)$ is the probability of being wrong in concluding that there is a statistical dependence of the dependent variable on the independent variable(s). For example, if $\mathrm{P}_{\mathrm{A}}=0.01$, there is only a $1 \%$ chance of being 
wrong in concluding that a statistical dependence exists (i.e., 99\% confidence) (Devore 2000).

\subsubsection{Regression Analysis}

In regression analysis, an equation is developed to describe one variable, the dependent variable, as a function of one or more independent variables (Devore, 2000; Johnson, 1994). The coefficient of determination $\left(R^{2}\right)$ is a measure of how well a regression model describes the data and is calculated as:

$$
\mathrm{R}^{2}=1-\frac{\mathrm{SS}_{\mathrm{Res}}}{\mathrm{SS}_{\text {Factor }}}
$$

If $\mathrm{R}^{2} \approx 1$, the curve predicted by the model is an acceptable description of the relationship between the independent and dependent variables; however, if $\mathrm{R}^{2}$ is near 0 , the dependent variable is poorly predicted by the independent variable.

ANOVA analyses can be applied to the regression equation to provide an additional measure of how well the regression describes the data set. The ability of the regression to predict values of the dependent variable is measured using the F-test statistic calculated in regression analysis, $F_{R}$. The regression F-test statistic is calculated as:

$$
\mathrm{F}_{\mathrm{R}}=\frac{\mathrm{MS} \mathrm{Reg}_{\mathrm{Res}}}{\mathrm{MS}_{\mathrm{Re}}}
$$

where $\mathrm{MS}_{\text {Reg }}$ and $\mathrm{MS}_{\mathrm{Res}}$ are the mean squares for the regression and residual, respectively. Calculation of $\mathrm{MS}_{\mathrm{Reg}}$ is similar to the $\mathrm{MS}_{\mathrm{Factor}}$ calculations presented in section 3.4.1. The residual mean square is calculated as:

$$
\mathrm{MS}_{\text {Res }}=\frac{\mathrm{SS}_{\text {Res }}}{\mathrm{DF}_{\text {Res }}}
$$


where $\mathrm{SS}_{\text {Res }}$, the residual sum of squares, and $\mathrm{DF}_{\text {Res, }}$, the residual degrees of freedom, are calculated by equations 3.19 and 3.20 , respectively.

$$
\begin{aligned}
& \mathrm{SS}_{\text {Res }}=\sum_{\mathrm{n}=1}^{\mathrm{N}}\left(\mathrm{y}_{\mathrm{n}}-\overline{\mathrm{y}}\right)^{2} \\
& \mathrm{DF}_{\text {Re s }}=\mathrm{N}-(\mathrm{k}+1)
\end{aligned}
$$

where $\mathrm{N}$ is the number of observations in the population, $\mathrm{y}_{\mathrm{n}}$ is the value of the dependent variable when the independent variable is held at level "n," $\bar{y}$ is the average of values of the dependent variable, and $\mathrm{k}$ is the regression model order (Hayter 1996).

If $F_{R} \approx 1$, the independent variable does not contribute significantly to the prediction of the dependent variable; however, if $F_{R} \gg 1$, the independent variable has a statistically significant influence on the determination of the dependent variable. The probability of being wrong in concluding that there is a statistically significant association between the dependent and independent variables is measured based on $\mathrm{P}$ values calculated in regression analysis $\left(\mathrm{P}_{\mathrm{R}}\right)$. Similar to the interpretation of $\mathrm{P}_{\mathrm{A}}$ data, when $\mathrm{P}_{\mathrm{R}}<0.01$, it can be concluded that an association exists between the dependent and independent variables.

\subsubsection{Incremental Regression Analysis}

Incremental regression analysis is performed to determine which model order best represents the relationship between the dependent and independent variables. In incremental analyses, each regression order is evaluated starting at first order and increasing stepwise to the highest order specified. If $\mathrm{DF}_{\text {Error }}=0, \mathrm{MS}_{\text {Error }}$ will be undefined (Equation 3.18); thus, $\mathrm{N}$ must be greater than " $\mathrm{k}+1$ " (Equation 3.20) and the highest regression order $(\mathrm{k})$ examined for each data set was determined as: 


$$
\text { Order }=\mathrm{N}-2
$$

In incremental analysis $F_{R}, P_{R}$ and $R^{2}$ values were calculated for each model order examined.

\subsubsection{Assumption Testing}

In the following section, methods are outlined which can be used to test the reasonableness of assumptions made in performing ANOVA and regression analyses. Two-way ANOVA is a parametric test performed under the following assumptions: 1) Normality - all samples are drawn from populations following a standard "bell" shaped Gaussian or "normal" distribution, and 2) Equal Variance - the deviations of observations of the dependent variable from the population mean are as close together as the observations themselves. Regression analysis is also a parametric test performed under the assumption that all samples and drawn from normally distributed populations. However, unlike ANOVA (assuming equal variance), in regression analysis it is assumed that the sample population has constant variance or the deviations of observation of the dependent variable from the population mean are constant, regardless of the value of the independent variable.

The statistical methods described in the previous sections (Two-way Analysis of Variance and Regression Analysis) are robust (Johnson, 1994; Hines and Montgomery, 1980); in other words, there is little effect on ANOVA or regression results when the assumptions are not satisfied. However, the following statistical procedures were performed to test the reasonableness of each of the assumptions made in performing ANOVA and regression analyses. 


\subsubsection{Kolmogorov-Smirnov Test}

The Kolmogorov-Smirnov test is used in ANOVA and regression analysis to test the assumption that all samples are drawn from populations following a normal distribution. The Kolmogorov-Smirnov test statistic $\left(\mathrm{k}_{\mathrm{sv}}\right)$ is calculated as the maximum deviation between the data and a representative model.

$$
\mathrm{k}_{\mathrm{sv}}=\max \left|\mathrm{Y}(\mathrm{x})-\mathrm{Y}^{\prime}(\mathrm{x})\right|
$$

where $\mathrm{Y}(\mathrm{x})$ is the value of the dependent variable when the value of the independent variable is " $\mathrm{x}$," and $\mathrm{Y}^{\prime}(\mathrm{x})$ is the value predicted by the representative model when the value of the independent variable is "x" (Gordon et al., 1992; Benjamin and Cornell, 1970). Birnbaum (1952) and Lindgren (1962) tabulated and presented critical values of $\mathrm{k}_{\mathrm{s}}$ as a function of population sample size for a $99 \%$ significance level. If the calculated value of $\mathrm{k}_{\mathrm{s}}$ is equal to or greater than the critical value, it is concluded that the population is not normally distributed. The probability of being incorrect in concluding that the data are not normally distributed is measured by $\mathrm{P}$ values calculated in the KolmogorovSmirnov test, $\mathrm{P}_{\mathrm{k}}$. For example, if $\mathrm{P}_{\mathrm{k}}=0.01$, there is a $1 \%$ chance of being wrong in concluding that the population is not normally distributed (i.e., 99\% confidence).

\subsubsection{Levene Median Test}

The Levene Median Test is used in ANOVA evaluations to test the assumption that all samples are drawn from populations containing equal variance. For each dependent variable, a one-way ANOVA is performed with the deviations of each observation from the population mean as the dependent variable and the sample observations as the independent variable. If the Levene Median test is determined to be statistically significant, then it is concluded that the population variance is not equal. The 
probability of being incorrect in concluding that the variance is not equal is measured by $\mathrm{P}$ values calculated from the Levene Median Test, $\mathrm{P}_{\mathrm{L}}$.

\subsubsection{Spearman Rank Correlation}

The Spearman Rank Correlation or the Rank Correlation is commonly used in regression analysis to test the assumption that all samples are drawn from populations containing constant variance. Values of the independent variables are ranked starting with " 1 " for the lowest value and increasing sequentially to the highest value. Each value is then replaced by it's respective rank. The same procedure is used to replace values of the dependent variable with ranked values. A hypothetical two-variable data set is presented in Table 3.9 .

Table 3.9 A hypothetical two-variable data set

\begin{tabular}{|l|l|l|l|l|l||}
\hline $\mathrm{x}$ & 10 & 5 & 15 & 30 & 20 \\
\hline $\mathrm{y}$ & 20 & 15 & 5 & 10 & 30 \\
\hline
\end{tabular}

Ranked values for the Spearman Rank Correlation are presented in Table 3.10. In performing the Spearman Rank Correlation, the data set presented in Table 3.9 would be replaced with ranked values as presented in Table 3.10.

Table 3.10 Ranked values for the Spearman Rank Correlation.

\begin{tabular}{|l|l|l|l|l|l||}
\hline $\mathrm{r}(\mathrm{x})$ & 2 & 1 & 3 & 5 & 4 \\
\hline $\mathrm{r}(\mathrm{y})$ & 4 & 3 & 1 & 2 & 5 \\
\hline
\end{tabular}

where $\mathrm{r}\left(\mathrm{x}_{\mathrm{i}}\right)$ is the rank of $\mathrm{x}_{\mathrm{i}}$ and $\mathrm{r}\left(\mathrm{y}_{\mathrm{i}}\right)$ is the rank of $\mathrm{y}_{\mathrm{i}}$. (Mood et al., 1974). Using the paired ranks, the correlation coefficient is calculated as:

$$
\mathrm{r}_{\mathrm{s}}=1-\frac{6 * \sum \mathrm{d}_{\mathrm{i}}^{2}}{\mathrm{~N}\left(\mathrm{~N}^{2}-1\right)}
$$


where $r_{s}$ is the Spearman correlation coefficient, $\mathrm{N}$ is the number of observations in the population, and $d_{i}$ is the difference between the ranks of the paired observations $\left(x_{i}, y_{i}\right)$, calculated as (Mood et al., 1974; Johnson, 1994):

$$
\mathrm{d}_{\mathrm{i}}=\mathrm{r}\left(\mathrm{x}_{\mathrm{i}}\right)-\mathrm{r}\left(\mathrm{y}_{\mathrm{i}}\right)
$$

The probability of being incorrect in concluding that the variance is not constant is measured by $\mathrm{P}$ values calculated from the Spearman Rank correlation coefficient, $\mathrm{P}_{\mathrm{s}}$. 


\section{CHAPTER 4.0}

\section{RESULTS AND PRELIMINARY DATA REDUCTION}

\subsection{METAL WORKING FLUID KINEMATIC VISCOSITY}

A plot of kinematic viscosity versus oil concentration for the synthetic and waste MW fluids is presented in Figure 4.1. For the synthetic MW fluid, the viscosity increased linearly with oil concentration up to $\sim 34 \%$ oil. At this point ( $34 \%$ oil) the viscosity versus oil concentration relationship exhibited an exponential increase. Similarly, for the waste MW fluid, viscosity increased linearly with oil concentration up to $40 \%$ oil. At oil concentrations greater than $40 \%$ oil, viscosity increased exponentially.

\subsection{TUBULAR ULTRAFILTRATION}

The tubular UF experiments were conducted using the synthetic MW fluid described in section 3.1.1. Permeate flux data presented in this section were temperature corrected from 29.4 to $43.3{ }^{\circ} \mathrm{C}$ in order to facilitate a comparison between the tubular and HSRUF systems (see section 3.2.3). Results from the discrete tubular UF experiments are presented in the following sections.

\subsubsection{7 $\mathrm{m} \cdot \mathrm{s}^{-1}-0.40 \mathrm{cSt}(30 \mathrm{gpm}-1 \% \mathrm{MW}$ Fluid)}

Permeate flux versus time for the $3.7 \mathrm{~m} \cdot \mathrm{s}^{-1}-0.40 \mathrm{cSt}$ experiment is presented in Figure 4.2. An initial decrease in permeate flux was observed over the first twenty minutes of operation, due to the formation of the concentration boundary layer at the membrane surface, at which point quasi steady-state permeate flux was achieved. Distinct step-wise increases in flux were observed as P was increased from 104 to 372 $\mathrm{kPa}$ (15 to $54 \mathrm{psi}$ ). Immediately after each pressure increase, a small decrease in permeate flux was observed after which the flux stabilized. This phenomenon was 


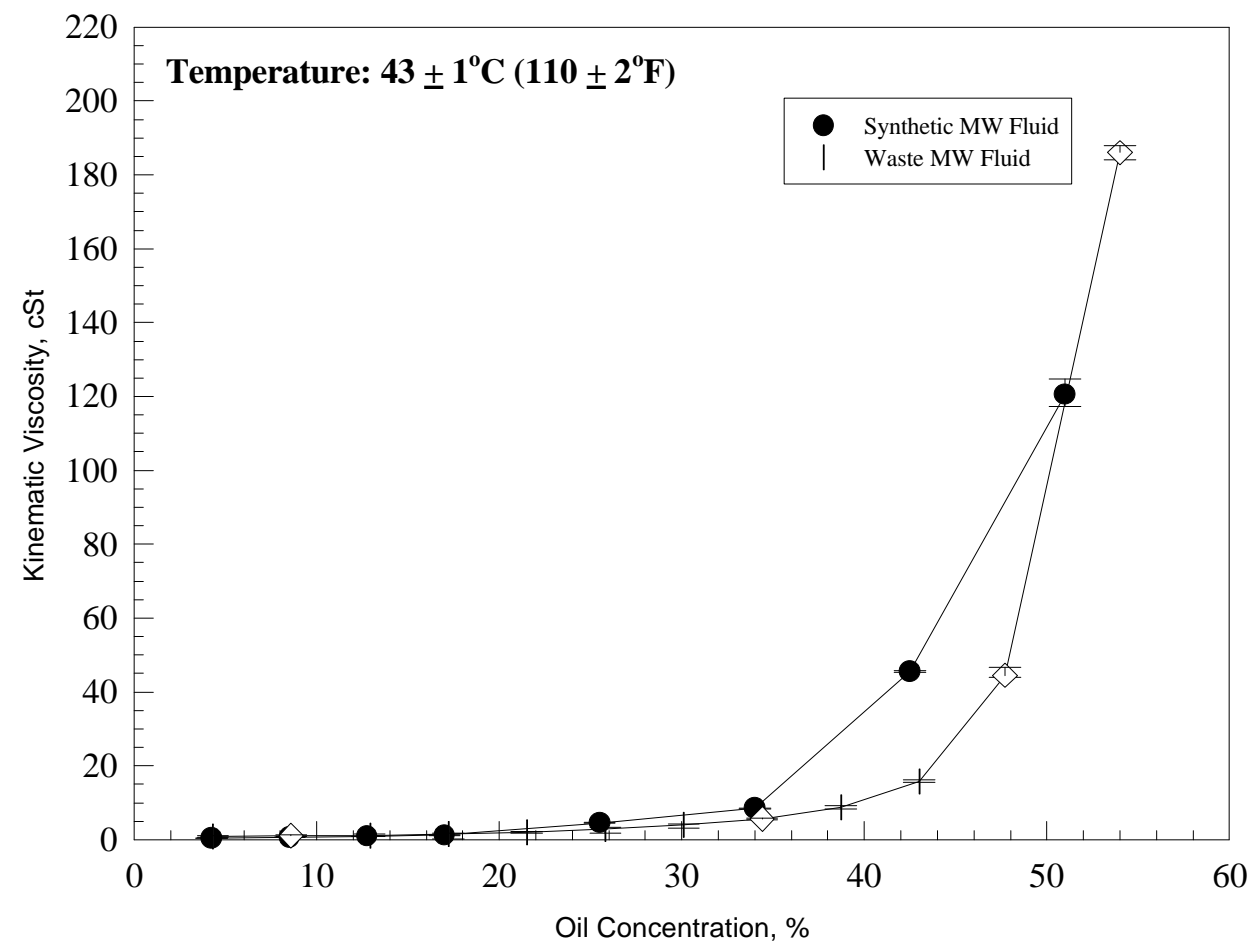

Figure 4.1 Kinematic viscosity versus oil concentration for the synthetic and waste MW fluids.

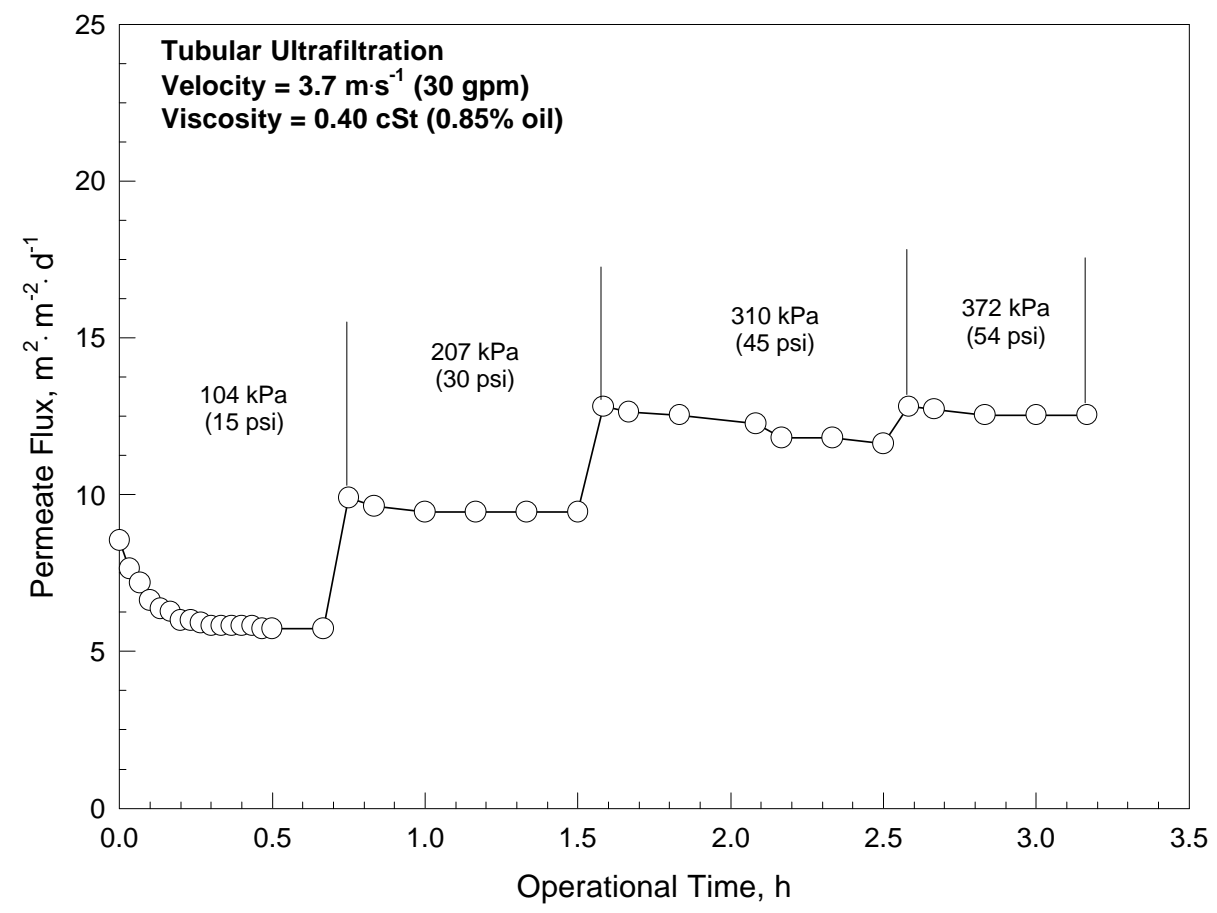

Figure 4.2 Permeate flux versus operational time for the $3.7 \mathrm{~m} \cdot \mathrm{s}^{-1}-0.40 \mathrm{cSt}$ experiment. 
attributed to a delay in stabilization of the concentration boundary layer (Viadero and Reed 1999). Average steady state permeate flux versus average transmembrane pressure for the $3.7 \mathrm{~m} \cdot \mathrm{s}^{-1}-0.40 \mathrm{cSt}$ experiment is presented in Figure 4.3. A limiting flux was not observed over the applied pressure range studied in this experiment; however, the average flux data diverged from linear behavior at higher pressures due to a shift from pressure-dependent to pressure-independent operating conditions.

The permeate $\mathrm{pH}$ ranged from 6.32 to 7.84 and averaged 6.89. The concentrate $\mathrm{pH}$ ranged from 6.67 to 7.66 and averaged 6.93. Permeate and concentrate $\mathrm{pH}$ both varied greatly throughout the run. The permeate conductivity ranged from 16.3 to 25.2 $\mu \mathrm{S} \cdot \mathrm{cm}^{-1}$ and averaged $19.0 \mu \mathrm{S} \cdot \mathrm{cm}^{-1}$. The concentrate conductivity ranged from 78.6 to 93.8 $\mu \mathrm{S} \cdot \mathrm{cm}^{-1}$ and averaged $86.6 \mu \mathrm{S} \cdot \mathrm{cm}^{-1}$. Permeate and concentrate conductivity both remained relatively constant and concentrate conductivity was greater than permeate conductivity throughout the entire run. Distinct step-wise changes in permeate and concentrate $\mathrm{pH}$ and conductivity were not observed as applied pressure was increased.

\subsection{2 $2.5 \mathrm{~m} \cdot \mathrm{s}^{-1}-0.40 \mathrm{cSt}(20 \mathrm{gpm}-1 \% \mathrm{MW}$ Fluid $)$}

Permeate flux versus time for the $2.5 \mathrm{~m} \cdot \mathrm{s}^{-1}-0.40 \mathrm{cSt}$ experiment is presented in Figure 4.4. An initial decrease in permeate flux was observed over the first half-hour of operation, due to the formation of the concentration boundary layer at the membrane surface, at which point quasi steady-state permeate flux was achieved. Distinct step-wise increases in flux were observed as $\mathrm{P}$ was increased from 104 to $517 \mathrm{kPa}$ (15 to $75 \mathrm{psi}$ ). Immediately after each pressure increase, a small decrease in permeate flux was observed 


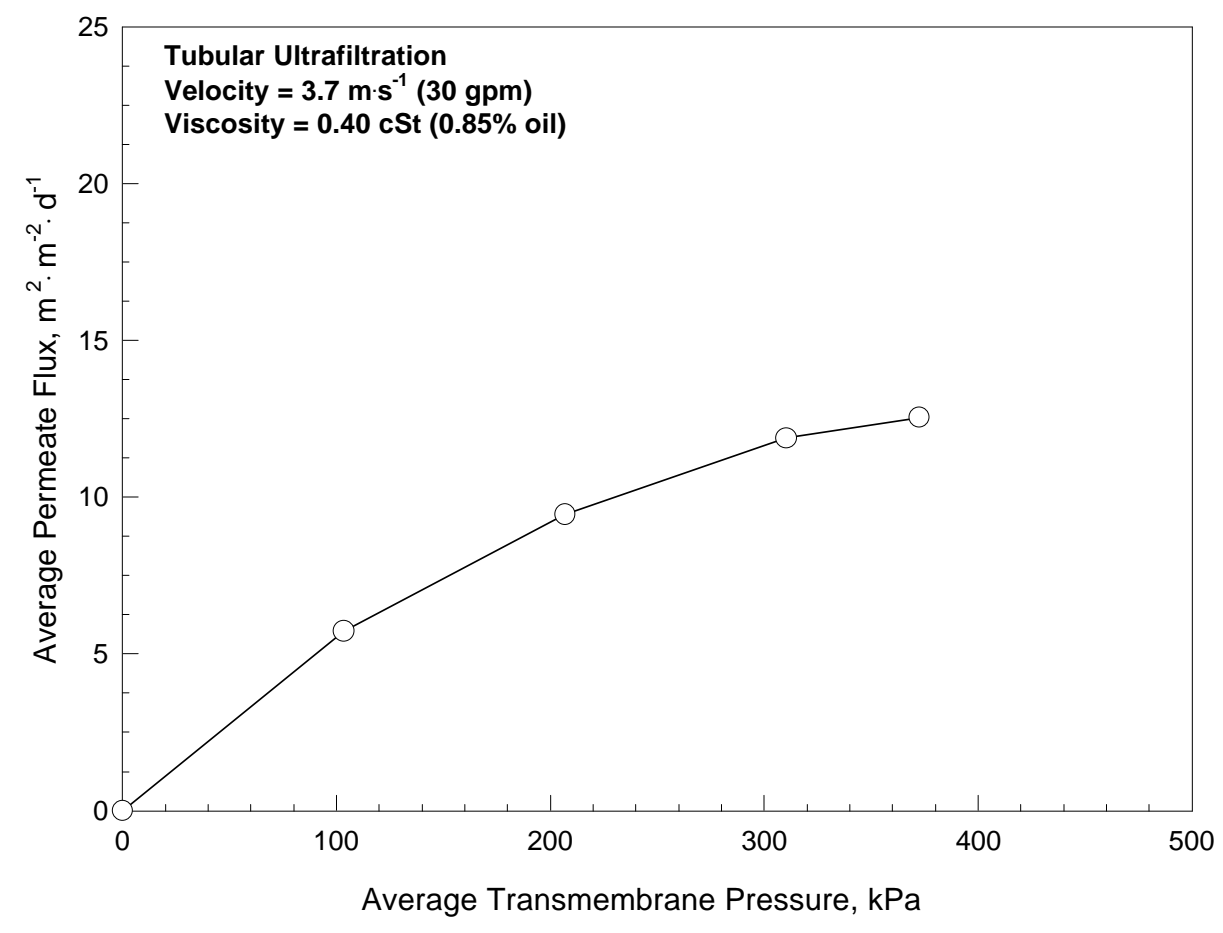

Figure 4.3 Average steady state permeate flux versus average transmembrane pressure for the $3.7 \mathrm{~m} \cdot \mathrm{s}^{-1}-0.40 \mathrm{cSt}$ experiment.

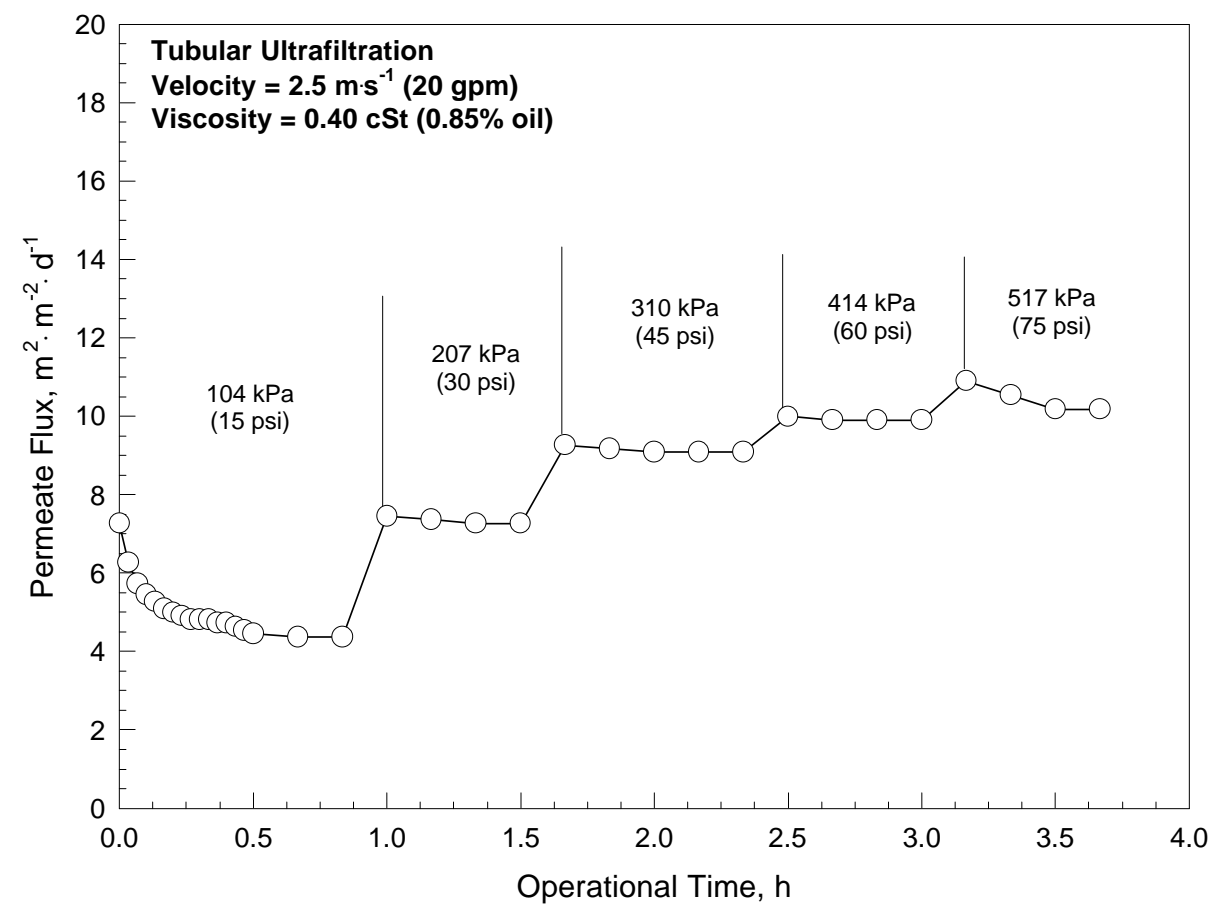

Figure 4.4 Permeate flux versus operational time for the $2.5 \mathrm{~m} \cdot \mathrm{s}^{-1}-0.40 \mathrm{cSt}$ experiment. 
after which the flux stabilized. This phenomenon was attributed to a delay in stabilization of the concentration boundary layer (Viadero and Reed 1999). Average steady state permeate flux versus average transmembrane pressure for the $2.5 \mathrm{~m} \cdot \mathrm{s}^{-1}-0.40$ cSt experiment is presented in Figure 4.5. A limiting flux was not observed over the applied pressure range studied in this experiment; however, the average flux data diverged from linear behavior at higher pressures due to a shift from pressure-dependent to pressure-independent operating conditions.

The permeate $\mathrm{pH}$ ranged from 6.23 to 7.85 and averaged 7.10. The concentrate $\mathrm{pH}$ ranged from 6.61 to 7.70 and averaged 7.06. Both permeate and concentrate $\mathrm{pH}$ varied greatly throughout the run. The permeate conductivity ranged from 7.45 to 13.3 $\mu \mathrm{S} \cdot \mathrm{cm}^{-1}$ and averaged $9.15 \mu \mathrm{S} \cdot \mathrm{cm}^{-1}$. The concentrate conductivity ranged from 43.5 to $50.7 \mu \mathrm{S} \cdot \mathrm{cm}^{-1}$ and averaged $46.4 \mu \mathrm{S} \cdot \mathrm{cm}^{-1}$. Permeate and concentrate conductivity remained relatively constant and permeate was less than concentrate conductivity throughout the entire run. Distinct step-wise changes in permeate and concentrate $\mathrm{pH}$ and conductivity were not observed as applied pressure was increased.

\subsection{3 $1.2 \mathrm{~m} \cdot \mathrm{s}^{-1}-0.40 \mathrm{cSt}(10 \mathrm{gpm}-1 \% \mathrm{MW}$ Fluid $)$}

Permeate flux versus time for the $1.2 \mathrm{~m} \cdot \mathrm{s}^{-1}-0.40 \mathrm{cSt}$ experiment is presented in Figure 4.6. An initial decrease in permeate flux was observed over the first halfhour of operation, due to the formation of the concentration boundary layer at the membrane surface, at which point quasi steady-state permeate flux was achieved. Distinct step-wise increases in flux were observed as $P$ was increased from 35 to 517 kPa (5 to 75 psi). Average steady state permeate flux versus average transmembrane pressure for the $1.2 \mathrm{~m} \cdot \mathrm{s}^{-1}-0.40 \mathrm{cSt}$ experiment is presented in Figure 4.7. A limiting flux was not observed 


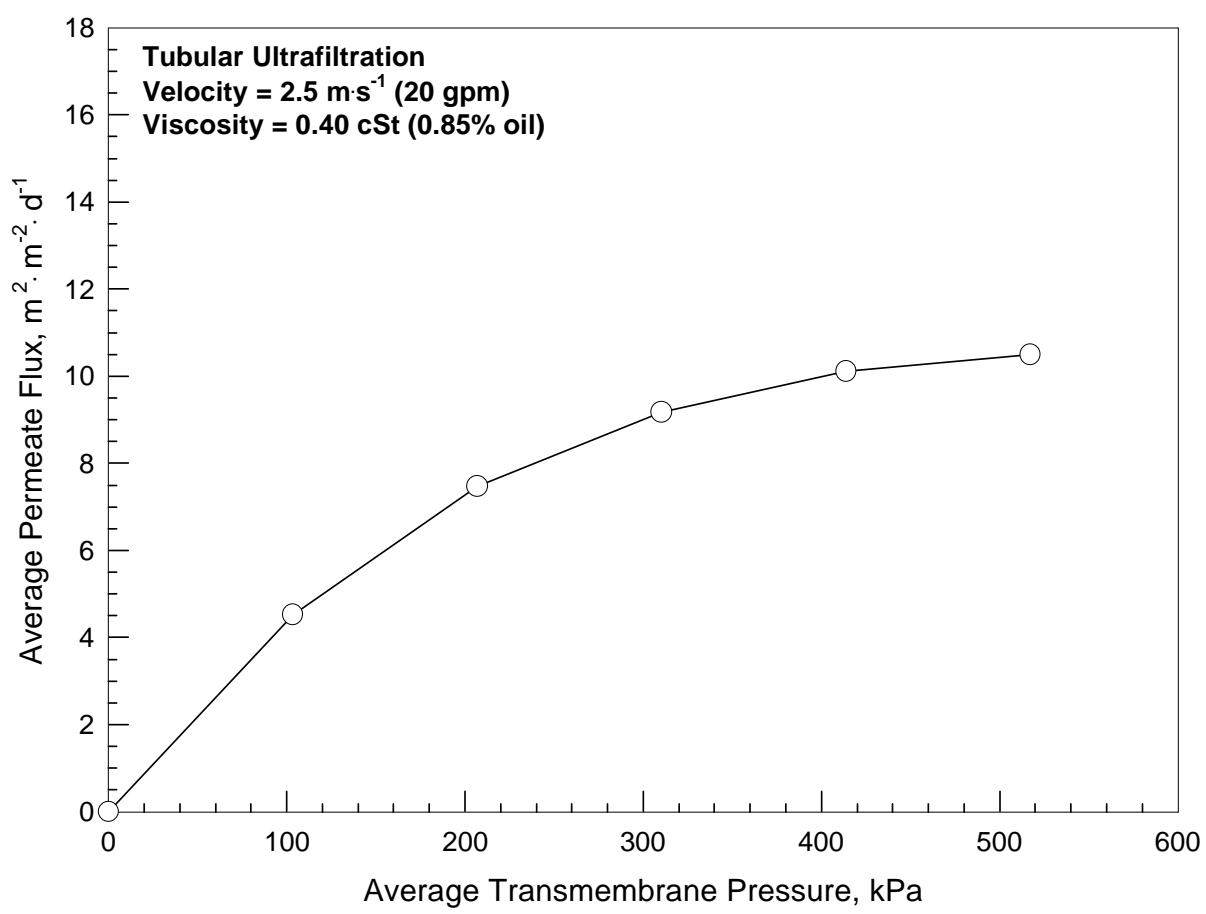

Figure 4.5 Average steady state permeate flux versus average transmembrane pressure for the $2.5 \mathrm{~m} \cdot \mathrm{s}^{-1}-0.40 \mathrm{cSt}$ experiment.

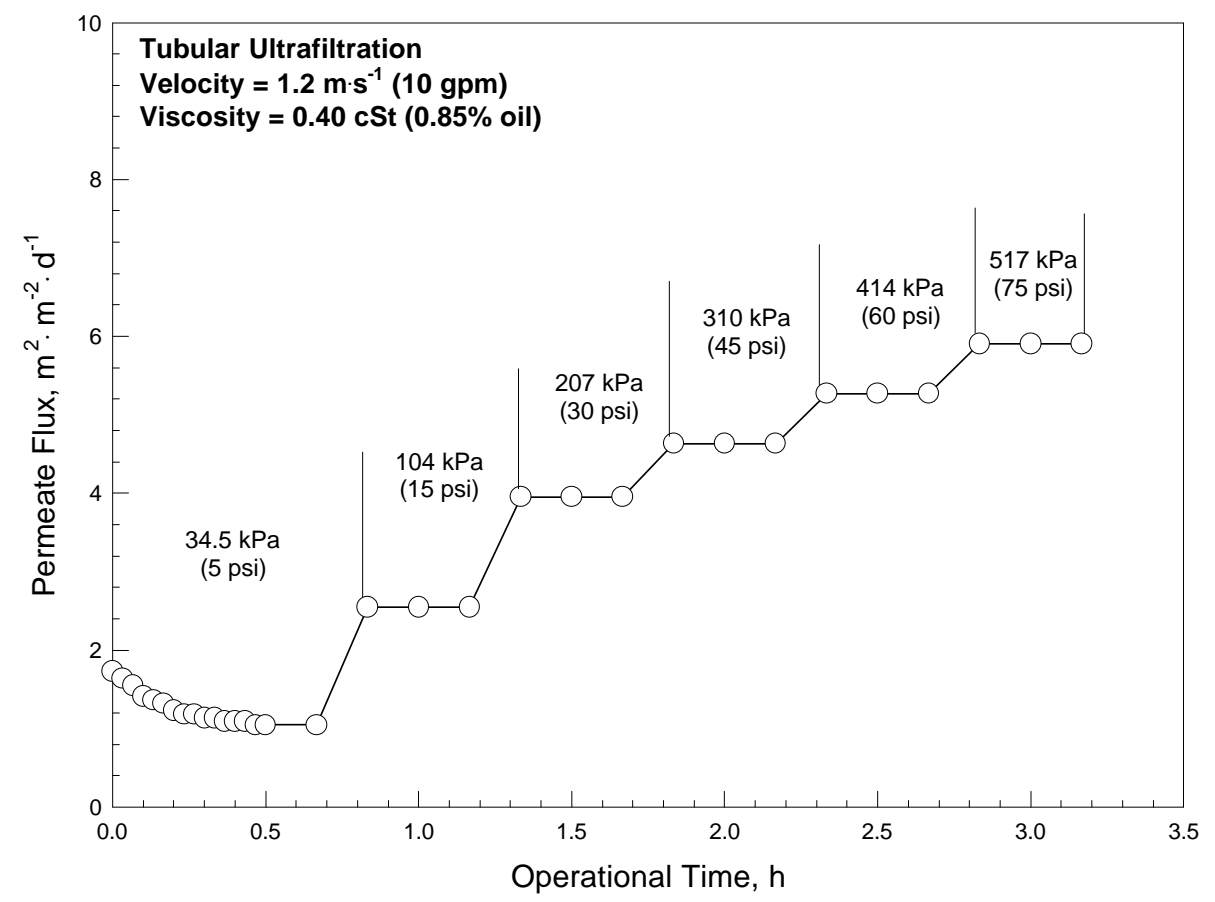

Figure4.6 Permeate flux versus operational time for the $1.2 \mathrm{~m} \cdot \mathrm{s}^{-1}-0.40 \mathrm{cSt}$ experiment. 


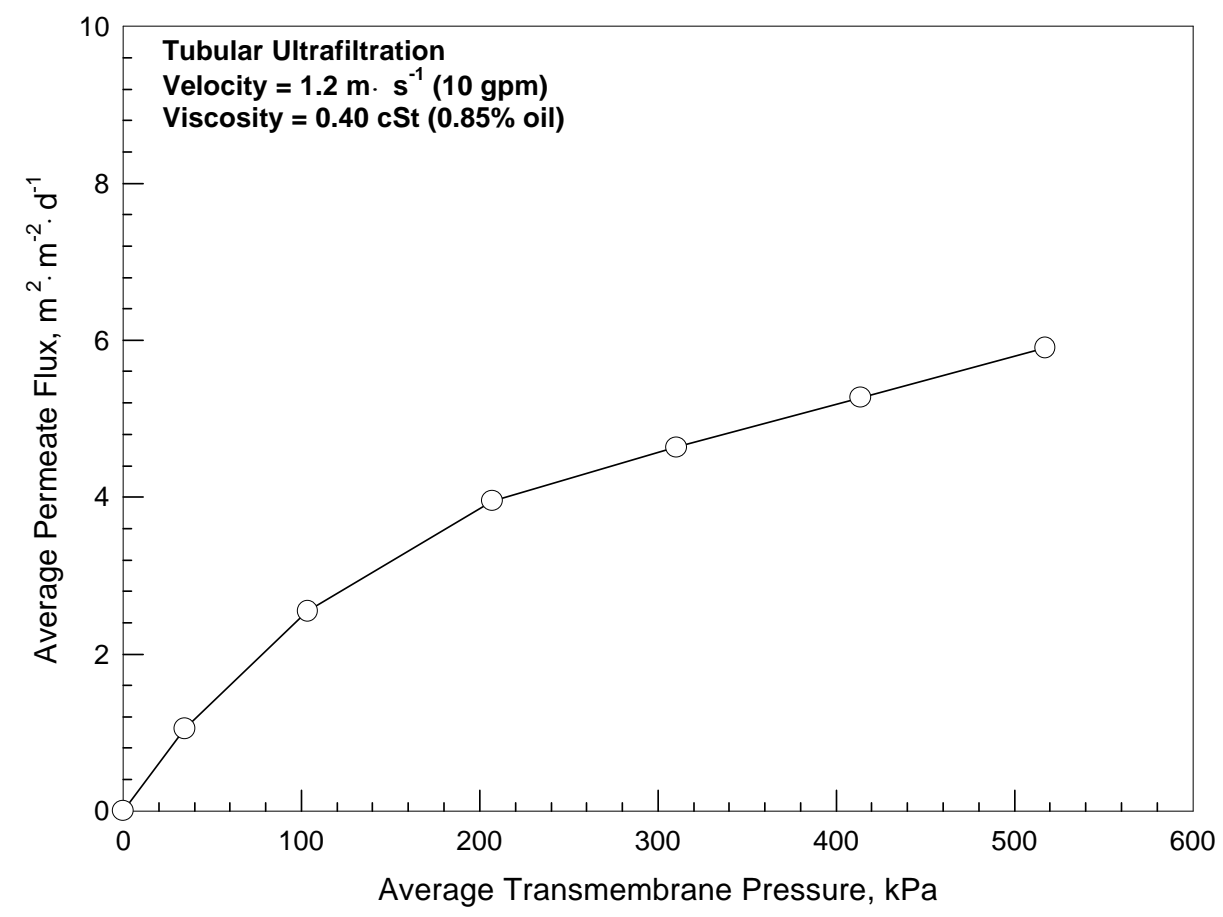

Figure 4.7 Average steady state permeate flux versus average transmembrane pressure for the $1.2 \mathrm{~m} \cdot \mathrm{s}^{-1}-0.40 \mathrm{cSt}$ experiment.

over the applied pressure range studied in this experiment; however, the average flux data diverged from linear behavior at higher pressures due to a shift from pressure-dependent to pressure-independent operating conditions.

The permeate $\mathrm{pH}$ ranged from 6.75 to 7.82 and averaged 7.09. The concentrate

$\mathrm{pH}$ ranged from 6.82 to 7.22 and averaged 6.98. Permeate and concentrate $\mathrm{pH}$ were both relatively constant throughout the entire run. The permeate conductivity ranged from 1.60 to $17.2 \mu \mathrm{S} \cdot \mathrm{cm}^{-1}$ and averaged $5.19 \mu \mathrm{S} \cdot \mathrm{cm}^{-1}$. The concentrate conductivity ranged from 19.6 to $21.6 \mu \mathrm{S} \cdot \mathrm{cm}^{-1}$ and averaged $20.6 \mu \mathrm{S} \cdot \mathrm{cm}^{-1}$. Permeate and concentrate conductivity remained relatively constant and permeate conductivity was less than concentrate conductivity throughout the entire run. Distinct step-wise changes in permeate and concentrate $\mathrm{pH}$ and conductivity were not observed as applied pressure was increased.

\subsection{2 m-s ${ }^{-1}-0.40 \mathrm{cSt}$ (5 gpm-1\% MW Fluid)}


Permeate flux versus time for the $0.62 \mathrm{~m} \cdot \mathrm{s}^{-1}-0.40 \mathrm{cSt}$ experiment is presented in Figure 4.8. An initial decrease in permeate flux was observed over the first half-hour of operation, due to the formation of the concentration boundary layer at the membrane surface, at which point quasi steady-state permeate flux was achieved. Immediately after each pressure increase, a small decrease in permeate flux was observed after which the flux stabilized. This phenomenon was attributed to a delay in stabilization of the concentration boundary layer (Viadero and Reed 1999). Average steady state permeate flux versus average transmembrane pressure for the $0.62 \mathrm{~m} \cdot \mathrm{s}^{-1}-0.40 \mathrm{cSt}$ experiment is presented in Figure 4.9. A limiting permeate flux of $2.8 \mathrm{~m}^{3} \cdot \mathrm{m}^{-2} \cdot \mathrm{d}^{-1}$ was observed at the 207 to $310 \mathrm{kPa}$ (30 to $45 \mathrm{psi}$ ) conditions. The experiment was stopped after the $310 \mathrm{kPa}$ pressure condition was examined, due to the fact that a limiting permeate flux was already achieved. Any additional increase in transmembrane pressure would only result in further consolidation of the concentration boundary layer, thus increasing the potential for membrane fouling (Cheryan 1998).

The permeate $\mathrm{pH}$ ranged from 5.68 to 8.11 and averaged 7.29. The concentrate $\mathrm{pH}$ ranged from 7.07 to 7.62 and averaged 7.42. Permeate and concentrate $\mathrm{pH}$ were both relatively constant throughout the entire run. The permeate conductivity ranged from 14.9 to $50.3 \mu \mathrm{S} \cdot \mathrm{cm}^{-1}$ and averaged $24.5 \mu \mathrm{S} \cdot \mathrm{cm}^{-1}$. The concentrate conductivity ranged from 78.3 to $87.3 \mu \mathrm{S} \cdot \mathrm{cm}^{-1}$ and averaged $82.0 \mu \mathrm{S} \cdot \mathrm{cm}^{-1}$. Permeate and concentrate conductivity remained relatively constant and permeate conductivity was less than concentrate conductivity throughout the entire run. Distinct step-wise changes in 


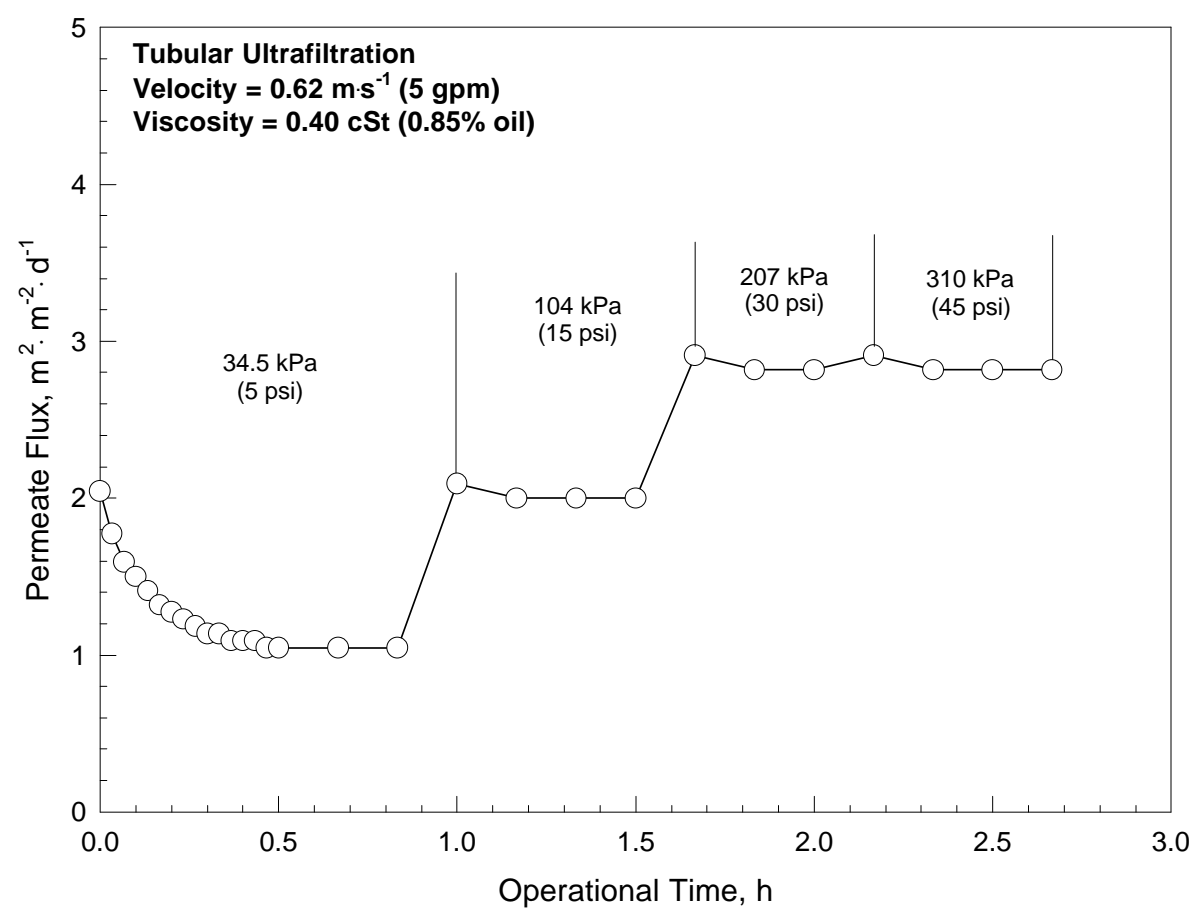

Figure 4.8 Permeate flux versus operational time for the $0.62 \mathrm{~m} \cdot \mathrm{s}^{-1}-0.40 \mathrm{cSt}$ experiment.

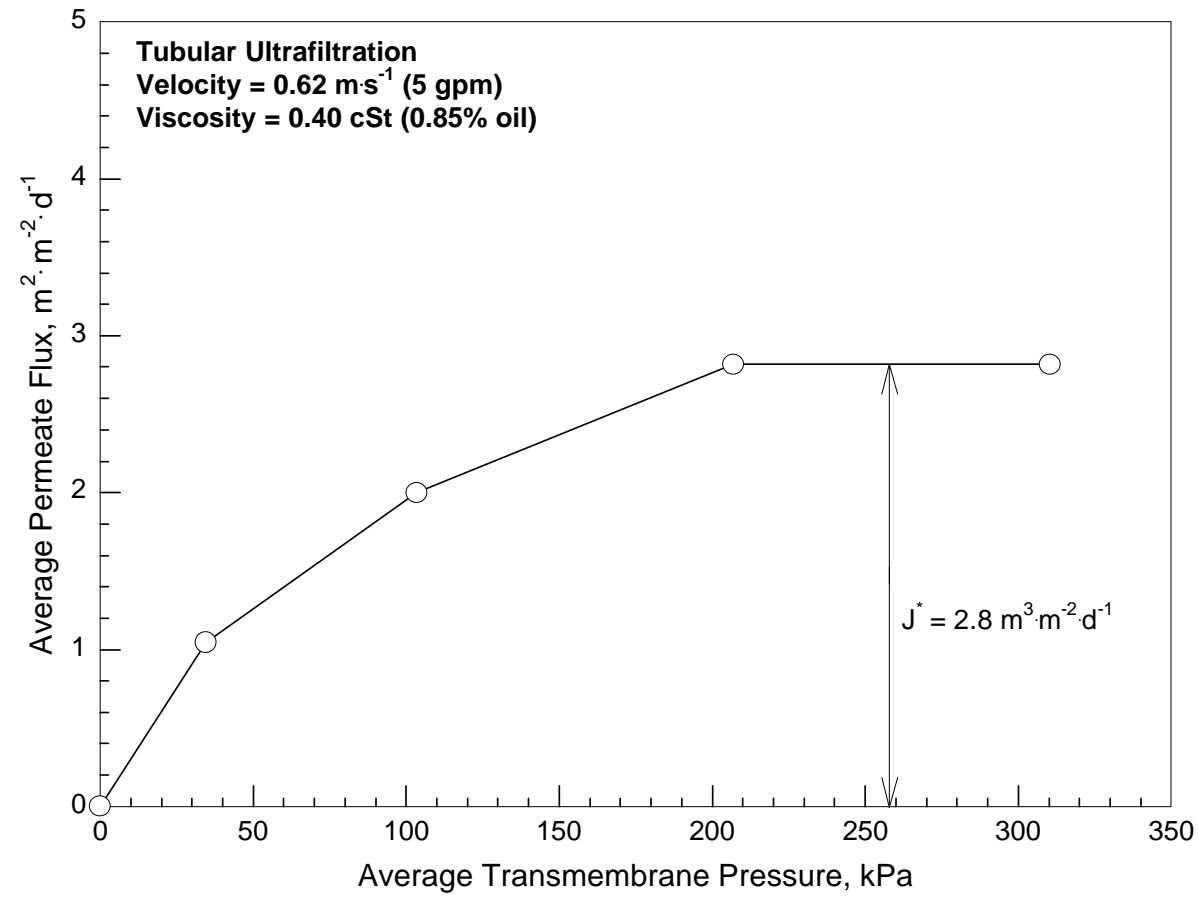

Figure 4.9 Average steady state permeate flux versus average transmembrane pressure for the $0.62 \mathrm{~m} \cdot \mathrm{s}^{-1}-0.40 \mathrm{cSt}$ experiment. 
permeate and concentrate $\mathrm{pH}$ and conductivity were not observed as applied pressure was increased.

\subsubsection{7 m-s ${ }^{-1}-0.46 \mathrm{cSt}(30 \mathrm{gpm}-5 \%$ MW Fluid)}

Permeate flux versus time for the $3.7 \mathrm{~m} \cdot \mathrm{s}^{-1}-0.46 \mathrm{cSt}$ experiment is presented in Figure 4.10. An initial decrease in permeate flux was observed over the first fifty minutes of operation, due to the formation of the concentration boundary layer at the membrane surface, at which point quasi steady-state permeate flux was achieved. Distinct step-wise increases in flux were observed as P was increased from 104 to 362 $\mathrm{kPa}$ (15 to $52.5 \mathrm{psi}$ ). Immediately after each pressure increase, a small decrease in permeate flux was observed after which the flux stabilized. This phenomenon was attributed to a delay in stabilization of the concentration boundary layer (Viadero and Reed 1999). Average steady state permeate flux versus average transmembrane pressure for the $3.7 \mathrm{~m} \cdot \mathrm{s}^{-1}-0.46 \mathrm{cSt}$ experiment is presented in Figure 4.11. A limiting flux was not observed over the applied pressure range studied in this experiment; however, the average flux data diverged from linear behavior at higher pressures due to a shift from pressure-dependent to pressure-independent operating conditions.

The permeate $\mathrm{pH}$ ranged from 7.11 to 7.80 and averaged 7.32. The concentrate $\mathrm{pH}$ ranged from 7.21 to 7.97 and averaged 7.50. Permeate and concentrate $\mathrm{pH}$ both varied greatly throughout the run. The permeate conductivity ranged from 120 to 135 $\mu \mathrm{S} \cdot \mathrm{cm}^{-1}$ and averaged $127 \mu \mathrm{S} \cdot \mathrm{cm}^{-1}$. The concentrate conductivity ranged from 242 to 256 $\mu \mathrm{S} \cdot \mathrm{cm}^{-1}$ and averaged $249 \mu \mathrm{S} \cdot \mathrm{cm}^{-1}$. Permeate and concentrate conductivity both remained relatively constant throughout the entire run. Concentrate conductivity was greater than permeate conductivity throughout the run. Distinct step-wise changes in 


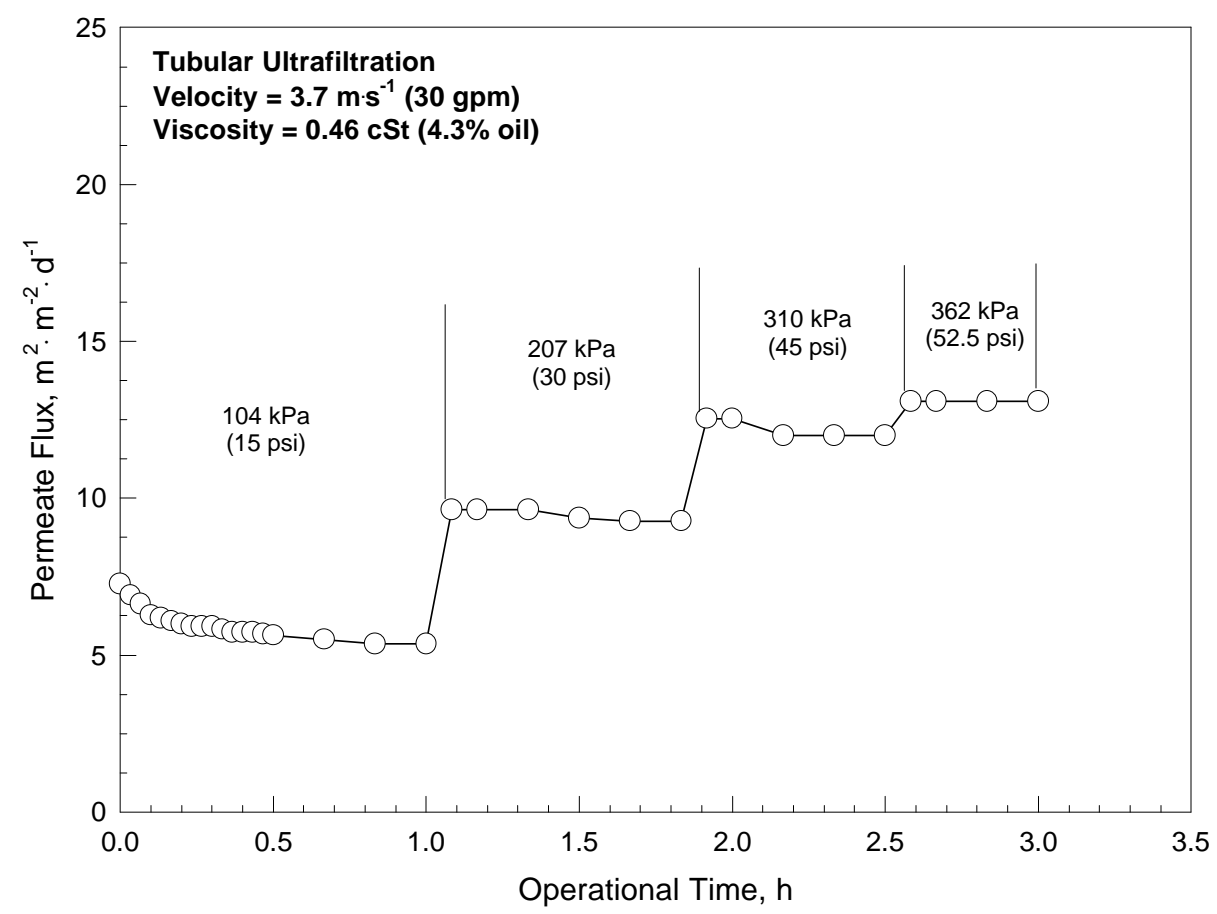

Figure 4.10 Permeate flux versus operational time for the $3.7 \mathrm{~m} \cdot \mathrm{s}^{-1}-0.46 \mathrm{cSt}$ experiment.

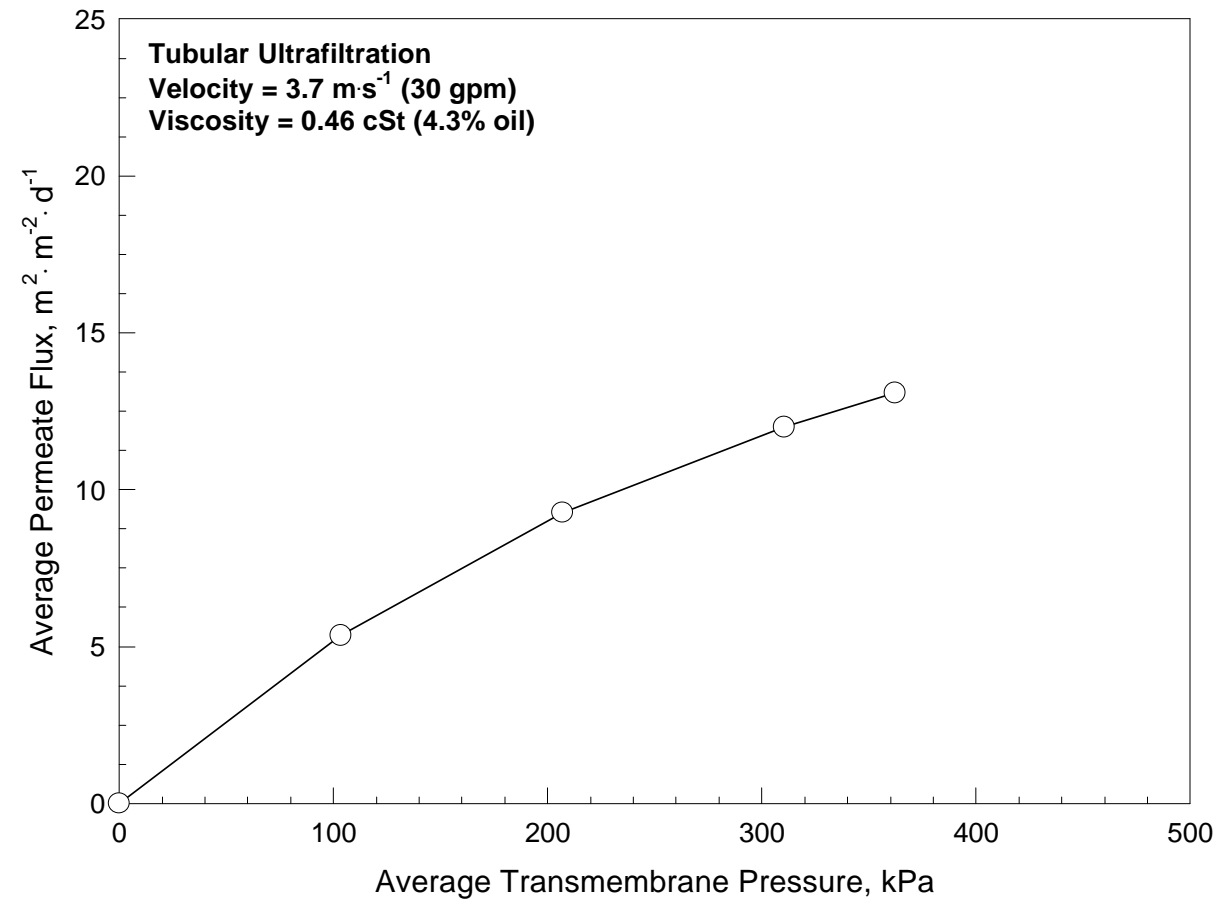

Figure 4.11 Average steady state permeate flux versus average transmembrane pressure for the $3.7 \mathrm{~m} \cdot \mathrm{s}^{-1}-\mathbf{0 . 4 6} \mathrm{cSt}$ experiment. 
permeate and concentrate $\mathrm{pH}$ and conductivity were not observed as applied pressure was increased.

\subsubsection{5 m-s $\mathrm{s}^{-1}-0.46 \mathrm{cSt}-(20 \mathrm{gpm}-5 \%$ MW Fluid)}

Permeate flux versus time for the $2.5 \mathrm{~m} \cdot \mathrm{s}^{-1}-0.46 \mathrm{cSt}$ experiment is presented in Figure 4.12. An initial decrease in permeate flux was observed over the first ten minutes of operation, due to the formation of the concentration boundary layer at the membrane surface, at which point quasi steady-state permeate flux was achieved. Distinct step-wise increases in flux were observed as $\mathrm{P}$ was increased from 104 to $507 \mathrm{kPa}$ (15 to $73.5 \mathrm{psi}$ ). Immediately after each pressure increase, a small decrease in permeate flux was observed after which the flux stabilized. This phenomenon was attributed to a delay in stabilization of the concentration boundary layer (Viadero and Reed 1999). Average steady state permeate flux versus average transmembrane pressure for the $2.5 \mathrm{~m} \cdot \mathrm{s}^{-1}-0.46$ cSt experiment is presented in Figure 4.13. A limiting flux was not observed over the applied pressure range studied in this experiment; however, the average flux data diverged from linear behavior at higher pressures due to a shift from pressure-dependent to pressure-independent operating conditions.

The permeate $\mathrm{pH}$ ranged from 7.12 to 7.70 and averaged 7.29. The concentrate $\mathrm{pH}$ ranged from 7.20 to 7.49 and averaged 7.30. Both permeate and concentrate $\mathrm{pH}$ were relatively constant throughout the run. The permeate conductivity ranged from 94.4 to $117 \mu \mathrm{S} \cdot \mathrm{cm}^{-1}$ and averaged $99.6 \mu \mathrm{S} \cdot \mathrm{cm}^{-1}$. The concentrate conductivity ranged from 213 to $228 \mu \mathrm{S} \cdot \mathrm{cm}^{-1}$ and averaged $221 \mu \mathrm{S} \cdot \mathrm{cm}^{-1}$. Permeate and concentrate conductivity remained relatively constant and permeate conductivity was less than concentrate 


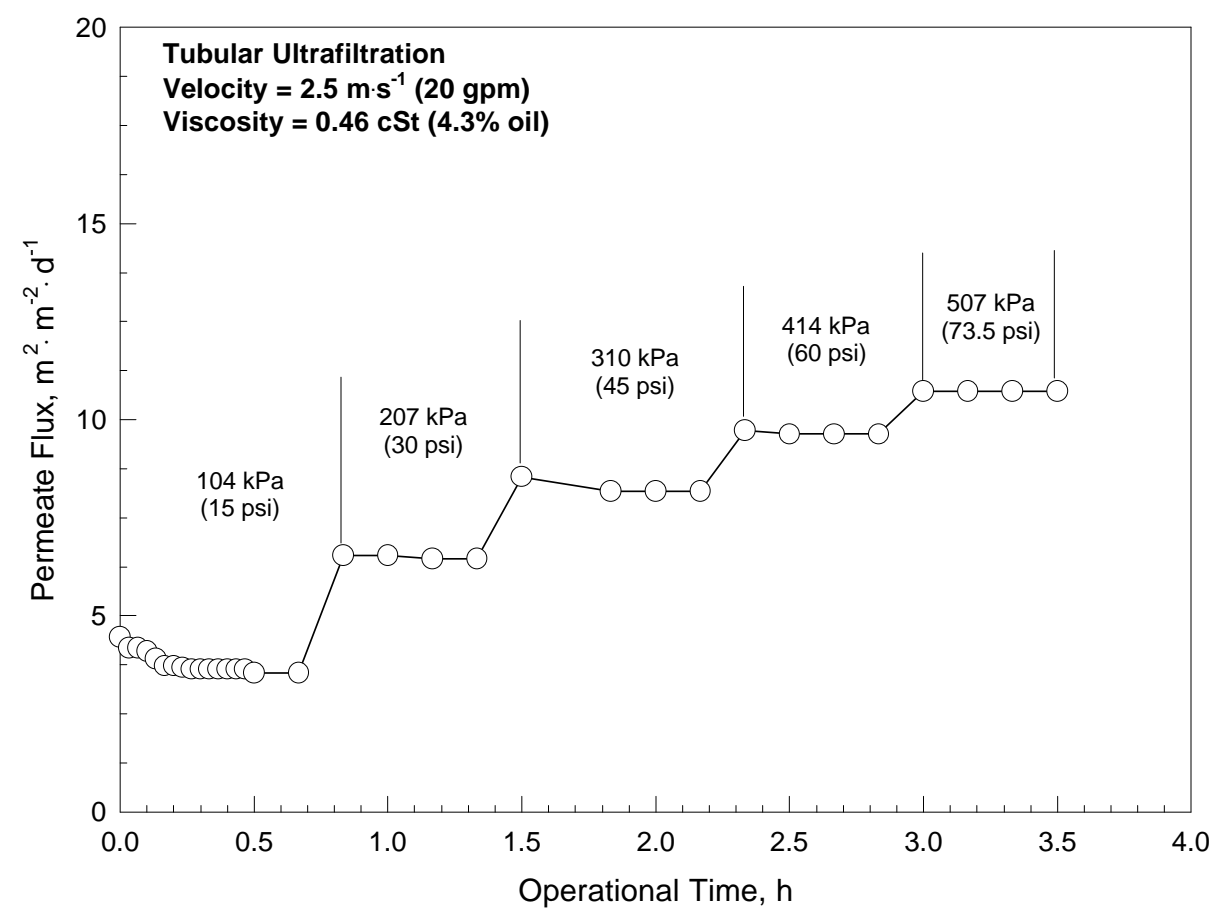

Figure 4.12 Permeate flux versus operational time for the $2.5 \mathrm{~m} \cdot \mathrm{s}^{-1}-0.46 \mathrm{cSt}$ experiment.

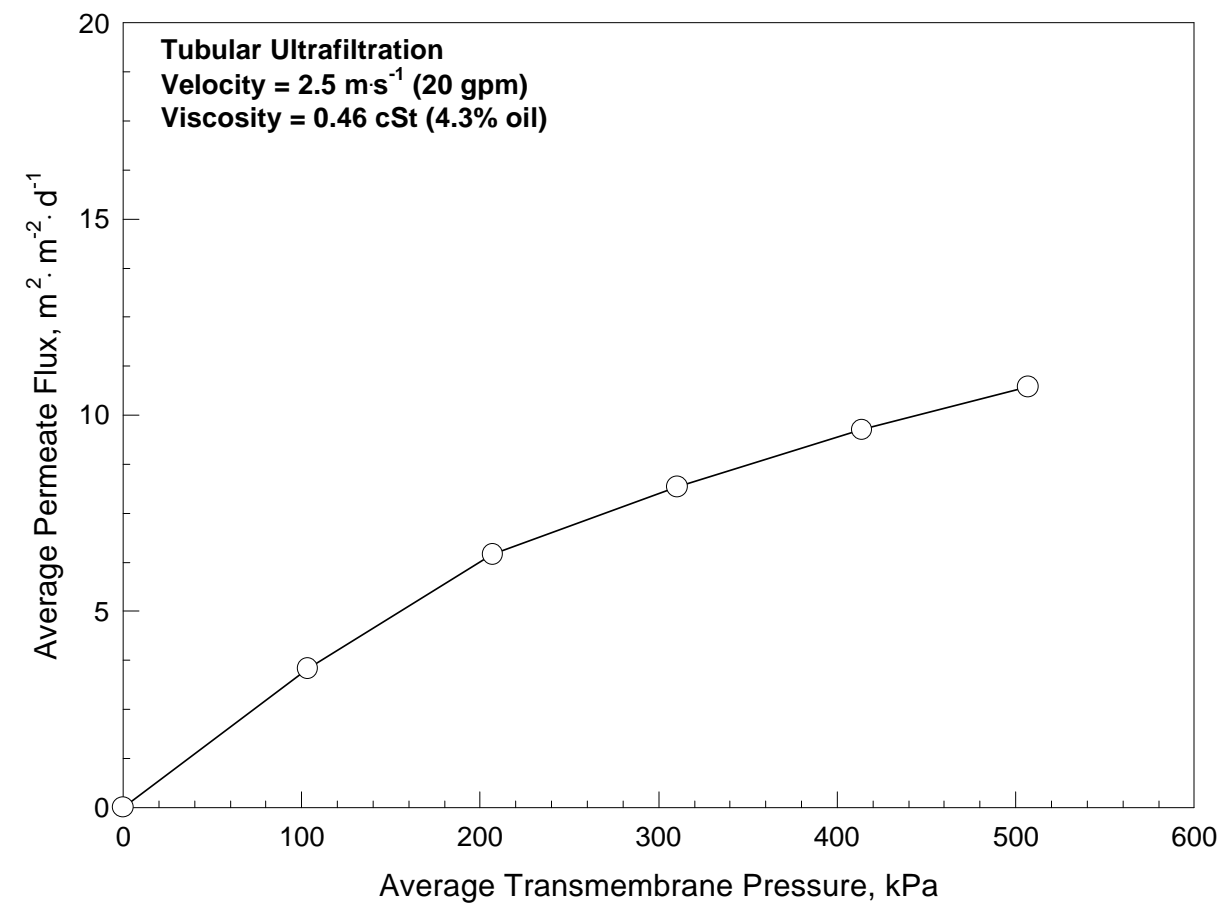

Figure 4.13 Average steady state permeate flux versus average transmembrane pressure for the $2.5 \mathrm{~m} \cdot \mathrm{s}^{-1}-0.46 \mathrm{cSt}$ experiment. 
conductivity throughout the entire run. Distinct step-wise changes in permeate and concentrate $\mathrm{pH}$ and conductivity were not observed as applied pressure was increased.

\subsection{7 $1.2 \mathrm{~m} \cdot \mathrm{s}^{-1}-\mathbf{0 . 4 6} \mathrm{cSt}(10 \mathrm{gpm}-5 \%$ MW Fluid)}

Permeate flux versus time for the $1.2 \mathrm{~m} \cdot \mathrm{s}^{-1}-0.46 \mathrm{cSt}$ experiment is presented in Figure 4.14. An initial decrease in permeate flux was observed over the first half-hour of operation, due to the formation of the concentration boundary layer at the membrane surface, at which point quasi steady-state permeate flux was achieved. Immediately after each pressure increase, a small decrease in permeate flux was observed after which the flux stabilized. This phenomenon was attributed to a delay in stabilization of the concentration boundary layer (Viadero and Reed 1999). Average steady state permeate flux versus average transmembrane pressure for the $1.2 \mathrm{~m} \cdot \mathrm{s}^{-1}-0.46 \mathrm{cSt}$ experiment is presented in Figure 4.15. A limiting permeate flux of $2.7 \mathrm{~m}^{3} \cdot \mathrm{m}^{-2} \cdot \mathrm{d}^{-1}$ was observed at the 310 to $414 \mathrm{kPa}$ (45 to $60 \mathrm{psi}$ ) conditions. The experiment was stopped after the $414 \mathrm{kPa}$ pressure condition was examined, due to the fact that a limiting permeate flux was already achieved. Any additional increase in transmembrane pressure would only result in further consolidation of the concentration boundary layer, thus increasing the potential for membrane fouling (Cheryan 1998).

The permeate $\mathrm{pH}$ ranged from 7.15 to 7.87 and averaged 7.34. The concentrate $\mathrm{pH}$ ranged from 7.23 to 7.61 and averaged 7.38. Permeate and concentrate $\mathrm{pH}$ were both relatively constant throughout the entire run. The permeate conductivity ranged from 48.8 to $146 \mu \mathrm{S} \cdot \mathrm{cm}^{-1}$ and averaged $116 \mu \mathrm{S} \cdot \mathrm{cm}^{-1}$. The concentrate conductivity ranged from 208 to $250 \mu \mathrm{S} \cdot \mathrm{cm}^{-1}$ and averaged $232 \mu \mathrm{S} \cdot \mathrm{cm}^{-1}$. Permeate and concentrate conductivity remained relatively constant and permeate conductivity was less than 


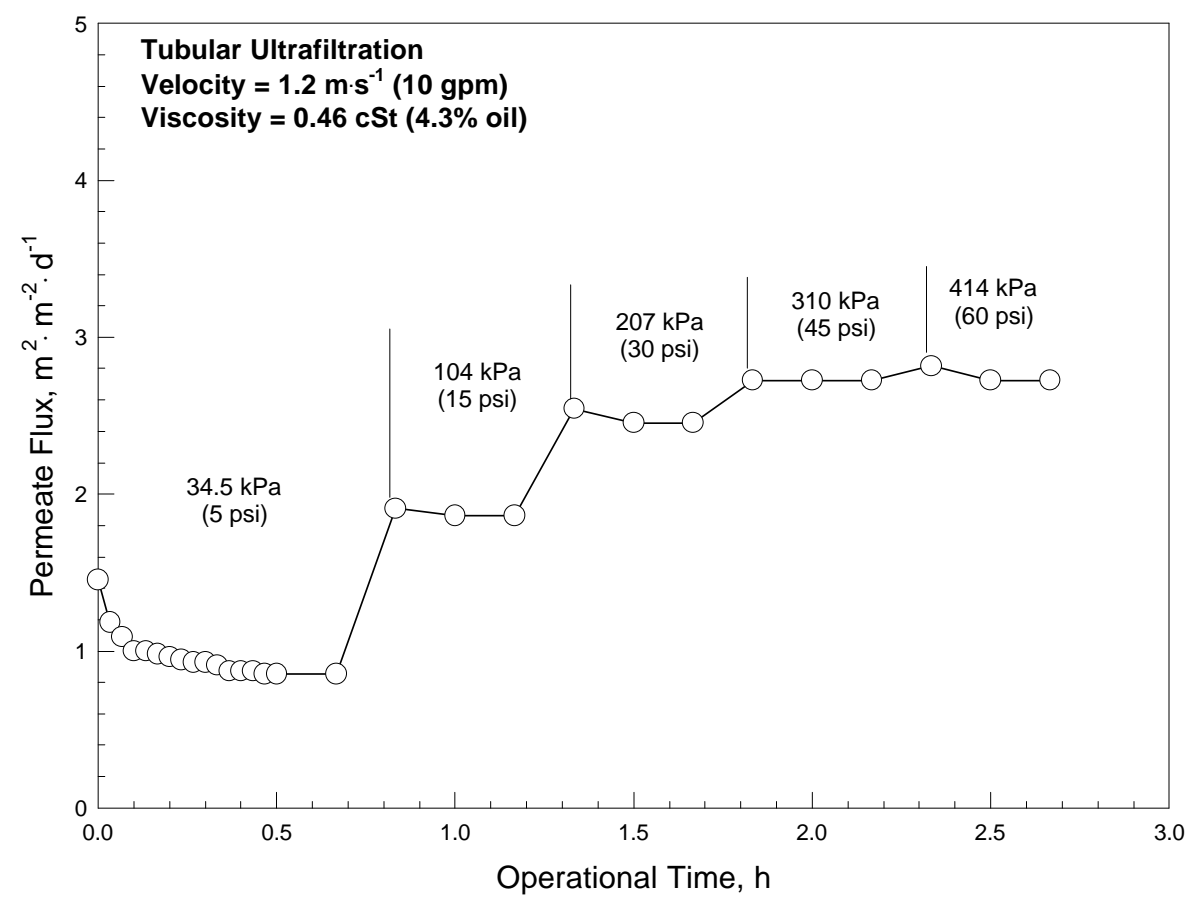

Figure 4.14 Permeate flux versus operational time for the $1.2 \mathrm{~m} \cdot \mathrm{s}^{-1}-0.46 \mathrm{cSt}$ experiment.

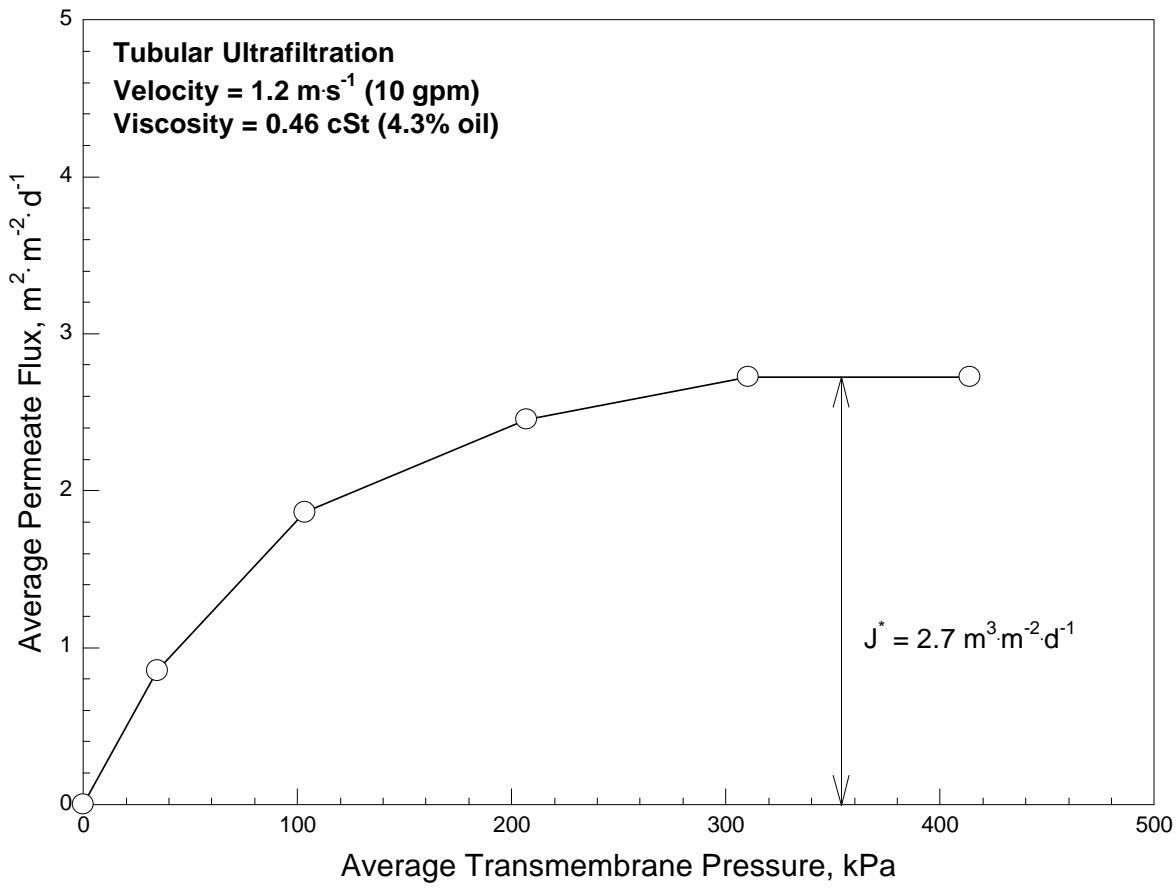

Figure 4.15 Average steady state permeate flux versus average transmembrane pressure for the $1.2 \mathrm{~m} \cdot \mathrm{s}^{-1}-0.46 \mathrm{cSt}$ experiment. 
concentrate conductivity throughout the entire run. Distinct step-wise changes in permeate and concentrate $\mathrm{pH}$ and conductivity were not observed as applied pressure was increased.

\subsection{8 $0.62 \mathrm{~m} \cdot \mathrm{s}^{-1}-0.46 \mathrm{cSt}-(5 \mathrm{gpm}-5 \%$ MW Fluid)}

Permeate flux versus time for the $0.62 \mathrm{~m} \cdot \mathrm{s}^{-1}-0.46 \mathrm{cSt}$ experiment is presented in Figure 4.16. An initial decrease in permeate flux was observed over the first ten minutes of operation, due to the formation of the concentration boundary layer at the membrane surface, at which point quasi steady-state permeate flux was achieved. Immediately after each pressure increase, a small decrease in permeate flux was observed after which the flux stabilized. This phenomenon was attributed to a delay in stabilization of the concentration boundary layer (Viadero and Reed 1999). Average steady state permeate flux versus average transmembrane pressure for the $0.62 \mathrm{~m} \cdot \mathrm{s}^{-1}-0.46 \mathrm{cSt}$ experiment is presented in Figure 4.17. A limiting permeate flux of $1.7 \mathrm{~m}^{3} \cdot \mathrm{m}^{-2} \cdot \mathrm{d}^{-1}$ was observed at the 310 to $414 \mathrm{kPa}$ (45 to $60 \mathrm{psi}$ ) conditions. The experiment was stopped after the $414 \mathrm{kPa}$ pressure condition was examined, due to the fact that a limiting permeate flux was already achieved. Any additional increase in transmembrane pressure would only result in further consolidation of the concentration boundary layer, thus increasing the potential for membrane fouling (Cheryan 1998).

The permeate $\mathrm{pH}$ ranged from 6.74 to 7.84 and averaged 7.41 . The concentrate $\mathrm{pH}$ ranged from 7.31 to 7.61 and averaged 7.45. Both the permeate and concentrate $\mathrm{pH}$ remained relatively constant throughout the entire run. The permeate conductivity ranged from 36.2 to $146 \mu \mathrm{S} \cdot \mathrm{cm}^{-1}$ and averaged $122 \mu \mathrm{S} \cdot \mathrm{cm}^{-1}$. The concentrate conductivity ranged from 229 to $252 \mu \mathrm{S} \cdot \mathrm{cm}^{-1}$ and averaged $238 \mu \mathrm{S} \cdot \mathrm{cm}^{-1}$. A net increase was observed 


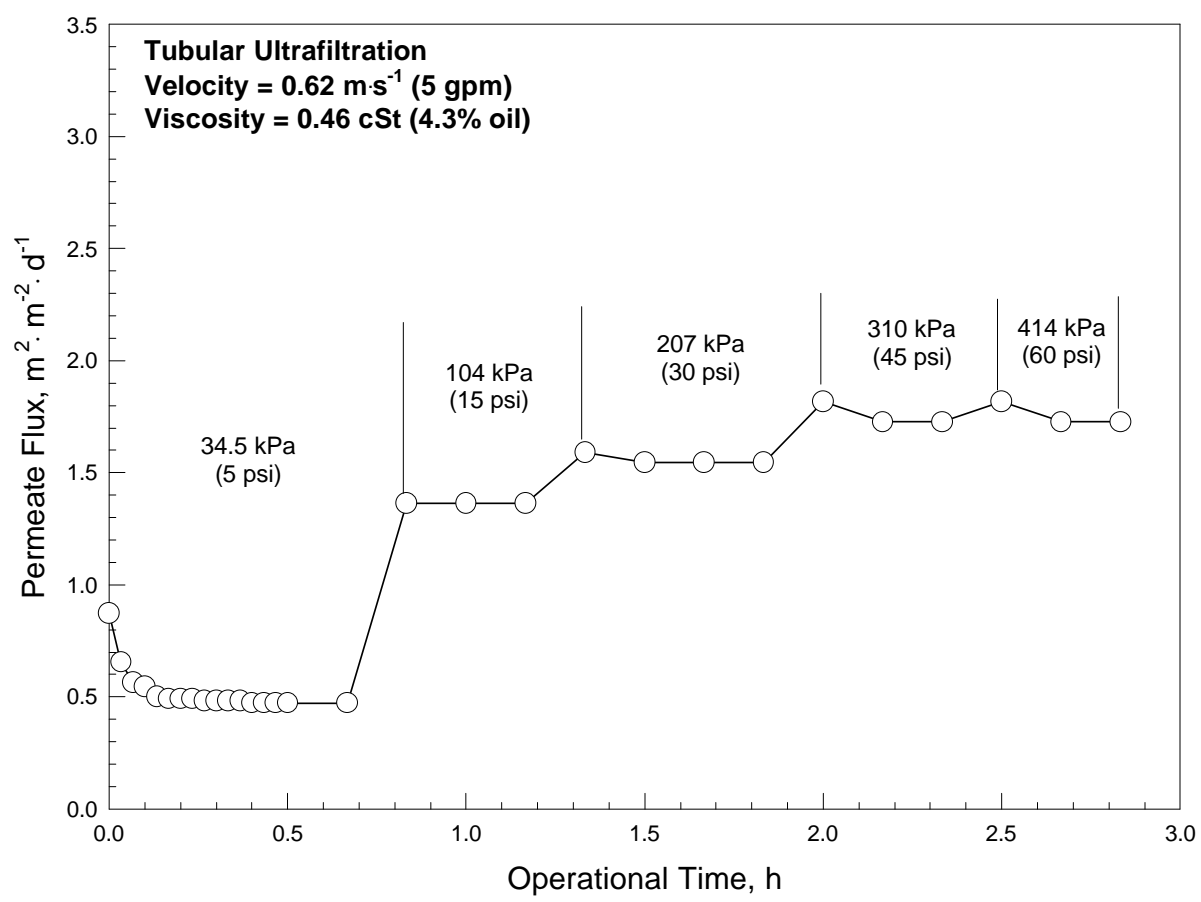

Figure 4.16 Permeate flux verus operational time for the $0.62 \mathrm{~m} \cdot \mathrm{s}^{-1}-0.46 \mathrm{cSt}$ experiment.

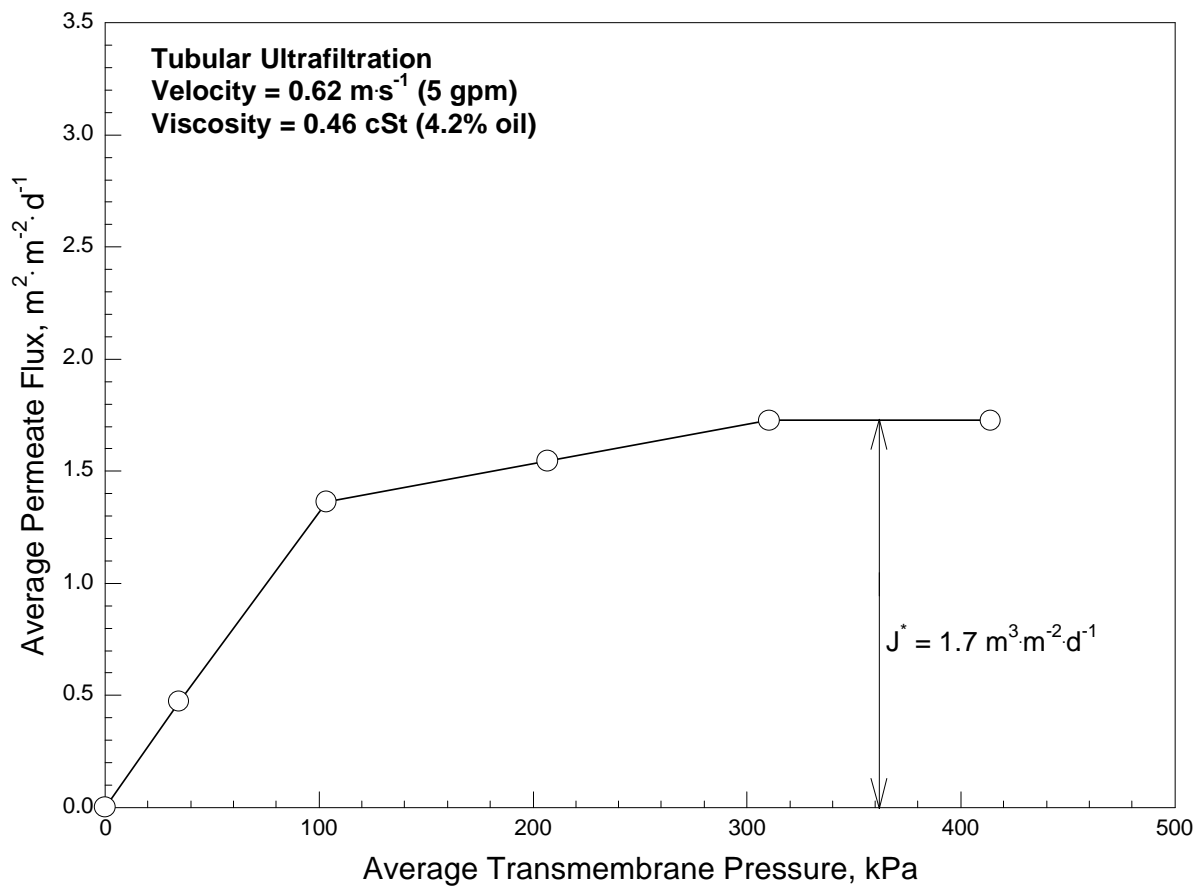

Figure 4.17 Average steady state permeate flux versus average transmembrane pressure for the $0.62 \mathrm{~m} \cdot \mathrm{s}^{-1}-0.46 \mathrm{cSt}$ experiment. 
In permeate conductivity over the first half-hour of the run, however, remained constant throughout the remainder of the run. Concentrate conductivity remained relatively constant and was greater than permeate conductivity throughout the entire run. Distinct step-wise changes in permeate and concentrate $\mathrm{pH}$ and conductivity were not observed as applied pressure was increased.

\subsubsection{7 $\mathrm{m} \cdot \mathrm{s}^{-1}-\mathbf{0 . 6 4} \mathrm{cSt}(30 \mathrm{gpm}-10 \% \mathrm{MW}$ Fluid)}

Permeate flux versus time for the $3.7 \mathrm{~m} \cdot \mathrm{s}^{-1}-0.64 \mathrm{cSt}$ experiment is presented in Figure 4.18. An initial decrease in permeate flux was observed over the first halfhour of operation, due to the formation of the concentration boundary layer at the membrane surface, at which point quasi steady-state permeate flux was achieved. Distinct step-wise increases in flux were observed as $P$ was increased from 104 to $372 \mathrm{kPa}(15$ to $54 \mathrm{psi})$. Average steady state permeate flux versus average transmembrane pressure for the $3.7 \mathrm{~m} \cdot \mathrm{s}^{-1}-0.64 \mathrm{cSt}$ experiment is presented in Figure 4.19. A limiting flux was not observed over the applied pressure range studied in this experiment; however, the average flux data diverged from linear behavior at higher pressures due to a shift from pressure-dependent to pressure-independent operating conditions.

The permeate $\mathrm{pH}$ ranged from 6.92 to 7.52 and averaged 7.12. The concentrate $\mathrm{pH}$ ranged from 7.06 to 7.44 and averaged 7.19. A net decrease was observed in both permeate and concentrate $\mathrm{pH}$ throughout the run. The permeate conductivity ranged from 248 to $265 \mu \mathrm{S} \cdot \mathrm{cm}^{-1}$ and averaged $258 \mu \mathrm{S} \cdot \mathrm{cm}^{-1}$. The concentrate conductivity ranged from 369 to $498 \mu \mathrm{S} \cdot \mathrm{cm}^{-1}$ and averaged $442 \mu \mathrm{S} \cdot \mathrm{cm}^{-1}$. Permeate conductivity remained relatively constant and was less than concentrate conductivity throughout the entire run. A net decrease was observed in concentrate conductivity throughout the run. Distinct 


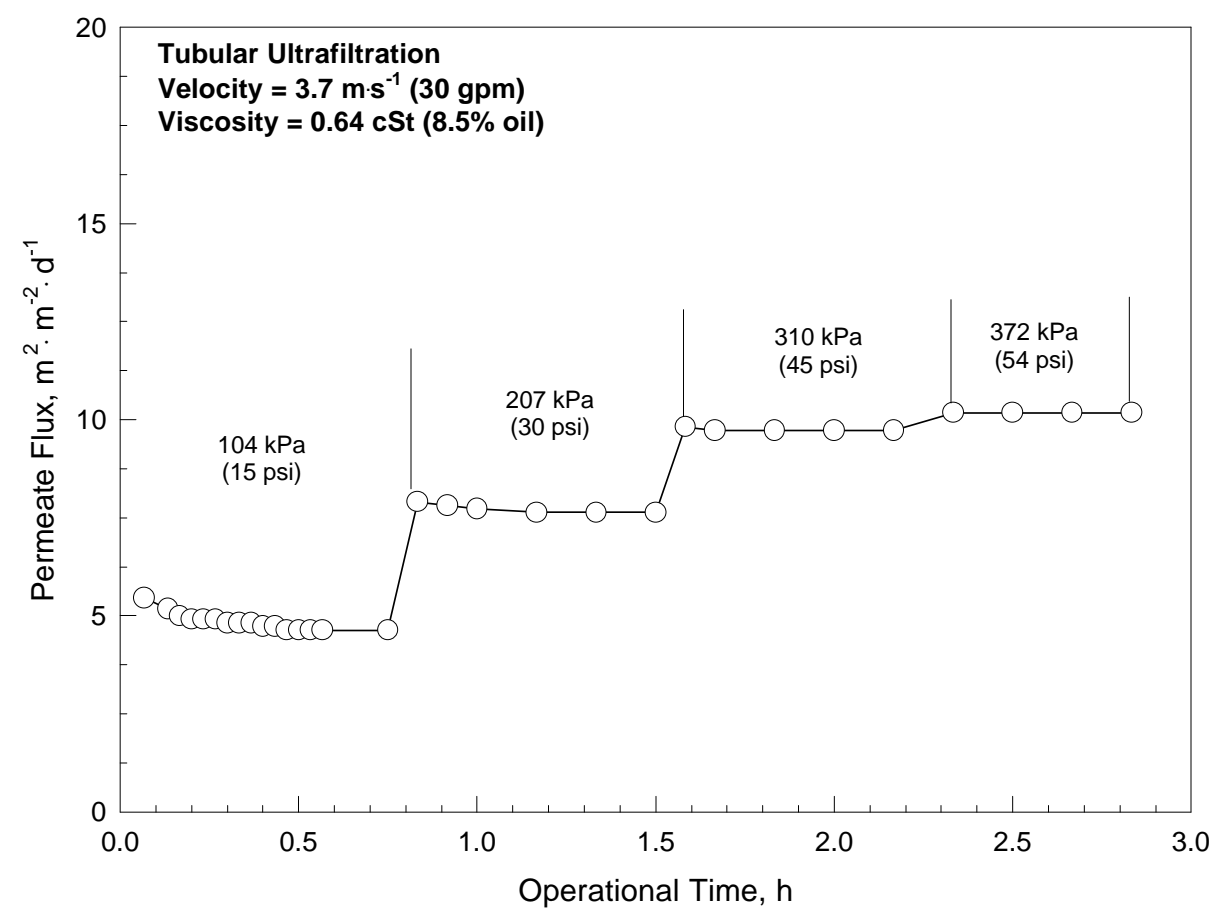

Figure 4.18 Permeate flux versus operational time for the $3.7 \mathrm{~m} \cdot \mathrm{s}^{-1}-0.64 \mathrm{cSt}$ experiment.

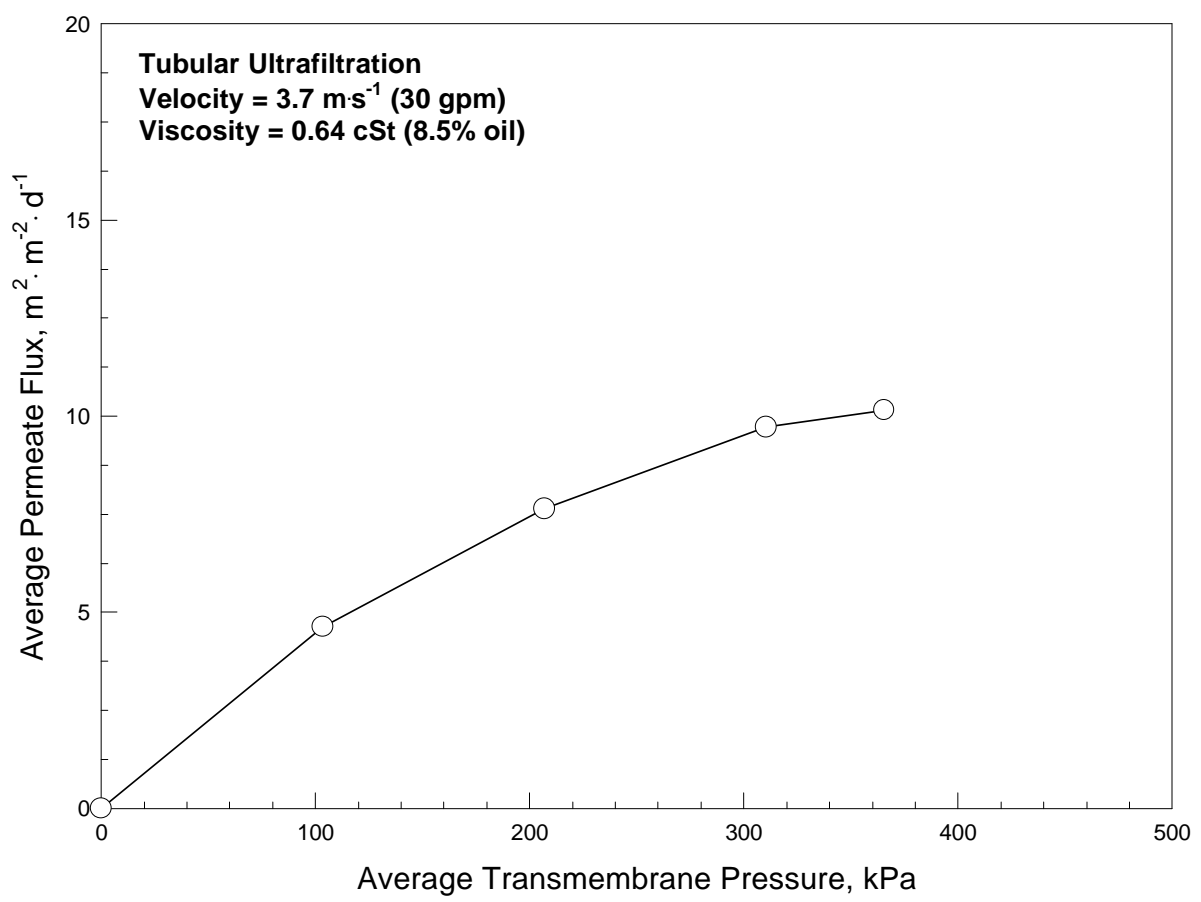

Figure 4.19 Average steady state permeate flux versus average transmembrane pressure for the $3.7 \mathrm{~m} \cdot \mathrm{s}^{-1}-0.64 \mathrm{cSt}$ experiment. 
Step-wise changes in permeate and concentrate $\mathrm{pH}$ and conductivity were not observed as applied pressure was increased.

\subsubsection{0 $2.5 \mathrm{~m} \cdot \mathrm{s}^{-1}-0.64 \mathrm{cSt}(20 \mathrm{gpm}-10 \%$ MW Fluid)}

Permeate flux versus time for the $2.5 \mathrm{~m} \cdot \mathrm{s}^{-1}-0.64 \mathrm{cSt}$ experiment is presented in Figure 4.20. An initial decrease in permeate flux was observed over the first twenty-five minutes of operation, due to the formation of the concentration boundary layer at the membrane surface, at which point quasi steady-state permeate flux was achieved. Distinct step-wise increases in flux were observed as $\mathrm{P}$ was increased from 104 to 504 $\mathrm{kPa}$ (15 to $73 \mathrm{psi}$ ). Average steady state permeate flux versus average transmembrane pressure for the $2.5 \mathrm{~m} \cdot \mathrm{s}^{-1}-0.64 \mathrm{cSt}$ experiment is presented in Figure 4.21. A limiting flux was not observed over the applied pressure range studied in this experiment; however, the average flux data diverged from linear behavior at higher pressures due to a shift from pressure-dependent to pressure-independent operating conditions.

The permeate $\mathrm{pH}$ ranged from 7.14 to 7.89 and averaged 7.36. The concentrate $\mathrm{pH}$ ranged from 7.28 to 7.68 and averaged 7.41. Both permeate and concentrate $\mathrm{pH}$ were relatively constant throughout the run. The permeate conductivity ranged from 101 to $119 \mu \mathrm{S} \cdot \mathrm{cm}^{-1}$ and averaged $111 \mu \mathrm{S} \cdot \mathrm{cm}^{-1}$. The concentrate conductivity ranged from 154 to $196 \mu \mathrm{S} \cdot \mathrm{cm}^{-1}$ and averaged $176 \mu \mathrm{S} \cdot \mathrm{cm}^{-1}$. Permeate and concentrate conductivity remained relatively constant and permeate was less than concentrate conductivity throughout the entire run. Distinct step-wise changes in permeate and concentrate $\mathrm{pH}$ and conductivity were not observed as applied pressure was increased. 


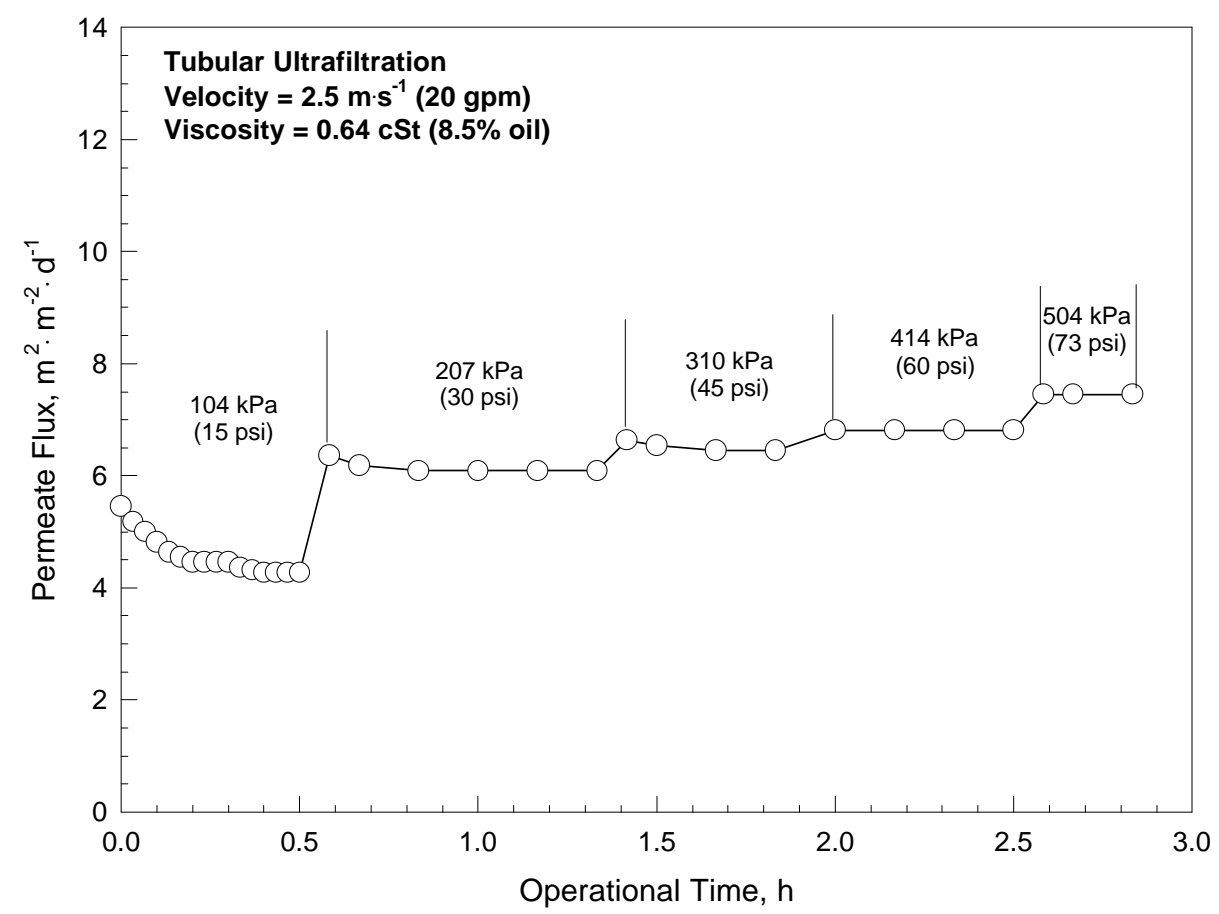

Figure 4.20 Permeate flux versus operational time for the $2.5 \mathrm{~m} \cdot \mathrm{s}^{-1}-0.64 \mathrm{cSt}$ experiment.

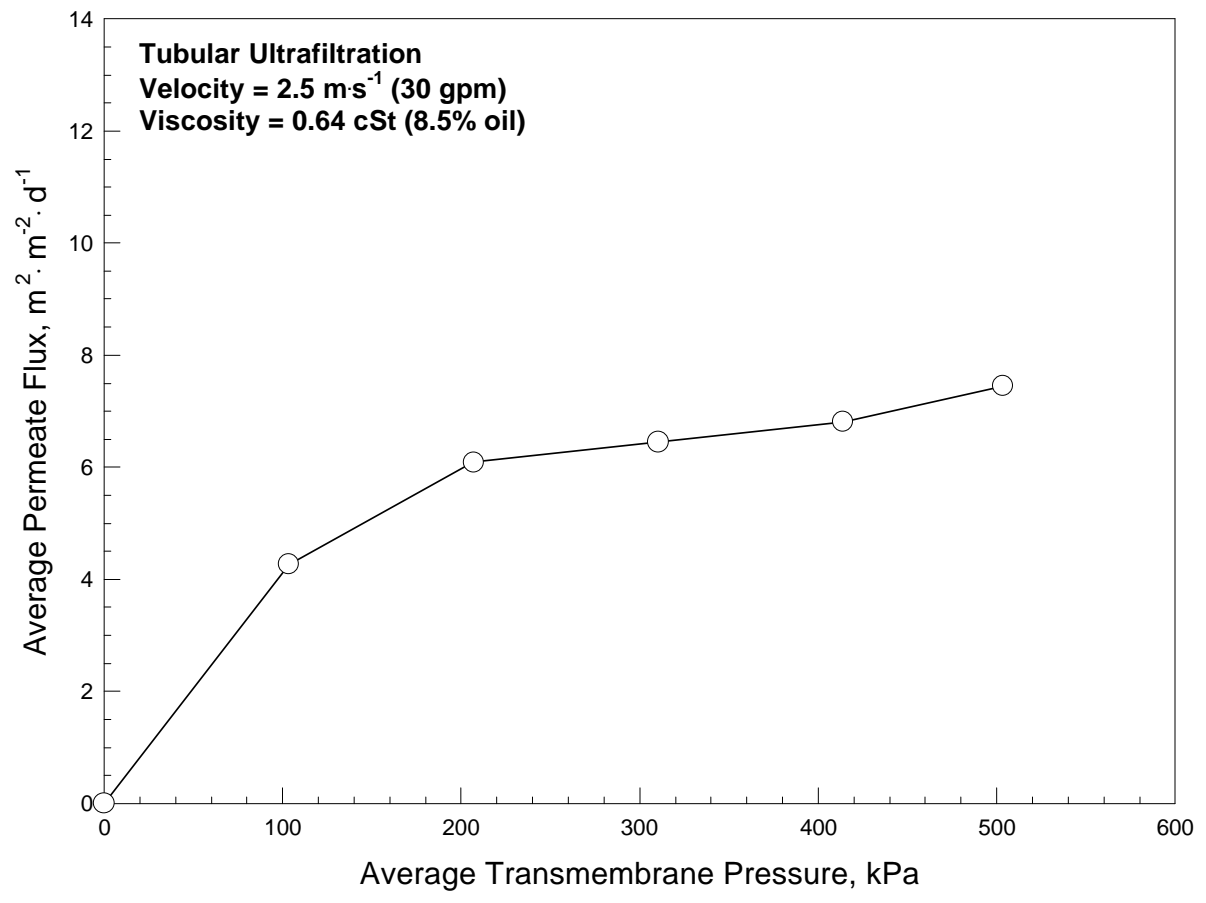

Figure 4.21 Average steady state permeate flux versus average transmembrane pressure for the $2.5 \mathrm{~m} \cdot \mathrm{s}^{-1}-0.64 \mathrm{cSt}$ experiment. 


\subsubsection{1 $1.2 \mathrm{~m} \cdot \mathrm{s}^{-1}-0.64 \mathrm{cSt}(10 \mathrm{gpm}-10 \% \mathrm{MW}$ Fluid)}

Permeate flux versus time for the $1.2 \mathrm{~m} \cdot \mathrm{s}^{-1}-0.64 \mathrm{cSt}$ experiment is presented in Figure 4.22. An initial decrease in permeate flux was observed over the first twenty minutes of operation, due to the formation of the concentration boundary layer at the membrane surface, at which point quasi steady-state permeate flux was achieved. Immediately after the transition from 104 to $207 \mathrm{kPa}$ (15 to $30 \mathrm{psi}$ ) and 207 to $310 \mathrm{kPa}$ (30 to 45 psi) conditions, a small increase in permeate flux was observed after which the flux dropped and stabilized at the value observed during the previous pressure condition. This phenomenon was attributed to a delay in stabilization of the concentration boundary layer (Viadero and Reed 1999). Average steady state permeate flux versus average transmembrane pressure for the $1.2 \mathrm{~m} \cdot \mathrm{s}^{-1}-0.64 \mathrm{cSt}$ experiment is presented in Figure 4.23. A limiting permeate flux of $2.1 \mathrm{~m}^{3} \cdot \mathrm{m}^{-2} \cdot \mathrm{d}^{-1}$ was observed at the 207 to $310 \mathrm{kPa}$ (30 to 45 psi) conditions. The experiment was stopped after the $310 \mathrm{kPa}$ pressure condition was examined, due to the fact that a limiting permeate flux was already achieved. Any additional increase in transmembrane pressure would only result in further consolidation of the concentration boundary layer, thus increasing the potential for membrane fouling (Cheryan 1998).

The permeate $\mathrm{pH}$ ranged from 7.20 to 7.86 and averaged 7.42. The concentrate $\mathrm{pH}$ ranged from 7.20 to 7.62 and averaged 7.38. Permeate and concentrate $\mathrm{pH}$ were both relatively constant throughout the entire run. The permeate conductivity ranged from 59.5 to $245 \mu \mathrm{S} \cdot \mathrm{cm}^{-1}$ and averaged $213 \mu \mathrm{S} \cdot \mathrm{cm}^{-1}$. The concentrate conductivity ranged from 350 to $374 \mu \mathrm{S} \cdot \mathrm{cm}^{-1}$ and averaged $358 \mu \mathrm{S} \cdot \mathrm{cm}^{-1}$. Permeate and concentrate conductivity remained relatively constant and permeate conductivity was less than 


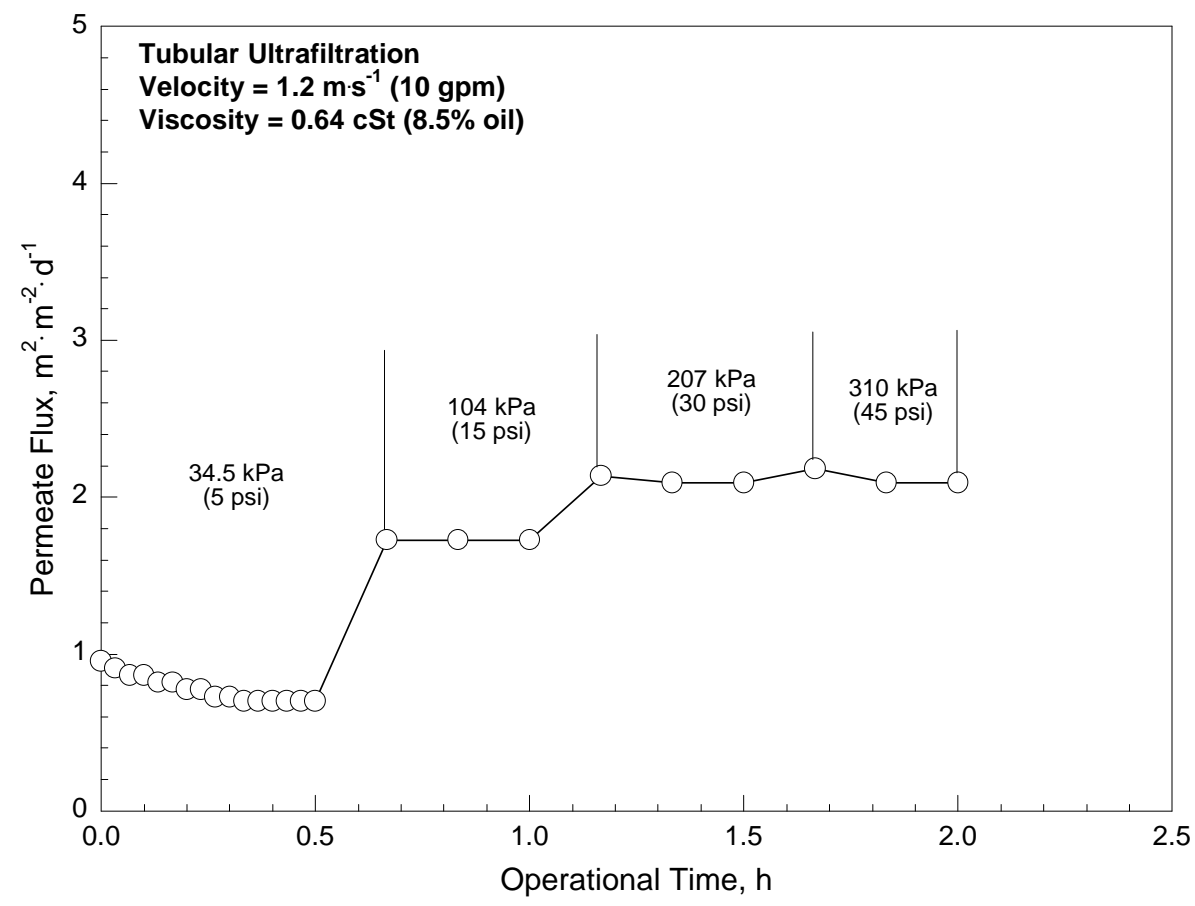

Figure 4.22 Permeate flux versus operational time for the $1.2 \mathrm{~m} \cdot \mathrm{s}^{-1}-0.64 \mathrm{cSt}$ experiment.

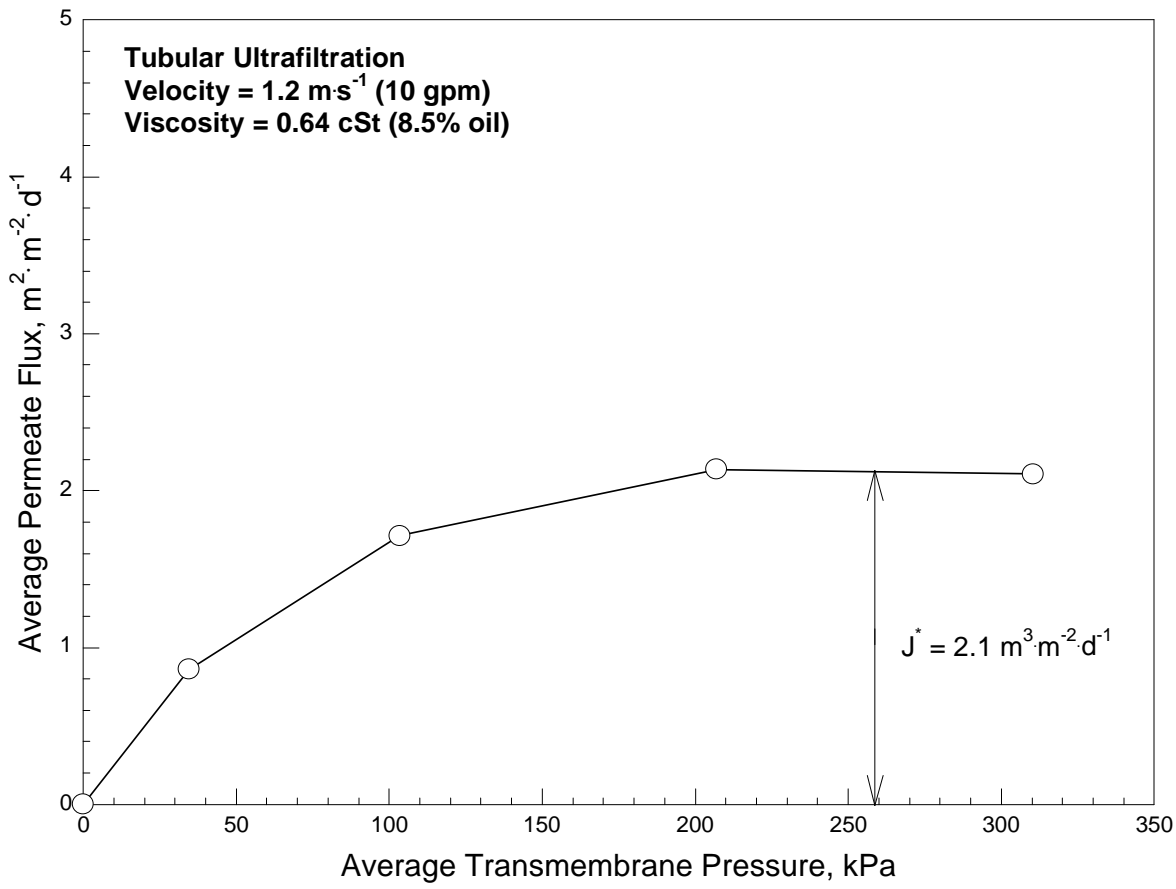

Figure 4.23 Average steady state permeate flux versus average transmembrane pressure for the $1.2 \mathrm{~m} \cdot \mathrm{s}^{-1}-0.64 \mathrm{cSt}$ experiment. 
concentrate conductivity throughout the entire run. Distinct step-wise changes in permeate and concentrate $\mathrm{pH}$ and conductivity were not observed as applied pressure was increased.

\subsection{2 $\mathrm{m} \cdot \mathrm{s}^{-1}-0.64 \mathrm{cSt}-(5 \mathrm{gpm}-10 \%$ MW Fluid)}

Permeate flux versus time for the $0.62 \mathrm{~m} \cdot \mathrm{s}^{-1}-0.64 \mathrm{cSt}$ experiment is presented in Figure 4.24. An initial decrease in permeate flux was observed over the first twenty minutes of operation, due to the formation of the concentration boundary layer at the membrane surface, at which point quasi steady-state permeate flux was achieved. Average steady state permeate flux versus average transmembrane pressure for the 0.62 $\mathrm{m} \cdot \mathrm{s}^{-1}-0.64 \mathrm{cSt}$ experiment is presented in Figure 4.25. A limiting permeate flux of 1.1 $\mathrm{m}^{3} \cdot \mathrm{m}^{-2} \cdot \mathrm{d}^{-1}$ was observed at the 104 to $207 \mathrm{kPa}$ (15 to $\left.30 \mathrm{psi}\right)$ conditions. The experiment was stopped after the $207 \mathrm{kPa}$ pressure condition was examined, due to the fact that a limiting permeate flux was already achieved. Any additional increase in transmembrane pressure would only result in further consolidation of the concentration boundary layer, thus increasing the potential for membrane fouling (Cheryan 1998).

The permeate $\mathrm{pH}$ ranged from 6.94 to 7.76 and averaged 7.47. The concentrate $\mathrm{pH}$ ranged from 7.35 to 7.61 and averaged 7.48. A net decrease was observed in permeate and concentrate $\mathrm{pH}$ throughout the entire run. The permeate conductivity ranged from 16.2 to $54.8 \mu \mathrm{S} \cdot \mathrm{cm}^{-1}$ and averaged $44.6 \mu \mathrm{S} \cdot \mathrm{cm}^{-1}$. The concentrate conductivity ranged from 51.7 to $67.2 \mu \mathrm{S} \cdot \mathrm{cm}^{-1}$ and averaged $58.2 \mu \mathrm{S} \cdot \mathrm{cm}^{-1}$. Permeate and concentrate conductivity remained relatively constant throughout the entire run. Distinct step-wise changes in permeate and concentrate $\mathrm{pH}$ and conductivity were not observed as applied pressure was increased. 


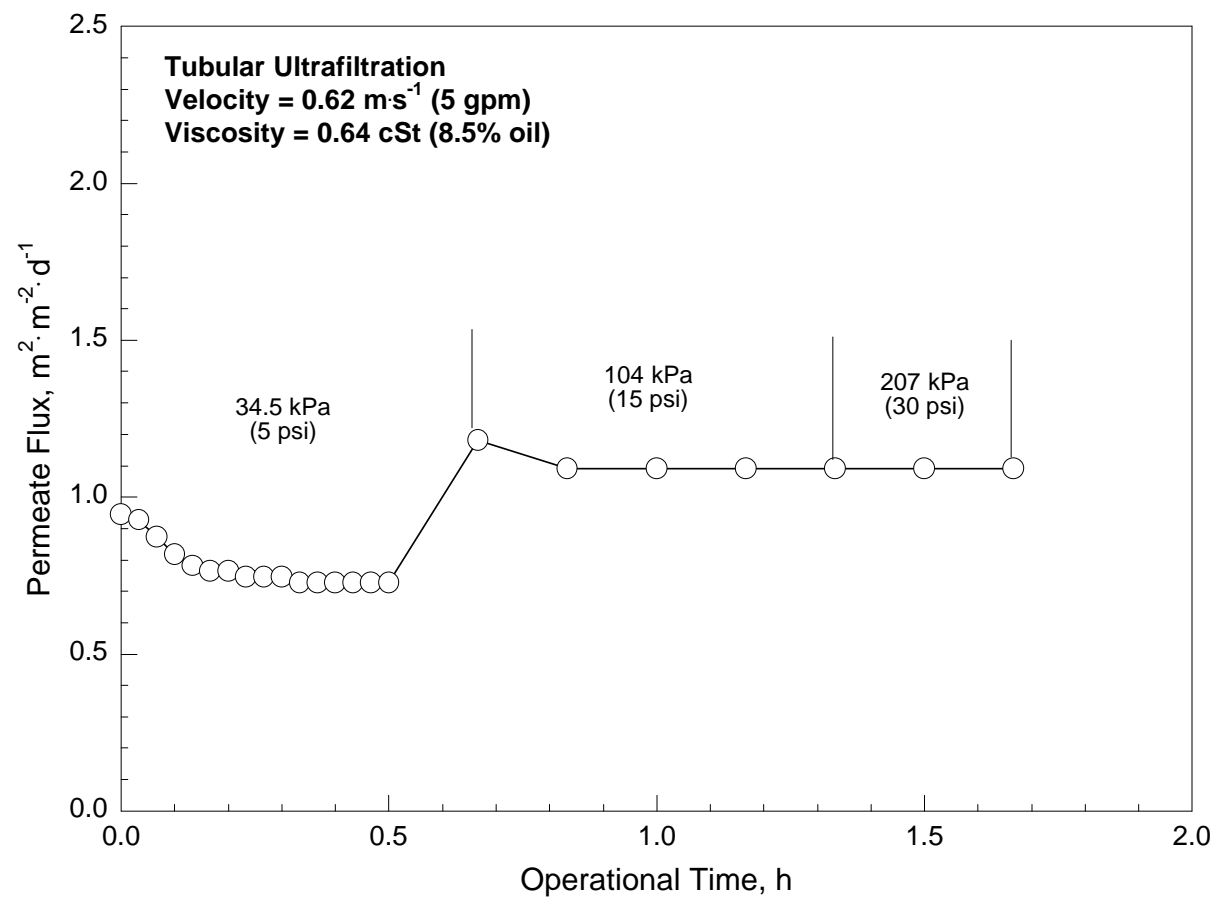

Figure 4.24 Permeate flux versus operational time for the $0.62 \mathrm{~m} \cdot \mathrm{s}^{-1}-0.64 \mathrm{cSt}$ experiment.

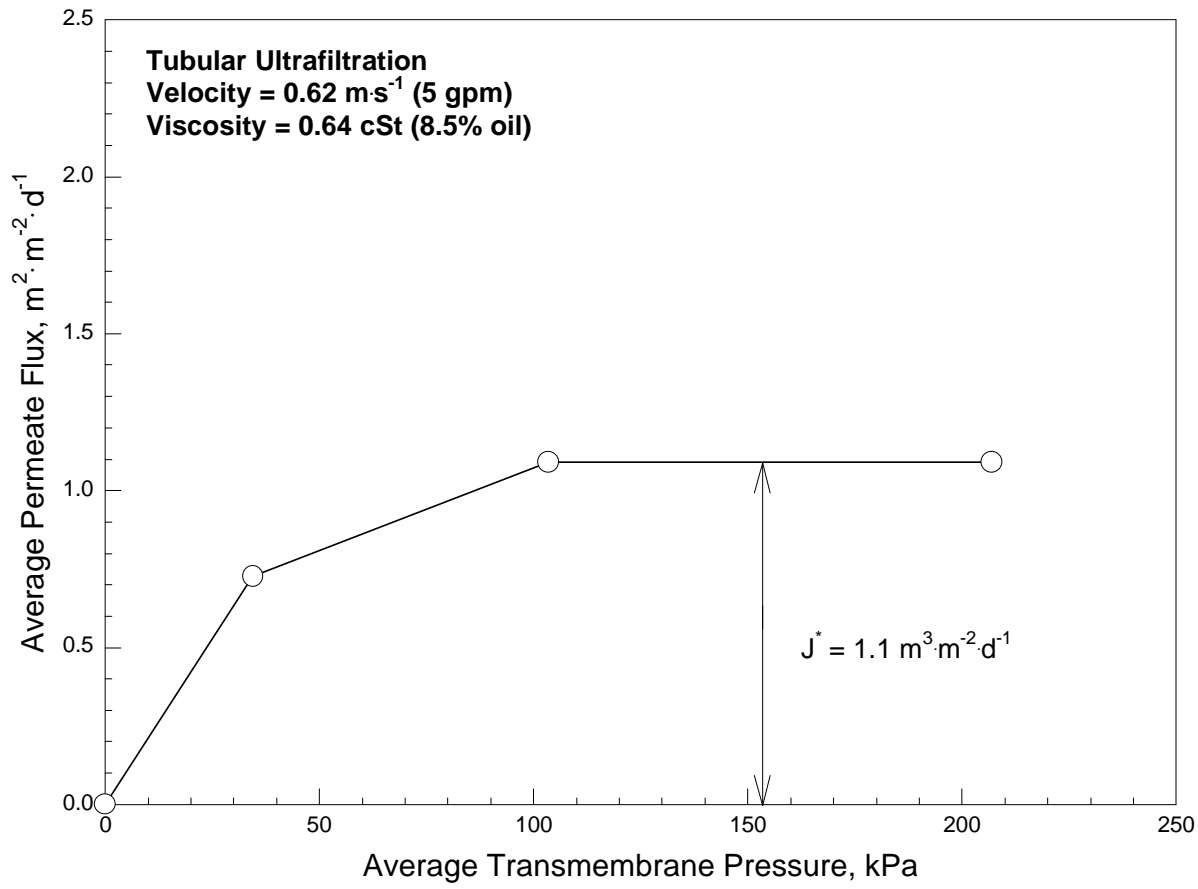

Figure 4.25 Average steady state permeate flux versus average transmembrane pressure for the $0.62 \mathrm{~m} \cdot \mathrm{s}^{-1}-0.64 \mathrm{cSt}$ experiment. 


\subsubsection{7 $\mathrm{m} \cdot \mathrm{s}^{-1}-0.94 \mathrm{cSt}(30 \mathrm{gpm}-15 \%$ MW Fluid)}

Permeate flux versus time for the $3.7 \mathrm{~m} \cdot \mathrm{s}^{-1}-0.94 \mathrm{cSt}$ experiment is presented in Figure 4.26. An initial decrease in permeate flux was observed over the first twentyminutes of operation, due to the formation of the concentration boundary layer at the membrane surface, at which point quasi steady-state permeate flux was achieved. Distinct step-wise increases in flux were observed as P was increased from 104 to 372 $\mathrm{kPa}$ (15 to $54 \mathrm{psi}$ ). Average steady state permeate flux versus average transmembrane pressure for the $3.7 \mathrm{~m} \cdot \mathrm{s}^{-1}-0.94 \mathrm{cSt}$ experiment is presented in Figure 4.27. A limiting flux was not observed over the applied pressure range studied in this experiment; however, the average flux data diverged from linear behavior at higher pressures due to a shift from pressure-dependent to pressure-independent operating conditions.

The permeate $\mathrm{pH}$ ranged from 6.82 to 7.44 and averaged 7.09. The concentrate $\mathrm{pH}$ ranged from 6.86 to 7.37 and averaged 7.14. A net decrease was observed in both permeate and concentrate $\mathrm{pH}$ throughout the run. The permeate conductivity ranged from 190 to $212 \mu \mathrm{S} \cdot \mathrm{cm}^{-1}$ and averaged $201 \mu \mathrm{S} \cdot \mathrm{cm}^{-1}$. The concentrate conductivity ranged from 260 to $326 \mu \mathrm{S} \cdot \mathrm{cm}^{-1}$ and averaged $284 \mu \mathrm{S} \cdot \mathrm{cm}^{-1}$. Permeate and concentrate conductivity remained relatively constant and permeate was less than concentrate conductivity throughout the entire run. Distinct step-wise changes in permeate and concentrate $\mathrm{pH}$ and conductivity were not observed as applied pressure was increased.

\subsubsection{4 $2.5 \mathrm{~m} \cdot \mathrm{s}^{-1}-0.94 \mathrm{cSt}(20 \mathrm{gpm}-15 \%$ MW Fluid)}

Permeate flux versus time for the $2.5 \mathrm{~m} \cdot \mathrm{s}^{-1}-0.94 \mathrm{cSt}$ experiment is presented in Figure 4.28. An initial decrease in permeate flux was observed over the first twenty minutes of operation, due to the formation of the concentration boundary layer at the 


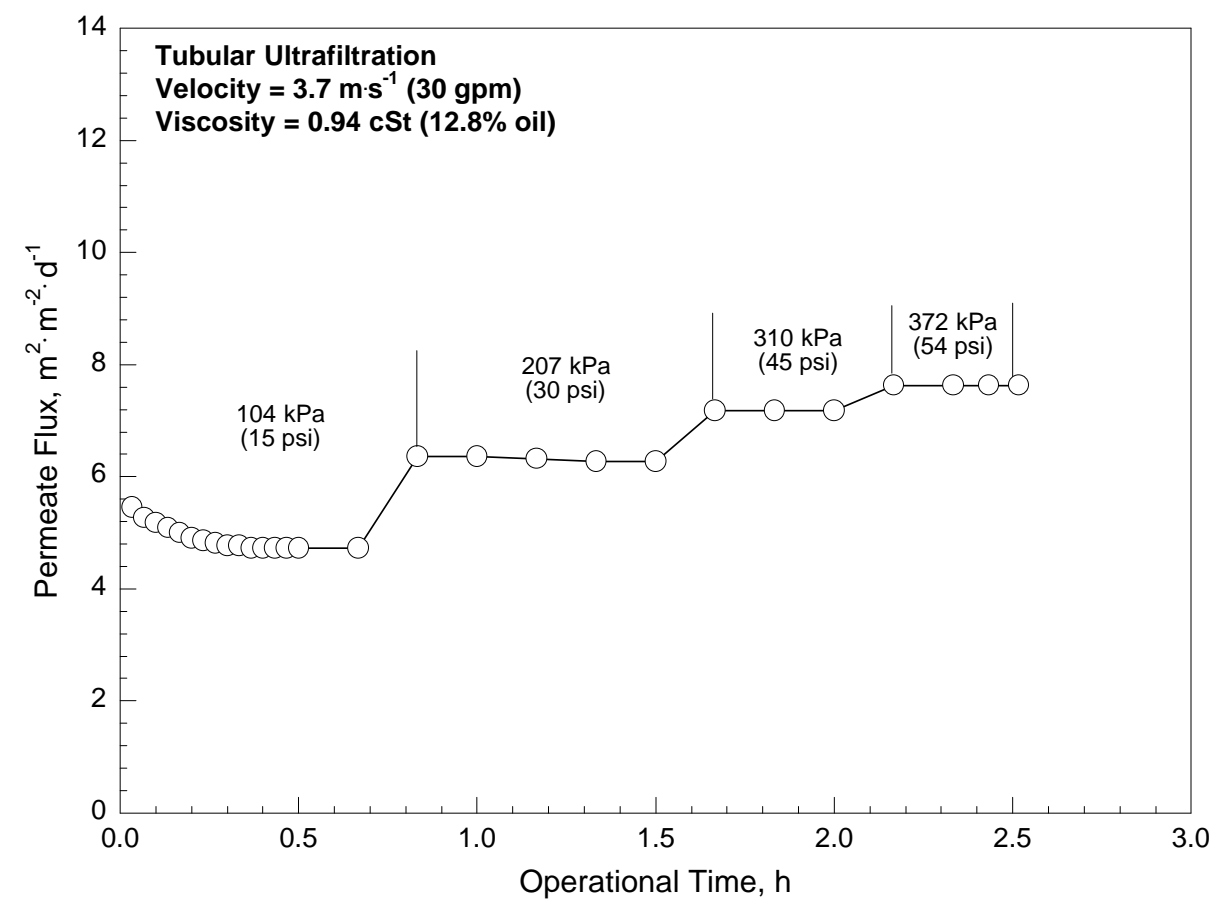

Figure 4.26 Permeate flux versus operational time for the $3.7 \mathrm{~m} \cdot \mathrm{s}^{-1}-0.94 \mathrm{cSt}$ experiment.

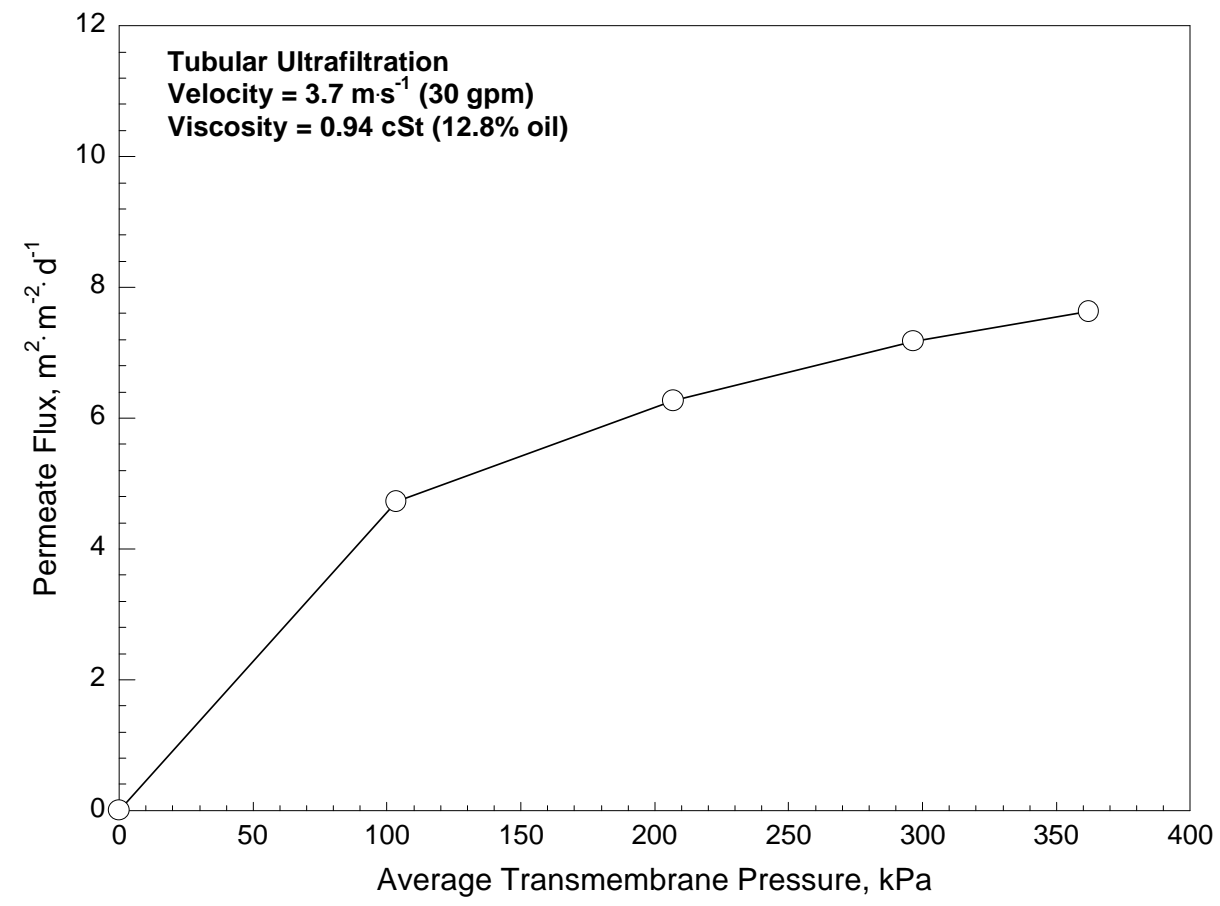

Figure 4.27 Average steady state permeate flux versus average transmembrane pressure for the $3.7 \mathrm{~m} \cdot \mathrm{s}^{-1}-0.94 \mathrm{cSt}$ experiment. 


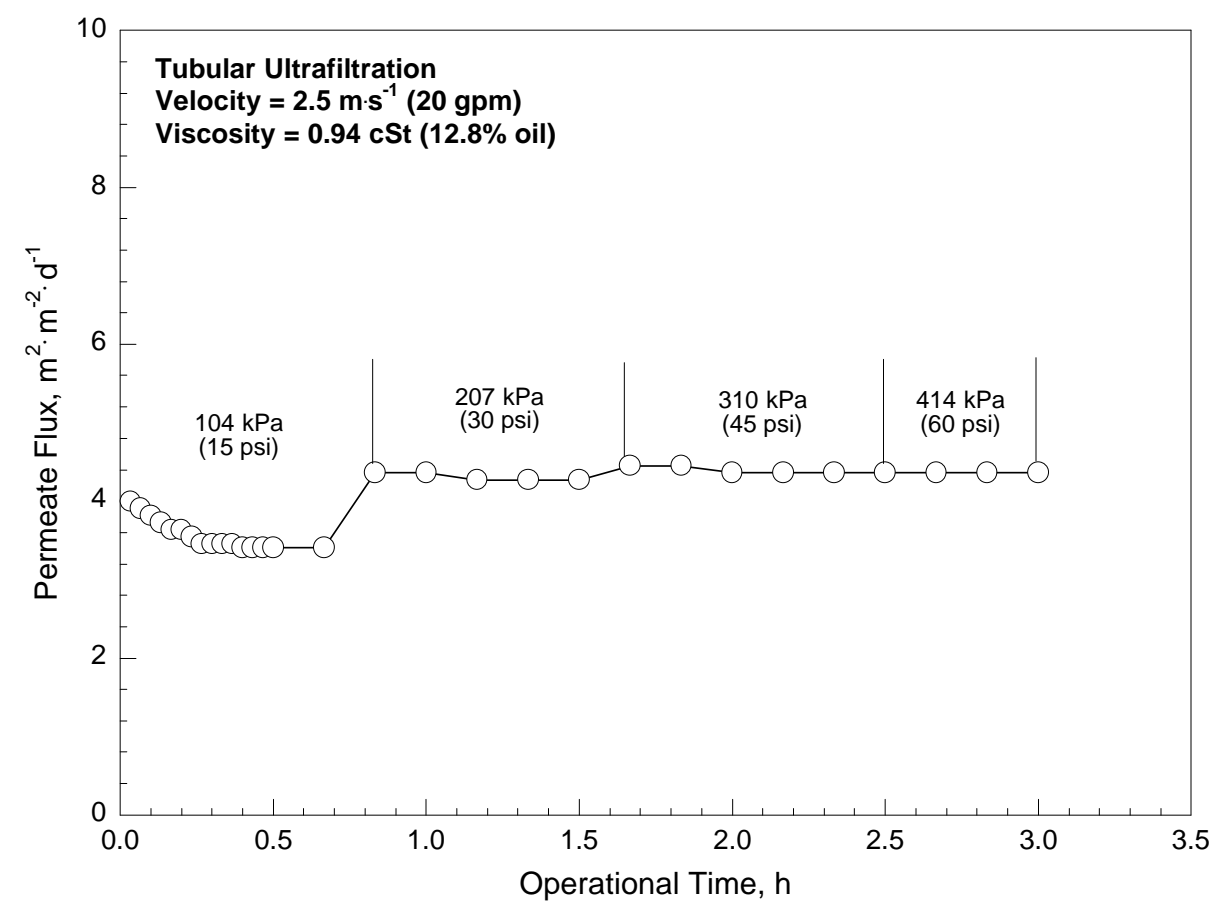

\section{Figure 4.28 Permeate flux versus operational time for the $2.5 \mathrm{~m} \cdot \mathrm{s}^{-1}-0.94 \mathrm{cSt}$ experiment.}

membrane surface, at which point quasi steady-state permeate flux was achieved. Average steady state permeate flux versus average transmembrane pressure for the 2.5 $\mathrm{m} \cdot \mathrm{s}^{-1}-0.94 \mathrm{cSt}$ experiment is presented in Figure 4.29. A limiting permeate flux of 4.4 $\mathrm{m}^{3} \cdot \mathrm{m}^{-2} \cdot \mathrm{d}^{-1}$ was observed at the 310 to $414 \mathrm{kPa}$ (45 to $60 \mathrm{psi}$ ) conditions. The experiment was stopped after the $414 \mathrm{kPa}$ pressure condition was examined, due to the fact that a limiting permeate flux was already achieved. Any additional increase in transmembrane pressure would only result in further consolidation of the concentration boundary layer, thus increasing the potential for membrane fouling (Cheryan 1998).

The permeate $\mathrm{pH}$ ranged from 6.59 to 7.60 and averaged 7.03. The concentrate $\mathrm{pH}$ ranged from 6.73 to 7.68 and averaged 7.03. A net decrease was observed in both permeate and concentrate $\mathrm{pH}$, throughout the run. The permeate conductivity ranged from 329 to $396 \mu \mathrm{S} \cdot \mathrm{cm}^{-1}$ and averaged $365 \mu \mathrm{S} \cdot \mathrm{cm}^{-1}$. The concentrate conductivity ranged 


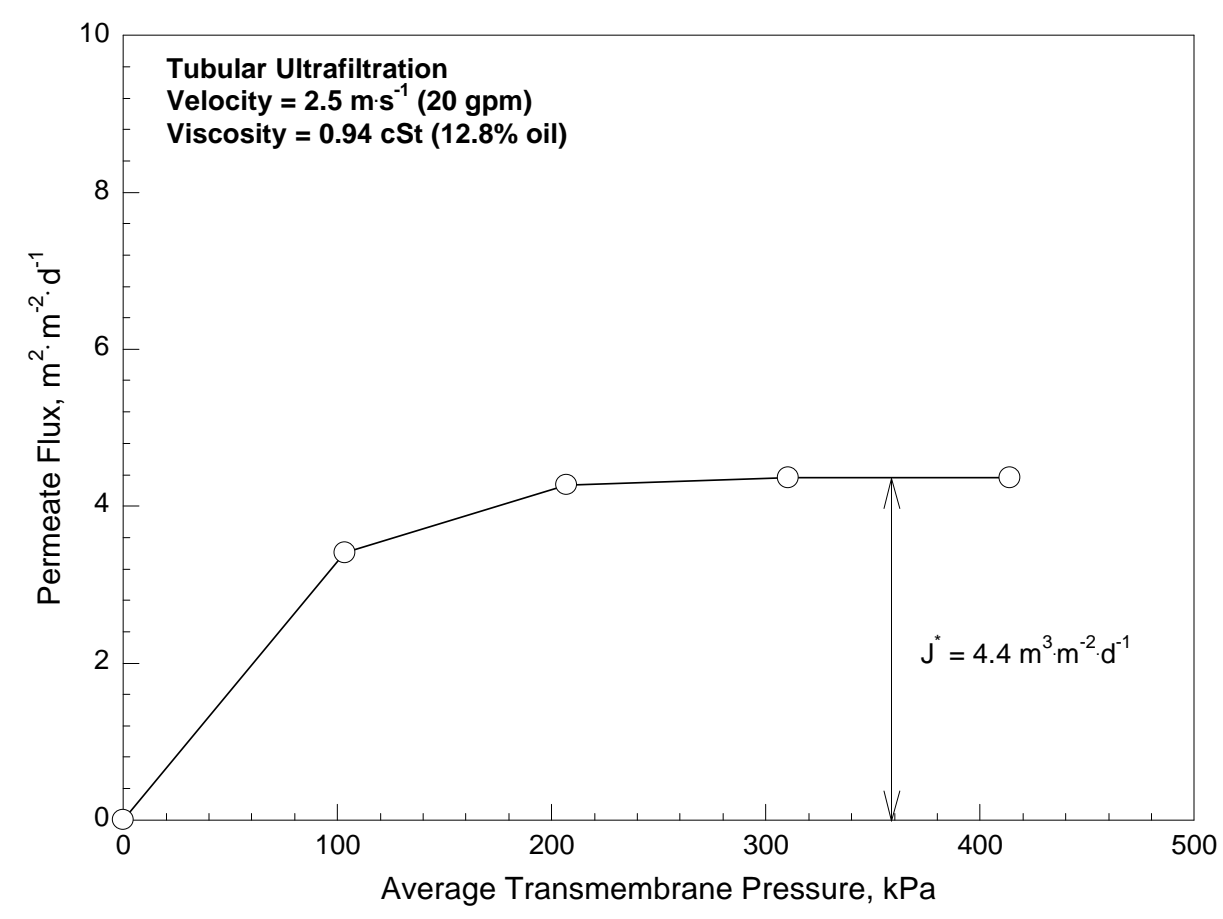

Figure 4.29 Average steady state permeate flux versus average transmembrane pressure for the $2.5 \mathrm{~m} \cdot \mathrm{s}^{-1}-0.94 \mathrm{cSt}$ experiment.

from 424 to $581 \mu \mathrm{S} \cdot \mathrm{cm}^{-1}$ and averaged $522 \mu \mathrm{S} \cdot \mathrm{cm}^{-1}$. A net decrease was observed in permeate conductivity, however, a net increase was observed in the concentrate conductivity. Permeate conductivity was less than concentrate conductivity throughout the entire run. Distinct step-wise changes in permeate and concentrate $\mathrm{pH}$ and conductivity were not observed as applied pressure was increased.

\subsubsection{2 $\mathrm{m} \cdot \mathrm{s}^{-1}-0.94 \mathrm{cSt}(10 \mathrm{gpm}-15 \%$ MW Fluid $)$}

Permeate flux versus time for the $1.2 \mathrm{~m} \cdot \mathrm{s}^{-1}-0.94 \mathrm{cSt}$ experiment is presented in Figure 4.30. An initial decrease in permeate flux was observed over the first twenty-five minutes of operation, due to the formation of the concentration boundary layer at the membrane surface, at which point quasi steady-state permeate flux was achieved. Average steady state permeate flux versus average transmembrane pressure for the 1.23 $\mathrm{m} \cdot \mathrm{s}^{-1}-0.94 \mathrm{cSt}$ experiment is presented in Figure 4.31. A limiting permeate flux of 1.5 


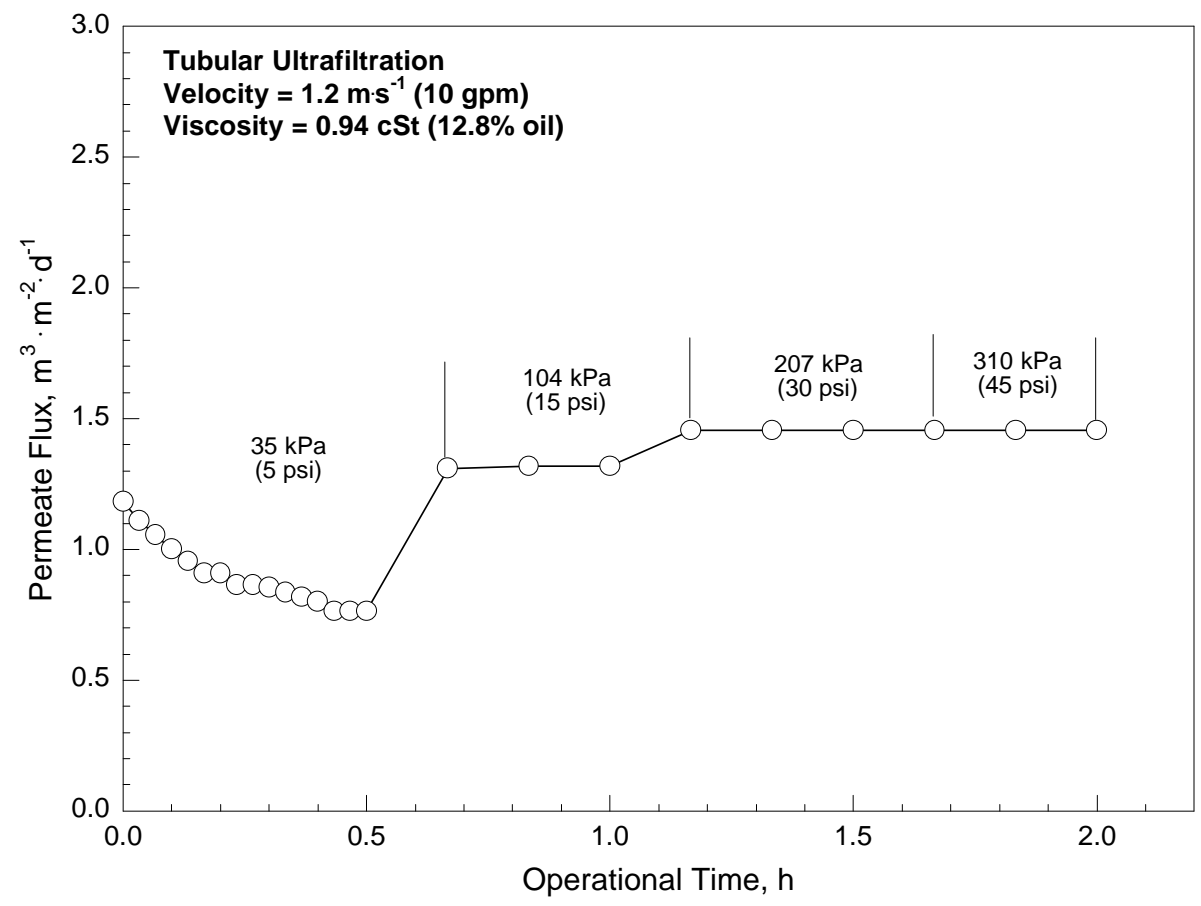

Figure 4.30 Permeate flux versus operational time for the $1.2 \mathrm{~m} \cdot \mathrm{s}^{-1}-0.94 \mathrm{cSt}$ experiment.

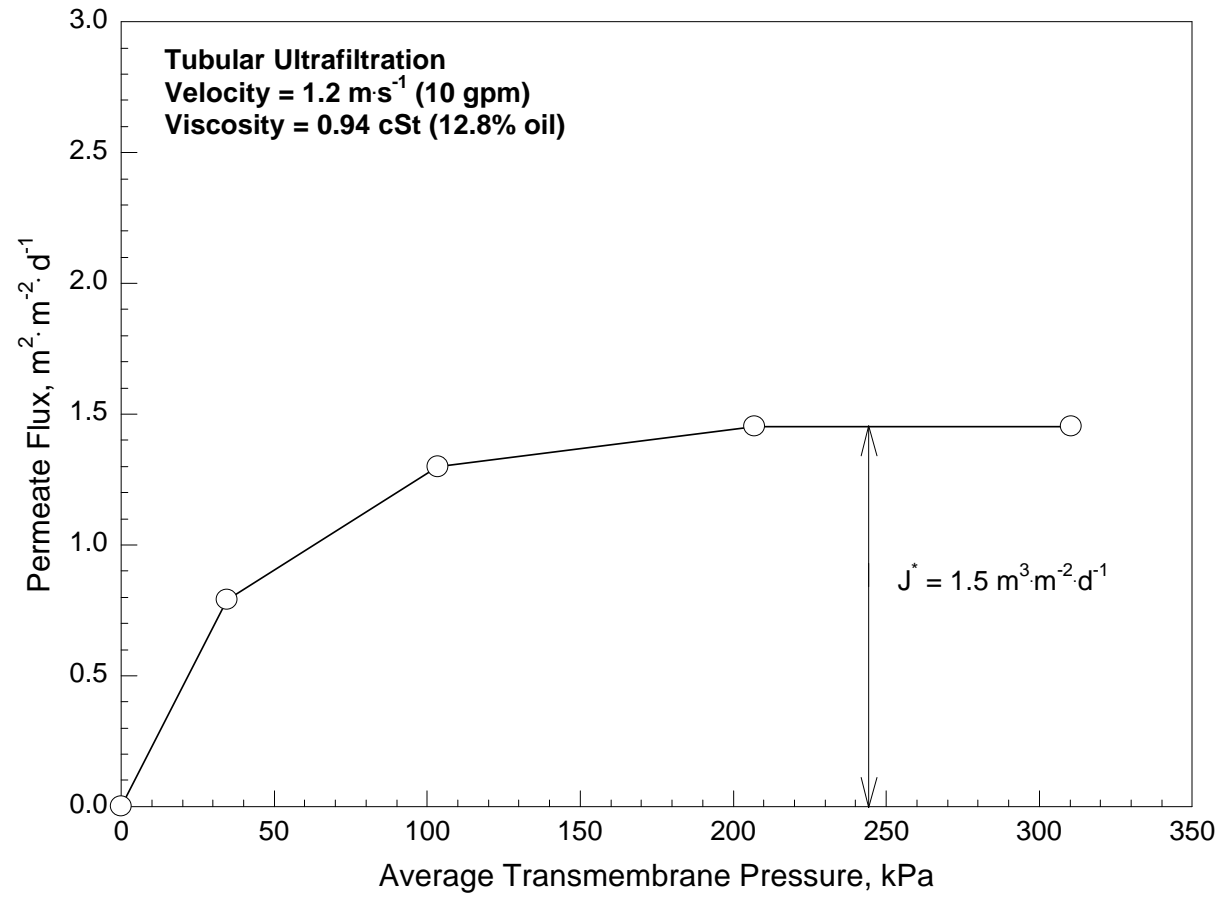

Figure 4.31 Average steady state permeate flux versus average transmembrane pressure for the $1.2 \mathrm{~m} \cdot \mathrm{s}^{-1}-0.94 \mathrm{cSt}$ experiment. 
$\mathrm{m}^{3} \cdot \mathrm{m}^{-2} \cdot \mathrm{d}^{-1}$ was observed at the 207 to $310 \mathrm{kPa}$ (30 to $45 \mathrm{psi}$ ) conditions. The experiment was stopped after the $310 \mathrm{kPa}$ pressure condition was examined, due to the fact that a limiting permeate flux was already achieved. Any additional increase in transmembrane pressure would only result in further consolidation of the concentration boundary layer, thus increasing the potential for membrane fouling (Cheryan 1998).

The permeate $\mathrm{pH}$ ranged from 7.32 to 7.72 and averaged 7.53. The concentrate $\mathrm{pH}$ ranged from 7.40 to 7.64 and averaged 7.53. Permeate and concentrate $\mathrm{pH}$ were both relatively constant throughout the entire run. The permeate conductivity ranged from 233 to $430 \mu \mathrm{S} \cdot \mathrm{cm}^{-1}$ and averaged $375 \mu \mathrm{S} \cdot \mathrm{cm}^{-1}$. The concentrate conductivity ranged from 525 to $604 \mu \mathrm{S} \cdot \mathrm{cm}^{-1}$ and averaged $560 \mu \mathrm{S} \cdot \mathrm{cm}^{-1}$. Permeate and concentrate conductivity remained relatively constant and permeate conductivity was less than concentrate conductivity throughout the entire run. Distinct step-wise changes in permeate and concentrate $\mathrm{pH}$ and conductivity were not observed as applied pressure was increased.

\subsection{2 $\mathrm{m} \cdot \mathrm{s}^{-1}-0.94 \mathrm{cSt}(5 \mathrm{gpm}-15 \%$ MW Fluid)}

Permeate flux versus time for the $0.62 \mathrm{~m} \cdot \mathrm{s}^{-1}-0.94 \mathrm{cSt}$ experiment is presented in Figure 4.32. An initial decrease in permeate flux was observed over the first half-hour of operation, due to the formation of the concentration boundary layer at the membrane surface, at which point quasi steady-state permeate flux was achieved. Average steady state permeate flux versus average transmembrane pressure for the $0.62 \mathrm{~m} \cdot \mathrm{s}^{-1}-0.94 \mathrm{cSt}$ experiment is presented in Figure 4.33. A limiting permeate flux of $0.50 \mathrm{~m}^{3} \cdot \mathrm{m}^{-2} \cdot \mathrm{d}^{-1}$ was observed at the 103 to $207 \mathrm{kPa}$ (15 to $30 \mathrm{psi}$ ) conditions. The experiment was stopped after the $207 \mathrm{kPa}$ pressure condition was examined, due to the fact that a limiting permeate flux was already achieved. Any additional increase in transmembrane pressure 


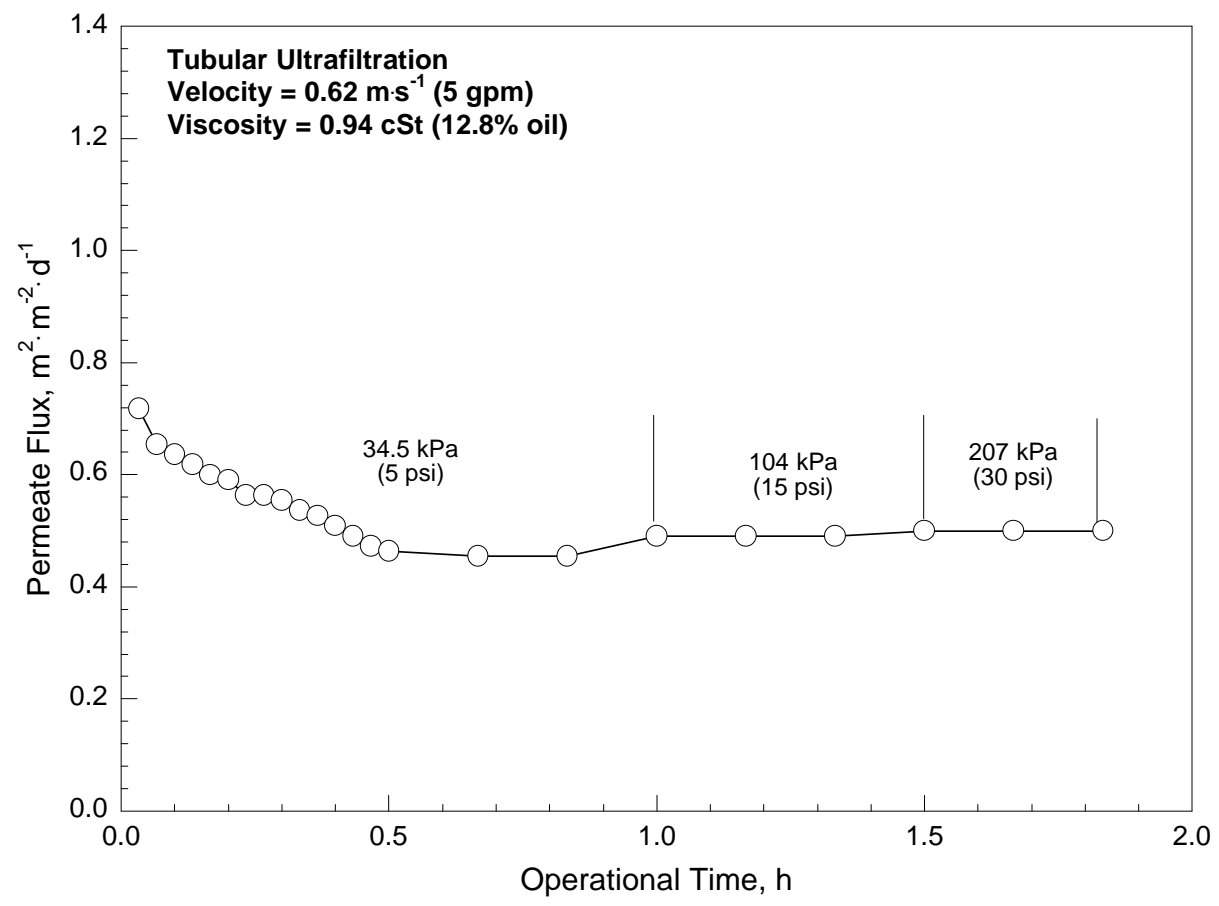

Figure 4.32 Permeate flux versus operational time for the $0.62 \mathrm{~m} \cdot \mathrm{s}^{-1}-0.94 \mathrm{cSt}$ experiment.

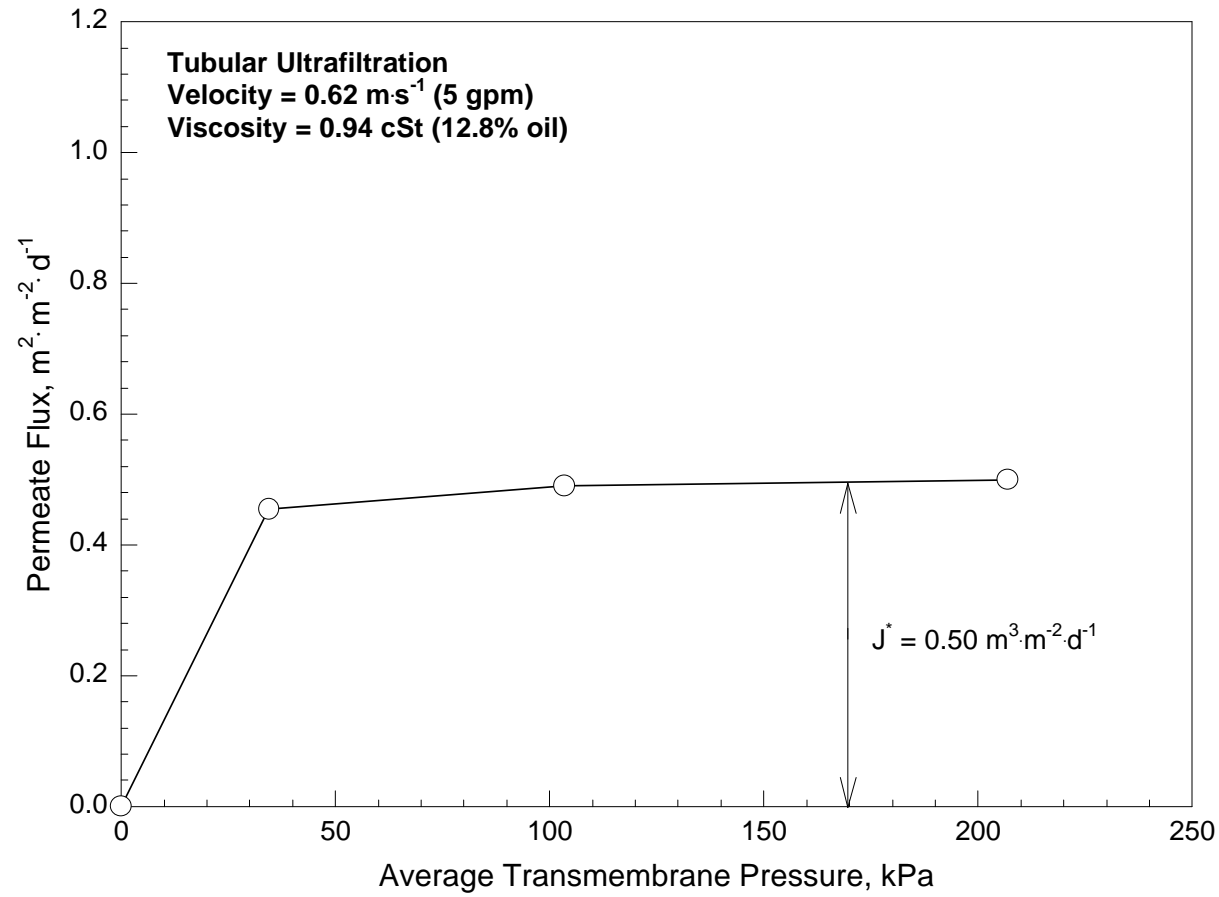

Figure 4.33 Average steady state permeate flux versus average transmembrane pressure for the $0.62 \mathrm{~m} \cdot \mathrm{s}^{-1}-0.94 \mathrm{cSt}$ experiment. 
would only result in further consolidation of the concentration boundary layer, thus increasing the potential for membrane fouling (Cheryan 1998).

The permeate $\mathrm{pH}$ ranged from 7.12 to 7.67 and averaged 7.36. The concentrate $\mathrm{pH}$ ranged from 7.05 to 7.72 and averaged 7.35. Both the permeate and concentrate $\mathrm{pH}$ remained relatively constant throughout the entire run. The permeate conductivity ranged from 17.4 to $90.2 \mu \mathrm{S} \cdot \mathrm{cm}^{-1}$ and averaged $77.9 \mu \mathrm{S} \cdot \mathrm{cm}^{-1}$. The concentrate conductivity ranged from 89.1 to $107 \mu \mathrm{S} \cdot \mathrm{cm}^{-1}$ and averaged $97.2 \mu \mathrm{S} \cdot \mathrm{cm}^{-1}$. A net increase was observed in permeate conductivity over the first half-hour of the run, however, remained constant throughout the remainder of the run. Concentrate conductivity remained relatively constant and was greater than permeate conductivity throughout the entire run. Distinct step-wise changes in permeate and concentrate $\mathrm{pH}$ and conductivity were not observed as applied pressure was increased.

\subsubsection{7 $\mathrm{m} \cdot \mathrm{s}^{-1}-1.2 \mathrm{cSt}(30 \mathrm{gpm}-20 \%$ MW Fluid)}

Permeate flux versus time for the $3.7 \mathrm{~m} \cdot \mathrm{s}^{-1}-1.2 \mathrm{cSt}$ experiment is presented in Figure 4.34. An initial decrease in permeate flux was observed over the first fifty minutes of operation, due to the formation of the concentration boundary layer at the membrane surface, at which point quasi steady-state permeate flux was achieved. Distinct step-wise increases in flux were observed as P was increased from 104 to 352 $\mathrm{kPa}$ (15 to $51 \mathrm{psi}$ ). Average steady state permeate flux versus average transmembrane pressure for the $3.7 \mathrm{~m} \cdot \mathrm{s}^{-1}-1.2 \mathrm{cSt}$ experiment is presented in Figure 4.35. A limiting flux was not observed over the applied pressure range studied in this experiment; however, the average flux data diverged from linear behavior at higher pressures due to a shift from pressure-dependent to pressure-independent operating conditions. 


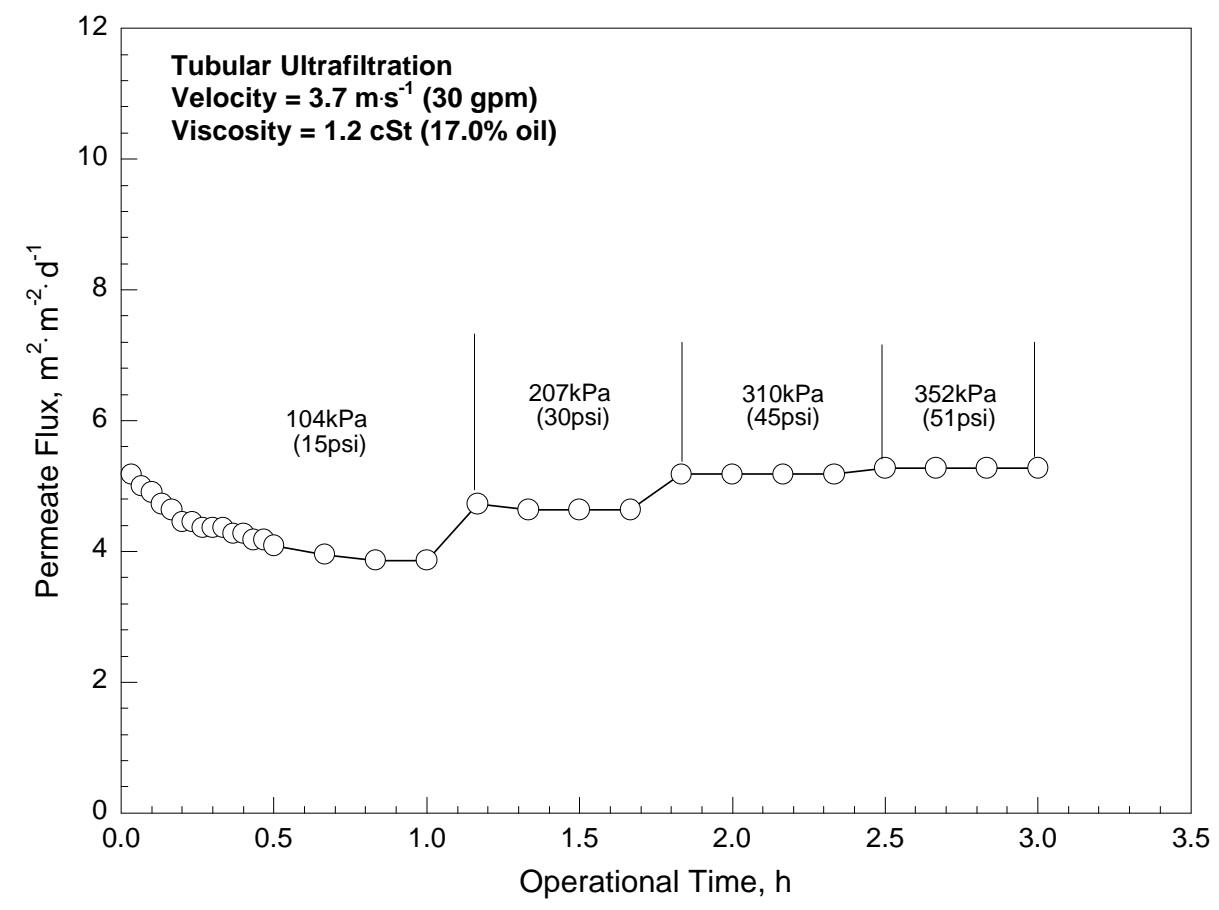

Figure 4.34 Permeate flux versus operational time for the $3.7 \mathrm{~m} \cdot \mathrm{s}^{-1}-1.2 \mathrm{cSt}$ experiment.

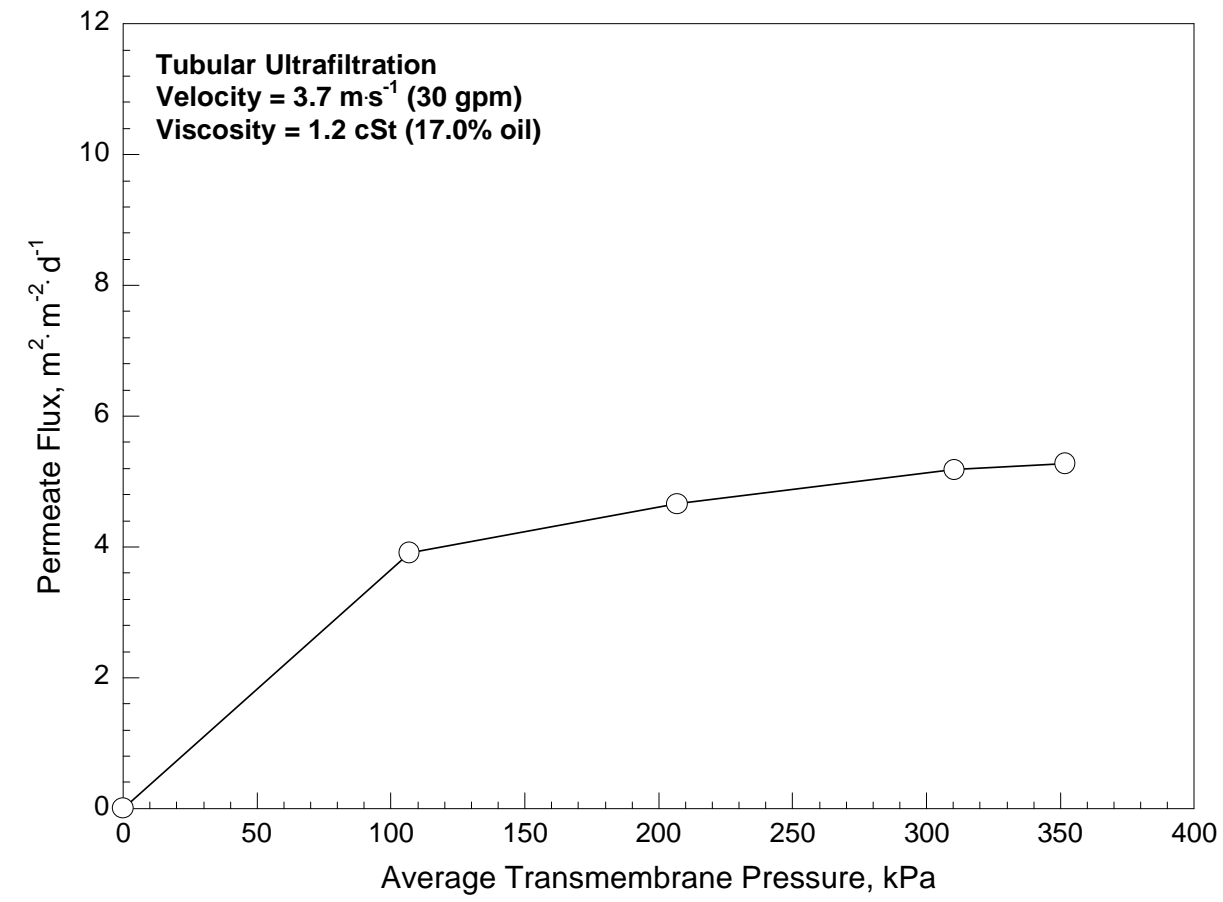

Figure 4.35 Average steady state permeate flux versus average transmembrane pressure for the $3.7 \mathrm{~m} \cdot \mathrm{s}^{-1}-1.2 \mathrm{cSt}$ experiment. 
The permeate $\mathrm{pH}$ ranged from 7.23 to 7.69 and averaged 7.39. The concentrate $\mathrm{pH}$ ranged from 7.38 to 7.90 and averaged 7.53. Both permeate and concentrate $\mathrm{pH}$ were relatively constant throughout the run. The permeate conductivity ranged from 460 to $516 \mu \mathrm{S} \cdot \mathrm{cm}^{-1}$ and averaged $490 \mu \mathrm{S} \cdot \mathrm{cm}^{-1}$. The concentrate conductivity ranged from 476 to $669 \mu \mathrm{S} \cdot \mathrm{cm}^{-1}$ and averaged $606 \mu \mathrm{S} \cdot \mathrm{cm}^{-1}$. Permeate conductivity remained relatively constant, however a net increase was observed in the concentrate conductivity. Permeate conductivity was less than concentrate conductivity throughout the entire run. Distinct step-wise changes in permeate and concentrate $\mathrm{pH}$ and conductivity were not observed as applied pressure was increased.

\subsubsection{8 $2.5 \mathrm{~m} \cdot \mathrm{s}^{-1}-1.2 \mathrm{cSt}(20 \mathrm{gpm}-20 \%$ MW Fluid)}

Permeate flux versus time for the $2.5 \mathrm{~m} \cdot \mathrm{s}^{-1}-1.2 \mathrm{cSt}$ experiment is presented in Figure 4.36. An initial decrease in permeate flux was observed over the first twenty-five minutes of operation, due to the formation of the concentration boundary layer at the membrane surface, at which point quasi steady-state permeate flux was achieved. Average steady state permeate flux versus average transmembrane pressure for the 2.5 $\mathrm{m} \cdot \mathrm{s}^{-1}-1.2 \mathrm{cSt}$ experiment is presented in Figure 4.37. A limiting permeate flux of 2.5 $\mathrm{m}^{3} \cdot \mathrm{m}^{-2} \cdot \mathrm{d}^{-1}$ was observed at the 207 to $310 \mathrm{kPa}$ (30 to $45 \mathrm{psi}$ ) conditions. The experiment was stopped after the $310 \mathrm{kPa}$ pressure condition was examined, due to the fact that a limiting permeate flux was already achieved. Any additional increase in transmembrane pressure would only result in further consolidation of the concentration boundary layer, thus increasing the potential for membrane fouling (Cheryan 1998).

The permeate $\mathrm{pH}$ ranged from 6.81 to 7.65 and averaged 7.42. The concentrate $\mathrm{pH}$ ranged from 7.35 to 7.54 and averaged 7.45 . Both permeate and concentrate $\mathrm{pH}$ were 


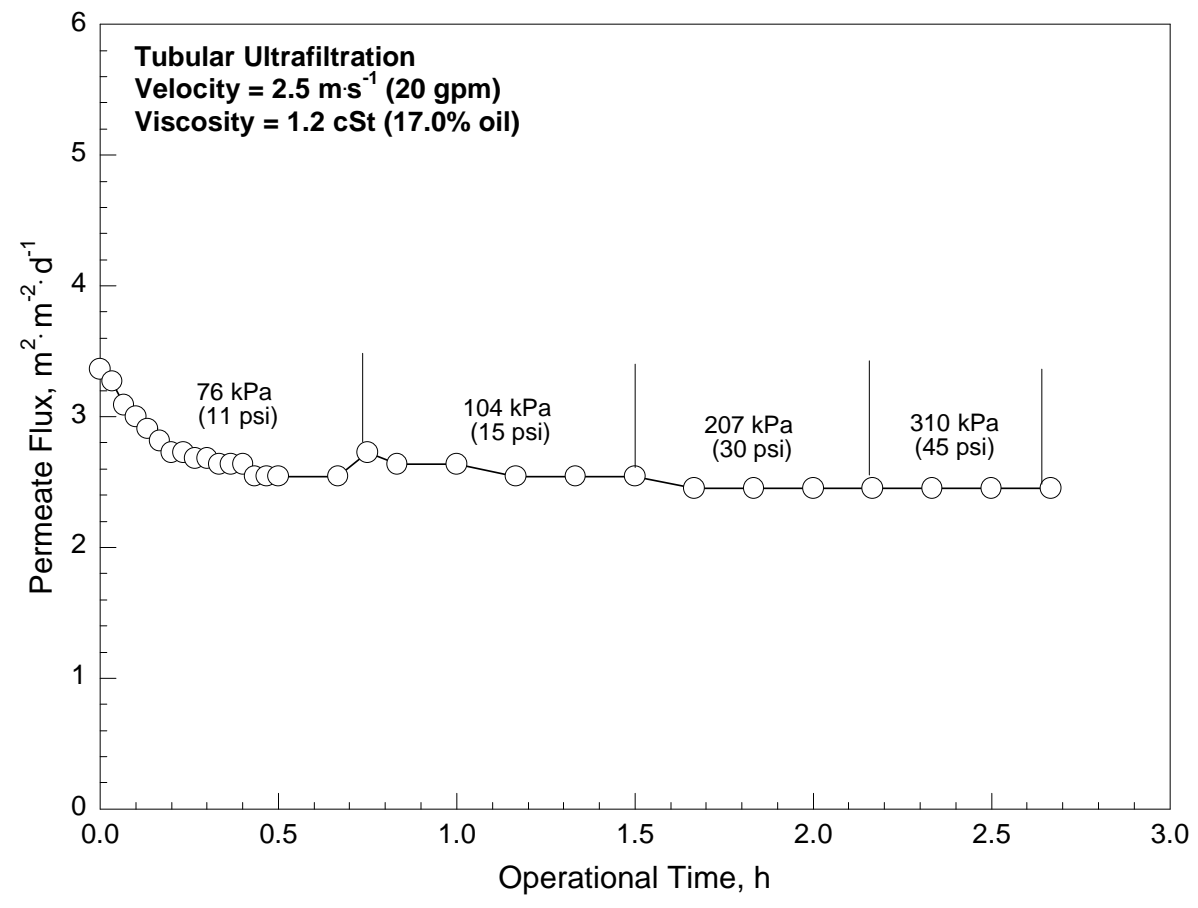

Figure 4.36 Permeate flux versus operational time for the $2.5 \mathrm{~m} \cdot \mathrm{s}^{-1}-1.2 \mathrm{cSt}$ experiment.

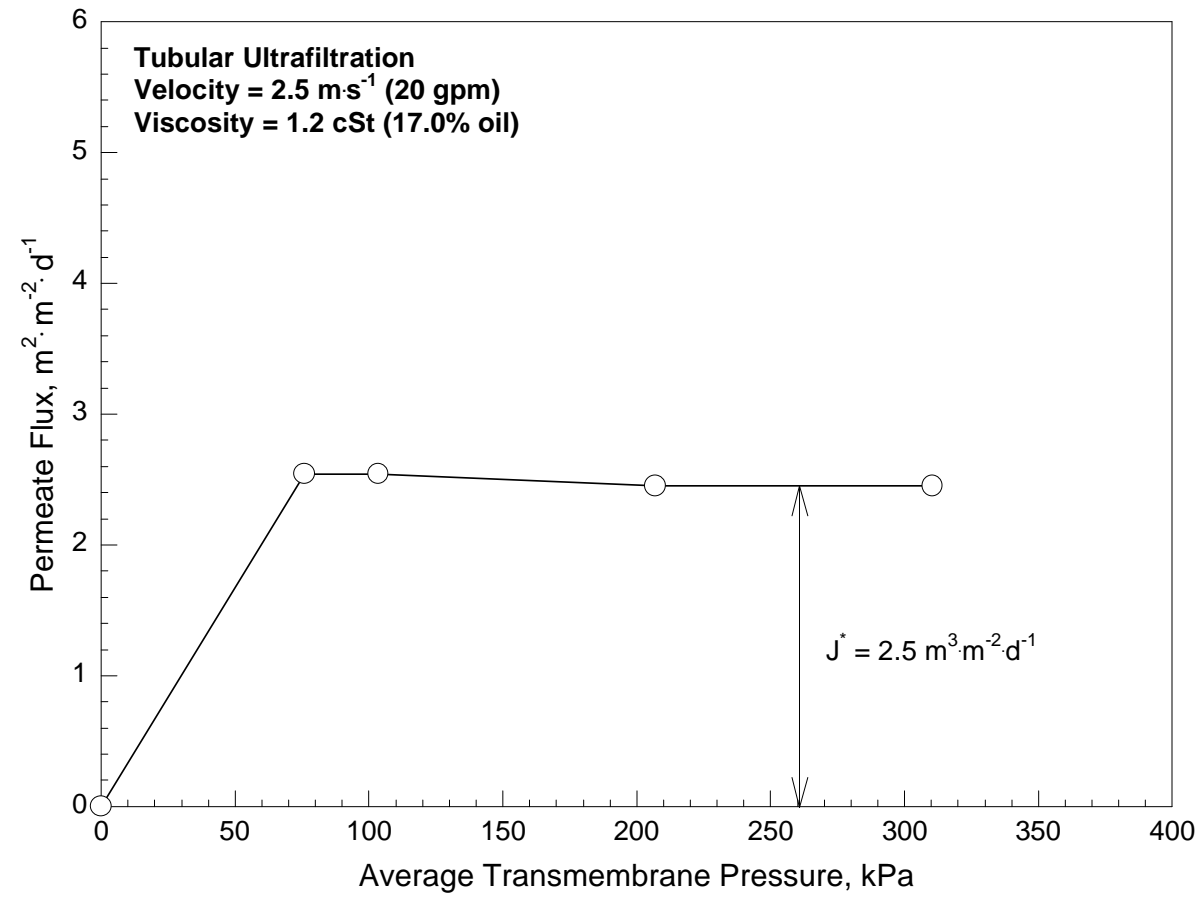

Figure 4.37 Average steady state permeate flux versus average transmembrane pressure for the $2.5 \mathrm{~m} \cdot \mathrm{s}^{-1}-1.2 \mathrm{cSt}$ experiment. 
relatively constant throughout the run. The permeate conductivity ranged from 435 to $581 \mu \mathrm{S} \cdot \mathrm{cm}^{-1}$ and averaged $528 \mu \mathrm{S} \cdot \mathrm{cm}^{-1}$. The concentrate conductivity ranged from 522 to $693 \mu \mathrm{S} \cdot \mathrm{cm}^{-1}$ and averaged $611 \mu \mathrm{S} \cdot \mathrm{cm}^{-1}$. A net decrease was observed in permeate conductivity, however, a net increase was observed in the concentrate conductivity. Permeate conductivity was less than concentrate conductivity throughout the entire run. Distinct step-wise changes in permeate and concentrate $\mathrm{pH}$ and conductivity were not observed as applied pressure was increased.

\subsubsection{2 $\mathrm{m} \cdot \mathrm{s}^{-1}-1.2 \mathrm{cSt}(10 \mathrm{gpm}-20 \%$ MW Fluid)}

Permeate flux versus time for the $1.2 \mathrm{~m} \cdot \mathrm{s}^{-1}-1.2 \mathrm{cSt}$ experiment is presented in Figure 4.38. An initial decrease in permeate flux was observed over the first fifteen minutes of operation, due to the formation of the concentration boundary layer at the membrane surface, at which point quasi steady-state permeate flux was achieved. Average steady state permeate flux versus average transmembrane pressure for the 1.2 $\mathrm{m} \cdot \mathrm{s}^{-1}-1.2 \mathrm{cSt}$ experiment is presented in Figure 4.39. A limiting permeate flux of 0.89 $\mathrm{m}^{3} \cdot \mathrm{m}^{-2} \cdot \mathrm{d}^{-1}$ was observed at the 104 to $207 \mathrm{kPa}$ (15 to $30 \mathrm{psi}$ ) conditions. The experiment was stopped after the $207 \mathrm{kPa}$ pressure condition was examined, due to the fact that a limiting permeate flux was already achieved. Any additional increase in transmembrane pressure would only result in further consolidation of the concentration boundary layer, thus increasing the potential for membrane fouling (Cheryan 1998).

The permeate $\mathrm{pH}$ ranged from 7.36 to 7.64 and averaged 7.48. The concentrate $\mathrm{pH}$ ranged from 7.40 to 7.64 and averaged 7.53. Permeate and concentrate $\mathrm{pH}$ were both relatively constant throughout the entire run. The permeate conductivity ranged from 337 to $540 \mu \mathrm{S} \cdot \mathrm{cm}^{-1}$ and averaged $485 \mu \mathrm{S} \cdot \mathrm{cm}^{-1}$. The concentrate conductivity ranged from 535 


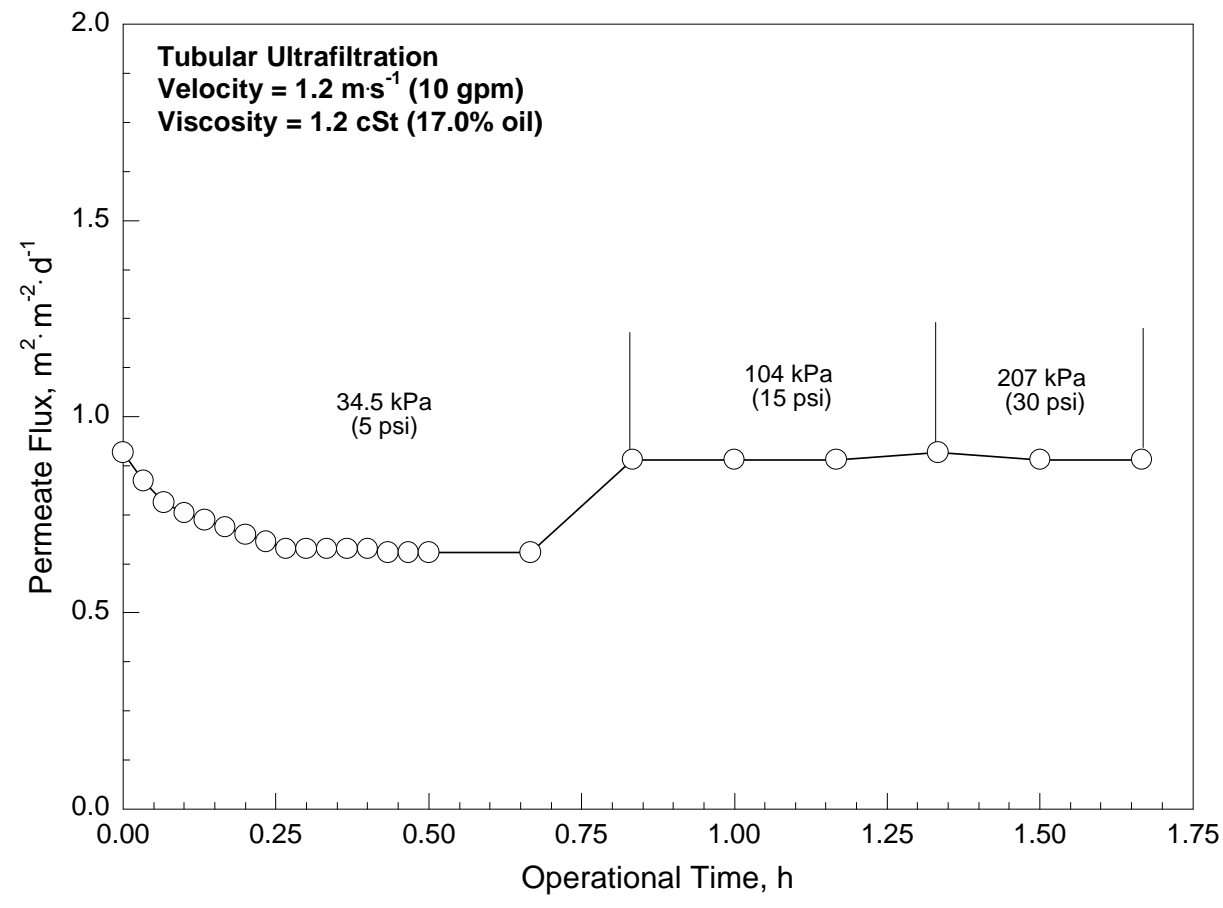

Figure 4.38 Permeate flux versus operational time for the $1.2 \mathrm{~m} \cdot \mathrm{s}^{-1}-1.2 \mathrm{cSt}$ experiment.

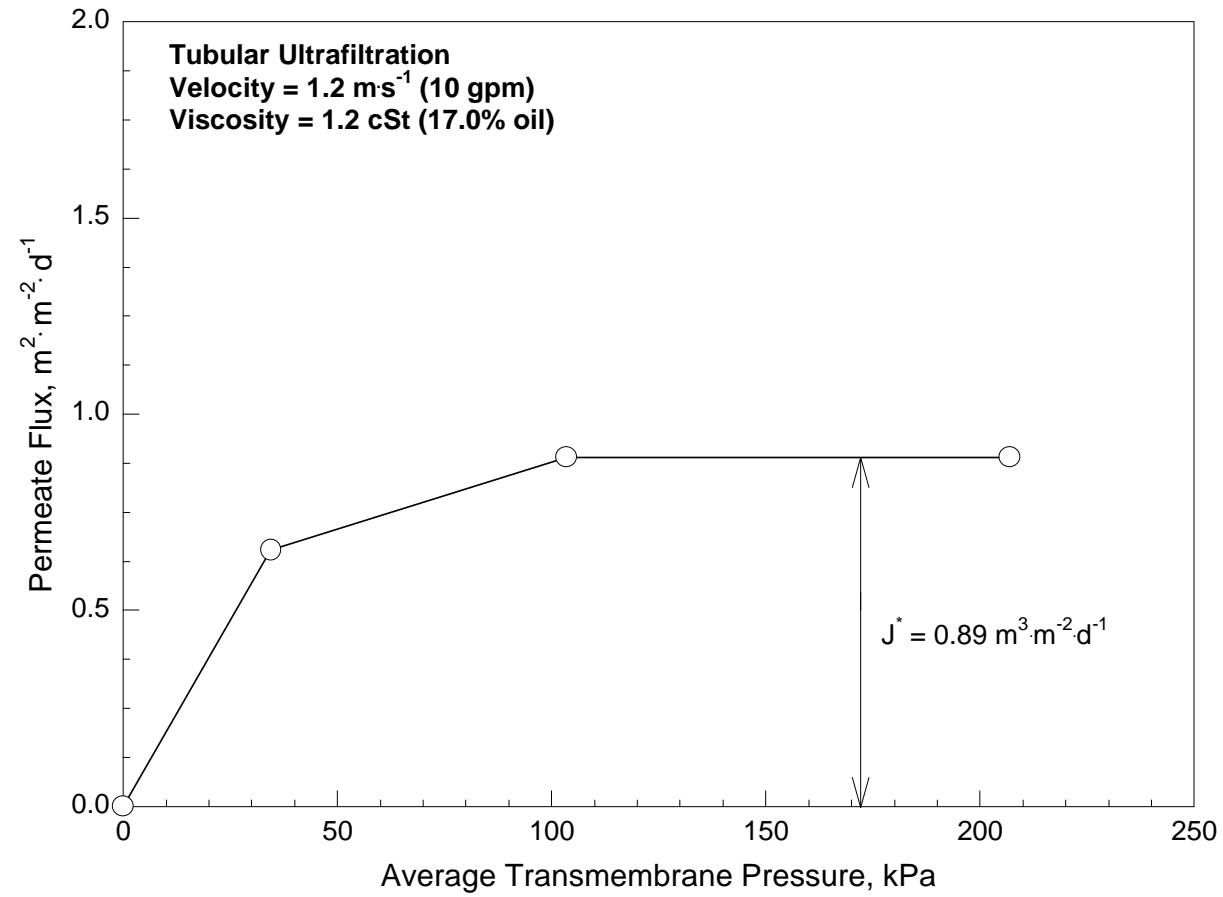

Figure 4.39 Average steady state permeate flux versus average transmembrane pressure for the $1.2 \mathrm{~m} \cdot \mathrm{s}^{-1}-1.2 \mathrm{cSt}$ experiment. 
to $607 \mu \mathrm{S} \cdot \mathrm{cm}^{-1}$ and averaged $578 \mu \mathrm{S} \cdot \mathrm{cm}^{-1}$. Permeate and concentrate conductivity remained relatively constant and permeate conductivity was less than concentrate conductivity throughout the entire run. Distinct step-wise changes in permeate and concentrate $\mathrm{pH}$ and conductivity were not observed as applied pressure was increased.

\subsection{2 $\mathrm{m} \cdot \mathrm{s}^{-1}-1.2 \mathrm{cSt}(5 \mathrm{gpm}-20 \%$ MW Fluid)}

Permeate flux versus time for the $0.62 \mathrm{~m} \cdot \mathrm{s}^{-1}-1.2 \mathrm{cSt}$ experiment is presented in Figure 4.40. An initial decrease in permeate flux was observed over the first twenty-five minutes of operation, due to the formation of the concentration boundary layer at the membrane surface, at which point quasi steady-state permeate flux was achieved. Immediately after the transition from 104 to $207 \mathrm{kPa}$ (15 to $30 \mathrm{psi}$ ), a small increase in permeate flux was observed after which the flux dropped and stabilized at the value observed during the $104 \mathrm{kPa}$ condition. This phenomenon was attributed to a delay in stabilization of the concentration boundary layer (Viadero and Reed 1999). Average steady state permeate flux versus average transmembrane pressure for the $0.62 \mathrm{~m} \cdot \mathrm{s}^{-1}-1.2$ cSt experiment is presented in Figure 4.41. A limiting permeate flux of $0.29 \mathrm{~m}^{3} \cdot \mathrm{m}^{-2} \cdot \mathrm{d}^{-1}$ was observed at the 104 to $207 \mathrm{kPa}$ (15 to $30 \mathrm{psi}$ ) conditions. The experiment was stopped after the $207 \mathrm{kPa}$ pressure condition was examined, due to the fact that a limiting permeate flux was already achieved. Any additional increase in transmembrane pressure would only result in further consolidation of the concentration boundary layer, thus increasing the potential for membrane fouling (Cheryan 1998).

The permeate $\mathrm{pH}$ ranged from 7.03 to 7.58 and averaged 7.41. The concentrate $\mathrm{pH}$ ranged from 7.14 to 7.40 and averaged 7.30. Permeate and concentrate $\mathrm{pH}$ were both relatively constant throughout the entire run. The permeate conductivity ranged from 


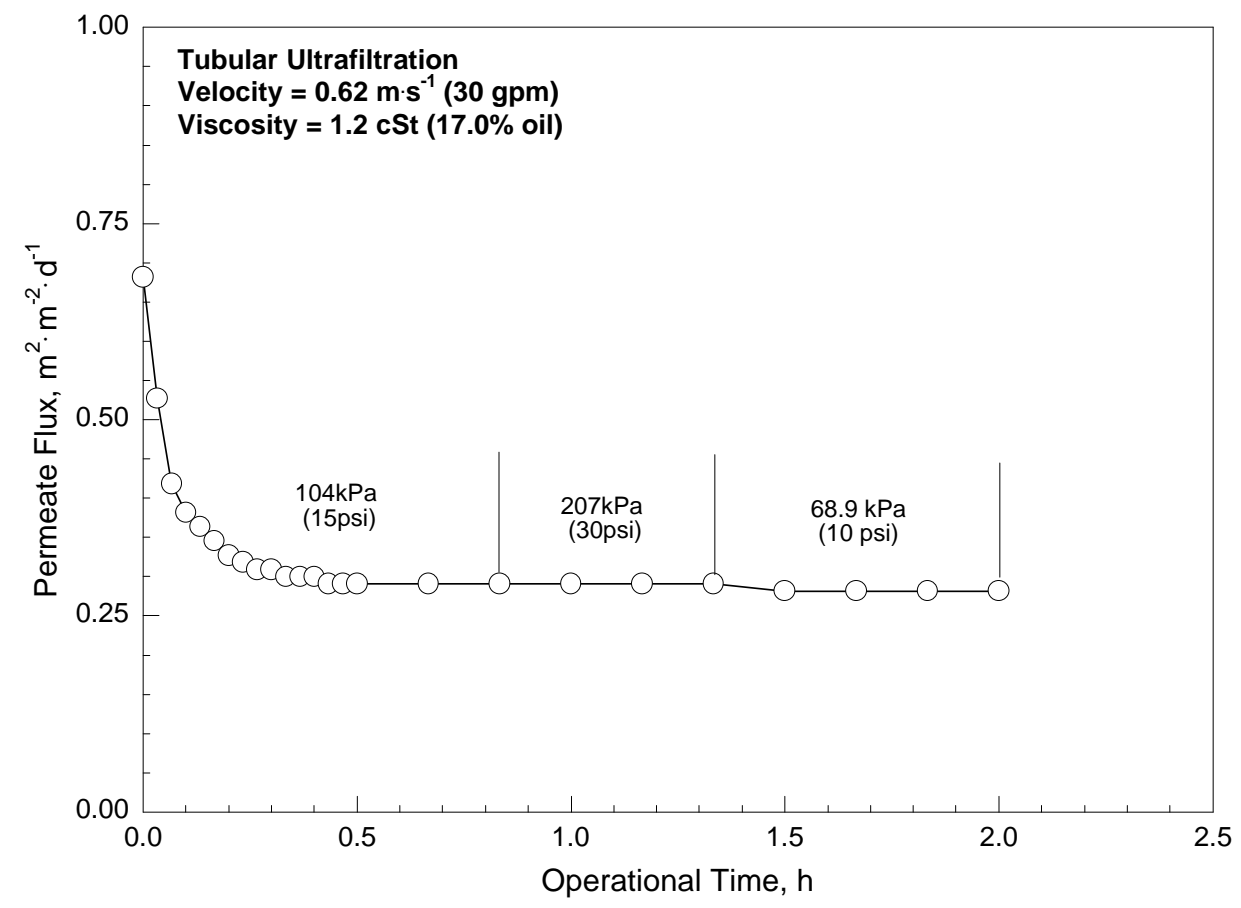

Figure 4.40 Permeate flux versus operational time for the $0.62 \mathrm{~m} \cdot \mathrm{s}^{-1}-1.2 \mathrm{cSt}$ experiment.

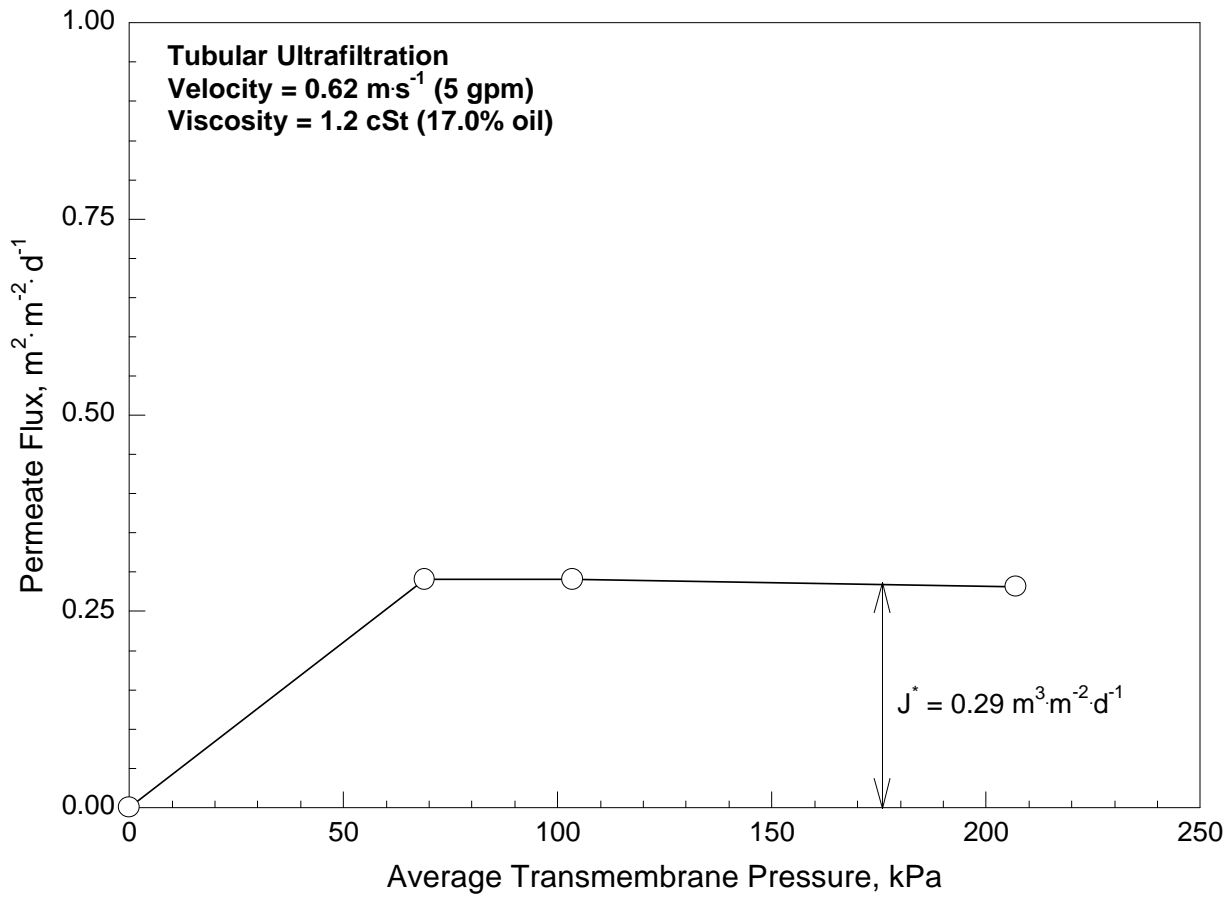

Figure 4.41 Average steady state permeate flux versus average transmembrane pressure for the $0.62 \mathrm{~m} \cdot \mathrm{s}^{-1}-1.2 \mathrm{cSt}$ experiment. 
16.5 to $171 \mu \mathrm{S} \cdot \mathrm{cm}^{-1}$ and averaged $137 \mu \mathrm{S} \cdot \mathrm{cm}^{-1}$. The concentrate conductivity ranged from 159 to $186 \mu \mathrm{S} \cdot \mathrm{cm}^{-1}$ and averaged $174 \mu \mathrm{S} \cdot \mathrm{cm}^{-1}$. Permeate and concentrate conductivity were both relatively constant and permeate conductivity was less than concentrate conductivity throughout the entire run. Distinct step-wise changes in permeate and concentrate $\mathrm{pH}$ and conductivity were not observed as applied pressure was increased.

\subsubsection{7 $\mathrm{m} \cdot \mathrm{s}^{-1}-4.5 \mathrm{cSt}(30 \mathrm{gpm}-30 \%$ MW Fluid)}

Permeate flux versus time for the $3.7 \mathrm{~m} \cdot \mathrm{s}^{-1}-4.5 \mathrm{cSt}$ experiment is presented in Figure 4.42. An initial decrease in permeate flux was observed over the first twenty minutes of operation, due to the formation of the concentration boundary layer at the membrane surface, at which point quasi steady-state permeate flux was achieved. Distinct step-wise increases in flux were observed as $\mathrm{P}$ was increased from 117 to 310 $\mathrm{kPa}$ (17 to $45 \mathrm{psi}$ ). Average steady state permeate flux versus average transmembrane pressure for the $3.7 \mathrm{~m} \cdot \mathrm{s}^{-1}-4.5 \mathrm{cSt}$ experiment is presented in Figure 4.43. A limiting flux was not observed over the applied pressure range studied in this experiment; however, the average flux data diverged from linear behavior at higher pressures due to a shift from pressure-dependent to pressure-independent operating conditions.

The permeate $\mathrm{pH}$ ranged from 7.15 to 7.54 and averaged 7.42. The concentrate $\mathrm{pH}$ ranged from 7.05 to 7.38 and averaged 7.26. Permeate and concentrate $\mathrm{pH}$ both remained relatively constant throughout the run. The permeate conductivity ranged from 169 to $1010 \mu \mathrm{S} \cdot \mathrm{cm}^{-1}$ and averaged $863 \mu \mathrm{S} \cdot \mathrm{cm}^{-1}$. The concentrate conductivity ranged from 1100 to $1160 \mu \mathrm{S} \cdot \mathrm{cm}^{-1}$ and averaged $1140 \mu \mathrm{S} \cdot \mathrm{cm}^{-1}$. Permeate and concentrate conductivity were both relatively constant, and permeate conductivity was less than 


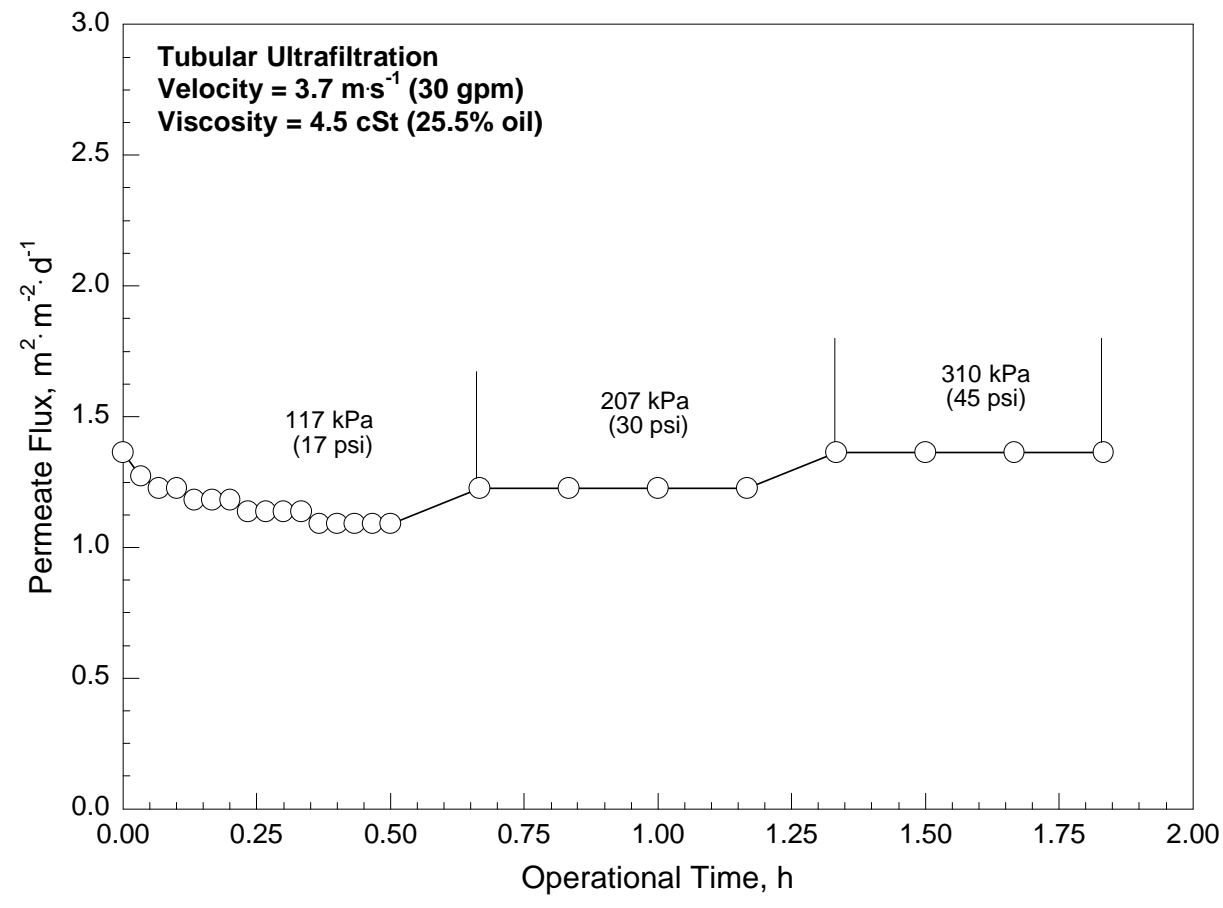

Figure 4.42 Permeate flux versus operational time for the $3.7 \mathrm{~m} \cdot \mathrm{s}^{-1}-4.5 \mathrm{cSt}$ experiment.

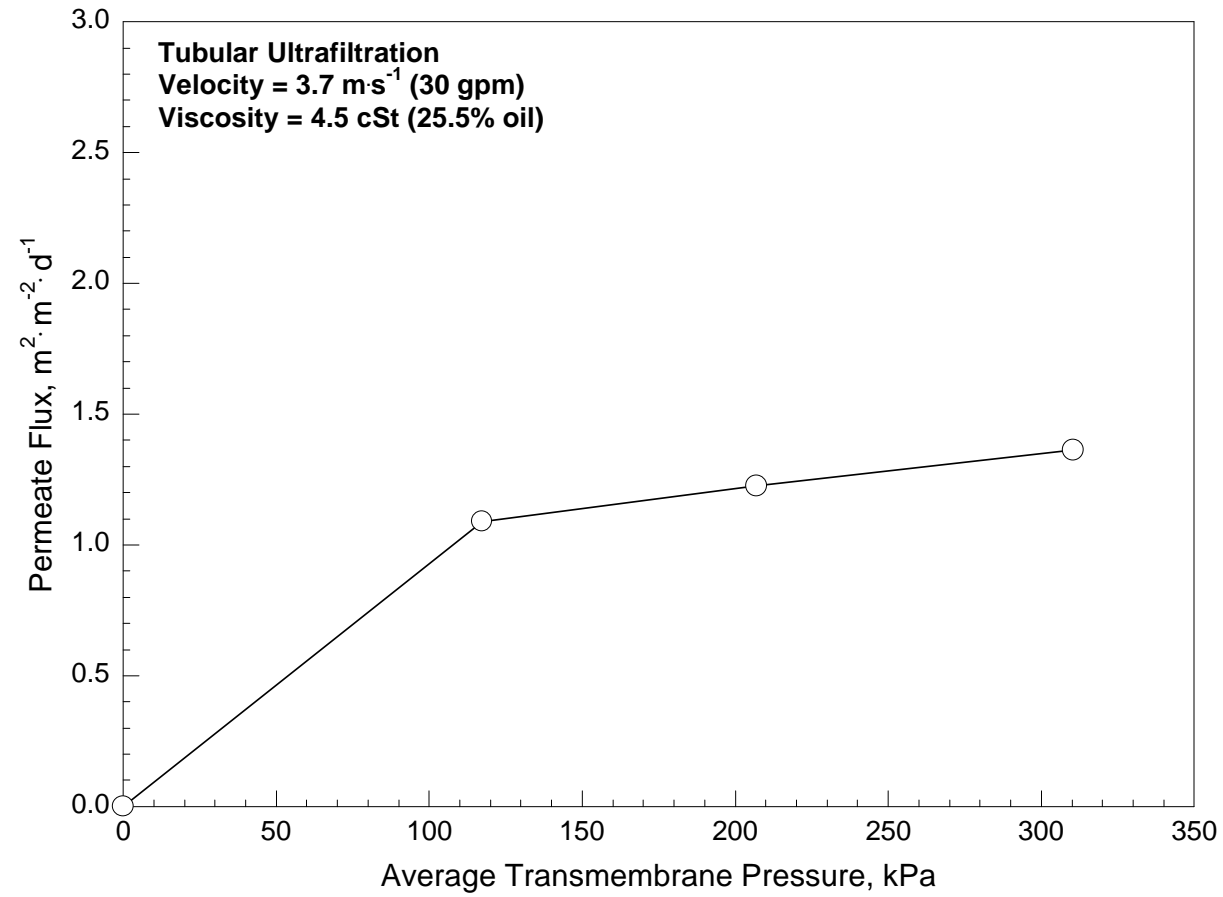

Figure 4.43 Average steady state permeate flux versus average transmembrane pressure for the $3.7 \mathrm{~m} \cdot \mathrm{s}^{-1}-4.5 \mathrm{cSt}$ experiment. 
concentrate conductivity throughout the entire run. Distinct step-wise changes in permeate and concentrate $\mathrm{pH}$ and conductivity were not observed as applied pressure was increased.

\subsubsection{2 $2.5 \mathrm{~m} \cdot \mathrm{s}^{-1}-4.5 \mathrm{cSt}(20 \mathrm{gpm}-30 \%$ MW Fluid $)$}

Permeate flux versus time for the $2.5 \mathrm{~m} \cdot \mathrm{s}^{-1}-4.5 \mathrm{cSt}$ experiment is presented in Figure 4.44. An initial decrease in permeate flux was observed over the first forty minutes of operation, due to the formation of the concentration boundary layer at the membrane surface, at which point quasi steady-state permeate flux was achieved. Immediately after the transition from 104 to $207 \mathrm{kPa}$ (15 to $30 \mathrm{psi}$ ), a small increase in permeate flux was observed after which the flux dropped and stabilized at the value observed during the $104 \mathrm{kPa}$ condition. This phenomenon was attributed to a delay in stabilization of the concentration boundary layer (Viadero and Reed 1999). Average steady state permeate flux versus average transmembrane pressure for the $2.5 \mathrm{~m} \cdot \mathrm{s}^{-1}-4.5$ cSt experiment is presented in Figure 4.45. A limiting permeate flux of $0.79 \mathrm{~m}^{3} \cdot \mathrm{m}^{-2} \cdot \mathrm{d}^{-1}$ was observed at the 104 to $207 \mathrm{kPa}$ (15 to $30 \mathrm{psi}$ ) conditions. The experiment was stopped after the $207 \mathrm{kPa}$ pressure condition was examined, due to the fact that a limiting permeate flux was already achieved. Any additional increase in transmembrane pressure would only result in further consolidation of the concentration boundary layer, thus increasing the potential for membrane fouling (Cheryan 1998).

The permeate $\mathrm{pH}$ ranged from 7.35 to 7.65 and averaged 7.50. The concentrate $\mathrm{pH}$ ranged from 7.34 to 7.45 and averaged 7.41. Permeate and concentrate $\mathrm{pH}$ were both relatively constant throughout the entire run. The permeate conductivity ranged from 702 to $925 \mu \mathrm{S} \cdot \mathrm{cm}^{-1}$ and averaged $813 \mu \mathrm{S} \cdot \mathrm{cm}^{-1}$. The concentrate conductivity ranged from 855 


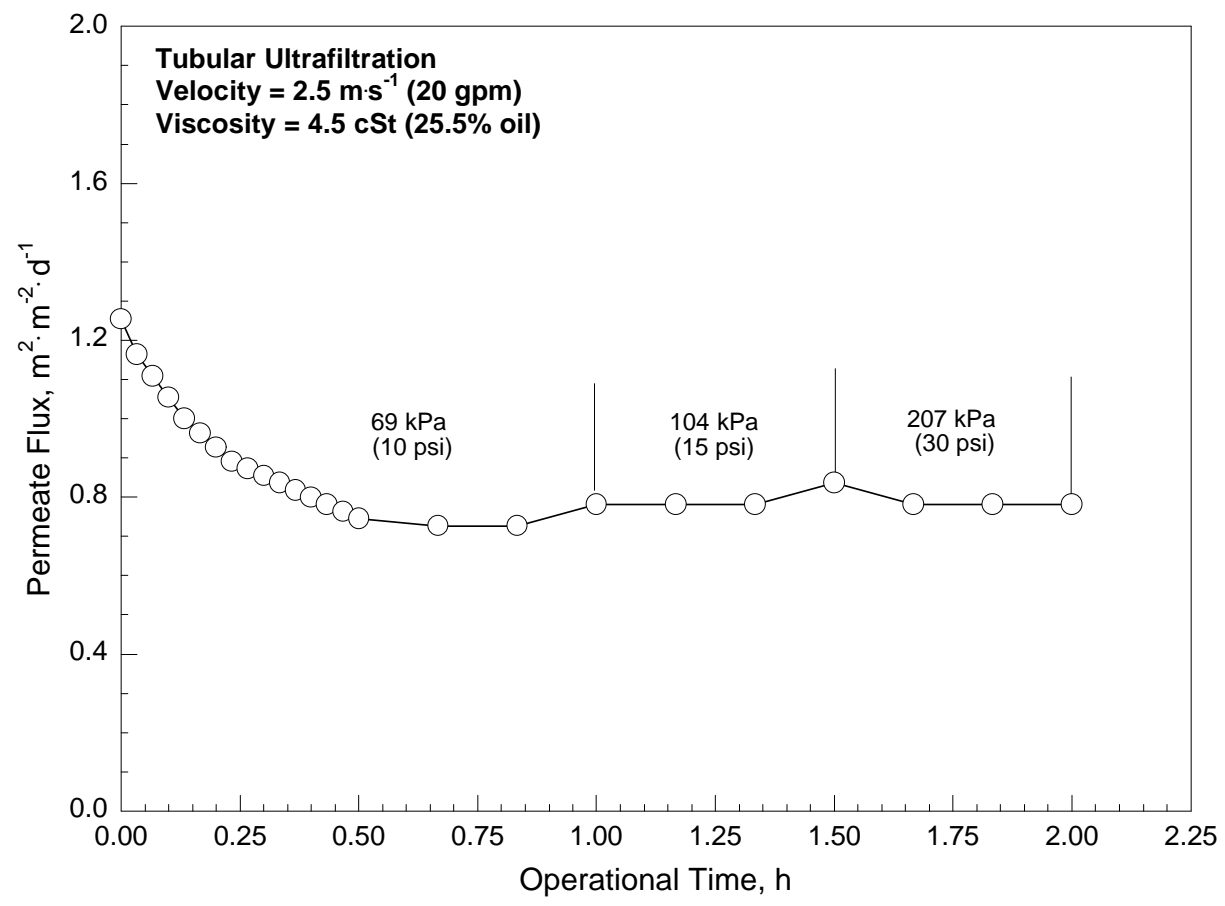

Figure 4.44 Permeate flux versus operational time for the $2.5 \mathrm{~m} \cdot \mathrm{s}^{-1}-4.5 \mathrm{cSt}$ experiment.

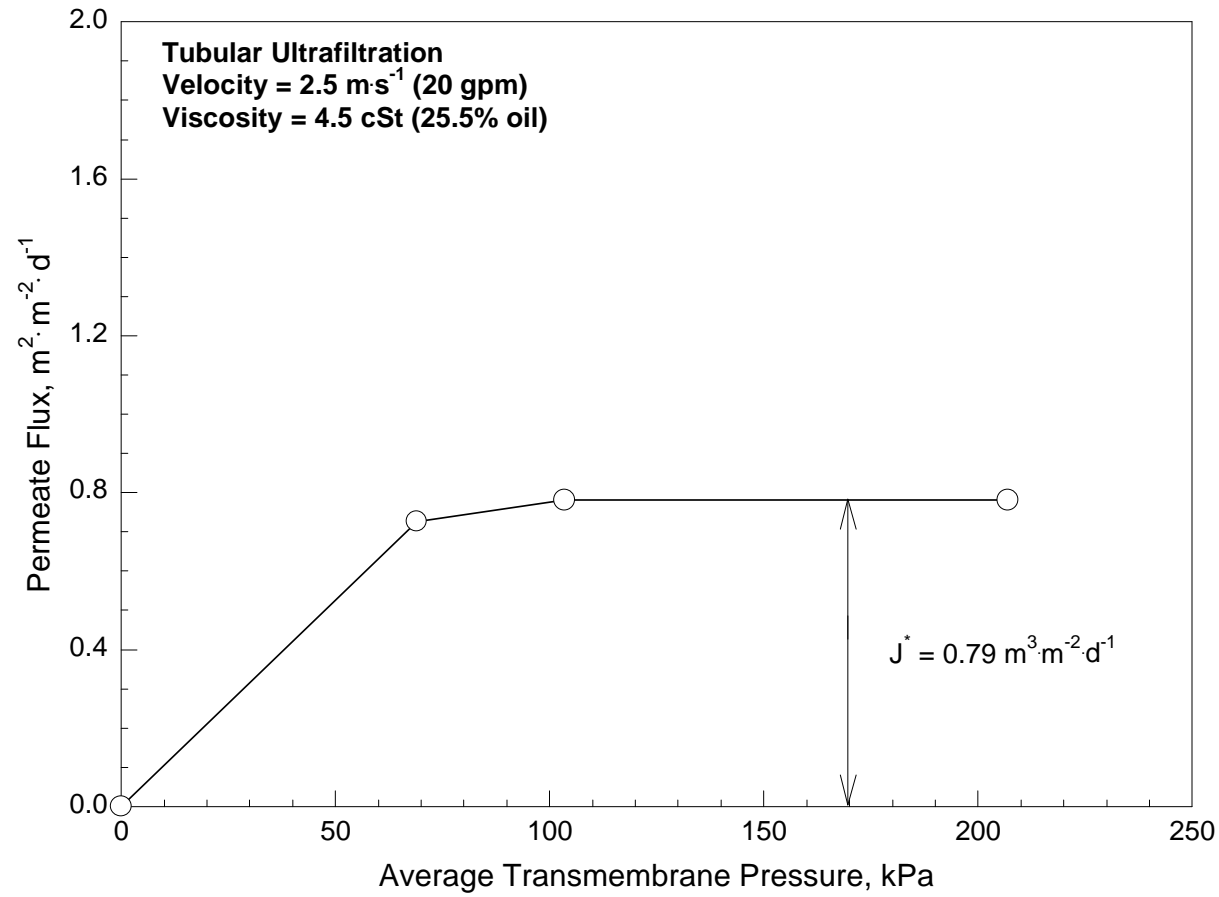

Figure 4.45 Average steady state permeate flux versus average transmembrane pressure for the $2.5 \mathrm{~m} \cdot \mathrm{s}^{-1}-4.5 \mathrm{cSt}$ experiment. 
to $909 \mu \mathrm{S} \cdot \mathrm{cm}^{-1}$ and averaged $880 \mu \mathrm{S} \cdot \mathrm{cm}^{-1}$. Permeate and concentrate conductivity were both relatively constant throughout the entire run.

\subsubsection{2 $\mathrm{m} \cdot \mathrm{s}^{-1}-4.5 \mathrm{cSt}(10 \mathrm{gpm}-30 \%$ MW Fluid $)$}

Permeate flux versus time for the $1.2 \mathrm{~m} \cdot \mathrm{s}^{-1}-4.5 \mathrm{cSt}$ experiment is presented in Figure 4.46. An initial decrease in permeate flux was observed over the first twenty minutes of operation, due to the formation of the concentration boundary layer at the membrane surface, at which point quasi steady-state permeate flux was achieved. Immediately after the transition from 104 to $207 \mathrm{kPa}$ (15 to $30 \mathrm{psi}$ ), a small increase in permeate flux was observed after which the flux dropped and stabilized at the value observed during the $104 \mathrm{kPa}$ condition. This phenomenon was attributed to a delay in stabilization of the concentration boundary layer (Viadero and Reed 1999). Average steady state permeate flux versus average transmembrane pressure for the $1.2 \mathrm{~m} \cdot \mathrm{s}^{-1}-4.5$ cSt experiment is presented in Figure 4.47. A limiting permeate flux of $0.34 \mathrm{~m}^{3} \cdot \mathrm{m}^{-2} \cdot \mathrm{d}^{-1}$ was observed at the 104 to $207 \mathrm{kPa}$ (15 to $30 \mathrm{psi}$ ) conditions. The experiment was stopped after the $207 \mathrm{kPa}$ pressure condition was examined, due to the fact that a limiting permeate flux was already achieved. Any additional increase in transmembrane pressure would only result in further consolidation of the concentration boundary layer, thus increasing the potential for membrane fouling (Cheryan 1998).

The permeate $\mathrm{pH}$ ranged from 7.23 to 7.92 and averaged 7.53. The concentrate $\mathrm{pH}$ ranged from 7.24 to 7.68 and averaged 7.42. Permeate and concentrate $\mathrm{pH}$ were both relatively constant throughout the entire run. The permeate conductivity ranged from 18.5 to $189 \mu \mathrm{S} \cdot \mathrm{cm}^{-1}$ and averaged $131 \mu \mathrm{S} \cdot \mathrm{cm}^{-1}$. The concentrate conductivity ranged from 208 to $211 \mu \mathrm{S} \cdot \mathrm{cm}^{-1}$ and averaged $210 \mu \mathrm{S} \cdot \mathrm{cm}^{-1}$. Permeate and concentrate 


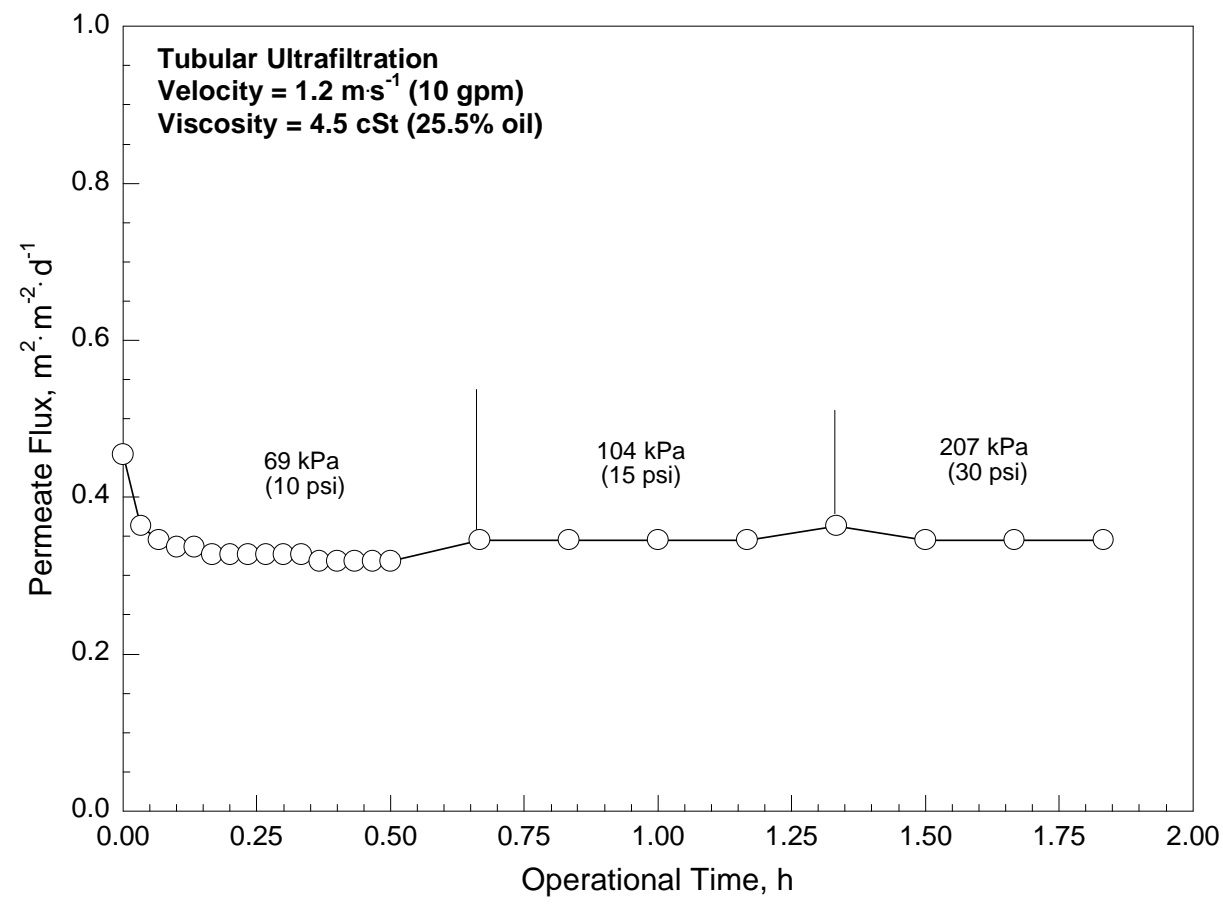

Figure 4.46 Permeate flux versus operational time for the $1.2 \mathrm{~m} \cdot \mathrm{s}^{-1}-4.5 \mathrm{cSt}$ experiment.

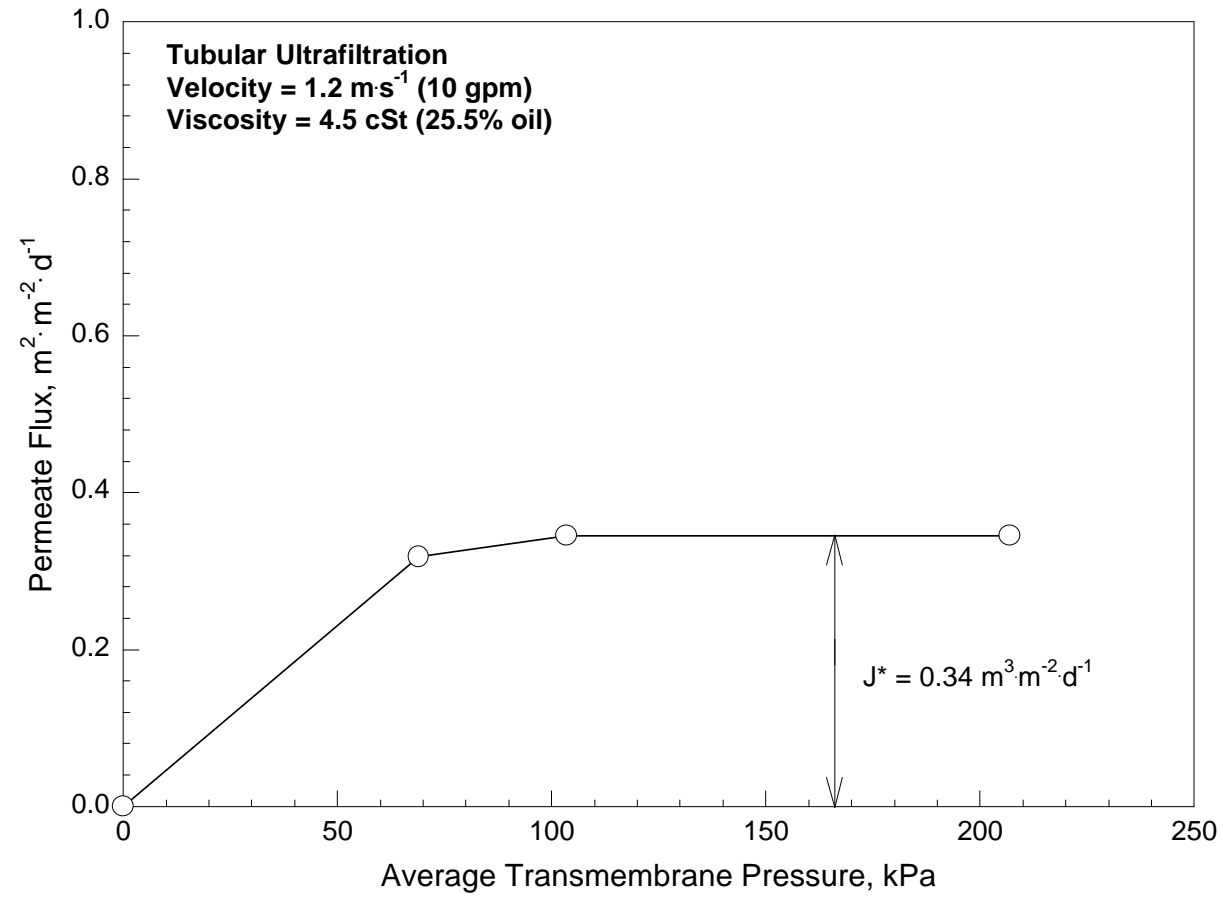

Figure 4.47 Average steady state permeate flux versus average transmembrane pressure for the $1.2 \mathrm{~m} \cdot \mathrm{s}^{-1}-4.5 \mathrm{cSt}$ experiment. 
conductivity were both relatively constant and permeate conductivity was less than concentrate conductivity throughout the entire run. Distinct step-wise changes in permeate and concentrate $\mathrm{pH}$ and conductivity were not observed as applied pressure was increased.

\subsection{2 m-s $\mathrm{s}^{-1}-4.5 \mathrm{cSt}(5 \mathrm{gpm}-30 \%$ MW Fluid)}

Permeate flux versus operational time for the $0.62 \mathrm{~m} \cdot \mathrm{s}^{-1}-4.5 \mathrm{cSt}$ experiment is presented in Figure 4.48. An initial decrease in permeate flux was observed over the first twenty minutes of operation, due to the formation of the concentration boundary layer at the membrane surface, at which point quasi steady-state permeate flux was achieved. Average steady state permeate flux versus average transmembrane pressure for the 0.62 $\mathrm{m} \cdot \mathrm{s}^{-1}-4.5 \mathrm{cSt}$ experiment is presented in Figure 4.49. A limiting permeate flux of 0.13 $\mathrm{m}^{3} \cdot \mathrm{m}^{-2} \cdot \mathrm{d}^{-1}$ was observed at all of the pressure conditions examined in this experiment $(9$, 104 and $207 \mathrm{kPa} ; 10,15$ and $30 \mathrm{psi}$ ). Thus, the entire experiment was conducted under pressure-independent operating conditions. Only one permeate flux measurement was taken at the highest (207 $\mathrm{kPa} ; 30 \mathrm{psi}$ ) pressure condition, because the permeate flux had been completely steady for an hour. The experiment was stopped after the $207 \mathrm{kPa}$ pressure condition was examined, due to the fact that a limiting permeate flux was already achieved. Any additional increase in transmembrane pressure would only result in further consolidation of the concentration boundary layer, thus increasing the potential for membrane fouling (Cheryan 1998).

The permeate $\mathrm{pH}$ ranged from 6.44 to 6.88 and averaged 6.60. The concentrate $\mathrm{pH}$ ranged from 7.30 to 7.74 and averaged 7.50. Permeate and concentrate $\mathrm{pH}$ were both relatively constant and permeate $\mathrm{pH}$ was less than concentrate $\mathrm{pH}$ throughout the entire 


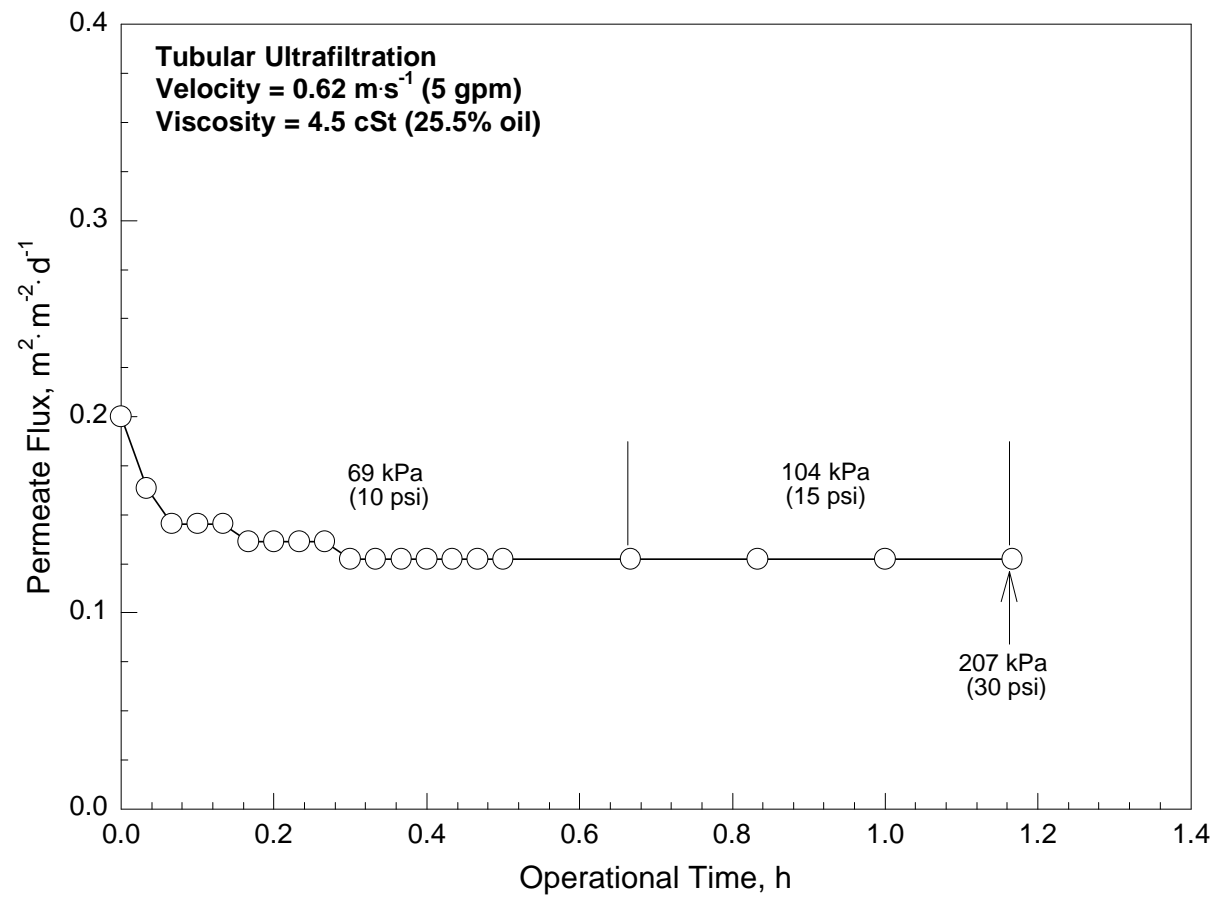

Figure 4.48 Permeate flux versus operational time for the $0.62 \mathrm{~m} \cdot \mathrm{s}^{-1}-4.5 \mathrm{cSt}$ experiment.

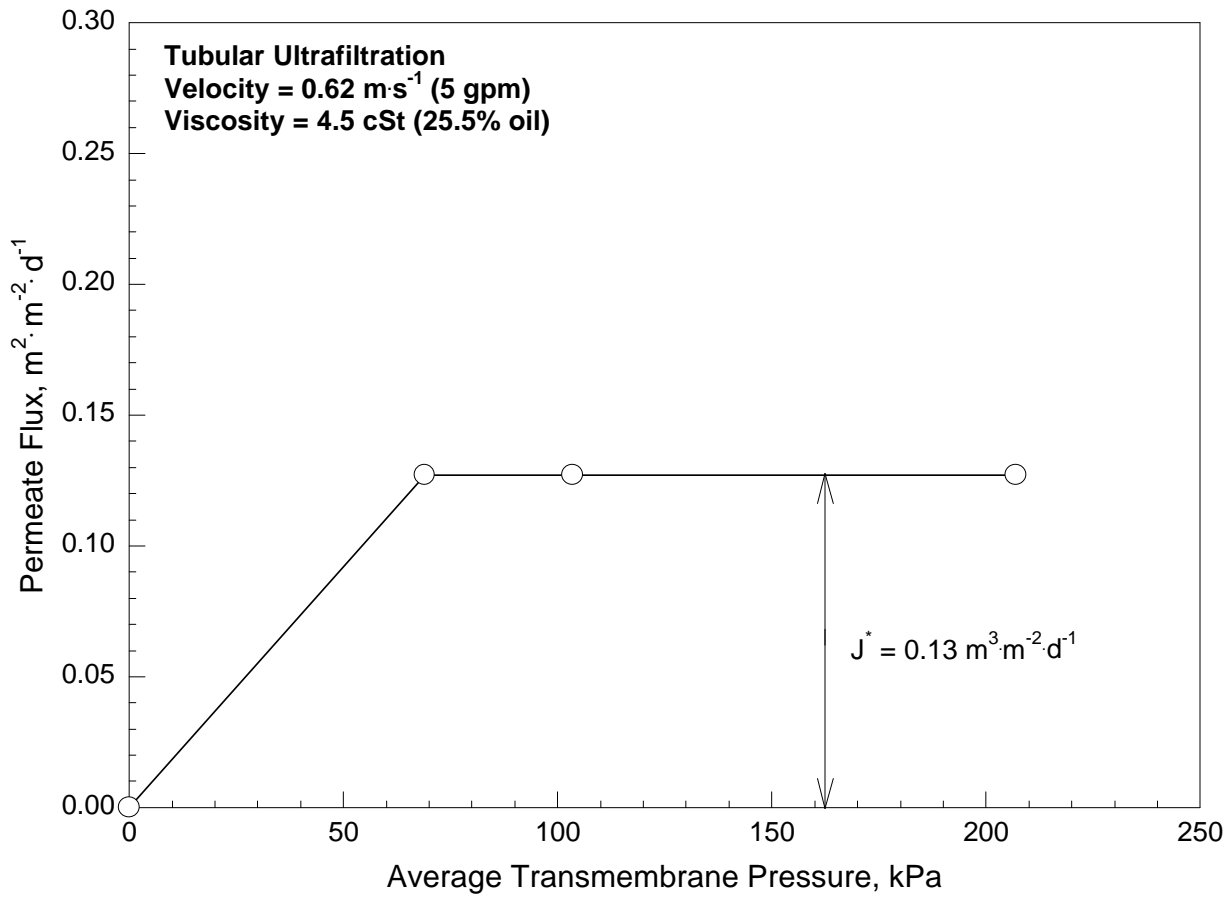

Figure 4.49 Average steady state permeate flux versus average transmembrane pressure for the $0.62 \mathrm{~m} \cdot \mathrm{s}^{-1}-4.5 \mathrm{cSt}$ experiment. 
run. The permeate conductivity ranged from 40.3 to $153 \mu \mathrm{S} \cdot \mathrm{cm}^{-1}$ and averaged 93.9 $\mu \mathrm{S} \cdot \mathrm{cm}^{-1}$. The concentrate conductivity ranged from 874 to $912 \mu \mathrm{S} \cdot \mathrm{cm}^{-1}$ and averaged 895 $\mu \mathrm{S} \cdot \mathrm{cm}^{-1}$. Permeate and concentrate conductivity were both relatively constant and permeate conductivity was less than concentrate conductivity throughout the entire run. Distinct step-wise changes in permeate and concentrate $\mathrm{pH}$ and conductivity were not observed as applied pressure was increased.

\subsection{HIGH-SHEAR ROTARY ULTRAFILTRATION}

\subsection{1 $12 \mathrm{~m} \cdot \mathrm{s}^{-1}-0.46 \mathrm{cSt}(1750 \mathrm{rpm}-5 \% \mathrm{MW}$ Fluid)}

Permeate flux versus time for the $12 \mathrm{~m} \cdot \mathrm{s}^{-1}-0.46 \mathrm{cSt}$ experiment is presented in Figure 4.50. Permeate flux was stable at each applied pressure condition. The system was operated over an applied pressure range of 172 to $517 \mathrm{kPa}$ ( 25 to $75 \mathrm{psig}$ ). The average permeate backpressure was $76.6 \mathrm{kPa}(11.1 \mathrm{psi})$ at an average relative velocity of $12 \mathrm{~m} \cdot \mathrm{s}^{-1}(1750 \mathrm{rpm})$. The average permeate flux versus average transmembrane pressure for the $12 \mathrm{~m} \cdot \mathrm{s}^{-1}-0.46 \mathrm{cSt}$ experiment is presented in Figure 4.51. A limiting flux was not observed over the applied pressure range studied in this experiment; however, the average flux data diverged from linear behavior at higher pressures due to a shift from pressure-dependent to pressure-independent operating conditions.

The permeate $\mathrm{pH}$ ranged from 8.01 to 8.31 and averaged 8.16. The concentrate $\mathrm{pH}$ ranged from 7.67 to 8.25 and averaged 7.94. Permeate $\mathrm{pH}$ was constant in this experiment. A net decrease in concentrate $\mathrm{pH}$ was observed with time. Additionally, permeate $\mathrm{pH}$ was greater than concentrate $\mathrm{pH}$ throughout the experiment. The permeate conductivity ranged from 131 to $181 \mu \mathrm{S} \cdot \mathrm{cm}^{-1}$ and averaged $153 \mu \mathrm{S} \cdot \mathrm{cm}^{-1}$. The concentrate conductivity ranged from 211 to $278 \mu \mathrm{S} \cdot \mathrm{cm}^{-1}$ and averaged $254 \mu \mathrm{S} \cdot \mathrm{cm}^{-1}$. A net increase 


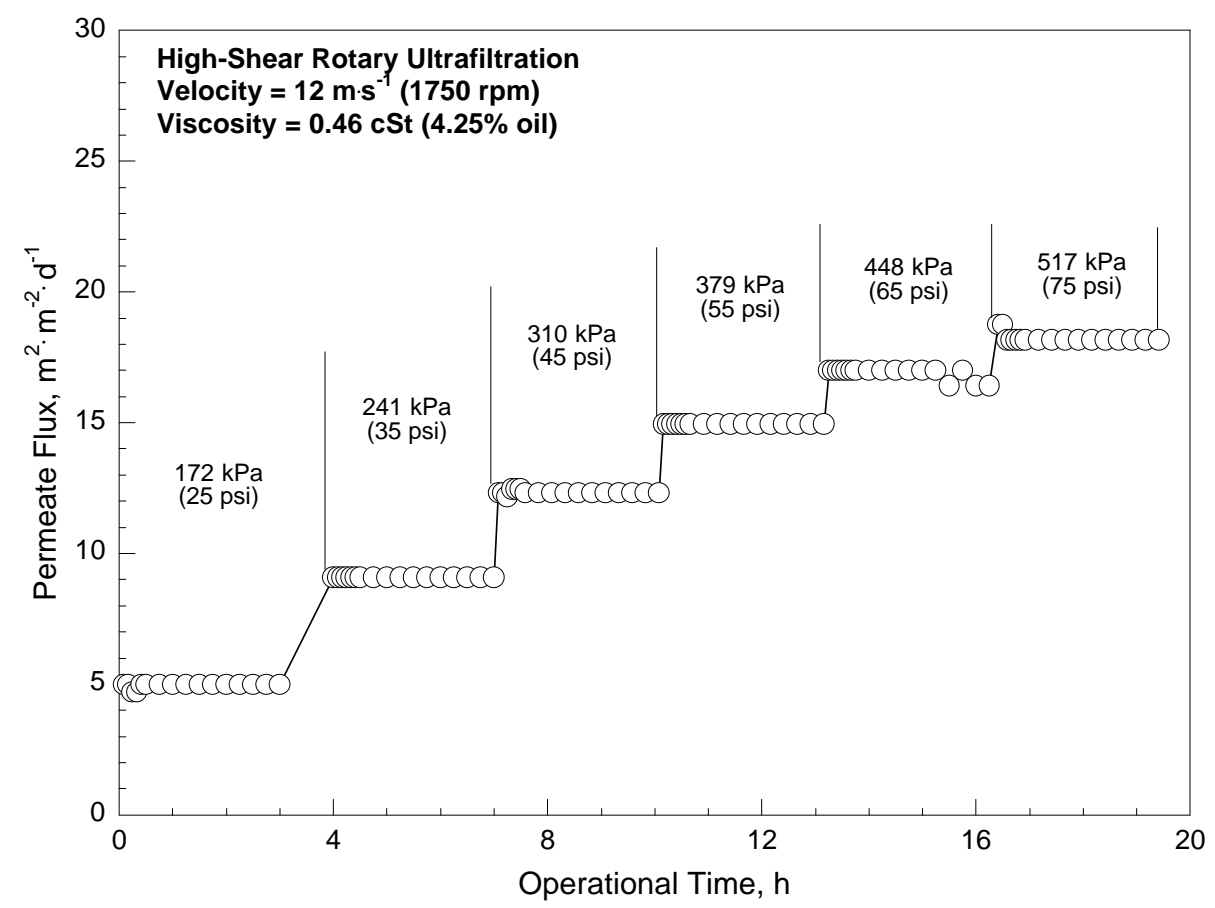

Figure 4.50 Permeate flux versus operational time for the $12 \mathrm{~m} \cdot \mathrm{s}^{-1}-0.46 \mathrm{cSt}$ experiment.

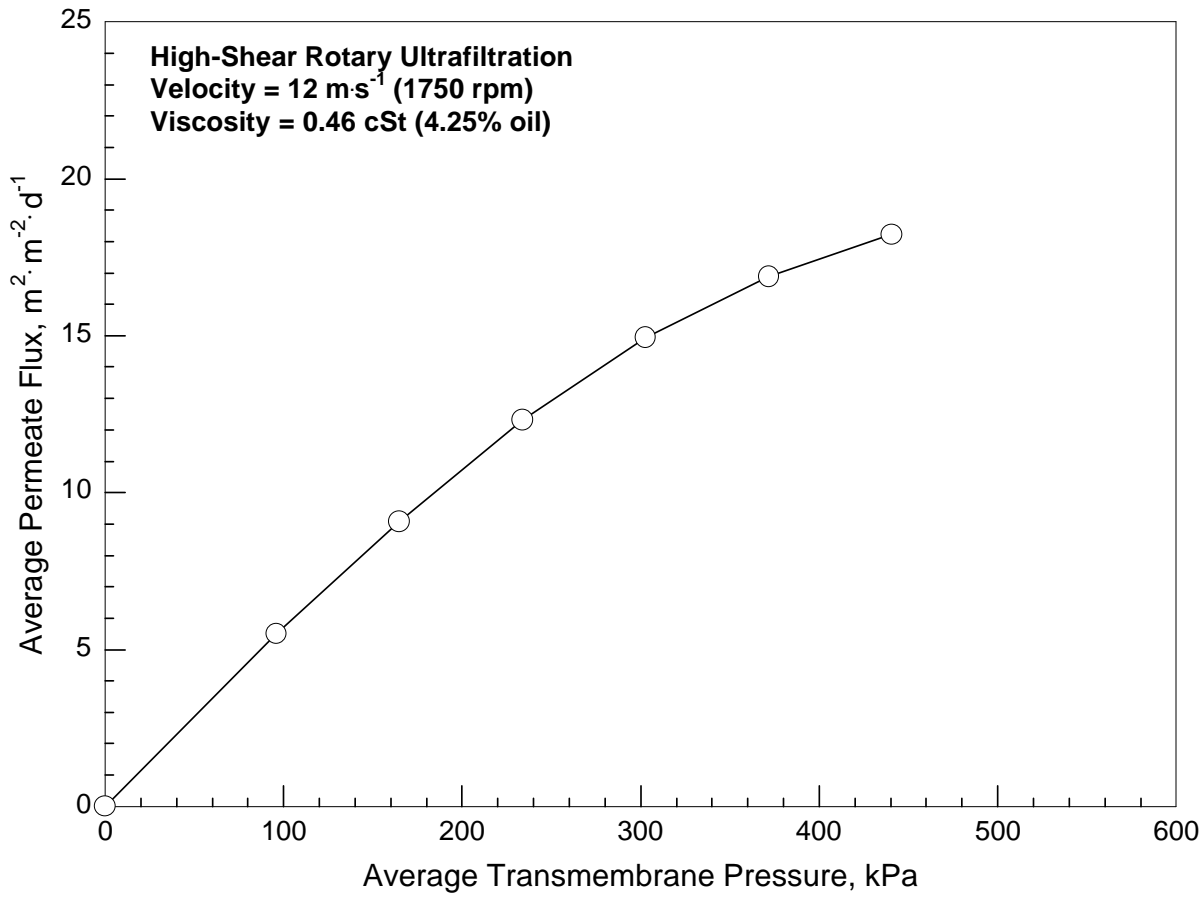

Figure 4.51 Average steady state permeate flux versus average transmembrane pressure for the $12 \mathrm{~m} \cdot \mathrm{s}^{-1}-0.46 \mathrm{cSt}$ experiment. 
in concentrate conductivity and a net decrease in permeate conductivity over time was observed. Distinct step-wise changes in permeate and concentrate $\mathrm{pH}$ and conductivity were not observed as applied pressure was increased.

\subsection{2 $10 \mathrm{~m} \cdot \mathrm{s}^{-1}-0.46 \mathrm{cSt}(1450 \mathrm{rpm}-5 \% \mathrm{MW}$ Fluid)}

Permeate flux versus time for the $10 \mathrm{~m} \cdot \mathrm{s}^{-1}-0.46 \mathrm{cSt}$ experiment is presented in Figure 4.52. Permeate flux was stable at each applied pressure condition. The system was operated over an applied pressure range of 172 to $517 \mathrm{kPa}$ ( 25 to $75 \mathrm{psig}$ ). The average permeate backpressure was $52.4 \mathrm{kPa}(7.6 \mathrm{psi})$ at an average relative velocity of $10 \mathrm{~m} \cdot \mathrm{s}^{-1}(1450 \mathrm{rpm})$. The average permeate flux versus average transmembrane pressure for the $10 \mathrm{~m} \cdot \mathrm{s}^{-1}-0.46 \mathrm{cSt}$ experiment is presented in Figure 4.53. A limiting flux was not observed over the applied pressure range studied in this experiment; however, the average flux data diverged from linear behavior at higher pressures due to a shift from pressure-dependent to pressure-independent operating conditions.

The permeate $\mathrm{pH}$ ranged from 7.97 to 8.28 and averaged 8.12. The concentrate $\mathrm{pH}$ ranged from 7.50 to 7.92 and averaged 7.61. Permeate $\mathrm{pH}$ was constant. Concentrate $\mathrm{pH}$ decreased over the first $1 \mathrm{hr}$. then remained constant at $\sim 7.6$. Permeate $\mathrm{pH}$ was greater than concentrate $\mathrm{pH}$ throughout this experiment. The permeate conductivity ranged from 123 to $140 \mu \mathrm{S} \cdot \mathrm{cm}^{-1}$ and averaged $126 \mu \mathrm{S} \cdot \mathrm{cm}^{-1}$. The concentrate conductivity ranged from 165 to $200 \mu \mathrm{S} \cdot \mathrm{cm}^{-1}$ and averaged $188 \mu \mathrm{S} \cdot \mathrm{cm}^{-1}$. Permeate and concentrate conductivity were constant throughout this experiment. Distinct step-wise changes in permeate and concentrate $\mathrm{pH}$ and conductivity were not observed as applied pressure was increased 


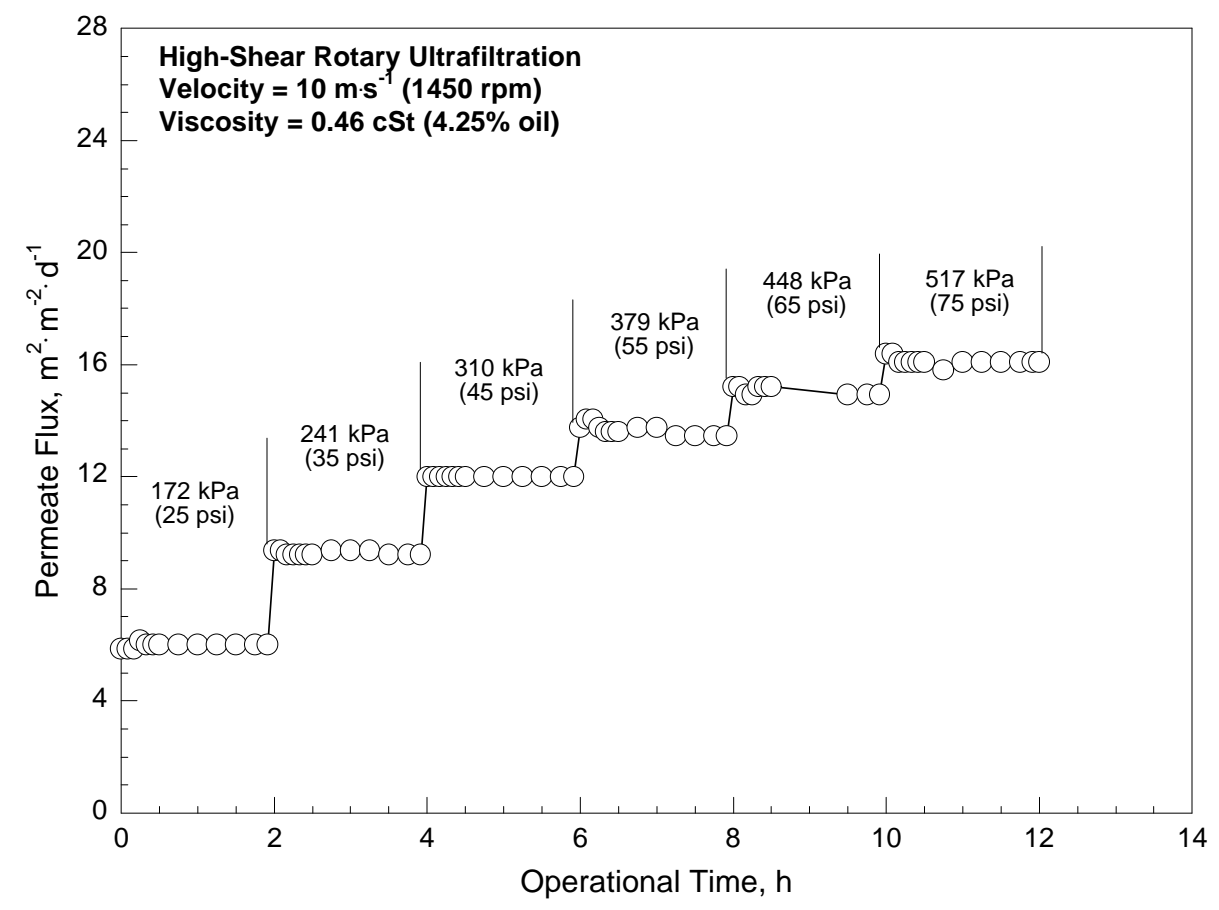

Figure 4.52 Permeate flux versus operational time for the $10 \mathrm{~m} \cdot \mathrm{s}^{-1}-0.46 \mathrm{cSt}$ experiment.

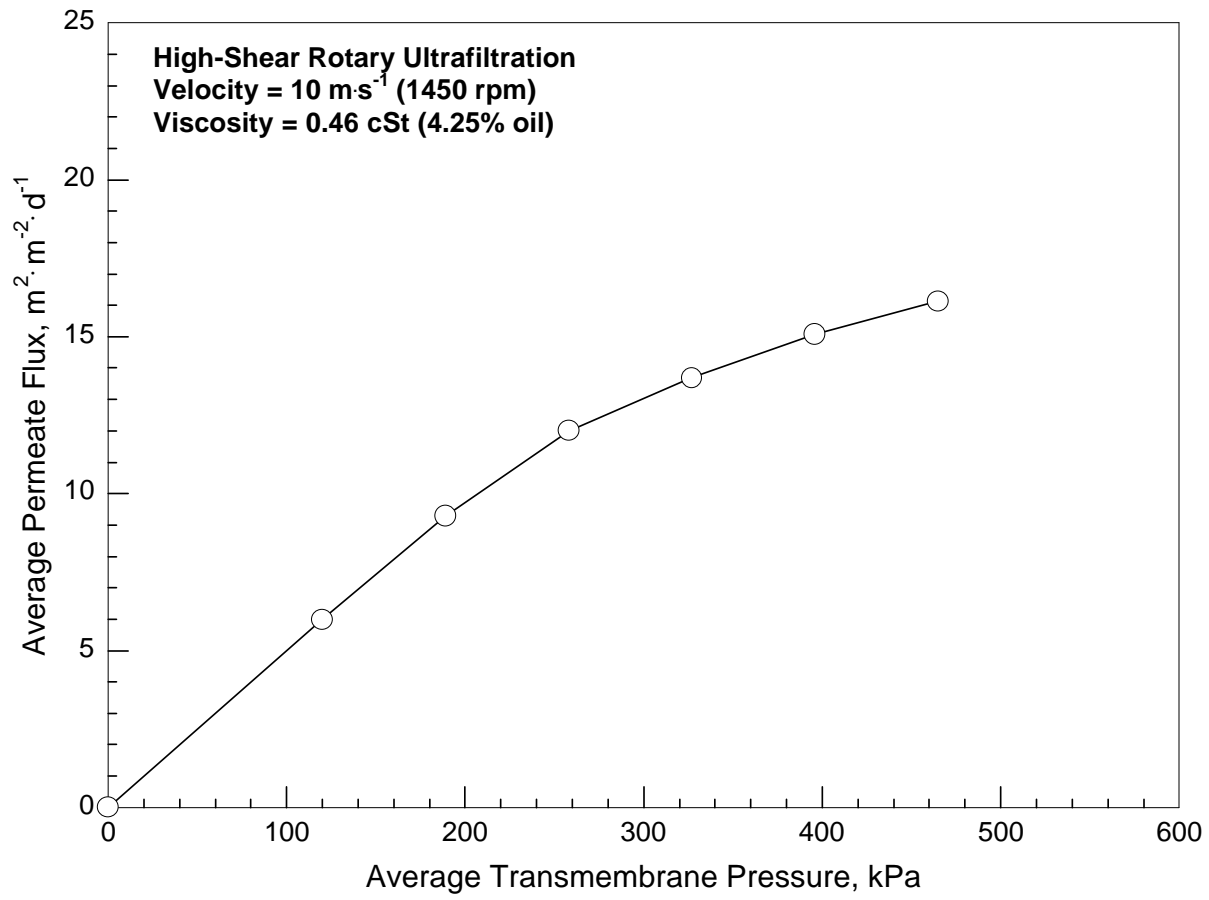

Figure 4.53 Average steady state permeate flux versus average transmembrane pressure for the $10 \mathrm{~m} \cdot \mathrm{s}^{-1}-0.46 \mathrm{cSt}$ experiment. 


\subsection{3 $8 \mathrm{~m} \cdot \mathrm{s}^{-1}-0.46 \mathrm{cSt}(1150 \mathrm{rpm}-5 \%$ MW Fluid)}

Permeate flux versus time for the $8 \mathrm{~m} \cdot \mathrm{s}^{-1}-0.46 \mathrm{cSt}$ experiment is presented in Figure 4.54. The system was operated over an applied pressure range of 172 to $517 \mathrm{kPa}$ (25 to $75 \mathrm{psig}$ ). The average permeate backpressure was $33.1 \mathrm{kPa}(4.8 \mathrm{psi}$ ) at an average relative velocity of $8 \mathrm{~m} \cdot \mathrm{s}^{-1}(1150 \mathrm{rpm})$. At each applied pressure condition, permeate flux was observed to increase then drop off to a constant value. This phenomenon was attributed to a delay in stabilization of the concentration boundary layer (Viadero and Reed 1999). Permeate flux at the 172 and $241 \mathrm{kPa}$ (25 and $35 \mathrm{psig}$ ) applied pressure conditions was initially steady for $\sim 15 \mathrm{~min}$. at 9.1 and $12 \mathrm{~m}^{3} \cdot \mathrm{m}^{-2} \cdot \mathrm{d}^{-1}\left(223\right.$ and $302 \mathrm{gal} \cdot \mathrm{ft}^{-}$ $\left.{ }^{2} \cdot \mathrm{d}^{-1}\right)$, respectively. The permeate flux at the 172 and $241 \mathrm{kPa}$ applied pressure conditions then decreased nominally to constant values of 8.8 and $12 \mathrm{~m}^{3} \cdot \mathrm{m}^{-2} \cdot \mathrm{d}^{-1}\left(215\right.$ and $294 \mathrm{gal} \cdot \mathrm{ft}^{-}$ $\left.{ }^{2} \cdot \mathrm{d}^{-1}\right)$, respectively. Similar initial increases in permeate flux were observed at the 310 to $517 \mathrm{kPa}$ (45 to $75 \mathrm{psig}$ ) applied pressure condition. However, the initial period of high permeate flux was shorter as the applied pressure was increased. At 448 and $517 \mathrm{kPa}$ (65 and 75 psig) applied pressure conditions, the initial increase in permeate flux was minimal. It is hypothesized that the initially higher permeate flux followed by a decrease at each applied pressure was due to the consolidation of the solute boundary layer at the membrane surface. The average permeate flux versus average transmembrane pressure for the $8 \mathrm{~m} \cdot \mathrm{s}^{-1}-0.46 \mathrm{cSt}$ experiment is presented in Figure 4.55. A limiting flux of 13 $\mathrm{m}^{3} \cdot \mathrm{m}^{-2} \cdot \mathrm{d}^{-1}\left(320 \mathrm{gal} \cdot \mathrm{ft}^{-2} \cdot \mathrm{d}^{-1}\right)$ was observed at the 310 to $517 \mathrm{kPa}$ (45 to $\left.75 \mathrm{psig}\right)$ applied pressure conditions.

The permeate $\mathrm{pH}$ ranged from 7.95 to 8.27 and averaged 8.12. The concentrate $\mathrm{pH}$ ranged from 7.50 to 8.17 and averaged 7.72. Permeate $\mathrm{pH}$ remained constant 


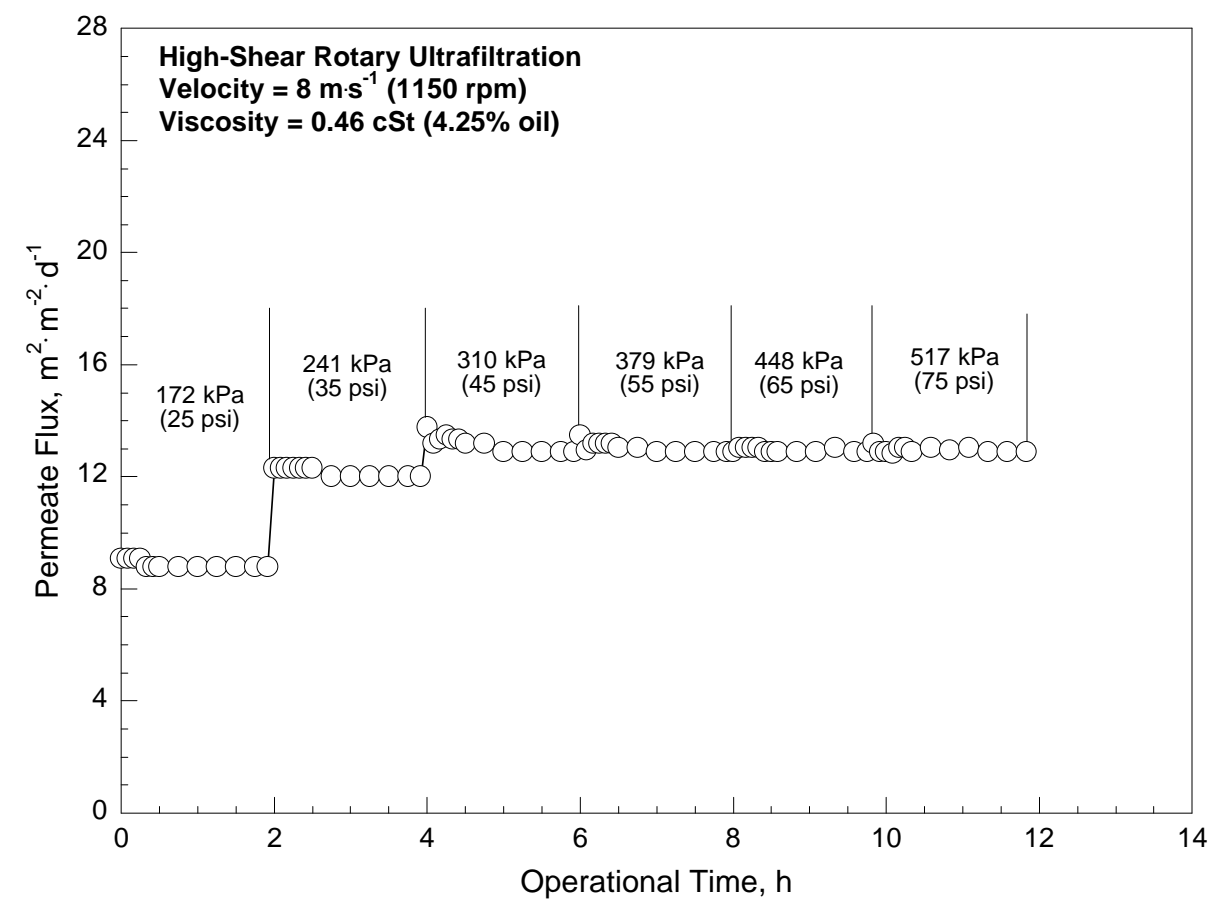

Figure 4.54 Permeate flux versus operational time for the $8 \mathrm{~m} \cdot \mathrm{s}^{-1}-0.46 \mathrm{cSt}$ experiment.

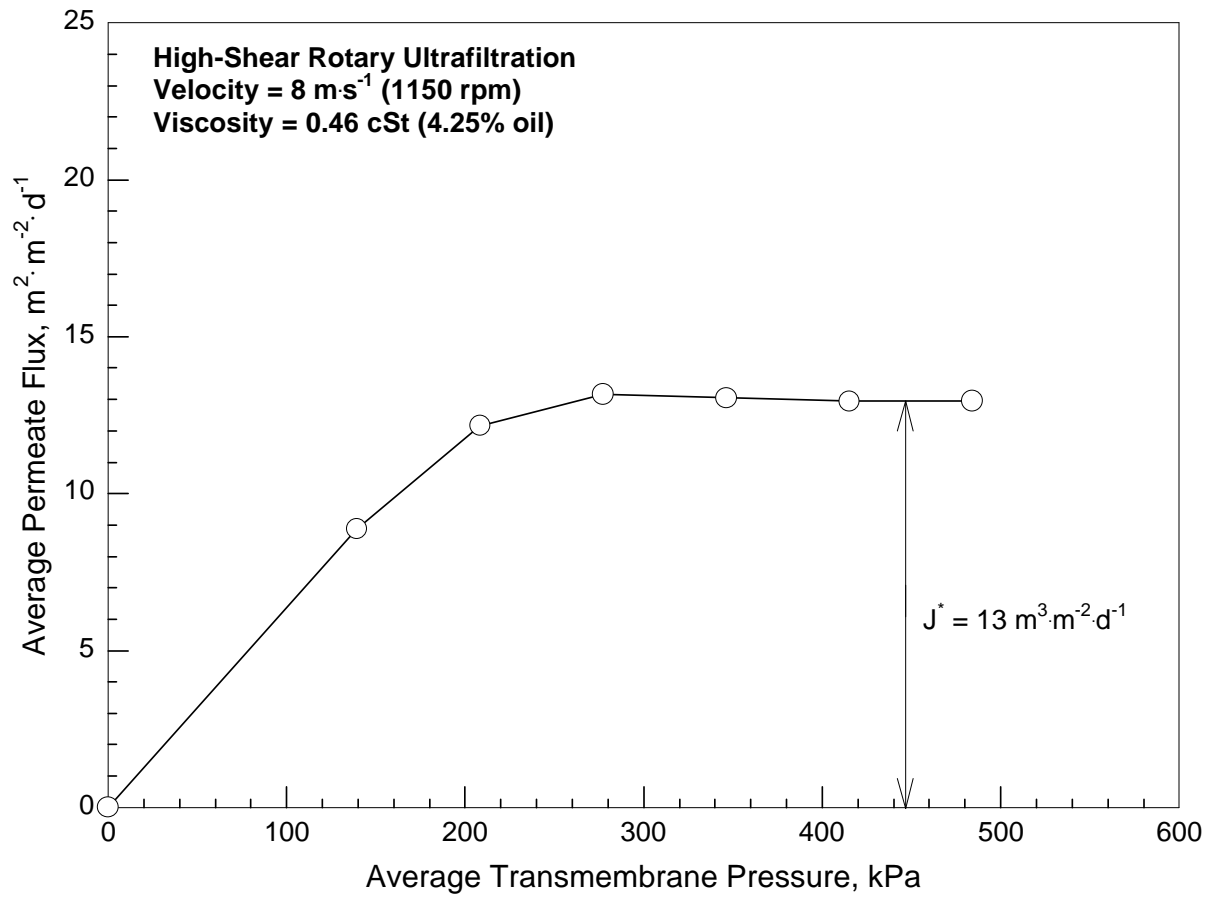

Figure 4.55 Average steady state permeate flux versus average transmembrane pressure for the $8 \mathrm{~m} \cdot \mathrm{s}^{-1}-0.46 \mathrm{cSt}$ experiment. 
throughout this experiment. Concentrate $\mathrm{pH}$ decreased over the first $4.5 \mathrm{hr}$. then varied between $\sim 7.5$ and 7.7 through the end of the experiment. Permeate $\mathrm{pH}$ was greater than concentrate $\mathrm{pH}$ throughout this experiment. The permeate conductivity ranged from 99 to $137 \mu \mathrm{S} \cdot \mathrm{cm}^{-1}$ and averaged $115 \mu \mathrm{S} \cdot \mathrm{cm}^{-1}$. The concentrate conductivity ranged from 169 to

$202 \mu \mathrm{S} \cdot \mathrm{cm}^{-1}$ and averaged $189 \mu \mathrm{S} \cdot \mathrm{cm}^{-1}$. Permeate and concentrate conductivity were constant throughout this experiment. Distinct step-wise changes in permeate and concentrate $\mathrm{pH}$ and conductivity were not observed as applied pressure was increased.

\subsection{4 $12 \mathrm{~m} \cdot \mathrm{s}^{-1}-\mathbf{0 . 6 4} \mathrm{cSt}(1750 \mathrm{rpm}-10 \%$ MW Fluid)}

Permeate flux versus time for the $12 \mathrm{~m} \cdot \mathrm{s}^{-1}-0.64 \mathrm{cSt}$ experiment is presented in Figure 4.56. Permeate flux was stable at each applied pressure condition. The system was operated over an applied pressure range of 172 to $517 \mathrm{kPa}$ ( 25 to $75 \mathrm{psig}$ ). The average permeate backpressure was $76.6 \mathrm{kPa}(11.1 \mathrm{psi})$ at an average relative velocity of $12 \mathrm{~m} \cdot \mathrm{s}^{-1}(1750 \mathrm{rpm})$. The average permeate flux versus average transmembrane pressure for the $12 \mathrm{~m} \cdot \mathrm{s}^{-1}-0.64 \mathrm{cSt}$ experiment is presented in Figure 4.57. A limiting flux of 16 $\mathrm{m}^{3} \cdot \mathrm{m}^{-2} \cdot \mathrm{d}^{-1}\left(400 \mathrm{gal} \cdot \mathrm{ft}^{-2} \cdot \mathrm{d}^{-1}\right)$ was observed at the 448 and $517 \mathrm{kPa}(65$ and $75 \mathrm{psig})$ applied pressure conditions.

The permeate $\mathrm{pH}$ ranged from 7.97 to 8.28 and averaged 8.05. The concentrate $\mathrm{pH}$ ranged from 7.31 to 7.78 and averaged 7.52. Permeate and concentrate $\mathrm{pH}$ was constant and the permeate $\mathrm{pH}$ was greater than the concentrate $\mathrm{pH}$ throughout the $10 \%$ MW Fluid-1750 rpm experiment. The permeate conductivity ranged from 245 to 291 $\mu \mathrm{S} \cdot \mathrm{cm}^{-1}$ and averaged $272 \mu \mathrm{S} \cdot \mathrm{cm}^{-1}$. The concentrate conductivity ranged from 242 to 343 $\mu \mathrm{S} \cdot \mathrm{cm}^{-1}$ and averaged $299 \mu \mathrm{S} \cdot \mathrm{cm}^{-1}$. Permeate conductivity was constant. A net increase in concentrate conductivity was observed. Permeate conductivity was greater than 


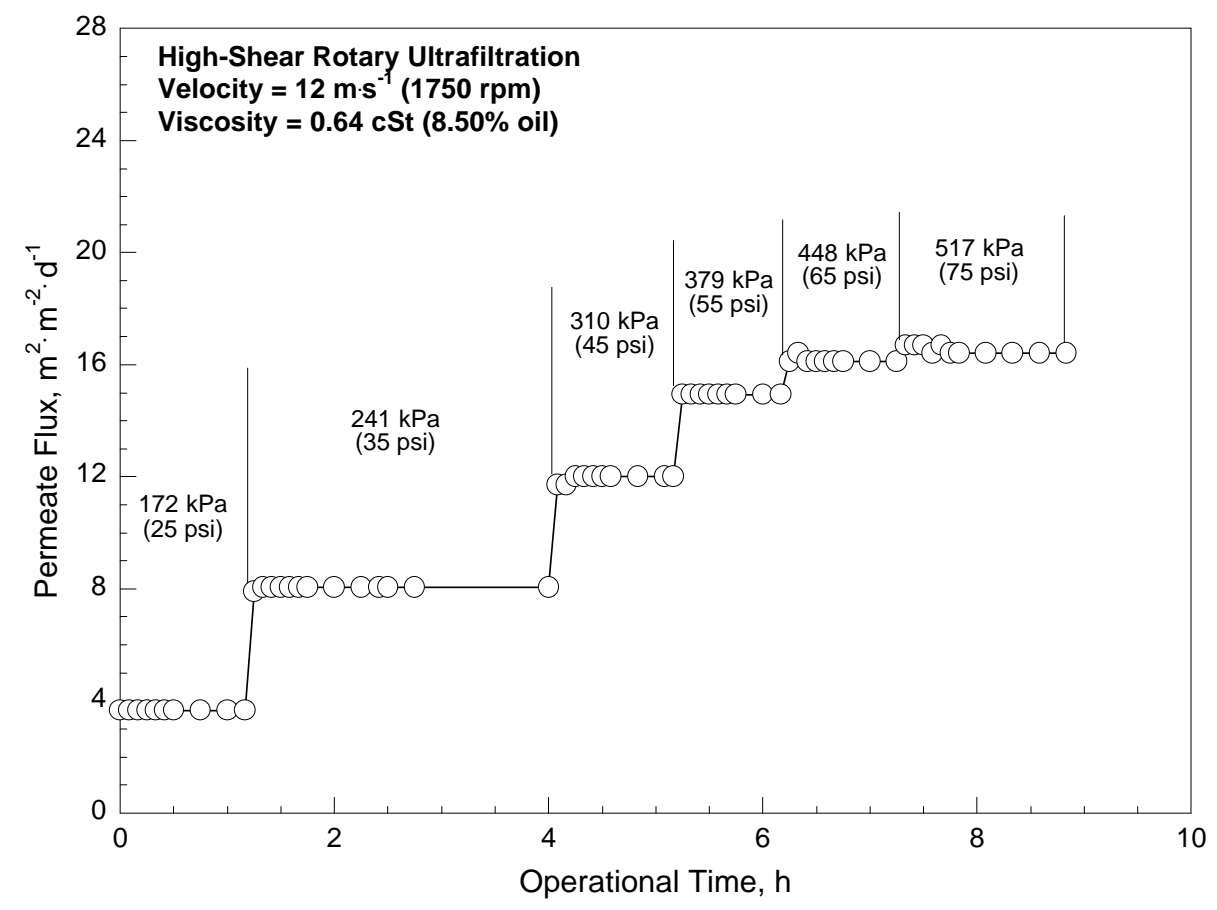

Figure 4.56 Permeate flux versus operational time for the $12 \mathrm{~m} \cdot \mathrm{s}^{-1}-0.64 \mathrm{cSt}$ experiment.

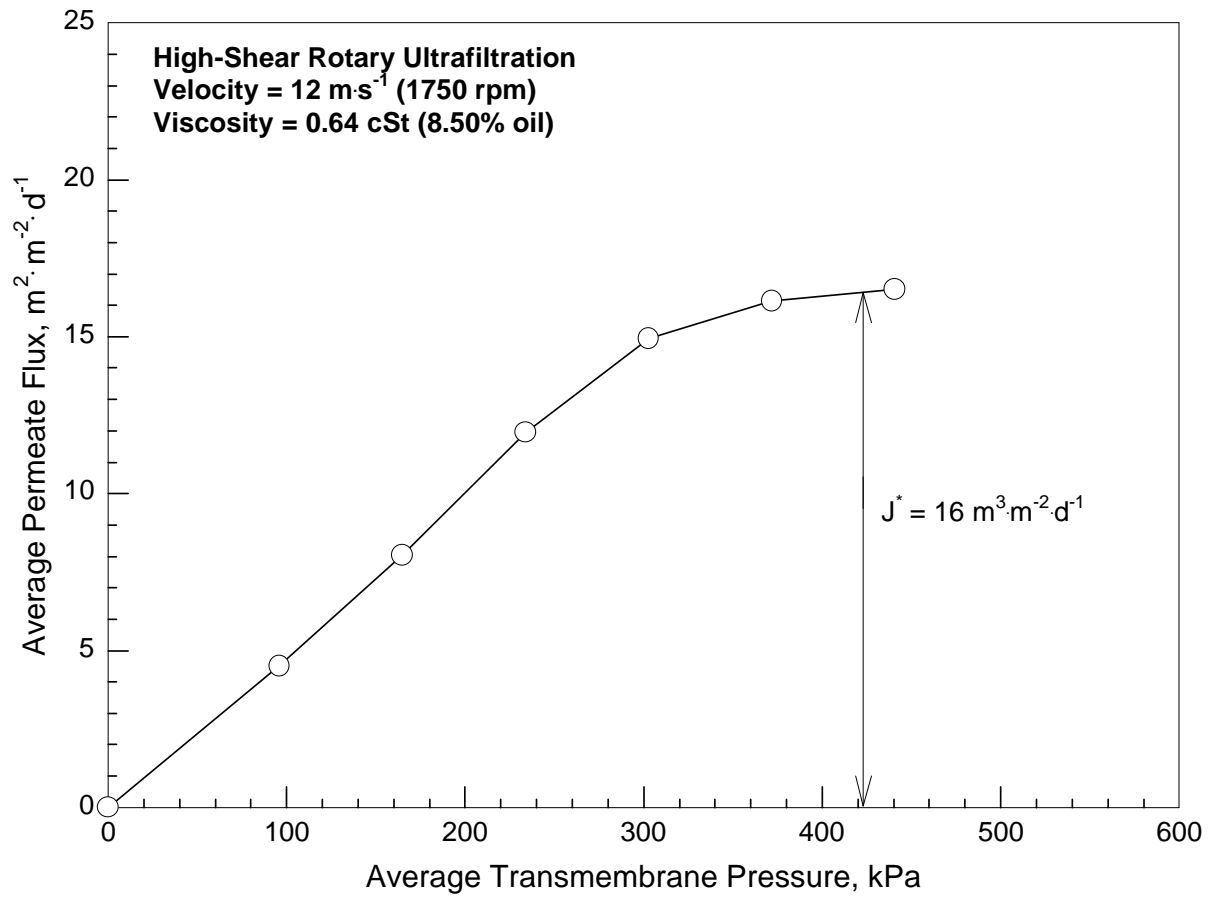

Figure 4.57 Average steady state permeate flux versus average transmembrane pressure for the $12 \mathrm{~m} \cdot \mathrm{s}^{-1}-0.64 \mathrm{cSt}$ experiment. 
concentrate conductivity through $\sim 3.5 \mathrm{hr}$. then concentrate conductivity exceeded permeate conductivity through the end of the experiment. Distinct step-wise changes in permeate and concentrate $\mathrm{pH}$ and conductivity were not observed as applied pressure was increased.

\subsection{5 $10 \mathrm{~m} \cdot \mathrm{s}^{-1}-\mathbf{0 . 6 4} \mathrm{cSt}(1450 \mathrm{rpm}-10 \%$ MW Fluid)}

Permeate flux versus time for the $10 \mathrm{~m} \cdot \mathrm{s}^{-1}-0.64 \mathrm{cSt}$ experiment is presented in Figure 4.58. Permeate flux was stable at each applied pressure condition. The system was operated over an applied pressure range of 172 to $517 \mathrm{kPa}$ ( 25 to $75 \mathrm{psig}$ ). The average permeate backpressure was $52.4 \mathrm{kPa}(7.6 \mathrm{psi})$ at an average relative velocity of $10 \mathrm{~m} \cdot \mathrm{s}^{-1}(1450 \mathrm{rpm})$. Due to operational problems with the feed pump, it was not possible to maintain a steady applied pressure at $448 \mathrm{kPa}$ (65 psig). Average permeate flux versus average transmembrane pressure for the $10 \mathrm{~m} \cdot \mathrm{s}^{-1}-0.64 \mathrm{cSt}$ experiment is presented in Figure 4.59. A limiting flux of $14 \mathrm{~m}^{3} \cdot \mathrm{m}^{-2} \cdot \mathrm{d}^{-1}\left(330 \mathrm{gal} \cdot \mathrm{ft}^{-2} \cdot \mathrm{d}^{-1}\right)$ was observed at the 310 to $517 \mathrm{kPa}$ (45 to $75 \mathrm{psig}$ ) applied pressure conditions.

The permeate $\mathrm{pH}$ ranged from 7.98 to 8.24 and averaged 8.08. The concentrate $\mathrm{pH}$ ranged from 7.51 to 7.83 and averaged 7.69. Permeate and concentrate $\mathrm{pH}$ were constant. Permeate $\mathrm{pH}$ was greater than concentrate $\mathrm{pH}$ throughout the experiment. Permeate conductivity ranged from 240 to $301 \mu \mathrm{S} \cdot \mathrm{cm}^{-1}$ and averaged $274 \mu \mathrm{S} \cdot \mathrm{cm}^{-1}$. Concentrate conductivity ranged from 261 to $331 \mu \mathrm{S} \cdot \mathrm{cm}^{-1}$ and averaged $305 \mu \mathrm{S} \cdot \mathrm{cm}^{-1}$. A net decrease in permeate conductivity and a net increase in concentrate conductivity were observed. Permeate conductivity was greater than concentrate conductivity throughout the first $\sim 2 \mathrm{hr}$. of operation at which point the concentrate conductivity surpassed the permeate conductivity. The increase in concentrate conductivity occurred approximately 


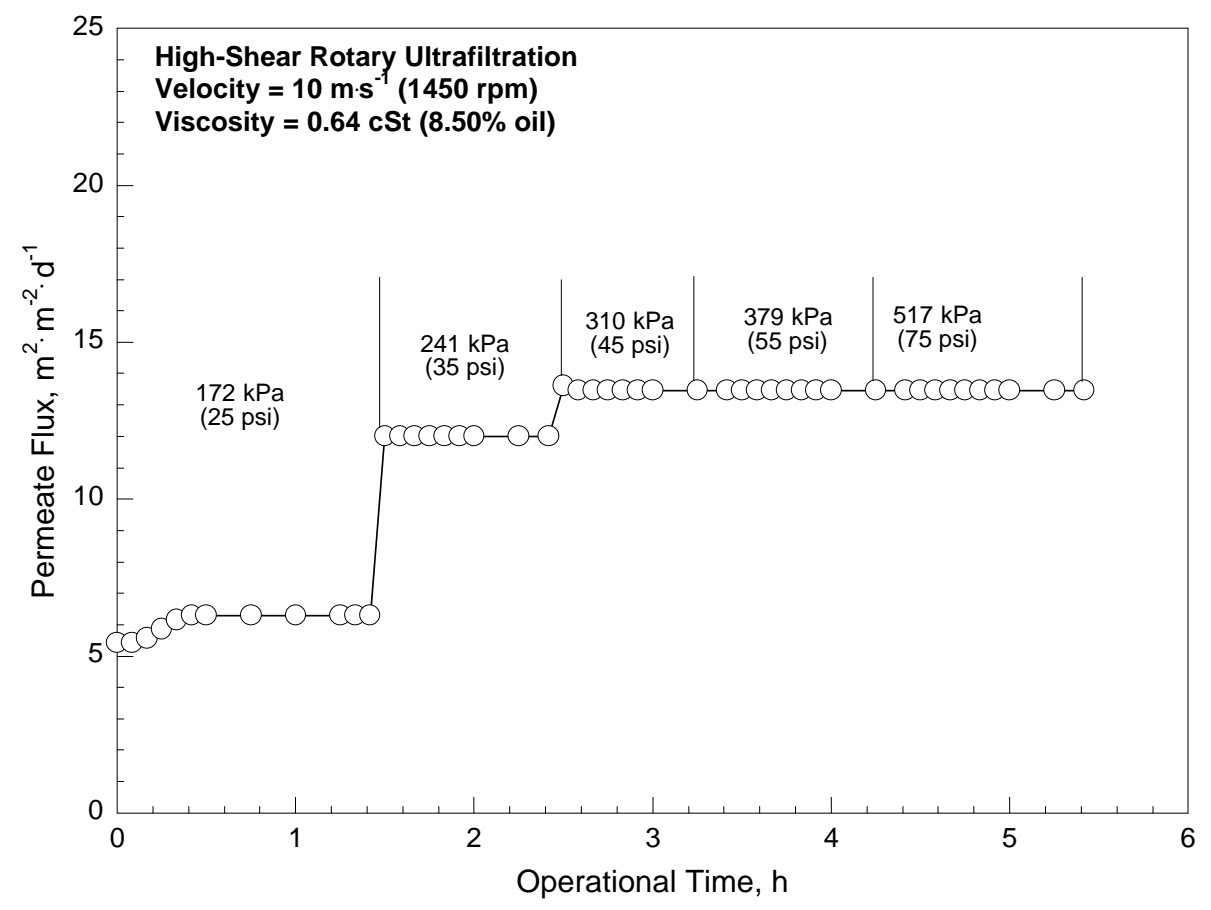

Figure 4.58 Permeate flux versus operational time for the $10 \mathrm{~m} \cdot \mathrm{s}^{-1}-0.64 \mathrm{cSt}$ experiment.

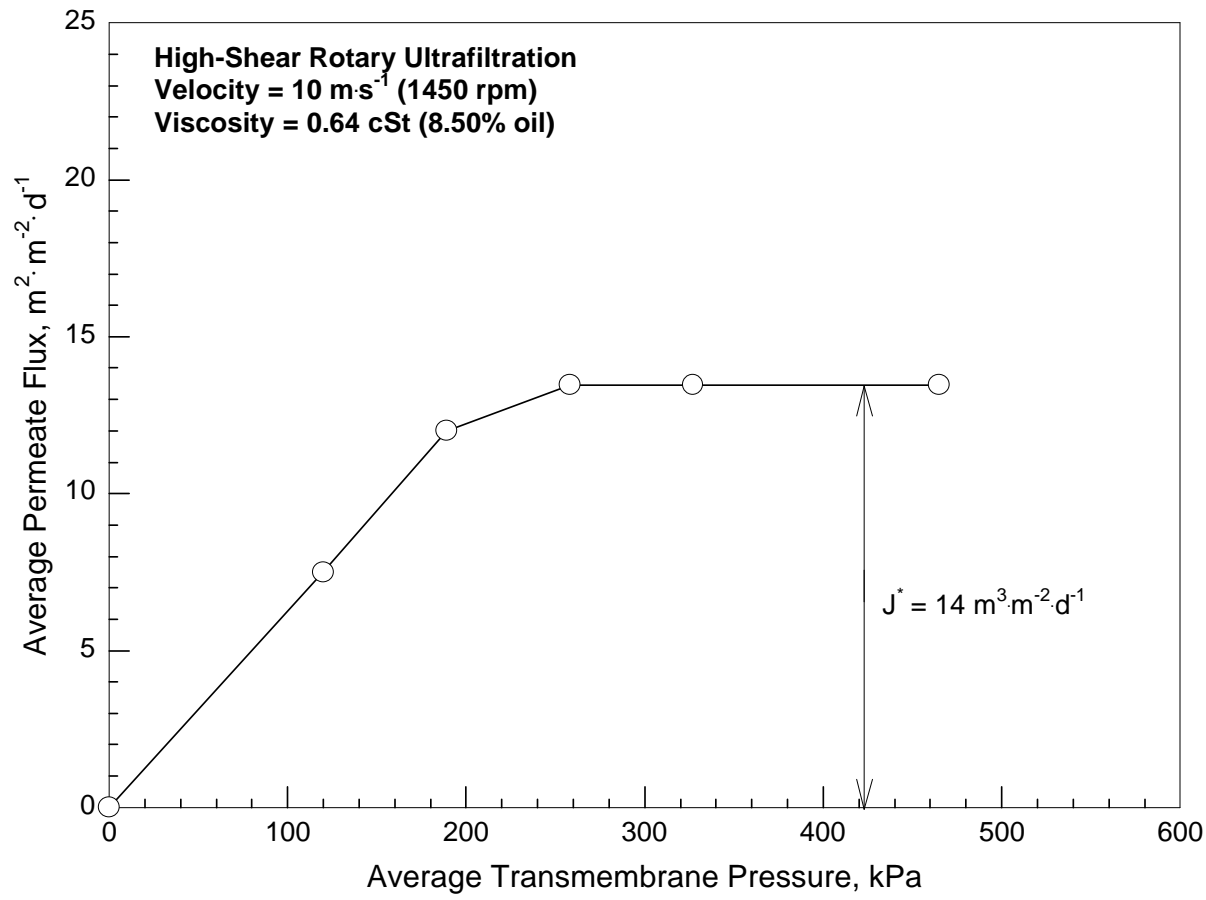

Figure 4.59 Average steady state permeate flux versus average transmembrane pressure for the $10 \mathrm{~m} \cdot \mathrm{s}^{-1}-0.64 \mathrm{cSt}$ experiment. 
in the middle of the $241 \mathrm{kPa}$ (35 psig) applied pressure condition and continued through and operational time of $\sim 3.5 \mathrm{hr}$. At this point in time the concentrate conductivity reached a constant value of $331 \mu \mathrm{S} \cdot \mathrm{cm}^{-1}$. Distinct step-wise changes in permeate and concentrate $\mathrm{pH}$ and conductivity were not observed as applied pressure was increased.

\subsection{6 $8 \mathrm{~m} \cdot \mathrm{s}^{-1}-0.64 \mathrm{cSt}(1150 \mathrm{rpm}-10 \% \mathrm{MW}$ Fluid)}

Permeate flux versus time for the $8 \mathrm{~m} \cdot \mathrm{s}^{-1}-0.64 \mathrm{cSt}$ experiment is presented in Figure 4.60. Permeate flux was stable at each applied pressure condition. The system was operated over an applied pressure range of 172 to $517 \mathrm{kPa}$ ( 25 to $75 \mathrm{psig}$ ). The average permeate backpressure was $33.1 \mathrm{kPa}$ (4.8 psi) at an average relative velocity of 8 $\mathrm{m} \cdot \mathrm{s}^{-1}(1150 \mathrm{rpm})$. Due to operational problems with the feed pump, it was not possible to maintain a steady applied pressure at $448 \mathrm{kPa}$ (65 psig). Average permeate flux versus average transmembrane pressure for the $8 \mathrm{~m} \cdot \mathrm{s}^{-1}-0.64 \mathrm{cSt}$ experiment is presented in Figure 4.61. A limiting flux of $9.1 \mathrm{~m}^{3} \cdot \mathrm{m}^{-2} \cdot \mathrm{d}^{-1}\left(223 \mathrm{gal} \cdot \mathrm{ft}^{-2} \cdot \mathrm{d}^{-1}\right)$ was observed at the 379 to $517 \mathrm{kPa}$ (55 to $75 \mathrm{psig}$ ) applied pressure conditions.

The permeate $\mathrm{pH}$ ranged from 7.85 to 8.34 and averaged 8.02 . The concentrate $\mathrm{pH}$ ranged from 7.46 to 7.97 and averaged 7.64. A net decrease in both permeate and concentrate $\mathrm{pH}$ was observed. The permeate $\mathrm{pH}$ was greater than the concentrate $\mathrm{pH}$ throughout this experiment. The permeate conductivity ranged from 230 to $282 \mu \mathrm{S} \cdot \mathrm{cm}^{-1}$ and averaged $262 \mu \mathrm{S} \cdot \mathrm{cm}^{-1}$. The concentrate conductivity ranged from 245 to $333 \mu \mathrm{S} \cdot \mathrm{cm}^{-1}$ and averaged $300 \mu \mathrm{S} \cdot \mathrm{cm}^{-1}$. A net decrease in permeate conductivity and a net increase in concentrate conductivity were observed. Permeate conductivity was greater than concentrate conductivity through $\sim 1.25 \mathrm{hr}$. (in the middle of the $241 \mathrm{kPa}$ (35 psig) condition). Concentrate conductivity then exceeded permeate conductivity through the 


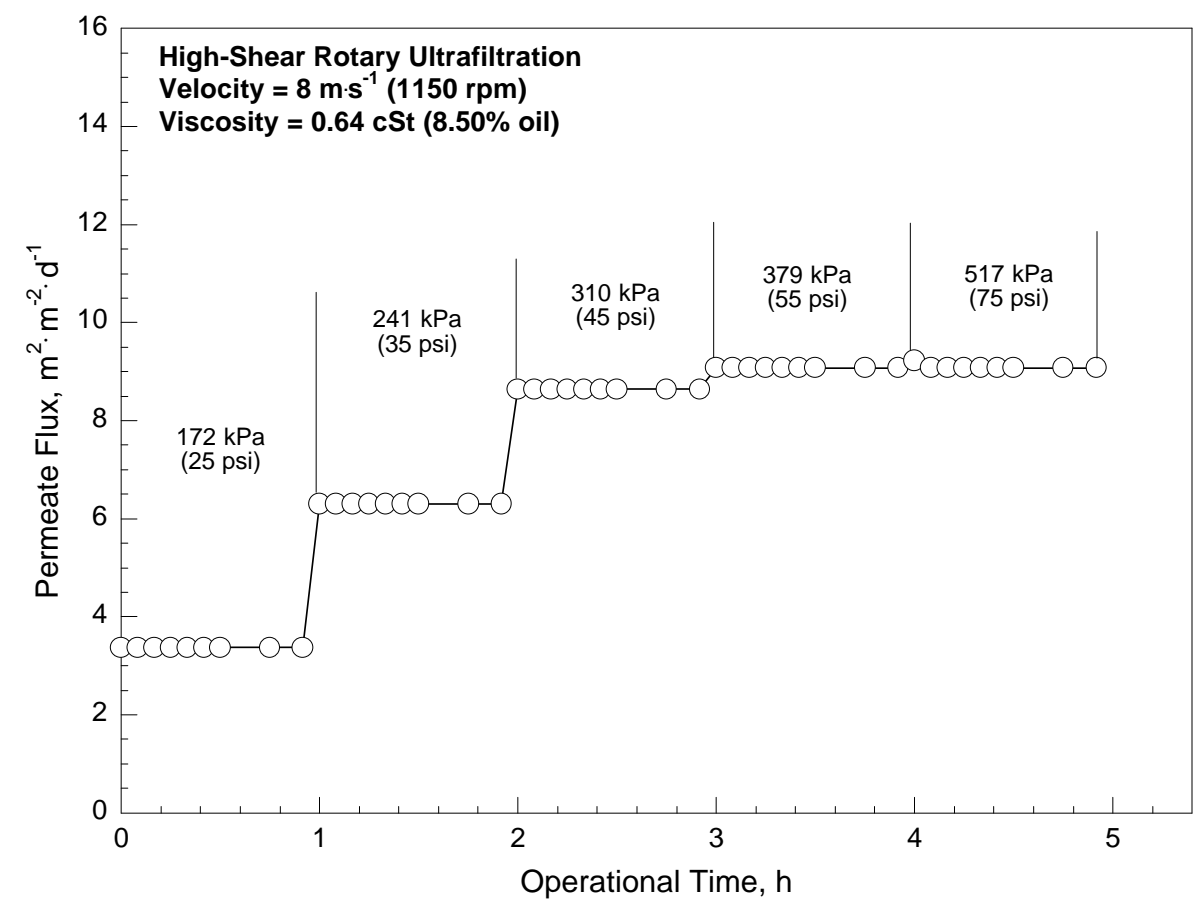

Figure 4.60 Permeate flux versus operational time for the $8 \mathrm{~m} \cdot \mathrm{s}^{-1}-0.64 \mathrm{cSt}$ experiment.

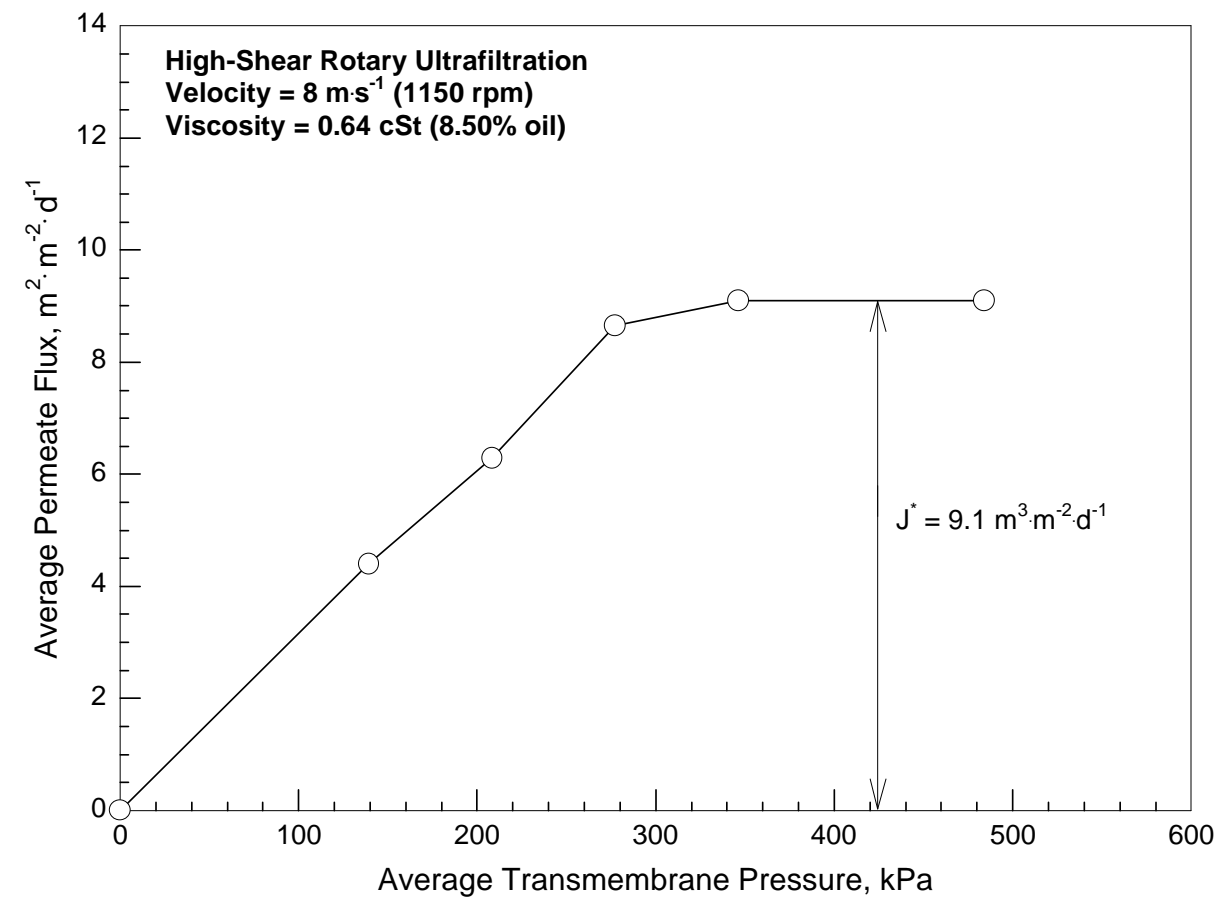

Figure 4.61 Average steady state permeate flux versus operational time for the 8 $\mathrm{m} \cdot \mathrm{s}^{-1}-\mathbf{0 . 6 4} \mathrm{cSt}$ experiment. 
end of this experiment. Distinct step-wise changes in permeate and concentrate $\mathrm{pH}$ and conductivity were not observed as applied pressure was increased.

\subsection{7 $12 \mathrm{~m} \cdot \mathrm{s}^{-1}-\mathbf{0 . 9 4} \mathrm{cSt}(1750 \mathrm{rpm}-15 \% \mathrm{MW}$ Fluid $)$}

Permeate flux versus time for the $12 \mathrm{~m} \cdot \mathrm{s}^{-1}-0.94 \mathrm{cSt}$ experiment is presented in Figure 4.62. Permeate flux was stable at each applied pressure condition. The system was operated over an applied pressure range of 172 to $483 \mathrm{kPa}$ (25 to $70 \mathrm{psig}$ ). The average permeate backpressure was $76.6 \mathrm{kPa}(11.1 \mathrm{psi})$ at an average relative velocity of $12 \mathrm{~m} \cdot \mathrm{s}^{-1}(1750 \mathrm{rpm})$. The average permeate flux versus average transmembrane pressure for the $12 \mathrm{~m} \cdot \mathrm{s}^{-1}-0.94 \mathrm{cSt}$ experiment is presented in Figure 4.63. A limiting flux of 12 $\mathrm{m}^{3} \cdot \mathrm{m}^{-2} \cdot \mathrm{d}^{-1}\left(305 \mathrm{gal} \cdot \mathrm{ft}^{-2} \cdot \mathrm{d}^{-1}\right)$ was observed at the 448 to $482 \mathrm{kPa}$ (65 to $\left.70 \mathrm{psig}\right)$ applied pressure conditions.

The permeate $\mathrm{pH}$ ranged from 5.83 to 7.19 and averaged 6.31. The concentrate $\mathrm{pH}$ ranged from 5.95 to 7.36 and averaged 6.34. A net decrease in both permeate and concentrate $\mathrm{pH}$ was observed in this experiment. Permeate and concentrate $\mathrm{pH}$ values were approximately equal and followed the same general trend (i.e., both permeate and concentrate $\mathrm{pH}$ decreased through $\sim 3 \mathrm{hr}$. then increased through the end of the experiment). The permeate conductivity ranged from 362 to $520 \mu \mathrm{S} \cdot \mathrm{cm}^{-1}$ and averaged $448 \mu \mathrm{S} \cdot \mathrm{cm}^{-1}$. The concentrate conductivity ranged from 459 to $564 \mu \mathrm{S} \cdot \mathrm{cm}^{-1}$ and averaged $534 \mu \mathrm{S} \cdot \mathrm{cm}^{-1}$. Permeate conductivity was greater than concentrate conductivity through $\sim 1 \mathrm{hr}$. Concentrate conductivity exceeded permeate conductivity from $\sim 1 \mathrm{hr}$. through the end of the experiment. Distinct step-wise changes in permeate and concentrate $\mathrm{pH}$ and conductivity were not observed as applied pressure was increased. 


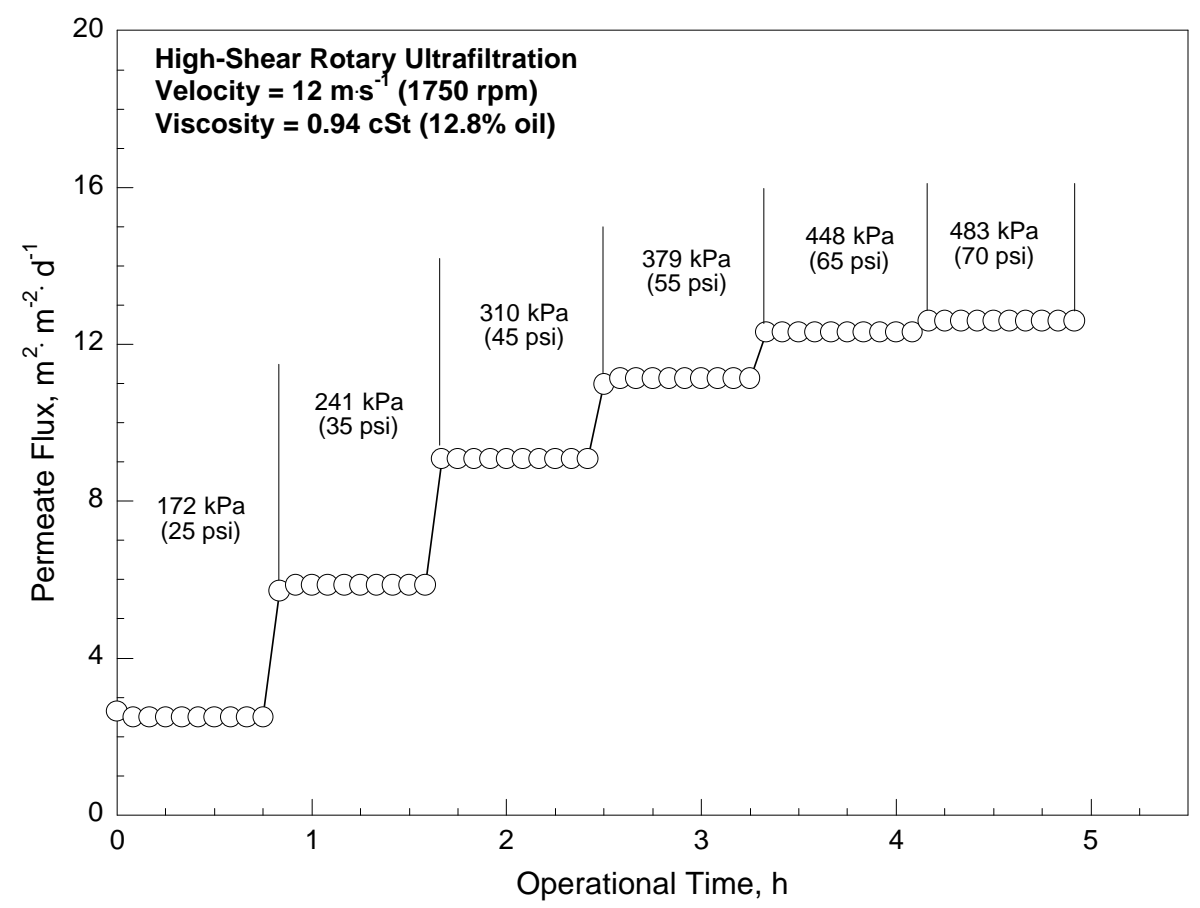

Figure 4.62 Permeate flux versus operational time for the $12 \mathrm{~m} \cdot \mathrm{s}^{-1}-0.94 \mathrm{cSt}$ experiment.

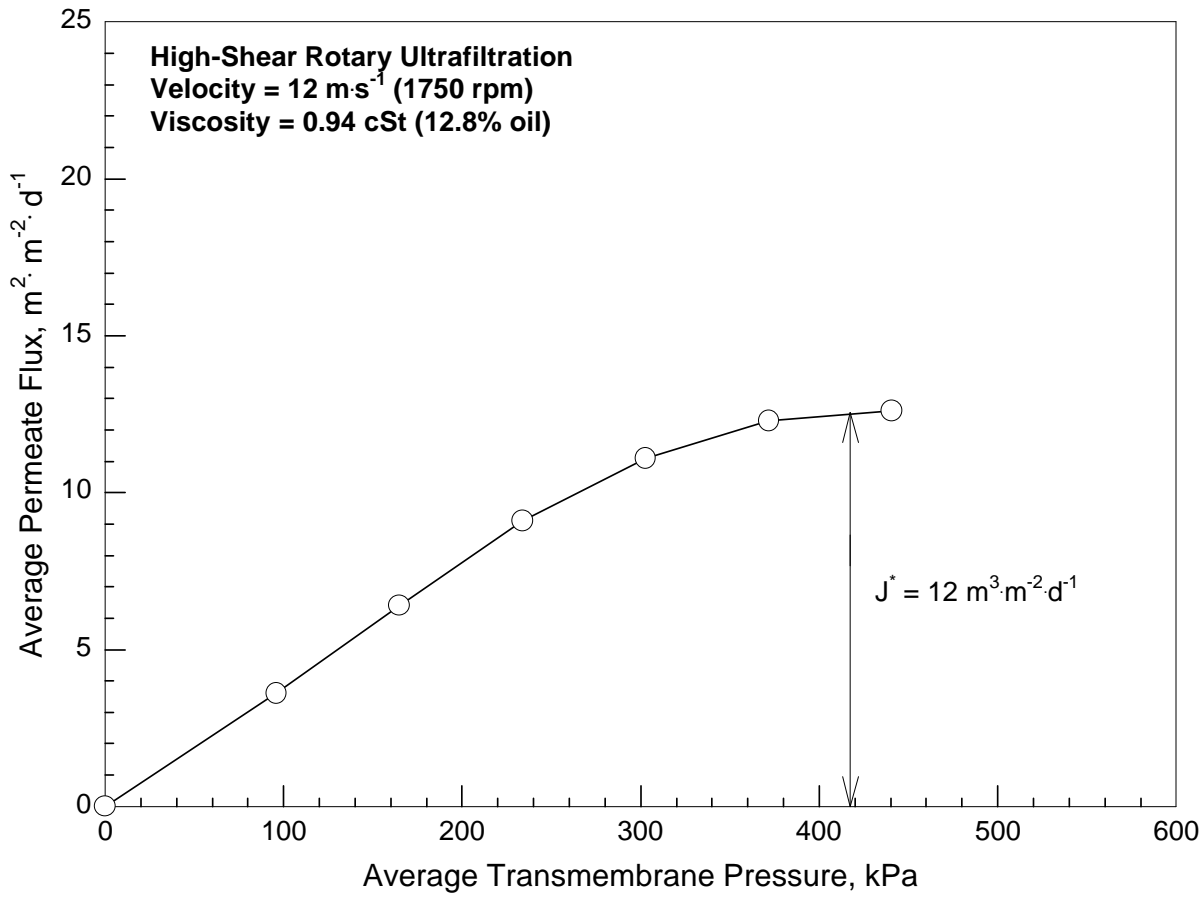

Figure 4.63 Average steady state permeate flux versus average transmembrane pressure for the $12 \mathrm{~m} \cdot \mathrm{s}^{-1}-0.94 \mathrm{cSt}$ experiment. 


\subsection{8 $10 \mathrm{~m} \cdot \mathrm{s}^{-1}-\mathbf{- 0 . 9 4} \mathrm{cSt}(1450 \mathrm{rpm}-15 \%$ MW Fluid)}

Permeate flux versus time for the $10 \mathrm{~m} \cdot \mathrm{s}^{-1}-0.94 \mathrm{cSt}$ experiment is presented in Figure 4.64. Permeate flux was stable at each applied pressure condition. The system was operated over an applied pressure range of 172 to $483 \mathrm{kPa}$ (25 to $70 \mathrm{psig}$ ). The average permeate backpressure was $52.4 \mathrm{kPa}(7.6 \mathrm{psi})$ at an average relative velocity of $10 \mathrm{~m} \cdot \mathrm{s}^{-1}(1450 \mathrm{rpm})$. The average permeate flux versus average transmembrane pressure for the $10 \mathrm{~m} \cdot \mathrm{s}^{-1}-0.94 \mathrm{cSt}$ experiment is presented in Figure 4.65. A limiting flux of 11 $\mathrm{m}^{3} \cdot \mathrm{m}^{-2} \cdot \mathrm{d}^{-1}\left(266 \mathrm{gal} \cdot \mathrm{ft}^{-2} \cdot \mathrm{d}^{-1}\right)$ was observed at the 379 to $483 \mathrm{kPa}$ (55 to $\left.70 \mathrm{psig}\right)$ applied pressure conditions.

The permeate $\mathrm{pH}$ ranged from 6.10 to 6.58 and averaged 6.30. The concentrate $\mathrm{pH}$ ranged from 6.10 to 7.01 and averaged 6.30. The permeate and concentrate $\mathrm{pH}$ were approximately equal in this experiment and followed the same general trend (i.e., a decrease in both permeate and concentrate $\mathrm{pH}$ was observed through the first $1 \mathrm{hr}$. then the permeate and concentrate $\mathrm{pH}$ then remained constant at $\sim 6.30$ from $1 \mathrm{hr}$. through the remainder of the experiment). The permeate conductivity ranged from 348 to $534 \mu \mathrm{S} \cdot \mathrm{cm}^{-}$ ${ }^{1}$ and averaged $433 \mu \mathrm{S} \cdot \mathrm{cm}^{-1}$. The concentrate conductivity ranged from 394 to 579 $\mu \mathrm{S} \cdot \mathrm{cm}^{-1}$ and averaged $514 \mu \mathrm{S} \cdot \mathrm{cm}^{-1}$. A net decrease in permeate conductivity and a net increase in concentrate conductivity were observed. Permeate conductivity was greater than concentrate conductivity through $\sim 1.5 \mathrm{hr}$. The concentrate conductivity then exceeded the permeate conductivity from $\sim 1.5 \mathrm{hr}$. through the end of the experiment. Distinct step-wise changes in permeate and concentrate $\mathrm{pH}$ and conductivity were not observed as applied pressure was increased. 


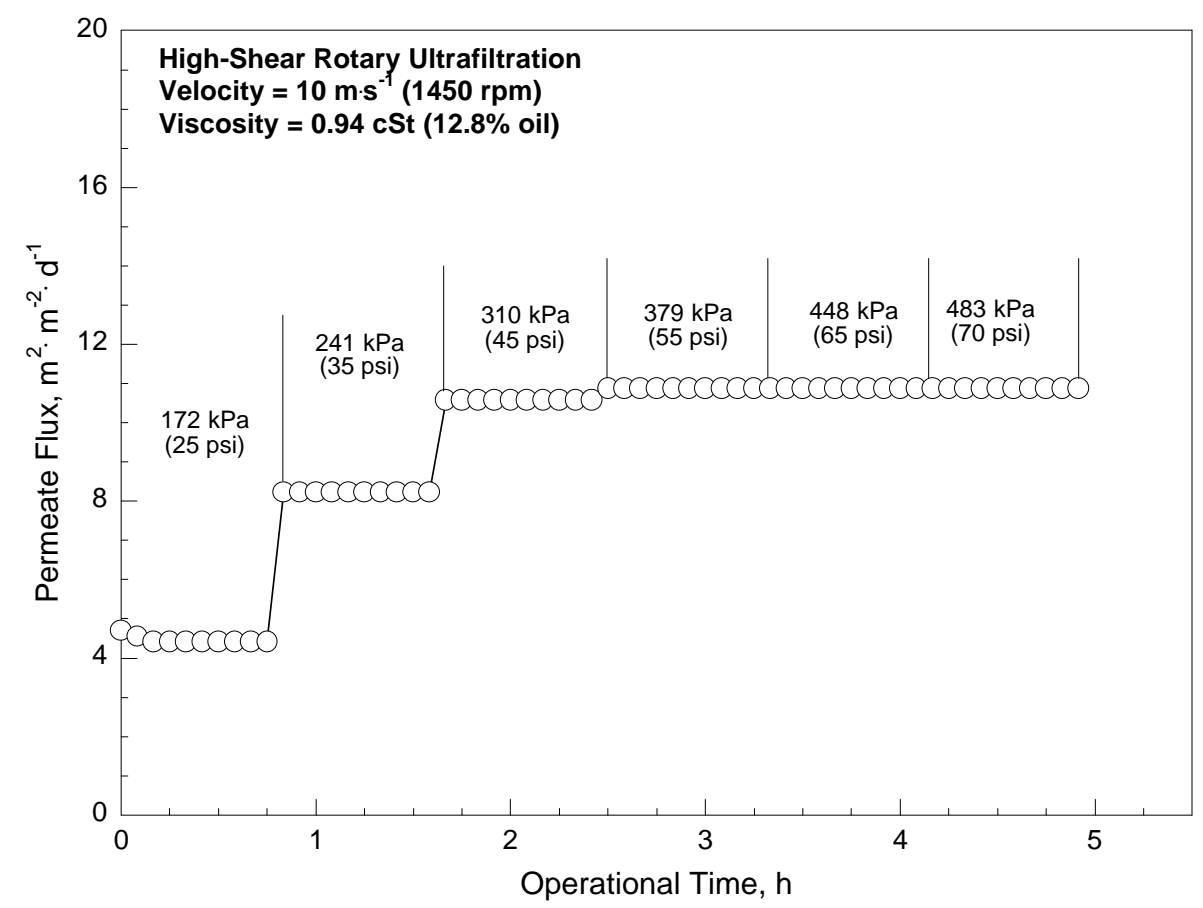

Figure 4.64 Permeate flux versus operational time for the $10 \mathrm{~m} \cdot \mathrm{s}^{-1}-0.94 \mathrm{cSt}$ experiment.

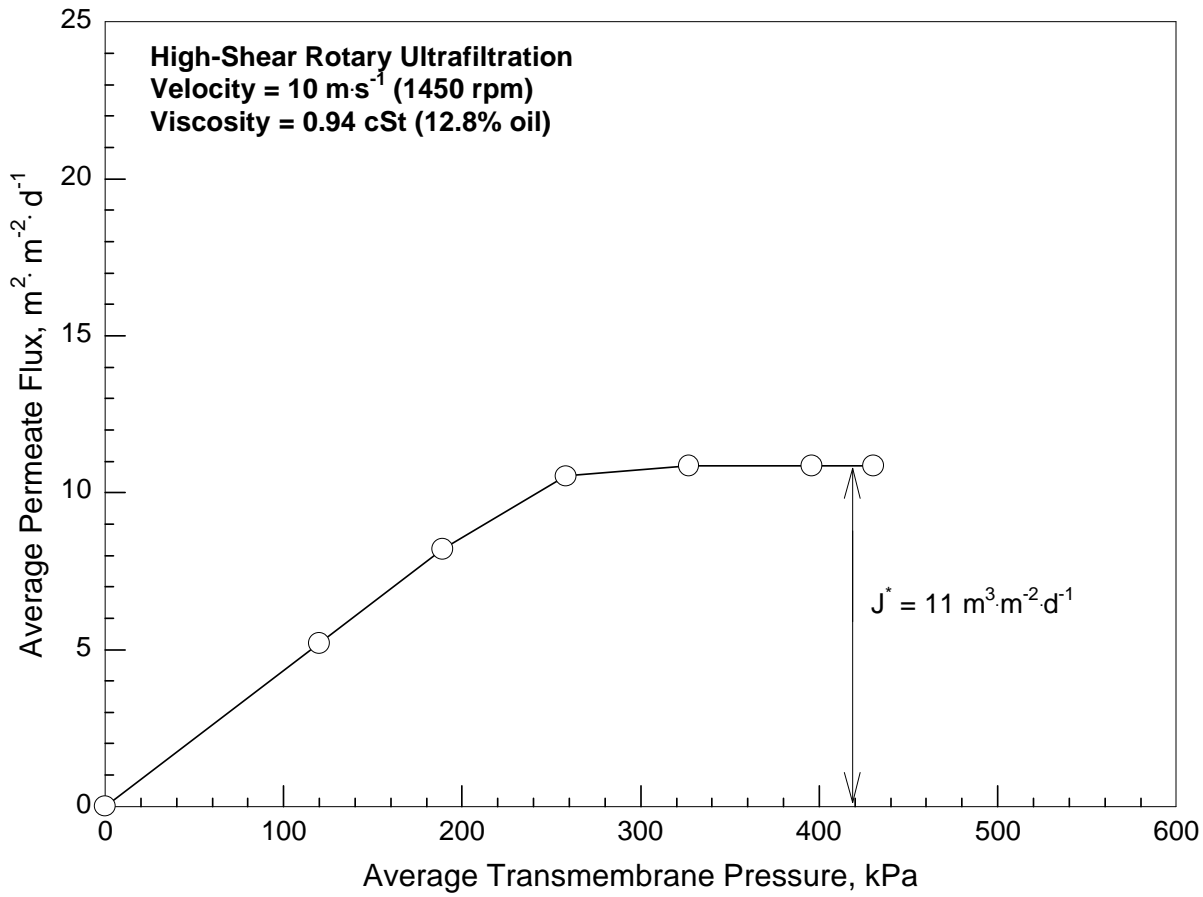

Figure 4.65 Average steady state permeate flux versus average transmembrane pressure for the $10 \mathrm{~m} \cdot \mathrm{s}^{-1}-0.94 \mathrm{cSt}$ experiment. 


\subsection{9 $8 \mathrm{~m} \cdot \mathrm{s}^{-1}-0.94 \mathrm{cSt}(1150 \mathrm{rpm}-15 \%$ MW Fluid)}

Permeate flux versus time for the $8 \mathrm{~m} \cdot \mathrm{s}^{-1}-0.94 \mathrm{cSt}$ experiment is presented in Figure 4.66. Permeate flux was stable at each applied pressure condition. The system was operated over an applied pressure range of 104 to $483 \mathrm{kPa}$ (15 to $70 \mathrm{psig}$ ). The average permeate backpressure was $33.1 \mathrm{kPa}(4.8 \mathrm{psi})$ at an average relative velocity of 8 $\mathrm{m} \cdot \mathrm{s}^{-1}(1150 \mathrm{rpm})$. The average permeate flux versus average transmembrane pressure for the $8 \mathrm{~m} \cdot \mathrm{s}^{-1}-0.94 \mathrm{cSt}$ experiment is presented in Figure 4.67. A limiting flux of $7.8 \mathrm{~m}^{3} \cdot \mathrm{m}^{-}$ ${ }^{2} \cdot \mathrm{d}^{-1}\left(190 \mathrm{gal} \cdot \mathrm{ft}^{-2} \cdot \mathrm{d}^{-1}\right)$ was observed at the 241 to $483 \mathrm{kPa}$ (35 to $70 \mathrm{psig}$ ) applied pressure conditions.

The permeate $\mathrm{pH}$ ranged from 5.84 to 7.60 and averaged 6.33. The concentrate $\mathrm{pH}$ ranged from 5.89 to 7.54 and averaged 6.42 . The permeate and concentrate $\mathrm{pH}$ were approximately equal and followed the same general decreasing trend with time. Permeate conductivity ranged from 282 to $540 \mu \mathrm{S} \cdot \mathrm{cm}^{-1}$ and averaged $395 \mu \mathrm{S} \cdot \mathrm{cm}^{-1}$. Concentrate conductivity ranged from 346 to $583 \mu \mathrm{S} \cdot \mathrm{cm}^{-1}$ and averaged $527 \mu \mathrm{S} \cdot \mathrm{cm}^{-1}$. A net decrease in permeate conductivity and a net increase in concentrate conductivity were observed in this experiment. Permeate conductivity was greater than concentrate conductivity through $\sim 1.75 \mathrm{hr}$. Concentrate conductivity then exceeded the permeate conductivity from $\sim 1.75 \mathrm{hr}$. through the end of the experiment. Distinct step-wise changes in permeate and concentrate $\mathrm{pH}$ and conductivity were not observed as applied pressure was increased.

\subsubsection{0 $12 \mathrm{~m} \cdot \mathrm{s}^{-1}-1.2 \mathrm{cSt}(1750 \mathrm{rpm}-20 \%$ MW Fluid)}

Permeate flux versus time for the $12 \mathrm{~m} \cdot \mathrm{s}^{-1}-1.2 \mathrm{cSt}$ experiment is presented in Figure 4.68. Permeate flux was stable at each applied pressure condition. The system 


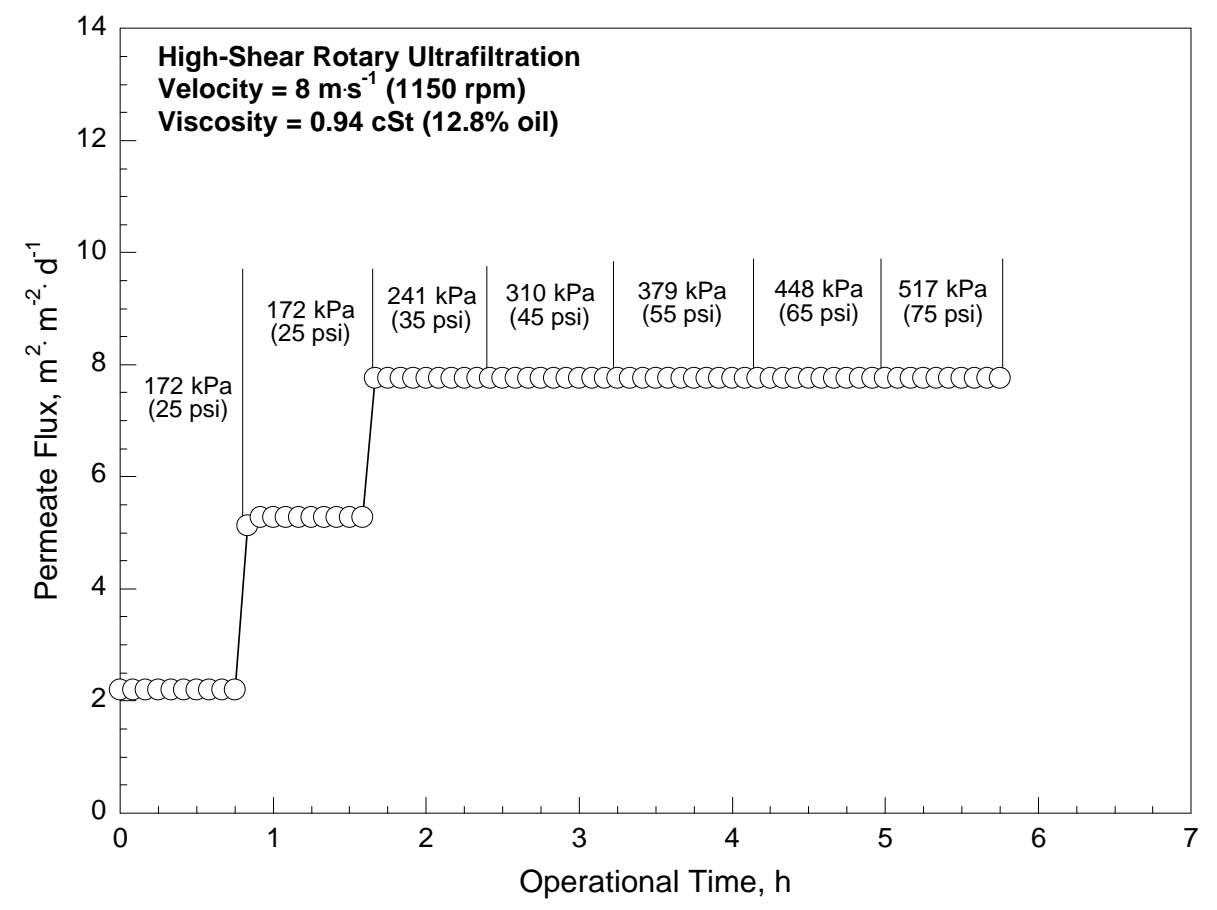

Figure 4.66 Permeate flux versus operational time for the $8 \mathrm{~m} \cdot \mathrm{s}^{-1}-0.94 \mathrm{cSt}$ experiment.

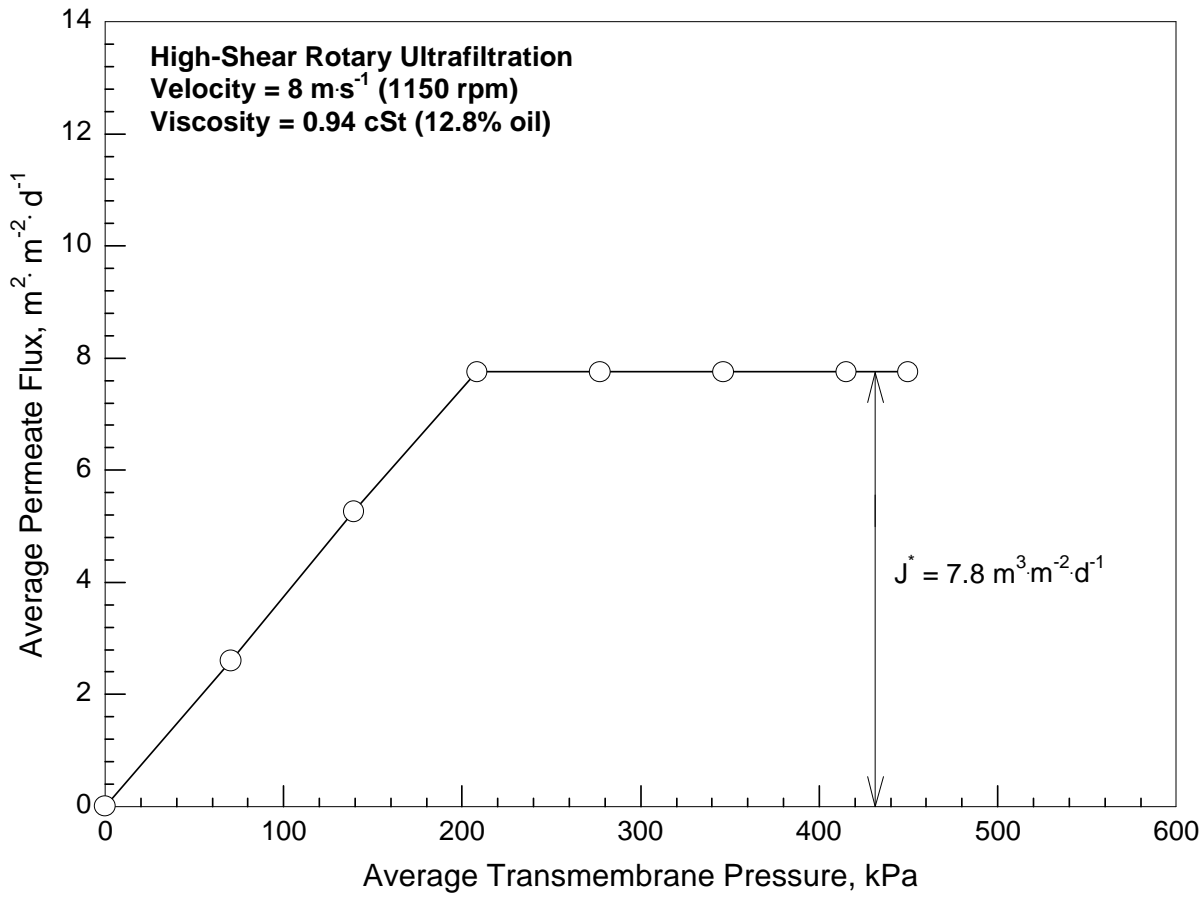

Figure 4.67 Average steady state permeate flux versus average transmembrane pressure for the $8 \mathrm{~m} \cdot \mathrm{s}^{-1}-0.94 \mathrm{cSt}$ experiment. 


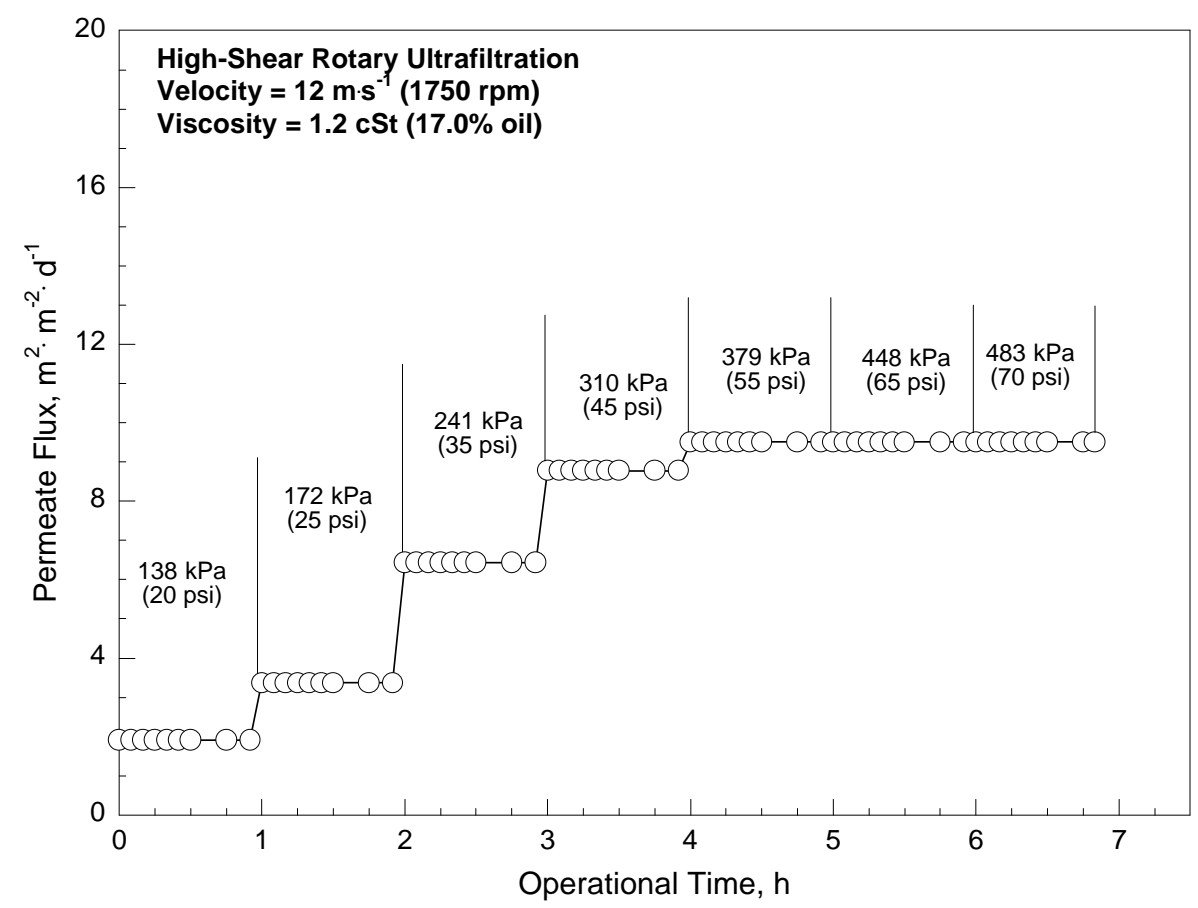

\section{Figure 4.68 Permeate flux versus operational time for the $12 \mathrm{~m} \cdot \mathrm{s}^{-1}-1.2 \mathrm{cSt}$ experiment.}

was operated over an applied pressure range of 138 to $483 \mathrm{kPa}$ (20 to $70 \mathrm{psig}$ ). The average permeate backpressure was $76.6 \mathrm{kPa}(11.1 \mathrm{psi})$ at an average relative velocity of $12 \mathrm{~m} \cdot \mathrm{s}^{-1}(1750 \mathrm{rpm})$. The average permeate flux versus average transmembrane pressure for the $12 \mathrm{~m} \cdot \mathrm{s}^{-1}-1.2 \mathrm{cSt}$ experiment is presented in Figure 4.69. A limiting flux of 9.5 $\mathrm{m}^{3} \cdot \mathrm{m}^{-2} \cdot \mathrm{d}^{-1}\left(233 \mathrm{gal} \cdot \mathrm{ft}^{-2} \cdot \mathrm{d}^{-1}\right)$ was observed at the 379 to $483 \mathrm{kPa}$ (55 to $\left.70 \mathrm{psig}\right)$ applied pressure conditions.

The permeate $\mathrm{pH}$ ranged from 7.53 to 8.17 and averaged 7.76 . The concentrate $\mathrm{pH}$ ranged from 7.63 to 7.75 and averaged 7.69. A decrease in permeate $\mathrm{pH}$ from 8.17 to 7.80 was observed over the first $1 \mathrm{hr}$. Permeate $\mathrm{pH}$ then remained constant at $\sim 7.75$ through the end of the experiment. Concentrate $\mathrm{pH}$ was constant at $\sim 7.75$. The permeate conductivity ranged from 431 to $726 \mu \mathrm{S} \cdot \mathrm{cm}^{-1}$ and averaged $569 \mu \mathrm{S} \cdot \mathrm{cm}^{-1}$. The concentrate conductivity ranged from 481 to $708 \mu \mathrm{S} \cdot \mathrm{cm}^{-1}$ and averaged $634 \mu \mathrm{S} \cdot \mathrm{cm}^{-1}$. A net decrease 


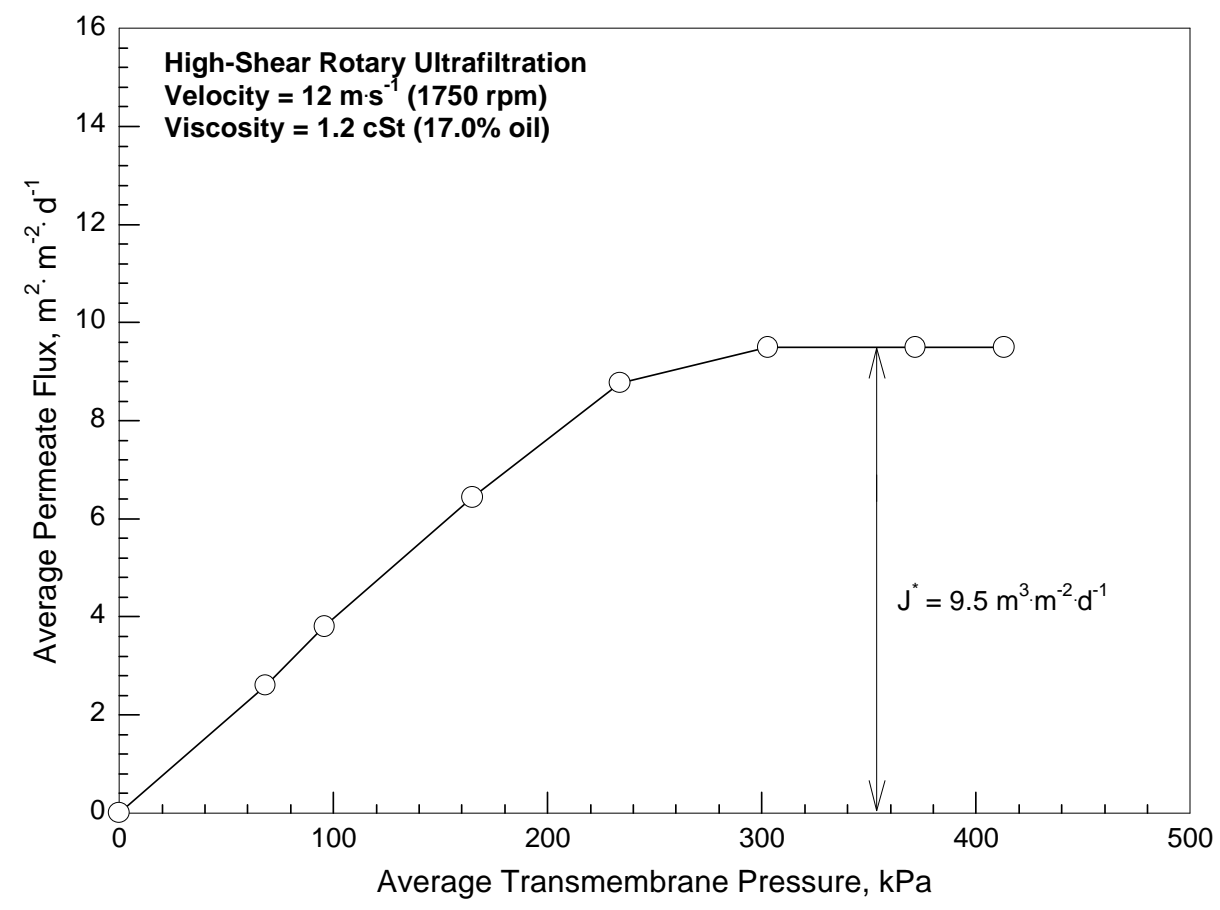

Figure 4.69 Average steady state permeate flux versus average transmembrane pressure for the $12 \mathrm{~m} \cdot \mathrm{s}^{-1}-1.2 \mathrm{cSt}$ experiment.

in permeate conductivity and a net increase in concentrate conductivity were observed. Permeate conductivity was greater than concentrate conductivity through $\sim 3 \mathrm{hr}$. The concentrate conductivity then exceeded the permeate conductivity through the end of the experiment. Distinct step-wise changes in permeate and concentrate $\mathrm{pH}$ and conductivity were not observed as applied pressure was increased.

\subsubsection{1 $10 \mathrm{~m} \cdot \mathrm{s}^{-1}-1.2 \mathrm{cSt}(1450 \mathrm{rpm}-20 \%$ MW Fluid)}

Permeate flux versus time for the $10 \mathrm{~m} \cdot \mathrm{s}^{-1}-1.2 \mathrm{cSt}$ experiment is presented in Figure 4.70. Permeate flux was stable at each applied pressure condition. The system was operated over an applied pressure range of 104 to $483 \mathrm{kPa}$ (15 to $70 \mathrm{psig}$ ). The average permeate backpressure was $52.4 \mathrm{kPa}(7.6 \mathrm{psi})$ at an average relative velocity of $10 \mathrm{~m} \cdot \mathrm{s}^{-1}(1450 \mathrm{rpm})$. The average permeate flux versus average transmembrane pressure for the $10 \mathrm{~m} \cdot \mathrm{s}^{-1}-1.2 \mathrm{cSt}$ experiment is presented in Figure 4.71. A limiting flux of 6.5 


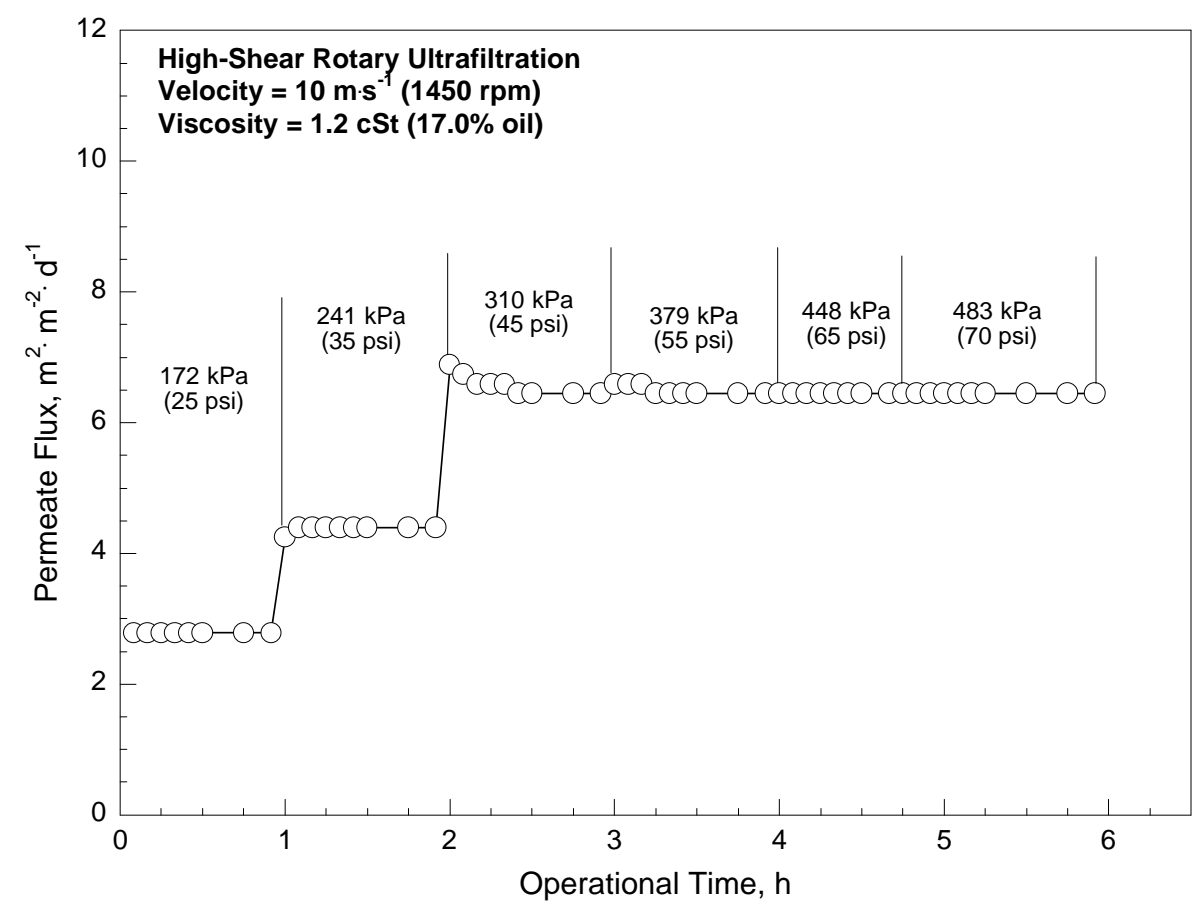

Figure 4.70 Permeate flux versus operational time for the $10 \mathrm{~m} \cdot \mathrm{s}^{-1}-1.2 \mathrm{cSt}$ experiment.

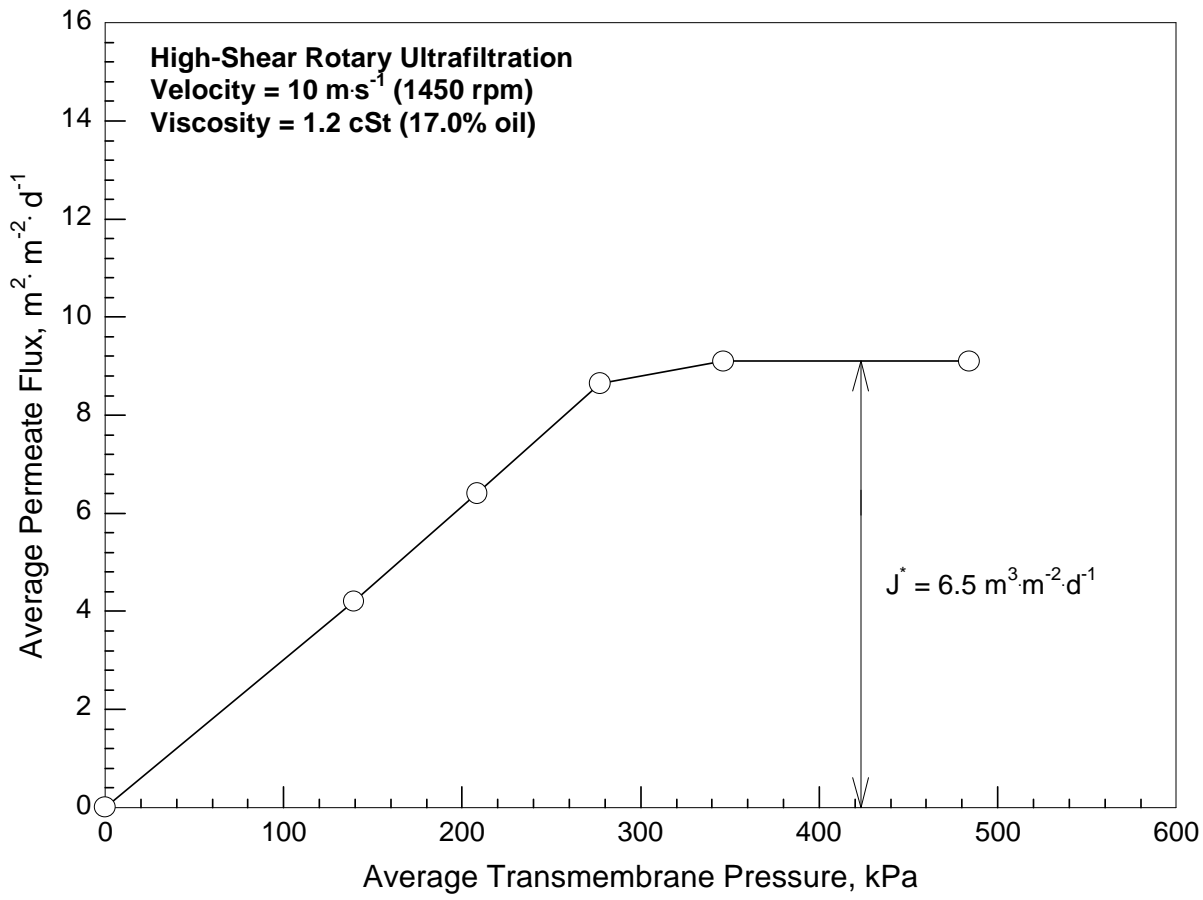

Figure 4.71 Average steady state permeate flux versus average transmembrane pressure for the $10 \mathrm{~m} \cdot \mathrm{s}^{-1}-1.2 \mathrm{cSt}$ experiment. 
$\mathrm{m}^{3} \cdot \mathrm{m}^{-2} \cdot \mathrm{d}^{-1}\left(159 \mathrm{gal} \cdot \mathrm{ft}^{-2} \cdot \mathrm{d}^{-1}\right)$ was observed at the 241 to $483 \mathrm{kPa}$ (35 to $\left.70 \mathrm{psig}\right)$ applied pressure conditions.

The permeate $\mathrm{pH}$ ranged from 7.68 to 8.24 and averaged 7.84 . The concentrate $\mathrm{pH}$ ranged from 7.41 to 7.79 and averaged 7.56. The permeate $\mathrm{pH}$ was greater than the concentrate $\mathrm{pH}$ throughout the entire experiment. A decrease in both permeate and concentrate $\mathrm{pH}$ was observed from 0 to $2 \mathrm{hr}$. The permeate and concentrate $\mathrm{pH}$ were then constant through the end of the experiment. The permeate conductivity ranged from 506 to $992 \mu \mathrm{S} \cdot \mathrm{cm}^{-1}$ and averaged $792 \mu \mathrm{S} \cdot \mathrm{cm}^{-1}$. The concentrate conductivity ranged from 616 to $939 \mu \mathrm{S} \cdot \mathrm{cm}^{-1}$ and averaged $805 \mu \mathrm{S} \cdot \mathrm{cm}^{-1}$. A net decrease in permeate conductivity and a net increase in concentrate conductivity was observed in this experiment. Permeate conductivity was greater than concentrate conductivity through $\sim 3.5 \mathrm{hr}$. Concentrate conductivity then exceeded permeate conductivity from $\sim 3.5 \mathrm{hr}$. through the end of the experiment. Distinct step-wise changes in permeate and concentrate $\mathrm{pH}$ and conductivity were not observed as applied pressure was increased.

\subsubsection{2 $8 \mathrm{~m} \cdot \mathrm{s}^{-1}-1.2 \mathrm{cSt}(1150 \mathrm{rpm}-20 \% \mathrm{MW}$ Fluid)}

Permeate flux versus time for the $8 \mathrm{~m} \cdot \mathrm{s}^{-1}-1.2 \mathrm{cSt}$ experiment is presented in Figure 4.72. Permeate flux was stable at each applied pressure condition. The system was operated over an applied pressure range of 104 to $483 \mathrm{kPa}$ (15 to $70 \mathrm{psig}$ ). The average permeate backpressure was $33.1 \mathrm{kPa}(4.8 \mathrm{psi})$ at an average relative velocity of 8 $\mathrm{m} \cdot \mathrm{s}^{-1}(1150 \mathrm{rpm})$. The average permeate flux versus average transmembrane pressure for the $8 \mathrm{~m} \cdot \mathrm{s}^{-1}-1.2 \mathrm{cSt}$ experiment is presented in Figure 4.73 . A limiting flux of $5.0 \mathrm{~m}^{3} \cdot \mathrm{m}^{-}$ ${ }^{2} \cdot \mathrm{d}^{-1}\left(122 \mathrm{gal} \cdot \mathrm{ft}^{-2} \cdot \mathrm{d}^{-1}\right)$ was observed at the 172 to $483 \mathrm{kPa}$ (25 to $70 \mathrm{psig}$ ) applied pressure conditions. 


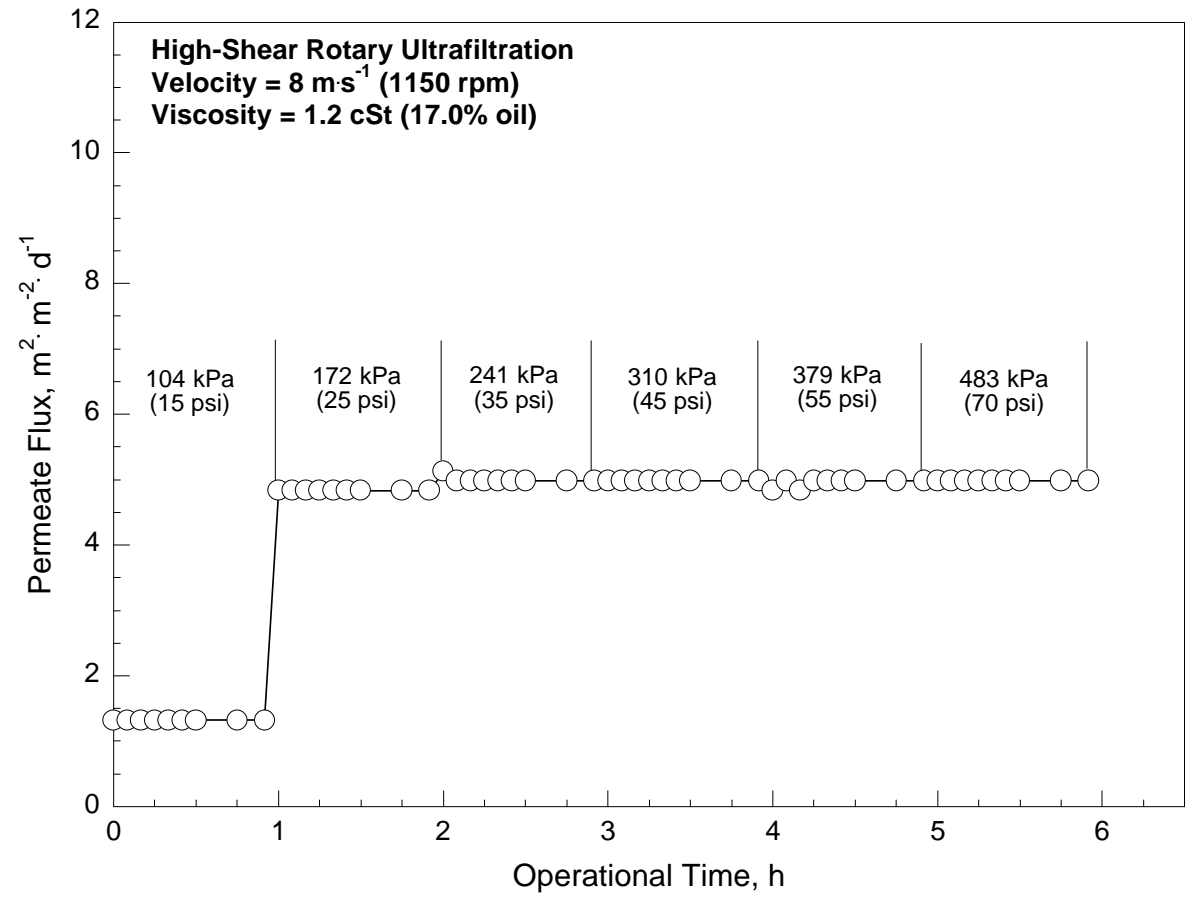

Figure 4.72 Permeate flux versus operational time for the $8 \mathrm{~m} \cdot \mathrm{s}^{-1}-1.2 \mathrm{cSt}$ experiment.

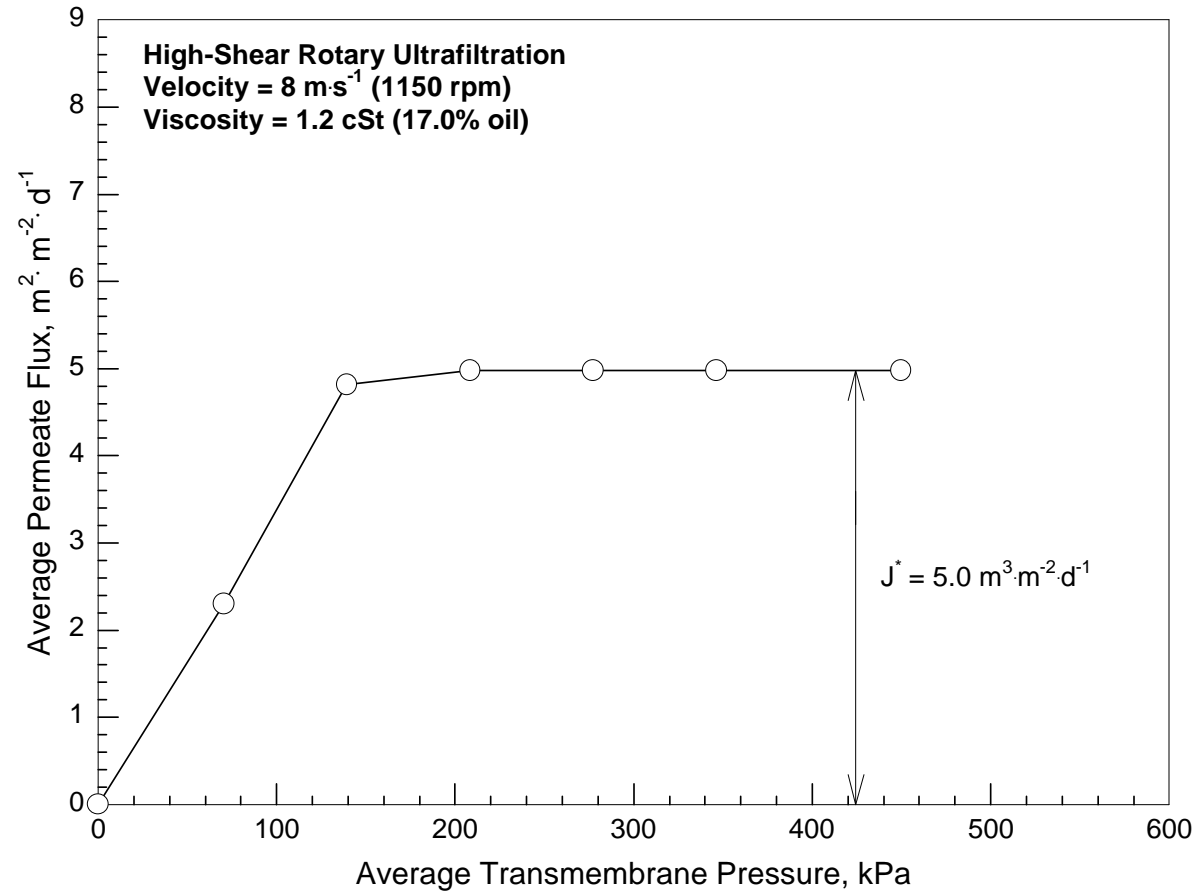

Figure 4.73 Average steady state permeate flux versus average transmembrane pressure for the $8 \mathrm{~m} \cdot \mathrm{s}^{-1}-1.2 \mathrm{cSt}$ experiment. 
The permeate $\mathrm{pH}$ ranged from 7.10 to 7.97 and averaged 7.36. The concentrate $\mathrm{pH}$ ranged from 6.67 to 7.55 and averaged 7.04 . The permeate $\mathrm{pH}$ was generally greater than the concentrate conductivity in this experiment. At $\sim 6 \mathrm{hr}$. concentrate $\mathrm{pH}$ exceeded permeate $\mathrm{pH}$. A net decrease in both permeate and concentrate $\mathrm{pH}$ was observed. The permeate conductivity ranged from 409 to $702 \mu \mathrm{S} \cdot \mathrm{cm}^{-1}$ and averaged $527 \mu \mathrm{S} \cdot \mathrm{cm}^{-1}$. The concentrate conductivity ranged from 390 to $706 \mu \mathrm{S} \cdot \mathrm{cm}^{-1}$ and averaged $600 \mu \mathrm{S} \cdot \mathrm{cm}^{-1}$. A net decrease in permeate conductivity and a net increase in concentrate conductivity were observed in this experiment. Permeate conductivity was greater than concentrate conductivity through $\sim 2 \mathrm{hr}$. The concentrate conductivity then exceeded the permeate conductivity from $\sim 2 \mathrm{hr}$. through the end of the experiment. Distinct step-wise changes in permeate and concentrate $\mathrm{pH}$ and conductivity were not observed as applied pressure was increased.

\subsubsection{3 $12 \mathrm{~m} \cdot \mathrm{s}^{-1}-4.5 \mathrm{cSt}(1750 \mathrm{rpm}-30 \%$ MW Fluid)}

Permeate flux versus time for the $12 \mathrm{~m} \cdot \mathrm{s}^{-1}-4.5 \mathrm{cSt}$ experiment is presented in Figure 4.74. Permeate flux was stable at each applied pressure condition. The system was operated over an applied pressure range of 172 to $483 \mathrm{kPa}$ ( 25 to $70 \mathrm{psig}$ ). The average permeate backpressure was $76.6 \mathrm{kPa}(11.1 \mathrm{psi})$ at an average relative velocity of $12 \mathrm{~m} \cdot \mathrm{s}^{-1}(1750 \mathrm{rpm})$. The average permeate flux versus average transmembrane pressure

for the $12 \mathrm{~m} \cdot \mathrm{s}^{-1}-4.5 \mathrm{cSt}$ experiment is presented in Figure 4.75. A limiting flux of 4.0 $\mathrm{m}^{3} \cdot \mathrm{m}^{-2} \cdot \mathrm{d}^{-1}\left(97 \mathrm{gal} \cdot \mathrm{ft}^{-2} \cdot \mathrm{d}^{-1}\right)$ was observed at the 172 to $483 \mathrm{kPa}$ (25 to $70 \mathrm{psig}$ ) applied pressure conditions.

The permeate $\mathrm{pH}$ ranged from 6.20 to 7.41 and averaged 6.74. The concentrate $\mathrm{pH}$ ranged from 5.91 to 7.25 and averaged 6.57. Permeate $\mathrm{pH}$ was greater than the 


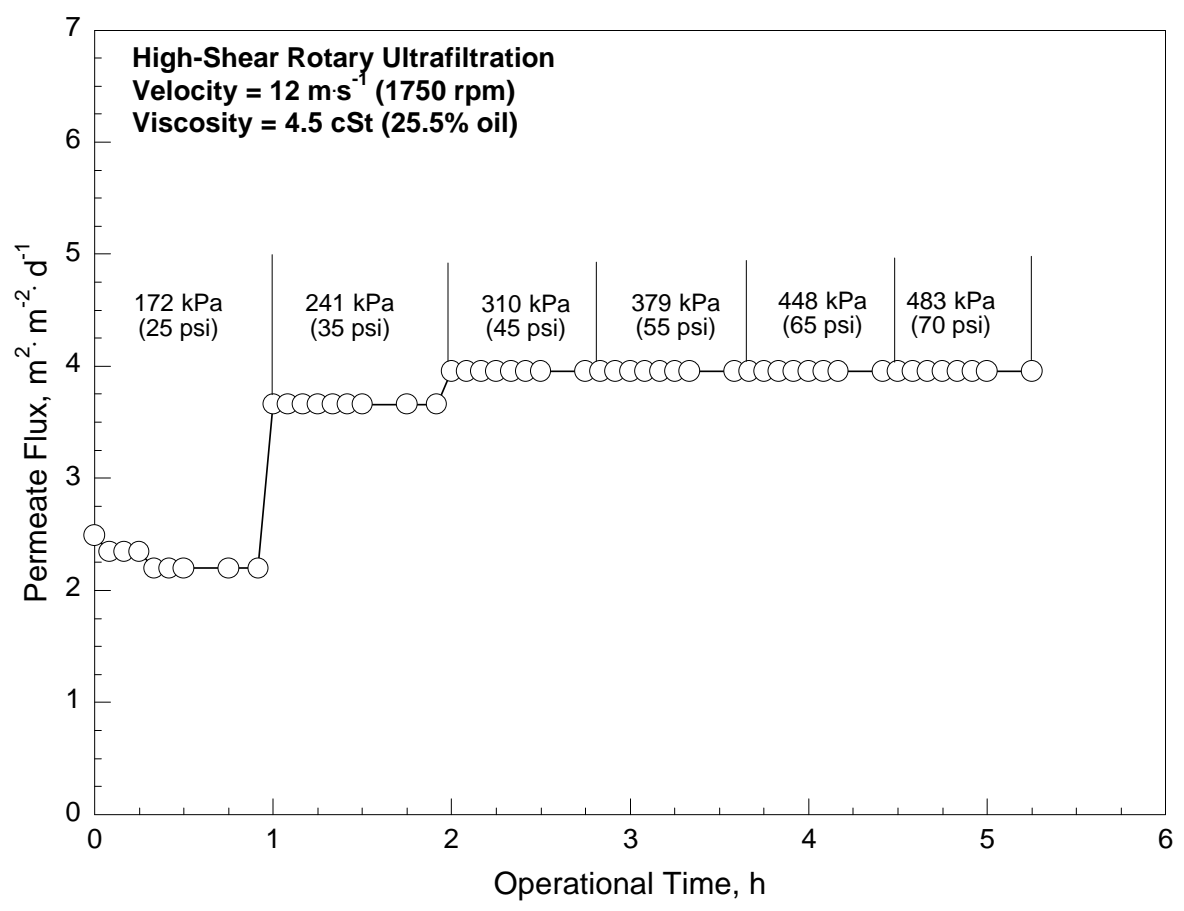

Figure 4.74 Permeate flux versus operational time for the $12 \mathrm{~m} \cdot \mathrm{s}^{-1}-4.5 \mathrm{cSt}$ experiment.

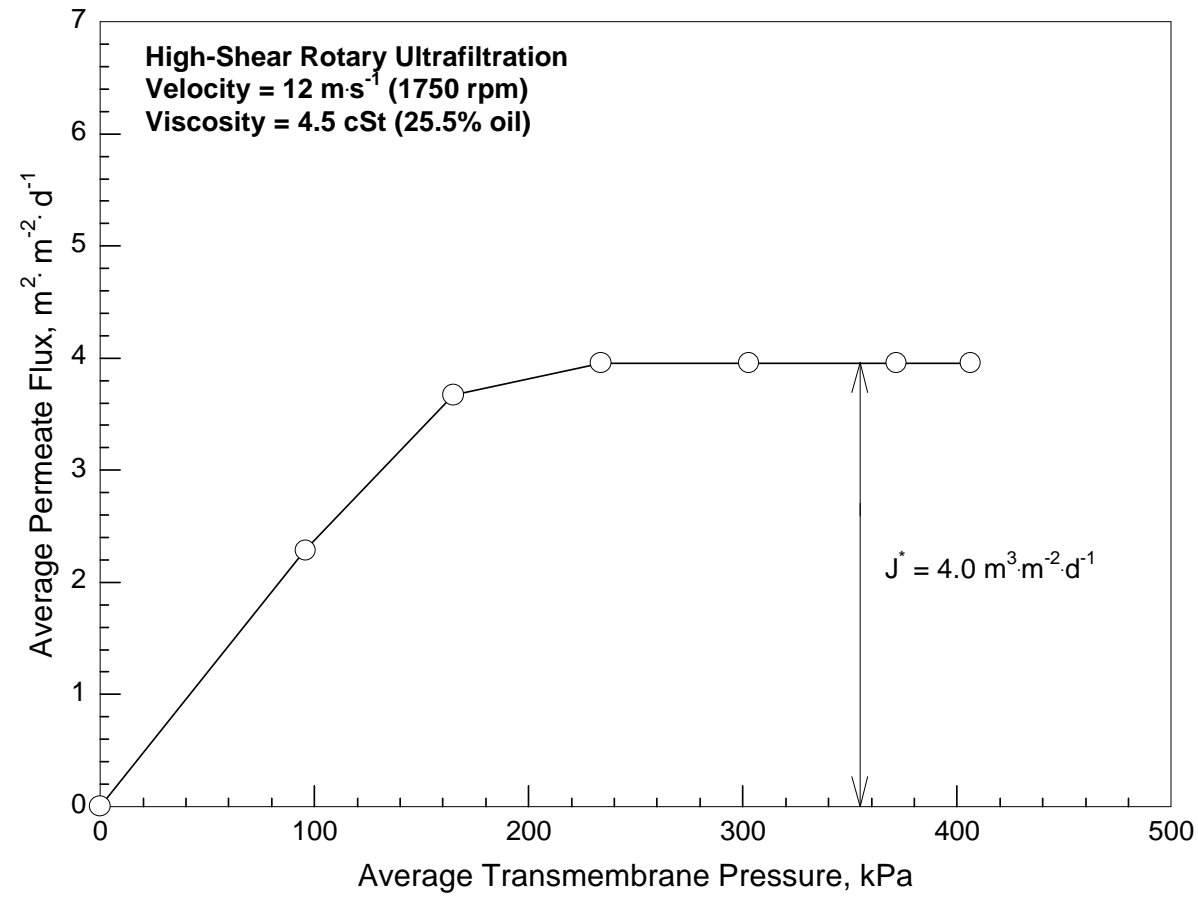

Figure 4.75 Average steady state permeate flux versus average transmembrane pressure for the $12 \mathrm{~m} \cdot \mathrm{s}^{-1}-4.5 \mathrm{cSt}$ experiment. 
concentrate $\mathrm{pH}$ throughout the $30 \% \mathrm{MW}$ Fluid-1750 rpm experiment. Both permeate and concentrate $\mathrm{pH}$ followed the same general trend in this experiment: $\mathrm{pH}$ decreased through $1 \mathrm{hr}$. then increased from $1 \mathrm{hr}$. through the end of the experiment. Additionally, permeate and concentrate $\mathrm{pH}$ values were approximately equal from $2.5 \mathrm{hr}$. through the end of the experiment. The permeate conductivity ranged from 868 to $1164 \mu \mathrm{S} \cdot \mathrm{cm}^{-1}$ and averaged $965 \mu \mathrm{S} \cdot \mathrm{cm}^{-1}$. The concentrate conductivity ranged from 880 to $976 \mu \mathrm{S} \cdot \mathrm{cm}^{-1}$ and averaged $936 \mu \mathrm{S} \cdot \mathrm{cm}^{-1}$. A net decrease in permeate conductivity was observed; however, concentrate conductivity was constant throughout the entire experiment. Permeate conductivity was greater than concentrate conductivity through $\sim 2.75 \mathrm{hr}$. The concentrate conductivity then exceeded the permeate conductivity from $\sim 2.75 \mathrm{hr}$. through the end of the experiment. Distinct step-wise changes in permeate and concentrate $\mathrm{pH}$ and conductivity were not observed as applied pressure was increased.

\subsubsection{4 $10 \mathrm{~m} \cdot \mathrm{s}^{-1}-4.5 \mathrm{cSt}(1450 \mathrm{rpm}-30 \%$ MW Fluid)}

Permeate flux versus time for the $10 \mathrm{~m} \cdot \mathrm{s}^{-1}-4.5 \mathrm{cSt}$ experiment is presented in Figure 4.76. The system was operated over an applied pressure range of 172 to $483 \mathrm{kPa}$ ( 25 to $70 \mathrm{psig}$ ). The average permeate backpressure was $52.4 \mathrm{kPa}(7.6 \mathrm{psi})$ at an average relative velocity of $10 \mathrm{~m} \cdot \mathrm{s}^{-1}(1450 \mathrm{rpm})$. At the $241 \mathrm{kPa}$ (35 psig) applied pressure condition, permeate flux decreased from an initial maximum value of 4.08 to $3.6 \mathrm{~m}^{3} \cdot \mathrm{m}^{-}$ ${ }^{2} \cdot \mathrm{d}^{-1}\left(100\right.$ to $\left.88 \mathrm{gal} \cdot \mathrm{ft}^{-2} \cdot \mathrm{d}^{-1}\right)$. This phenomenon was attributed to a delay in stabilization of the concentration boundary layer (Viadero and Reed 1999). Permeate flux was stable at each other applied pressure condition. The average permeate flux versus average transmembrane pressure for the $10 \mathrm{~m} \cdot \mathrm{s}^{-1}-4.5 \mathrm{cSt}$ experiment is presented in Figure 4.77. 


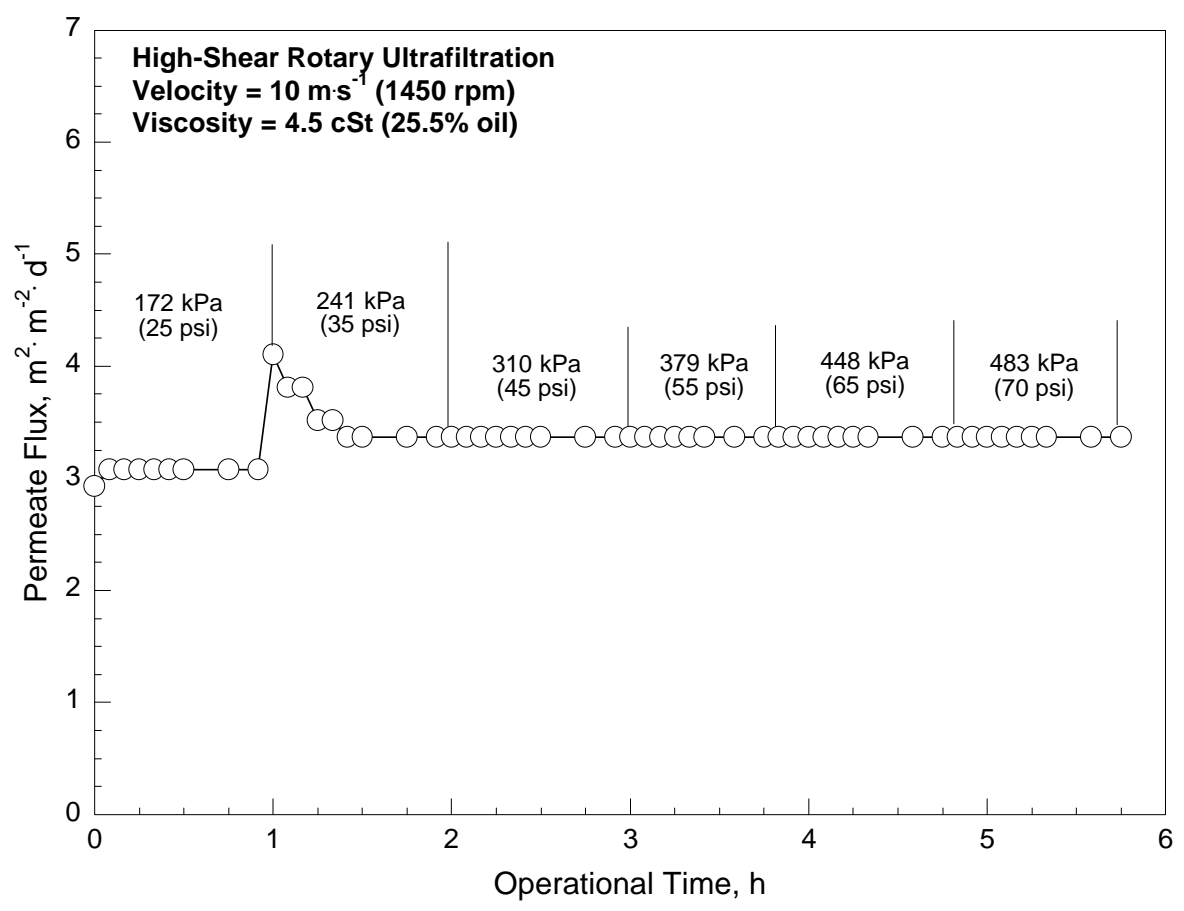

Figure 4.76 Permeate flux versus operational time for the $10 \mathrm{~m} \cdot \mathrm{s}^{-1}-4.5 \mathrm{cSt}$ experiment.

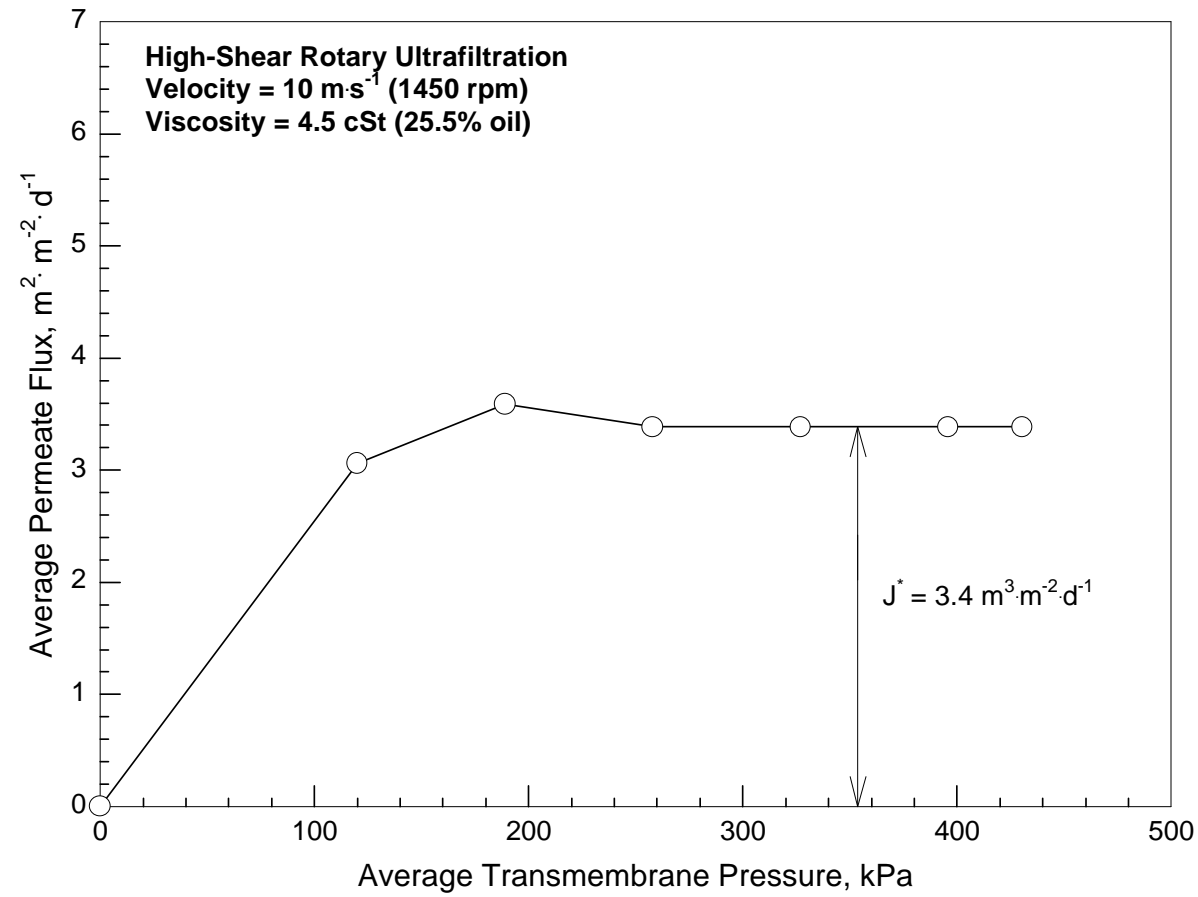

Figure 4.77 Average steady state permeate flux versus average transmembrane pressure for the $10 \mathrm{~m} \cdot \mathrm{s}^{-1}-4.5 \mathrm{cSt}$ experiment. 
A limiting flux of $3.4 \mathrm{~m}^{3} \cdot \mathrm{m}^{-2} \cdot \mathrm{d}^{-1}\left(83 \mathrm{gal} \cdot \mathrm{ft}^{-2} \cdot \mathrm{d}^{-1}\right)$ was observed at the 241 to $483 \mathrm{kPa}$ (35 to 70 psig) applied pressure conditions.

The permeate $\mathrm{pH}$ ranged from 6.11 to 7.80 and averaged 6.48. The concentrate $\mathrm{pH}$ ranged from 5.91 to 7.15 and averaged 6.30. The $\mathrm{pH}$ probe broke at $\sim 4 \mathrm{hr}$. Consequently, $\mathrm{pH}$ measurements were not made from $\sim 4 \mathrm{hr}$. through the end of this experiment. Permeate $\mathrm{pH}$ was greater than the concentrate $\mathrm{pH}$ from 0 to $\sim 4 \mathrm{hr}$. Permeate and concentrate $\mathrm{pH}$ decreased through $\sim 1 \mathrm{hr}$. then were both constant at $\sim 6.20$ through $\sim 4$ hr. The permeate conductivity ranged from 815 to $1001 \mu \mathrm{S} \cdot \mathrm{cm}^{-1}$ and averaged 945 $\mu \mathrm{S} \cdot \mathrm{cm}^{-1}$. The concentrate conductivity ranged from 833 to $986 \mu \mathrm{S} \cdot \mathrm{cm}^{-1}$ and averaged 891 $\mu \mathrm{S} \cdot \mathrm{cm}^{-1}$. A net decrease in permeate conductivity and a net increase in concentrate conductivity were observed throughout the experiment. Permeate conductivity was greater than concentrate conductivity through $\sim 4.25 \mathrm{hr}$. The concentrate conductivity then exceeded the permeate conductivity from $\sim 4.25 \mathrm{hr}$. through the end of the $10 \mathrm{~m} \cdot \mathrm{s}^{-1}$ $4.47 \mathrm{cSt}$ experiment. Distinct step-wise changes in permeate and concentrate $\mathrm{pH}$ and conductivity were not observed as applied pressure was increased.

\subsubsection{5 $8 \mathrm{~m} \cdot \mathrm{s}^{-1}-4.5 \mathrm{cSt}(1150 \mathrm{rpm}-30 \%$ MW Fluid)}

Permeate flux versus time for the $8 \mathrm{~m} \cdot \mathrm{s}^{-1}-4.5 \mathrm{cSt}$ experiment is presented in Figure 4.78. Permeate flux was stable at each applied pressure condition. The system was operated over an increasing applied pressure range of 104 to $483 \mathrm{kPa}$ (15 to $70 \mathrm{psig}$ ). The average permeate backpressure was $33.1 \mathrm{kPa}(4.8 \mathrm{psi})$ at an average relative velocity of $8 \mathrm{~m} \cdot \mathrm{s}^{-1}(1150 \mathrm{rpm})$. The permeate flux at each applied pressure was constant. The average permeate flux versus average transmembrane pressure for the $8 \mathrm{~m} \cdot \mathrm{s}^{-1}-4.5 \mathrm{cSt}$ experiment is presented in Figure 4.79. A limiting flux of $2.3 \mathrm{~m}^{3} \cdot \mathrm{m}^{-2} \cdot \mathrm{d}^{-1}\left(57 \mathrm{gal} \cdot \mathrm{ft}^{-2} \cdot \mathrm{d}^{-1}\right)$ 


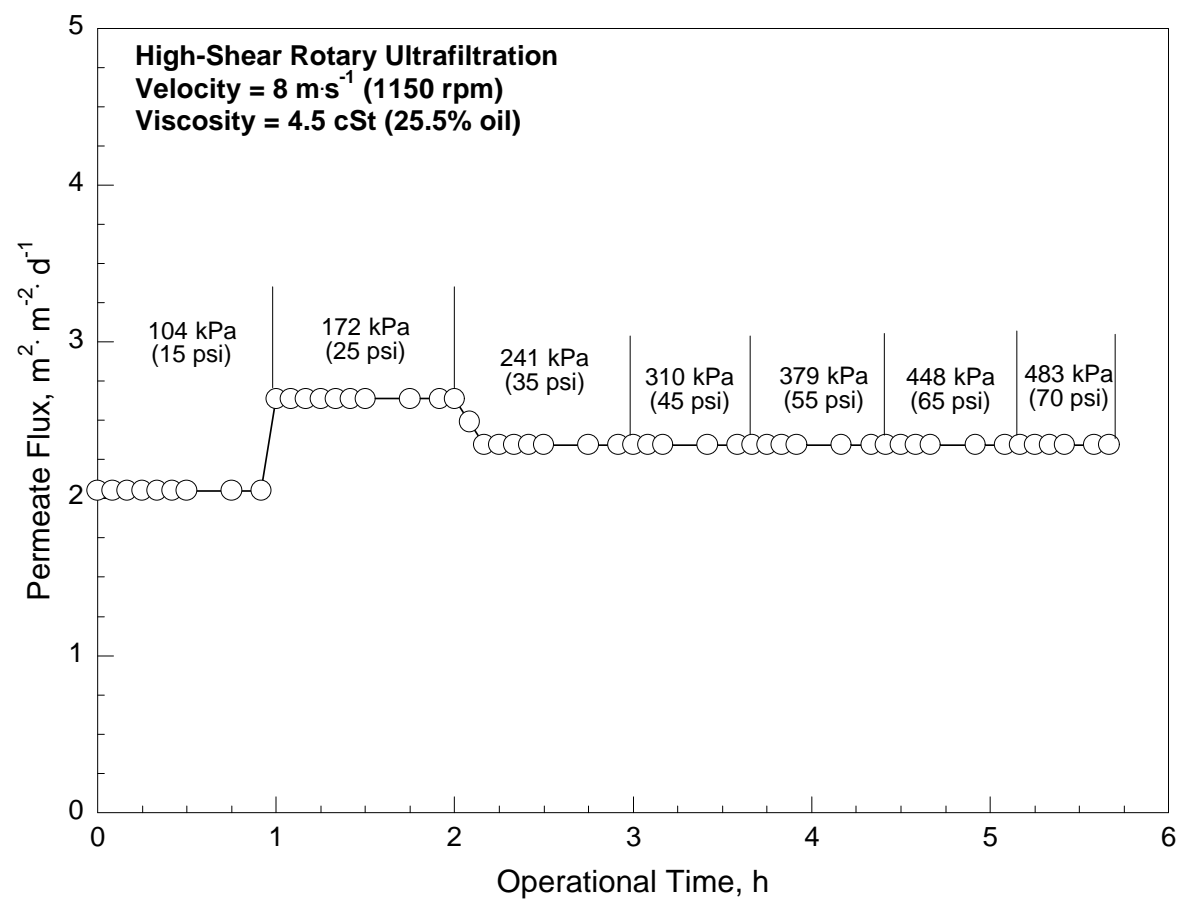

Figure 4.78 Permeate flux versus operational time for the $8 \mathrm{~m} \cdot \mathrm{s}^{-1}-4.5 \mathrm{cSt}$ experiment.

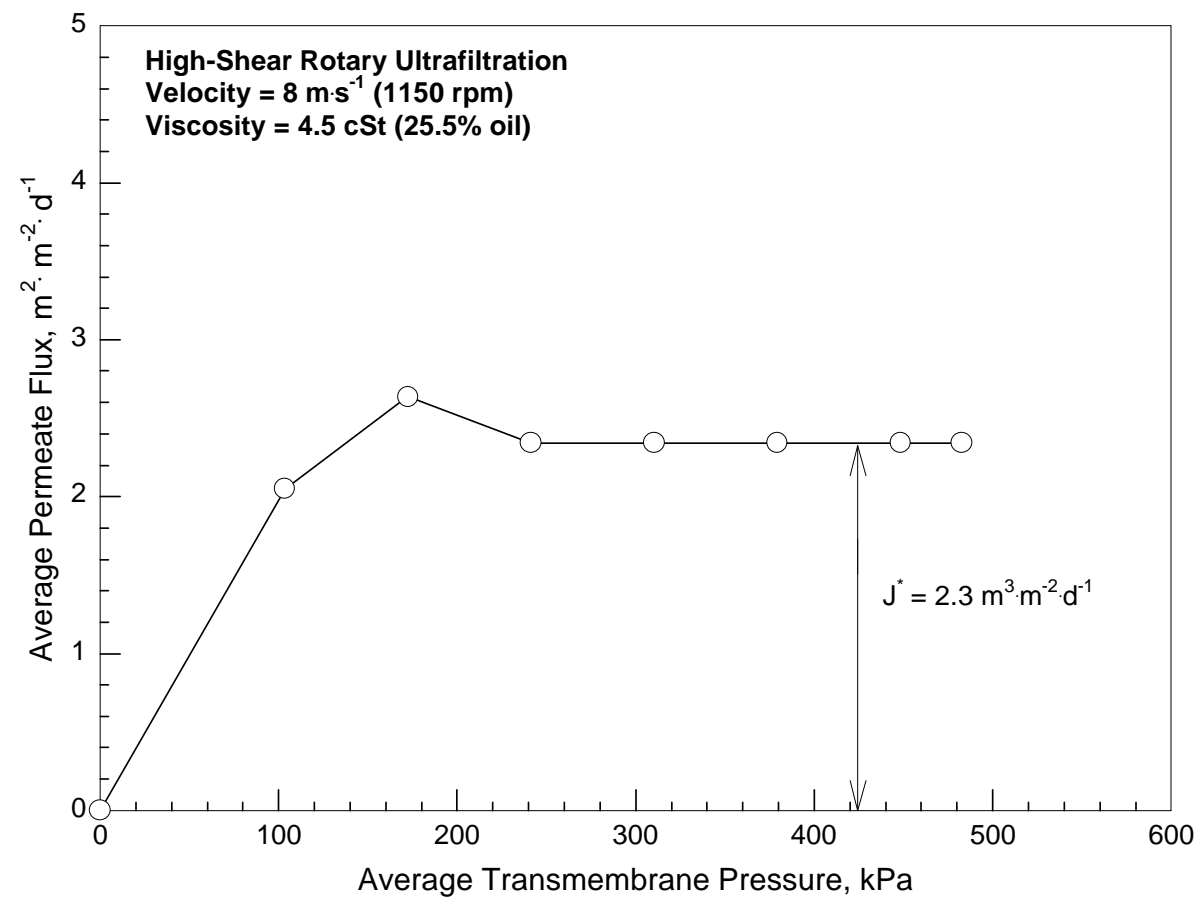

Figure 4.79 Average steady state permeate flux versus average transmembrane pressure for the $8 \mathrm{~m} \cdot \mathrm{s}^{-1}-4.5 \mathrm{cSt}$ experiment. 
was observed at the 310 to $483 \mathrm{kPa}$ (45 to $70 \mathrm{psig}$ ) applied pressure conditions. The average permeate flux at the $172 \mathrm{kPa}(25 \mathrm{psig})$ applied pressure was $2.7 \mathrm{~m}^{3} \cdot \mathrm{m}^{-2} \cdot \mathrm{d}^{-1}(65$ $\mathrm{gal} \cdot \mathrm{ft}^{-2} \cdot \mathrm{d}^{-1}$ ) which was greater than the limiting permeate flux of $57 \mathrm{gal} \cdot \mathrm{ft}^{-2} \cdot \mathrm{d}^{-1}$.

The permeate $\mathrm{pH}$ ranged from 7.64 to 7.85 and averaged 7.76. Permeate and concentrate $\mathrm{pH}$ were not measured through the first $3.5 \mathrm{hr}$. due to operational problems with the $\mathrm{pH}$ probe. A $\mathrm{pH}$ meter and probe were borrowed from another laboratory and $\mathrm{pH}$ was measured from $3.5 \mathrm{hr}$. thorough the end of the experiment. The concentrate $\mathrm{pH}$ ranged from 7.41 to 7.69 and averaged 7.51. Permeate $\mathrm{pH}$ was greater than the concentrate $\mathrm{pH}$ throughout the $8 \mathrm{~m} \cdot \mathrm{s}^{-1}-4.47 \mathrm{cSt}$ experiment. Both permeate and concentrate $\mathrm{pH}$ remained constant from 3.5 through the end of the experiment. The permeate conductivity ranged from 755 to $1101 \mu \mathrm{S} \cdot \mathrm{cm}^{-1}$ and averaged $863 \mu \mathrm{S} \cdot \mathrm{cm}^{-1}$. The concentrate conductivity ranged from 812 to $961 \mu \mathrm{S} \cdot \mathrm{cm}^{-1}$ and averaged $871 \mu \mathrm{S} \cdot \mathrm{cm}^{-1}$. A net decrease in permeate conductivity was observed. Concentrate conductivity was constant in this experiment. Permeate conductivity was greater than concentrate conductivity through $\sim 2.5$ to $3 \mathrm{hr}$. The concentrate conductivity then exceeded the permeate conductivity from $3 \mathrm{hr}$. through the end of the experiment. Distinct step-wise changes in permeate and concentrate $\mathrm{pH}$ and conductivity were not observed as applied pressure was increased.

\subsubsection{6 $12 \mathrm{~m} \cdot \mathrm{s}^{-1}-8.1 \mathrm{cSt}(1750 \mathrm{rpm}-40 \%$ MW Fluid)}

Permeate flux versus time for the $12 \mathrm{~m} \cdot \mathrm{s}^{-1}-8.1 \mathrm{cSt}$ experiment is presented in Figure 4.80. Permeate flux was stable at each applied pressure condition. The system was operated over an applied pressure range of 172 to $483 \mathrm{kPa}$ (25 to $70 \mathrm{psig}$ ). The average permeate backpressure was $76.6 \mathrm{kPa}(11.1 \mathrm{psi})$ at an average relative velocity of 


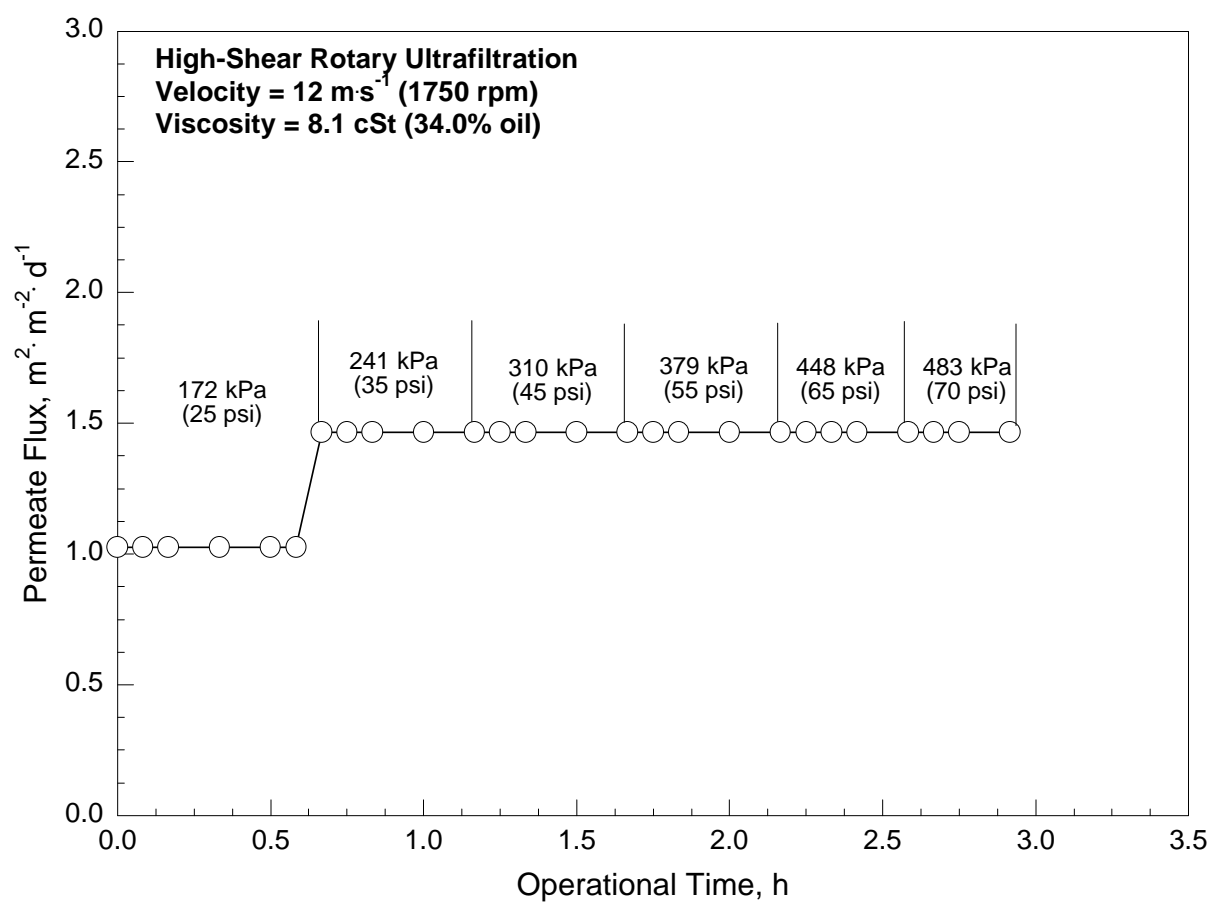

\section{Figure 4.80 Permeate flux versus operational time for the $12 \mathrm{~m} \cdot \mathrm{s}^{-1}-8.1 \mathrm{cSt}$ experiment.}

$12 \mathrm{~m} \cdot \mathrm{s}^{-1}(1750 \mathrm{rpm})$. The average permeate flux versus average transmembrane pressure for the $12 \mathrm{~m} \cdot \mathrm{s}^{-1}-8.1 \mathrm{cSt}$ experiment is presented in Figure 4.81. A limiting flux of 1.5 $\mathrm{m}^{3} \cdot \mathrm{m}^{-2} \cdot \mathrm{d}^{-1}\left(36 \mathrm{gal} \cdot \mathrm{ft}^{-2} \cdot \mathrm{d}^{-1}\right)$ was observed at the 241 to $483 \mathrm{kPa}$ (35 to $70 \mathrm{psig}$ ) applied pressure conditions.

The permeate $\mathrm{pH}$ ranged from 7.33 to 7.68 and averaged 7.45. The concentrate $\mathrm{pH}$ ranged from 7.17 to 7.39 and averaged 7.24. Permeate $\mathrm{pH}$ was greater than the concentrate $\mathrm{pH}$ throughout the $12 \mathrm{~m} \cdot \mathrm{s}^{-1}-8.12 \mathrm{cSt}$ experiment. Permeate and concentrate $\mathrm{pH}$ followed the same trend in this experiment: $\mathrm{pH}$ decreased through $0.75 \mathrm{hr}$., increased through $1.25 \mathrm{hr}$., decreased through $1.75 \mathrm{hr}$., and then remained constant. The permeate conductivity ranged from 1058 to $1340 \mu \mathrm{S} \cdot \mathrm{cm}^{-1}$ and averaged $1174 \mu \mathrm{S} \cdot \mathrm{cm}^{-1}$. The concentrate conductivity ranged from 1003 to $1267 \mu \mathrm{S} \cdot \mathrm{cm}^{-1}$ and averaged $1150 \mu \mathrm{S} \cdot \mathrm{cm}^{-1}$. A net decrease in permeate conductivity was observed. The concentrate conductivity was 


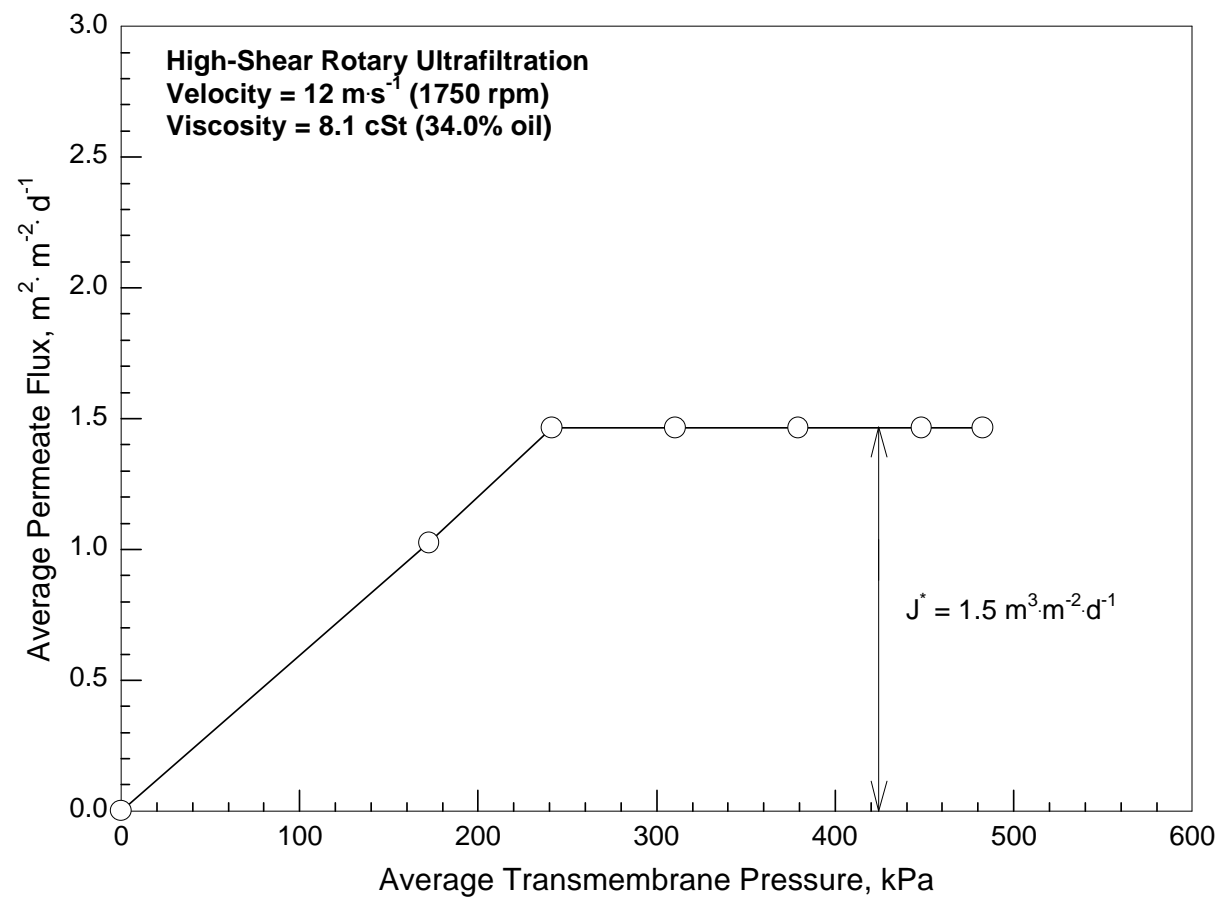

Figure 4.81 Average steady state permeate flux versus average transmembrane pressure for the $12 \mathrm{~m} \cdot \mathrm{s}^{-1}-8.1 \mathrm{cSt}$ experiment.

constant. Permeate conductivity was greater than concentrate conductivity through $\sim 1.5$ hr. The concentrate conductivity then generally exceeded the permeate conductivity from $\sim 1.5 \mathrm{hr}$. through the end of the experiment. Distinct step-wise changes in permeate and concentrate $\mathrm{pH}$ and conductivity were not observed as applied pressure was increased.

\subsubsection{7 $10 \mathrm{~m} \cdot \mathrm{s}^{-1}-8.1 \mathrm{cSt}(1450 \mathrm{rpm}-40 \%$ MW Fluid)}

Permeate flux versus time for the $10 \mathrm{~m} \cdot \mathrm{s}^{-1}-8.1 \mathrm{cSt}$ experiment is presented in Figure 4.82. Permeate flux was stable at each applied pressure condition. The system was operated over an applied pressure range of 138 to $483 \mathrm{kPa}$ (20 to $70 \mathrm{psig}$ ). The average permeate backpressure was $52.4 \mathrm{kPa}(7.6 \mathrm{psi})$ at an average relative velocity of $10 \mathrm{~m} \cdot \mathrm{s}^{-1}(1450 \mathrm{rpm})$. The average permeate flux versus average transmembrane pressure for $10 \mathrm{~m} \cdot \mathrm{s}^{-1}-8.1 \mathrm{cSt}$ experiment is presented in Figure 4.83. A limiting flux of $1.2 \mathrm{~m}^{3} \cdot \mathrm{m}$ 


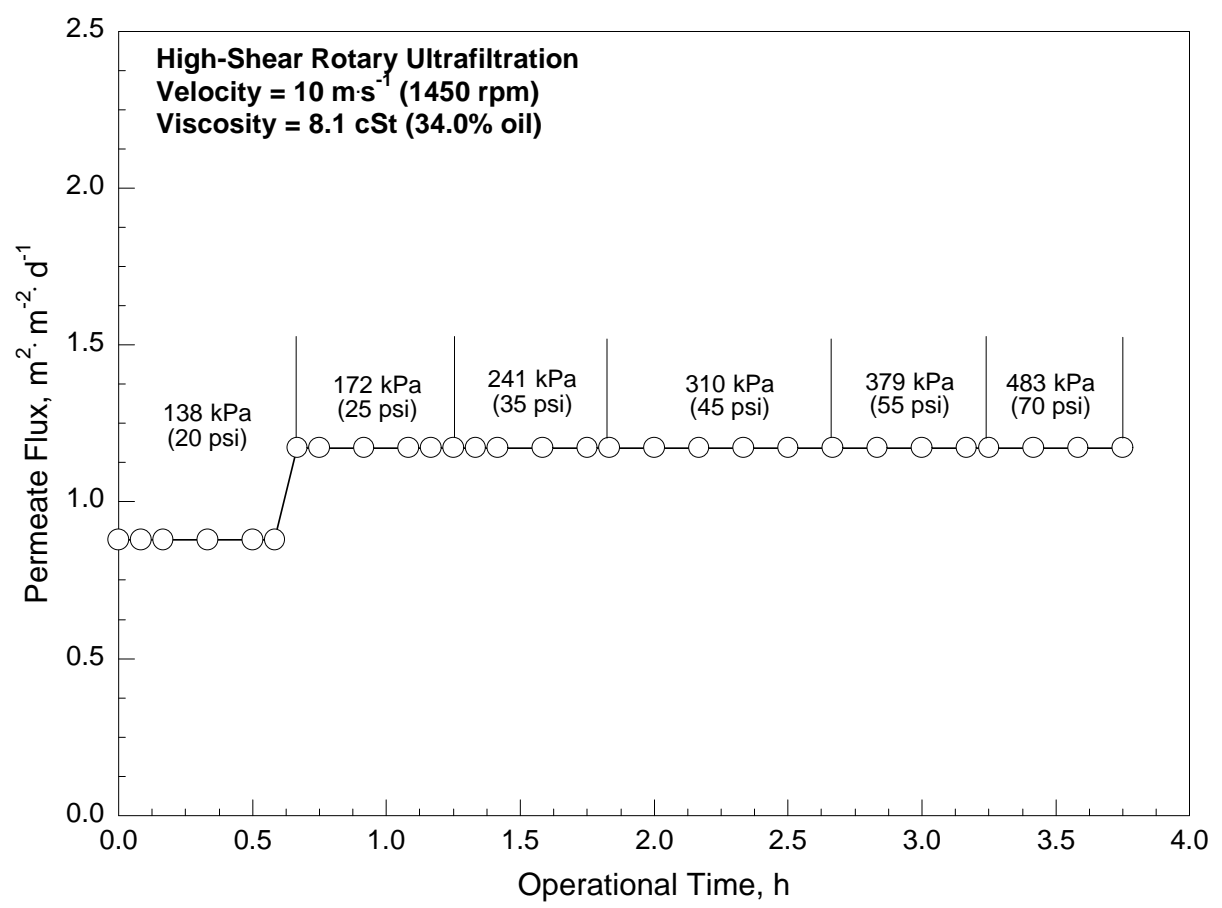

Figure 4.82 Permeate flux versus operational time for the $10 \mathrm{~m} \cdot \mathrm{s}^{-1}-8.1 \mathrm{cSt}$ experiment.

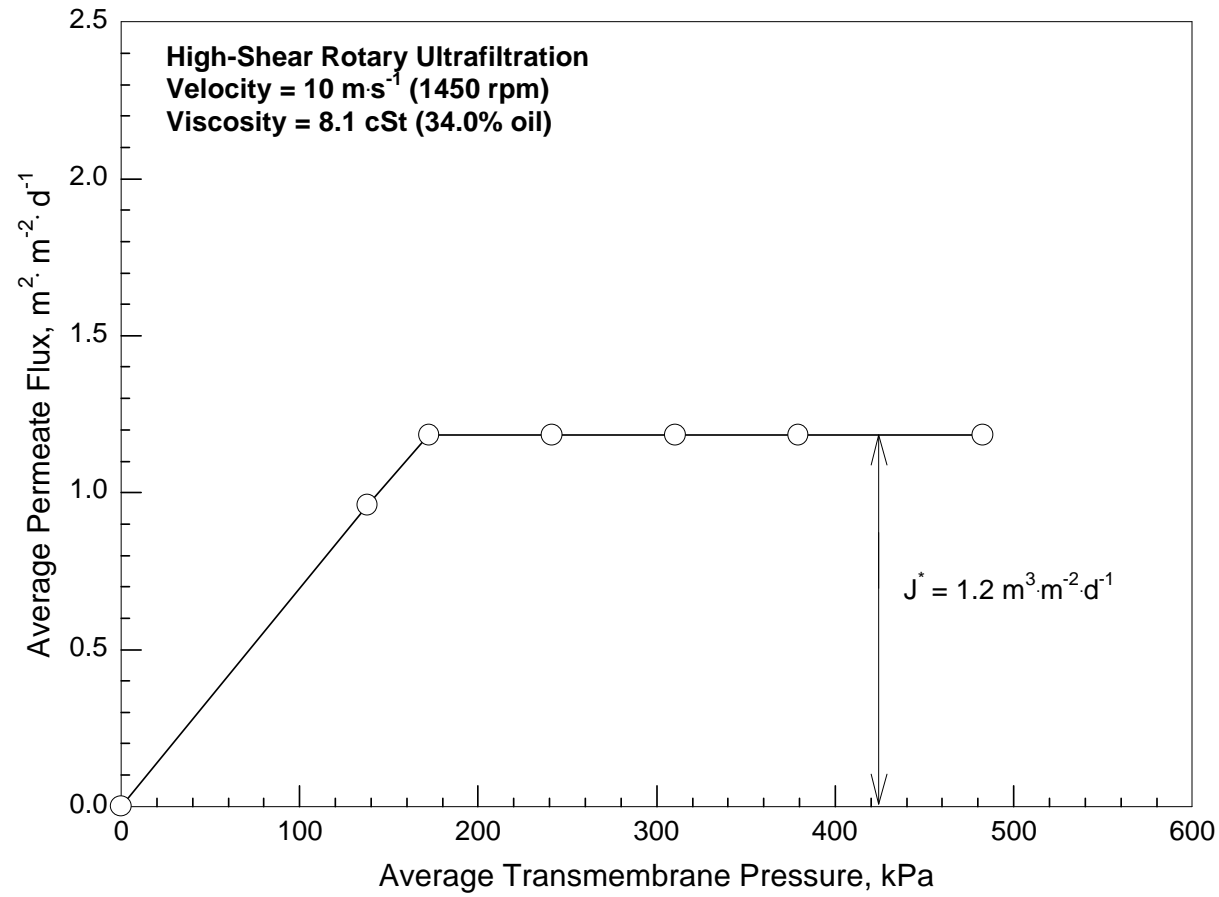

Figure 4.83 Average steady state permeate flux versus average transmembrane pressure for the $10 \mathrm{~m} \cdot \mathrm{s}^{-1}-8.1 \mathrm{cSt}$ experiment. 
${ }^{-2} \cdot \mathrm{d}^{-1}\left(29 \mathrm{gal} \cdot \mathrm{ft}^{-2} \cdot \mathrm{d}^{-1}\right)$ was observed at the 172 to $483 \mathrm{kPa}$ (25 to $70 \mathrm{psig}$ ) applied pressure conditions.

The permeate $\mathrm{pH}$ ranged from 6.74 to 7.10 and averaged 6.92. The concentrate $\mathrm{pH}$ ranged from 6.59 to 7.10 and averaged 6.94. Permeate and concentrate $\mathrm{pH}$ were not measured 0 to $\sim 1.75$ due to operational problems with the $\mathrm{pH}$ probe. $\mathrm{A} \mathrm{pH}$ meter and probe were borrowed from another laboratory and $\mathrm{pH}$ was measured from $\sim 1.75 \mathrm{hr}$. thorough the end of the experiment. Permeate and concentrate $\mathrm{pH}$ values were approximately equal and a net increase in both permeate and concentrate $\mathrm{pH}$ was observed. The permeate conductivity ranged from 1061 to $1333 \mu \mathrm{S} \cdot \mathrm{cm}^{-1}$ and averaged $1184 \mu \mathrm{S} \cdot \mathrm{cm}^{-1}$. The concentrate conductivity ranged from 1069 to $1243 \mu \mathrm{S} \cdot \mathrm{cm}^{-1}$ and averaged $1149 \mu \mathrm{S} \cdot \mathrm{cm}^{-1}$. A net decrease in both permeate and concentrate conductivity was observed throughout the $10 \mathrm{~m} \cdot \mathrm{s}^{-1}-8.12 \mathrm{cSt}$ experiment. Permeate conductivity was greater than concentrate conductivity through $\sim 1.5 \mathrm{hr}$. then both permeate and concentrate conductivities converged at a constant value of $\sim 1070 \mu \mathrm{S} \cdot \mathrm{cm}^{-1}$. Distinct stepwise changes in permeate and concentrate $\mathrm{pH}$ and conductivity were not observed as applied pressure was increased.

\subsubsection{8 $8 \mathrm{~m} \cdot \mathrm{s}^{-1}-8.1 \mathrm{cSt}(1150 \mathrm{rpm}-40 \%$ MW Fluid)}

Permeate flux versus time for the $8 \mathrm{~m} \cdot \mathrm{s}^{-1}-8.1 \mathrm{cSt}$ experiment is presented in Figure 4.84. Permeate flux was stable at each applied pressure condition. The system was operated over an applied pressure range of 104 to $483 \mathrm{kPa}$ (15 to $70 \mathrm{psig}$ ). The average permeate backpressure was $33.1 \mathrm{kPa}(4.8 \mathrm{psi})$ at an average relative velocity of 8 $\mathrm{m} \cdot \mathrm{s}^{-1}(1150 \mathrm{rpm})$. The average permeate flux versus average transmembrane pressure for the $8 \mathrm{~m} \cdot \mathrm{s}^{-1}-8.1 \mathrm{cSt}$ experiment is presented in Figure 4.85 . A limiting flux of $0.90 \mathrm{~m}^{3} \cdot \mathrm{m}$ 


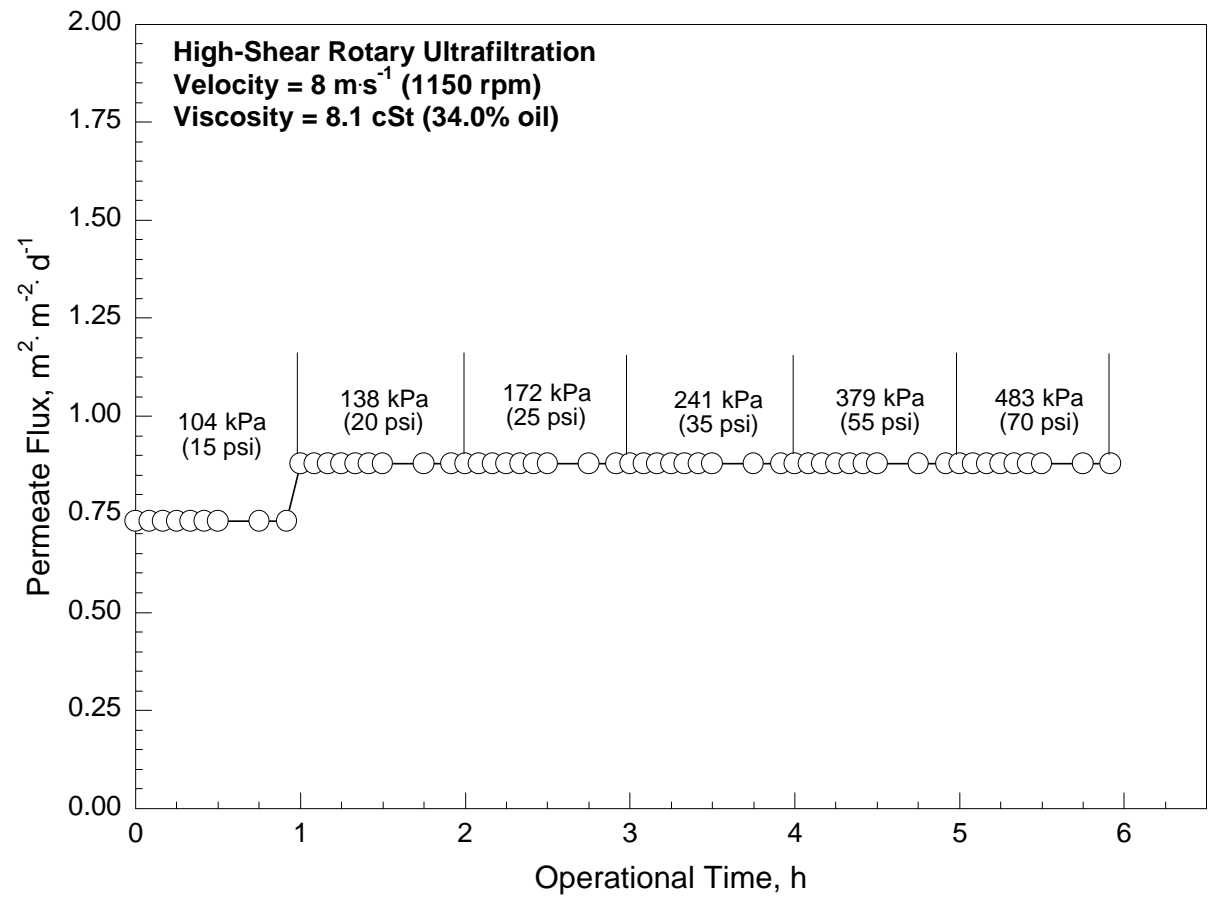

Figure 4.84 Permeate flux versus operational time for the $8 \mathrm{~m} \cdot \mathrm{s}^{-1}-8.1 \mathrm{cSt}$ experiment.

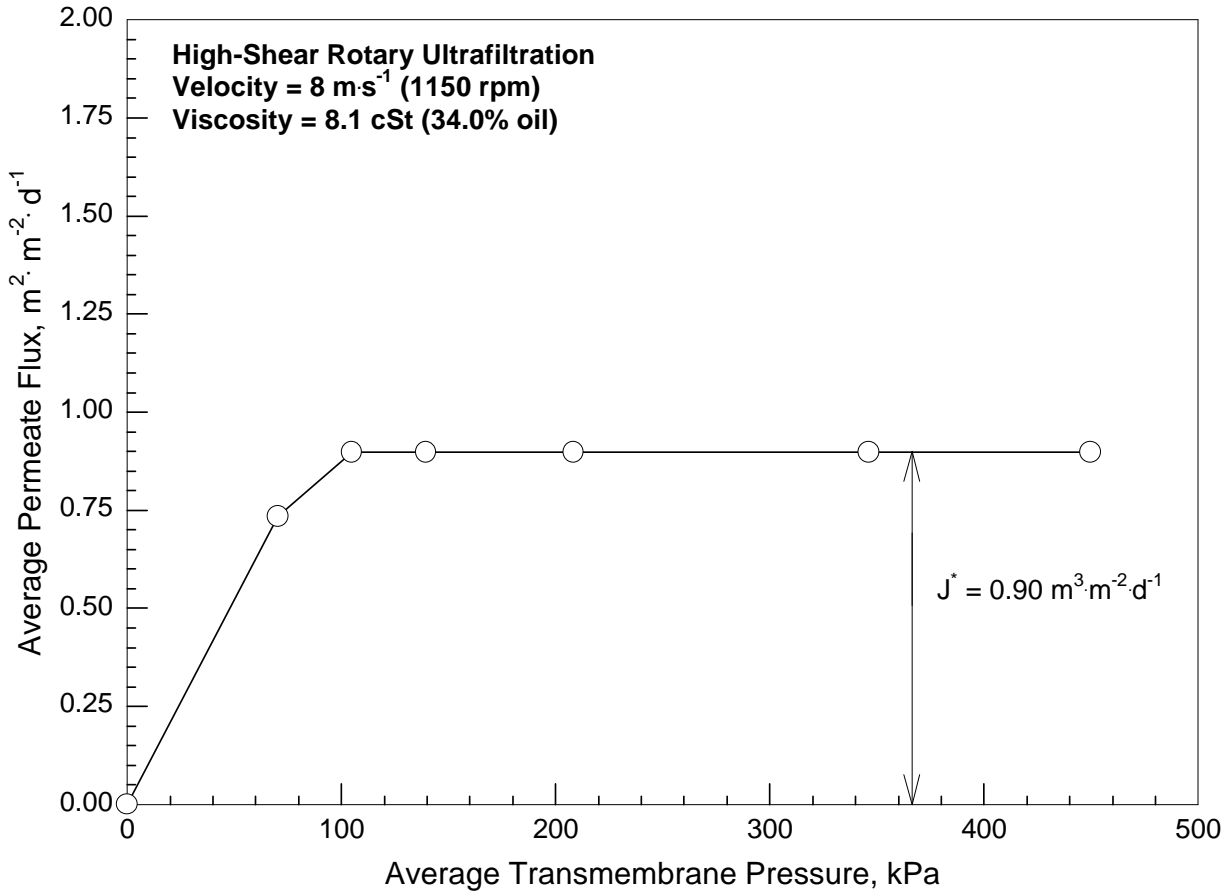

Figure 4.85 Average steady state permeate flux versus average transmembrane pressure for the $8 \mathrm{~m} \cdot \mathrm{s}^{-1}-8.1 \mathrm{cSt}$ experiment. 
${ }^{-2} \cdot \mathrm{d}^{-1}\left(22 \mathrm{gal} \cdot \mathrm{ft}^{-2} \cdot \mathrm{d}^{-1}\right)$ was observed at the 138 to $483 \mathrm{kPa}$ (20 to $70 \mathrm{psig}$ ) applied pressure conditions.

The permeate $\mathrm{pH}$ ranged from 7.60 to 8.10 and averaged 7.73 . The concentrate $\mathrm{pH}$ ranged from 7.48 to 7.73 and averaged 7.59. A net decrease in permeate $\mathrm{pH}$ was observed and concentrate $\mathrm{pH}$ remained constant. The permeate conductivity ranged from 348 to $471 \mu \mathrm{S} \cdot \mathrm{cm}^{-1}$ and averaged $404 \mu \mathrm{S} \cdot \mathrm{cm}^{-1}$. The concentrate conductivity ranged from 383 to $433 \mu \mathrm{S} \cdot \mathrm{cm}^{-1}$ and averaged $400 \mu \mathrm{S} \cdot \mathrm{cm}^{-1}$. A net decrease in permeate conductivity was observed. Concentrate conductivity was constant. Permeate conductivity was greater than concentrate conductivity through $\sim 2.5 \mathrm{hr}$. Concentrate conductivity then exceeded permeate conductivity from $\sim 2.5 \mathrm{hr}$. through the end of the experiment. Distinct step-wise changes in permeate and concentrate $\mathrm{pH}$ and conductivity were not observed as applied pressure was increased.

\subsubsection{9 $12 \mathrm{~m} \cdot \mathrm{s}^{-1}-45.6 \mathrm{cSt}$ (1750 rpm -50\% MW Fluid)}

Permeate flux versus time for the $12 \mathrm{~m} \cdot \mathrm{s}^{-1}-45.6 \mathrm{cSt}$ experiment is presented in Figure 4.86. Permeate flux was stable at each applied pressure condition. The system was operated over an applied pressure range of 173 to $483 \mathrm{kPa}$ ( 25 to $70 \mathrm{psig}$ ). The average permeate backpressure was $76.6 \mathrm{kPa}(11.1 \mathrm{psi})$ at an average relative velocity of $12 \mathrm{~m} \cdot \mathrm{s}^{-1}(1750 \mathrm{rpm})$. The average permeate flux versus average transmembrane pressure for the $12 \mathrm{~m} \cdot \mathrm{s}^{-1}-45.6 \mathrm{cSt}$ experiment is presented in Figure 4.87. A limiting flux of 1.5 $\mathrm{m}^{3} \cdot \mathrm{m}^{-2} \cdot \mathrm{d}^{-1}\left(36 \mathrm{gal} \cdot \mathrm{ft}^{-2} \cdot \mathrm{d}^{-1}\right)$ was observed at the 241 to $483 \mathrm{kPa}$ (35 to $70 \mathrm{psig}$ ) applied pressure conditions.

The permeate $\mathrm{pH}$ ranged from 6.58 to 7.25 and averaged 6.86. The concentrate $\mathrm{pH}$ ranged from 6.44 to 7.21 and averaged 6.70 . The permeate conductivity ranged from 


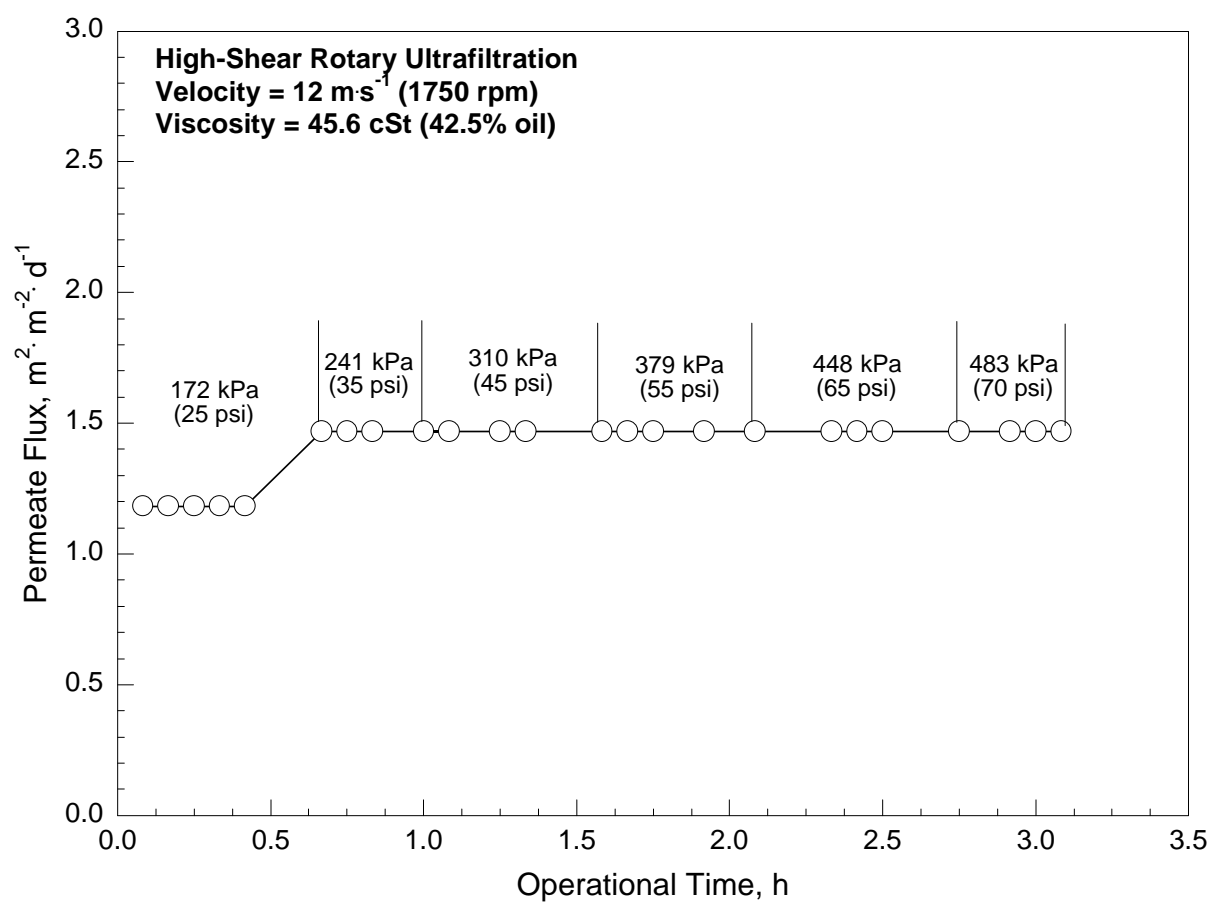

Figure 4.86 Permeate flux versus operational time for the $12 \mathrm{~m} \cdot \mathrm{s}^{-1}-45.6 \mathrm{cSt}$ experiment.

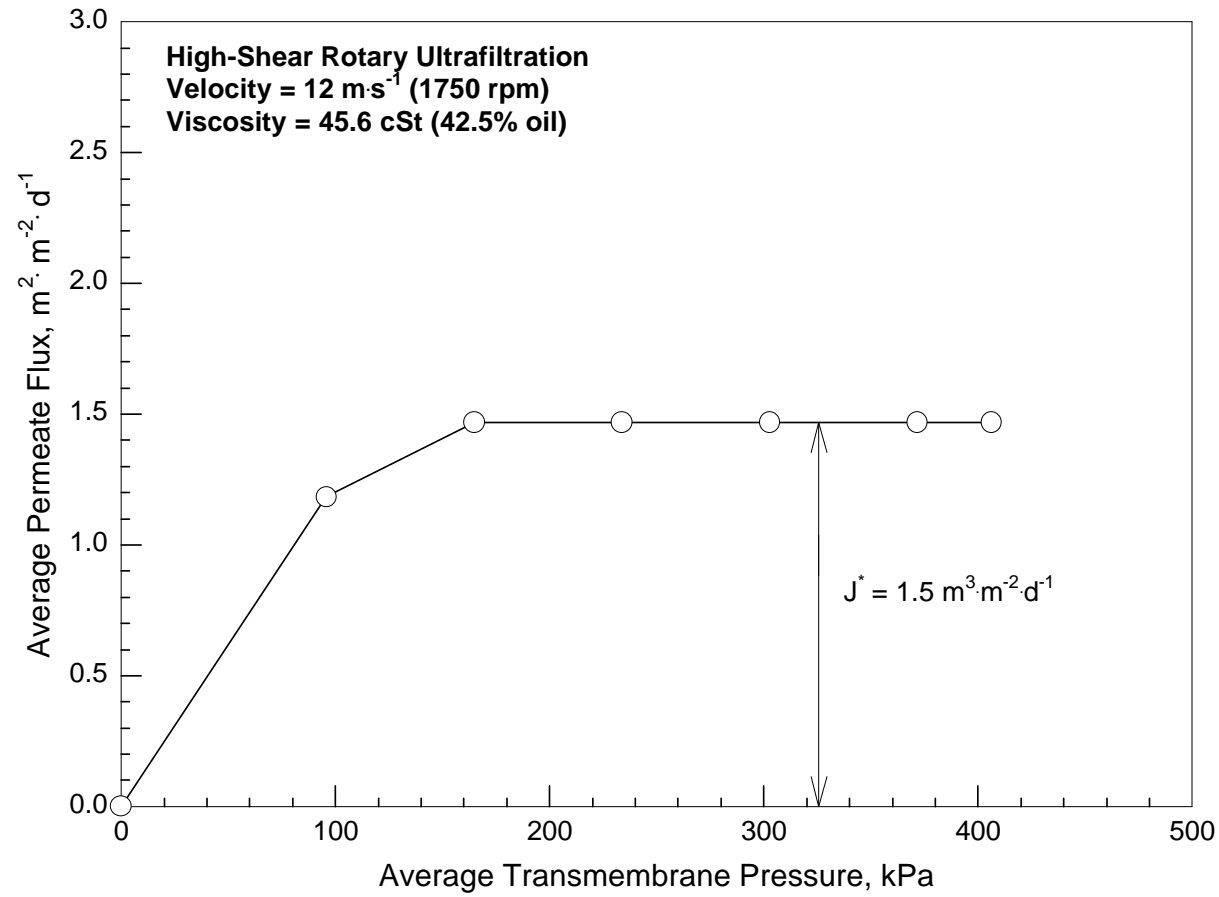

Figure 4.87 Average steady state permeate flux versus average transmembrane pressure for the $12 \mathrm{~m} \cdot \mathrm{s}^{-1}-45.6 \mathrm{cSt}$ experiment. 
1385 to $1586 \mu \mathrm{S} \cdot \mathrm{cm}^{-1}$ and averaged $1460 \mu \mathrm{S} \cdot \mathrm{cm}^{-1}$. The concentrate conductivity ranged from 1281 to $2080 \mu \mathrm{S} \cdot \mathrm{cm}^{-1}$ and averaged $1882 \mu \mathrm{S} \cdot \mathrm{cm}^{-1}$. Distinct step-wise changes in permeate and concentrate $\mathrm{pH}$ and conductivity were not observed as applied pressure was increased.

\subsubsection{0 $10 \mathrm{~m} \cdot \mathrm{s}^{-1}-45.6 \mathrm{cSt}(1450 \mathrm{rpm}-50 \%$ MW Fluid)}

Permeate flux versus time for the $10 \mathrm{~m} \cdot \mathrm{s}^{-1}-45.6 \mathrm{cSt}$ experiment is presented in Figure 4.88. Permeate flux was stable at each applied pressure condition. The system was operated over an applied pressure range of 138 to $483 \mathrm{kPa}$ (20 to $70 \mathrm{psig}$ ). The average permeate backpressure was $52.4 \mathrm{kPa}(7.6 \mathrm{psi})$ at an average relative velocity of $10 \mathrm{~m} \cdot \mathrm{s}^{-1}(1450 \mathrm{rpm})$. The average permeate flux versus average transmembrane pressure for the $10 \mathrm{~m} \cdot \mathrm{s}^{-1}-45.6 \mathrm{cSt}$ experiment is presented in Figure 4.89. A limiting flux of 1.0 $\mathrm{m}^{3} \cdot \mathrm{m}^{-2} \cdot \mathrm{d}^{-1}\left(25 \mathrm{gal} \cdot \mathrm{ft}^{-2} \cdot \mathrm{d}^{-1}\right)$ was observed at all applied pressure conditions.

The permeate $\mathrm{pH}$ ranged from 6.34 to 6.59 and averaged 6.43. The concentrate $\mathrm{pH}$ ranged from 6.32 to 6.67 and averaged 6.44 . The permeate conductivity ranged from 1148 to $2407 \mu \mathrm{S} \cdot \mathrm{cm}^{-1}$ and averaged $1910 \mu \mathrm{S} \cdot \mathrm{cm}^{-1}$. The concentrate conductivity ranged from 1350 to $1541 \mu \mathrm{S} \cdot \mathrm{cm}^{-1}$ and averaged $1439 \mu \mathrm{S} \cdot \mathrm{cm}^{-1}$. Distinct step-wise changes in permeate and concentrate $\mathrm{pH}$ and conductivity were not observed as applied pressure was increased.

\subsubsection{1 $8 \mathrm{~m} \cdot \mathrm{s}^{-1}-45.6 \mathrm{cSt}(1150 \mathrm{rpm}-50 \%$ MW Fluid)}

Permeate flux versus time for the $8 \mathrm{~m} \cdot \mathrm{s}^{-1}-45.6 \mathrm{cSt}$ experiment is presented in Figure 4.90. Permeate flux was stable at each applied pressure condition. The system was operated over an applied pressure range of 104 to $483 \mathrm{kPa}$ (15 to $70 \mathrm{psig}$ ). The average permeate backpressure was $33.1 \mathrm{kPa}$ (4.8 psi) at an average relative velocity of 8 


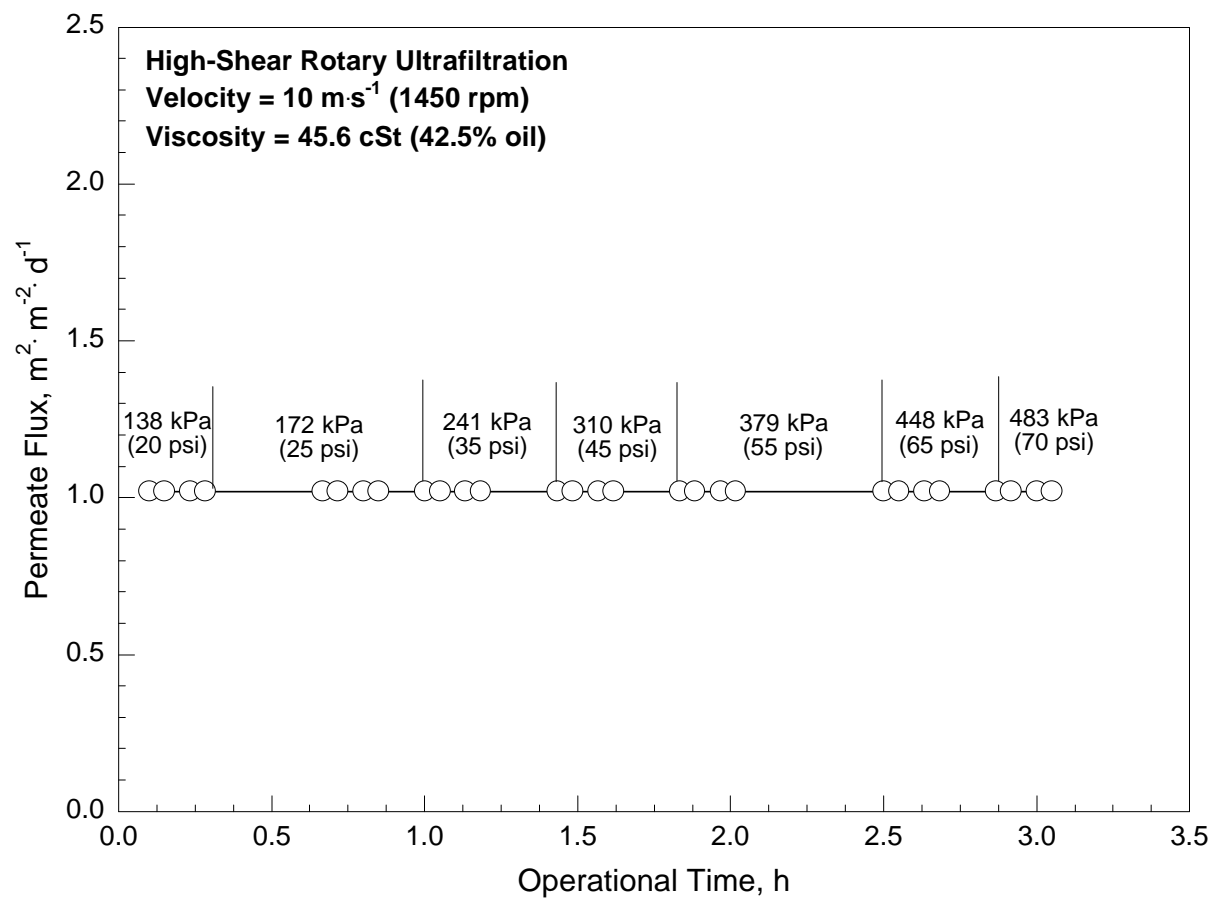

Figure 4.88 Permeate flux versus operational time for the $10 \mathrm{~m} \cdot \mathrm{s}^{-1}-45.6 \mathrm{cSt}$ experiment.

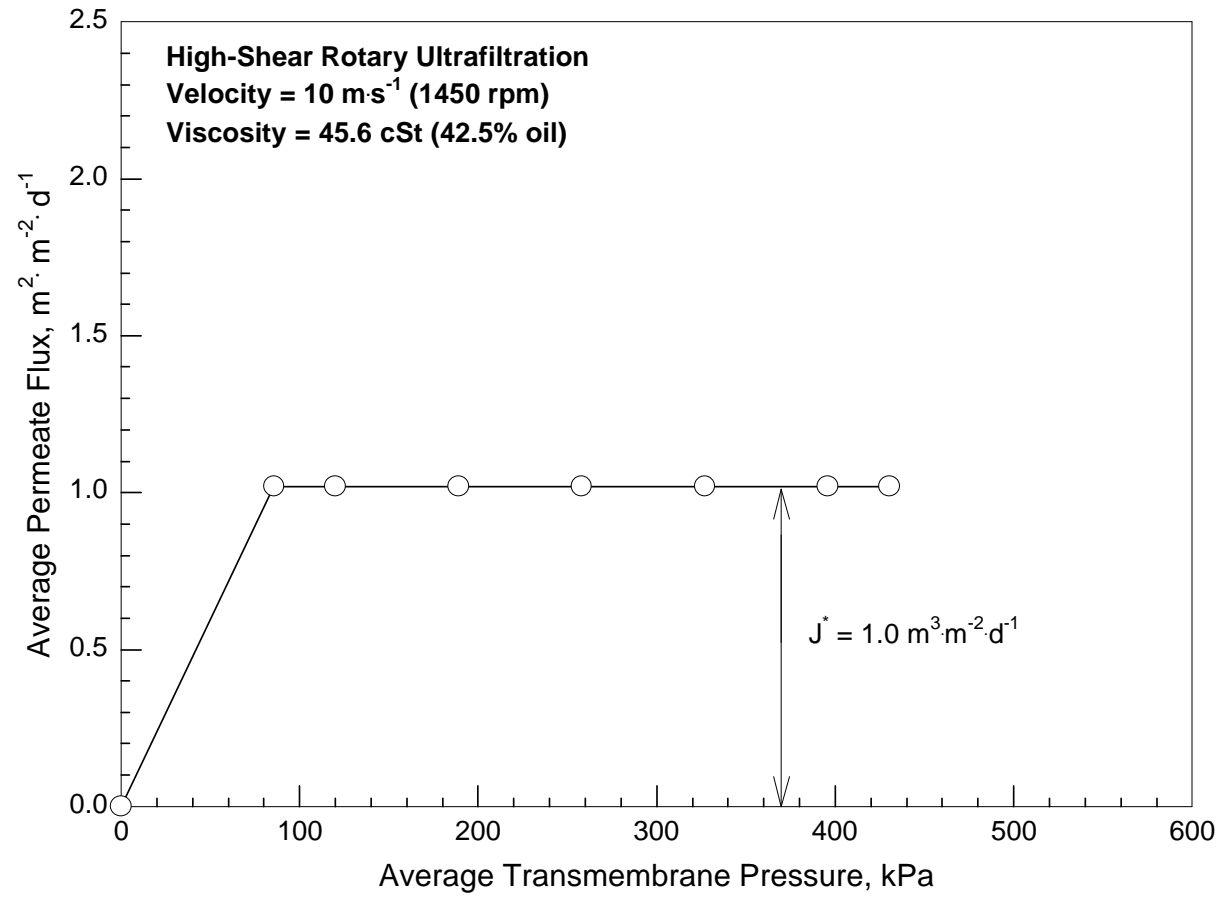

Figure 4.89 Average steady state permeate flux versus average transmembrane pressure for the $10 \mathrm{~m} \cdot \mathrm{s}^{-1}-45.6 \mathrm{cSt}$ experiment. 


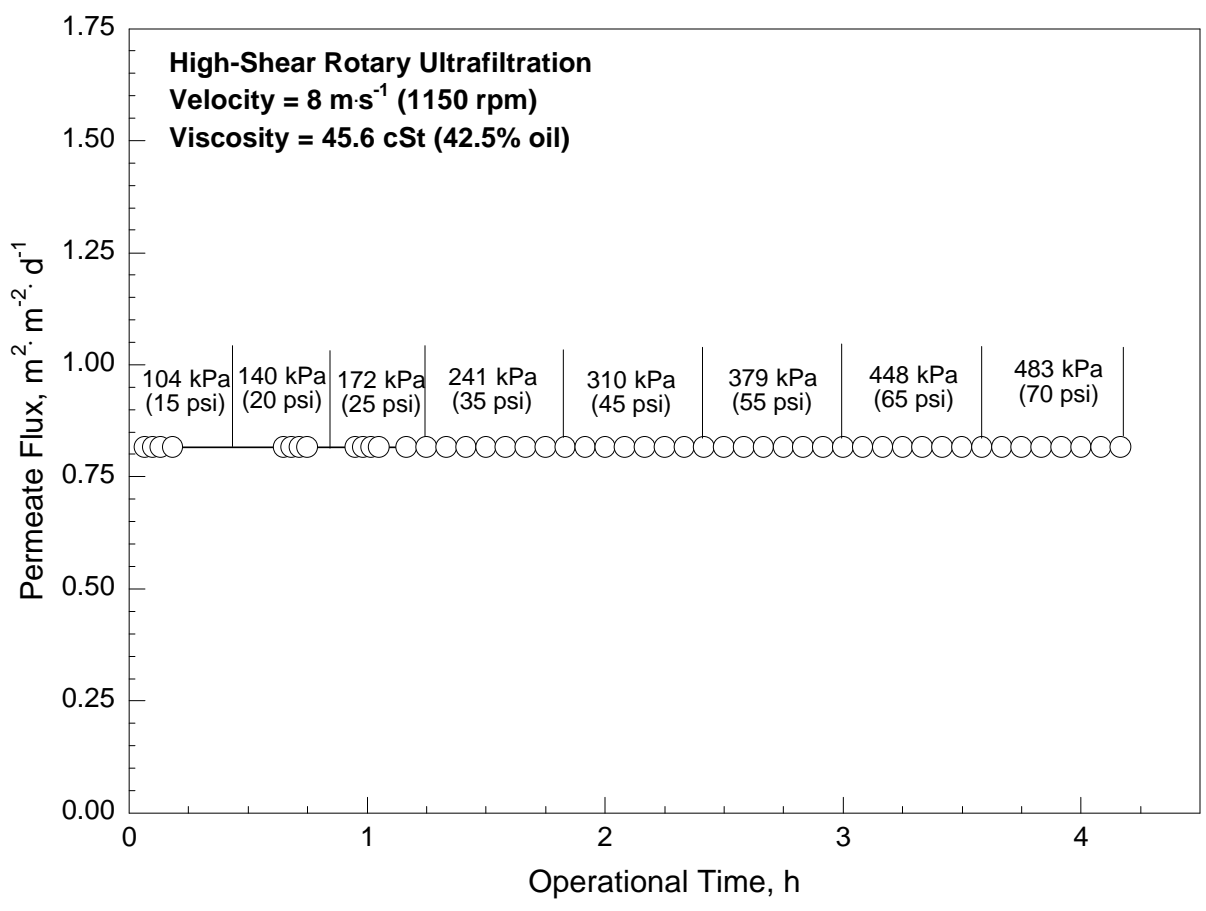

\section{Figure 4.90 Permeate flux versus operational time for the $8 \mathrm{~m} \cdot \mathrm{s}^{-1}-45.6 \mathrm{cSt}$ experiment.}

$\mathrm{m} \cdot \mathrm{s}^{-1}(1150 \mathrm{rpm})$. The average permeate flux versus average transmembrane pressure for the $8 \mathrm{~m} \cdot \mathrm{s}^{-1}-45.6 \mathrm{cSt}$ experiment is presented in Figure 4.91. A limiting flux of $0.81 \mathrm{~m}^{3} \cdot \mathrm{m}$ ${ }^{-2} \cdot \mathrm{d}^{-1}\left(20 \mathrm{gal} \cdot \mathrm{ft}^{-2} \cdot \mathrm{d}^{-1}\right)$ was observed at all applied pressure conditions.

The permeate $\mathrm{pH}$ ranged from 6.39 to 7.00 and averaged 6.63. The concentrate $\mathrm{pH}$ ranged from 6.29 to 7.34 and averaged 6.56 . The permeate conductivity ranged from 1273 to $1786 \mu \mathrm{S} \cdot \mathrm{cm}^{-1}$ and averaged $1573 \mu \mathrm{S} \cdot \mathrm{cm}^{-1}$. The concentrate conductivity ranged from 1148 to $1321 \mu \mathrm{S} \cdot \mathrm{cm}^{-1}$ and averaged $1199 \mu \mathrm{S} \cdot \mathrm{cm}^{-1}$. Distinct step-wise changes in permeate and concentrate $\mathrm{pH}$ and conductivity were not observed as applied pressure was increased.

\subsubsection{2 $12 \mathrm{~m} \cdot \mathrm{s}^{-1}-121 \mathrm{cSt}(1750 \mathrm{rpm}-60 \% \mathrm{MW}$ Fluid)}

Permeate flux versus time for the $12 \mathrm{~m} \cdot \mathrm{s}^{-1}-121 \mathrm{cSt}$ experiment is presented in Figure 4.92. Permeate flux was stable at each applied pressure condition. The system 


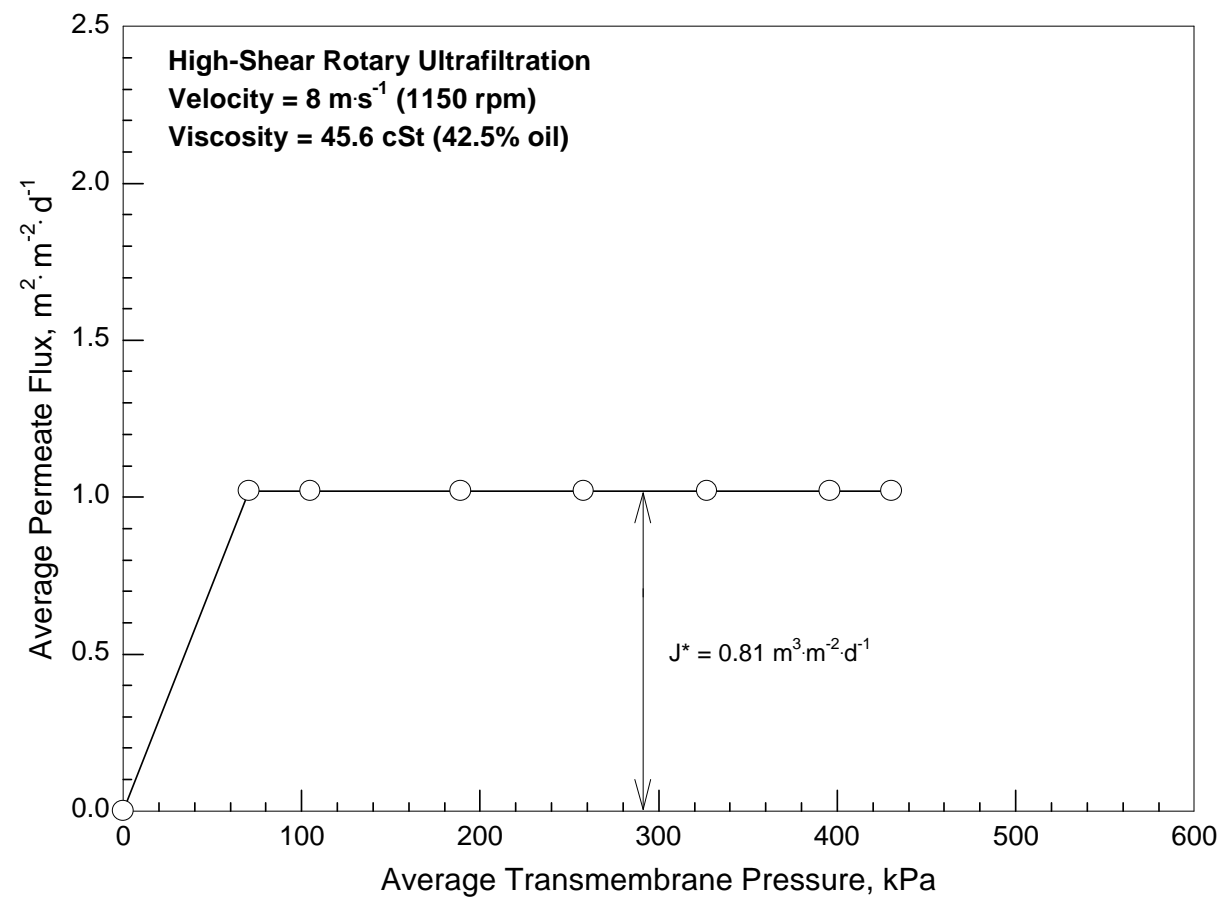

Figure 4.91 Average steady state permeate flux versus average transmembrane pressure for the $8 \mathrm{~m} \cdot \mathrm{s}^{-1}-45.6 \mathrm{cSt}$ experiment.

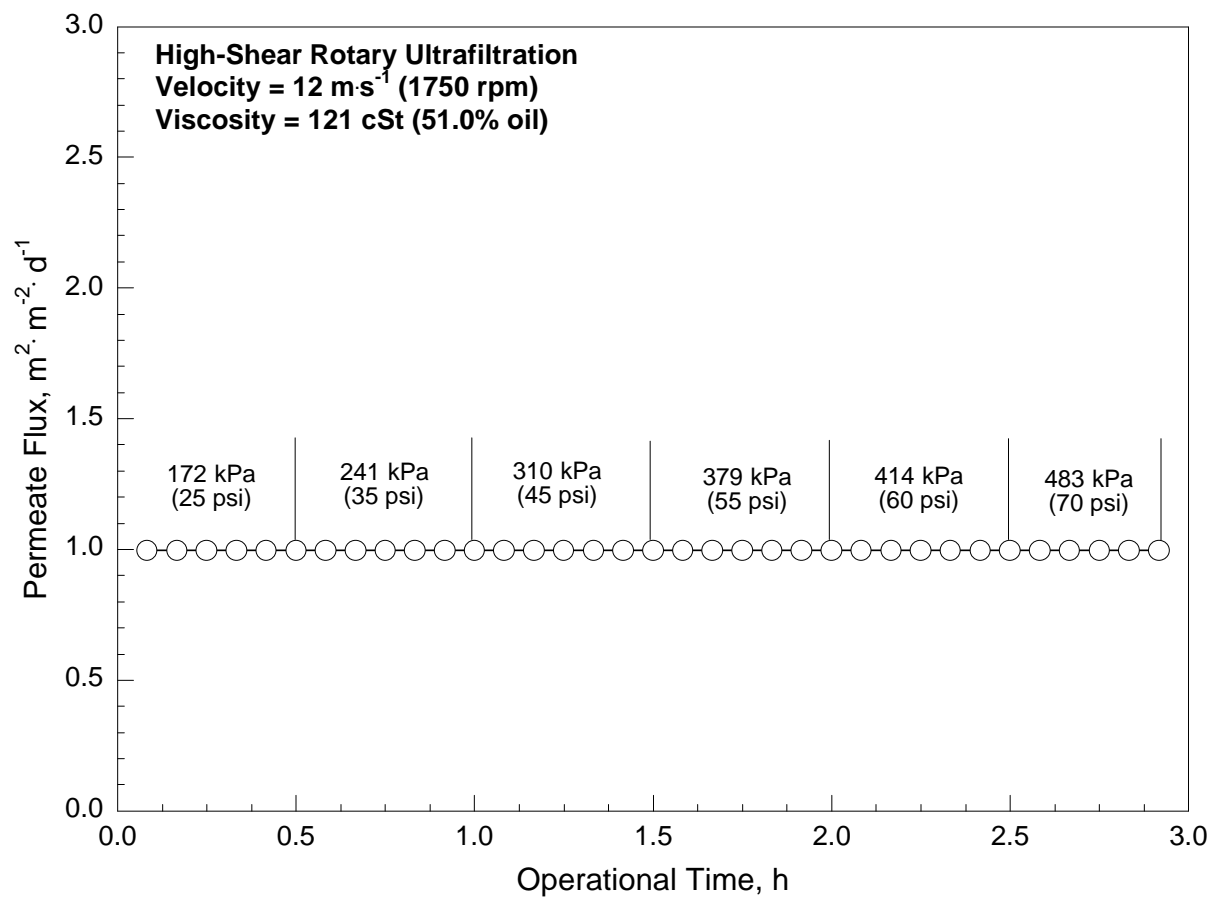

Figure 4.92 Permeate flux versus operational time for the $12 \mathrm{~m} \cdot \mathrm{s}^{-1}-121 \mathrm{cSt}$ experiment . 
was operated over an applied pressure range of 172 to $483 \mathrm{kPa}$ (25 to $70 \mathrm{psig}$ ). The average permeate backpressure was $76.6 \mathrm{kPa}(11.1 \mathrm{psi})$ at an average relative velocity of $12 \mathrm{~m} \cdot \mathrm{s}^{-1}(1750 \mathrm{rpm})$. The average permeate flux versus average transmembrane pressure for the $12 \mathrm{~m} \cdot \mathrm{s}^{-1}-121 \mathrm{cSt}$ experiment is presented in Figure 4.93. A limiting flux of 1.0 $\mathrm{m}^{3} \cdot \mathrm{m}^{-2} \cdot \mathrm{d}^{-1}\left(24 \mathrm{gal} \cdot \mathrm{ft}^{-2} \cdot \mathrm{d}^{-1}\right)$ was observed at all applied pressure conditions.

The permeate $\mathrm{pH}$ ranged from 7.60 to 8.10 and averaged 7.73 . The concentrate $\mathrm{pH}$ ranged from 7.48 to 7.73 and averaged 7.59. The permeate conductivity ranged from 348 to $471 \mu \mathrm{S} \cdot \mathrm{cm}^{-1}$ and averaged $404 \mu \mathrm{S} \cdot \mathrm{cm}^{-1}$. The concentrate conductivity ranged from 383 to $433 \mu \mathrm{S} \cdot \mathrm{cm}^{-1}$ and averaged $400 \mu \mathrm{S} \cdot \mathrm{cm}^{-1}$. Distinct step-wise changes in permeate and concentrate $\mathrm{pH}$ and conductivity were not observed as applied pressure was increased.

\subsubsection{3 $10 \mathrm{~m} \cdot \mathrm{s}^{-1}-121 \mathrm{cSt}(1450 \mathrm{rpm}-60 \% \mathrm{MW}$ Fluid $)$}

Permeate flux versus time for the $10 \mathrm{~m} \cdot \mathrm{s}^{-1}-121 \mathrm{cSt}$ experiment is presented in Figure 4.94. Permeate flux was stable at each applied pressure condition. The system was operated over an applied pressure range of 138 to $483 \mathrm{kPa}$ (20 to $70 \mathrm{psig}$ ). The average permeate backpressure was $52.4 \mathrm{kPa}(7.6 \mathrm{psi})$ at an average relative velocity of $10 \mathrm{~m} \cdot \mathrm{s}^{-1}(1450 \mathrm{rpm})$. The average permeate flux versus average transmembrane pressure for the $12 \mathrm{~m} \cdot \mathrm{s}^{-1}-121 \mathrm{cSt}$ experiment is presented in Figure 4.95. A limiting flux of 0.85 $\mathrm{m}^{3} \cdot \mathrm{m}^{-2} \cdot \mathrm{d}^{-1}\left(21 \mathrm{gal} \cdot \mathrm{ft}^{-2} \cdot \mathrm{d}^{-1}\right)$ was observed at all applied pressure conditions.

The permeate $\mathrm{pH}$ ranged from 7.60 to 8.10 and averaged 7.73 . The concentrate $\mathrm{pH}$ ranged from 7.48 to 7.73 and averaged 7.59 . The permeate conductivity ranged from 348 to $471 \mu \mathrm{S} \cdot \mathrm{cm}^{-1}$ and averaged $404 \mu \mathrm{S} \cdot \mathrm{cm}^{-1}$. The concentrate conductivity ranged from

383 to $433 \mu \mathrm{S} \cdot \mathrm{cm}^{-1}$ and averaged $400 \mu \mathrm{S} \cdot \mathrm{cm}^{-1}$. Distinct step-wise changes in permeate 


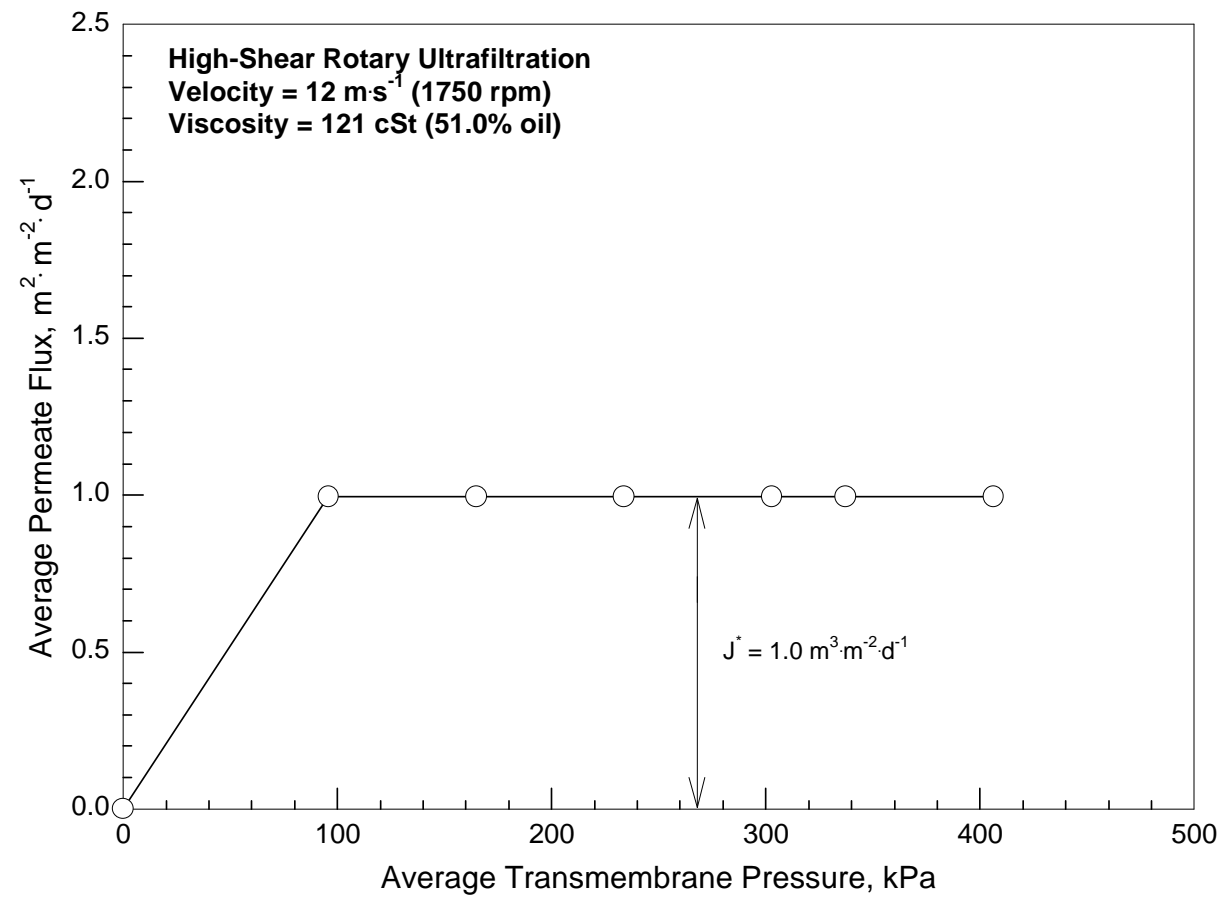

Figure 4.93 Average steady state permeate flux versus average transmembrane pressure for the $12 \mathrm{~m} \cdot \mathrm{s}^{-1}-121 \mathrm{cSt}$ experiment .

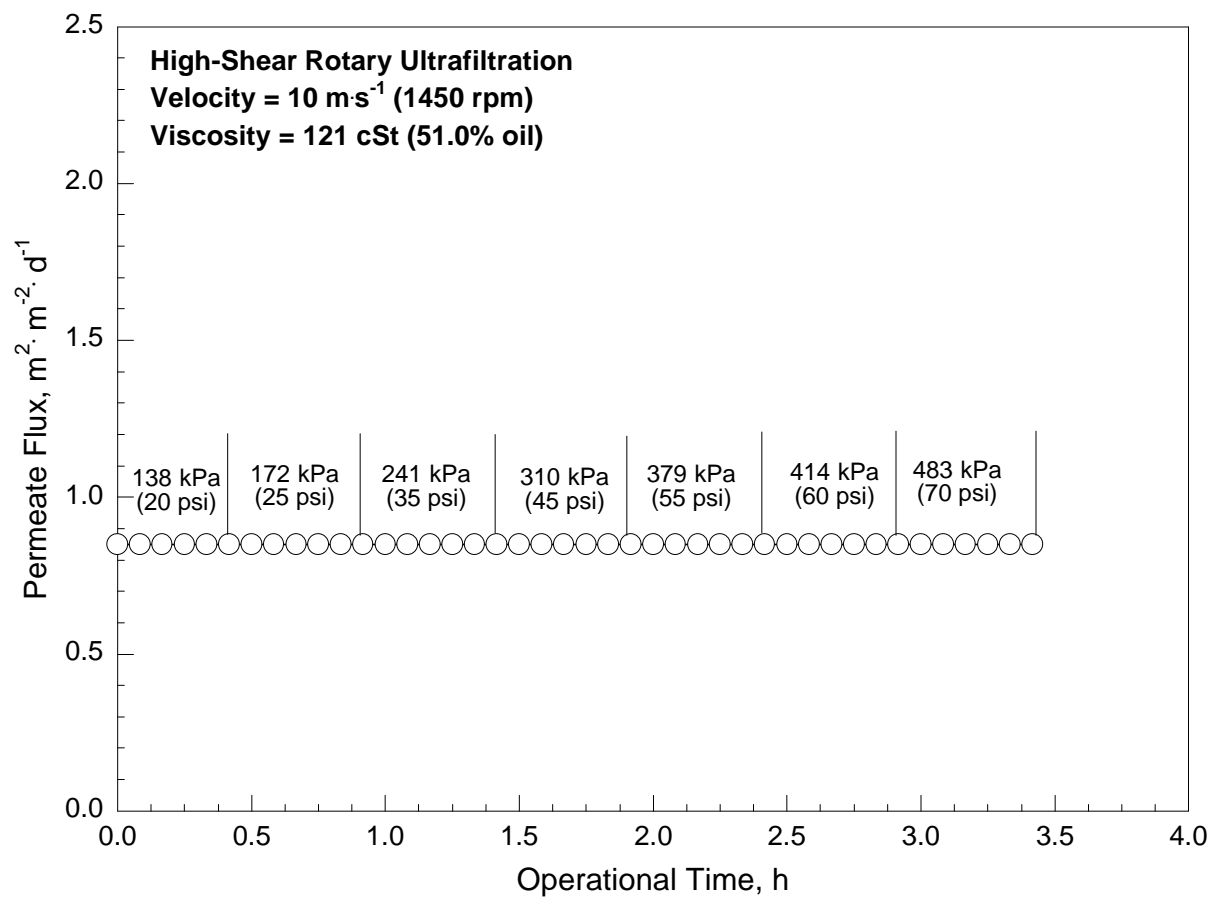

Figure 4.94 Permeate flux versus operational time for the $10 \mathrm{~m} \cdot \mathrm{s}^{-1}-121 \mathrm{cSt}$ experiment. 


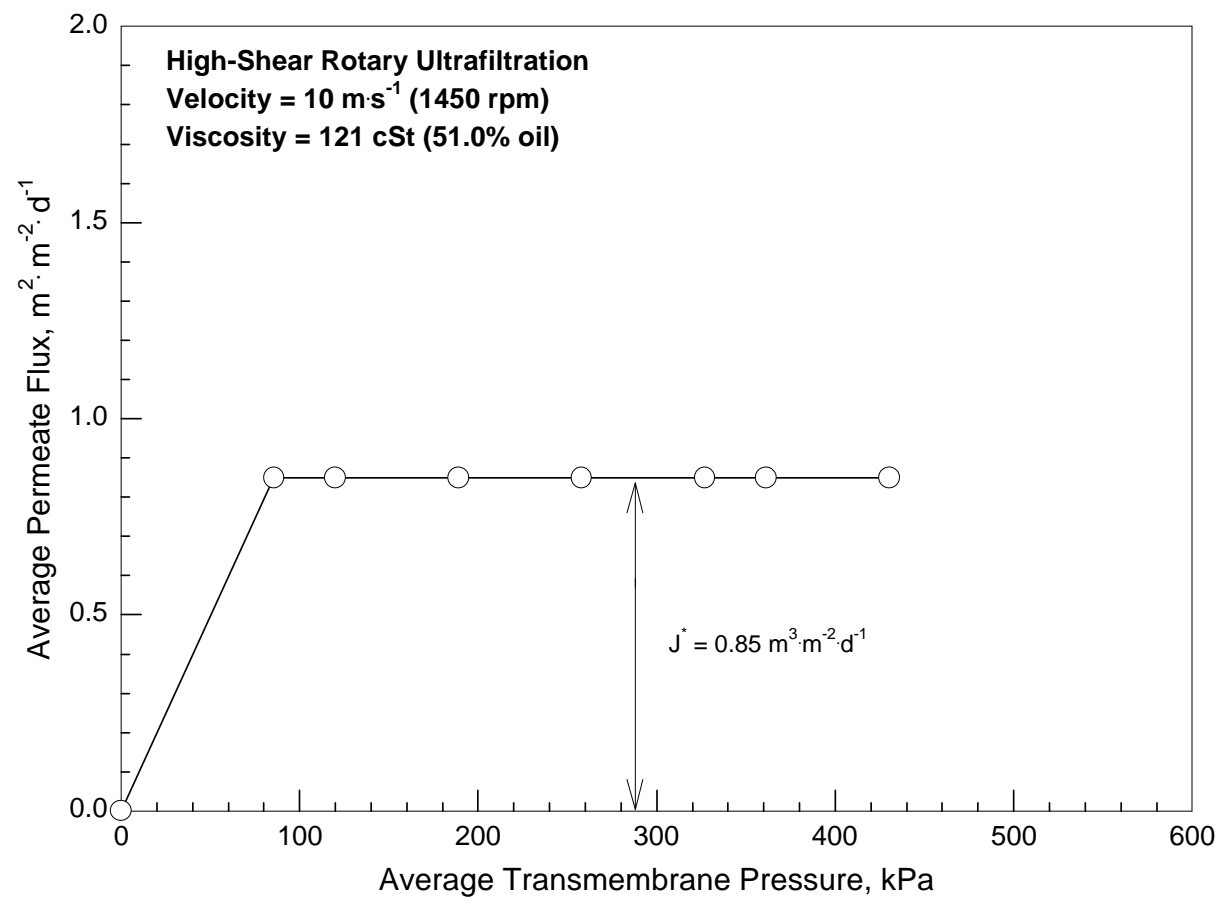

Figure 4.95 Average steady state permeate flux versus average transmembrane pressure for the $10 \mathrm{~m} \cdot \mathrm{s}^{-1}-121 \mathrm{cSt}$ experiment .

and concentrate $\mathrm{pH}$ and conductivity were not observed as applied pressure was increased.

\subsubsection{4 $8 \mathrm{~m} \cdot \mathrm{s}^{-1}-121 \mathrm{cSt}(1150 \mathrm{rpm}-60 \%$ MW Fluid)}

Permeate flux versus time for the $8 \mathrm{~m} \cdot \mathrm{s}^{-1}-121 \mathrm{cSt}$ experiment is presented in Figure 4.96. Permeate flux was stable at each applied pressure condition. The system was operated over an applied pressure range of 104 to $379 \mathrm{kPa}$ (15 to $55 \mathrm{psig}$ ). The average permeate backpressure was $33.1 \mathrm{kPa}(4.8 \mathrm{psi})$ at an average relative velocity of 8 $\mathrm{m} \cdot \mathrm{s}^{-1}(1150 \mathrm{rpm})$. The average permeate flux versus average transmembrane pressure for the $8 \mathrm{~m} \cdot \mathrm{s}^{-1}-121 \mathrm{cSt}$ experiment is presented in Figure 4.97. A limiting flux of $0.58 \mathrm{~m}^{3} \cdot \mathrm{m}^{-}$ ${ }^{2} \cdot \mathrm{d}^{-1}\left(14 \mathrm{gal} \cdot \mathrm{ft}^{-2} \cdot \mathrm{d}^{-1}\right)$ was observed at all applied pressure conditions.

The permeate $\mathrm{pH}$ ranged from 7.60 to 8.10 and averaged 7.73. The concentrate $\mathrm{pH}$ ranged from 7.48 to 7.73 and averaged 7.59. The permeate conductivity ranged from 


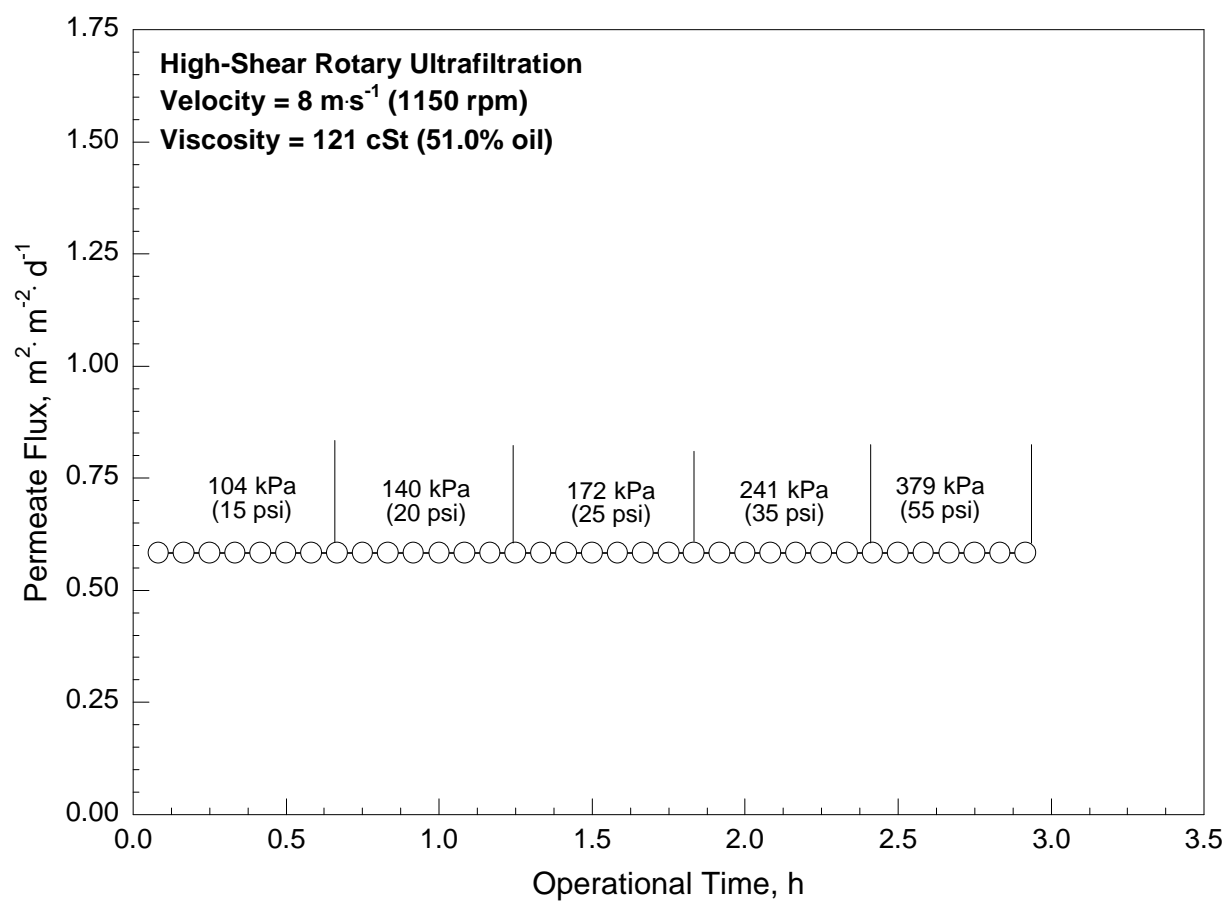

Figure 4.96 Permeate flux versus operational time for the $8 \mathrm{~m} \cdot \mathrm{s}^{-1}-121 \mathrm{cSt}$ experiment.

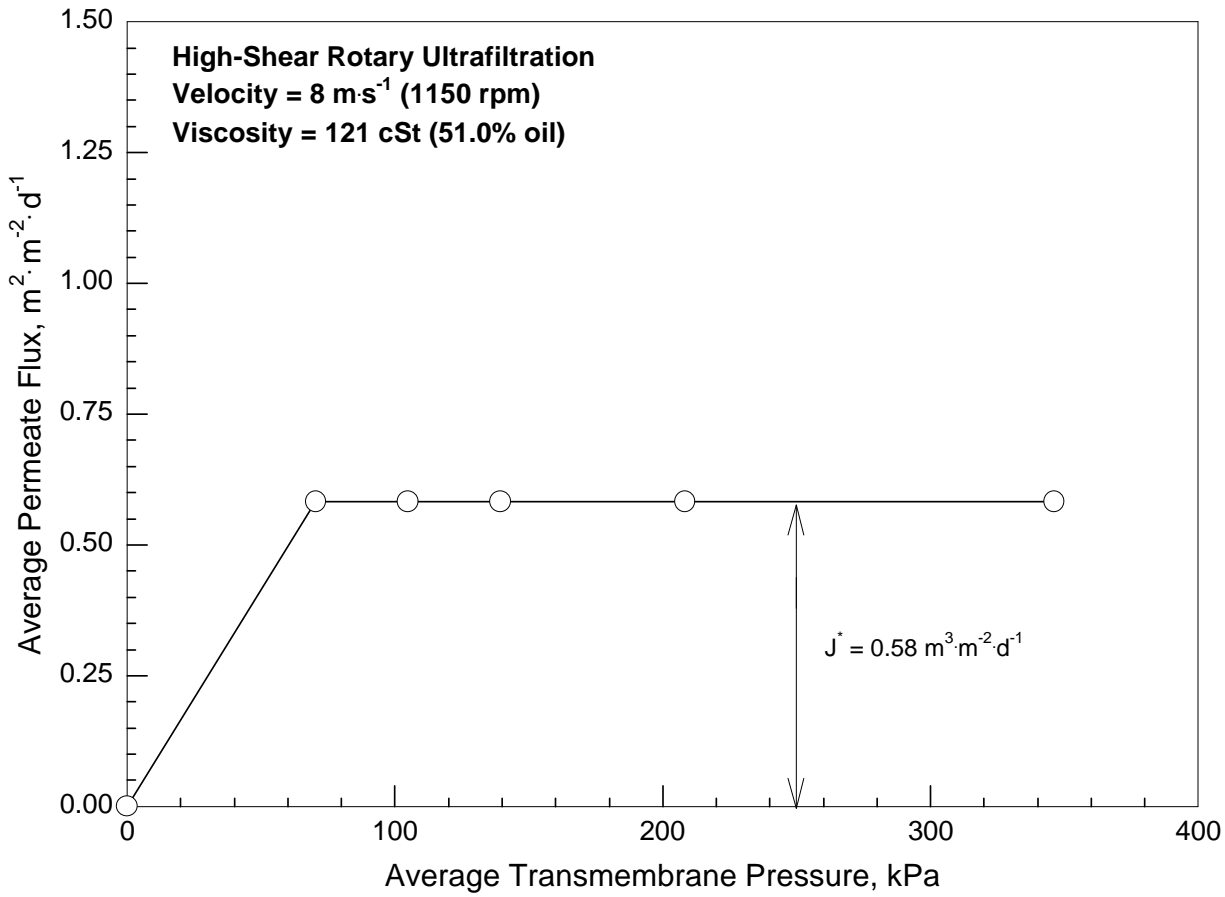

Figure 4.97 Average steady state permeate flux versus average transmembrane pressure for the $8 \mathrm{~m} \cdot \mathrm{s}^{-1}-121 \mathrm{cSt}$ experiment . 
348 to $471 \mu \mathrm{S} \cdot \mathrm{cm}^{-1}$ and averaged $404 \mu \mathrm{S} \cdot \mathrm{cm}^{-1}$. The concentrate conductivity ranged from 383 to $433 \mu \mathrm{S} \cdot \mathrm{cm}^{-1}$ and averaged $400 \mu \mathrm{S} \cdot \mathrm{cm}^{-1}$. Distinct step-wise changes in permeate and concentrate $\mathrm{pH}$ and conductivity with applied pressure were not observed in any of the experiments conducted using either the tubular or HSRUF systems; thus, these parameters were excluded from any further data reduction.

\subsection{WASTE METAL WORKING FLUID EXPERIMENT}

Permeate flux versus operational time for the waste MW fluid experiment is presented in Figure 4.98. The average permeate flux versus feed oil concentration for the waste MW fluid experiment is presented in Figure 4.99. Permeate flux decreased with time and feed oil concentration throughout the run. The run was stopped due to a low permeate flux $\left(\sim 1.2 \mathrm{~m}^{3} \cdot \mathrm{m}^{-2} \cdot \mathrm{d}^{-1}\right)$ at a final feed oil concentration of $\sim 70 \%$. The permeate $\mathrm{pH}$ ranged from 6.94 to 7.47 and averaged 7.27. The average permeate $\mathrm{pH}$ values during semi-batch and batch operation were 7.25 and 7.35 , respectively. The concentrate $\mathrm{pH}$ ranged from 6.86 to 7.91 and averaged 7.26. The average concentrate $\mathrm{pH}$ values during semi-batch and batch operation were 7.26 and 7.29 , respectively. 


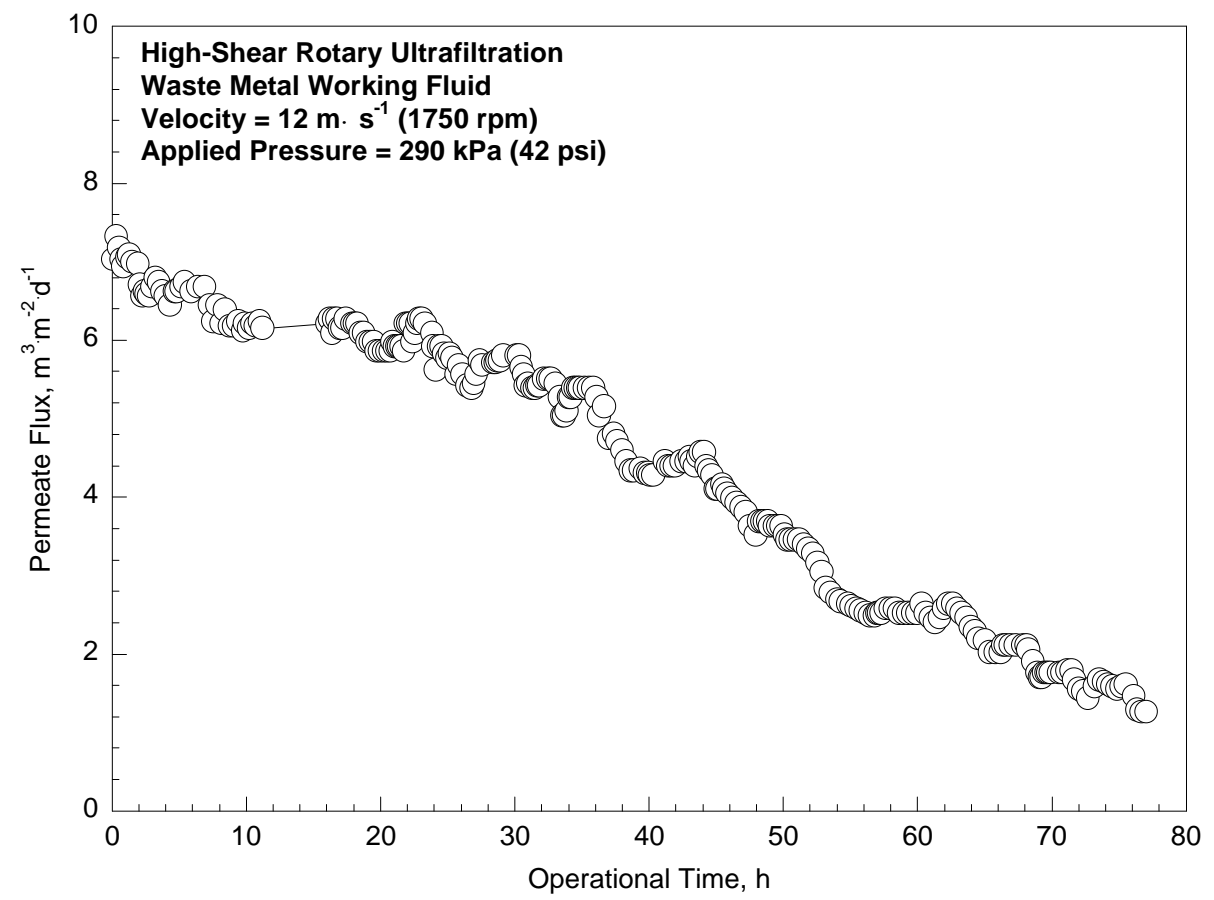

Figure 4.98 Permeate flux versus operational time for the waste MW fluid experiment.

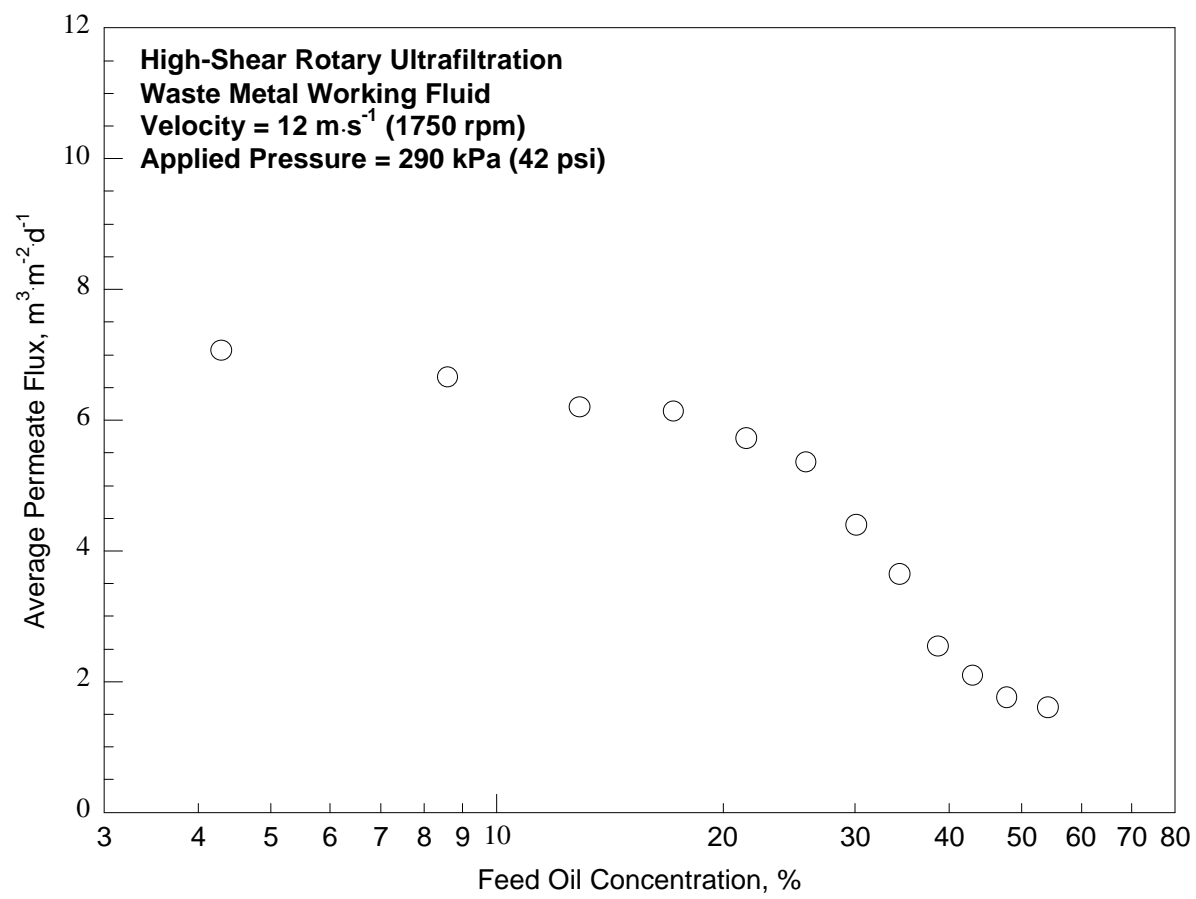

Figure 4.99 Permeate flux versus feed oil concentration for the waste MW fluid experiment. 


\section{CHAPTER 5.0 \\ DISCUSSION}

\subsection{THE EFFECT OF OPERATING PARAMETERS ON PERMEATE FLUX}

\subsubsection{Tubular Ultrafiltration}

As presented previously in Chapter 4, quasi steady-state permeate flux values at each pressure condition were averaged and plotted versus average transmembrane pressure for each discrete $v-\eta$ experiment. Average permeate flux versus average transmembrane pressure for each experiment conducted at average relative velocities of $3.7 \mathrm{~m} \cdot \mathrm{s}^{-1}$ and feed kinematic viscosities of $4.5 \mathrm{cSt}$ are presented in Figures 5.1 and 5.2, respectively. In general, permeate flux increased with average transmembrane pressure, $\mathrm{P}$, in experiments conducted at high $\mathrm{v}$ and low $\eta\left(e . g ., 3.7 \mathrm{~m} \cdot \mathrm{s}^{-1}-0.40 \mathrm{cSt}\right.$ experiment as presented Figure 5.1). A pressure-independent permeate flux was not achieved in experiments conducted at $0.40 \mathrm{cSt}$, though the average flux data diverged from linear behavior at higher pressures due to a shift from pressure-dependent to pressureindependent operational conditions. However, pressure-independent permeate flux values were achieved in experiments conducted at low $\mathrm{v}$ and higher $\eta\left(e . g ., 0.62 \mathrm{~m} \cdot \mathrm{s}^{-1}-4.5\right.$ cSt experiment as presented in Figure 5.2).

In the data set as a whole, lower permeate flux was observed as $\mathrm{v}$ was decreased and $\eta$ was increased. Additionally, the transition from pressure-dependent to pressureindependent operational conditions occurred at lower pressures as $\mathrm{v}$ was decreased and $\eta$ was increased due to a greater build-up of rejected oil droplets at the membrane surface (Fane and Fell 1984; Lee et al. 1984). According to Cheryan (1998), most macromolecular solutions will display non-Newtonian behavior at sufficiently high solute concentrations (viscosity). However, it has been shown that the concentration- 


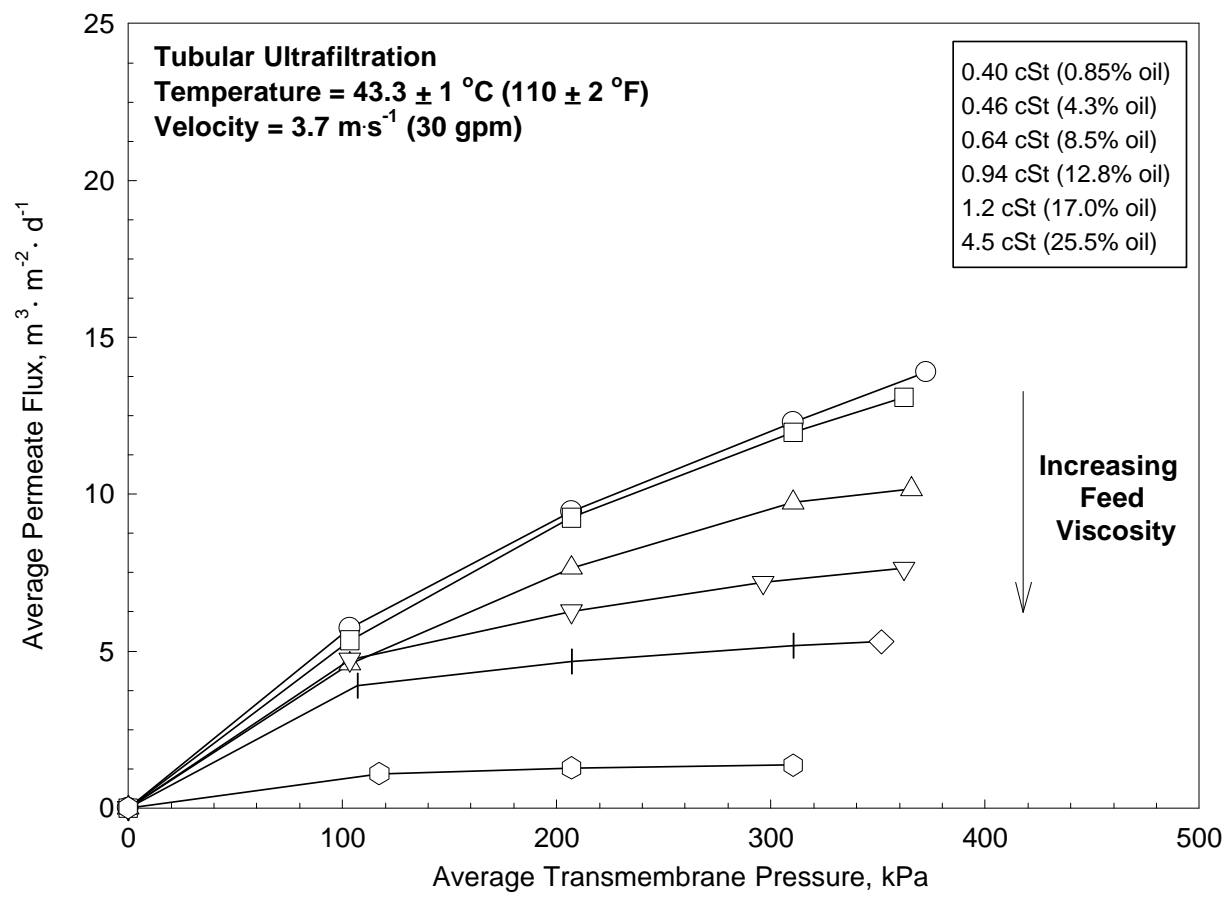

Figure 5.1 Average steady state permeate flux versus average transmembrane pressure for experiments conducted at $3.7 \mathrm{~m} \cdot \mathrm{s}^{-1}$.

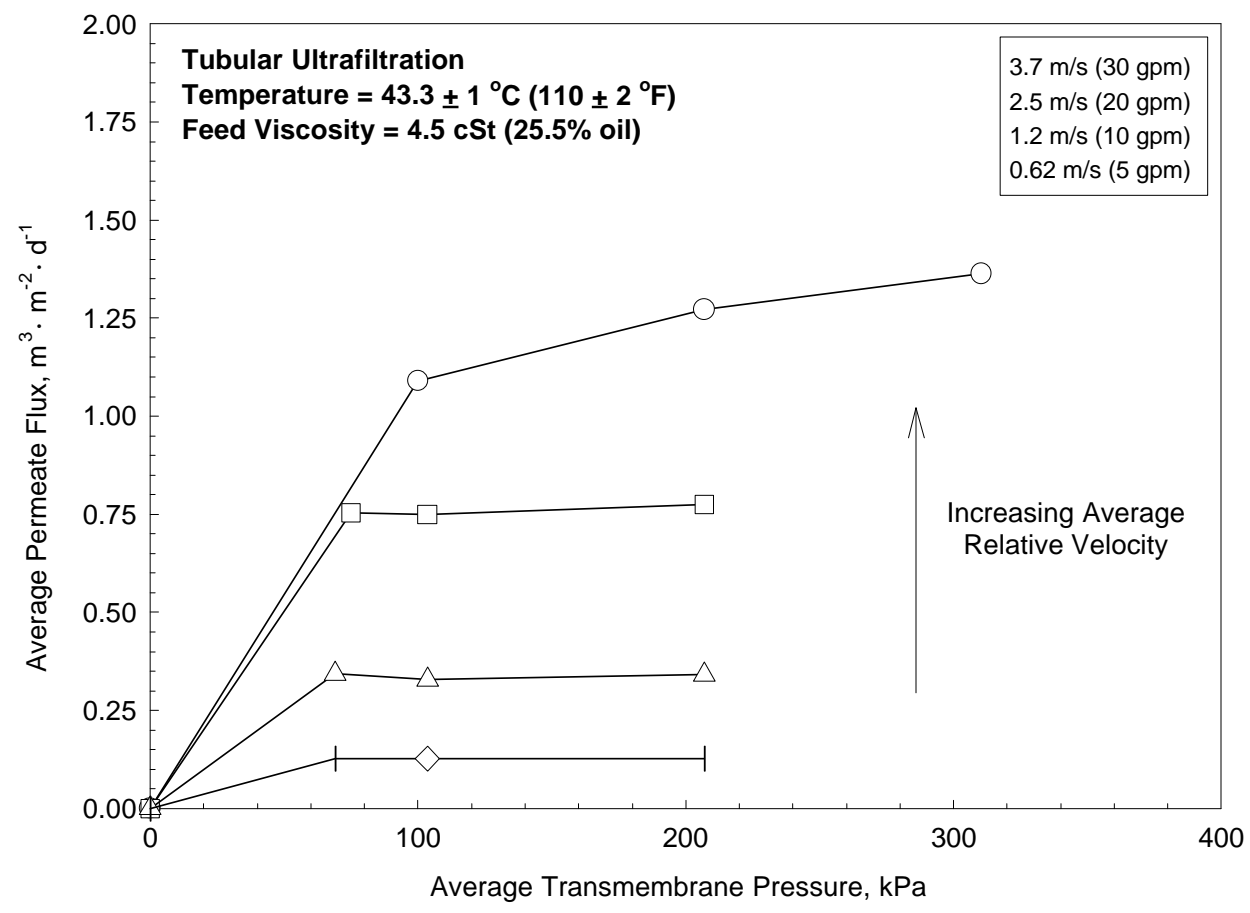

Figure 5.2 Average steady state permeate flux versus average transmembrane pressure for experiments conducted at $4.5 \mathrm{cSt}$. 
dependence of viscosity has a greater effect than both non-Newtonian behavior and solute diffusivity on permeate flux in UF processes (Pritchard et al. 1995); thus, the influences of non-Newtonian behavior and solute diffusivity were not examined in this study.

\subsubsection{High-Shear Rotary Ultrafiltration}

Average permeate flux versus average transmembrane pressure for each experiment conducted at an average relative velocity of $12 \mathrm{~m} \cdot \mathrm{s}^{-1}$ and a feed kinematic viscosity of $4.5 \mathrm{cSt}$ are presented in Figures 5.3 and 5.4, respectively. In general, permeate flux increased with $\mathrm{P}$ in experiments conducted at high $\mathrm{v}$ and low $\eta$ (e.g., 12 $\mathrm{m} \cdot \mathrm{s}^{-1}-0.46 \mathrm{cSt}$ experiment as presented in Figure 5.3). A pressure-independent permeate flux was not achieved in any of the experiments conducted at $0.46,0.64$, and $0.94 \mathrm{cSt}$, though the average flux data diverged from linear behavior at higher pressures due to a shift from pressure-dependent to pressure-independent operational conditions. However, pressure-independent permeate flux values were achieved in experiments conducted at low $\mathrm{v}$ and high $\eta\left(\right.$ e.g., $0.62 \mathrm{~m} \cdot \mathrm{s}^{-1}-8.1 \mathrm{cSt}$ experiment as presented in Figure 5.4). In the data set as a whole, lower permeate flux was observed as $\mathrm{v}$ was decreased and $\eta$ was increased. Additionally, the transition from pressure-dependent to pressure-independent operational conditions occurred at lower pressures as $\mathrm{v}$ was decreased and $\eta$ was increased due to a greater build-up of rejected oil droplets at the membrane surface (Fane and Fell, 1984; and Lee et al., 1984). 


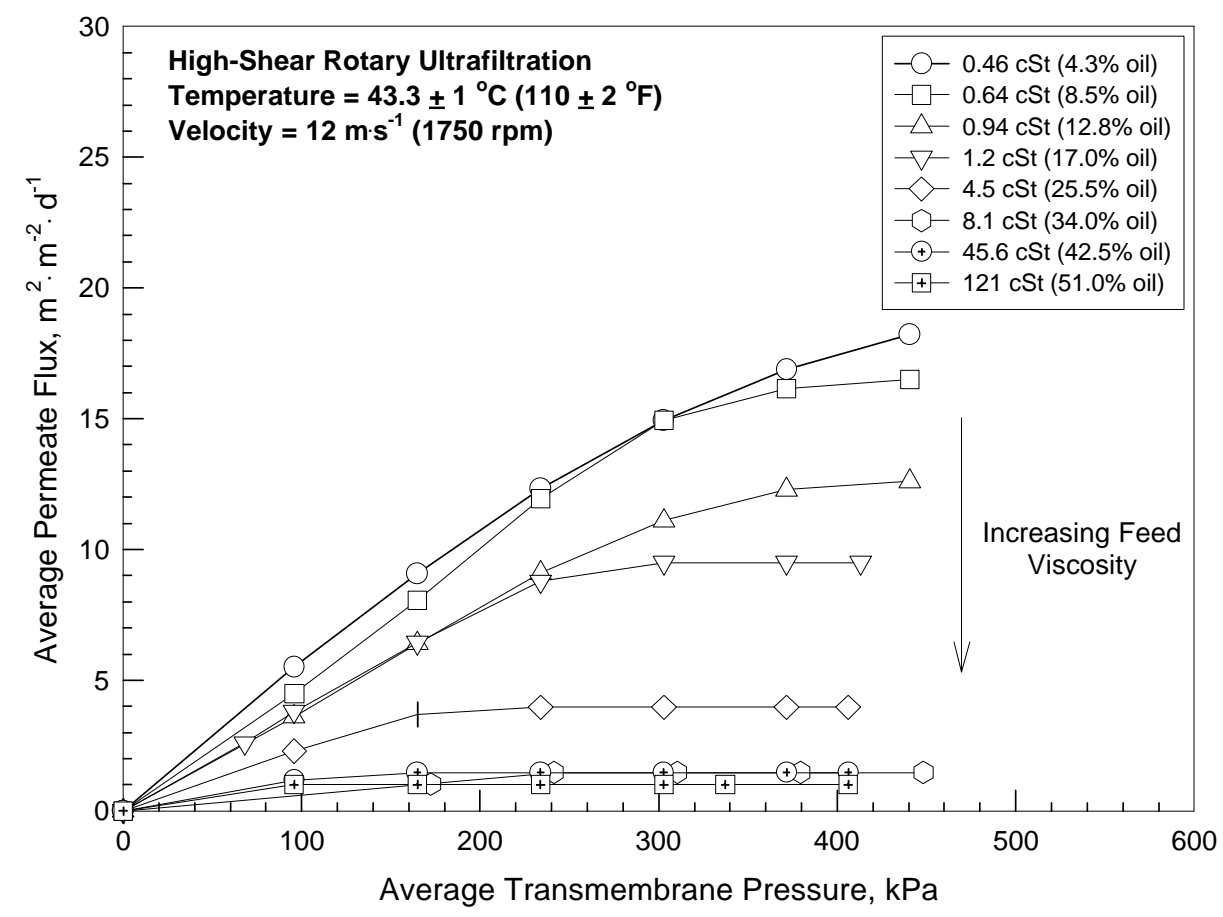

Figure 5.3 Average steady state permeate flux versus average transmembrane pressure for experiments conducted at $12 \mathrm{~m} \cdot \mathrm{s}^{-1}$.

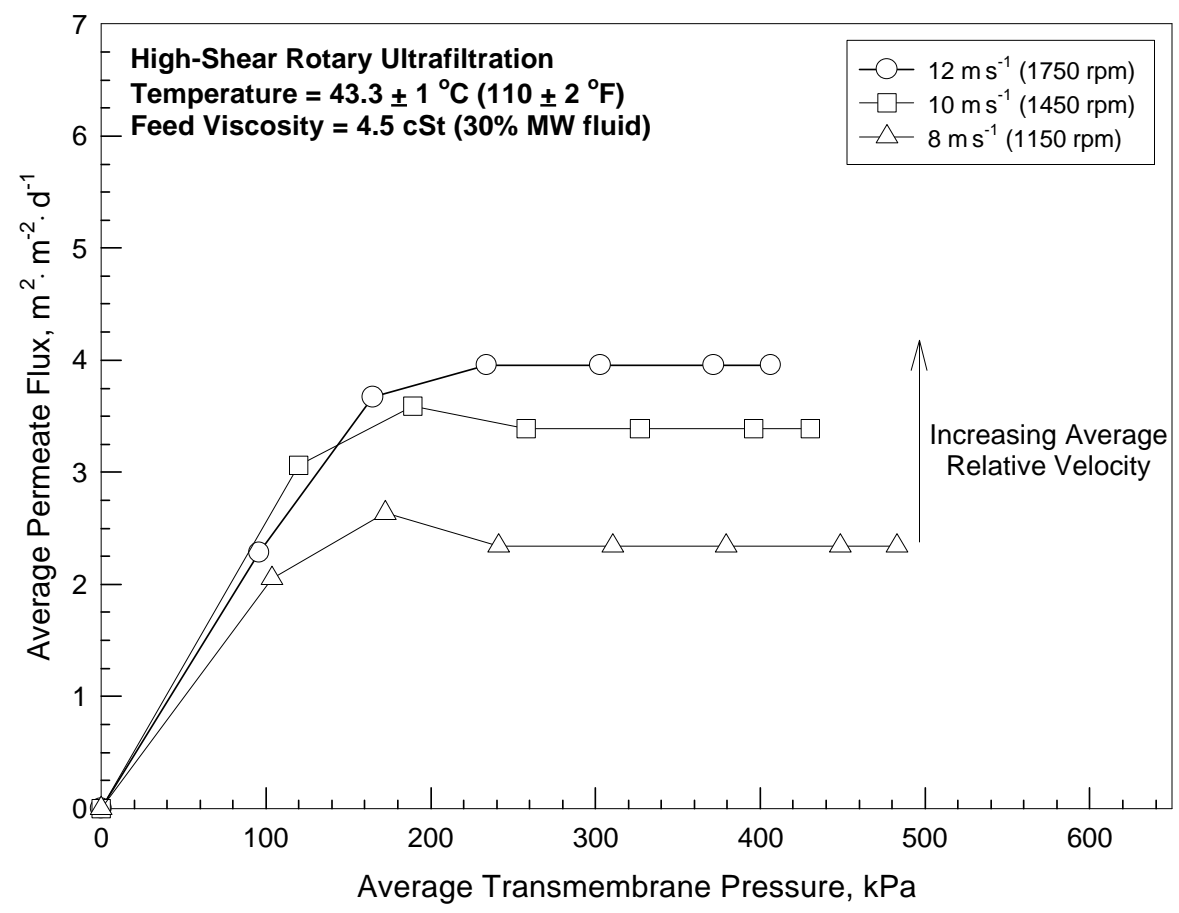

Figure 5.4 Average steady state permeate flux versus average transmembrane pressure for experiments conducted at $4.5 \mathrm{cSt}$. 


\subsection{MASS TRANSFER-THE THIN-FILM MODEL}

\subsubsection{Gel Layer Oil Concentration}

\subsubsection{Tubular Ultrafiltration}

Average permeate flux values presented previously in Chapter 4 were plotted versus the $\log$ of oil concentration, $\mathrm{OC}$, for each $\mathrm{P} / \mathrm{v}$ condition to study the prediction of limiting flux data using the thin film model. Semi-log plots of average permeate flux versus feed oil concentration for experiments conducted at $2.5,1.2$ and $0.62 \mathrm{~m} \cdot \mathrm{s}^{-1}$ are presented in Figures 5.5, 5.6, and 5.7, respectively. A limiting permeate flux was not reached in any experiments conducted at $3.7 \mathrm{~m} \cdot \mathrm{s}^{-1}$; thus, $3.7 \mathrm{~m} \cdot \mathrm{s}^{-1}$ data were excluded from further analysis even though divergence from a linear flux-pressure relationship was observed. The gel layer concentration was estimated by extrapolating the linear portion (i.e., pressure-independent data) of each data set to $\mathrm{J}^{*}=0$. Gel layer oil concentrations $\left(\mathrm{OC}_{\mathrm{gel}}\right)$ and mass transfer coefficients $\left(\mathrm{k}_{\mathrm{s}}\right)$ were determined for each $\mathrm{v} / \mathrm{P}$ experiment using the tubular UF system and are presented in Table 5.1. No more than two pressureindependent fluxes were attained in the $104 \mathrm{kPa}$ (15 psi) pressure conditions examined at each velocity. Conditions at which three or more pressure-independent data points were attained were considered to be most representative of limiting flux behavior; thus, 104 $\mathrm{kPa}$ data were excluded from further analysis.

For the tubular UF system, $\mathrm{OC}_{\text {gel }}$ ranged from 29 to $32 \%$ oil and averaged $31 \%$ oil. A single-factor analysis of variance (ANOVA) was conducted with $\mathrm{P}$ as the independent variable and $\mathrm{OC}_{\mathrm{gel}}$ as the dependent variable to ascertain the dependence of $\mathrm{OC}_{\mathrm{gel}}$ on $\mathrm{P}$ and $\mathrm{v}$. Similar ANOVA analyses were conducted with $\mathrm{v}$ as the independent 


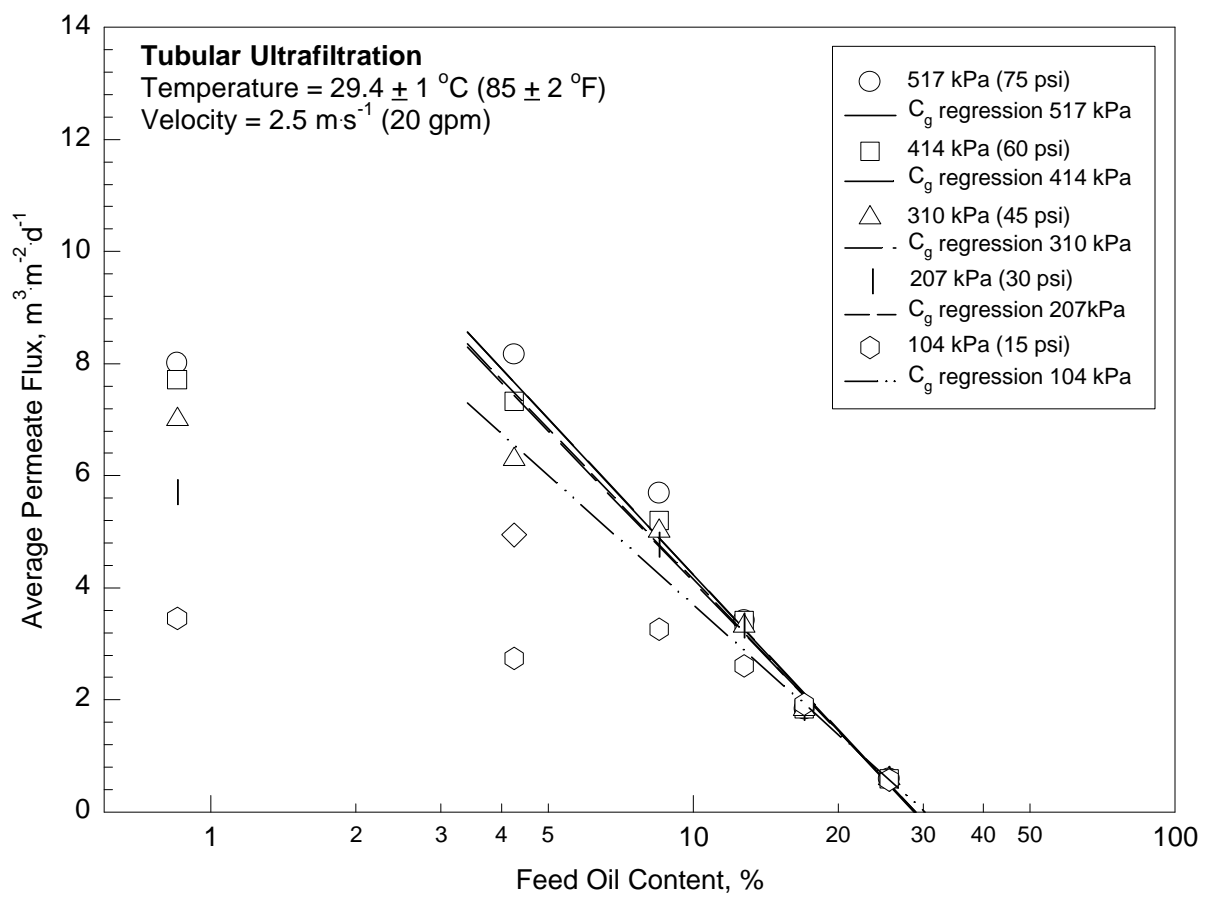

Figure 5.5 Average steady state permeate flux versus feed oil concentration for experiments conducted at $2.5 \mathrm{~m} \cdot \mathrm{s}^{-1}$.

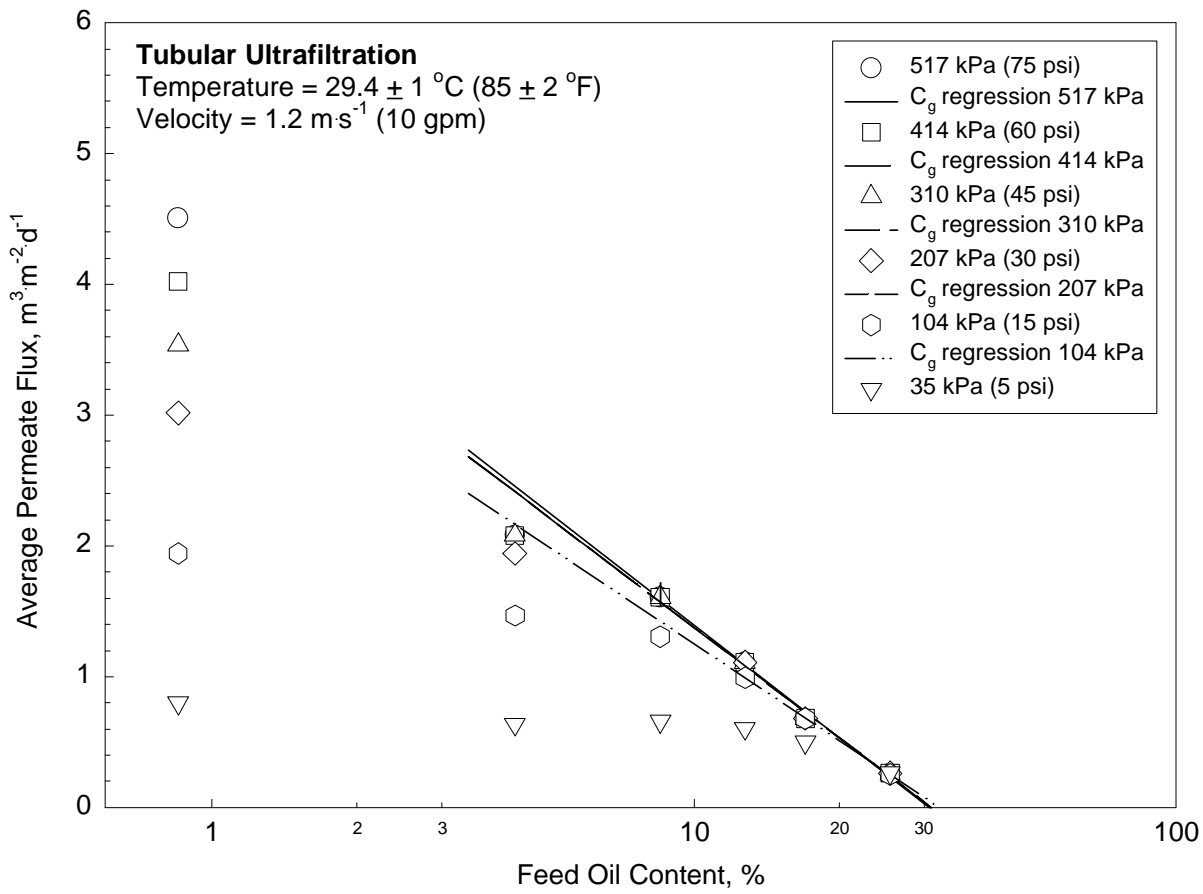

Figure 5.6 Average steady state permeate flux versus feed oil concentration for experiments conducted at $1.2 \mathrm{~m} \cdot \mathrm{s}^{-1}$. 


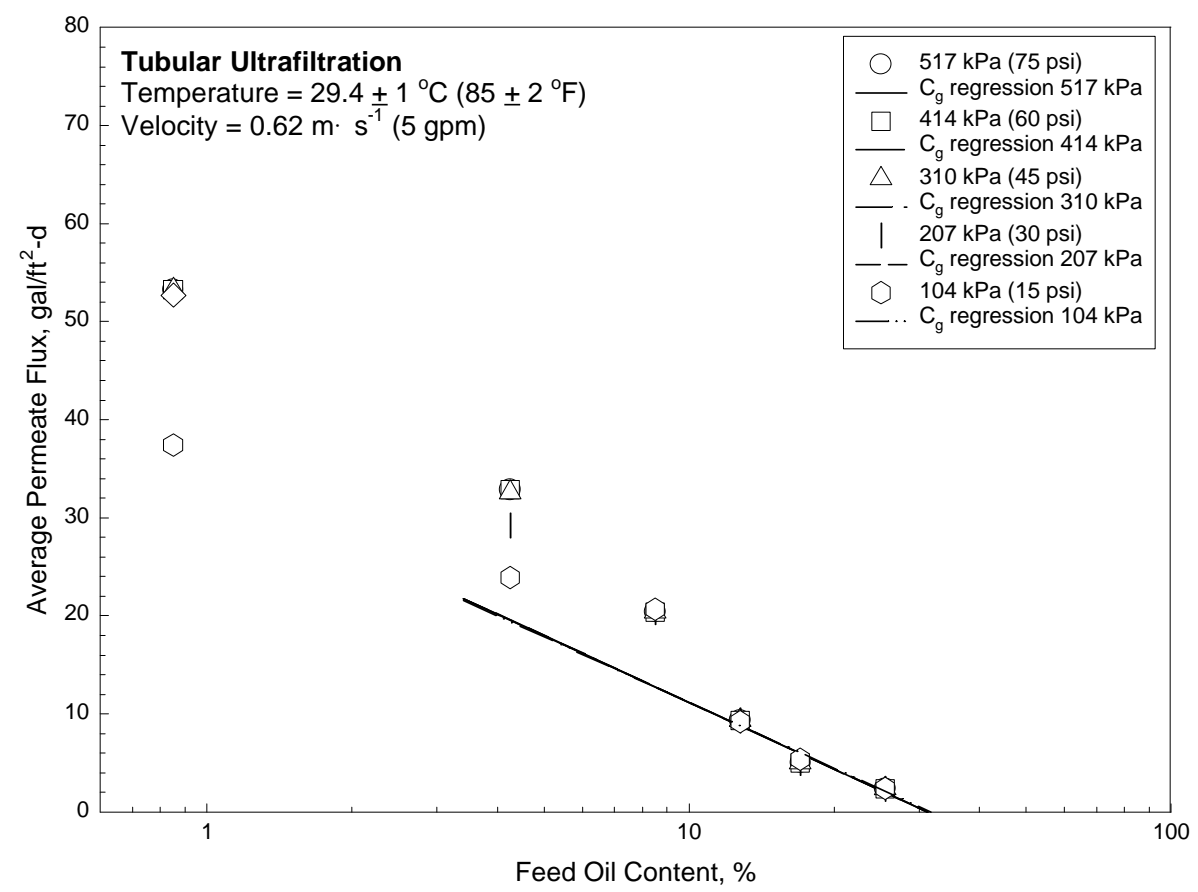

Figure 5.7 Average steady state permeate flux versus feed oil concentration for experiments conducted at $0.62 \mathrm{~m} \cdot \mathrm{s}^{-1}$.

variable and $\mathrm{OC}_{\text {gel }}$ as the dependent variable. In each case, $\mathrm{F}_{\mathrm{A}} \approx 1$ and $\mathrm{P}_{\mathrm{A}}>0.01\left(\mathrm{~F}_{\mathrm{A}}=3\right.$

and $8 ; \mathrm{P}_{\mathrm{A}}=0.18$ and 0.21 for $\mathrm{P}$ and $\mathrm{v}$, respectively); thus, it was concluded that $\boldsymbol{O C}_{\text {gel }}$ was not statistically dependent on either operating parameter in the tubular UF system.

Table 5.1 Gel layer oil concentrations and mass transfer coefficients for each v/P combination examined in the tubular unit.

\begin{tabular}{|c|c|c|c|c|c|c|}
\hline \multirow{2}{*}{$\begin{array}{c}\text { Average Relative } \\
\text { Velocity (v), } \rightarrow \\
\text { Average } \\
\text { Transmembrane } \\
\text { Pressure (P), } \\
\downarrow\end{array}$} & \multicolumn{2}{|c|}{$\begin{array}{c}0.62 \mathrm{~m} \cdot \mathrm{s}^{-1} \\
(5 \mathrm{gpm})\end{array}$} & \multicolumn{2}{|c|}{$\begin{array}{l}1.2 \mathrm{~m} \cdot \mathrm{s}^{-1} \\
(10 \mathrm{gpm})\end{array}$} & \multicolumn{2}{|c|}{$\begin{array}{l}2.5 \mathrm{~m} \cdot \mathrm{s}^{-1} \\
(20 \mathrm{gpm})\end{array}$} \\
\hline & $\begin{array}{l}\mathrm{OC}_{\text {gel, }}, \\
\% \text { oil }\end{array}$ & $\begin{array}{c}\mathbf{k}_{\mathrm{s}}, \\
\mathrm{m}^{3} \cdot \mathbf{m}^{-2} \cdot \mathbf{d}^{-1}\end{array}$ & $\begin{array}{l}\mathrm{OC}_{\text {gel, }} \\
\% \text { oil }\end{array}$ & $\begin{array}{c}\mathbf{k}_{\mathrm{s}}, \\
\mathbf{m}^{3} \cdot \mathbf{m}^{-2} \cdot \mathbf{d}^{-1}\end{array}$ & $\begin{array}{l}\mathrm{OC}_{\text {gel, }} \\
\% \text { oil }\end{array}$ & $\begin{array}{c}k_{s}, \\
m^{3} \cdot m^{-2} \cdot d^{-1}\end{array}$ \\
\hline 207 kPa (30 psi) & 31 & 0.94 & 31 & 2.8 & 29 & 9.0 \\
\hline 310 kPa (45 psi) & 31 & 0.94 & 31 & 2.8 & 29 & 8.9 \\
\hline 414 kPa (60 psi) & 31 & 0.94 & 31 & 2.8 & 29 & 9.2 \\
\hline 517 kPa (75 psi) & 31 & 0.94 & 31 & 2.9 & 29 & 10.9 \\
\hline Average & 31 & 0.94 & 31 & 2.8 & 29 & 9.5 \\
\hline
\end{tabular}




\subsubsection{High-Shear Rotary Ultrafiltration}

Average permeate flux values were plotted versus $\log (\mathrm{OC})$ for each $\mathrm{P} / \mathrm{v}$ condition to examine application of the thin film model. Only experiments conducted at feed kinematic viscosities of 0.46 to $8.1 \mathrm{cSt}$ (5 to $40 \%$ MW fluid) were included in the initial application of the thin-film model. Semi-log plots of average permeate flux versus feed oil concentration for experiments conducted at 12,10 , and $8 \mathrm{~m} \cdot \mathrm{s}^{-1}$ are presented in Figures 5.8, 5.9, and 5.10, respectively. The gel layer concentration was estimated by extrapolating the linear portion (i.e., pressure-independent data) of each plot to $\mathrm{J}^{*}=0$. Gel layer oil concentrations and mass transfer coefficients were determined for each v/P experiment using the HSRUF system and are presented in Table 5.2. No more than two pressure-independent fluxes were attained in the $173 \mathrm{kPa}$ (25 psi) pressure conditions examined at each velocity. Further, only two pressure-independent fluxes were attained at the 241 and $310 \mathrm{kPa}$ pressure conditions conducted at $12 \mathrm{~m} \cdot \mathrm{s}^{-1}$; thus, these data were excluded from further analysis.

Table 5.2. Gel layer oil concentrations and mass transfer coefficients for each v/P combination examined in the HSRUF unit.

\begin{tabular}{|c|c|c|c|c|c|c|}
\hline \multirow{2}{*}{$\begin{array}{c}\text { Average Relative } \\
\text { Velocity (v), } \rightarrow \\
\text { Average } \\
\text { Transmembrane } \\
\text { Pressure (P), } \\
\downarrow\end{array}$} & \multicolumn{2}{|c|}{$\begin{array}{c}8 \mathrm{~m} \cdot \mathrm{s}^{-1} \\
(1150 \mathrm{rpm})\end{array}$} & \multicolumn{2}{|c|}{$\begin{array}{c}10 \mathrm{~m} \cdot \mathrm{s}^{-1} \\
(1450 \mathrm{rpm})\end{array}$} & \multicolumn{2}{|c|}{$\begin{array}{c}12 \mathrm{~m} \cdot \mathrm{s}^{-1} \\
(1750 \mathrm{rpm})\end{array}$} \\
\hline & $\begin{array}{l}\mathrm{OC}_{\mathrm{gel}}, \\
\% \text { oil }\end{array}$ & $\begin{array}{c}\mathbf{k}_{\mathrm{s}} \\
\mathbf{m}^{3} \cdot \mathbf{m}^{-2} \cdot \mathbf{d}^{-1}\end{array}$ & $\begin{array}{l}\text { OC }_{\text {gel, }} \\
\% \text { oil }\end{array}$ & $\begin{array}{c}k_{s}, \\
m^{3} \cdot m^{-2} \cdot d^{-1}\end{array}$ & $\begin{array}{l}\mathrm{OC}_{\text {gel, }} \\
\% \text { oil }\end{array}$ & $\begin{array}{c}k_{s}, \\
m^{3} \cdot m^{-2} \cdot d^{-1}\end{array}$ \\
\hline $241 \mathrm{kPa}$ (35 psi) & 42 & 6.92 & 40 & 7.77 & $2-{ }^{1}$ & 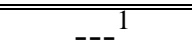 \\
\hline $310 \mathrm{kPa}(45 \mathrm{psi})$ & 40 & 6.92 & 36 & 7.65 & $-\mathrm{C}^{\mathrm{T}}$ & $-{ }^{1}$ \\
\hline $379 \mathrm{kPa}(55 \mathrm{psi})$ & 40 & 6.88 & 37 & 7.57 & 38 & 11.7 \\
\hline $448 \mathrm{kPa}(65 \mathrm{psi})$ & 40 & 6.88 & 37 & 9.52 & 38 & 11.4 \\
\hline $517 \mathrm{kPa}(75 \mathrm{psi})$ & 40 & 6.92 & 37 & 9.52 & 38 & 11.7 \\
\hline Average & 40 & 6.90 & 37 & 8.41 & 38 & 11.6 \\
\hline
\end{tabular}

${ }^{1}$ Only two pressure-independent data points observed. 


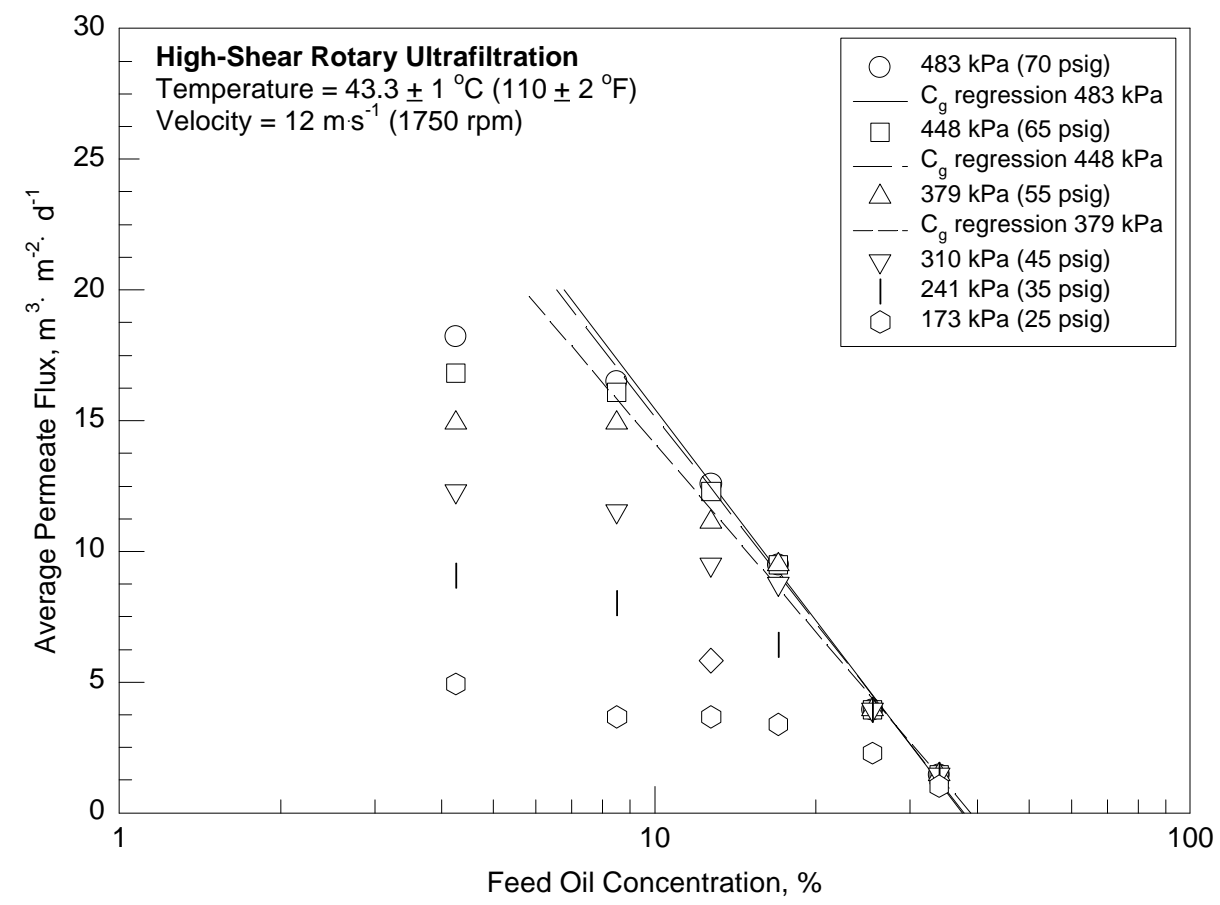

Figure 5.8 Average steady state permeate flux versus feed oil concentration for experiments conducted at $12 \mathrm{~m} \cdot \mathrm{s}^{-1}$.

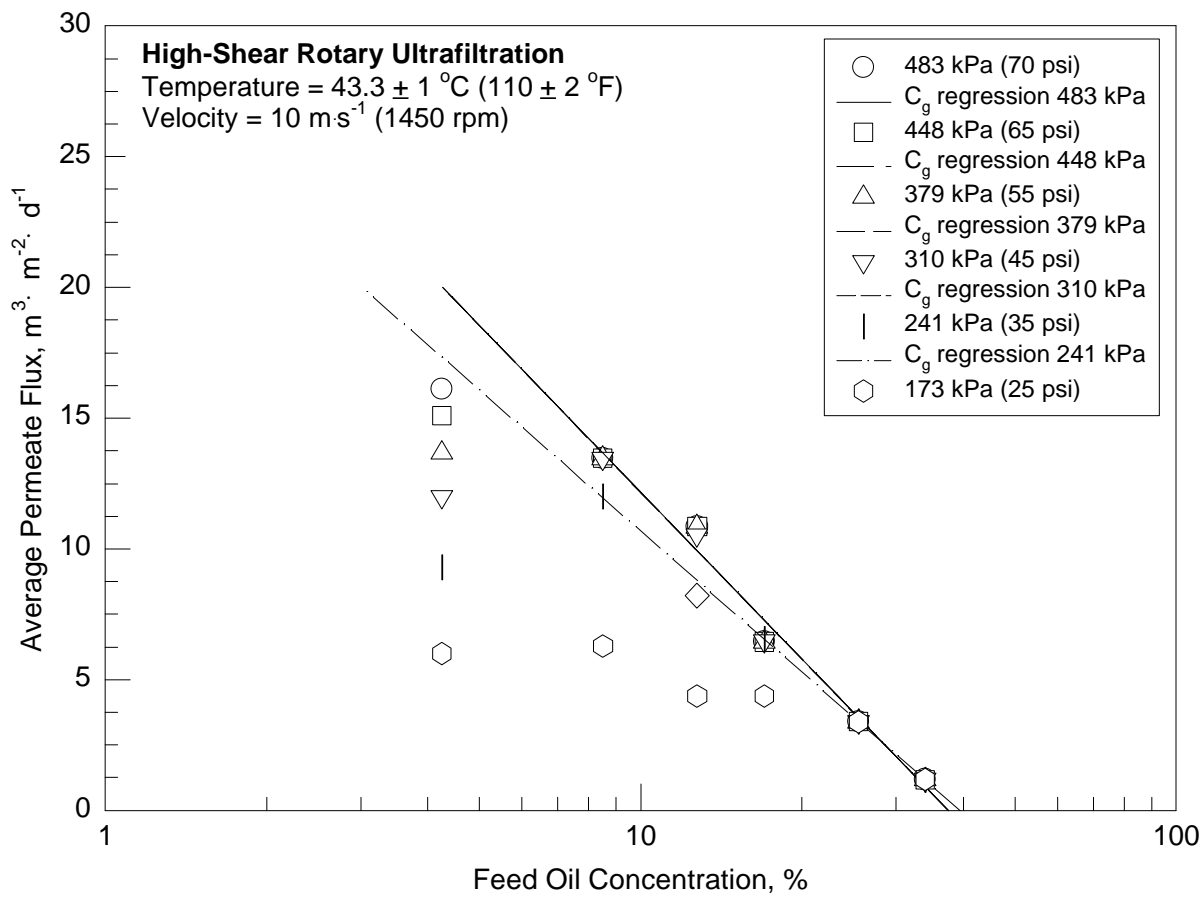

Figure 5.9 Average steady state permeate flux versus feed oil concentration for experiments conducted at $10 \mathrm{~m} \cdot \mathrm{s}^{-1}$. 


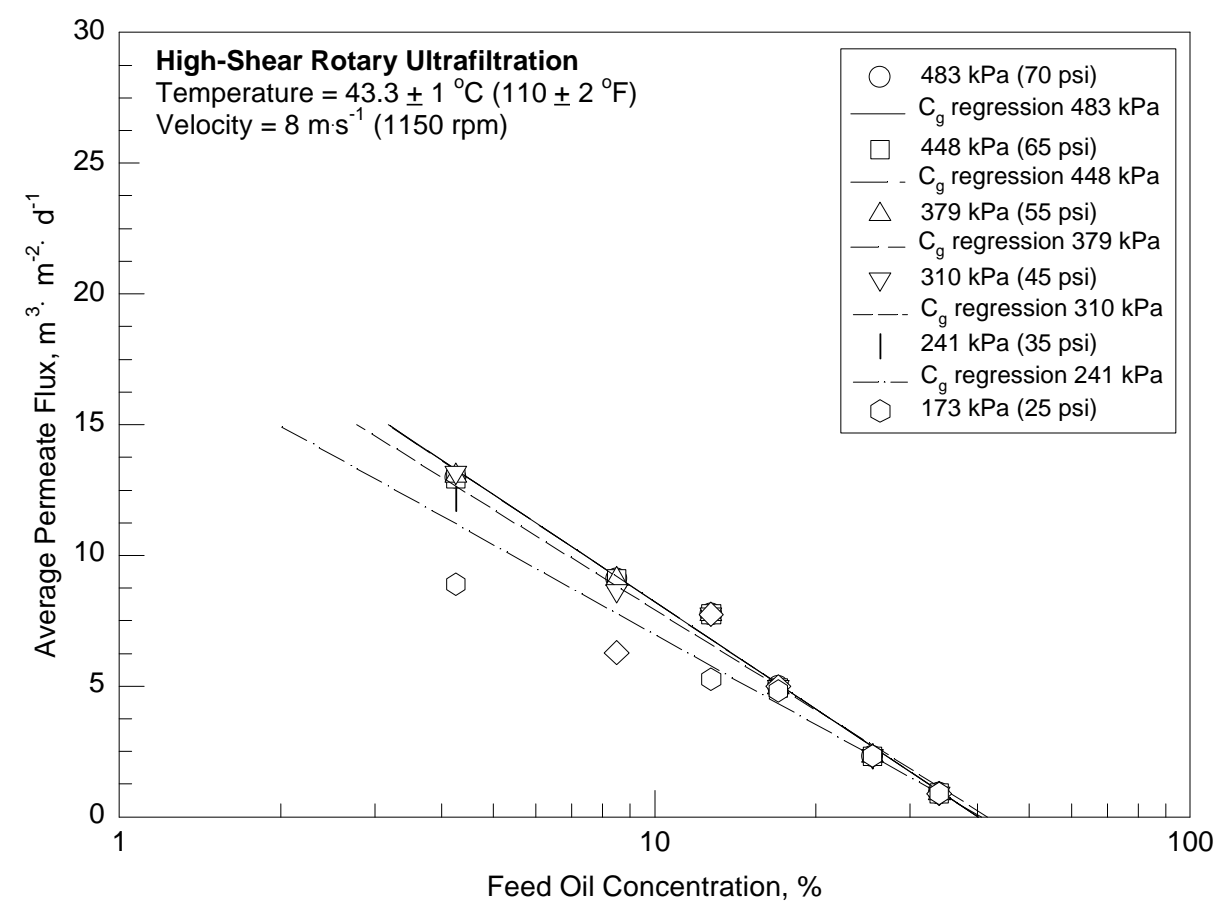

\section{Figure 5.10 Average steady state permeate flux versus feed oil concentration for experiments conducted at $8 \mathrm{~m} \cdot \mathrm{s}^{-1}$.}

In the HSRUF system, $\mathrm{OC}_{\text {gel }}$ ranged from 37 to $42 \%$ oil and averaged $39 \%$ oil. A single-factor analysis of variance (ANOVA) was conducted with $\mathrm{P}$ as the independent variable and $\mathrm{OC}_{\mathrm{gel}}$ as the dependent variable to ascertain the dependence of $\mathrm{OC}_{\mathrm{gel}}$ on $\mathrm{P}$ and $\mathrm{v}$. Similar ANOVA analyses were conducted with $\mathrm{v}$ as the independent variable and $\mathrm{OC}_{\mathrm{gel}}$ as the dependent variable. In each case, $\mathrm{F}_{\mathrm{A}} \approx 1$ and $\mathrm{P}_{\mathrm{A}}>0.01\left(\mathrm{~F}_{\mathrm{A}}=3\right.$ and $1 ; \mathrm{P}_{\mathrm{A}}=$ 0.15 and 0.45 for $\mathrm{P}$ and $\mathrm{v}$, respectively); thus, it was concluded that $\boldsymbol{O C}_{\text {gel }}$ was not statistically dependent on either operating parameter in the HSRUF system.

Average gel layer oil concentrations from this study were in close agreement with values of $35 \%$ and $40 \%$ reported by Lipp et al. (1988) and Lee et al. (1984) for the treatment of synthetic oil-in-water emulsions. In direct concentration experiments using the HSRUF system, Reed et al. (1997b) reported an $\mathrm{OC}_{\mathrm{gel}}$ of $\sim 50 \%$ for a ceramic membrane and 58 to $59 \%$ oil for a polyvinylidene fluoride (PVDF) membrane. The 
membranes examined by Reed et al. were constructed of the same materials and had the same average pore sizes as the tubular PVDF and the rotary ceramic membranes examined in this study. The MW fluid used in this study was a synthetic version of the waste mill coolant used by Reed et al. Thus, the discrepancy between the $39 \% \mathrm{OC}_{\mathrm{gel}}$ calculated in this study and $\mathrm{OC}_{\mathrm{gel}}$ reported by Reed et al. was most likely due to the differences between the freshly prepared synthetic emulsions and waste MW fluids. In particular, a waste MW fluid is typically less stable than a freshly prepared emulsion; thus, the repulsive force between oil droplets is typically lower and the "closest packed" arrangement of oil droplets is higher for a waste MW fluid. Additionally, solids present in waste emulsions could potentially affect solute boundary layer properties (e.g., decrease solute boundary layer thickness and thus lower the resistance to hydraulic flow) (Viadero and Reed 1999).

In the thin-film model the gel layer concentration is defined as a physico-chemical property of the feed solution, which is independent of operating parameters. However, the average gel layer oil concentrations determined for the two units examined in this study were substantially different from one another $(31 \%$ and $39 \%$ for the tubular and HSRUF systems, respectively), for an identical feed solution (synthetic MW fluid). It is hypothesized that elevated hydraulic turbulence in the HSRUF system $\left(\operatorname{Re}_{\max }=180,000\right.$, as opposed to $\operatorname{Re}_{\text {max }}=90,000$ in the tubular system) resulted in a wider particle size distribution and a subsequent closer packed arrangement of oil droplets at the membrane surface. Thus, an increased gel layer oil concentration was observed during HSRUF operation and it was determined that the gel layer was not a true physico-chemical property of the synthetic MW fluid examined in this study. Consequently, the validity of 
the thin-film model in describing data obtained from the ultrafiltration of oily feed streams is in question.

\subsubsection{Mass Transfer Coefficient}

\subsubsection{Tubular Ultrafiltration}

Mass transfer coefficients $\left(\mathrm{k}_{\mathrm{s}}\right)$ for each $\mathrm{v} / \mathrm{P}$ experiment were presented previously in Table 5.1. The mass transfer coefficient was calculated as the negative slope of the regression line fitted to pressure-independent data on the semi-log plots of permeate flux versus OC (presented previously in Figures 5.5, 5.6, and 5.7). A single-factor ANOVA was conducted with transmembrane pressure as the independent variable and $\mathrm{k}_{\mathrm{s}}$ as the dependent variable. An $\mathrm{F}_{\mathrm{A}}$ of 4 and a $\mathrm{P}_{\mathrm{A}}$ of 0.18 were determined. Thus, it was concluded that $\boldsymbol{k}_{s}$ was not dependent on $\boldsymbol{P}$, which was expected since mass transfer coefficients were calculated using pressure-independent data. Similar ANOVA analyses were performed with $\mathrm{v}$ and $\mathrm{k}_{\mathrm{s}}$ as the independent and dependent variables, respectively. An $\mathrm{F}_{\mathrm{A}}$ of 81 and a $\mathrm{P}_{\mathrm{A}}$ of 0.05 were determined; thus, it was concluded that a statistical relationship existed between average relative velocity and the mass transfer coefficient in the tubular $\boldsymbol{U F}$ system. Increasing $\mathrm{v}$ results in enhanced turbulence at the membrane surface, therefore the thickness of the concentration boundary layer is minimized. Consequently, the mass transfer coefficient is increased.

A $\mathrm{k}_{\mathrm{s}}$ of $\sim 1.1 \mathrm{~m}^{3} \cdot \mathrm{m}^{-2} \cdot \mathrm{d}^{-1}\left(\sim 28 \mathrm{gal} \cdot \mathrm{ft}^{-2} \cdot \mathrm{d}^{-1}\right)$ was calculated from data presented by Goldsmith et al. (1974) for the treatment of an oily wastewater using a conventional tubular UF system at a cross-flow velocity of $3.75 \mathrm{~m} \cdot \mathrm{s}^{-1}$ (30 gpm). The mass transfer coefficient calculated from Goldsmith's data was comparable to the $\mathrm{k}_{\mathrm{s}}$ values of $\sim 1 \mathrm{~m}^{3} \cdot \mathrm{m}$ ${ }^{-2} \cdot \mathrm{d}^{-1}\left(\sim 25 \mathrm{gal} \cdot \mathrm{ft}^{-2} \cdot \mathrm{d}^{-1}\right)$, determined from the experiments conducted in this study at a 
cross-flow velocity of $0.62 \mathrm{~m} \cdot \mathrm{s}^{-1}$. Overall, the mass transfer coefficient ranged from $\sim 1$ to $9 \mathrm{~m}^{3} \cdot \mathrm{m}^{-2} \cdot \mathrm{d}^{-1}$ (23 to $216 \mathrm{gal} \cdot \mathrm{ft}^{-2} \cdot \mathrm{d}^{-1}$ ) for data collected from the tubular UF system.

$\mathrm{J}^{*}$ was predicted using the thin-film model (Equation 2.27) with a constant $\mathrm{OC}_{\mathrm{gel}}$ of $31 \%$ and average $\mathrm{k}_{\mathrm{s}}$ values determined at each $\mathrm{v}$ using the tubular UF system (as summarized previously in Table 5.1). Plots of experimental data and thin-film model predictions of limiting permeate flux versus feed oil concentration for the tubular UF system are presented in Figure 5.11. For each v, model predictions deviate from experimental data at low oil concentrations $(\leq 10 \%$ oil), due to operation near pressuredependent conditions. The $\mathrm{R}^{2}$ values were $0.85,0.96$, and 0.98 for model predictions at cross-flow velocities of $0.62,1.23$, and $2.47 \mathrm{~m} \cdot \mathrm{s}^{-1}$, respectively. Thus, it was concluded that the thin-film model adequately described the limiting flux data collected during tubular UF operation. The lowest $\mathrm{R}^{2}$ value (0.85) was observed for model predictions at a cross-flow velocity of $0.62 \mathrm{~m} \cdot \mathrm{s}^{-1}$, due to the low permeate flux values observed in experiments conducted at $0.62 \mathrm{~m} \cdot \mathrm{s}^{-1}$. Predicted values did not deviate substantially from the observed flux values in comparison to other cross-flow velocities; however, the relationships of the deviations to the actual flux values were more substantial.

\subsubsection{High-Shear Rotary Ultrafiltration}

Mass transfer coefficients $\left(\mathrm{k}_{\mathrm{s}}\right)$ for each $\mathrm{v} / \mathrm{P}$ experiment were presented previously in Table 5.2. The mass transfer coefficient was calculated as the negative slope of the regression line fitted to pressure-independent data on the semi-log plots of permeate flux versus OC (presented previously in Figures 5.8, 5.9, and 5.10). A single-factor ANOVA was conducted with transmembrane pressure as the independent variable and $\mathrm{k}_{\mathrm{s}}$ as the dependent variable. An $\mathrm{F}_{\mathrm{A}}$ of 6 and a $\mathrm{P}_{\mathrm{A}}$ of 0.09 were determined. As with tubular $\mathrm{UF}$, it 


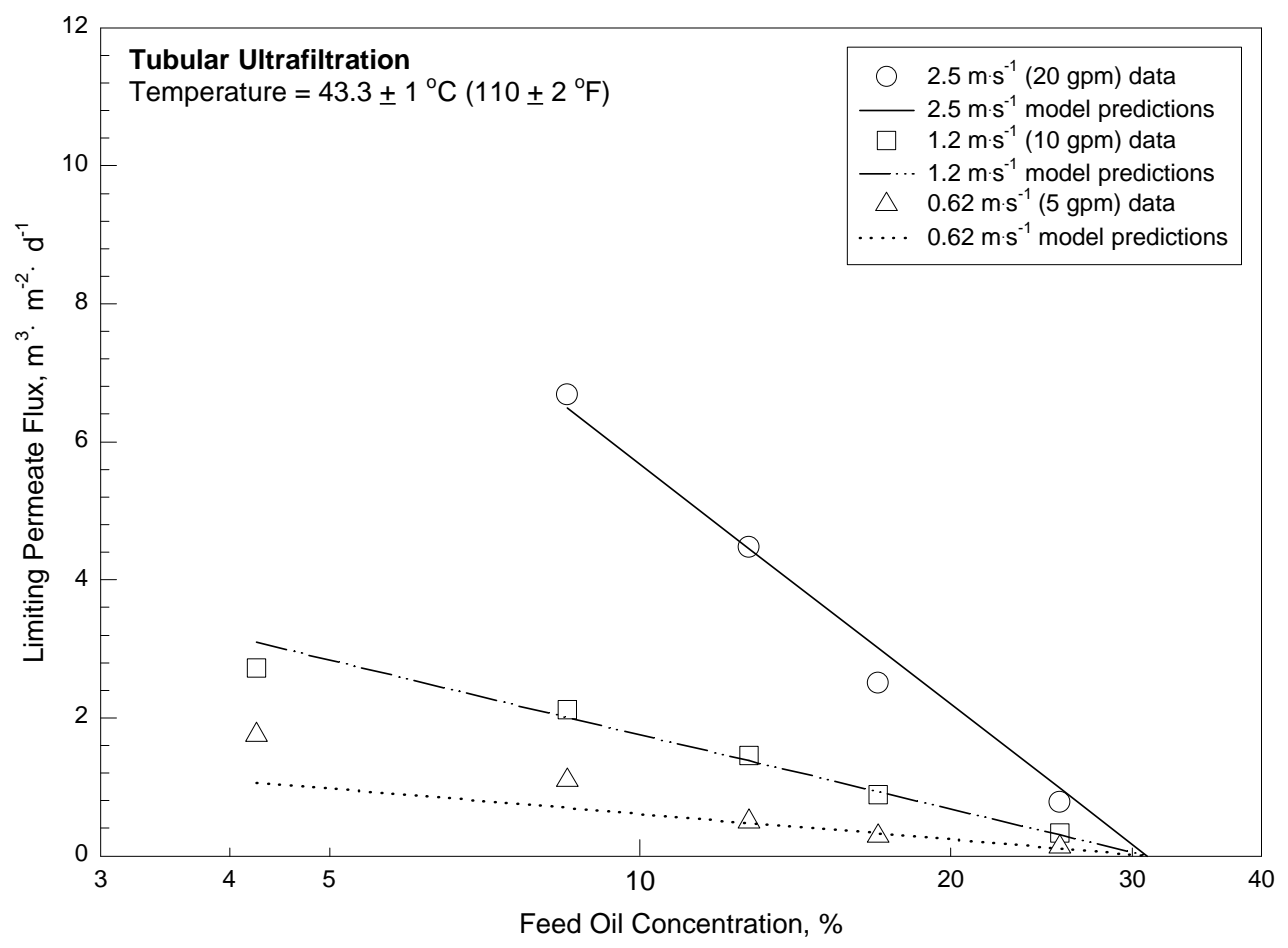

Figure 5.11 Plots of experimental data and thin-film model predictions of limiting flux versus feed oil concentration for the tubular UF system.

was concluded that $\boldsymbol{k}_{s}$ was not dependent on P in the HSRUF system. Similar ANOVA analyses were performed with $\mathrm{v}$ and $\mathrm{k}_{\mathrm{s}}$ as the independent and dependent variables, respectively. An $\mathrm{F}_{\mathrm{A}}$ of 24 and a $\mathrm{P}_{\mathrm{A}}$ of 0.04 were determined; thus, it was concluded that a statistical relationship existed between average relative velocity and the mass transfer coefficient in the HSRUF system.

The HSRUF system was more effective than conventional membrane separation systems at attaining a high $\mathrm{k}_{\mathrm{s}} \cdot \quad \mathrm{A} \mathrm{k}_{\mathrm{s}}$ of $1.14 \mathrm{~m}^{3} \cdot \mathrm{m}^{-2} \cdot \mathrm{d}^{-1}\left(\sim 28 \mathrm{gal} \cdot \mathrm{ft}^{-2} \cdot \mathrm{d}^{-1}\right)$ was calculated from data presented by Goldsmith et al. (1974) for the treatment of an oily wastewater using a conventional tubular UF system at a cross-flow velocity of $3.75 \mathrm{~m} \cdot \mathrm{s}^{-1}\left(\sim 12 \mathrm{ft} \cdot \mathrm{s}^{-1}\right)$. Further, the mass transfer coefficients determined for the tubular UF system examined in this study ranged from $\sim 1$ to $9 \mathrm{~m}^{3} \cdot \mathrm{m}^{-2} \cdot \mathrm{d}^{-1}$ (23 to $\left.216 \mathrm{gal} \cdot \mathrm{ft}^{-2} \cdot \mathrm{d}^{-1}\right)$. In comparison, average $\mathrm{k}_{\mathrm{s}}$ values determined from HSRUF data ranged from 7 to $10 \mathrm{~m}^{3} \cdot \mathrm{m}^{-2} \cdot \mathrm{d}^{-1}$ (171 to $246 \mathrm{gal} \cdot \mathrm{ft}$ 
$\left.{ }^{-2} \cdot \mathrm{d}^{-1}\right)$. The mass transfer characteristics observed in the HSRUF system were attributed to the decoupling of the recirculation flow and the hydraulic conditions at the membrane surface. "Cleaning energy" in the HSRUF system is conveyed directly to the membrane surface through rotation and the thickness of the solute boundary layer was effectively minimized, resulting in a higher $\mathrm{k}_{\mathrm{s}}$ when compared with the conventional tubular UF system.

Additionally, $\mathrm{k}_{\mathrm{s}}$ determined using synthetic emulsions was greater than reported for waste emulsions in the HSRUF system. Reed et al. (1997b) presented $\mathrm{k}_{\mathrm{s}}$ values ranging from 1.41 to $1.86 \mathrm{~m}^{3} \cdot \mathrm{m}^{-2} \cdot \mathrm{d}^{-1}$ (35 to $46 \mathrm{gal} \cdot \mathrm{ft}^{-2} \cdot \mathrm{d}^{-1}$ ) using the HSRUF system equipped with $0.11 \mu \mathrm{m}$ ceramic and $100,000 \mathrm{MWCO}$ polymeric membranes, for the treatment of a waste version of the synthetic MW fluid investigated in this study. The experimental conditions used by Reed et al. were comparable to the $12 \mathrm{~m} \cdot \mathrm{s}^{-1}$ (1750 rpm) conditions in this study. However, the average $\mathrm{k}_{\mathrm{s}}$ of $11.6 \mathrm{~m}^{3} \cdot \mathrm{m}^{-2} \cdot \mathrm{d}^{-1}\left(285 \mathrm{gal} \cdot \mathrm{ft}^{-2} \cdot \mathrm{d}^{-1}\right)$ calculated at $12 \mathrm{~m} \cdot \mathrm{s}^{-1}$ in this study was $\sim 6$ to 8 times greater than that reported by Reed et al. for the treatment of a waste MW fluid. Since the stability and thus the repulsive force between oil droplets is typically greater in a synthetic MW fluid than in a waste $\mathrm{O} / \mathrm{W}$ emulsion, it is hypothesized that oil droplets were more easily removed from the membrane surface in the case of the synthetic emulsion. Consequently, a higher $\mathrm{k}_{\mathrm{s}}$ was determined for the synthetic MW fluid in the HSRUF system. However, future research on the ultrafiltration of synthetic and waste MW fluids must be conducted in order to support this hypothesis. 
The limiting flux was predicted using Equation 2.27 with the constant $\mathrm{OC}_{\text {gel }}$ of $39 \%$ and $\mathrm{k}_{\mathrm{s}}$ values determined at each average relative velocity examined in the HSRUF system. Plots of experimental data and thin-film model predictions of limiting permeate flux versus feed oil concentration for the HSRUF system are presented in Figure 5.12. To quantify how well the model predicted the limiting flux data for a given feed oil concentration, a coefficient of determination was calculated at each membrane rotational speed. The value of $\mathrm{R}^{2}$ was $0.98,0.97$, and 0.93 at 12,10 , and $8 \mathrm{~m} \cdot \mathrm{s}^{-1}$, respectively; thus, it was concluded that the limiting permeate flux data for experiments conducted at 0.46 to 8.1 cSt were well described by the thin-film model.

\subsubsection{Hydraulic Turbulence}

\subsubsection{Tubular Ultrafiltration}

Plots of limiting permeate flux versus Reynolds number $(\mathrm{Re})$ for experiments conducted at 4.3 through $26 \%$ oil ( 0.46 through $4.5 \mathrm{cSt})$ in the tubular UF system are presented in Figure 5.13. The power law relationship presented previously in Equation 2.9 was used as a means to judge the effect of hydraulic turbulence on the limiting permeate flux in the tubular UF unit and as a basis for comparison with other studies. The exponent, $\alpha$, in the power law relationship was calculated as the slope of the regression line fitted to $\log \left(\mathrm{J}^{*}\right)$ versus $\log (\mathrm{Re})$ data. An $\alpha$ of 0.63 was calculated at $4.3 \%$ oil $(0.46 \mathrm{cSt})$, while $\alpha$ ranged from 1.30 to 1.58 and averaged 1.44 for data collected at 8.5 through $26 \%$ oil ( 0.64 through $4.5 \mathrm{cSt}$ ). In experiments conducted at $4.3 \%$ oil, there were only two pressure-independent fluxes. Conditions at which three or more pressure- 


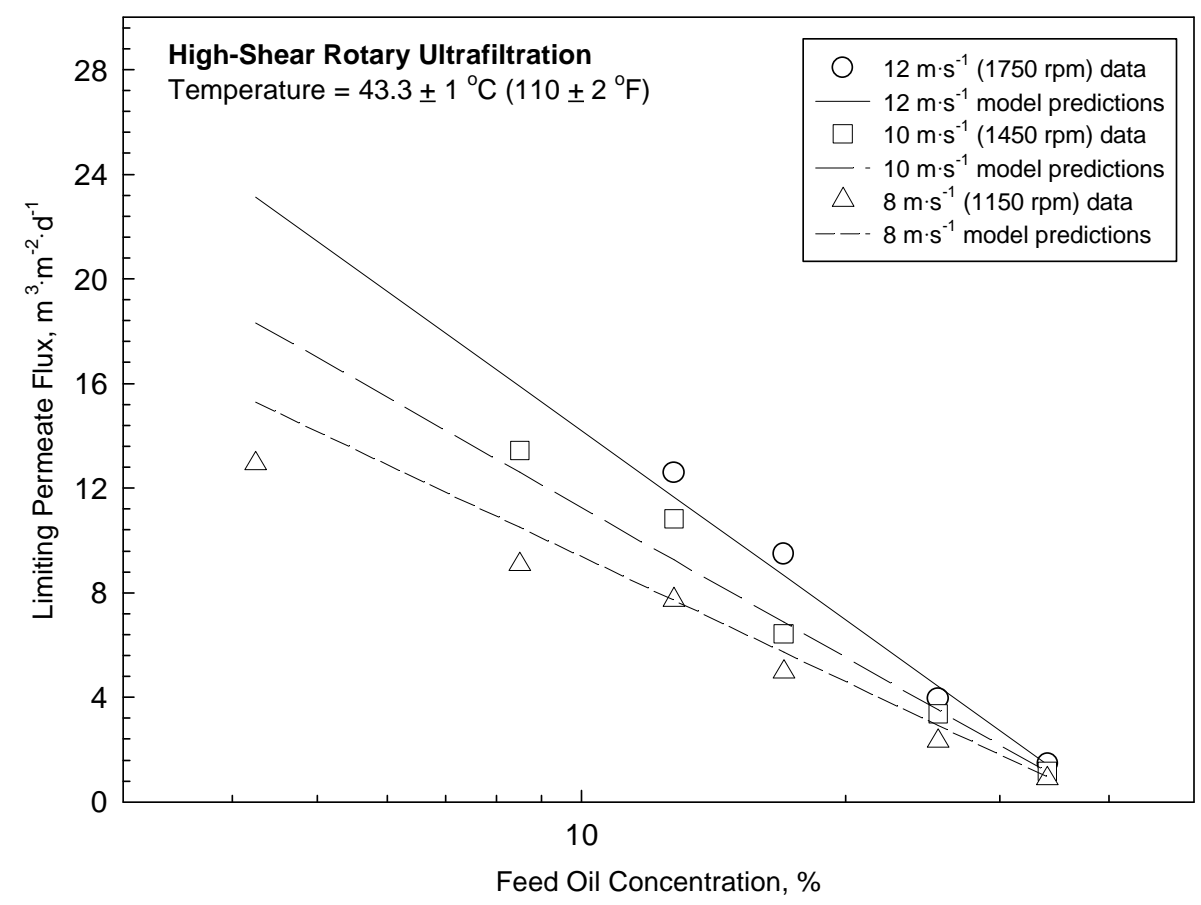

Figure 5.12 Experimental data and thin-film model predictions of limiting permeate flux versus feed oil concentration for the high-shear rotary unit.

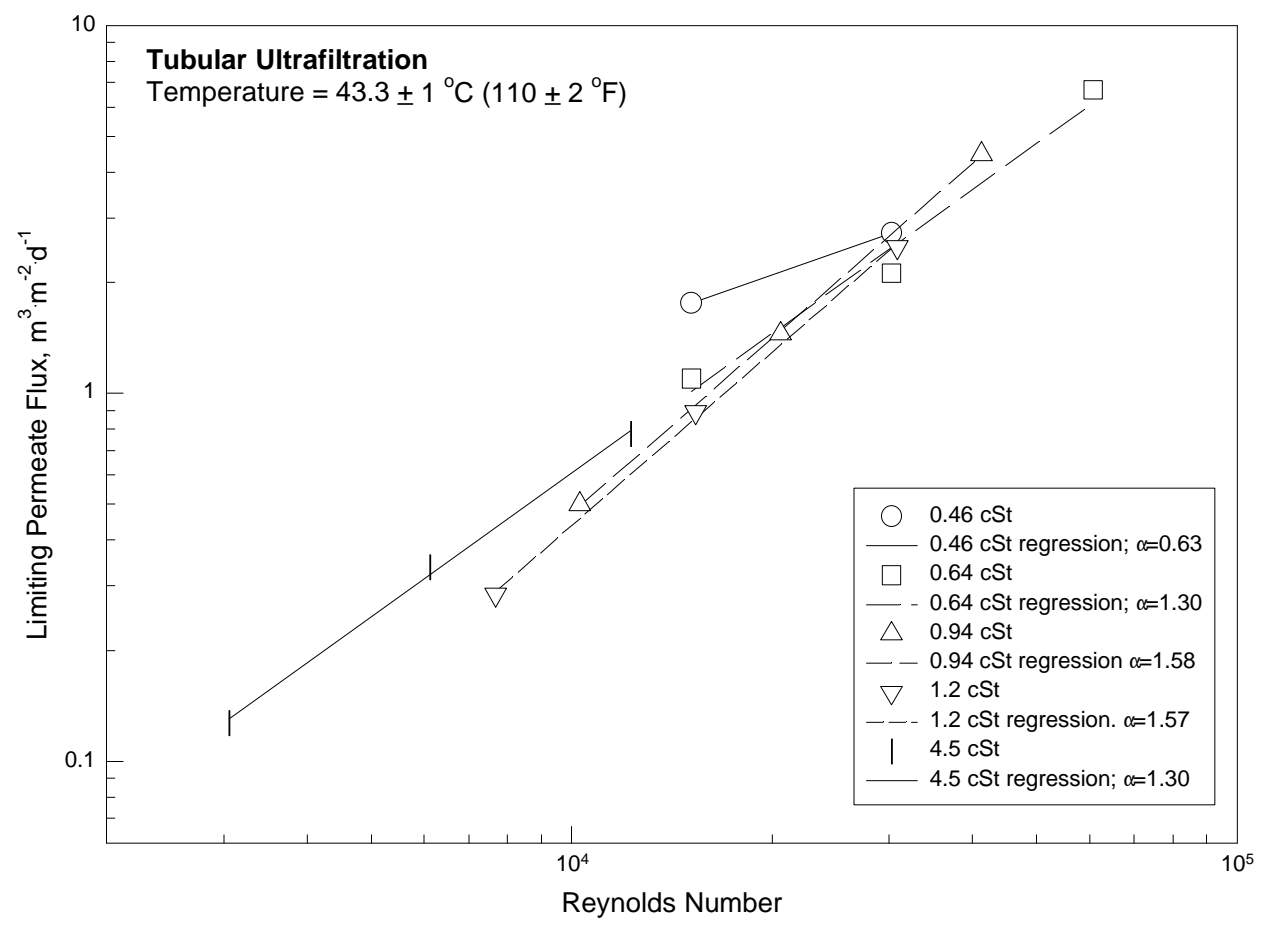

Figure 5.13 Limiting permeate flux versus Reynolds Number from experiments conducted using the tubular UF system. 
independent data points were attained were considered to be most representative of limiting flux behavior; thus, the $4.3 \%$ oil data were excluded from further analysis.

Values of $\alpha$ observed in the tubular UF experiments conducted in this study, 0.63 to 1.58 , were in close agreement with other data presented in the literature. Cheryan (1998) reported typical $\alpha$ values for conventional tubular UF systems of 0.8 to 1.2 for turbulent flow conditions. Similarly, Goldsmith et al. (1974) reported an $\alpha$ value of 1.2 in the tubular UF of a $1.7 \%$ oily waste for $1.0 \times 10^{5} \leq \operatorname{Re} \leq 1.5 \times 10^{5}$.

\subsubsection{High-Shear Rotary Ultrafiltration}

Plots of limiting permeate flux versus average radial Reynolds number $\left(\mathrm{Re}_{\mathrm{r} \text {-avg }}\right.$; see Equation 2.7) for experiments conducted at 4.3 through $34 \%$ oil $(0.46$ through $8.1 \mathrm{cSt}$ ) in the HSRUF system are presented in Figure 5.14. The exponent, $\alpha$, ranged from 1.4 at 4.3 and $8.5 \%$ oil ( 0.46 and $0.64 \mathrm{cSt})$ to 1.5 at 13 through $34 \%$ oil $(0.94$ through $8.1 \mathrm{cSt})$. A distinct break in $\mathrm{J}^{*}$ versus $\mathrm{Re}_{\mathrm{r}-\text { avg }}$ data was observed at $2 \times 10^{5} \leq \mathrm{Re}_{\mathrm{r}-\mathrm{avg}} \leq 4 \times 10^{5}$. According to Ketola and McGrew (1968), hydraulic flow conditions are turbulent for $\operatorname{Re}_{\mathrm{r}}$

$>2 \times 10^{5}$ in a rotary disk membrane system in which the stationary turbulence promoter was a solid plate. Thus, it was hypothesized that the break in $\mathrm{J}^{*}$ versus $\mathrm{Re}_{\mathrm{r}-\mathrm{avg}}$ data was due to a shift from turbulent to laminar hydraulic flow conditions as the oil concentration was increased. However, in this study the criteria presented by Ketola and McGrew was used only as an approximate guideline for the cut-off between laminar and turbulent hydraulic flow due to the presence of the "wagon wheel" turbulence promoters in the HSRUF system.

Values of $\alpha$ observed in the tubular and HSRUF experiments examined in this study were in close agreement with each other. In the tubular UF experiments, $\alpha$ 


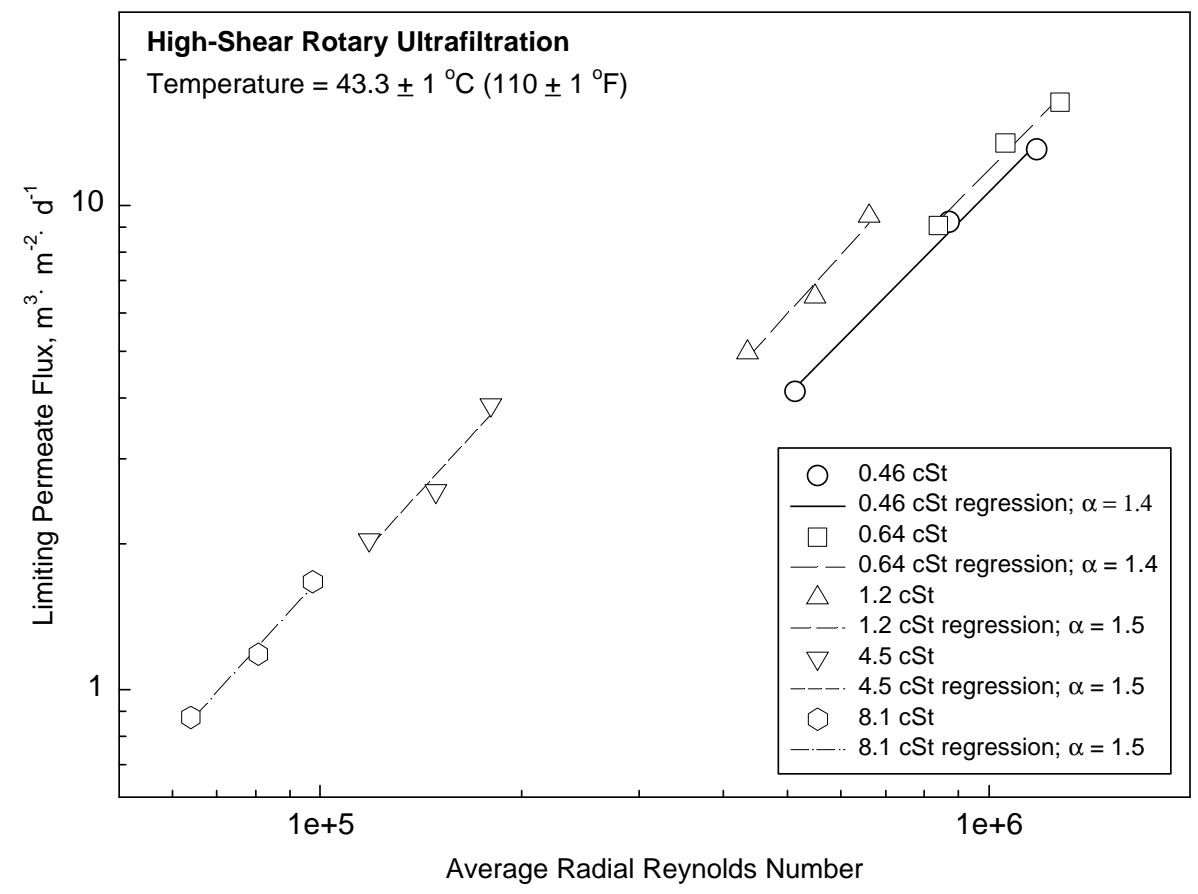

Figure 5.14 Limiting permeate flux versus average radial Reynolds Number from experiments conducted using the HSRUF system.

decreases from 1.57 to 1.30 at 17 to $26 \%$ oil (1.2 to $4.5 \mathrm{cSt}$ ), respectively. However, in the HSRUF system, $\alpha$ was sustained at 1.4 to 1.5 throughout the range of oil concentrations examined due to a more effective decrease in solute boundary layer thickness as a result of membrane rotation-induced hydraulic turbulence.

Further, a higher net permeate flux was observed in the HSRUF than in the tubular UF system at each oil concentration examined. Limiting flux values of $\sim 9$ to 16 $\mathrm{m}^{3} \cdot \mathrm{m}^{-2} \cdot \mathrm{d}^{-1}$ were observed in the HSRUF system at a feed oil concentration of $0.85 \%$ as compared to values of $\sim 0.8$ to $5 \mathrm{~m}^{3} \cdot \mathrm{m}^{-2} \cdot \mathrm{d}^{-1}$ observed in the tubular UF system. Additionally, a substantial decrease in permeate flux was observed as the oil concentration was increased in the tubular system; whereas, oil concentration had less of an affect on permeate flux in the HSRUF unit. Thus, it was possible to reach oil concentrations in the HSRUF system which were unattainable with the conventional 
tubular UF module due to the efficient delivery of cleaning energy to the membrane surface through the effective decoupling of feed pressurization from recirculation/hydraulic turbulence. In tubular UF, increases in viscosity associated with higher oil concentrations make it difficult to maintain the cross-flow velocity necessary to effectively scour the membrane of accumulated emulsified-oil particles, and a decrease in permeate flux is observed. Thus, it is envisioned that conventional tubular and HSRUF modules may be operated in conjunction as a hybrid separation system for treatment applications where high feed concentrations are desired.

$\mathrm{J}^{*}$ and thus the $\alpha$ value in Equation 2.9 are functions of physical and chemical properties of the feed solution (e.g., diffusivity and viscosity) and system operating conditions/characteristics (e.g., temperature, the extent of membrane fouling, etc.). Reed et al. (1997b) reported an $\alpha$ value of 0.90 determined in a membrane rotational speed excursion $\left(1,750\right.$ to $1,000 \mathrm{rpm} ; 12$ to $\left.7 \mathrm{~m} \cdot \mathrm{s}^{-1}\right)$ using a $17 \%$ waste oil solution in the HSRUF system. The experimental conditions reported by Reed et al. were comparable to the $17 \%$ oil $(1.2 \mathrm{cSt})$ experiments conducted in this study in which an $\alpha$ value of 1.5 was determined. Since the MW fluid used in this study was a virgin version of the waste studied by Reed et al., it was hypothesized that the "age" of the MW fluid affected the performance of the HSRUF system. In the case of the synthetic MW fluid, it was easier to scour oil droplets from the membrane surface as hydraulic turbulence was increased because of the greater repulsive forces between oil droplets, as presented earlier in section 5.2.2.2 when considering the mass transfer coefficient. Thus, the $\alpha$ values determined in this study using the HSRUF system were greater and limiting flux changed 
more rapidly with hydraulic turbulence than that reported by Reed et al. for the waste emulsion.

Additionally, the rotational excursion in the study reported by Reed et al. was conducted in the middle of a direct concentration experiment. As a result, other factors such as membrane fouling may have also contributed to lower $\mathrm{J}^{*}$ and $\alpha$ values reported by Reed et al. Thus, pilot-scale experiments must be conducted using actual waste streams to properly establish the $\mathrm{J}^{*}-\mathrm{Re}$ relationship, while the general relationships determined in this study may be used as a basis for comparison between the tubular and HSRUF systems as well as other membrane separation systems under controlled conditions.

\subsubsection{Two Region Limiting Flux}

Six additional experiments were conducted using the HSRUF to examine application of the thin-film model to high feed oil concentrations. Feed kinematic viscosities of 45.6 and $121 \mathrm{cSt}$ (50 and 60\% MW fluid) were examined at each of the average relative velocities $\left(8,10\right.$, and $\left.12 \mathrm{~m} \cdot \mathrm{s}^{-1}\right)$. Semi-logarithmic plots of $\mathrm{J}^{*}$ versus OC for each average relative velocity examined in the HSRUF synthetic MW fluid experiments are presented in Figure 5.15. Linear regression analyses were conducted to ascertain changes in limiting flux with concentration. A summary of regression parameters for the data presented in Figure 5.15 is presented in Table 5.3. At oil concentrations less than $34 \%$, the $\mathrm{J}^{*}$ versus OC relationship was linear (Region 1). Based upon Region 1 data, an $\mathrm{OC}_{\mathrm{gel}}$ of $39 \%$ oil was determined for all membrane rotational speeds as presented earlier. In contrast, a gel layer oil concentration in excess of $100 \%$ was ascertained at each of the three rotational speeds for OC $\geq 34 \%$ oil (Region 2). Thus, 


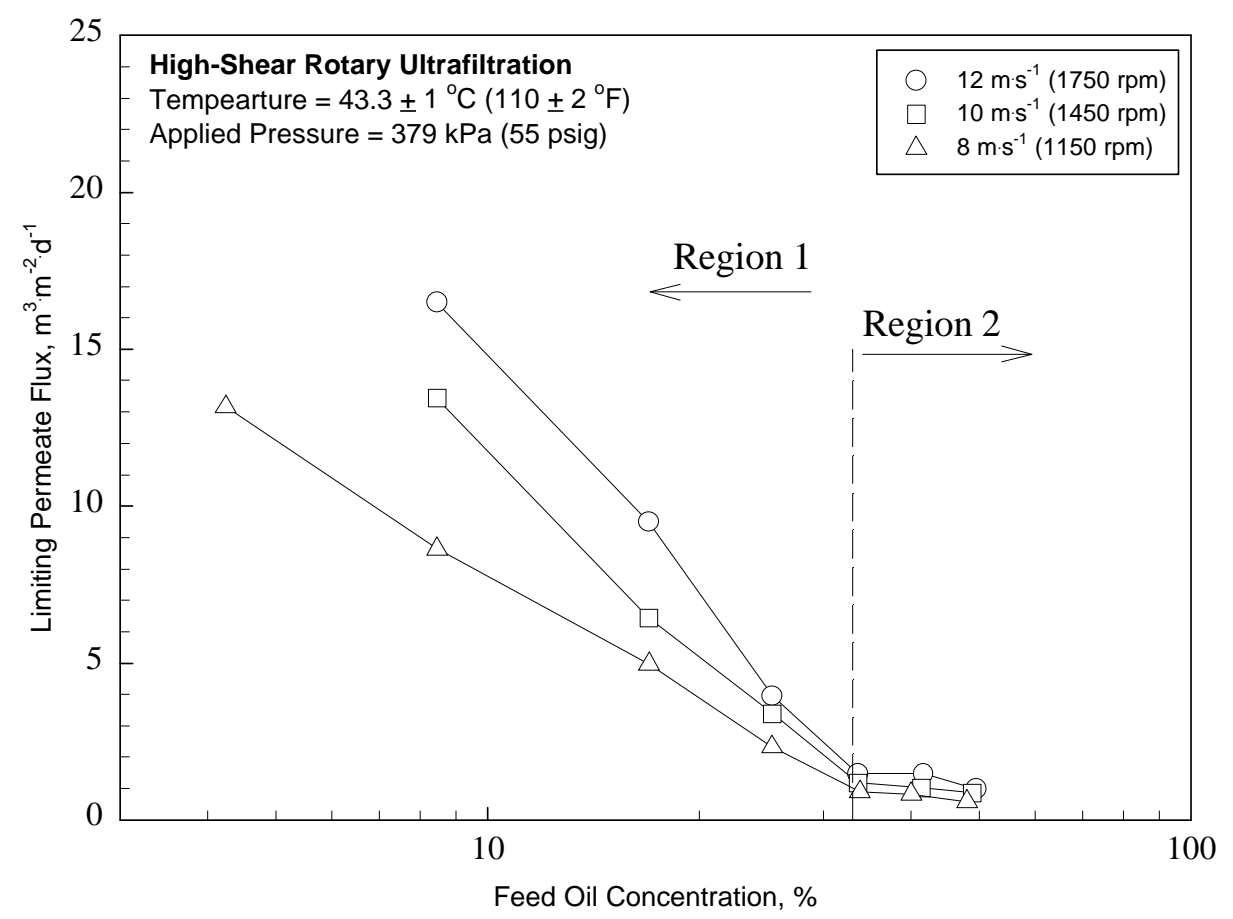

Figure 5.15 Limiting permeate flux versus feed oil concentration for the high-shear rotary synthetic metal-working fluid experiments conducted at $379 \mathrm{kPa}$.

a two region limiting flux was ascertained as oil concentration was increased in the HSRUF system. Due to the limitations of conventional UF technologies, $25.5 \%$ oil was the highest feed concentration examined in the tubular UF experiments; thus, a two region limiting flux was not ascertained in the tubular UF system. Reports of a two region limiting flux are sparse in the literature due to: (1) a lack of controlled studies in which the phenomena was observed and (2) a limited ability to attain high solute concentrations in conventional UF modules (Cheryan 1998).

Table 5.3 Summary of regression parameters for HSRUF synthetic MW fluid experiments.

\begin{tabular}{|c|l|c|}
\hline & \multicolumn{2}{|c|}{ Slope, $\mathbf{~ m}^{\mathbf{3}} \cdot \%$ oil $^{-\mathbf{2}} \cdot \mathbf{d}^{\mathbf{- 1}}\left(\mathbf{r}^{\mathbf{2}}\right)$} \\
\hline Average Relative Velocity, $\mathbf{~ m} \cdot \mathbf{s}^{\mathbf{- 1}}$ & Region 1 & Region 2 $^{*}$ \\
\hline 12 & $-26(0.99)$ & $-2.89(0.70)$ \\
\hline 10 & $-21(0.99)$ & $-2.0(0.99)$ \\
\hline 8 & $-14(0.99)$ & $-2.7(0.94)$ \\
\hline
\end{tabular}

See Figure 5.15 for graphical identification of regions 1 and 2. 
A semi-logarithmic plot of $\mathrm{J}^{*}$ versus OC for data collected using the waste MW fluid is presented in Figure 5.16. Permeate flux data were pressure dependent below 26\% oil; thus, these data are not presented in Figure 5.16. A summary of regression parameters for the waste MW fluid experiment is presented in Table 5.4. At oil contents ranging from 26 to $43 \%, \mathrm{~J}^{*}$ decreased linearly with $\mathrm{OC}$ (Region 1) and an $\mathrm{OC}_{\mathrm{gel}}$ of $58 \%$ oil was determined. However, at oil contents $>43 \%$ (Region 2 ), the rate of change in $\mathrm{J}^{*}$ with $\mathrm{OC}$ decreased in comparison to Region 1 and an $\mathrm{OC}_{\mathrm{gel}}$ in excess of $100 \%$ was determined, as observed previously for experiments conducted using the synthetic MW fluid.

Table 5.4 Summary of regression parameters for the HSRUF waste MW fluid experiment.

\begin{tabular}{|c|c|c|}
\hline ( & \multicolumn{2}{|c|}{ Slope, $\mathrm{m}^{3} \cdot \%$ oil $\cdot \mathrm{m}^{-2} \cdot \mathrm{d}^{-1}\left(\mathrm{r}^{2}\right)$} \\
\hline Average Relative Velocity, $\mathrm{m} \cdot \mathrm{s}^{-1}$ & Region $1^{*}$ & Region $2^{*}$ \\
\hline 12 & $-15.6(0.99)$ & $-4.83(0.93)$ \\
\hline
\end{tabular}

See Figure 5.16 for graphical identification of regions 1 and 2.

\subsubsection{Gel Layer Oil Concentration}

When the thin film theory is applied to Region 1 data, a gross underestimate of the HSRUF system's treatment capacity results, while an equivocal result of $\mathrm{OC}_{\mathrm{gel}}>$ $100 \%$ oil is ascertained when the thin film theory is applied to Region 2 data. In the thin film theory, the gel layer is idealized as the "closest-packed" arrangement of either rigid spherical or hexagonal particles; thus, a theoretical gel layer concentration of $\sim 74 \%$ solids is ascertained (Cheryan 1998; Blatt 1970). While emulsified oil droplets are macromolecular in size, the deformable nature of such a system makes it difficult to obtain meaningful results by applying the thin film theory. For example, an $85 \%$ oil solution (100\% base MW fluid) is homogeneous by appearance (i.e., no phase separation occurs). Therefore, one may assert that $85 \%$ oil is the closest packed arrangement of oil 


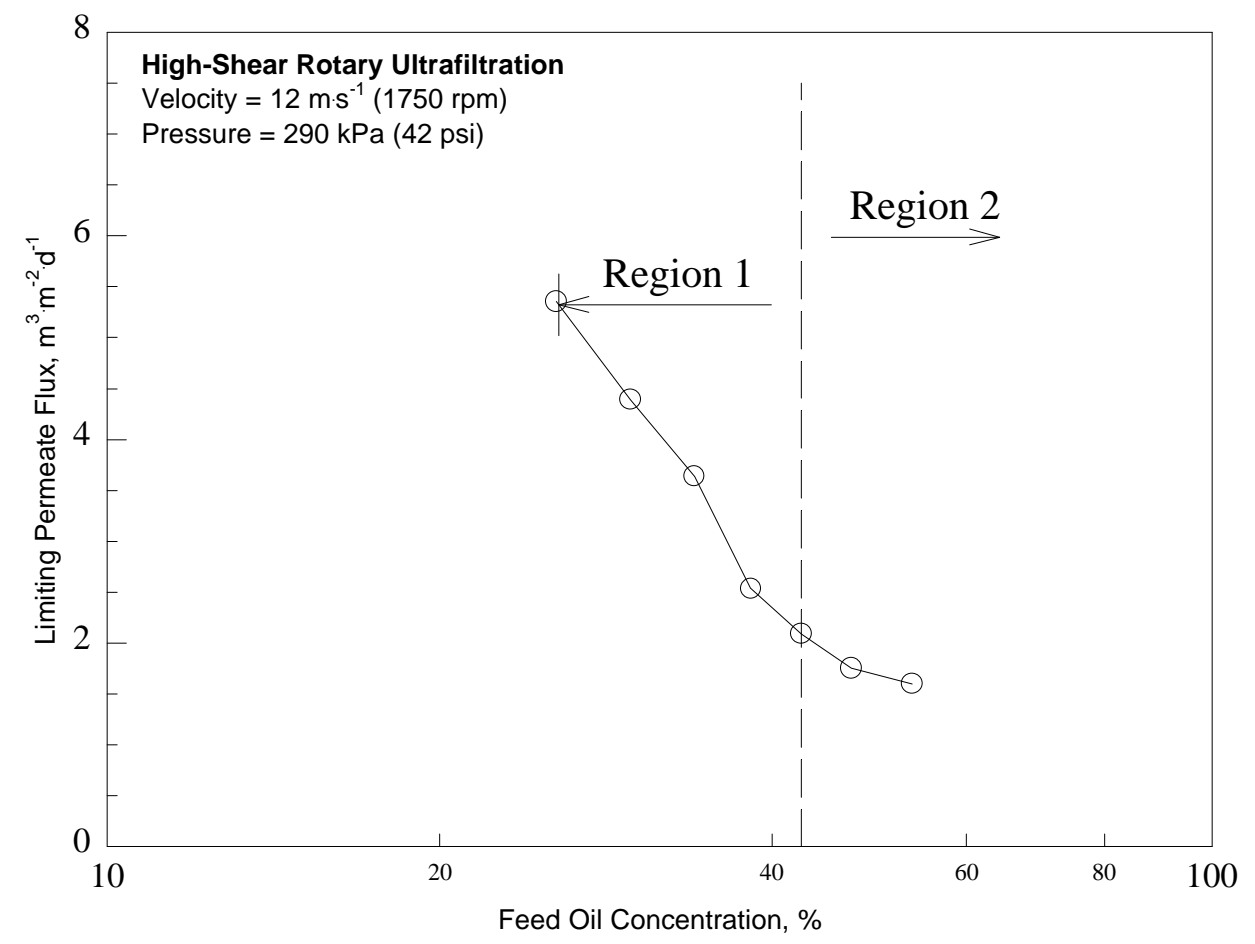

Figure 5.16 Limiting permeate flux versus feed oil concentration for the high-shear rotary waste metal-working fluid experiment.

droplets possible without phase separation. Furthermore, it can be argued that one could remove the low molecular weight additives from the MW fluid using UF to obtain oil concentrations $>85 \%$; however, phase separation would likely ensue and a continuous free-oil phase would result due to the removal of low molecular weight emulsifying agents. This theory was not verified experimentally in the HSRUF system to avoid inadvertently oil-wetting (and thus destroying) the membrane, which would preclude future experimentation.

\subsubsection{Mass Transfer Coefficient}

While the coefficients presented previously in Equations 2.25 and 2.26 were derived for a tubular membrane module, the functional relationships between variables can be used to draw analogous cause-effect relationships and a corresponding interpretation of physical phenomena in the HSRUF system. Unlike other studies on the 
separation of oil-in-water emulsions using membrane filtration (Lipp et al. 1988), it was not possible to make in situ measurements of the physico-chemical properties of the polarization layer in this study due to physical constraints imposed by the presence of the stationary shear elements and the rotating membrane.

In Section 5.2.2.2, it was shown that a statistically significant relationship existed between the mass transfer coefficient and the average relative velocity in the HSRUF synthetic MW fluid experiments. Thus, correspondingly higher limiting fluxes were observed at incrementally higher membrane rotational speeds in both Regions 1 and 2, as presented earlier in Figure 5.15. However, since the transition from Region 1 to Region 2 was observed at the same oil concentration for each of the three membrane rotational speeds, the influence of membrane rotation on the formation of the two region limiting flux was considered to be minimal. Further, Pritchard et al. (1995) reported that the influence of changes in solute diffusivity on the solute mass transfer coefficient are generally much less significant than changes in viscosity (which in turn have an influence on hydraulic turbulence and consequently, the thickness of the solute concentration boundary layer). Thus, further analyses were focused on the role of viscosity.

\subsubsection{Viscosity}

Kinematic viscosity versus oil concentration for the synthetic and waste MW fluids was presented previously in Figure 4.1. The transition to a two region limiting flux was observed at 34 and $43 \%$ oil in experiments conducted using the synthetic and waste MW fluids, respectively. In experiments conducted using the synthetic MW fluid, a rapid increase in the MW fluid kinematic viscosity was observed at oil concentrations in excess of $34 \%$ (6.6 cSt per $\%$ oil at concentrations $\geq 34 \%$ compared to $0.19 \mathrm{cSt}$ per $\%$ oil at 
concentrations < 34\%.) However, permeate flux in Region 2 decreased at a slower rate relative to Region 1 (see Table 5.3) at each membrane rotational speed for synthetic MW fluids. Similar observations were made in the waste MW fluid experiment in which MW fluid viscosity increased rapidly (16 cSt/\% oil) at concentrations $\geq 43 \%$ oil while permeate flux decreased slower relative to data observed at concentrations less than $43 \%$ (see Table 5.4).

As presented previously in section 2.4.2, the solute mass transfer coefficient has been reported as a function of solution properties for laminar and turbulent hydraulic conditions in tubular UF separation systems:

Turbulent flow:

$$
\mathrm{k}_{\mathrm{s}}=0.023\left(\frac{\mathrm{D}^{0.67} \mathrm{v}^{0.8}}{\mathrm{~d}_{\mathrm{h}}^{0.2} \eta^{0.47}}\right)
$$

Laminar flow:

$$
\mathrm{k}_{\mathrm{s}}=1.86\left(\frac{\mathrm{D}^{0.67} \mathrm{v}^{0.33}}{\mathrm{~d}_{\mathrm{h}}^{0.33} \mathrm{~L}^{0.33}}\right)
$$

Under conditions of turbulent hydraulic flow, the limiting flux decreases as the oil concentration is increased due to a corresponding increase in viscosity as presented in Equation 5.1. However, as the MW fluid concentration is increased, a corresponding increase in MW fluid viscosity is observed; thus, a transition from turbulent to laminar flow can occur due to a decrease in the Reynolds number (Equation 2.7). Under laminar flow conditions, the mass transfer coefficient is not a function of viscosity (Equation 5.2). Additionally, the slower flux decrease (observed in Region 2; Figure 5.15) and rapid viscosity increase (Figure 4.1 ) were observed at $\sim 35 \%$ oil. Consequently, this point 
( 35\% oil) was interpreted as a transition from turbulent to laminar flow conditions, as

reported in work performed on conventional membrane separation modules by Pritchard et al. (1995) and Cheryan (1998).

Due to the presence of the stationary shear elements, the exact transition from laminar to turbulent hydraulic flow in the HSRUF system is not known. Based on the viscosity data presented earlier in Figure 4.1, the average, maximum, and minimum radial Reynolds numbers for the synthetic MW fluid at 12,10 , and $8 \mathrm{~m} \cdot \mathrm{s}^{-1}$ and the waste MW fluid at $12 \mathrm{~m} \cdot \mathrm{s}^{-1}$ were calculated according to Equation 2.7 and are summarized in Table 5.5 .

While exact agreement between the transitional $\mathrm{Re}_{\mathrm{r}}$ calculated for the waste and synthetic MW fluids was not obtained, the average radial Reynolds number at the transition from Region 1 to Region 2 was below the transitional $\mathrm{Re}_{\mathrm{r}}$ of $2.0 \times 10^{5}$ reported for a solid plate turbulence promoter (Ketola and McGrew 1968). A more detailed study of the fluid mechanics in the HSRUF vessel must be conducted; however, based on these preliminary results, it is believed that the effect of the turbulence promoters was to enhance hydraulic turbulence in the HSRUF system. Consequently, the transition from laminar to turbulent hydraulic flow occurred at lower $\mathrm{Re}_{\mathrm{r}}$ in the HSRUF system than reported for a system operated using a solid plate turbulence promoter.

Table 5.5 Summary of maximum, average, and minimum radial Re at transitional oil concentrations for HSRUF synthetic and waste MW fluid experiments.

\begin{tabular}{|c|c|c|c|c|c||}
\hline $\begin{array}{c}\text { Transitional oil } \\
\text { concentration, } \\
\text { \% }\end{array}$ & $\begin{array}{c}\text { Transitional } \\
\text { viscosity, cSt }\end{array}$ & $\begin{array}{c}\text { Average } \\
\text { relative } \\
\text { velocity, m-s } \mathbf{s}^{-1}\end{array}$ & $\mathbf{R e}_{\text {r-max }}$ & $\mathbf{R e}_{\text {r-avg }}$ & $\mathbf{R e}_{\text {r-min }}$ \\
\hline \hline $43 \%$ (waste) & 16 & 12 & $9.4 \times 10^{4}$ & $5.1 \times 10^{4}$ & $6.8 \times 10^{3}$ \\
\hline $34 \%$ (synthetic) & 8.2 & 12 & $1.8 \times 10^{5}$ & $9.7 \times 10^{4}$ & $1.3 \times 10^{4}$ \\
\hline $34 \%$ (synthetic) & 8.2 & 10 & $1.5 \times 10^{5}$ & $8.1 \times 10^{4}$ & $1.1 \times 10^{4}$ \\
\hline $34 \%$ (synthetic) & 8.2 & 8 & $1.2 \times 10^{5}$ & $6.4 \times 10^{4}$ & $8.6 \times 10^{3}$ \\
\hline
\end{tabular}




\subsubsection{Consequences of Two Region Limiting Flux}

While the interpretation of a theoretically attainable oil concentration in excess of $100 \%$ is ambiguous, it is still feasible from a technical point of view to attain oil concentrations in the HSRUF system which are in excess of the performance capabilities of conventional UF separation modules. For example, Zaidi et al. (1992) reported a flux of 1.9 to $2.9 \mathrm{~m}^{3} \cdot \mathrm{m}^{-2} \cdot \mathrm{d}^{-1}\left(47\right.$ to $\left.71 \mathrm{gal} \cdot \mathrm{ft}^{-2} \cdot \mathrm{d}^{-1}\right)$ for the treatment of oilfield brine $(\sim 20 \%$ oil) in a hollow fiber UF system. Also, Reed et al. (1997a) reported a flux of $2.6 \mathrm{~m}^{3} \cdot \mathrm{m}^{-2} \cdot \mathrm{d}^{-1}$ $\left(63 \mathrm{gal} \cdot \mathrm{ft}^{-2} \cdot \mathrm{d}^{-1}\right)$ in a tubular UF module for the treatment a $20 \%$ emulsified oil wastewater. In contrast, a permeate flux ranging from $\sim 4 \mathrm{~m}^{3} \cdot \mathrm{m}^{-2} \cdot \mathrm{d}^{-1}\left(98 \mathrm{gal} \cdot \mathrm{ft}^{-2} \cdot \mathrm{d}^{-1}\right)$ at $8 \mathrm{~m} \cdot \mathrm{s}^{-1}$ to $\sim 7$ $\mathrm{m}^{3} \cdot \mathrm{m}^{-2} \cdot \mathrm{d}^{-1}\left(172 \mathrm{gal} \cdot \mathrm{ft}^{-2} \cdot \mathrm{d}^{-1}\right)$ at $12 \mathrm{~m} \cdot \mathrm{s}^{-1}$ was observed in the treatment of the synthetic MW fluid at $20 \%$ oil in the HSRUF system. Additionally, permeate flux values of 0.58 and $1.0 \mathrm{~m}^{3} \cdot \mathrm{m}^{-2} \cdot \mathrm{d}^{-1}\left(14\right.$ to $\left.25 \mathrm{gal} \cdot \mathrm{ft}^{-2} \cdot \mathrm{d}^{-1}\right)$ were observed at 8 and $12 \mathrm{~m} \cdot \mathrm{s}^{-1}$, respectively, in the HSRUF of a synthetic MW fluid at $51 \%$ oil; however, a similar flux of $1.3 \mathrm{~m}^{3} \cdot \mathrm{m}^{-2} \cdot \mathrm{d}^{-1}$ was observed at $3.7 \mathrm{~m} \cdot \mathrm{s}^{-1}$ and $26 \%$ oil in the tubular UF system. Thus, it was possible to concentrate oil beyond the typical operating limitations of conventional UF modules while maintaining an acceptable permeate flux.

Since capital and operating costs of membrane separation systems typically scale directly as a function of permeate flux ( Pickering and Wiesner 1993), it can be asserted that it is feasible to produce highly concentrated solutions at acceptable permeation rates while minimizing operating costs associated with pumping and membrane rotation in the HSRUF system. In Region 2, increased membrane rotational speed had little effect on the rate of flux decline; thus, operation at the minimum pressure necessary to attain limiting flux conditions while decreasing membrane rotational speed is a viable HSRUF 
system operating strategy in Region 2. However, since membrane fouling is more likely to occur at elevated feed concentrations (Cheryan 1998; Fane and Fell 1987), it is necessary to balance the cost savings of operation at lower membrane rotational speeds against the decrease in fouling potential and resulting increase in membrane process life cycle attained through operation at elevated membrane rotational speeds, as reported by Reed et al. (1997b).

Although the thin-film model adequately describes pressure-independent permeate flux behavior at low feed oil concentrations, a two-region limiting flux was observed as the feed concentration is increased. The resultant two-region flux corresponded to gel layer concentrations in excess of $100 \%$ oil, which is physically impossible; thus, the thin-film model is not adequate to describe the performance of membrane treatment applications where high feed concentrations are encountered. An alternative approach to permeate flux modeling is necessary to include a more diverse range of UF applications.

\subsection{SERIES RESISTANCES IN ULTRAFILTRATION PROCESSES}

Unlike the thin-film model, both the pressure-dependent and pressureindependent regions can be predicted using the resistance-in-series (RIS) approach. The RIS model was presented previously in section 2.4.3:

$$
J=\frac{P}{R_{m}{ }^{\prime}+\Phi \cdot P}
$$

Flux versus pressure data for the tubular and HSRUF units were fitted to Equation 5.3 using non-linear regression analysis (SigmaPlot ${ }^{\circledR}$ Version 5.0.5). To aid in understanding the data reduction process, a data reduction flow chart is presented in Figure 5.17. 


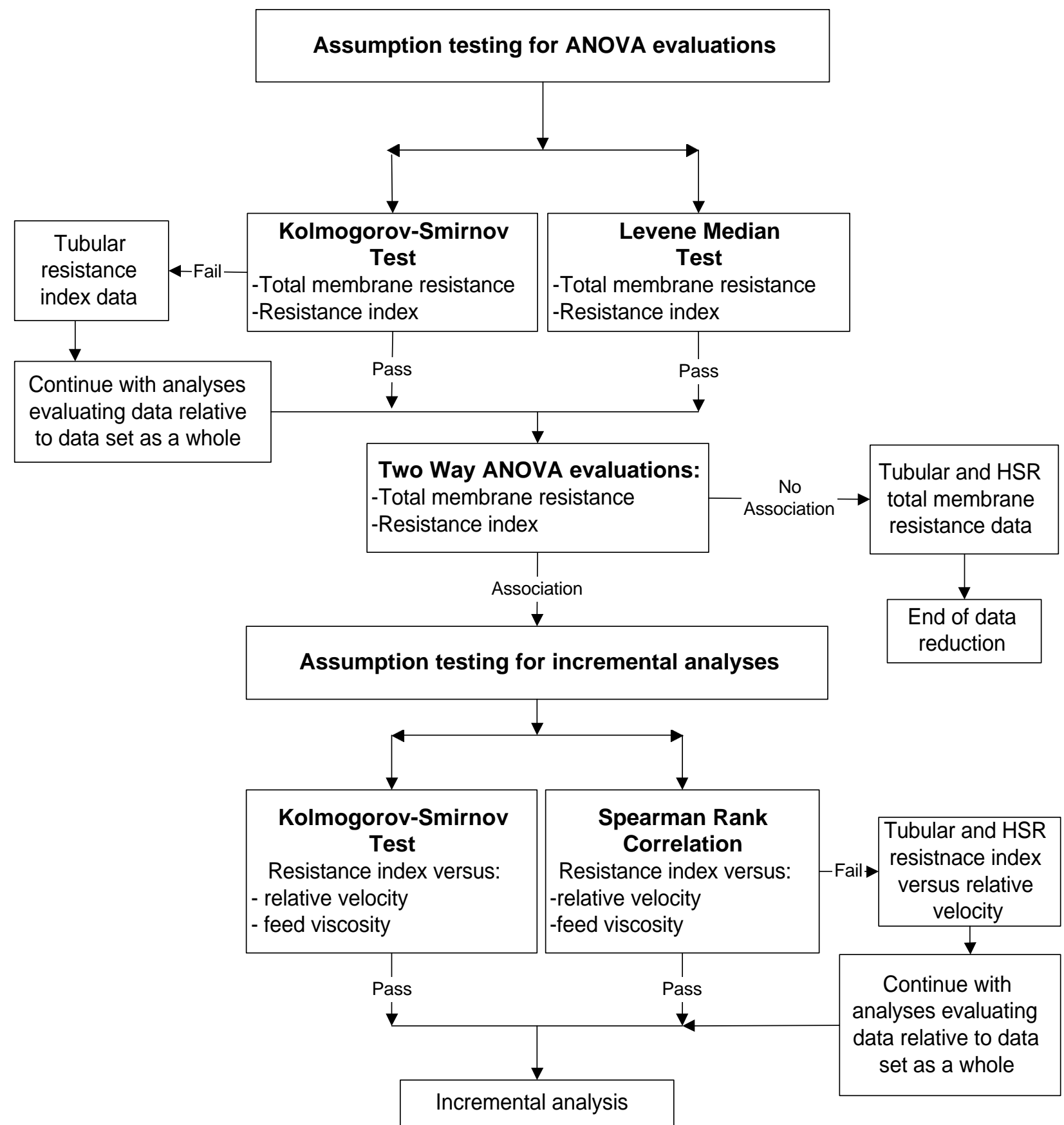

Figure 5.17 Data reduction flow chart for modified RIS model development. 


\subsubsection{Total Membrane Resistance}

As presented previously in section 2.4 .3 , total membrane resistance, $\mathrm{R}_{\mathrm{m}}{ }^{\prime}$, is a combination of the intrinsic membrane and fouling resistances:

$$
\mathrm{R}_{\mathrm{m}}{ }^{\prime}=\mathrm{R}_{\mathrm{m}}+\mathrm{R}_{\mathrm{f}}
$$

In the resistance-in-series approach, the total membrane resistance is inversely proportional to the permeate flux, as presented in Equation 5.3. Total membrane resistance values were determined for each discrete velocity/viscosity combination examined in the tubular and HSRUF systems by fitting Equation 5.3 to permeate flux versus transmembrane pressure data from the synthetic MW fluid.

\subsubsection{Tubular Ultrafiltration}

Velocity relative to a stationary reference frame (v) was calculated for each crossflow velocity examined in the tubular UF unit as a basis for comparison with the HSRUF system (see [Tubular] Experimental Apparatus, Section 3.1.1). Total membrane resistance values ranged from 10 to $1243 \mathrm{kPa} \cdot \mathrm{h} \cdot \mathrm{m}^{-1}$ for the tubular UF system. In the treatment of synthetic MW fluids, Nazzal and Wiesner (1996) reported $\mathrm{R}_{\mathrm{m}}{ }^{\prime}$ values of 24 to $240 \mathrm{kPa} \cdot \mathrm{h} \cdot \mathrm{m}^{-1}$ using a ceramic tubular membrane and Lipp et al. (1988) reported an $\mathrm{R}_{\mathrm{m}}{ }^{\prime}$ of $\sim 170 \mathrm{kPa} \cdot \mathrm{h} \cdot \mathrm{m}^{-1}$ using stirred UF cells. Similarly, Chiang and Cheryan (1986) reported an average $\mathrm{R}_{\mathrm{m}}{ }^{\prime}$ value of $\sim 864 \mathrm{kPa} \cdot \mathrm{h} \cdot \mathrm{m}^{-1}$, in the ultrafiltration of skimmilk using a hollow fiber module.

Total membrane resistance versus average relative velocity for each feed kinematic viscosity examined in the tubular UF unit is presented in Figure 5.18. A twofactor ANOVA was conducted (SigmaStat ${ }^{\circledR}$ Version 2.03) on tubular UF data with $\mathrm{v}$ and $\eta$ as the independent variables and $R_{m}{ }^{\prime}$ as the dependent variable to assess the 


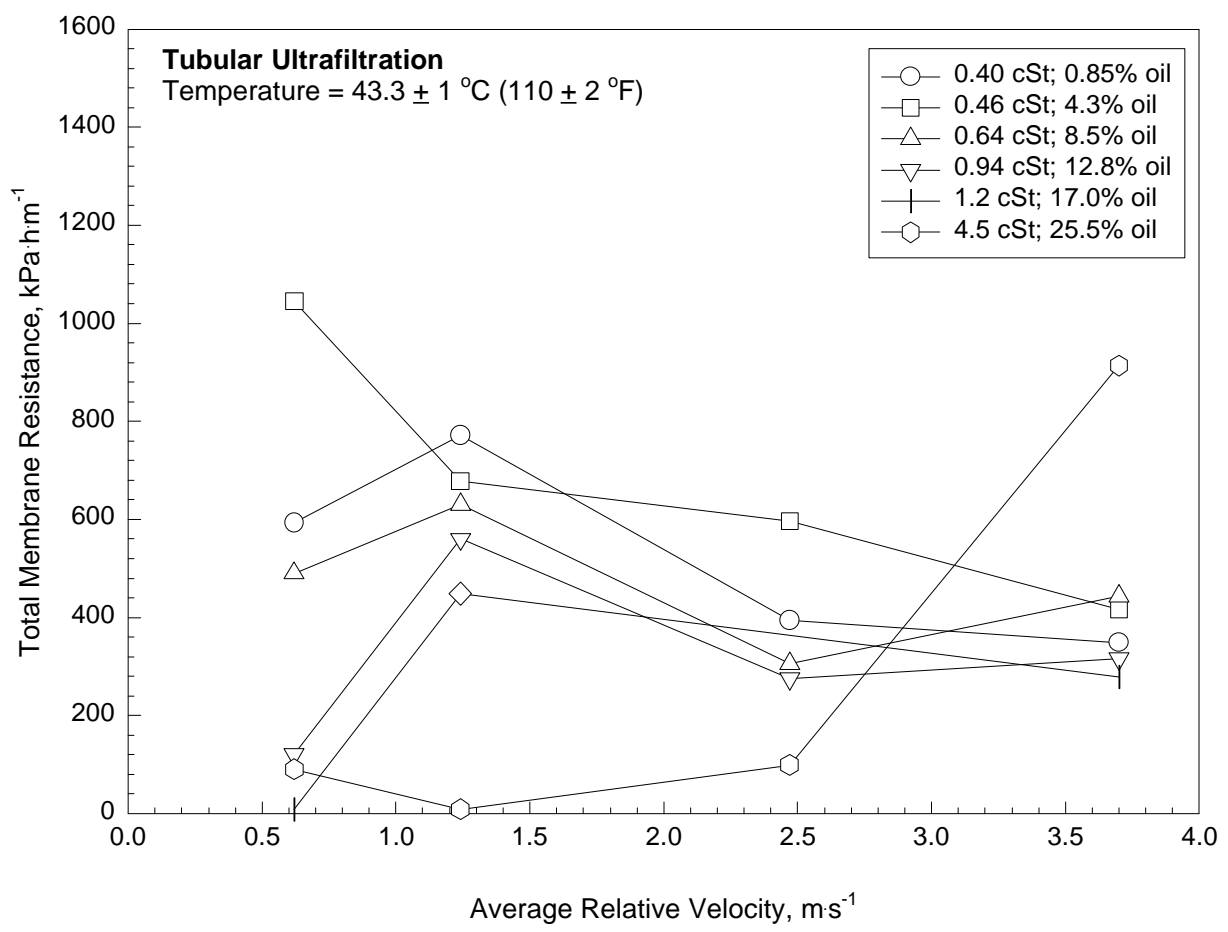

Figure 5.18 Total membrane resistance versus average relative velocity for each feed viscosity examined in the tubular UF unit.

dependence of total membrane resistance on feed viscosity and average relative velocity as well as testing for interactions between the independent variables (see Figure 5.17). Prior to performing ANOVA evaluations, the Kolmogorov-Smirnov and Levene Median tests were applied to check the normality and equal variance assumptions, respectively. $\mathrm{P}_{\mathrm{k}}$ and $\mathrm{P}_{\mathrm{L}}$ calculated from tubular UF resistance index data were 0.011 and 1.000, respectively. Both $\mathrm{P}$-values were greater than 0.01 (i.e., 99\% confidence); thus, it was concluded that the populations were normally distributed with equal variance and application of two-way ANOVA evaluation on tubular UF total membrane resistance data was valid.

$\mathrm{F}_{\mathrm{A}}$ and $\mathrm{P}_{\mathrm{A}}$ values calculated from the two-way ANOVA performed on tubular total membrane resistance versus velocity data were 1 and 0.524 , respectively. Similarly, $\mathrm{F}_{\mathrm{A}}$ and $\mathrm{P}_{\mathrm{A}}$ values calculated from the two-way ANOVA performed on tubular total membrane resistance versus viscosity data were 2 and 0.152 , respectively. For velocity 
and viscosity data, $\mathrm{F}_{\mathrm{A}} \approx 1$ and $\mathrm{P}_{\mathrm{A}}>0.01$; thus, it was concluded that $\boldsymbol{R}_{\boldsymbol{m}}{ }^{\prime}$ was not statistically dependent on feed viscosity or average relative velocity in the tubular UF system. Chiang and Cheryan (1986) also reported $\mathrm{R}_{\mathrm{m}}{ }^{\prime}$ to be independent of cross-flow velocity (average relative velocity) and feed concentration (viscosity) in the treatment of skimmilk using a hollow fiber UF module.

The average $\mathrm{R}_{\mathrm{m}}{ }^{\prime}$ value for all discrete experiments conducted with the tubular UF unit was $498 \mathrm{kPa} \cdot \mathrm{h} \cdot \mathrm{m}^{-1}$ and the intrinsic resistance of the virgin membrane, $\mathrm{R}_{\mathrm{m}}$, was determined to be $186 \mathrm{kPa} \cdot \mathrm{h} \cdot \mathrm{m}^{-1}$ (ascertained using clean water prior to the initiation of MW fluid experiments). The average fouling resistance, $\mathrm{R}_{\mathrm{F}}$, was calculated to be 312 $\mathrm{kPa} \cdot \mathrm{h} \cdot \mathrm{m}^{-1}$ (Equation 5.4), which corresponded to $63 \%$ of the average total membrane resistance; thus, the fouling and intrinsic resistances provided approximately equal contributions to the total membrane resistance. As presented previously, $\mathrm{R}_{\mathrm{m}}{ }^{\prime}$ was not a function of either cross-flow velocity or feed viscosity; thus, reversible fouling was determined to be a major factor controlling the fouling resistance.

\subsubsection{High-Shear Rotary Ultrafiltration}

As with the tubular UF unit, velocity relative to a stationary reference frame (v) was calculated for each membrane rotational speed examined in the HSRUF system as a basis for comparison of the two, membrane separation systems (see [High-Shear Rotary] Experimental Apparatus, Section 3.2.1). Total membrane resistance values ranged from 123 to $701 \mathrm{kPa} \cdot \mathrm{h} \cdot \mathrm{m}^{-1}$ in the HSRUF system and were slightly lower than $\mathrm{R}_{\mathrm{m}}{ }^{\prime}$ values observed in the tubular UF system; however, HSRUF values more closely agreed with data presented in the literature, as presented in section 5.3.1.1. 
Total membrane resistance versus average relative velocity for each feed kinematic viscosity examined in the HSRUF unit is presented in Figure 5.19. As presented previously with the tubular UF unit, a two-factor ANOVA was conducted (SigmaStat ${ }^{\circledR}$ Version 2.03) to assess the dependence of total membrane resistance on feed viscosity and average relative velocity in the HSRUF system. $\mathrm{P}_{\mathrm{k}}$ and $\mathrm{P}_{\mathrm{L}}$ calculated from HSRUF total membrane resistance data were 0.2 and 1.0, respectively. Both P-values were greater than 0.01 (i.e., $99 \%$ confidence); thus, it was concluded that the populations were normally distributed with equal variance and application of two-way ANOVA evaluation on HSRUF total membrane resistance data was valid.

$\mathrm{F}_{\mathrm{A}}$ and $\mathrm{P}_{\mathrm{A}}$ values calculated from the two-way ANOVA performed on HSRUF total membrane resistance versus velocity data were 1.0 and 0.426 , respectively. Similarly, $\mathrm{F}_{\mathrm{A}}$ and $\mathrm{P}_{\mathrm{A}}$ values calculated from the two-way ANOVA performed on HSRUF total membrane resistance versus viscosity data were 2.0 and 0.114 , respectively. As with the tubular UF unit, $\mathrm{F}_{\mathrm{A}} \approx 1$ and $\mathrm{P}_{\mathrm{A}}>0.01$; thus, it was concluded that $\boldsymbol{R}_{\boldsymbol{m}}{ }^{\prime}$ was not statistically dependent on feed viscosity or average relative velocity in the HSRUF system.

The average $\mathrm{R}_{\mathrm{m}}{ }^{\prime}$ (of all discrete $\mathrm{v} / \eta$ experiments) was $358 \mathrm{kPa} \cdot \mathrm{h} \cdot \mathrm{m}^{-1}$. The intrinsic resistance of the virgin membrane, $R_{m}$, was determined to be $314 \mathrm{kPa} \cdot \mathrm{h} \cdot \mathrm{m}^{-1}$ (ascertained using clean water prior to initiating MW fluid experiments). Thus, the average $\mathrm{R}_{\mathrm{f}}$ value was $43 \mathrm{kPa} \cdot \mathrm{h} \cdot \mathrm{m}^{-1}$ (Equation 5.4), which corresponded to $12 \%$ of the average total membrane resistance. As presented previously, fouling was responsible for $63 \%$ of the total membrane resistance in the tubular UF unit; thus, fouling was much less prevalent in the HSRUF system. As a result of membrane rotation induced hydraulic 


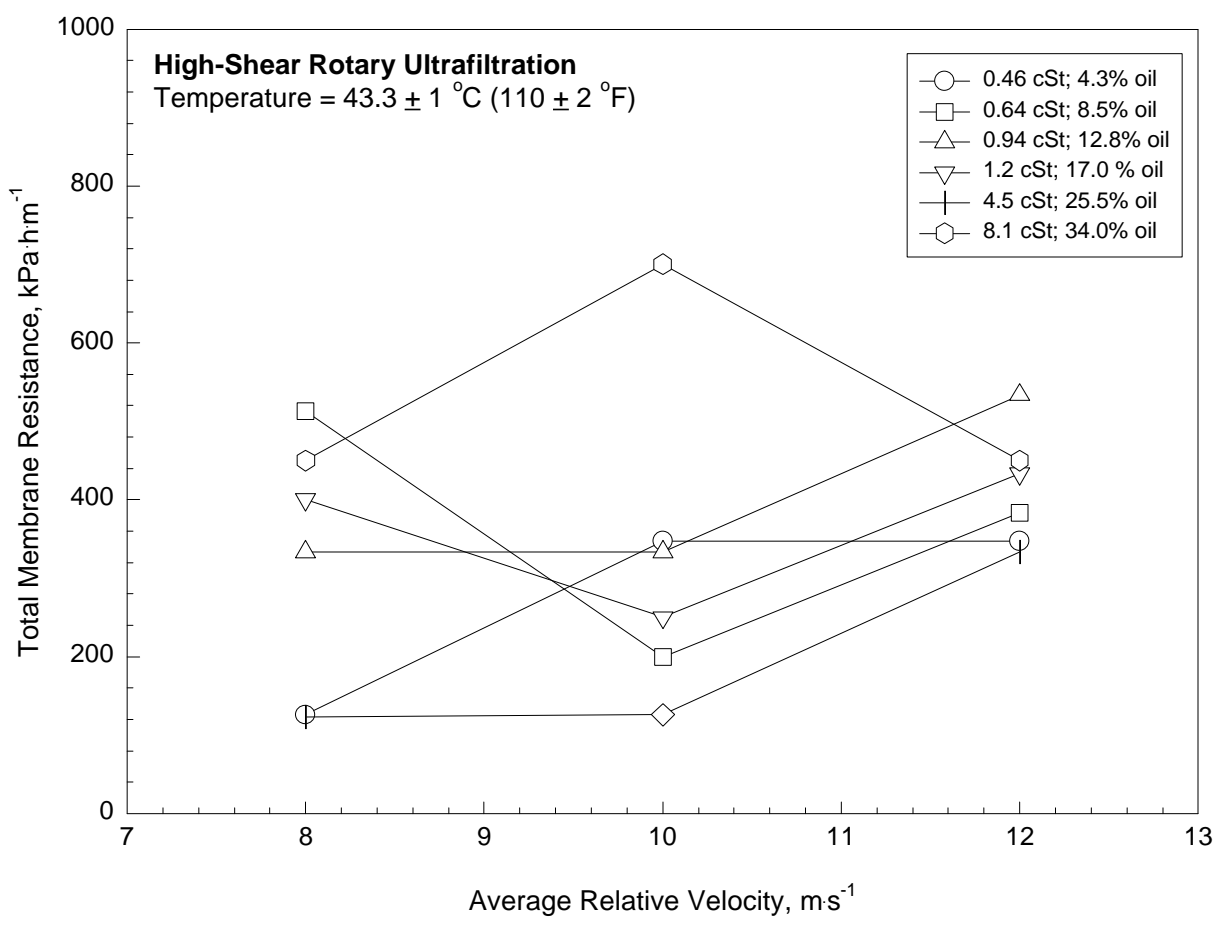

Figure 5.19 Total membrane resistance versus average relative velocity for each feed viscosity examined in the high-shear rotary UF unit.

turbulence in the HSRUF system, fewer solute droplets accumulated on the membrane surface, effectively minimizing the potential for membrane pore plugging and solute adsorption.

\subsubsection{Resistance Index}

As presented previously in section 2.4 .3 , the resistance index, $\Phi$, times the average transmembrane pressure is equal to the polarization resistance

$$
\mathrm{R}_{\mathrm{p}}=\Phi \cdot \mathrm{P}
$$

Further, the resistance index is described as a function of system mass transfer properties (e.g., feed viscosity and hydraulic turbulence). In the resistance-in-series approach, the product of the resistance index and the transmembrane pressure (polarization resistance) is inversely proportional to the permeate flux, as presented in Equation 5.3. As with the total membrane resistance, resistance index values were determined for each discrete 
velocity/viscosity combination examined in the tubular and HSRUF systems by fitting Equation 5.3 to permeate flux versus transmembrane pressure data from the synthetic MW fluid experiments.

\subsubsection{Tubular Ultrafiltration}

Resistance index values ranged from 1 to 246 in the tubular UF system. Chiang and Cheryan (1986) reported $\Phi$ values ranging from 21 to $120 \mathrm{~h} \cdot \mathrm{m}^{-1}$ in the UF treatment of skim milk using a hollow fiber module. Further, resistance index values ranging from 5 to $30 \mathrm{~h} \cdot \mathrm{m}^{-1}$ were calculated in the treatment of a reactive-dye wastewater in a tubular UF module (Wu et al., 1998).

Resistance index versus average relative velocity for each feed kinematic viscosity examined in the tubular UF unit is presented in Figure 5.20. In the tubular UF system, $\Phi$ appeared to decrease with $v$ and increase with $\eta$ over the ranges examined in this study. A two-way ANOVA was conducted with $\eta$ and $v$ as the independent variables and $\Phi$ as the dependent variable to assess the dependence of the resistance index on feed viscosity and average relative velocity as well as testing for interactions between the independent variables. The assumptions of normally distributed populations with equal variance were checked by performing the Kolmogorov-Smirnov and Levene Median tests, respectively (Figure 5.17).

The tubular UF $\Phi$ data passed the Levene Median test $\left(\mathrm{P}_{\mathrm{L}}>0.01\right)$ but failed the Kolmogorov-Smirnov test $\left(\mathrm{P}_{\mathrm{k}}=0.002\right)$. The low $\mathrm{P}_{\mathrm{k}}$ calculated from the tubular UF data was attributed to the small number of observations in the sample populations $(\mathrm{N}=4$ and 6 for $\mathrm{v}$ and $\eta$ data, respectively; Hayter, 1996). However, ANOVA procedures are robust and there is little effect on results when the assumptions are not satisfied (Johnson, 1994; 


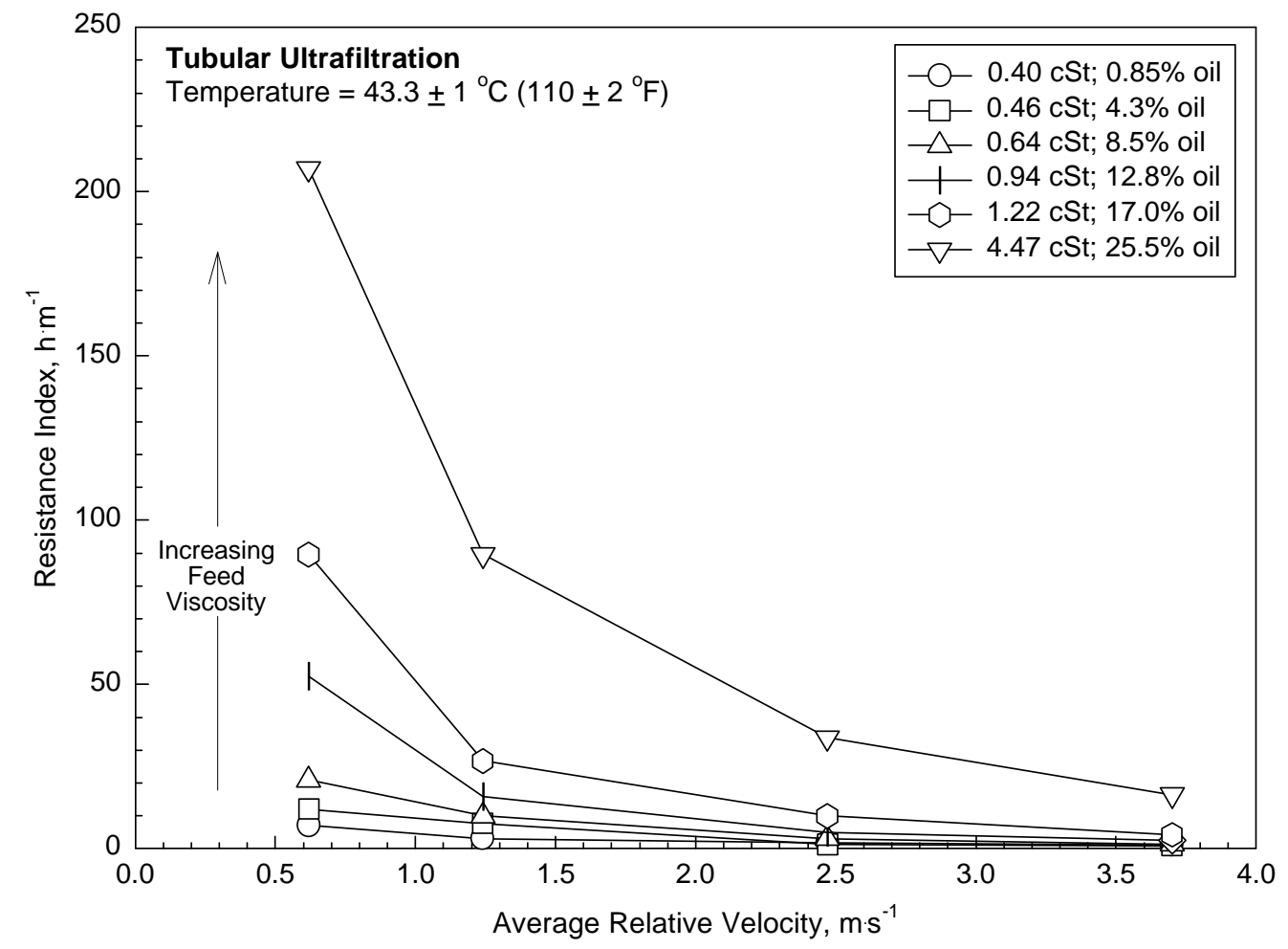

Figure 5.20 Resistance Index versus average relative velocity for each feed viscosity examined in the tubular UF unit.

Hines and Montgomery, 1980); thus, tubular UF data were included in further data reduction and application of two-way ANOVA evaluation was valid.

$\mathrm{F}_{\mathrm{A}}$ and $\mathrm{P}_{\mathrm{A}}$ values calculated from the two-way ANOVA performed on tubular resistance index versus velocity data were 4 and 0.017 , respectively. Similarly, $\mathrm{F}_{\mathrm{A}}$ and $\mathrm{P}_{\mathrm{A}}$ values calculated from the two-way ANOVA performed on tubular resistance index versus viscosity data were 5 and 0.020 , respectively. For the tubular UF data, $\mathrm{F}_{\mathrm{A}}>1$; however, $\mathrm{P}_{\mathrm{A}}$ values were greater than 0.01 . The highest $\mathrm{P}_{\mathrm{A}}$ value was 0.02 , which corresponded to a $2 \%$ chance of being wrong in concluding that tubular UF viscosity has a statistically significant influence on the resistance index (i.e., 98\% confidence). Since $\mathrm{F}_{\mathrm{A}}>1, \mathrm{P}_{\mathrm{A}} \leq$ 0.02 were accepted; thus, it was concluded that the resistance index was statistically 
dependent on average relative velocity and feed viscosity in the tubular UF system. Chiang and Cheryan (1986) also determined the resistance index to be a function of both cross-flow velocity (average relative velocity) and feed concentration (viscosity) using a hollow fiber UF module for the filtration of skimmilk.

Resistance index values for each discrete average relative velocity/feed viscosity experiment corresponded to polarization resistances of 30.8 to $6.4 \times 10^{4} \mathrm{kPa} \cdot \mathrm{h} \cdot \mathrm{m}^{-1}$ for the range of transmembrane pressures examined in this study (Equation 5.5). $R_{p}$ values were lowest and permeate flux was highest under conditions of high average relative velocity and low feed viscosity. When compared with the average total membrane resistance of $498 \mathrm{kPa} \cdot \mathrm{h} \cdot \mathrm{m}^{-1}$, it was determined that polarization was the predominant rate controlling resistance in the tubular UF system.

\subsubsection{High-Shear Rotary Ultrafiltration}

Resistance index values ranged from 0.5 to $20 \mathrm{~h} \cdot \mathrm{m}^{-1}$ in the HSRUF system, which were lower than values observed in the tubular UF system and other data presented in the literature, as presented in section 5.3.2.1. Resistance index versus average relative velocity for each feed kinematic viscosity examined in the HSRUF system is presented in Figure 5.21. As presented previously for the tubular UF unit, a two-factor ANOVA was conducted (SigmaStat ${ }^{\circledR}$ Version 2.03) to assess the dependence of the resistance index on feed viscosity and average relative velocity in the HSRUF system. $\mathrm{P}_{\mathrm{k}}$ and $\mathrm{P}_{\mathrm{L}}$ calculated from HSRUF resistance index data were 0.2 and 1.0, respectively. Both P-values were greater than 0.01 (i.e., $99 \%$ confidence); thus, it was concluded that the populations were normally distributed with equal variance and application of two-way ANOVA evaluation on HSRUF resistance index data was valid. 


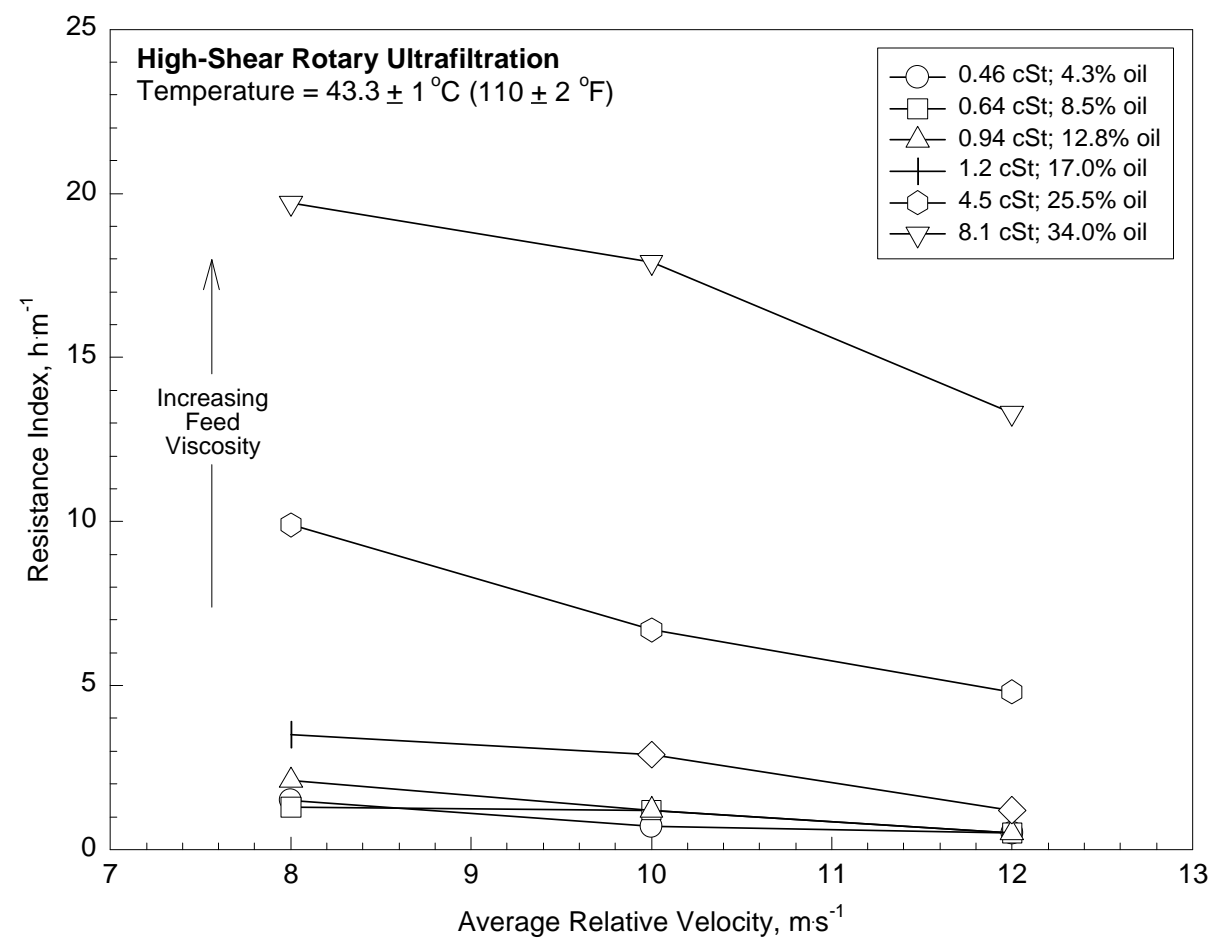

Figure 5.21 Resistance Index versus average relative velocity for each feed viscosity examined in the high-shear rotary unit .

$\mathrm{F}_{\mathrm{A}}$ and $\mathrm{P}_{\mathrm{A}}$ values calculated from the two-way ANOVA performed on HSRUF resistance index versus velocity data were 7 and 0.01 , respectively. Similarly, $\mathrm{F}_{\mathrm{A}}$ and $\mathrm{P}_{\mathrm{A}}$ values calculated from the two-way ANOVA performed on HSRUF resistance index versus viscosity data were 71 and 0.001 , respectively. $\mathrm{F}_{\mathrm{A}}>1$ and $\mathrm{P}_{\mathrm{A}}<0.01$; thus, it was concluded that $\Phi$ was statistically dependent on average relative velocity and feed viscosity in the HSRUF system. $\Phi$ values corresponded to polarization resistances ranging from 13.5 to $1.17 \times 10^{4} \mathrm{kPa} \cdot \mathrm{h} \cdot \mathrm{m}^{-1}$ for the range of transmembrane pressures examined in this study (Equation 5.5). $R_{p}$, values were lowest and permeate flux was highest under conditions of low feed viscosity and high average relative velocity. As presented previously for the tubular UF unit, it was determined that polarization was the predominant rate controlling resistance in the HSRUF system for an average total membrane resistance of $358 \mathrm{kPa} \cdot \mathrm{h} \cdot \mathrm{m}^{-1}$. 


\subsubsection{Modification of the Resistance in Series Model}

Since $\Phi$ was a function of both $\mathrm{v}$ and $\eta$ for both the tubular and HSRUF units as presented in Figures 5.20 and 5.21, respectively, efforts were focused on developing an explicit form of the resistance index in terms of cross-flow velocity and feed viscosity. Cheryan (1998) presented the use of a power law model to represent the relationship between viscosity, shear rate/shear stress; thus, the relationships between $\Phi, \mathrm{v}$ and $\eta$ were represented by a power series model for the tubular and HSRUF membrane separation systems:

$$
\mathrm{y}=\sum_{\mathrm{n}=0}^{\mathrm{z}} \mathrm{C}_{\mathrm{n}} \mathrm{x}^{\mathrm{n}}
$$

where $\mathrm{y}$ is the dependent variable, $\mathrm{x}$ is the independent variable, $\mathrm{C}_{\mathrm{n}}$ is a constant and $\mathrm{z}$ is the order of the model. The model order must be determined individually for each data set to achieve acceptable predictive ability without significantly increasing model complexity; thus, incremental regression evaluations were performed (SigmaStat ${ }^{\circledR}$ Version 2.03) on $\Phi$ versus $\eta$ and $\Phi$ versus v data for both the tubular and HSRUF units (see data reduction flow chart presented previously in Figure 5.17). The number of observations in each population, degrees of freedom (Equation 3.10), and the highest model order examined (Equation 3.21) for each of the independent variables are presented in Table 5.6.

Table 5.6 Number of observations, degrees of freedom, and highest model order examined for each dependent variable.

\begin{tabular}{|c|c|c|c|}
\hline Variable & N & DF & Highest Model Order \\
\hline $\begin{array}{c}\text { Feed Viscosity } \\
\text { (both tubular and HSR) }\end{array}$ & 6 & 5 & 4 \\
\hline Tubular Velocity & 4 & 3 & 2 \\
\hline HSRUF Velocity & 3 & 2 & 1 \\
\hline
\end{tabular}


Prior to performing the incremental analysis, the Kolmogorov-Smirnov test was conducted to check for normally distributed populations, and the Spearman rank correlation was calculated to check for constant variance. In every case, $\Phi$ versus $\eta$ data for the tubular and HSRUF units passed both the Kolmogorov-Smirnov and Spearman rank correlation tests $\left(\mathrm{P}_{\mathrm{k}}\right.$ and $\left.\mathrm{P}_{\mathrm{s}}>0.01\right)$; however, $\Phi$ versus $\mathrm{v}$ data for both units passed the Kolmogorov-Smirnov test $\left(\mathrm{P}_{\mathrm{k}}>0.01\right)$ but failed the Spearman rank correlation test $\left(\mathrm{P}_{\mathrm{s}}<0.01\right)$. The low $\mathrm{P}_{\mathrm{s}}$ values were attributed to the low number of observations in the sample populations $(\mathrm{N}=4$ and 3 for the tubular and HSRUF units, respectively; see Table 5.6). However, regression analyses are robust and there is little effect on results when the assumptions are not satisfied (Johnson, 1994; Hines and Montgomery, 1980); thus, tubular and HSRUF $\Phi$ versus v data were included in further analysis.

\subsubsection{Tubular Ultrafiltration}

Incremental regression results for the tubular UF $\Phi$ versus $v$ data at each MW fluid viscosity examined in the tubular UF unit are presented in Tables 5.7 through 5.12. Second order was the highest model order examined due to the number of observations present in the tubular UF $\Phi$ versus v data set $(\mathrm{N}=4$; see Table 5.6).

Table 5.7 Incremental analysis on tubular $\Phi$ versus v data at 4.5 cSt (25.5\% Oil).

\begin{tabular}{|c|c|c|c|}
\hline Model Order & $\mathbf{R}^{\mathbf{2}}$ & $\mathbf{F}_{\mathbf{R}}$ & $\mathbf{P}_{\mathbf{R}}$ \\
\hline \hline $\mathbf{1}$ & 0.786 & 7 & 0.113 \\
\hline $\mathbf{2}$ & 0.960 & 12 & 0.179 \\
\hline
\end{tabular}

Table 5.8 Incremental analysis on tubular $\Phi$ versus v data at 1.2 cSt (17.0\% Oil).

\begin{tabular}{|c|c|c|c|}
\hline \hline Model Order & $\mathbf{R}^{\mathbf{2}}$ & $\mathbf{F}_{\mathbf{R}}$ & $\mathbf{P}_{\mathbf{R}}$ \\
\hline \hline $\mathbf{1}$ & 0.688 & 4 & 0.170 \\
\hline $\mathbf{2}$ & 0.993 & 5 & 0.262 \\
\hline
\end{tabular}


Table 5.9 Incremental analysis on tubular $\Phi$ versus v data at 0.94 cSt (12.8\% Oil).

\begin{tabular}{|c|c|c|c|}
\hline \hline Model Order & $\mathbf{R}^{\mathbf{2}}$ & $\mathbf{F}_{\mathbf{R}}$ & $\mathbf{P}_{\mathbf{R}}$ \\
\hline \hline $\mathbf{1}$ & 0.686 & 4 & 0.172 \\
\hline $\mathbf{2}$ & 0.992 & 6 & 0.249 \\
\hline
\end{tabular}

Table 5.10 Incremental analysis on tubular $\Phi$ versus v data at $0.64 \mathrm{cSt}(8.5 \%$ Oil).

\begin{tabular}{|c|c|c|c|}
\hline Model Order & $\mathbf{R}^{\mathbf{2}}$ & $\mathbf{F}_{\mathbf{R}}$ & $\mathbf{P}_{\mathbf{R}}$ \\
\hline \hline $\mathbf{1}$ & 0.820 & 9 & 0.094 \\
\hline $\mathbf{2}$ & 0.980 & 25 & 0.126 \\
\hline
\end{tabular}

Table 5.11 Incremental analysis on tubular $\Phi$ versus v data at 0.46 cSt (4.3\% Oil).

\begin{tabular}{|c|c|c|c|}
\hline Model Order & $\mathbf{R}^{\mathbf{2}}$ & $\mathbf{F}_{\mathbf{R}}$ & $\mathbf{P}_{\mathbf{R}}$ \\
\hline \hline $\mathbf{1}$ & 0.869 & 13 & 0.068 \\
\hline $\mathbf{2}$ & 0.998 & 249 & 0.040 \\
\hline
\end{tabular}

Table 5.12 Incremental analysis on tubular $\Phi$ versus $v$ data at $0.40 \mathrm{cSt}(0.85 \%$ Oil $)$.

\begin{tabular}{|c|c|c|c|}
\hline \hline Model Order & $\mathbf{R}^{\mathbf{2}}$ & $\mathbf{F}_{\mathbf{R}}$ & $\mathbf{P}_{\mathbf{R}}$ \\
\hline \hline $\mathbf{1}$ & 0.745 & 6 & 0.137 \\
\hline $\mathbf{2}$ & 0.932 & 7 & 0.233 \\
\hline
\end{tabular}

For all tubular UF $\Phi$ versus v data, the first order $F_{R}>1$; however, first order $R^{2}<$

1. Second order $F_{R}$ increased over first order values and second order $R^{2} \approx 1$. Further, $P_{R}$ values were greater than 0.01 for all first and second order models; thus, model selections were based on $\mathrm{F}_{\mathrm{R}}$ and $\mathrm{R}^{2}$ values. It was concluded that the second order regression was the lowest order model which produced a high level of predictive ability $\left(\mathrm{R}^{2} \approx 1\right)$ with an acceptable $\mathrm{F}_{\mathrm{R}}(>1)$ and the following general model was postulated:

$$
\Phi=\mathrm{A}_{1}+\mathrm{A}_{2} \mathrm{v}+\mathrm{A}_{3} \mathrm{v}^{2}
$$

where $A_{1}, A_{2}$ and $A_{3}$ are curve fitting parameters.

Incremental regression results for $\Phi$ versus $\eta$ data at each average relative velocity examined in the tubular UF unit are presented in Tables 5.13 through 5.16. Fourth order was the highest model order examined due to the number of observations present in the tubular UF $\Phi$ versus $\eta$ data set $(\mathrm{N}=6$; see Table 5.6). 
Table 5.13 Incremental analysis on tubular $\Phi$ versus $\eta$ data at $3.7 \mathrm{~m} \mathrm{~s}^{-1}$ (30 gpm).

\begin{tabular}{|c|c|c|c|}
\hline \hline Model Order & $\mathbf{R}^{\mathbf{2}}$ & $\mathbf{F}_{\mathbf{R}}$ & $\mathbf{P}_{\mathbf{R}}$ \\
\hline \hline $\mathbf{1}$ & 0.998 & 1733 & $<0.001$ \\
\hline $\mathbf{2}$ & 0.998 & 652 & $<0.001$ \\
\hline $\mathbf{3}$ & 1.000 & 2995 & $<0.001$ \\
\hline $\mathbf{4}$ & 1.000 & 1607 & 0.016 \\
\hline
\end{tabular}

Table 5.14 Incremental analysis on tubular $\Phi$ versus $\eta$ data at $2.5 \mathrm{~m} \mathrm{~s}^{-1}(20 \mathrm{gpm})$.

\begin{tabular}{|c|c|c|c|}
\hline \hline Model Order & $\mathbf{R}^{\mathbf{2}}$ & $\mathbf{F}_{\mathbf{R}}$ & $\mathbf{P}_{\mathbf{R}}$ \\
\hline \hline $\mathbf{1}$ & 0.993 & 597 & $<0.001$ \\
\hline $\mathbf{2}$ & 0.995 & 327 & $<0.001$ \\
\hline $\mathbf{3}$ & 0.999 & 749 & 0.001 \\
\hline $\mathbf{4}$ & 0.999 & 475 & 0.029 \\
\hline
\end{tabular}

Table 5.14 Incremental analysis on tubular $\Phi$ versus $\eta$ data at $1.2 \mathrm{~m} \mathrm{~s}^{-1}(10 \mathrm{gpm})$.

\begin{tabular}{|c|c|c|c|}
\hline \hline Model Order & $\mathbf{R}^{\mathbf{2}}$ & $\mathbf{F}_{\mathbf{R}}$ & $\mathbf{P}_{\mathbf{R}}$ \\
\hline \hline $\mathbf{1}$ & 0.995 & 820 & $<0.001$ \\
\hline $\mathbf{2}$ & 0.997 & 596 & $<0.001$ \\
\hline $\mathbf{3}$ & 0.998 & 383 & 0.003 \\
\hline $\mathbf{4}$ & 0.999 & 424 & 0.031 \\
\hline
\end{tabular}

Table 5.15 Incremental analysis on tubular $\Phi$ versus $\eta$ data at $0.62 \mathrm{~m} \mathrm{~s}^{-1}(5 \mathrm{gpm})$.

\begin{tabular}{|c|c|c|c|}
\hline \hline Model Order & $\mathbf{R}^{\mathbf{2}}$ & $\mathbf{F}_{\mathbf{R}}$ & $\mathbf{P}_{\mathbf{R}}$ \\
\hline \hline $\mathbf{1}$ & 0.951 & 78 & $<0.001$ \\
\hline $\mathbf{2}$ & 0.995 & 286 & $<0.001$ \\
\hline $\mathbf{3}$ & 1.000 & 4162 & $<0.001$ \\
\hline $\mathbf{4}$ & 1.000 & 1583 & 0.016 \\
\hline
\end{tabular}

For all tubular UF $\Phi$ versus $\eta$ data $F_{R} \gg 1$ and for all first order models, $P_{R}$ values were less than 0.01 and $\mathrm{R}^{2} \approx 1$. Further, a decrease in most $\mathrm{F}_{\mathrm{R}}$ values was observed from first to second order models; thus, it was concluded that the first order regression was the lowest order model which produced a high level of predictive ability $\left(\mathrm{R}^{2} \approx 1\right)$ with an acceptable $\mathrm{F}_{\mathrm{R}}(>1)$ and $\mathrm{P}_{\mathrm{R}}(<0.01)$ and the following general model was postulated:

$$
\Phi=\mathrm{B}_{1}+\mathrm{B}_{2} \eta
$$

where $\mathrm{B}_{1}$ and $\mathrm{B}_{2}$ are curve fitting parameters. Combining Equations 5.7 and 5.8 through matrix multiplication (see data reduction flow chart; Figure 5.17): 


$$
\begin{aligned}
& \Phi_{\mathrm{T}}=\left[\begin{array}{c}
\mathrm{A}_{1} \\
\mathrm{~A}_{2} \mathrm{v} \\
\mathrm{A}_{3} \mathrm{v}^{2}
\end{array}\right] \times\left[\begin{array}{lll}
\mathrm{B}_{1} & \mathrm{~B}_{2} \eta & 0 \eta^{2}
\end{array}\right] \\
& \Phi_{\mathrm{T}}=\mathrm{A}_{1} \mathrm{~B}_{1}+\mathrm{A}_{1} \mathrm{~B}_{2} \eta+\mathrm{A}_{2} \mathrm{~B}_{1} \mathrm{v}+\mathrm{A}_{2} \mathrm{~B}_{2} \mathrm{v} \eta+\mathrm{A}_{3} \mathrm{~B}_{1} \mathrm{v}^{2}+\mathrm{A}_{3} \mathrm{~B}_{2} \mathrm{v}^{2} \eta
\end{aligned}
$$

where $\Phi_{\mathrm{T}}$ is the resistance index for the tubular UF system. The fourth and sixth terms in Equation 5.10 (containing both $\mathrm{v}$ and $\eta$ ) are defined as interaction terms, because they represent the combined effects of more than one parameter on the tubular UF resistance index. When common terms are combined in Equation 5.10, the following expression is obtained:

$$
\Phi_{\mathrm{T}}=\mathrm{C}_{1}+\mathrm{C}_{2} \mathrm{v}+\mathrm{C}_{3} \mathrm{v}^{2}+\mathrm{C}_{4} \eta+\mathrm{C}_{5} \mathrm{v} \eta+\mathrm{C}_{6} \mathrm{v}^{2} \eta
$$

where $\mathrm{C}_{1}, \mathrm{C}_{2}, \mathrm{C}_{3}, \mathrm{C}_{4}, \mathrm{C}_{5}$, and $\mathrm{C}_{6}$ are curve fitting parameters.

\subsubsection{High-Shear Rotary Ultrafiltration}

Only first order models were applied to the HSRUF $\Phi$ versus v data, due to the number of observations in the sample population $(\mathrm{N}=3$; see table 5.6); thus, incremental analyses were not performed and the following general first order model was postulated:

$$
\Phi=\mathrm{D}_{1}+\mathrm{D}_{2} \mathrm{~V}
$$

where $D_{1}$ and $D_{2}$ are curve fitting parameters.

Incremental regression results for $\Phi$ versus $\eta$ data at each average relative velocity examined in the HSRUF unit are presented in Tables 5.17 through 5.19. Fourth order were the highest model order examined due to the number of observations present in the HSRUF $\Phi$ versus $\eta$ data set $(\mathrm{N}=6$; see Table 5.6). 
Table 5.17 Incremental analysis on HSR $\Phi$ versus $\eta$ data at $12 \mathrm{~m} \mathrm{~s}^{-1}$ (1750 rpm).

\begin{tabular}{|c|c|c|c||}
\hline Model Order & $\mathbf{R}^{\mathbf{2}}$ & $\mathbf{F}_{\mathbf{R}}$ & $\mathbf{P}_{\mathbf{R}}$ \\
\hline \hline $\mathbf{1}$ & 0.969 & 159 & $<0.001$ \\
\hline $\mathbf{2}$ & 0.999 & 1539 & $<0.001$ \\
\hline $\mathbf{4}$ & 0.999 & 1120 & $<0.001$ \\
\hline
\end{tabular}

Table 5.18 Incremental analysis on HSR $\Phi$ versus $\eta$ data at $10 \mathrm{~m} \mathrm{~s}^{-1}(1450 \mathrm{rpm})$.

\begin{tabular}{|c|c|c|c||}
\hline \hline Model Order & $\mathbf{R}^{\mathbf{2}}$ & $\mathbf{F}_{\mathbf{R}}$ & $\mathbf{P}_{\mathbf{R}}$ \\
\hline \hline $\mathbf{1}$ & 0.970 & 162 & $<0.001$ \\
\hline $\mathbf{2}$ & 0.992 & 234 & $<0.001$ \\
\hline $\mathbf{4}$ & 0.997 & 304 & $<0.001$ \\
\hline
\end{tabular}

Table 5.19 Incremental analysis on HSR $\Phi$ versus $\eta$ data at $8 \mathrm{~m} \mathrm{~s}^{-1}(1150 \mathrm{rpm})$.

\begin{tabular}{||c|c|c|c||}
\hline Model Order & $\mathbf{R}^{\mathbf{2}}$ & $\mathbf{F}_{\mathbf{R}}$ & $\mathbf{P}_{\mathbf{R}}$ \\
\hline \hline $\mathbf{1}$ & 0.996 & 1340 & $<0.001$ \\
\hline $\mathbf{2}$ & 0.997 & 758 & $<0.001$ \\
\hline $\mathbf{3}$ & 0.998 & 607 & $<0.001$ \\
\hline $\mathbf{4}$ & 0.999 & 373 & 0.003 \\
\hline
\end{tabular}

For all model orders examined at each average relative velocity, $\mathrm{F}_{\mathrm{R}}>>1, \mathrm{P}_{\mathrm{R}}<0.01$ and $\mathrm{R}^{2} \approx 1$; thus, model selection was based solely on the minimization of complexity. It was concluded that first order was the lowest order model that produced a high level of predictive ability $\left(\mathrm{R}^{2} \approx 1\right)$ while maintaining acceptable $\mathrm{F}_{\mathrm{R}}$ and $\mathrm{P}_{\mathrm{R}}$ values and the following general model was postulated for HSRUF $\Phi$ versus $\eta$ data:

$$
\Phi=\mathrm{E}_{1}+\mathrm{E}_{2} \eta
$$

where $E_{1}$ and $E_{2}$ are curve fitting parameters. Combining Equations 5.12 and 5.13 through matrix multiplication, yields the following (see data reduction flow chart; Figure 5.17):

$$
\Phi_{\mathrm{R}}=\left[\begin{array}{c}
\mathrm{D}_{1} \\
\mathrm{D}_{2} \mathrm{v}
\end{array}\right] \times\left[\begin{array}{ll}
\mathrm{E}_{1} & \mathrm{E}_{2} \eta
\end{array}\right]
$$




$$
\Phi_{\mathrm{R}}=\mathrm{D}_{1} \mathrm{E}_{1}+\mathrm{D}_{1} \mathrm{E}_{2} \eta+\mathrm{D}_{2} \mathrm{E}_{1} \mathrm{v}+\mathrm{D}_{2} \mathrm{E}_{2} \mathrm{v} \eta
$$

where $\Phi_{\mathrm{R}}$ is the resistance index for the HSRUF unit. Combining terms in Equation 5.15, yields the following equation for the HSRUF resistance index:

$$
\Phi_{\mathrm{R}}=\mathrm{F}_{1}+\mathrm{F}_{2} \mathrm{v}+\mathrm{F}_{3} \eta+\mathrm{F}_{4} \mathrm{v} \eta
$$

where $F_{1}, F_{2}, F_{3}$ and $F_{4}$ are curve fitting parameters.

\subsubsection{Interaction Analyses}

Regression analyses were performed on the tubular and HSRUF resistance index models proposed in Equations 5.11 and 5.16, respectively, to test the contribution of each "interaction term" to the predictive ability of the models. Similar to incremental regression analyses, $R^{2}, F_{R}$ and $P_{R}$ values were calculated for the tubular and HSRUF models with and without each of the interaction terms. Regression results were then compared as a basis for model selection such that a balance between model predictive ability and lack of complexity was achieved.

\subsubsection{Tubular Ultrafiltration}

Results of regression interaction analysis performed on the tubular UF resistance index model (Equation 5.11) is presented in Table 5.20.

Table 5.20 Results from regression interaction analysis of the tubular UF model.

\begin{tabular}{|c|c|c|c|}
\hline $\begin{array}{c}\text { Regression parameter } \rightarrow \\
\text { Interaction terms included in analyses } \downarrow\end{array}$ & $\mathbf{R}^{\mathbf{2}}$ & $\mathbf{F}_{\mathbf{R}}$ & $\mathbf{P}_{\mathbf{R}}$ \\
\hline $\mathrm{C}_{5} \mathrm{v} \eta$ and $\mathrm{C}_{6} \mathrm{v}^{2} \eta$ (All interactions) & 0.943 & 60 & $<0.001$ \\
\hline $\mathrm{C}_{5} \mathrm{v} \eta$ only & 0.840 & 25 & $<0.001$ \\
\hline $\mathrm{C}_{6} \mathrm{v}^{2} \eta$ only & 0.756 & 15 & $<0.001$ \\
\hline No interactions & 0.611 & 11 & $<0.001$ \\
\hline
\end{tabular}

In each case, $F_{R}$ and $P_{R}$ values were acceptable $\left(F_{R}>>1\right.$ and $\left.P_{R}<0.01\right)$. The $R^{2}$ value calculated with the inclusion of all interaction terms in the tubular UF model was 0.943 
$(\approx 1)$; however, in each other case $(<2$ interaction terms $), \mathrm{R}^{2}<<1$. Further, $\mathrm{F}_{\mathrm{R}}$ values decreased with each interaction term omitted. Thus, it was concluded that all interaction terms were necessary and the final general form of the tubular UF resistance index remained unchanged (as presented in Equation 5.11). Substituting Equation 5.11 for $\Phi$ in Equation 5.3, the following general form of the modified RIS model for the tubular UF unit was obtained:

$$
\mathrm{J}_{\mathrm{T}}=\frac{\Delta \mathrm{P}}{\mathrm{R}_{\mathrm{m}^{\prime}{ }^{\prime}(\mathrm{T})}+\left[\mathrm{C}_{1}+\mathrm{C}_{2} \mathrm{v}+\mathrm{C}_{3} \mathrm{v}^{2}+\mathrm{C}_{4} \eta+\mathrm{C}_{5} \mathrm{v} \eta+\mathrm{C}_{6} \mathrm{v}^{2} \eta\right] \Delta \mathrm{P}}
$$

where $J_{T}$ is the predicted tubular UF permeate flux and $R_{m}^{\prime}(T)$ is the average total membrane resistance determined for the tubular UF system. Equation 5.17 was fitted to experimental data collected using the tubular UF system (SigmaStat ${ }^{\circledR}$ Version 2.03) to determine the values of the curve fitting parameters $\left(\mathrm{C}_{1}, \mathrm{C}_{2}, \mathrm{C}_{3}, \mathrm{C}_{4}, \mathrm{C}_{5}, \mathrm{C}_{6}\right)$ and the modified RIS model for the tubular UF system was obtained:

$$
\mathrm{J}_{\mathrm{T}}=\frac{\Delta \mathrm{P}}{17.7+\left[-1.3+0.85 \mathrm{v}-0.14 \mathrm{v}^{2}+4.4 \eta-2.8 \mathrm{v} \eta+0.43 \mathrm{v}^{2} \eta\right] \Delta \mathrm{P}}
$$

Modified RIS model results and experimental data versus average transmembrane pressure for tubular UF experiments conducted at $3.7,2.5,1.2$, and $0.62 \mathrm{~m} \cdot \mathrm{s}^{-1}(30,20,10$, and $5 \mathrm{gpm}$ ) are presented in Figures 5.22, 5.23, 5.24, and 5.25, respectively.

\subsubsection{High-Shear Rotary Ultrafiltration}

Results of regression interaction analyses performed on the HSRUF resistance index model (Equation 5.16) are presented in Table 5.21. Since $F_{R}>1, P_{R}<0.01$ and $\mathrm{R}^{2} \approx 1$, selection was based solely on model complexity; thus, the interaction term was omitted and the following general model was obtained: 


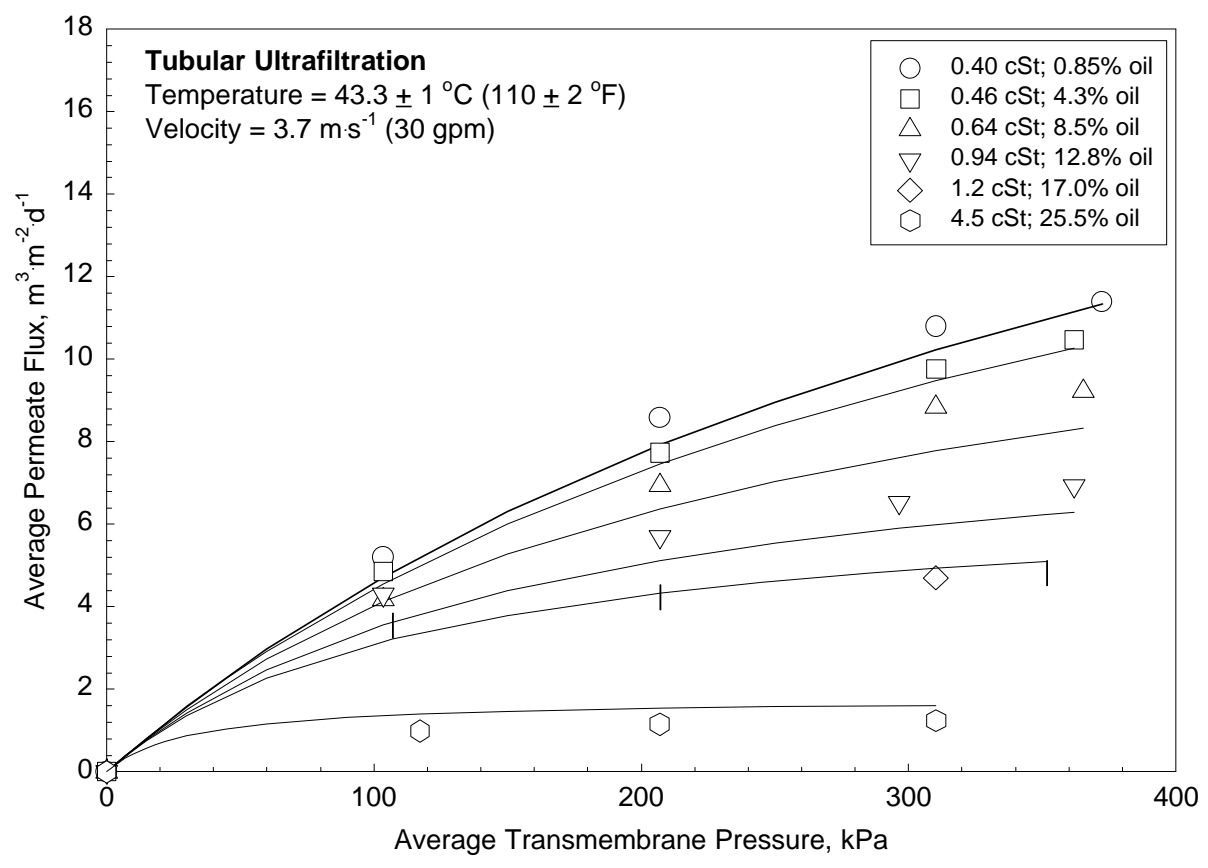

Figure 5.22 Permeate flux versus average transmembrane pressure for experiments conducted at $3.7 \mathrm{~m} \cdot \mathrm{s}^{-1}$.

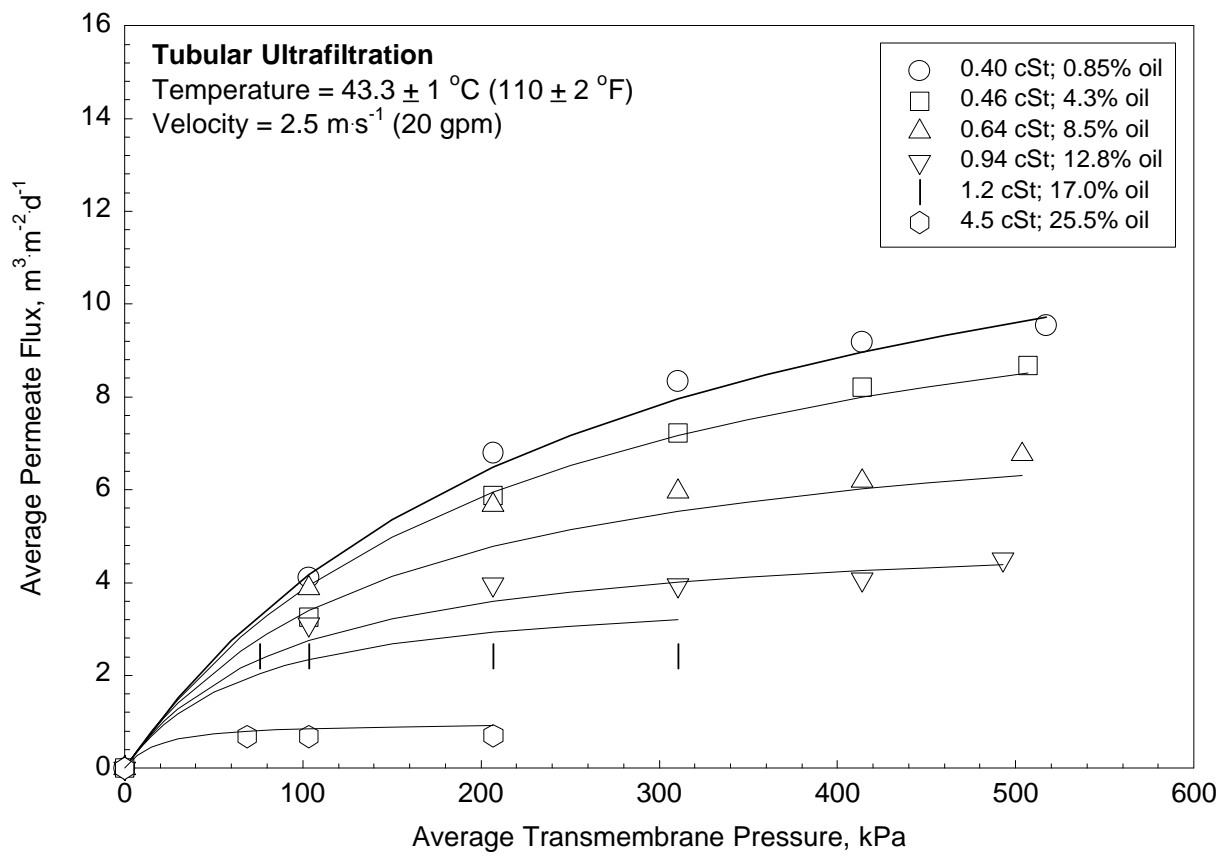

Figure 5.23 Permeate flux versus average transmembrane pressure for experiments conducted at $2.5 \mathrm{~m} \cdot \mathrm{s}^{-1}$. 


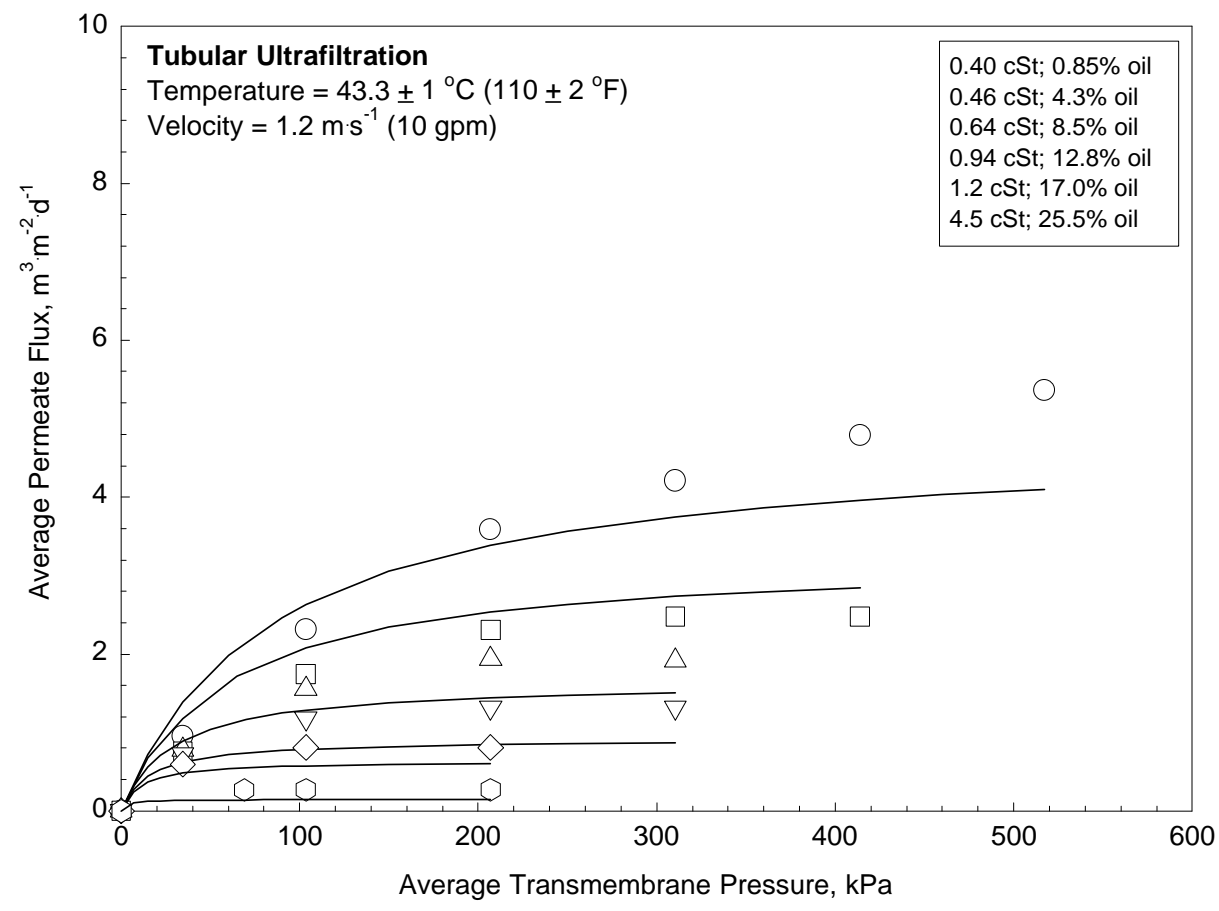

Figure 5.24 Permeate flux versus average transmembrane pressure for experiments conducted at $1.2 \mathrm{~m} \cdot \mathrm{s}^{-1}$.

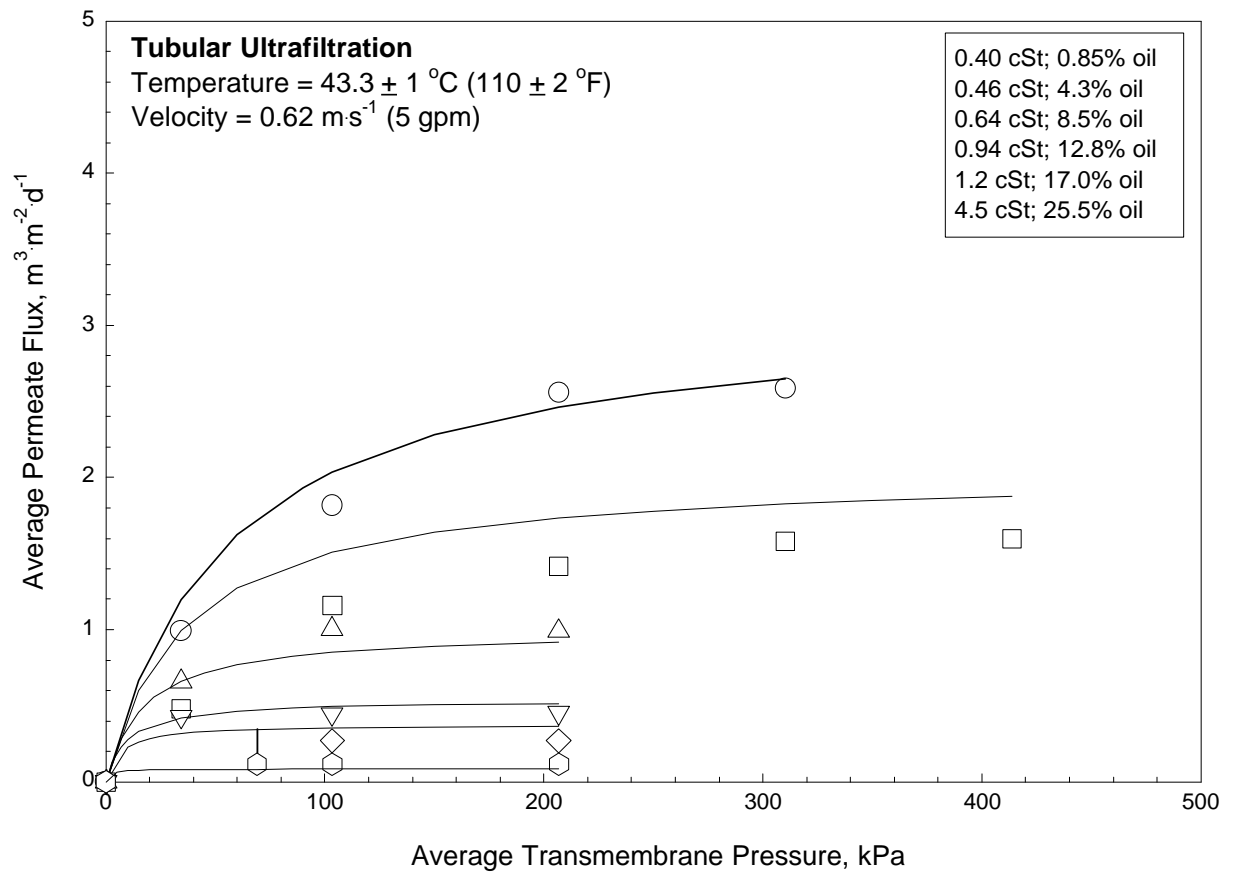

Figure 5.25 Permeate flux versus average transmembrane pressure for experiments conducted at $0.62 \mathrm{~m} \cdot \mathrm{s}^{-1}$. 


$$
\Phi_{\mathrm{R}}=\mathrm{H}_{1}+\mathrm{H}_{2} \mathrm{v}+\mathrm{H}_{3} \eta
$$

where $\mathrm{H}_{1}, \mathrm{H}_{2}$ and $\mathrm{H}_{3}$ are curve fitting parameters.

Table 5.21 Results from HSRUF regression interaction analyses.

\begin{tabular}{|c|c|c|c|}
\hline & $\mathbf{R}^{\mathbf{2}}$ & $\mathbf{F}_{\mathbf{R}}$ & $\mathbf{P}_{\mathbf{R}}$ \\
\hline With interaction term $\mathbf{( v \boldsymbol { \eta }})$ & 0.981 & 294 & $<0.001$ \\
\hline Without interaction term & 0.959 & 208 & $<0.001$ \\
\hline
\end{tabular}

Substituting Equation 5.19 for $\Phi$ in Equation 5.3, the following general form of the modified RIS model for the HSRUF unit was obtained:

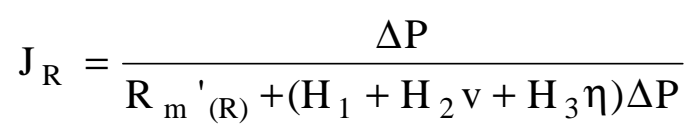

where $J_{R}$ is the predicted HSRUF permeate flux, and $R_{m}{ }^{\prime}(R)$ is the average total membrane resistance determined for the HSRUF system. Equation 5.20 was fitted to HSRUF experimental data (SigmaStat ${ }^{\circledR}$ Version 2.03) to determine the values of the curve fitting parameters $\left(\mathrm{H}_{1}, \mathrm{H}_{2}\right.$ and $\left.\mathrm{H}_{3}\right)$ and the following form of the modified RIS model was obtained:

$$
\mathrm{J}_{\mathrm{R}}=\frac{\Delta \mathrm{P}}{14.8+\left(6.5 \times 10^{-2}-6.8 \times 10^{-3} \mathrm{v}+6.8 \times 10^{-2} \eta\right) \Delta \mathrm{P}}
$$

Modified RIS model results and experimental data versus average transmembrane pressure for HSRUF experiments conducted at 12, 10, and $8 \mathrm{~m} \cdot \mathrm{s}^{-1}(1750,1450$ and 1150 rpm) are presented in Figures 5.26, 5.27, and 5.28, respectively.

\subsubsection{Significance of the Modified Resistance-In-Series Model.}

The relationship between permeate flux, transmembrane pressure, average relative velocity and feed viscosity for the tubular and HSRUF units (presented in Equations 5.18 and 5.21, respectively) adequately predicted both pressure-dependent and pressure-independent permeate flux behavior. Thus, the modified models may be 


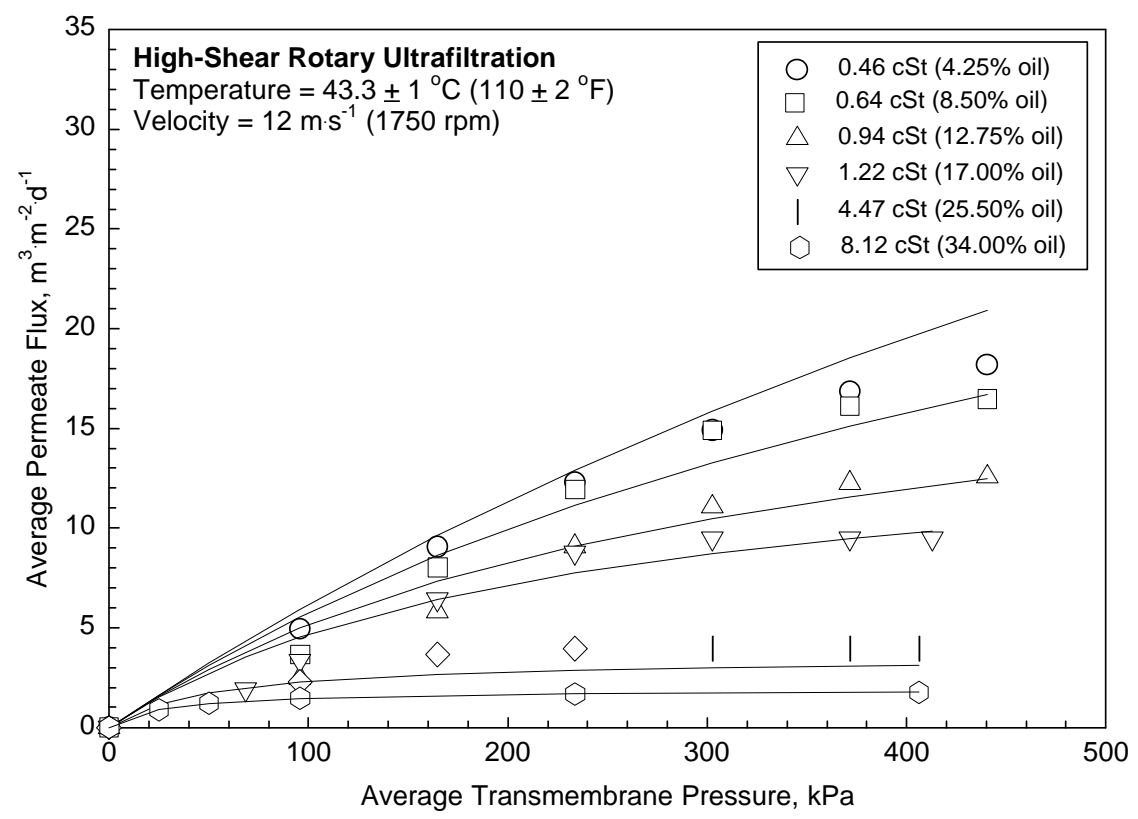

Figure 5.26 Permeate flux versus average transmembrane pressure for experiments conducted at 0.46 through $8.1 \mathrm{cSt}-12 \mathrm{~m} \cdot \mathrm{s}^{-1}$.

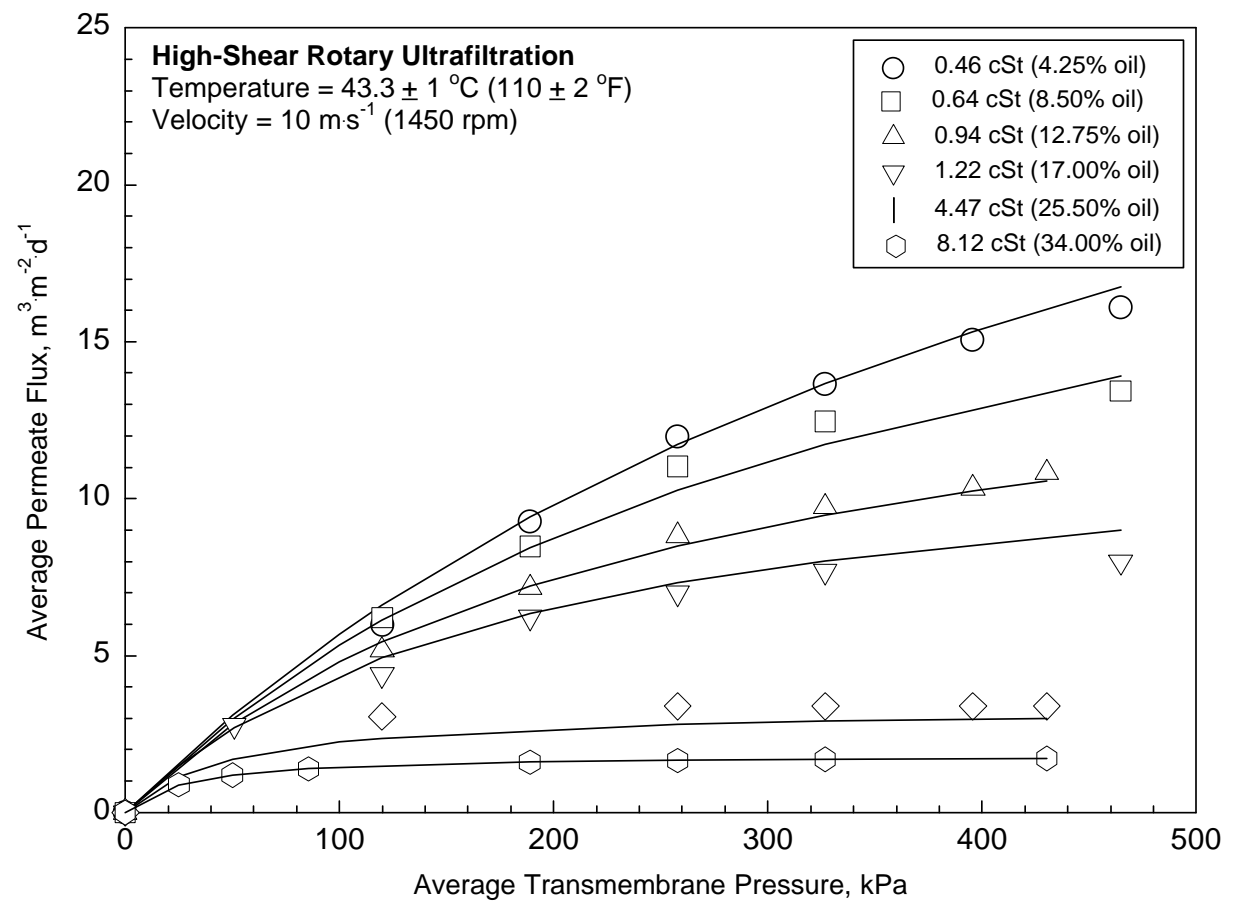

Figure 5.27 Permeate flux versus average transmembrane pressure for experiments conducted at 0.46 through $8.1 \mathrm{cSt}-10 \mathrm{~m} \cdot \mathrm{s}^{-1}$. 


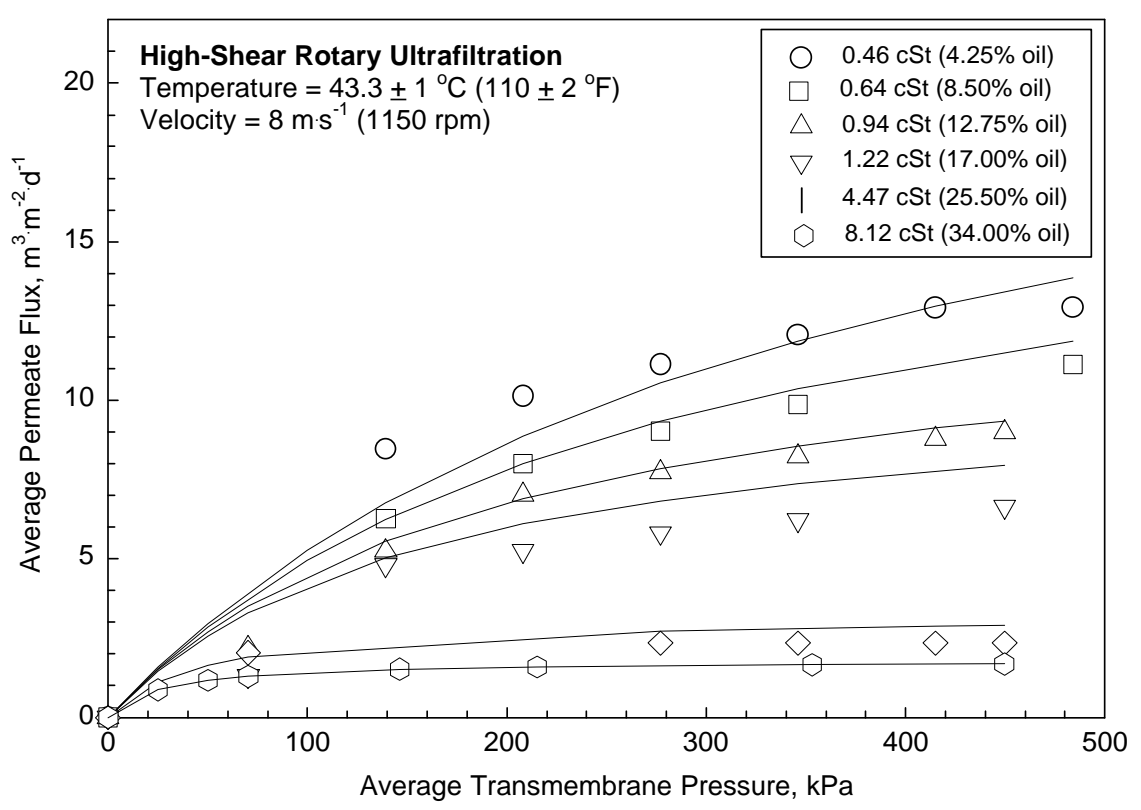

\section{Figure 5.28 Permeate flux versus average transmembrane pressure for experiments conducted at 0.46 through $8.1 \mathrm{cSt}-8 \mathrm{~m} \cdot \mathrm{s}^{-1}$.}

used to predict permeate flux at intermediate transmembrane pressure, average relative velocity and feed kinematic viscosity conditions within the experimental matrix, though prediction outside the range of experimental conditions examined in this study must be based upon an extrapolation of data.

As discussed in Section 5.2.2.2, the thin-film model adequately predicted data from experiments conducted at 0.46 to $8.1 \mathrm{cSt}$ ( 4.3 to $34 \%$ oil); however, when applied to experimental data collected at 45.6 and $121 \mathrm{cSt}$ (42.5 and 51\% oil), a two-region limiting flux was observed. The resultant two-region limiting flux corresponds to gel layer concentrations in excess of $100 \%$ oil, which is physically meaningless. Thus, in order to gauge the true success of the modified RIS model, the application to the 45.6 and $121 \mathrm{cSt}$ experimental data must be examined. Experimental data and model predictions of average permeate flux versus average transmembrane pressure for experiments conducted at 45.6 and $121 \mathrm{cSt}-12,10$ and $8 \mathrm{~m} \cdot \mathrm{s}^{-1}$ using the high-shear rotary unit are presented in 
Figures 5.29, 5.31 and 5.32, respectively. The modified RIS model does not predict 45.6 and $121 \mathrm{cSt}$ data as closely as other experiments examined in this study. The HSRUF modified RIS model was developed from experimental data collected at 0.46 to $8.1 \mathrm{cSt}$; thus, predictions of the data collected at 45.6 and $121 \mathrm{cSt}$ are based on an extrapolation of the experimental data. However, modified RIS model predictions at 45.6 and $121 \mathrm{cSt}$ are physically meaningful, whereas thin-film predictions at high viscosities are not (i.e., $\mathrm{OC}_{\mathrm{gel}}>100 \%$ oil). Further, the modified RIS model can be used to obtain a prediction of pressure-dependent data as well as an approximate description of permeate flux behavior at high feed concentrations, while the thin-film model can not.

\subsubsection{Waste Permeate Flux Prediction}

The modified RIS model developed in the previous section was applied to the data collected from the waste MW fluid experiment. Average permeate flux versus average transmembrane pressure for waste experimental data and modified RIS model predictions is presented in Figure 5.32. The relationship between permeate flux, transmembrane pressure, relative velocity and feed viscosity developed from treatment of the synthetic MW fluid adequately predicted the permeate flux behavior observed in the waste MW fluid experiment. The successful prediction of the waste permeate flux behavior was attributed to the use of viscosity as opposed to solute concentration in the modified RIS model. Thus, the model developed in this study can be used to examine permeate flux behavior in the HSRUF of a wide range of feed streams as long as a viscosity/concentration relationship is known. 


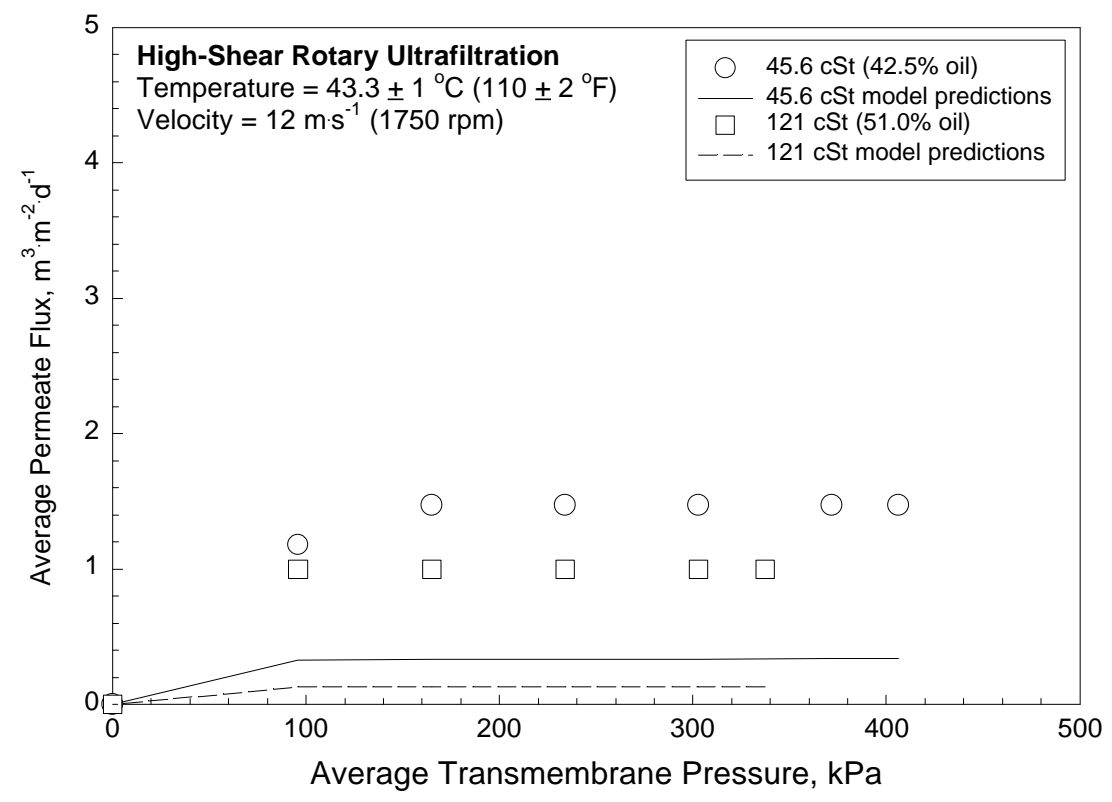

Figure 5.29 Permeate flux versus average transmembrane pressure for experiments conducted at 45.6 and $121 \mathrm{cSt}-12 \mathrm{~m} \cdot \mathrm{s}^{-1}$.

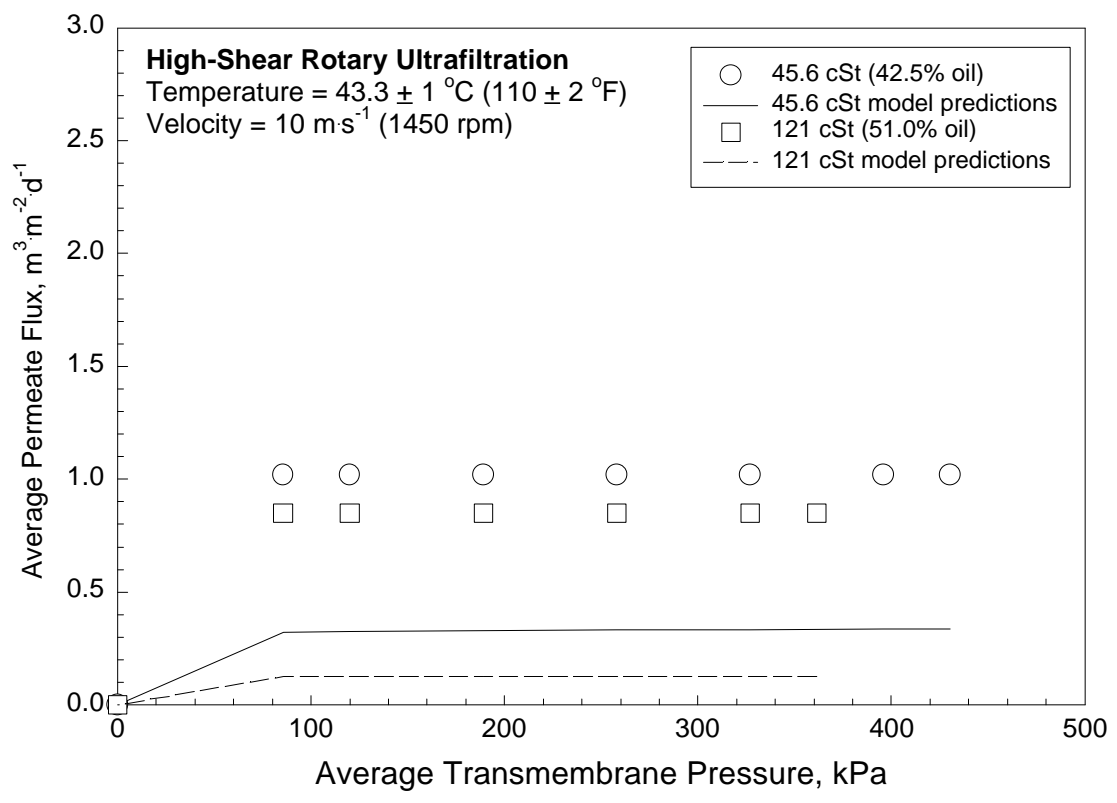

Figure 5.30 Permeate flux versus average transmembrane pressure for experiments conducted at 45.6 and $121 \mathrm{cSt}-10 \mathrm{~m} \cdot \mathrm{s}^{-1}$. 


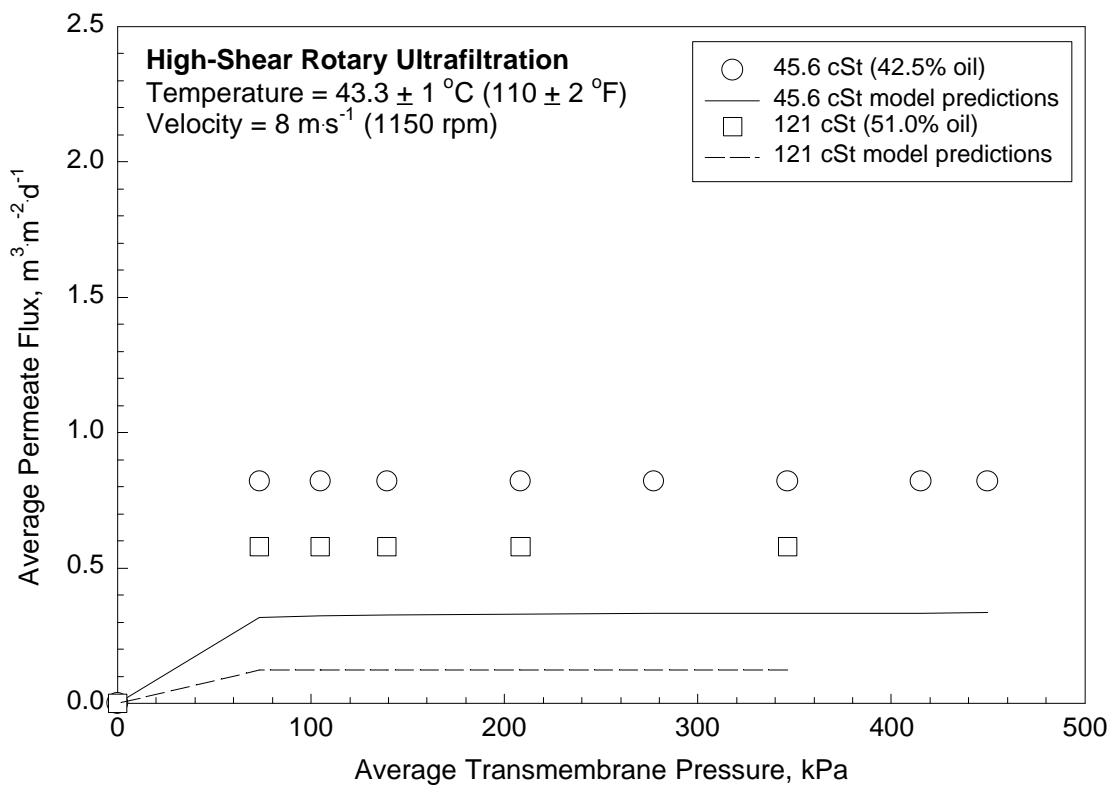

Figure 5.31 Permeate flux versus average transmembrane pressure for experiments conducted at 45.6 and $121 \mathrm{cSt}-8 \mathrm{~m} \cdot \mathrm{s}^{-1}$.

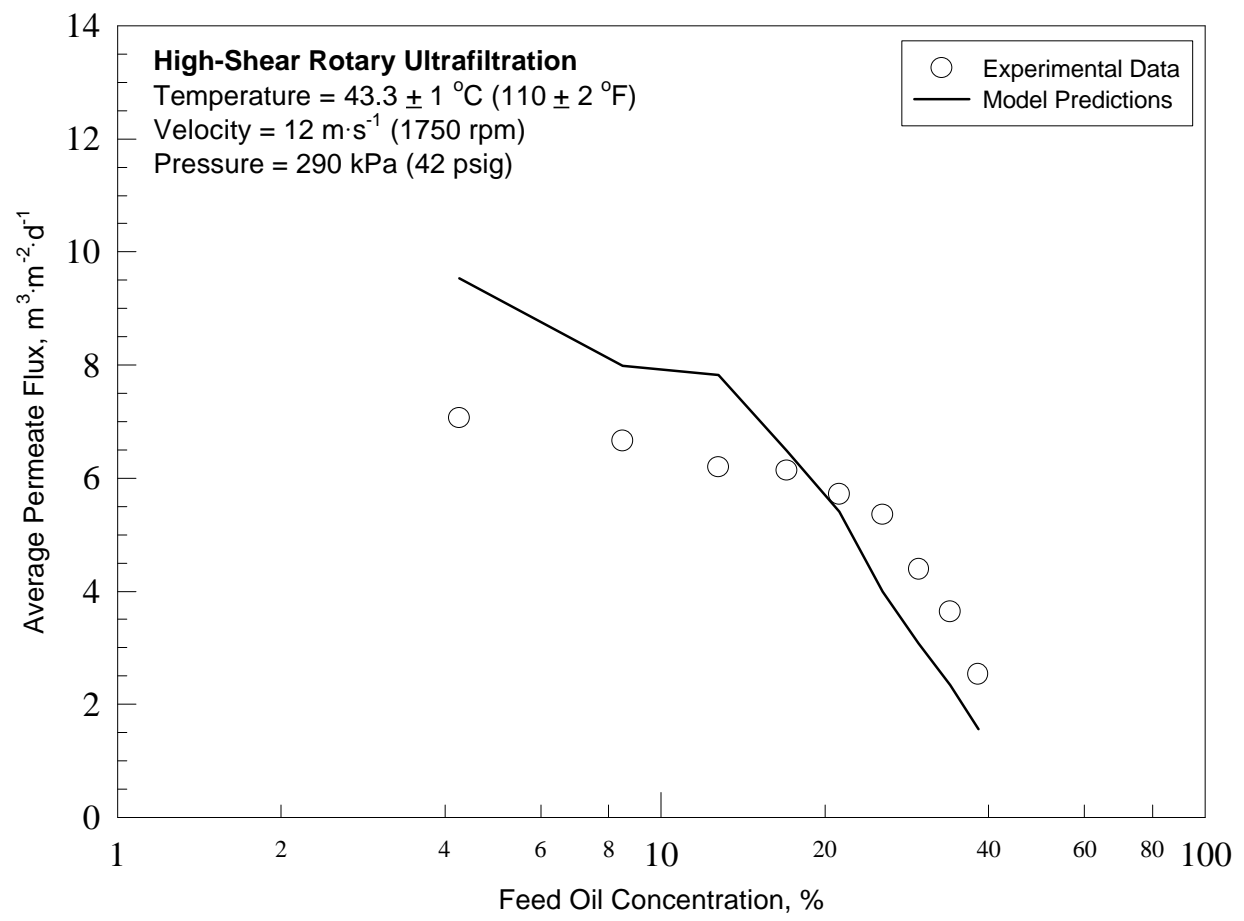

Figure 5.32 Average permeate flux versus feed oil concentration for the waste MW fluid experiment. 


\subsection{SET POINT OPERATING PRESSURE}

UF treatment processes in the field will likely be operated at the highest possible average relative velocities in order to minimize concentration polarization and subsequent flux decline. Thus, further efforts to minimize the polarization resistance were focused on optimizing the relationship between $\Delta \mathrm{P}$ and $\eta$. Cheryan (1998) presented a set point for UF operation, $\Delta \mathrm{P}_{\text {set }}$, where the pressure/polarization effects are balanced against total membrane resistance, which was presented previously in section 2.4 .3 as:

$$
\Delta \mathrm{P}_{\mathrm{set}}=\frac{\mathrm{R}_{\mathrm{m}}{ }^{\prime}}{\Phi}
$$

\subsubsection{Tubular Ultrafiltration}

The form of $\Phi$, for the tubular UF system, presented in Equation 5.18, was substituted into Equation 5.22 and the set point transmembrane pressure for the tubular UF system was given as:

$$
\Delta \mathrm{P}_{\operatorname{Set}(\mathrm{T})}=\frac{17.7}{\left[-1.3+0.85 \mathrm{v}-0.14 \mathrm{v}^{2}+4.4 \eta-2.8 \mathrm{v} \eta+0.43 \mathrm{v}^{2} \eta\right]}
$$

where $\Delta \mathrm{P}_{\text {set(T) }}$ is the set point operating pressure for the tubular UF system. The set point operating pressure versus feed kinematic viscosity for each average relative velocity examined in the tubular UF unit is presented in Figure 5.33. Full-scale tubular UF treatment processes will likely be operated at constant, elevated cross-flow velocities. Thus, based on the relationship between $\Delta \mathrm{P}_{\text {set }}$ and feed viscosity (Figure 5.33), by adjusting the average transmembrane pressure it is possible to maintain $\Delta \mathrm{P}_{\text {set }}$ as waste streams are concentrated. 


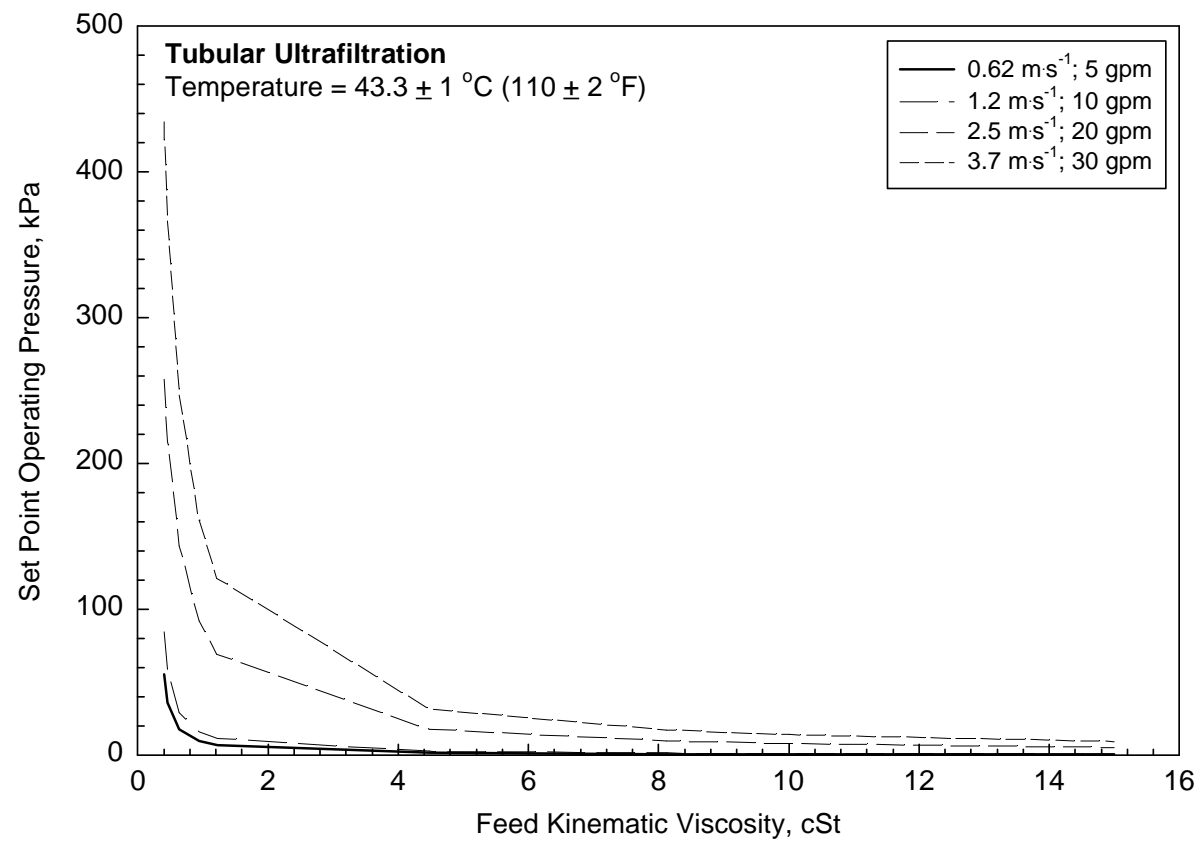

Figure 5.33 Set point operating pressure versus feed kinematic viscosity for each average relative velocity examined in the tubular unit.

\subsubsection{High-Shear Rotary Ultrafiltration}

The HSRUF form of $\Phi$ presented in Equations 5.21 was substituted into Equation 5.22 and the set point transmembrane pressure was given as:

$$
\Delta \mathrm{P}_{\mathrm{Set}(\mathrm{R})}=\frac{14.8}{\left[6.5 \times 10^{-2}-6.8 \times 10^{-3} \mathrm{v}+6.8 \times 10^{-2} \eta\right]}
$$

where $\Delta \mathrm{P}_{\text {set }(\mathrm{R})}$ is the set point operating pressure for the HSRUF system. Set point operating pressure versus feed kinematic viscosity for each average relative velocity examined in the HSRUF unit is presented in Figure 5.34. In the HSRUF system, it is possible to maintain $\Delta \mathrm{P}_{\text {set }}$ as a waste is concentrated because pressure and hydraulic turbulence can be varied independently; unlike conventional UF separation systems in which pressure and average relative velocity are generally coupled. 


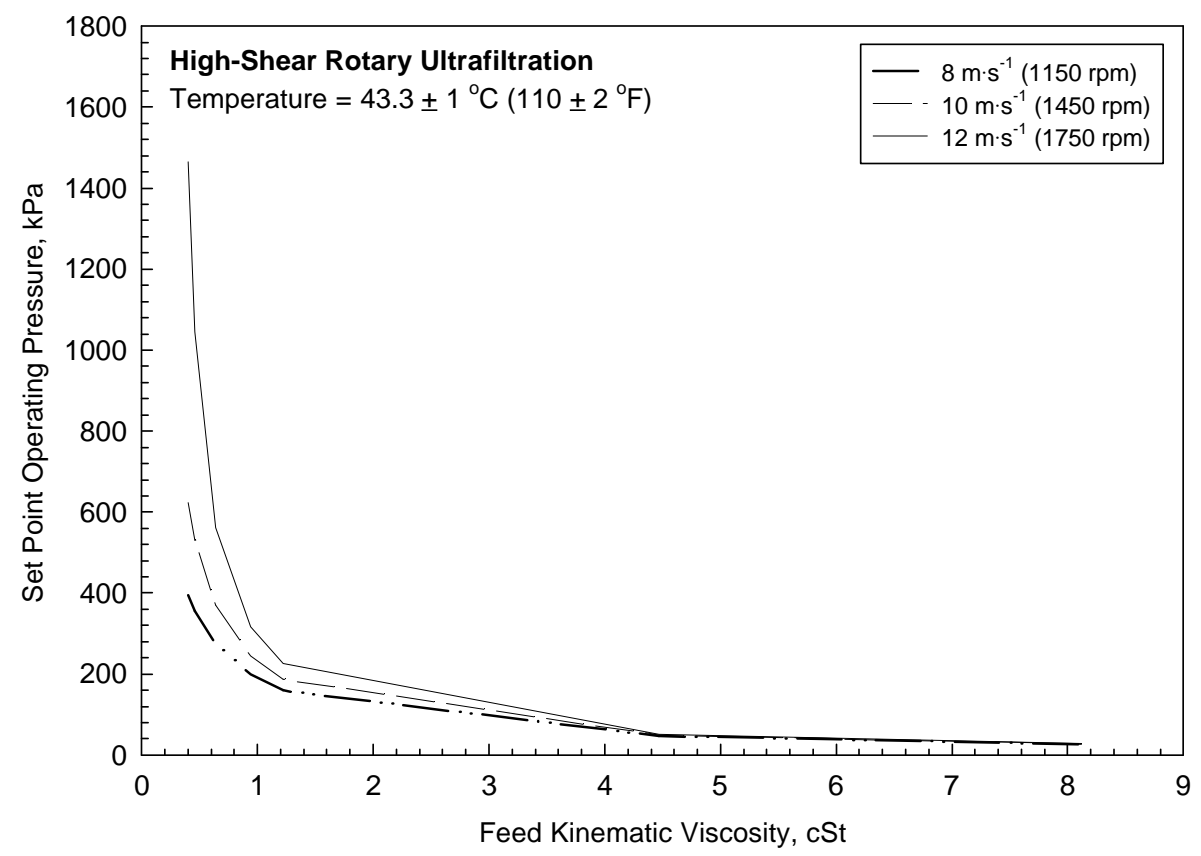

\section{Figure 5.34 Set point operating pressure versus feed kinematic viscosity for each average relative velocity examined in the high-shear rotary unit.}

\subsubsection{Significance of the Set Point Operating Pressure}

Based on the convergence of $\Delta \mathrm{P}_{\text {set }}$ with increasing feed viscosity for each average relative velocity examined in both the tubular and HSRUF units (Figures 5.33 and 5.34, respectively), an additional operational strategy for either unit would be to decrease average relative velocity while maintaining $\Delta \mathrm{P}_{\text {set }}$ as wastes are concentrated. Decreases in both transmembrane pressure and average relative velocity will minimize operational costs associated with pumping (tubular UF) or membrane rotation (HSRUF), while balancing between polarization effects and total membrane resistance. However, Reed et al. (1997a) reported an increase in permeate flux with cross-flow velocity (average relative velocity) in the tubular UF of a synthetic MW fluid using a polyvinylidane fluoride membrane. Similarly, Fane and Fell (1987) reported an increase in permeate flux with increasing cross-flow velocity in the tubular UF of a bacterial suspension. Operation at elevated average relative velocities is necessary to effectively minimize the 
potential for membrane fouling typical at high feed concentrations; however, velocityinduced hydraulic turbulence decreases as feed concentration is increased due to an increase in viscosity. Thus, the benefits of operating at lower average relative velocities must be weighed against the disadvantages of decreasing permeate flux.

\subsection{HYBRID MEMBRANE-MEMBRANE SYSTEMS}

The successful treatment of numerous types of waste streams using both tubular and HSRUF has been reported (Goldsmith et al. 1974; Lee et al. 1984; Chiang and Cheryan 1986; Fane and Fell 1987; Lipp et al. 1988; Reed et al. 1997a and b; Viadero and Reed 1999; Viadero et al. 1999 and 2000; Masciola et al. 2000). However, as discussed previously, in conventional cross-flow UF systems (tubular, hollow fiber, spiral wound, etc.) a substantial decline in permeate flux has been observed with increasing feed concentration due to a build up of rejected solute species at the membrane surface. In the HSRUF system, membrane rotation results in a decoupling of hydraulic cleaning action from recirculation/pressurization; thus, permeate flux decline with increasing feed concentration is minimized. It has been shown in this study that the HSRUF system is capable of treating highly concentrated emulsified oils, whereas the tubular UF system was more suited to the treatment of oils at lower concentrations. Thus, in treatment applications where high feed concentrations are desired, a dual treatment train is proposed, where a HSRUF system is used to treat tubular UF residual.

Presently, little is known about the performance and operating strategies of hybrid membrane-membrane separation systems. However, through the use of the general operating parameters in the tubular and HSRUF modified RIS models (Section 5.3), a technical comparison between the two modules can be performed as a first step in 
developing a hybrid tubular-HSRUF technical operating scheme. In hybrid operation it is envisioned that the tubular UF system is used to treat raw waste (low viscosities/concentrations) in a semi-batch configuration and the HSRUF system is used to treat tubular residual. Full-scale UF applications are typically operated at a constant pressure and velocity; thus, the development of a hybrid tubular-HSRUF operating scheme would be based on the determination of an optimum waste viscosity representing a transition from treatment with the tubular to treatment with the HSRUF system.

The final goal of this research was to develop a technical operating scheme for a hybrid tubular-HSRUF separation system using the modified RIS models also developed in this study. Based solely on technical considerations, the optimum point for transition between components of the hybrid tubular-HSRUF system would be the waste viscosity at which the permeate flux observed in the tubular system would drop below that observed in the HSRUF system. In the modified RIS models, the resistance index is the only term that is a function of operational parameters (velocity and viscosity; Equations 5.18 and 5.21); thus, the effects of operating parameters on permeate flux observed during tubular and HSRUF operation can be examined through the use of the modified forms of the resistance index. Further development of the technical operating scheme will be focused on the tubular and HSRUF modified resistance indices. 3-dimmensional plots of average relative velocity and feed kinematic viscosity versus resistance index for the tubular and HSRUF systems are presented in Figure 5.35. A matrix of set points representing an optimum technical transition from tubular to the HSRUF operation can be developed by determining velocity and corresponding viscosity values along the intersection of the two planes presented in Figure 5.35. The equation for the intersection 


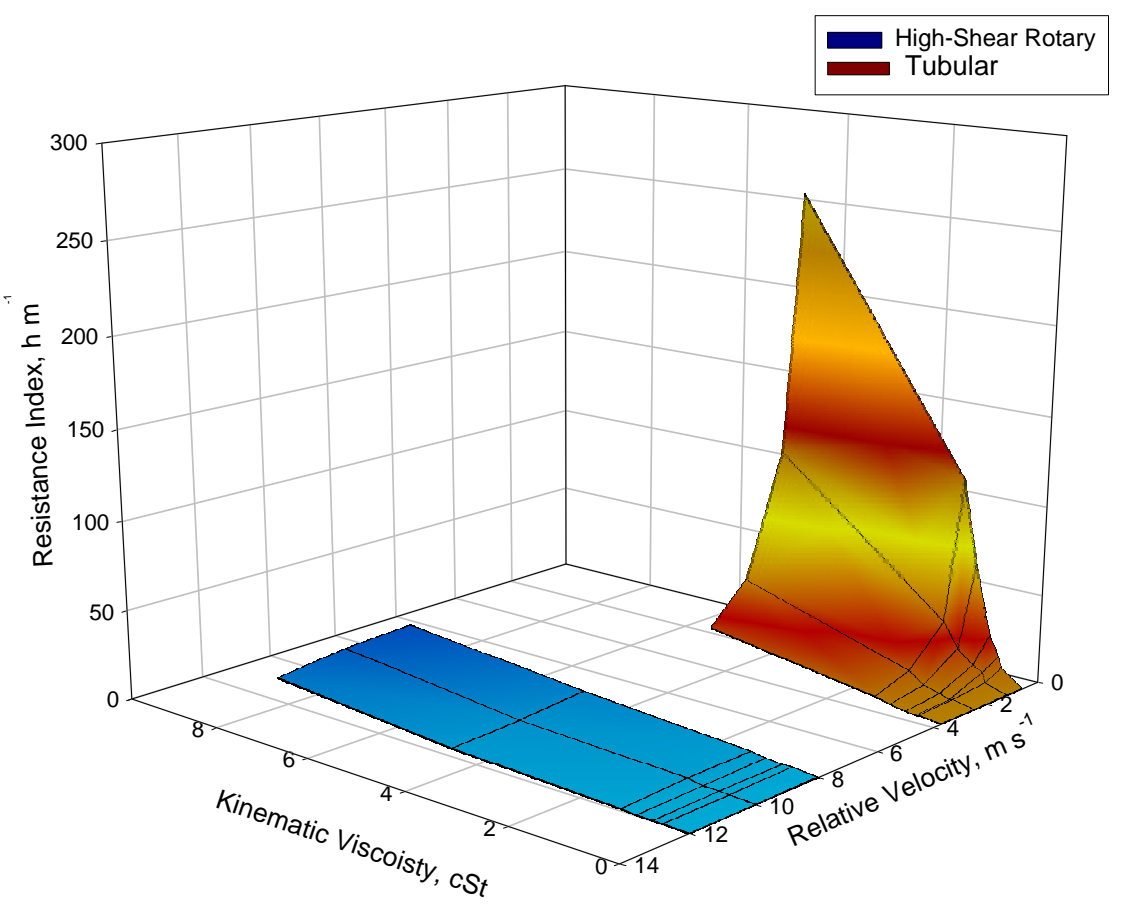

Figure 5.35 Plots of tubular and high-shear rotary resistance index versus feed kinematic viscosity versus average relative velocity.

line can be determined through the functional relationships developed between average relative velocity, feed kinematic viscosity and resistance index for the tubular and HSRUF systems presented previously as part of the modified RIS model development procedure (Equations 5.18 and 5.21). By equating the tubular and HSRUF resistance indices:

$$
\Phi_{\mathrm{T}}=\Phi_{\mathrm{R}}
$$

and substituting in the modified forms presented previously in Equations 5.18 and 5.21, the following relationship was developed:

$$
\begin{gathered}
-1.4+0.92 v-0.15 v^{2}+4.9 \eta+3.1 v \eta+0.48 v^{2} \eta= \\
6.5 \times 10^{-2}-6.8 \times 10^{-3} v+6.8 \times 10^{-2} \eta
\end{gathered}
$$

Solving for $\eta$ gives the equation of the intersection line in terms of feed kinematic viscosity: 


$$
\eta=\frac{-1.5+0.93 \mathrm{v}-0.15 \mathrm{v}^{2}}{-4.8+3.1 \mathrm{v}+0.48 \mathrm{v}^{2}}
$$

Feed kinematic viscosities were calculated according to Equation 5.27 for a range of average relative velocities corresponding to typical operating values encountered in waste MW fluid treatment facilities. A plot of feed kinematic viscosity versus average relative velocity is presented in Figure 5.36. The plot presented in Figure 5.36 corresponds to a set of operational parameters where the tubular and high-shear rotary resistance indices are equal. Since permeate flux is inversely proportional to the resistance index, the portion of Figure 5.36 where feed viscosity exceeds the $\Phi_{T}=\Phi_{R}$ plot represents a set of velocities and corresponding viscosities where the high-shear rotary permeate flux exceeds the tubular permeate flux. Thus, the $\Phi_{T}=\Phi_{R}$ plot provides a technical transition between the tubular and high-shear rotary units when operated in a hybrid scheme. Full-scale treatment facilities are commonly operated at constant velocities; thus, it is envisioned that values of velocity can be substituted into Equation 5.27 to obtain a corresponding feed viscosity providing a set point for transition between the two systems.

It can be observed from Figure 5.36 that the $\Phi_{\mathrm{T}}=\Phi_{\mathrm{R}}$ plot is asymptotic to an average relative velocity of $\sim 1.30 \mathrm{~m} \cdot \mathrm{s}^{-1}$; thus, a linear regression was applied to the $\Phi_{\mathrm{T}}=$ $\Phi_{\mathrm{R}}$ data to provide a more distinct cut-off between the two units. Conditions where $\boldsymbol{v}<$ $1.26 \mathrm{~m} \cdot \mathrm{s}^{-1}$ (the $\Phi_{T}=\Phi_{R}$ asymptote presented in Figure 5.36), and where $\eta$ is lower than the regression line presented in Figure 5.36, are favorable to tubular UF operation. In contrast, conditions where $v \geq 1.26 m \cdot s^{-1}$ or where $\eta$ is greater than the regression line presented in Figure 5.36 facilitate HSRUF operation. A plot of the tubular and HSRUF 


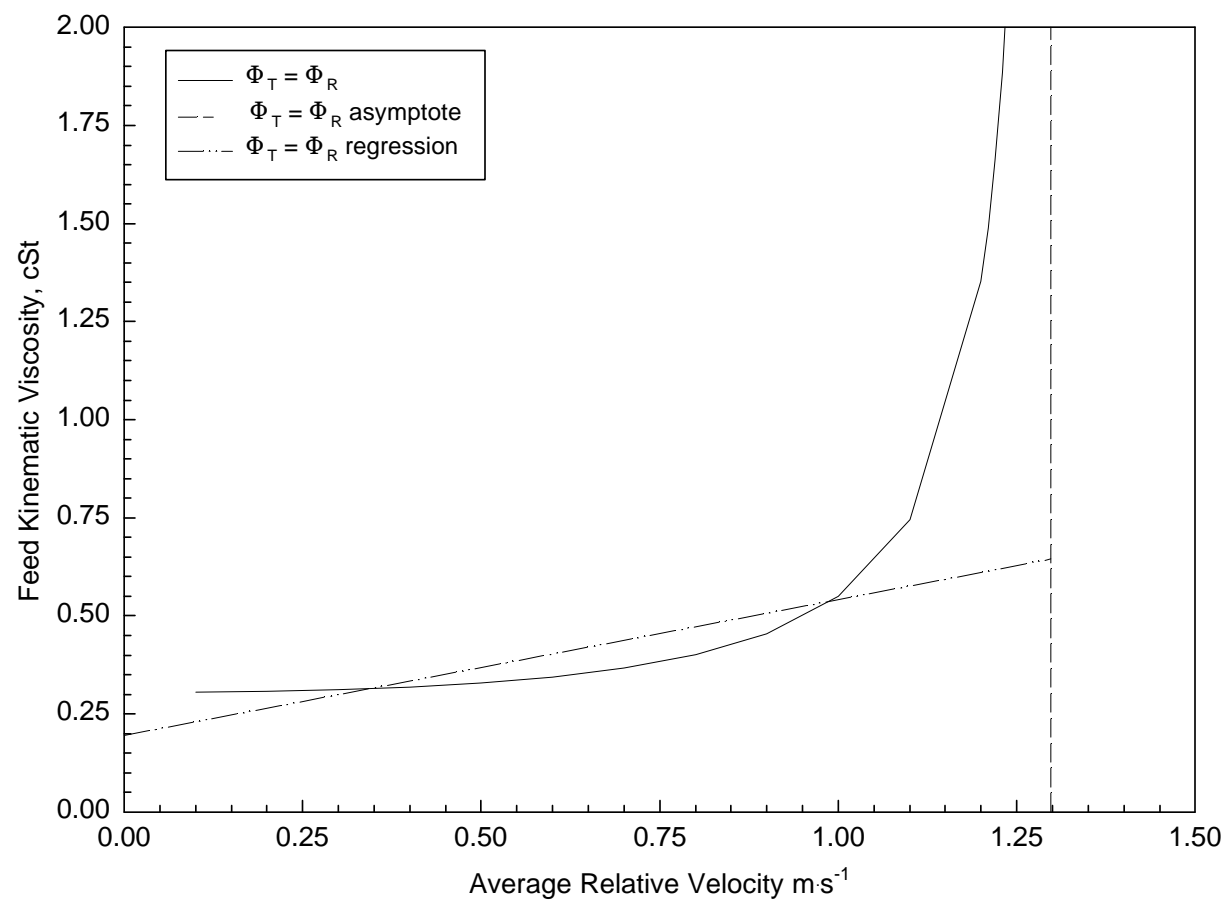

Figure 5.36 A plot of kinematic viscosity versus average relative velocity.

proposed operational regions are presented in Figure 5.37. The regression presented in Figure 5.36 can be expressed by the following equation:

$$
\eta=0.35 v+0.20
$$

For a constant velocity, the regression equation presented in Equation 5.28 can be used in place of Equation 5.27 to calculate a corresponding feed viscosity representing a transition between operation in the tubular and HSRUF units. 


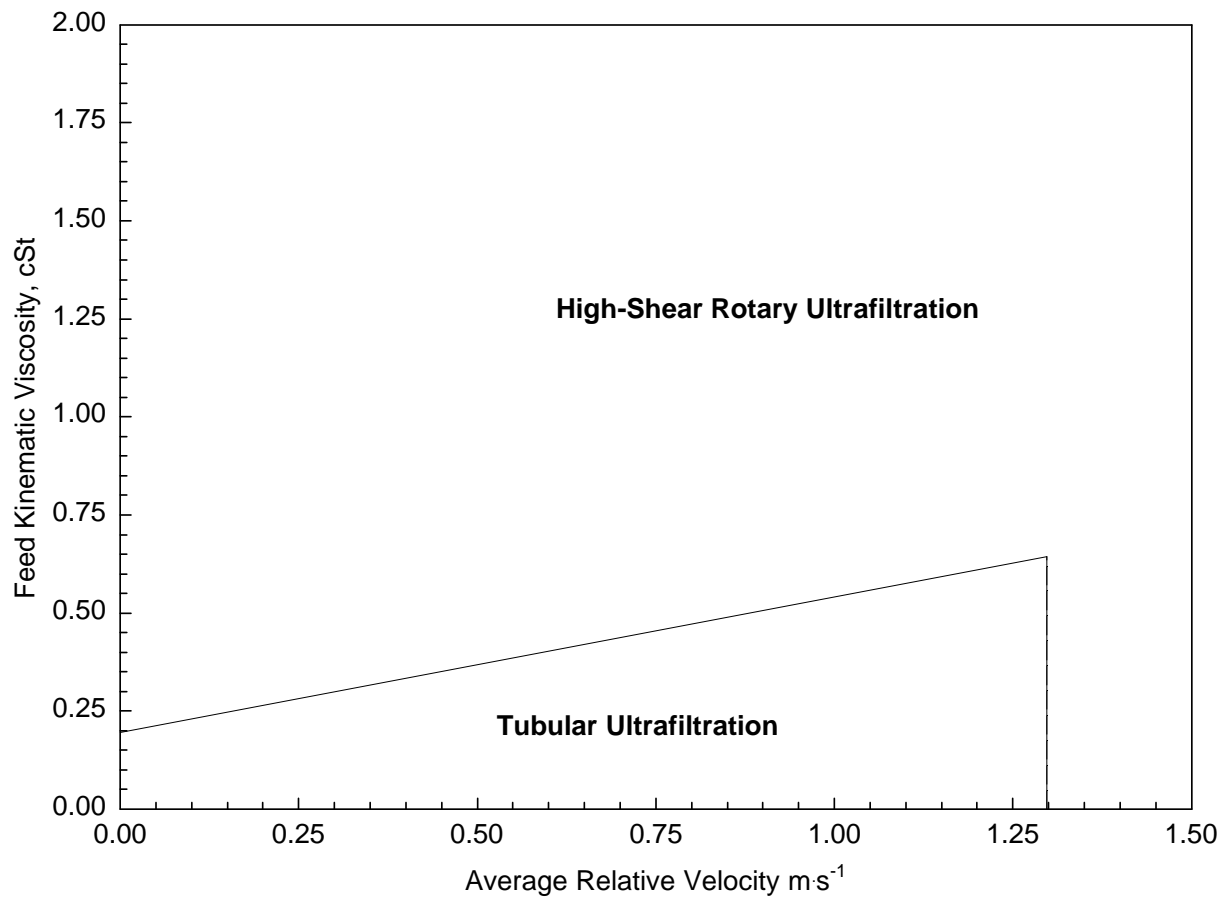

Figure 5.37 Proposed operational regions for the tubular and high-shear rotary systems. 


\section{CHAPTER 6.0 \\ CONCLUSIONS}

A parametric study was conducted to develop a more general modeling framework as a basis for comparison of ultrafiltration membrane modules and separation processes. Discrete experiments were performed to examine the effects of operating parameters on permeate flux in tubular and high-shear rotary (HSR) ultrafiltration (UF).

A synthetic MW fluid was selected as a surrogate feed stream due to its relevance in industrial process and treatment applications. The specific tasks addressed in this research were to:

1) Determine the ability of the thin-film model to predict permeate flux behavior in the tubular and HSRUF.

2) Investigate the formation of a two-phase limiting flux when applying the thinfilm model to high feed oil concentrations in HSRUF of a synthetic and waste MW fluid.

3) Apply the resistance-in-series model to data collected from the tubular and HSR treatment of a synthetic MW fluid in order to:

- Develop a more general form of the RIS model in terms of average relative velocity and feed kinematic viscosity,

- Examine application of the modified RIS model to high feed oil concentrations in the tubular and HSRUF of the synthetic and waste MW fluid.

4) Investigate application of the modified RIS model to the HSRUF a waste MW fluid.

5) Study the modified RIS models for use as a technical optimization of a tubular-HSRUF separation system by developing a procedure to determine a matrix of operational set points for transition between the two modules when operated in a hybrid scheme.

The goals of this research were satisfied through the following specific conclusions:

1) The thin-film model successfully described permeate flux behavior in the tubular and HSRUF systems at low feed oil concentrations $(\leq 34 \%$ oil $)$. 
However, gel layer concentrations for the tubular and HSRUF systems were substantially different from one another; thus, it was determined that the gel layer oil concentration was not a physico-chemical property of the synthetic MW fluid examined in this study.

2) Thin-film model predictions were physically meaningless $\left(\mathrm{C}_{\text {gel }}>100 \%\right.$ oil $)$ at higher feed oil concentrations (>34 and $43 \%$ oil for the synthetic and waste MW fluids, respectively) in both the synthetic and waste MW fluid experiments due to the formation of a two-region limiting permeate flux. The formation of a two-region flux was attributed to a transition from turbulent to laminar flow conditions when compared with similar trends in the relationship between MW fluid kinematic viscosity and oil concentration. Further, due to the fact that the gel layer oil concentration was not a physico-chemical property of the synthetic MW fluid (as is assumed in the thin-film model), it was determined that the thin-film model was invalid for application to processes treating oily feed streams. Although the thin-film model did not provide an accurate description of permeate flux behavior, the slow decrease in $\mathrm{J}^{*}$ at high oil concentrations is an advantage in that higher concentrations than predicted can be treated while maintaining an acceptable permeate flux in the HSRUF system.

3) The RIS model was modified by postulating an explicit form of the resistance index in terms of average relative velocity and feed viscosity. The modified RIS model was successful in predicting pressure-dependent and pressureindependent permeate flux behavior in the tubular and HSRUF of all synthetic 
MW fluid concentrations examined in this study. Thus, the modified model may be used to predict permeate flux behavior at operational conditions within the experimental matrix examined in this study. The model was not as accurate in predicting permeate flux in experiments conducted at 43 and $51 \%$ oil due to the fact that the model was developed using lower feed oil concentration data (4.3 to $34 \%$ oil); thus, predictions at higher feed concentrations were based on an extrapolation of the data. However, modified RIS predictions at high feed oil concentrations were physically significant, whereas thin-film model predictions were not. Thus, the modified RIS model can provide physically significant predictions of permeate flux behavior at operational parameters outside the experimental matrix examined in this study.

4) The modified RIS model developed from the synthetic MW fluid experiments was successful at predicting data collected from the waste MW fluid experiment. The model predictions followed the shape and were close to the actual values of the experimental data. Thus, the modified RIS model is capable of providing a physically significant prediction of waste streams other than the synthetic MW fluid from which it was developed. The use of viscosity as opposed to a more waste specific parameter, enables application of the modified RIS model to a wide range of feed streams. Further, the use of velocity as opposed to a more process or module specific variable enables application of the model to a wide range of UF modules; thus, future research is necessary to investigate the approach presented in this study as a means to 
describe a broader range of UF applications. Additionally, the modified RIS modeling approach developed in this study can be used as a means of comparison between UF applications as a first step in facilitating the design and optimization of hybrid membrane-membrane separation processes.

5) By equating the explicit forms of the tubular and HSRUF resistance indices, a relationship was developed between average relative velocity and feed viscosity for the two separation systems. The specific relationship developed can be used to provide a matrix of operational set points for transition between processes in a tubular-HSRUF hybrid separation system. The operating scheme presented in this study is a first attempt at describing a hybrid system and is based solely on technical considerations; thus, future research is necessary to further examine the technical and economic aspects of such processes.

The general form of the modified RIS model enables application of the procedures presented in this study to a wide range of membrane modules and UF applications; thus, the approach developed can be utilized as a means for comparison between separation processes. Additionally, the modeling approach developed is a general foundation for future investigations. The United States National Science Foundation (NSF) has recently defined hybrid separation schemes as an emerging focus area in interfacial, transport and separation process research. The first step in acquiring a better understanding of hybrid system performance is to develop an approach for comparison between modules, which may be included in such processes. Further, the framework developed in this study provides a means for process comparison; thus, the 
procedures and conclusions presented herein can be viewed as a steeping stone for future examination in areas, which are on the cutting edge of scientific and engineering research needs. 


\section{CHAPTER 7.0 \\ REFERENCES}

Belfort, G. (1988) "Membrane Modules: Comparison of Different Configurations Using Fluid Mechanics," Journal of Membrane Science, 35, 245.

Benjamin, J.R. and C.A. Cornell. (1970). Probability, Statistics, and Decision for Civil Engineers. McGraw-Hill Book Co., New York, NY.

Bennett, G. (1988). "The Removal of Oil From Wastewater by Air Flotation, A Review," CRC Critical Reviews in Environmental Control, 18 (3) 220.

Bhave, R. (1991). Inorganic Membranes: Synthesis, Characteristics, and Applications, Van Nostrand Reinhold, New York.

Bird, R., Stewart, W., and E. Lightfoot (1960). Transport Phenomena, John Wiley and Sons, New York.

Blatt, W. David, A., Michaels, A., and L. Nelson (1970). Membrane Science and Technology, J. Flinn, Ed., Plenum Press, New York.

Blank, R.G. and F.J. Brady (1991) Wastewater Treatment with Cross Flow Membrane Technology, Koch Membrane Systems, Inc., Wilmington, Ma.

Bodzek, M. and K. Konieczny (1992). "The Use of Ultrafiltration Membranes Made of Various Polymers in the Treatment of Oil-Emulsion Wastewaters," Waste Management, 12, 75-84.

Bowker, A. and Leiberman (1972). Engineering Statistics, $2^{\text {nd }}$ ed., Prentice Hall, Englewood Cliffs, New Jersey.

Burke, J. (1991). "Waste Treatment of Metal-Working Fluids, A Comparison of Three Common Methods," Lubrication Engineering, April, 238-246.

Canepa, P., Marignetti, N., Rognoni, U., and S. Calgari (1988). "Olive Mills Wastewater Treatment by Combined Membrane Processes," Water Resources, 22(12), 14911494.

Cheryan, M. (1977). "Mass Transfer Characteristics of Hollow Fiber Ultrafiltration of Soy Protein Systems," Journal of Food Process Engineering, 1, 269-287.

Cheryan, M. (1998). Ultrafiltration and Microfiltration Handbook. Technomic Publishing Co., Inc., Lancaster, PA. 
Cheryan, M. and N. Rajagopalan (1998). "Membrane Processing of Oily Streams. Wastewater Treatment and Waste Reduction," Journal of Membrane Science, 151, 13-28.

Chiang, B. and M. Cheryan (1986). "Ultrafiltration of skimmilk in hollow fibers," Journal of Food Science. 51(2) 340.

Dang, J.S., Clark, T.V., and D.V. Glenn (1992). "Treatment of Synthetic Hydraulic Fluids by Ultrafiltration and Biological Treatment: A Case History," in: Proceedings of the $47^{\text {th }}$ Purdue Industrial Waste Conference, West Lafayette, IN, Lewis Publishers, Chelsea, MI, 1992, p. 277.

Devore, J.L. (2000). Probability and Statistics for Engineering and the Sciences, $5^{\text {th }}$ Edition, Duxbury, Pacific Grove, CA.

Eringis, A., Brady, F., and R. Blank (1993). "Wastewater Treatment in the Die Casting Industry Using Cross-Flow Membrane Filtration," Transactions of the North American Die Casting Association.

Fane, A. and C. Fell (1987). "A Review of Fouling and Fouling Control in Ultrafiltration,” Desalination, 62, 117-136.

Goldsmith, R., Roberts, D., and D. Burre (1974). "Ultrafiltration of Soluble Oil Wastes," Journal Of The Water Pollution Control Federation, 46 (9), 2183-2192.

Gutman R. (1987). Membrane Filtration: The Technology of Pressure-Driven Membrane Processes, IOP Publishing, Ltd., Bristol, Great Britain.

Hayter, A.J. (1996). Probability and Statistics for Engineers and Scientists, PWS Publishing Co., Boston, MA.

Hines, W.A. and D.C. Montgomery (1980). Probability and Statistics in Engineering and Management Science. John Wiley \& Sons, New York, NY.

Johnson, R.A. (1994). Miller and Freund's Probability and Statistics for Engineers, $5^{\text {th }}$ Edition, Prentice Hall, Englewood Cliffs, NJ.

Jonsson, G. and C. Boesen (1984). "Polarization Phenomena in Membrane Processes," Synthetic Membrane Processes, G. Belfort, Ed. Academic Press, Inc., New York.

Jonsson, A. and G. Tragardh (1990). "Fundamental Principles of Ultrafiltration," Chemical Engineering Processes, 27, 67-81.

Ketola, H. and J. McGrew (1968). "Pressure, Frictional Resistance, and Flow Characteristics of the Partially Wetted Rotating Disk," Journal of Lubrication Technology, April, 395-404. 
Koltuniewicz, A.B. and R.W. Field (1996). "Process Factors During Removal of Oil-inWater Emulsions with Cross-Flow Microfiltration," Desalination, 105, 79-89.

Laemmle, J. (1992). Metalworking Lubricants, American Petroleum Institute.

Lee, S., Aurelle, Y., and H. Roques (1984). "Concentration Polarization, Membrane Fouling, And Cleaning In Ultrafiltration Of Soluble Oil,” Journal of Membrane Science, 19, 23-38.

Lipp, P., Lee, C., Fane, A., and C. Fell (1988). “A Fundamental Study Of The Ultrafiltration Of Oil-Water Emulsions," Journal of Membrane Science, 36, 161177.

MacNeil, J. and D. McCoy (1989). "Membrane Separation Technologies," Standard Handbook of Hazardous Waste Treatment and Disposal, McGraw-Hill Book Co., New York, 6.91-6.106.

Mahdi, S. and R. Skold (1991). "Membrane Filtration for the Recycling of Water-Based Synthetic Metalworking Fluids," Filtration and Separation, 28 (6), 407-414.

Masciola, D., Viadero C., and B. Reed (2000). "Effects of Operating Parameters in Tubular Ultrafiltration," ASCE Journal of Environmental Engineering, in press.

Mood, A.M., Graybill, F.A., and D.C. Boes (1974). Introduction to the Theory of Statistics, McGraw-Hill Book Co., New York, NY.

Mueller, J., Cen, Y., and R.H. Davis (1997) "Crossflow Microfiltration of Oily Water," Journal of Membrane Science, 129, 221-235.

Murkes, J. and C. Carlsson (1988). Crossflow Filtration, John Wiley and Sons, Ltd., Bath, Great Britain.

Nazzal, F. and M. Wiesner (1996). "Microfiltration of Oil-in-Water Emulsions," Water Environment Research, 68 (7), 1187-1191.

Perry, R. and D. Green (1997). Perry's Chemical Engineering Handbook, $7^{\text {th }}$ Ed., McGraw-Hill, New York.

Pope, J.M., Yao, S., and A.G. Fane (1996) "Quantitative Measurements of the Concentration Polarisation Layer Thickness in Membrane Filtration of Oil-Water Emulsions Using NMR Micro-Imaging," Journal of Membrane Science, 118, 247-257.

Pritchard, M. Howell, J., and R. Field (1995). "The Ultrafiltration of Viscous Fluids," Journal of Membrane Science, 102, 223. 
Reed, B., Lin, W., Dunn, C., Carriere, P., and G. Roark (1997a). "Treatment Of An Oil/Grease Wastewater Using Ultrafiltration: Pilot-Scale Results," Separation Science and Technology, 123 (12), 1234-1242.

Reed, V., Lin, W., Dunn, C., Carriere, P., and G. Roark (1997b) "Treatment of Concentrated Oily Wastes Using Centrifugal Membrane Separation (CMS)," ASCE Journal of Environmental Engineering, 123 (12), 1-9.

Sethi, S. and M. Wiesner (1997). "Modeling of Transient Permeate Flux in Cross-Flow Membrane Filtration Incorporating Multiple Particle Transport Mechanisms," Journal of Membrane Science, 136, 191.

Smith, A. and S. Circle (1972). Soybeans: Chemistry and Technology, Avi Publishing Co., New York.

Viadero, R., Masciola, D., Reed B., and R. Vaughan (2000) "Two-Phase Limiting Flux in High-Shear Rotary Ultrafiltration of Oil-in-Water Emulsions," Journal of Membrane Science, 175, 85-89.

Viadero, R., and B. Reed (1999). "Rotation and Concentration Effects in High Shear Ultrafiltration," ASCE Journal of Environmental Engineering, 125, 7, 638-646.

Viadero, R., Vaughan, R., and B. Reed (1999). "Study of series resistances in high-shear rotary Ultrafiltration," Journal of Membrane Science, 162, 199-211.

Weast, R. Ed. (1976) Handbook of Chemistry and Physics, CRC Press, Cleveland, OH.

Wu, J., Eiteman, M. and S. Law (1998). "Evaluation of Membrane Filtration and Ozonation Processes for Treatment of Reactive-Dye Wastewater," Journal of Environmental Engineering, 124(3), 272.

Zaidi, A., Simms, K., and S. Kok (1992). "The Use of Micro/Ultrafiltration for the Removal of Oil and Suspended Solids from Oilfield Brines," Water Science and Technology, 25(10), 163-176. 